\section{Pacific Northwest}

National Laboratory

Operated by Battelle for the

U.S. Department of Energy

\title{
A Catalog of Geologic Data for the Hanford Site
}

\author{
D. G. Horton \\ G. V. Last \\ T. J Gilmore \\ B. N. Bjornstad \\ R. D. Mackley
}

July 2005

Prepared for the U.S. Department of Energy

under Contract DE-AC05-76RL01830 


\title{
DISCLAIMER
}

This report was prepared as an account of work sponsored by an agency of the United States Government. Neither the United States Government nor any agency thereof, nor Battelle Memorial Institute, nor any of their employees, makes any warranty, express or implied, or assumes any legal liability or responsibility for the accuracy, completeness, or usefulness of any information, apparatus, product, or process disclosed, or represents that its use would not infringe privately owned rights. Reference herein to any specific commercial product, process, or service by trade name, trademark, manufacturer, or otherwise does not necessarily constitute or imply its endorsement, recommendation, or favoring by the United States Government or any agency thereof, or Battelle Memorial Institute. The views and opinions of authors expressed herein do not necessarily state or reflect those of the United States Government or any agency thereof.

\author{
PACIFIC NORTHWEST NATIONAL LABORATORY \\ operated by \\ BATTELLE \\ for the \\ UNITED STATES DEPARTMENT OF ENERGY \\ under Contract DE-AC05-76RL01830
}

Printed in the United States of America
Available to DOE and DOE contractors from the Office of Scientific and Technical Information,
P.O. Box 62, Oak Ridge, TN 37831-0062;
ph: (865) 576-8401
fax: $(865) 576-5728$
email: reports@adonis.osti.gov

\footnotetext{
Available to the public from the National Technical Information Service, U.S. Department of Commerce, 5285 Port Royal Rd., Springfield, VA 22161 ph: (800) 553-6847 fax: $(703) 605-6900$ email: orders@ntis.fedworld.gov online ordering: http://www.ntis.gov/ordering.htm
}

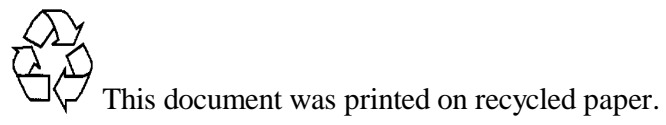


PNNL-13653, Rev. 2

\title{
A Catalog of Geologic Data for the Hanford Site
}

\author{
D. G. Horton \\ G. V. Last \\ T. J Gilmore \\ B. N. Bjornstad \\ R. D. Mackley
}

July 2005

Prepared for

the U.S. Department of Energy

under Contract DE-AC05-76RL01830

Pacific Northwest National Laboratory

Richland, Washington 99352 


\section{Summary}

The purpose of this report is to update the previous version of the geologic data catalog (Horton et al. 2002). The earlier catalog gathered sources of existing borehole geologic data for the Hanford Site, focusing on the 100, 200, and 300 Areas, with a particular emphasis on the 200 Areas. Over 2,600 boreholes were included in the previous catalog.

This revision of the geologic data catalog incorporates new boreholes drilled after September 2002 as well as other older wells, particularly from the 600 Area, omitted from the earlier catalogs. Additionally, a description of available borehole geophysical log data has been included in the catalog. This version of the geologic data catalog now contains 3,519 boreholes and is current with boreholes drilled as of November 2004. However, the data catalog (Appendix A) is by no means complete. Many individuals have been involved in geologic studies through the years, and the extent of unpublished data retained in their files is unknown. The geologic data catalog is estimated to represent the majority of borehole geologic data currently available from the Hanford Site, but will continued to evolve as additional boreholes and data are added to the database from other areas.

The data catalog in Appendix A identifies the majority of existing lithologic data available for the Hanford Site. Appendix B is an annotated bibliography of references given in Appendix A. Some, but not all of the references in Appendix B, describe the procedures used to collect the data and/or the pedigree of the data if known.

This geologic data catalog is not a database. Instead, it is a listing of the types of existing data and places where the data can be found (e.g., published documents, formal databases, or informal databases). Most of the data were generated during characterization and remediation activities by Bechtel Hanford, Inc.; the waste management and environmental programs of Rockwell Hanford Operations, Westinghouse Hanford Company, and Fluor Hanford, Inc.; the characterization and monitoring activities done by Pacific Northwest National Laboratory (PNNL); and studies done by independent contractors. The geologic data catalog is intended for the subject matter expert who has a need for existing geologic data. The data catalog also can be used to identify data gaps and technical needs 


\section{Contents}

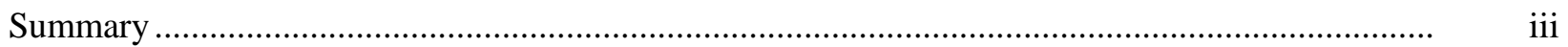

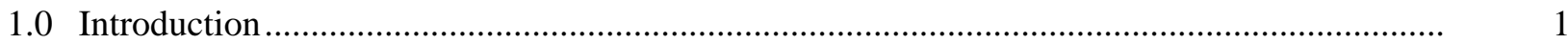

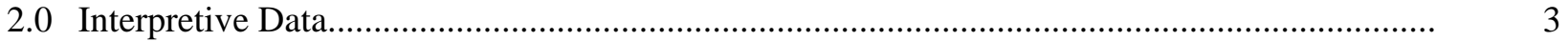

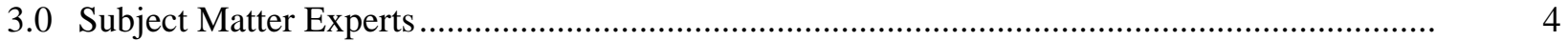

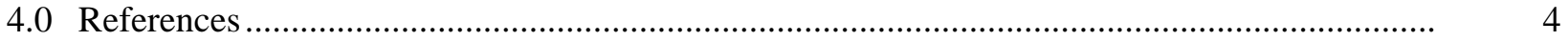

Appendix A - Geologic Data Catalog ….................................................................................. A.1

Appendix B - Bibliography of Geologic Data for the Hanford Site............................................. B.1

\section{Table}

1 Selected Sources of Recent Interpretive Data . 


\subsection{Introduction}

Site characterization and modeling activities to support the U.S. Department of Energy's (DOE) Hanford Site environmental restoration programs have been underway for several years and are expected to continue for many more years. Part of these characterization efforts is to describe the physical and chemical nature of the vadose zone and aquifer sediment. Although it is necessary to collect new data, voluminous existing data have been collected for various studies during the past 55 years. These existing data provide an essential starting point for future investigations and are critical for successful future site characterization and modeling efforts.

Unfortunately, existing data have not been consolidated into one library or database. Instead, existing data are found in several forms and locations. These include databases, published and unpublished reports, and individuals' technical files.

The Remediation Decision Support Task (formerly the Characterization of Systems Task) under DOE's Groundwater Remediation Project is responsible for establishing a consistent set of data and conceptual models to support Hanford Site efforts to estimate contaminant migration and impact (DeLamare 2000). As part of these efforts, the Remediation Decision Support Task assembled a series of catalogs to identify the depth and breadth of existing data and to facilitate access to those data. The preparation of these catalogs was aimed at helping develop a comprehensive, useable, and scientifically defensible database(s). These catalogs are "living documents" that continue to evolve as additional existing data are found and new data are collected.

Three separate catalogs have been prepared in parallel to document the existing vadose zone data. These included the borehole geologic data (this report), vadose-zone hydraulic-property data (Freeman et al. 2002), and geochemical data (Cantrell et al. 2003). These catalogs joined an already existing catalog on surface and borehole geophysical data (Last and Horton 2000) and a catalog on release mechanisms (Riley and LoPresti 2003).

The purpose of this report is to update the previous version of the geologic data catalog (Horton et al. 2002). The earlier catalog gathered sources of existing borehole geologic data for the Hanford Site, focusing on the 100, 200, and 300 Areas with a particular emphasis on the 200 Areas. Over 2,600 boreholes were included in the previous catalog. This new revision of the geologic data catalog incorporates new boreholes drilled after September 2002 as well as other older wells, particularly from the 600 Area, omitted from the earlier catalogs. Additionally, a description of available borehole geophysical log data has been included in the catalog. This version of the geologic data catalog now contains 3,519 boreholes and is current with boreholes drilled as of November 2004. However, the data catalog (Appendix A) is by no means complete. Many individuals have been involved in geologic studies through the years, and the extent of unpublished data retained in their files is unknown. The geologic data catalog is estimated to represent the majority of borehole geologic data currently available from the Hanford Site, but will continued to evolve as additional boreholes and data are added to the database. 
The data catalog (Appendix A) contains the majority of existing lithologic data available for the Hanford Site. The emphasis is on raw data needed to support performance assessments at varying scales and dimensions. The catalog identifies sources for the various geologic parameters needed to support the understanding of hydrologic and geochemical processes in the vadose zone, such as three-dimensional simulations of multiphase flow and contaminant sorption. A bibliography of data sources is included in Appendix B.

This geologic data catalog is not a database. Instead, it is a listing of the types of existing data and places where the data can be found (e.g., published documents, formal databases, or informal databases). Most of the data were generated during characterization and remediation activities by Bechtel Hanford, Inc.; the waste management and environmental programs of Rockwell Hanford Operations, Westinghouse Hanford Company, and Fluor Hanford, Inc.; the characterization and monitoring activities done by Pacific Northwest National Laboratory (PNNL); and studies done by independent contractors. The geologic data catalog is intended for the subject matter expert who has a need for existing geologic data. The data catalog also can be used to identify data gaps and technical needs.

Appendix A contains the catalog of borehole geologic data. Appendix B is an annotated bibliography of references given in Appendix A. Some, but not all of the references in Appendix B, describe the procedures used to collect the data and/or the pedigree of the data if known.

The scope of this catalog includes borehole geologic data collected from all geologic units penetrated by each borehole. This includes units within the unconfined aquifer as well as those in the vadose zone. This was done for completeness, because the geologic units that are below the water table in some areas are above the water table in other areas. It may be possible to extrapolate data from those areas below the water table to those areas above the water table. Also, in cases where there are no data from samples collected above the water table, data from below the water table may be the only available data. This catalog, however, is restricted to geologic data and does not include hydrologic information such as hydraulic conductivity, water table gradient, flow rate, flow direction, or groundwater chemical composition. The data types included in the catalog are lithology descriptions, borehole geophysics, rock composition (mineralogy and chemical composition), physical properties (grain size, density, cementation properties), and geologic structure.

The geologic data catalog includes only sources for raw data. It does not include interpretive data such as contacts between geologic units. The distinction between raw data and interpreted data is important and sometimes subtle. For example, laboratory-derived chemical data show the quantities of various elements in Hanford Site sediment and is considered raw data. The depth of a geologic contact based on the chemical data is interpretive data. 


\subsection{Interpretive Data}

Table 1 contains a list of selected sources for recent interpretive data. The conceptual model of the Hanford Site vadose zone continues to evolve as new data and interpretive methods are developed. As with the raw data, the information in Table 1 is incomplete. However, it represents many of the recently published (post-1990) interpretive data sets and more interpretive data may be available through the reference sections of the reports listed in Table 1.

Table 1. Selected Sources of Recent Interpretive Data

\begin{tabular}{|c|c|}
\hline Author and Year & Title and Document Number \\
\hline Last et al. 2004 & $\begin{array}{l}\text { Vadose Zone Hydrogeology Data Package for the } 2004 \text { Composite Analysis. PNNL-14702, } \\
\text { Rev. } 0 .\end{array}$ \\
\hline Reidel 2004 & $\begin{array}{l}\text { Geologic Data Package for } 2005 \text { Integrated Disposal Facility Waste Performance } \\
\text { Assessment. PNNL-14586. }\end{array}$ \\
\hline Bjornstad 2004 & Geologic Contacts Database for the 200 Areas of the Hanford Site. WMP-22817. \\
\hline $\begin{array}{l}\text { CH2M HILL Hanford Group, } \\
\text { Inc. 2003a }\end{array}$ & $\begin{array}{l}\text { Subsurface Conditions Description of the } C \text { and A-AX Waste Management Areas. } \\
\text { RPP-15808. }\end{array}$ \\
\hline $\begin{array}{l}\text { CH2M HILL Hanford Group, } \\
\text { Inc. } 2003 \mathrm{~b}\end{array}$ & Subsurface Conditions Description of the U Waste Management Area. RPP-15808. \\
\hline DOE 2002 & $\begin{array}{l}\text { Standardized Stratigraphic Nomenclature for Post-Ringold Formation Sediments Within the } \\
\text { Central Pasco Basin. DOE/RL-2002-39, Rev. 0. }\end{array}$ \\
\hline Williams et al. 2002 & $\begin{array}{l}\text { Revised Hydrogeology for the Suprabasalt Aquifer System, 200-West Area and Vicinity, } \\
\text { Hanford Site, Washington. PNNL-13858. }\end{array}$ \\
\hline $\begin{array}{l}\text { CH2M HILL Hanford Group, } \\
\text { Inc. 2002a }\end{array}$ & Field Investigation Report for Waste Management Area B-BX-BY. RPP-10098. \\
\hline $\begin{array}{l}\text { CH2M HILL Hanford Group, } \\
\text { Inc. } 2002 \text { b }\end{array}$ & Field Investigation Report for Waste Management Area S-SX. RPP-7884. \\
\hline Wood et al. 2001 & Subsurface Conditions Description of the T-TX-TY Waste Management Area. RPP-7123. \\
\hline Williams et al. 2000 & $\begin{array}{l}\text { Revised Hydrogeology for the Suprabasalt Aquifer System, 200-East Area and Vicinity, } \\
\text { Hanford Site, Washington. PNNL-12261. }\end{array}$ \\
\hline Slate 2000 & $\begin{array}{l}\text { Nature and Variability of the Plio-Pleistocene Unit in the 200-West Area of the Hanford } \\
\text { Site. BHI-01203. }\end{array}$ \\
\hline Reidel and Horton 1999 & $\begin{array}{l}\text { Geologic Data Package for the } 2001 \text { Immobilized Low-Activity Waste Performance } \\
\text { Assessment. PNNL-12257, Rev. 1. (Includes interpretations for the former Grout Treatment } \\
\text { Facility) }\end{array}$ \\
\hline Peterson et al. 1996 & $\begin{array}{l}\text { Conceptual Site Models for Groundwater Contamination at 100-BC-5, 100-KR-4, 100-HR-3, } \\
\text { and 100-FR-3 Operable Units. BHI-00197. }\end{array}$ \\
\hline Lindsey et al. 1994 & Geologic Setting of the Low-Level Burial Grounds, WHC-SD-EN-TI-290. \\
\hline Reidel and Fecht 1994a & Geologic Map of the Richland 1:100,000 Quadrangle, Washington. \\
\hline Reidel and Fecht $1994 b$ & Geologic Map of the Priest Rapids 1:100,000 Quadrangle, Washington. \\
\hline Hoffmann 1992 & Summary of the Geology of the 200-BP-1 Operable Unit. WHC-SD-EN-TI-037. \\
\hline Lindsey et al. 1992 & Geologic Setting of the 200-East Area: An Update. WHC-SD-EN-TI-012. \\
\hline Delaney et al. 1991 & $\begin{array}{l}\text { Geology and Hydrology of the Hanford Site: A Standardized Text for Use in Westinghouse } \\
\text { Hanford Company Documents and Reports. WHC-SD-ER-TI-003. }\end{array}$ \\
\hline Lindsey and Connelly 1991 & Geologic Setting of the 200-West Area: An Update. WHC-SD-EN-TI-008. \\
\hline Bjornstad 1990 & Geohydrology of the 218-W-5 Burial Ground, 200-West Area, Hanford Site. PNL-7336. \\
\hline
\end{tabular}




\subsection{Subject Matter Experts}

Many geoscientists have worked at the Hanford Site during the past 55 years. A quick scan of the bibliography will reveal the names of most of them. Most of these scientists have retired or taken employment at places other than the Hanford Site. However, several of these individuals remain at the Hanford Site and represent, in many cases, the individuals who collected some of the raw data and formulated the interpreted data. Those experts are acknowledged here so that other workers will know whom to contact for information:

- Bruce N. Bjornstad

- Mickie A. Chamness

- Karl R. Fecht

- Tyler J Gilmore

- Shannon M. Goodwin

- Duane G. Horton

- George V. Last

- Jon W. Lindberg

- Kevin A. Lindsey

- Rick McCain

- Steve P. Reidel

- Virginia A. Rohay

- Les Walker

- Dave C. Weekes

- Bruce A. Williams

It is recognized that many field geologists contributed to our knowledge of the Hanford Site over the past years. It is because of their work collecting samples, describing drill cuttings, and measuring geologic properties, that our understanding of the Hanford Site geology is as good as it is.

\subsection{References}

Bjornstad BN. 1990. Geohydrology of the 218-W-5 Burial Ground, 200 West Area, Hanford Site. PNL-7336, Pacific Northwest Laboratory, Richland, Washington.

Bjornstad BN. 2004. Geologic Contacts Database for the 200 Areas of the Hanford Site. WMP-22817, Rev. 0, Fluor Hanford, Richland, Washington.

Cantrell KJ, RJ Serne, and GV Last. 2003. Hanford Contaminant Distribution Coefficient Database and Users Guide. PNNL-13895, Rev. 1, Pacific Northwest National Laboratory, Richland, Washington. 
CH2M HILL Hanford Group. 2002a. Field Investigation Report for Waste Management Area B-BX-BY. RPP-10098, Rev. 0, CH2M HILL Hanford Group, Inc., Richland, Washington.

CH2M HILL Hanford Group. 2002b. Field Investigation Report for Waste Management Area S-SX. RPP-7884, Rev. 0, CH2M HILL Hanford Group, Inc., Richland, Washington.

CH2M HILL Hanford Group. 2003a. Subsurface Conditions Description of the C and A-AX Waste Management Areas. RPP-14430, Rev. 0, CH2M HILL Hanford Group, Inc., Richland, Washington.

CH2M HILL Hanford Group. 2003b. Subsurface Conditions Description of the U Waste Management Area. RPP-15808, Rev. 0, CH2M HILL Hanford Group, Inc., Richland, Washington.

DeLamare MA. 2000. Groundwater/Vadose Zone Integration Project Systems Engineering Management Plan. BHI-01347, Rev. 0, Bechtel Hanford, Inc., Richland, Washington.

Delaney CD, KA Lindsey, and SP Reidel. 1991. Geology and Hydrology of the Hanford Site: A Standardized Text for Use in Westinghouse Hanford Company Documents and Reports. WHC-SD-ENTI-003, Westinghouse Hanford Company, Richland, Washington.

DOE/RL-2002-39. 2002. Standardized Stratigraphic Nomenclature for Post-Ringold Formation Sediments Within the Central Pasco Basin. U.S. Department of Energy, Richland Operations, Richland, Washington.

Freeman EJ, R Khaleel, and PR Heller. 2002. A Catalog of Vadose Zone Hydraulic Properties for the Hanford Site. PNNL-13672, Rev. 1, Pacific Northwest National Laboratory, Richland, Washington.

Hoffmann KM. 1992. Summary of the Geology of the 200-BP-1 Operable Unit. WHC-SD-EN-TI-037, Westinghouse Hanford Company, Richland, Washington.

Horton DG, GV Last, TJ Gilmore, and BN Bjornstad. 2002. A Catalog of Geologic Data for the Hanford Site. PNNL-13653, Rev. 1, Pacific Northwest National Laboratory, Richland, Washington.

Last GV and DG Horton. 2000. Review of Geophysical Characterization Methods Used at the Hanford Site. PNNL-13149, Pacific Northwest National Laboratory, Richland, Washington.

Last GV, EJ Freeman, KJ Cantrell, MJ Fayer, GW Gee, WE Nichols, BN Bjornstad, and DG Horton. 2004. Vadose Zone Hydrogeology Data Package for the 2004 Composite Analysis. PNNL-14702, Rev. 0, Pacific Northwest National Laboratory, Richland, Washington.

Lindsey KA and MP Connelly. 1991. Geologic Setting of the 200-West Area: An Update. WHC-SDEN-TI-008, Westinghouse Hanford Company, Richland, Washington.

Lindsey KA, BN Bjornstad, JW Lindberg, and KM Hoffman. 1992. Geologic Setting of the 200-East Area: An Update. WHC-SD-EN-TI-012, Westinghouse Hanford Company, Richland, Washington. 
Lindsey KA, JL Slate, GK Jaeger, KJ Swett, and RB Mercer. 1994. Geologic Setting of the Low-Level Burial Grounds. WHC-SD-EN-TI-290, Westinghouse Hanford Company, Richland, Washington.

Peterson RE, RF Raidl, and CW Denslow. 1996. Conceptual Site Models for Groundwater Contamination at 100-BC-5, 100-KR-4, 100-HR-3, and 100-FR-3 Operable Units. BHI-0097, Rev. 0, Bechtel Hanford, Inc., Richland, Washington.

Reidel SP. 2004. Geologic Data Package for 2005 Integrated Disposal Facility Waste Performance Assessment. PNNL-14586, Pacific Northwest National Laboratory, Richland, Washington.

Reidel SP and KR Fecht. 1994a. Geologic Map of the Richland 1:100,000 Quadrangle, Washington. Open File Report 94-8, Washington Division of Geology and Earth Resources, Washington State Department of Natural Resources, Olympia, Washington.

Reidel SP and KR Fecht. 1994b. Geologic Map of the Priest Rapids 1:100,000 Quadrangle, Washington. Open File Report 94-13, Washington Division of Geology and Earth Resources, Washington State Department of Natural Resources, Olympia, Washington.

Reidel SP and DG Horton. 1999. Geologic Data Package for the 2001 Immobilized Low-Activity Waste Performance Assessment. PNNL-12257, Rev. 1, Pacific Northwest National Laboratory, Richland, Washington.

Riley RG and CA LoPresti. 2003. Data Catalog for Models Simulating Release of Contaminants from Hanford Site Waste Sources. PNNL-13666, Rev. 1, Pacific Northwest National Laboratory, Richland, Washington.

Slate JL. 2000. Nature and Variability of the Plio-Pleistocene Unit in the 200-West Area of the Hanford Site. BHI-01203, Bechtel Hanford, Inc., Richland, Washington.

Williams BA, BN Bjornstad, R Schalla, and WD Webber. 2000. Revised Hydrogeology for the Suprabasalt Aquifer System, 200-East Area and Vicinity, Hanford Site, Washington. PNNL-12261, Pacific Northwest National Laboratory, Richland, Washington.

Williams BA, BN Bjornstad, R Schalla, and WD Webber. 2002. Revised Hydrogeology for the Suprabasalt Aquifer System, 200-West Area and Vicinity, Hanford Site, Washington. PNNL-13858, Pacific Northwest National Laboratory, Richland, Washington.

Wood MI, TE Jones, R Schalla, BN Bjornstad, and FN Hodges. 2001. Subsurface Conditions Description of the T-TX-TY Waste Management Area. RPP-7123, Rev. 0, CH2M HILL Hanford Group, Richland, Washington. 


\section{Appendix A}

Geologic Data Catalog 


\section{Appendix A}

\section{Geologic Data Catalog}

The geologic data catalog presented in the tables that follow represents a static image of the catalog maintained as a MicroSoft Access database. The catalog is organized by area and well name (in rows). Thus, if there is interest in a certain location, data derived from wells near that location can be easily found. The corresponding well identification number (WIN or Well ID) from the Hanford Well Information System (HWIS) is also included as an additional means of well identification. Chamness and Merz (1993) document the well naming scheme used at the Hanford Site until about 2000. An understanding of the old well naming system provides insight into the location of the well, the relative age of the well, and the type of well (e.g., groundwater, vadose, piezometer). The current well naming system is different from the older system and is described in a Fluor Hanford, Inc. procedure (FHI 2004) for naming and numbering wells (and its predecessor, a Bechtel Hanford, Inc. procedure [BHI 2001]).

In addition to the well name, all water wells, borings, and dry wells are given a well identification number (Well ID). These numbers contain four digits, are alpha-numeric, and take the form A2763, A5892, C1483, etc. These numbers are assigned in alpha-numeric order as new wells or boreholes are drilled. If a borehole is drilled and not constructed as a well, it may have a well ID but no well name.

\section{A.1 Format and Content of the Geologic Data Catalog}

The types of raw data available for each well are grouped into ten different categories (columns):

- Descriptive Log Data (Log Type)

- Borehole Geophysical Log Data (GPX Log Runs)

- Particle Size Data (Sieved)

- Calcium Carbonate Data $\left(\mathrm{CaCO}_{3}\right)$

- Moisture Content Data (Moisture)

- Chemical Property Data (Chemical Properties)

- Physical Property Data (Physical Properties)

- Mineralogic Properties

- Geochronological Properties

- Archived Samples (Archived)

There is somewhat of an inequity in the seven categories of information. Whereas geophysical logs, particle size distribution, calcium carbonate content, and moisture content are given separate columns, all other information is contained in either the chemical property or physical property categories. This was done because data on particle size distribution and calcium carbonate and moisture contents are available for a large number of wells at the Hanford Site, whereas relatively few wells have chemical or physical 
property data. In addition, the catalog identifies the source and/or location of the data described in the previous columns. Brief descriptions of each of the general data types are provided in the following sections.

\section{A.1.1 Log Type}

The column labeled "Log Type" indicates what types of descriptive logs are available for a given well. Wells with a "D" in the column have an available driller's log. Wells with a "G" have an available geologist's log. All logs on the spreadsheet are available in the Hanford Well Information System (http://apweb02.rl.gov/cfroot/rapidweb/phmc/cp/hwisapp/) and/or the PNNL Well Log Library, located in the Sigma V building at 3110 Port of Benton Blvd., Richland, Washington. Some geologist's and driller's logs are located at both locations.

Generally, the types of information on the geologist's log includes a visual estimate of particle size distribution, sorting, grain roundness, general mineralogy of the gravel and sand fraction, color, reaction to $10 \% \mathrm{HCl}$, degree of consolidation and cementation, and any unusual characteristics. The type of information on driller's logs varies greatly from log to log but generally contains only a visual estimate of gravel, sand, and silt.

Entries of "As-built" or "Well Summary" in the Log Type column indicate that these types of summary documents are available. If a reference to HWIS (Hanford Well Information System) is given, the as-built or well summary is available at the HWIS web page (http://apweb02.rl.gov/cfroot/rapidweb/phmc/cp/hwisapp/). For many wells, the as-built diagrams and well summaries are available both at the HWIS web page and in the PNNL Well Log Library. The type of geologic data on the as-built or well summary is generally a summary of the driller's or the geologist's logs and limited to the type of lithology.

\section{A.1.2 GPX Log Runs}

This column contains information on available borehole geophysical logging data. A key to the types of logging runs is listed in Table A.1. This information was derived from three primary sources:

1. PNNL Log Database (http://boreholelogs.pnl.gov/)

2. Hanford Geophysical Logging Project Database (http://gj.em.doe.gov/hanf/)

3. Archived hard-copy reports located in the PNNL Well Log Library in room 2110, Sigma V building

Raw data, log headers, and interpreted logging reports are available from the first two sources in electronic format. 
The PNNL Log Database contains geophysical data for the 100, 200, 300, and 600 Areas. These logging data were collected by Westinghouse Hanford Company, Waste Management Federal Services, and Duratek between 1989 and 2002.

The Hanford Geophysical Logging Project Database primarily contains data collected from 2001 to present (a few 1991 and 1993 logging efforts are also available) for the 100, 200, and 600 Areas. Duratek, Mactec-ERS, and S.M. Stoller Corporation were the principal contractors.

Table A.1. Types of Borehole Geophysical Logs

\begin{tabular}{|c|c|c|}
\hline Symbol & Log Type & Description \\
\hline $\mathrm{CP}$ & Caliper Logs & $\begin{array}{l}\text { Records the diameter of the borehole or well casing. Changes in diameter are } \\
\text { related to fracturing or caving along the borehole wall and to well construction. }\end{array}$ \\
\hline DN & $\begin{array}{l}\text { Density Logs } \\
\text { (Gamma- } \\
\text { Gamma Logs) }\end{array}$ & $\begin{array}{l}\text { Records the back-scattered gamma-rays coming from the interaction of an active } \\
\text { source of gamma-rays with the formation and other borehole/well materials. } \\
\text { Changes can be related to the bulk density of the formation. }\end{array}$ \\
\hline GG & $\begin{array}{l}\text { Gross Gamma } \\
\text { Logs }\end{array}$ & $\begin{array}{l}\text { Records the total gamma radiation emitted by the formation surrounding the } \\
\text { borehole. The amount of radiation is related to lithology and/or the presence of } \\
\text { anthropogenic gamma-emitting radionuclides. }\end{array}$ \\
\hline MG & Magnetic Logs & $\begin{array}{l}\text { Records the natural magnetic field in the formation surrounding the borehole. } \\
\text { Changes relate to the magnetic reversals in basalt flows and the presence of } \\
\text { interbeds. }\end{array}$ \\
\hline MO & $\begin{array}{l}\text { Moisture Logs } \\
\text { (Neutron Logs) }\end{array}$ & $\begin{array}{l}\text { Records the number of neutrons impinging on a detector from a constant source } \\
\text { after interacting with surrounding particles (particularly hydrogen). When used in } \\
\text { the unsaturated zone, changes relate to the changes in moisture content. }\end{array}$ \\
\hline NT & Neutron Logs & $\begin{array}{l}\text { Records the number of neutrons impinging on a detector from a constant source, } \\
\text { after interacting with surrounding formation materials (particularly hydrogen). } \\
\text { When used in saturated conditions, changes relate to total porosity. }\end{array}$ \\
\hline OT & Other Logs & $\begin{array}{l}\text { This refers to other various logging types (e.g., 3D velocity, dual induction, dip } \\
\text { meter). }\end{array}$ \\
\hline SN & $\begin{array}{l}\text { Sonic Logs } \\
\text { (Acoustic Logs) }\end{array}$ & $\begin{array}{l}\text { Measures the travel time and attenuation of an acoustic signal. Useful in } \\
\text { determining relative porosities of different formations and/or how well the casing } \\
\text { has been cemented to the formation. }\end{array}$ \\
\hline SG & $\begin{array}{l}\text { Spectral- } \\
\text { Gamma Ray } \\
\text { Logs }\end{array}$ & $\begin{array}{l}\text { Measures the energy spectrum and intensity of gamma rays emitted from the } \\
\text { formation. Analysis of these data permit identification of gamma-emitting } \\
\text { radionuclides and their estimated concentrations. }\end{array}$ \\
\hline $\mathrm{TP}$ & $\begin{array}{l}\text { Temperature } \\
\text { Logs }\end{array}$ & $\begin{array}{l}\text { Record the water temperature in the borehole. These logs are useful for delineating } \\
\text { fluid levels, water-bearing zones, cement location, and formation temperatures. }\end{array}$ \\
\hline
\end{tabular}

Electronic files from previous compilations of geophysical logs were used to catalog pre-1992 geophysical logs archived in the Sigma V building (e.g., Chamness et al. 1991; Chamness et al. 1992). 


\section{A.1.3 Sieved}

The information in the "Sieved" column indicates whether particle size distribution analyses exist for samples from each well. Entries of "Virtual Library" in the column mean that the particle size distribution data are available in the Virtual Library database maintained by Fluor Hanford, Inc. and located at http://vlprod.rl.gov/vlib/app/index.cfm. A user name and password are required for access to the Virtual Library and can be obtained from Fluor Hanford, Inc. Laboratory particle size data in the Virtual Library was derived from the now abandoned ROCSAN Database. Field estimates in the Virtual Library were derived from information on geologist's logs.

Price and Fecht (1976) give a brief explanation of the procedures used to obtain data for the ROCSAN (a.k.a. ROC) database.

When using the particle size data in the Virtual Library database, the user should attempt to determine the method used to sample the borehole. Boreholes that were cored or sampled by cable tool with a drive barrel generally give much better information than boreholes sampled by cable tool with hard tool and bailer methods because the hard tool tends to alter the natural grain size distribution. The sampling method can usually be determined by examining the well logs in the PNNL or HWIS libraries. The old ROCSAN database would default to "drive barrel" as the drilling method if no specific method was entered. Thus, the older ROCSAN database is not a reliable way to determine sampling method; the sampling method is not yet part of the Virtual Library database.

Entries of "Y" (yes) in the "Sieved" column indicate that particle size distribution data are available in sources other than the Virtual Library database. The source of the data is indicated in the "Source" column, which can be cross-referenced to the bibliography.

For many of the wells, grain size distribution data are available for samples collected at 5-foot intervals. However, for other wells, grain size distribution data are available only for part of the borehole or for random, or preselected depths. The quantity of the sieve data for a specific well can only be determined by going to the Virtual Library or the referenced data source(s).

\section{A.1.4 $\mathrm{CaCO}_{3}$}

The information in the " $\mathrm{CaCO}_{3}$ " column indicates whether calcium carbonate concentration data exist for samples from each well. As with the "Sieved" column, entries of "Virtual Library" in the " $\mathrm{CaCO}_{3}$ " column mean that calcium carbonate concentration data are available in the Virtual Library database maintained by Fluor Hanford, Inc. The calcium carbonate information in the Virtual Library database was taken from the ROCSAN database. The calcium carbonate content of samples in the Virtual Library database was measured with a semiquantitative $\mathrm{CO}_{2}$ displacement method (e.g. Horwitz 1970). Qualitative estimates of calcium carbonate content as made by the geologists in the field are not included in the " $\mathrm{CaCO}_{3}$ " column. 
Entries of "Y" (yes) in the " $\mathrm{CaCO}_{3}$ " column indicate that data are available from sources other than the Virtual Library database. The source of the data is indicated in the "Source" column which can be cross-referenced to the bibliography.

Just as for the grain-size distribution data, data for calcium carbonate content are available for samples collected at 5-foot intervals for many of the wells. However, for other wells, calcium carbonate data are available only for part of the borehole or for random, or preselected depths. The quantity of the data can only be determined by going to the Virtual Library or the referenced data source.

\section{A.1.5 Moisture}

A "Y" (Yes) in the "Moisture" column means that moisture content data are available for samples from the specified well. If there is a reference in the "Source" column, the data are available through the reference. Some of the references describe the procedure used to collect the data or give the laboratory that measured the data so that some judgment of the data quality can be made. If no reference is given in the "Source" column, the data are available in the PNNL Well Log Library.

For many of the wells, moisture content is available for samples collected at 5-foot intervals. However, for other wells, moisture data are available only for part of the borehole or for random, or preselected depths. The quantity of the data can only be determined by going to the referenced data source or to the PNNL Well Log Library.

\section{A.1.6 Chemical Properties}

Information in the "Chemical Properties" column indicates what chemical property data are available for samples from each well. The types of data and their abbreviations used in this column are given in Table A.2. The sources of the data are indicated in the "Source" column, which can be cross-referenced to the bibliography. Annotations in the bibliography tell which data are contained in the source. Thus, if more than one data type and more than one source are listed, the bibliography can help the user to determine which source contains the desired data. For example, well 299-E17-21 has available sieve data and magnetic polarity data. A check of the bibliography shows that the sieve data are in Valenta et al. (2000) and the magnetic polarity data are in Reidel and Horton (1999). If more than one type of data is listed in the "Chemical Properties" column and if the annotations in the bibliography do not indicate that the source contains the desired data, then that data are available in the PNNL Well Log Library. 
Table A.2. Types of Chemical Property Data

\begin{tabular}{|c|c|}
\hline Abbreviation & Meaning \\
\hline $1: 1$ & $\begin{array}{l}\text { Chemical analysis of metals, and/or cations, and/or anions, and/or } \mathrm{pH} \text { of } 1: 1 \text { sediment to } \\
\text { water extract }\end{array}$ \\
\hline Aex & Chemical analysis of metals, cations, anions, and/or $\mathrm{pH}$ of $1: 1$ sediment to acid extract \\
\hline Alk & Alkalinity analysis of porewater or 1:1 water extract \\
\hline Alpha & Laboratory analysis of gross alpha or alpha spectrometry \\
\hline $\mathrm{Am}$ & Americium-241 \\
\hline An & Anions \\
\hline $\mathrm{ASiO}_{2}$ & Amorphous silica \\
\hline Beta & Laboratory analysis of gross beta or beta spectrometry \\
\hline $\mathrm{CN}$ & Analysis of cyanide \\
\hline CrVI & Analysis of hexavalent chromium \\
\hline Field $\mathrm{pH}$ & Field measurements of $\mathrm{pH}$ in geologist's $\operatorname{logs}$ \\
\hline Field Rad & Data are available in driller's logs for field measurements of radiation (GM counter) \\
\hline Field Rad/Temp & $\begin{array}{l}\text { Data are available in driller's logs for field measurements of radiation (GM counter) and/or } \\
\text { temperature }\end{array}$ \\
\hline Gamma & Laboratory analysis of gross gamma or gamma spectrometry \\
\hline GEA & Gamma energy analysis for radionuclides \\
\hline $\mathrm{H}-3$ & Laboratory analysis for tritium \\
\hline IC & Laboratory analysis of inorganic carbon \\
\hline Lab Rad & Laboratory measurements of soil radioactivity \\
\hline Metals & Laboratory analysis of metals usually by inductively coupled plasma or atomic absorption \\
\hline MiscMetals & Laboratory analysis of various miscellaneous metals or radioisotopes \\
\hline $\mathrm{NO}^{3}$ & Laboratory analysis for nitrate \\
\hline $\mathrm{N}$ & Laboratory analysis for nitrogen \\
\hline $\mathrm{P}$ & Laboratory analysis for phosphorus \\
\hline PID & Field measurements with a photoionization detector in geologist's logs \\
\hline $\mathrm{Pu}$ & One or more isotope of plutonium \\
\hline Pwater & Porewater composition \\
\hline Res & Resistivity \\
\hline Sr-90 & Laboratory analysis of Sr-90 \\
\hline StIso & Stable isotopes \\
\hline SVOA & Semi-volatile organic compounds \\
\hline
\end{tabular}


Table A.2. (contd)

\begin{tabular}{||l|l||}
\hline \multicolumn{1}{|c||}{ Abbreviation } & \multicolumn{1}{c||}{ Meaning } \\
\hline \hline TC & Total carbon \\
\hline TFe/Fe2 & Total iron and ferrous iron \\
\hline TOC & Total organic carbon \\
\hline VarMetals & Analysis of various metals by unspecified methods \\
\hline VOA & Volatile organic compounds \\
\hline Wet Chem & Digestate and/or leachate composition (metals and/or anions) \\
\hline XRF & Chemical analysis by x-ray fluorescence \\
\hline Y & Data available \\
\hline
\end{tabular}

In some cases, two sources may be listed that give the same information. For example, sieve data for boreholes in the tank farms are found in the Virtual Library database and in the series of reports by Price and Fecht (see bibliography, Appendix B).

If no source is given for the data, the data are found in the PNNL Well Log Library.

\section{A.1.7 Physical Properties}

The "Physical Properties" column indicates what physical property data are available for samples from each well. The "Physical Properties" column is used similar to the "Chemical Properties" column described above. The types of data and their abbreviations used in this column are given in Table A.3.

Table A.3. Types of Physical Property Data

\begin{tabular}{||l|l||}
\hline \multicolumn{1}{|c||}{ Abbreviation } & \multicolumn{1}{c||}{ Meaning } \\
\hline \hline$\%$ GSSC & $\begin{array}{l}\text { Percent gravel, sand, silt, and clay. These data require hydrometer analysis to differentiate } \\
\text { silt and clay. Most sources giving this type of information mention the use of hydrometer } \\
\text { analysis; some do not. Those that do not suggest the data may be suspect. If hydrometer } \\
\text { data were given but no calculation of percent clay and silt, the symbol \%GSS was still } \\
\text { applied. }\end{array}$ \\
\hline 15-Atm & $\begin{array}{l}\text { 15-Atmosphere moisture. This is the moisture content held in a soil against a pressure of } \\
225 \text { psi. }\end{array}$ \\
\hline AirPerm & Air permeability \\
\hline Bden & Bulk density \\
\hline CEC & Cation exchange capacity \\
\hline Cond & Electrical conductivity of 1:1 water extract or porewater \\
\hline Pden & Particle density \\
\hline Pip & Pipette analysis of grain size \\
\hline Por & Porosity \\
\hline SpG & Specific gravity \\
\hline
\end{tabular}




\section{A.1.8 Mineralogical Properties}

Information in the Mineralogical Properties column indicates the types of mineralogic data that are available. The types of data are defined in Table A.4.

Table A.4. Types of Mineralogic Data

\begin{tabular}{|l|l||}
\hline \multicolumn{1}{|c|}{ Abbreviation } & \multicolumn{1}{||}{ Meaning } \\
\hline \hline EM & Electron microprobe \\
\hline Min & Mineralogy by petrographic microscope \\
\hline PbC & Pebble count data \\
\hline PC & Point count modal composition \\
\hline SEM & Scanning electron microscopy \\
\hline TEM & Transmission electron microscope \\
\hline XRD & Mineralogy by x-ray diffraction \\
\hline
\end{tabular}

\section{A.1.9 Geochronological Properties}

Information in the Geochronological Properties column indicates the types of geochronological data that are available. The types of data are defined in Table A.5.

Table A.5. Types of Geochronologic Data

\begin{tabular}{|l|l||}
\hline \hline \multicolumn{1}{|c||}{ Abbreviation } & \multicolumn{1}{c|}{ Meaning } \\
\hline \hline C-14 & Carbon-14 age date \\
\hline Date & Radiometric or thermo luminescent age date \\
\hline Paleomag & Geomagnetic polarity \\
\hline
\end{tabular}

\section{A.1.10 Archived}

A "Y" (Yes) in the "Archived" column indicates that samples from the well are archived in the Geotechnical Sample Library in the 300 Area. A database of samples in the library is maintained by PNNL. The database specifies the well name and number, the depth, the drill method, the drill date, and the library location for each sample. In general, wells were sampled at 5-foot intervals. However, many wells were not sampled through their entire depth for various reasons. Access to the database and the Geotechnical Sample Library is through S. P. Reidel or D. G. Horton at PNNL. A few samples are flagged $\mathrm{Y}^{*}$ in the Archived column. The asterisk indicates that archived samples are available but they are potentially radiologically contaminated and they are archived in the 325 building laboratory. Archived samples are available to users who have a need for them. 


\section{A.1.11 Source}

The "Source" column gives the reference or references to the locations of the data listed in all other columns. A bibliography of data sources listed in this column can be found in Appendix B. Most references in the bibliography are annotated to indicate what types of data are in the referenced source. If the referenced data source described the procedures used for data collection or mentioned the laboratory that collected the data, that information is included in the annotations. This allows the data users to form an opinion about the quality of the data.

If no source is given, the log data are in the PNNL Well Log Library and/or the particle size data are in the Virtual Library database. References to as-built diagrams, well summary sheets, and the Hanford Well Information System (HWIS) indicate that these sources are available. The HWIS and/or the PNNL Well Log Library contains as-built diagrams and well summary sheets. The HWIS can be found at (http://apweb02.rl.gov/cfroot/rapidweb/phmc/cp/hwisapp/). Both as-built diagrams and well summary sheets mostly contain information about the well itself and may or may not include geologic information. These are good sources, however, to determine how a specific well was drilled and constructed.

Table A.6 contains the actual geologic data.

\section{A.2 References}

BHI. 2001. Naming, Numbering, and Tracking of Groundwater Resource Protection Well, GeoProbe, Geotechnical Soil Boring, and River Substrate and Aquifer Porewater Monitoring Tubes. Procedure 1.9, Rev. 2 in BHI-EE-01, Environmental Investigations Procedures, Bechtel Hanford, Inc., Richland, Washington.

Chamness MA and JK Merz. 1993. Hanford Wells. PNL-8800, Pacific Northwest Laboratory, Richland, Washington

Chamness MA, RE Lewis, SS Teel, and AW Pearson. 1991. T Plant Geologic and Geophysics Data Package for the 200 Aggregate Area Management Study. WHC-SD-EN-DP-022, Westinghouse Hanford Operations, Richland, Washington.

Chamness MA, RE Lewis, SS Teel, RW Brockman, DC Lanigan, and AW Pearson. 1992. PUREX Aggregate Area Management Study Geologic Data Package. WHC-SD-EN-DP-025, Westinghouse Hanford Operations, Richland, Washington.

Driscol FG. 1986. Groundwater and Wells. Second Edition. Johnson Filtration Systems Inc., St. Paul, Minnesota.

FHI. 2004. Naming, Numbering, and Tracking of Groundwater Resource Protection Well, GeoProbe, Geotechnical Soil Boring, and River Substrate and Aquifer Porewater Monitoring Tubes. Procedure GRP-EE-01-1.9, Rev. 0, Fluor Hanford, Richland, Washington. 
Horwitz W. 1970. Official Methods of Analysis of the Association of Official Analytical Chemists. $11^{\text {th }}$ Edition, Association of Official Analytical Chemists (now named AOAC International), Gaithersburg, Maryland, p. 139.

Price WH and KR Fecht. 1976. Geology of the 241-SX Tank Farm. ARH-LD-134, Atlantic Richfield Hanford Company, Richland, Washington.

Reidel SP and DG Horton. 1999. Geologic Data Package for the 2001 Immobilized Low-Activity Waste Performance Assessment. PNNL-12257, Rev. 1, Pacific Northwest National Laboratory, Richland, Washington.

Valenta MM, JR Moreno, MB Martin, RE Ferri, DG Horton, and SP Reidel. 2000. Particle Size Distribution Data From Existing Boreholes at the Immobilized Low-Activity Waste Site. PNNL-13328, Pacific Northwest National Laboratory, Richland, Washington. 
Table A.6. Geologic Data

\begin{tabular}{|c|c|c|c|c|c|c|c|c|c|c|c|c|c|c|c|}
\hline SortIndex & Area & Well Name & WellID & Log Type & GPX Log Runs & Sieved & $\mathrm{CaCO} 3$ & Moisture & $\begin{array}{l}\text { Chemical } \\
\text { Properties }\end{array}$ & $\begin{array}{c}\text { Physical } \\
\text { Properties }\end{array}$ & \begin{tabular}{|c|} 
Min \\
Properties
\end{tabular} & $\begin{array}{l}\text { Geochron } \\
\text { Properties }\end{array}$ & Archived & Source & Comments \\
\hline & $100-B / C$ & 199-B2-12 & A4550 & $\mathrm{D}, \mathrm{G}$, Well summary & GG, SG & $Y$ & & $\mathrm{Y}$ & & SpG, Bden & & & & $\begin{array}{l}\text { HWIS; Khaleel } \\
1999\end{array}$ & Aquifer test (slug test) \\
\hline 2 & \begin{tabular}{|l|l|}
2 & $100-B / C$
\end{tabular} & 199-B2-13 & A4551 & $\mathrm{D}, \mathrm{G}$, Well summary & GG, SG & & & & & & & & & HWIS & Aquifer test (slug test) \\
\hline 3 & $3 \mid 100-B / C$ & 199-B3-1 & A4552 & D, As-built & GG, OT, SG, TP & & & & & & & & & HWIS & \\
\hline & $4 \mid 100-B / C$ & 199-B3-2 & A9505 & D, As-built & GG, OT, SG, TP & & & & & & & & & HWIS & \\
\hline & $5 \mid 100-B / C$ & 199-B3-46 & A4553 & D, G, Well summary & GG, SG & & & & & & & & & $\begin{array}{l}\text { DOE 1994a; } \\
\text { HWIS }\end{array}$ & Aquifer test (slug test) \\
\hline & \begin{tabular}{l|l}
6 & $100-B / C$ \\
\end{tabular} & 199-B3-47 & A4554 & $\mathrm{D}, \mathrm{G}$, Well summary & GG, SG & & & & & & & & & HWIS & \\
\hline 7 & $7 \mid 100-B / C$ & 199-B3-48 & A5537 & $\mathrm{D}, \mathrm{G}$ & SG & & & & & & & & & & \\
\hline & $8100-B / C$ & 199-B4-1 & A4555 & D, As-built & GG, OT, SG, TP & & & & & & & & & HWIS & \\
\hline 9 & $\begin{array}{l}9100-\mathrm{B} / \mathrm{C} \\
\end{array}$ & 199-B4-10 & A5542 & $\mathrm{D}, \mathrm{G}$ & SG & & & & & & & & & & \\
\hline 10 & $0 \mid 100-B / C$ & 199-B4-11 & A9907 & D, G & & & & & & & & & & & \\
\hline 11 & $100-B / C$ & 199-B4-12 & A9908 & D, G & & & & & & & & & & & \\
\hline 12 & $2100-B / C$ & 199-B4-13 & A9909 & $\mathrm{D}, \mathrm{G}$ & & & & & & & & & & & \\
\hline 13 & $3 \mid 100-B / C$ & $199-B 4-2$ & A5539 & D, As-built & $\mathrm{DN}, \mathrm{GG}, \mathrm{NT}, \mathrm{OT}, \mathrm{TP}$ & & & & & & & & & HWIS & \\
\hline 14 & $4 \mid 100-B / C$ & 199-B4-3 & A4556 & D, As-built & DN, GG, NT, OT, TP & & & & & & & & & HWIS & \\
\hline 15 & $5 \mid 100-B / C$ & 199-B4-4 & A4557 & D, As-built & GG, OT, SG, TP & & & & & & & & & HWIS & \\
\hline 16 & $6 \mid 100-B / C$ & 199-B4-5 & A5540 & $\mathrm{D}, \mathrm{G}$ & GG, SG & & & & & & & & & & \\
\hline 17 & $7 \mid 100-B / C$ & 199-B4-6 & A4558 & D, G, As-built & GG & & & & & & & & & HWIS & \\
\hline 18 & $8100-B / C$ & 199-B4-7 & A5541 & D, G, As-built & GG & & & & & & & & & HWIS & \\
\hline 19 & $9100-B / C$ & 199-B4-8 & A4559 & D, G, As-built & GG, SG & & & & Field Rad & & & & & HWIS & \\
\hline 20 & $100-B / C$ & 199-B4-9 & A4560 & D, G, As-built & SG & $Y$ & & Y & Field Rad & SpG, Bden & & & & $\begin{array}{l}\text { HWIS, see R. } \\
\text { Khaleel or G. } \\
\text { Freemen for } \\
\text { sieve data }\end{array}$ & \\
\hline 21 & $100-B / C$ & 199-B5-1 & A4561 & D, As-built & GG, OT, TP & & & & & & & & & HWIS & \\
\hline$\frac{22}{22}$ & $100-B / C$ & $\frac{199-D-1}{199-B 5-2}$ & A4562 & D, G & GG, SG & & & & Field Rad & & & & & & \\
\hline 23 & $100-B / C$ & $199-\mathrm{B} 5-3$ & A5543 & D, G & SG & & & & Field Rad & & & & & & \\
\hline 24 & $100-B / C$ & 199-B5-4 & A5544 & D, G & SG & & & & Field Rad & & & & & & \\
\hline 25 & $100-B / C$ & 199-B8-1 & A5545 & D & & & & & & & & & & & \\
\hline 26 & f. $100-B / C$ & $\frac{199-B 8-1}{199-2}$ & A5546 & $\mathrm{D}$ & & & & & & & & & & & \\
\hline 27 & $100-B / C$ & 199-B8-3 & A5547 & $\mathrm{D}$ & & & & & & & & & & & \\
\hline 28 & $100-B / C$ & 199-B8-4 & A5548 & D & & & & & & & & & & & \\
\hline 29 & $100-B / C$ & 199-B8-5 & A5549 & D & & & & & & & & & & & \\
\hline 30 & $100-B / C$ & 199-B8-6 & A4563 & $\mathrm{D}, \mathrm{G}$, Well summary & GG, SG & & & & Field Rad & & & & & HWIS & \\
\hline 31 & $100-B / C$ & 199-B9-1 & A4564 & D. As-built & GG, OT, SG, TP & & & & & & & & & HWIS & \\
\hline 32 & $100-B / C$ & 199-B9-2 & A4565 & $\mathrm{D}, \mathrm{G}$, Well summary & GG, SG & $Y$ & & $\mathrm{Y}$ & Field Rad & SpG, Bden & & & & $\begin{array}{l}\text { HWIS, see R. } \\
\text { Khaleel or G. } \\
\text { Freemen for } \\
\text { sieve data }\end{array}$ & \\
\hline 33 & \begin{tabular}{l|l}
3 & $100-B / C$
\end{tabular} & 199-B9-3 & A4566 & $\mathrm{D}, \mathrm{G}$, Well summary & GG, SG & & & & Field Rad & & & & & HWIS & \\
\hline 34 & $100-B / C$ & 199-B9-4 & A5550 & Well summary & SG & & & & & & & & & & \\
\hline 35 & $100-B / C$ & $\begin{array}{l}\text { 116-B-1 vadose } \\
\text { borehole }\end{array}$ & & & & & & & \begin{tabular}{|l|} 
Field Rad, \\
Lab Rad, \\
Metals, \\
SVOA, VOA
\end{tabular} & & & & & DOE 1994b & \\
\hline
\end{tabular}




\begin{tabular}{|c|c|c|c|c|c|c|c|c|c|c|c|c|c|c|c|}
\hline SortIndex & \begin{tabular}{|l} 
Area \\
\end{tabular} & Well Name & WelllD & Log Type & GPX Log Runs & Sieved & $\mathrm{CaCO} 3$ & Moisture & $\begin{array}{l}\text { Chemical } \\
\text { Properties }\end{array}$ & $\begin{array}{l}\text { Physical } \\
\text { Properties }\end{array}$ & \begin{tabular}{|c|} 
Min \\
Properties
\end{tabular} & $\begin{array}{l}\text { Geochron } \\
\text { Properties }\end{array}$ & Archived & Source & Comments \\
\hline 36 & $6100-B / C$ & 116-B-6A; BH-1 & & G & & & & & & & & & & & Wells for in situ vitrification \\
\hline 37 & $7100-B / C$ & $\mathrm{BH}-2$ & & G & & & & & & & & & & & Wells for in situ vitrification \\
\hline 38 & $8100-B / C$ & $\mathrm{BH}-3$ & & G & & & & & & & & & & & Wells for in situ vitrification \\
\hline 39 & $9100-D / D R$ & 199-D2-2 & A5552 & & & & & & & & & & $\mathrm{Y}$ & & Abandoned \\
\hline 40 & $0100-D / D R$ & 199-D2-4 & A5554 & & & & & & & & & & $\mathrm{Y}$ & & \\
\hline 41 & $100-D / D R$ & 199-D2-5 & A4567 & D & GG, SG, TP & & & & & & & & $Y$ & HWIS & Decommissioned 3/2/98 \\
\hline 42 & \begin{tabular}{|l|l|l|l|l|} 
& $100-D / D R$ \\
\end{tabular} & 199-D2-6 & A4568 & G & GG, SG & & & & & & & & $\mathrm{Y}$ & DOE 1993a & Groundwater monitoring \\
\hline 43 & $3100-\mathrm{D} / \mathrm{DR}$ & 199-D2-8 & C3040 & G & & & & & & & & & & HWIS & \\
\hline 44 & 4 100-D/DR & 199-D3-1 & A5555 & D & & & & & & & & & & HWIS & Abandoned \\
\hline 45 & $5 \mid 100-D / D R$ & 199-D3-3 & C3312 & G & & & & & & & & & & $\begin{array}{l}\text { Martinez and } \\
\text { Weekes } 2002\end{array}$ & \\
\hline 46 & \begin{tabular}{|l|l|}
6 & $100-D / D R$ \\
\end{tabular} & 199-D3-4 & C3314 & G & & & & & & & & & & $\begin{array}{l}\text { Martinez and } \\
\text { Weekes } 2002\end{array}$ & \\
\hline 47 & 7 100-D/DR & 199-D4-1 & B2895 & G, Well summary & & & & & & & & & & & ISRM Monitoring \\
\hline 48 & 8 | $100-D / D R$ & 199-D4-10 & B8068 & G & & & & & & & & & & HWIS & ISRM injection \\
\hline 49 & $9100-D / D R$ & 199-D4-11 & B8069 & G & & & & & & & & & & HWIS & ISRM injection \\
\hline 50 & $100-D / D R$ & 199-D4-12 & B8070 & G & & & & & & & & & & HWIS & ISRM injection \\
\hline 51 & $100-D / D R$ & 199-D4-16 & B8110 & G & & & & & & & & & & HWIS & ISRM Monitoring; Westbay \\
\hline 52 & $2100-D / D R$ & 199-D4-17 & B8459 & G & & & & & & & & & & HWIS & ISRM Monitoring; Westbay \\
\hline 53 & $3100-\mathrm{D} / \mathrm{DR}$ & 199-D4-18 & B8460 & G & & & & & & & & & & HWIS & ISRM Monitoring; Westbay \\
\hline 54 & 1100-D/DR & 199-D4-19 & B8746 & G & & & & & & & & & & Lee 1999 & Groundwater monitoring \\
\hline 55 & 100-D/DR & $199-D 4-2$ & B8058 & G & & $\mathrm{Y}$ & & & & $\begin{array}{l}\text { Bden, Pden, } \\
\text { Por }\end{array}$ & & & & $\begin{array}{l}\text { Williams et al. } \\
2000\end{array}$ & ISRM Monitoring \\
\hline 56 & |100-D/DR & 199-D4-20 & B8750 & G & & & & & & & & & & Lee 1999 & Groundwater monitoring \\
\hline 57 & $700-\mathrm{D} / \mathrm{DR}$ & 199-D4-21 & B8755 & G & & & & & & & & & & Lee 1999 & Groundwater monitoring \\
\hline 58 & $100-D / D R$ & 199-D4-23 & B8779 & G & & & & & & & & & & HWIS & \\
\hline 59 & $100-\mathrm{D} / \mathrm{DR}$ & 199-D4-24 & B8975 & G & & & & & & & & & & $\begin{array}{l}\text { Laurenz and } \\
\text { Walker } 2001\end{array}$ & ISRM injection \\
\hline 60 & $100-\mathrm{D} / \mathrm{DR}$ & 199-D4-25 & B8976 & G & & & & & & & & & & $\begin{array}{l}\text { Laurenz and } \\
\text { Walker } 2001\end{array}$ & ISRM injection \\
\hline 61 & $100-D / D R$ & 199-D4-26 & B8977 & G & & & & & & & & & & $\begin{array}{l}\text { Laurenz and } \\
\text { Walker } 2001\end{array}$ & ISRM injection \\
\hline 62 & $2100-\mathrm{D} / \mathrm{DR}$ & 199-D4-27 & B8978 & G & & & & & & & & & & $\begin{array}{l}\text { Laurenz and } \\
\text { Walker } 2001\end{array}$ & ISRM injection \\
\hline
\end{tabular}




\begin{tabular}{|c|c|c|c|c|c|c|c|c|c|c|c|c|c|c|c|}
\hline SortIndex & Area & Well Name & WelliD & Log Type & GPX Log Runs & Sieved & $\mathrm{CaCO} 3$ & Moisture & $\begin{array}{l}\text { Chemical } \\
\text { Properties }\end{array}$ & $\begin{array}{c}\text { Physical } \\
\text { Properties }\end{array}$ & $\begin{array}{c}\text { Min } \\
\text { Properties }\end{array}$ & $\begin{array}{l}\text { Geochron } \\
\text { Properties }\end{array}$ & Archived & Source & Comments \\
\hline 63 & $3100-D / D R$ & 199-D4-28 & B8979 & G & & & & & & & & & & $\begin{array}{l}\text { Laurenz and } \\
\text { Walker } 2001\end{array}$ & ISRM injection \\
\hline 64 & 100-D/DR & 199-D4-29 & B8980 & G & & & & & & & & & & $\begin{array}{l}\text { Laurenz and } \\
\text { Walker } 2001\end{array}$ & ISRM injection \\
\hline 65 & |100-D/DR & 199-D4-3 & B8059 & G & & $\bar{Y}$ & & & & \begin{tabular}{|l} 
Bden, Pden, \\
Por
\end{tabular} & & & & $\begin{array}{l}\text { Williams et al. } \\
2000\end{array}$ & ISRM Monitoring \\
\hline 66 & |100-D/DR & 199-D4-31 & B8982 & G & & & & & & & & & & $\begin{array}{l}\text { Laurenz and } \\
\text { Walker } 2001\end{array}$ & ISRM injection \\
\hline 67 & 7100-D/DR & 199-D4-32 & B8983 & G & & & & & & & & & & $\begin{array}{l}\text { Laurenz and } \\
\text { Walker } 2001\end{array}$ & ISRM injection \\
\hline 68 & 100-D/DR & 199-D4-33 & B8984 & G & & & & & & & & & & $\begin{array}{l}\text { Laurenz and } \\
\text { Walker } 2001\end{array}$ & ISRM injection \\
\hline 69 & 100-D/DR & 199-D4-34 & B8985 & G & & & & & & & & & & $\begin{array}{l}\text { Laurenz and } \\
\text { Walker } 2001\end{array}$ & ISRM injection \\
\hline 70 & 100-D/DR & 199-D4-35 & B8986 & G & & & & & & & & & & $\begin{array}{l}\text { Laurenz and } \\
\text { Walker } 2001\end{array}$ & ISRM injection \\
\hline 71 & $100-D / D R$ & 199-D4-36 & B8987 & G & & & & & & & & & & $\begin{array}{l}\text { Laurenz and } \\
\text { Walker } 2001\end{array}$ & ISRM injection \\
\hline 72 & $100-D / D R$ & 199-D4-37 & B8988 & G & & & & & & & & & & $\begin{array}{l}\text { Laurenz and } \\
\text { Walker } 2001\end{array}$ & ISRM injection \\
\hline 73 & 100-D/DR & 199-D4-38 & B8989 & G & & & & & & & & & & $\begin{array}{l}\text { Laurenz and } \\
\text { Walker } 2001\end{array}$ & Groundwater monitoring \\
\hline 74 & 100-D/DR & 199-D4-39 & B8990 & G & & & & & & & & & & $\begin{array}{l}\text { Laurenz and } \\
\text { Walker } 2001\end{array}$ & Groundwater monitoring \\
\hline 75 & |100-D/DR & 199-D4-4 & B8060 & G & & $\mathrm{Y}$ & & & & $\begin{array}{l}\text { Bden, Pden, } \\
\text { Por }\end{array}$ & & & & HWIS & ISRM Monitoring \\
\hline 76 & 100-D/DR & 199-D4-40 & C3270 & G & & & & & & & & & & $\begin{array}{l}\text { Trice et al. 2001; } \\
\text { HWIS }\end{array}$ & \\
\hline 77 & $7100-D / D R$ & 199-D4-41 & C3271 & G & & & & & & & & & & $\begin{array}{l}\text { Trice et al. 2001; } \\
\text { HWIS }\end{array}$ & \\
\hline 78 & $100-D / D R$ & 199-D4-42 & C3272 & G & & & & & & & & & & $\begin{array}{l}\text { Trice et al. 2001; } \\
\text { HWIS }\end{array}$ & \\
\hline 79 & |100-D/DR & 199-D4-43 & C3273 & G & & & & & & & & & & $\begin{array}{l}\text { Trice et al. 2001; } \\
\text { HWIS }\end{array}$ & \\
\hline 80 & 100-D/DR & 199-D4-44 & C3274 & G & & & & & & & & & & $\begin{array}{l}\text { Trice et al. 2001; } \\
\text { HWIS }\end{array}$ & \\
\hline 81 & $100-D / D R$ & 199-D4-45 & C3275 & G & & & & & & & & & & $\begin{array}{l}\text { Trice et al. 2001; } \\
\text { HWIS }\end{array}$ & \\
\hline 82 & $100-D / D R$ & 199-D4-46 & C3276 & G & & & & & & & & & & $\begin{array}{l}\text { Trice et al. 2001; } \\
\text { HWIS }\end{array}$ & \\
\hline 83 & 100-D/DR & 199-D4-47 & C3277 & G & & & & & & & & & & $\begin{array}{l}\text { Trice et al. 2001; } \\
\text { HWIS }\end{array}$ & \\
\hline 84 & $100-D / D R$ & 199-D4-48 & C3278 & G & & & & & & & & & & $\begin{array}{l}\text { Trice et al. 2001; } \\
\text { HWIS }\end{array}$ & \\
\hline 85 & 100-D/DR & 199-D4-49 & C3279 & G & & & & & & & & & & $\begin{array}{l}\text { Trice et al. 2001; } \\
\text { HWIS }\end{array}$ & \\
\hline 86 & |100-D/DR & 199-D4-5 & B8061 & G & & $\mathrm{Y}$ & & & & $\begin{array}{l}\text { Bden, Pden, } \\
\text { Por }\end{array}$ & & & & HWIS & ISRM Monitoring \\
\hline 87 & $7100-D / D R$ & 199-D4-50 & C3280 & G & & & & & & & & & & $\begin{array}{l}\text { Trice et al. 2001; } \\
\text { HWIS }\end{array}$ & \\
\hline 88 & 100-D/DR & 199-D4-51 & C3281 & G & & & & & & & & & & $\begin{array}{l}\text { Trice et al. 2001; } \\
\text { HWIS }\end{array}$ & \\
\hline 89 & 100-D/DR & 199-D4-52 & C3282 & G & & & & & & & & & & $\begin{array}{l}\text { Trice et al. 2001; } \\
\text { HWIS }\end{array}$ & \\
\hline
\end{tabular}




\begin{tabular}{|c|c|c|c|c|c|c|c|c|c|c|c|c|c|c|c|}
\hline SortIndex & Area & Well Name & WellID & Log Type & GPX Log Runs & Sieved & $\mathrm{CaCO} 3$ & Moisture & $\begin{array}{l}\text { Chemical } \\
\text { Properties }\end{array}$ & $\begin{array}{c}\text { Physical } \\
\text { Properties }\end{array}$ & $\begin{array}{c}\text { Min } \\
\text { Properties } \\
\end{array}$ & $\begin{array}{l}\text { Geochron } \\
\text { Properties }\end{array}$ & Archived & Source & Comments \\
\hline & 100-D/DR & 199-D4-53 & C3283 & G & & & & & & & & & & $\begin{array}{l}\begin{array}{l}\text { Trice et al. 2001; } \\
\text { HWIS }\end{array} \\
\end{array}$ & \\
\hline 91 & 100-D/DR & 199-D4-54 & C3284 & G & & & & & & & & & & $\begin{array}{l}\text { Trice et al. 2001; } \\
\text { HWIS }\end{array}$ & \\
\hline 92 & 100-D/DR & 199-D4-55 & C3285 & G & & & & & & & & & & $\begin{array}{l}\text { Trice et al. 2001; } \\
\text { HWIS }\end{array}$ & \\
\hline 93 & 100-D/DR & 199-D4-56 & C3286 & G & & & & & & & & & & $\begin{array}{l}\text { Trice et al. 2001; } \\
\text { HWIS }\end{array}$ & \\
\hline 94 & 100-D/DR & 199-D4-57 & C3287 & G & & & & & & & & & & $\begin{array}{l}\text { Trice et al. 2001; } \\
\text { HWIS }\end{array}$ & \\
\hline 95 & 100-D/DR & 199-D4-58 & C3288 & G & & & & & & & & & & $\begin{array}{l}\text { Trice et al. 2001; } \\
\text { HWIS }\end{array}$ & \\
\hline 96 & 100-D/DR & 199-D4-59 & C3289 & G & & & & & & & & & & $\begin{array}{l}\text { Trice et al. 2001; } \\
\text { HWIS }\end{array}$ & \\
\hline 97 & 100-D/DR & 199-D4-6 & B8064 & G & & & & & & & & & & HWIS & ISRM Monitoring \\
\hline 98 & 100-D/DR & 199-D4-60 & C3290 & G & & & & & & & & & & $\begin{array}{l}\text { Trice et al. 2001; } \\
\text { HWIS }\end{array}$ & \\
\hline 99 & 100-D/DR & 199-D4-61 & C3291 & G & & & & & & & & & & $\begin{array}{l}\text { Trice et al. 2001; } \\
\text { HWIS }\end{array}$ & \\
\hline 100 & 100-D/DR & 199-D4-62 & C3292 & G & & & & & & & & & & $\begin{array}{l}\text { Trice et al. 2001; } \\
\text { HWIS }\end{array}$ & \\
\hline 101 & 100-D/DR & 199-D4-63 & C3293 & G & & & & & & & & & & $\begin{array}{l}\text { Trice et al. 2001; } \\
\text { HWIS }\end{array}$ & \\
\hline 102 & 100-D/DR & 199-D4-64 & C3294 & G & & & & & & & & & & $\begin{array}{l}\text { Trice et al. 2001; } \\
\text { HWIS }\end{array}$ & \\
\hline 103 & 100-D/DR & 199-D4-65 & C3295 & G & & & & & & & & & & $\begin{array}{l}\begin{array}{l}\text { Trice et al. 2001; } \\
\text { HWIS }\end{array} \\
\end{array}$ & \\
\hline 104 & 100-D/DR & 199-D4-66 & C3296 & G & & & & & & & & & & $\begin{array}{l}\text { Trice et al. 2001; } \\
\text { HWIS }\end{array}$ & \\
\hline 105 & 100-D/DR & 199-D4-67 & C3297 & G & & & & & & & & & & $\begin{array}{l}\text { Trice et al. 2001; } \\
\text { HWIS }\end{array}$ & \\
\hline 106 & 100-D/DR & 199-D4-68 & C3298 & G & & & & & & & & & & $\begin{array}{l}\text { Laurenz and } \\
\text { Walker } 2000\end{array}$ & \\
\hline 107 & 100-D/DR & 199-D4-69 & C3299 & G & & & & & & & & & & $\begin{array}{l}\text { Martinez and } \\
\text { Weekes } 2002\end{array}$ & \\
\hline 108 & 100-D/DR & 199-D4-7 & B8065 & G & & & & & & & & & & $\begin{array}{l}\text { Williams et al. } \\
2000\end{array}$ & ISRM injection \\
\hline 109 & 100-D/DR & 199-D4-70 & C3300 & G & & & & & & & & & & $\begin{array}{l}\text { Martinez and } \\
\text { Weekes } 2002\end{array}$ & \\
\hline 110 & 100-D/DR & 199-D4-71 & C3301 & G & & & & & & & & & & $\begin{array}{l}\text { Martinez and } \\
\text { Weekes } 2002\end{array}$ & \\
\hline 111 & 100-D/DR & 199-D4-72 & C3302 & G & & & & & & & & & & $\begin{array}{l}\text { Martinez and } \\
\text { Weekes } 2002\end{array}$ & \\
\hline 112 & 100-D/DR & 199-D4-73 & C3303 & G & & & & & & & & & & $\begin{array}{l}\text { Martinez and } \\
\text { Weekes } 2002\end{array}$ & \\
\hline 113 & 100-D/DR & 199-D4-74 & C3304 & G & & & & & & & & & & $\begin{array}{l}\text { Martinez and } \\
\text { Weekes } 2002\end{array}$ & \\
\hline
\end{tabular}




\begin{tabular}{|c|c|c|c|c|c|c|c|c|c|c|c|c|c|c|c|}
\hline SortIndex & Area & Well Name & WelliD & Log Type & GPX Log Runs & Sieved & $\mathrm{CaCO} 3$ & Moisture & $\begin{array}{l}\text { Chemical } \\
\text { Properties }\end{array}$ & $\begin{array}{c}\text { Physical } \\
\text { Properties }\end{array}$ & \begin{tabular}{|c|} 
Min \\
Properties
\end{tabular} & $\begin{array}{l}\text { Geochron } \\
\text { Properties }\end{array}$ & Archived & Source & Comments \\
\hline 114 & $100-D / D R$ & 199-D4-75 & $\mathrm{C} 3305$ & G & & & & & & & & & & $\begin{array}{l}\text { Martinez and } \\
\text { Weekes } 2002\end{array}$ & \\
\hline 115 & 100-D/DR & 199-D4-76 & C3306 & G & & & & & & & & & & $\begin{array}{l}\text { Martinez and } \\
\text { Weekes } 2002\end{array}$ & \\
\hline 116 & 100-D/DR & 199-D4-77 & C3307 & G & & & & & & & & & & $\begin{array}{l}\text { Martinez and } \\
\text { Weekes } 2002\end{array}$ & \\
\hline 117 & $100-D / D R$ & 199-D4-78 & C3308 & G & & & & & & & & & & $\begin{array}{l}\text { Martinez and } \\
\text { Weekes } 2002\end{array}$ & \\
\hline 118 & 100-D/DR & 199-D4-79 & C3309 & G & & & & & & & & & & $\begin{array}{l}\text { Martinez and } \\
\text { Weekes } 2002\end{array}$ & \\
\hline 119 & 100-D/DR & 199-D4-8 & B8066 & G & & & & & & & & & & $\begin{array}{l}\text { Williams et al. } \\
2000\end{array}$ & ISRM Monitoring \\
\hline 120 & 100-D/DR & 199-D4-80 & C3310 & G & & & & & & & & & & $\begin{array}{l}\text { Martinez and } \\
\text { Weekes } 2002\end{array}$ & \\
\hline 121 & 100-D/DR & 199-D4-81 & C3311 & G & & & & & & & & & & $\begin{array}{l}\text { Martinez and } \\
\text { Weekes } 2002\end{array}$ & \\
\hline 122 & $100-D / D R$ & 199-D4-82 & C3313 & G & & & & & & & & & & $\begin{array}{l}\text { Martinez and } \\
\text { Weekes } 2002\end{array}$ & \\
\hline 123 & $100-\mathrm{D} / \mathrm{DR}$ & 199-D4-83 & C3315 & G & & & & & & & & & & $\begin{array}{l}\text { Trice et al. 2001; } \\
\text { HWIS }\end{array}$ & \\
\hline 124 & $100-D / D R$ & 199-D4-84 & C3316 & G & & & & & & & & & & 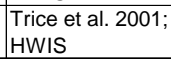 & \\
\hline 125 & $100-\mathrm{D} / \mathrm{DR}$ & 199-D4-85 & C3317 & G & & & & & & & & & & Trice et al. 2001 & \\
\hline 126 & 6100-D/DR & 199-D4-86 & C3318 & G & & & & & & & & & & Trice et al. 2001 & \\
\hline 127 & $7100-\mathrm{D} / \mathrm{DR}$ & 199-D4-87 & C3799 & G & & & & & & & & & & $\begin{array}{l}\text { Martinez and } \\
\text { Weekes } 2002\end{array}$ & \\
\hline 128 & 100-D/DR & 199-D4-88 & C3800 & G & & & & & & & & & & $\begin{array}{l}\text { Martinez and } \\
\text { Weekes } 2002\end{array}$ & \\
\hline 129 & 9100-D/DR & 199-D4-89 & C3801 & G & & & & & & & & & & $\begin{array}{l}\text { Martinez and } \\
\text { Weekes } 2002\end{array}$ & \\
\hline 130 & $100-\mathrm{D} / \mathrm{DR}$ & 199-D4-9 & B8067 & G & & & & & & & & & & $\begin{array}{l}\text { Williams et al. } \\
2000\end{array}$ & ISRM injection \\
\hline 131 & $100-D / D R$ & 199-D5-1 & A5556 & & & & & & & & & & $\mathrm{Y}$ & & Abandoned \\
\hline 132 & 100-D/DR & 199-D5-10 & A5565 & G & & & & & & & & & Y & HWIS & \\
\hline 133 & $100-D / D R$ & 199-D5-11 & A5566 & G & & & & & & & & & $\mathrm{Y}$ & HWIS & \\
\hline 134 & $100-\mathrm{D} / \mathrm{DR}$ & 199-D5-12 & A4569 & G & DN, GG, NT, SG, TP & & & & & & & & & DOE 1993a & Groundwater monitoring \\
\hline 135 & 100-D/DR & 199-D5-13 & A4570 & G & GG, SG & $\bar{Y}$ & Y & Y & & & & & $\mathrm{Y}$ & DOE 1993a & Groundwater monitoring \\
\hline
\end{tabular}




\begin{tabular}{|c|c|c|c|c|c|c|c|c|c|c|c|c|c|c|c|}
\hline SortIndex & Area & Well Name & WellID & Log Type & GPX Log Runs & Sieved & $\mathrm{CaCO} 3$ & Moisture & $\begin{array}{l}\text { Chemical } \\
\text { Properties }\end{array}$ & $\begin{array}{l}\text { Physical } \\
\text { Properties }\end{array}$ & \begin{tabular}{|c|} 
Min \\
Properties
\end{tabular} & $\begin{array}{l}\text { Geochron } \\
\text { Properties }\end{array}$ & Archived & Source & Comments \\
\hline 136 & $\begin{array}{l}6 \mid 100-D / D R \\
\end{array}$ & 199-D5-14 & A4571 & G & GG, SG & $\mathrm{Y}$ & & & & $\begin{array}{l}\text { Bden, Por, } \\
\text { SpG }\end{array}$ & & & Y & $\begin{array}{l}\text { DOE 1993a; } \\
\text { Khaleel 1999; } \\
\text { Peterson et al. } \\
1996\end{array}$ & Groundwater monitoring \\
\hline 137 & 7 100-D/DR & 199-D5-15 & A4572 & G & GG, SG & & & & & $\begin{array}{l}\text { Bden, Por, } \\
\text { SpG }\end{array}$ & & & $\mathrm{Y}$ & \begin{tabular}{|l|} 
DOE 1993a; \\
Peterson et al. \\
1996 \\
\end{tabular} & Groundwater monitoring \\
\hline 138 & $8100-D / D R$ & 199-D5-16 & A4573 & G & GG, SG & & & & & $\begin{array}{l}\text { Bden, Por, } \\
\text { SpG }\end{array}$ & & & $\mathrm{Y}$ & \begin{tabular}{|l} 
DOE 1993a; \\
Peterson et al. \\
1996
\end{tabular} & Groundwater monitoring \\
\hline 139 & $9100-\mathrm{D} / \mathrm{DR}$ & 199-D5-17 & A4574 & G & GG, SG & $Y$ & & & & $\begin{array}{l}\text { Bden, Por, } \\
\text { SpG }\end{array}$ & & & $\mathrm{Y}$ & $\begin{array}{l}\text { DOE 1993a; } \\
\text { Khaleel 1999; } \\
\text { Peterson et al. } \\
1996\end{array}$ & Groundwater monitoring \\
\hline 140 & $100-D / D R$ & 199-D5-18 & A4575 & G & GG, SG & & & & & $\begin{array}{l}\text { Bden, Por, } \\
\text { SpG }\end{array}$ & & & $Y$ & $\begin{array}{l}\text { DOE 1993a; } \\
\text { Peterson et al. } \\
\text { 1996 }\end{array}$ & Groundwater monitoring \\
\hline 141 & $100-D / D R$ & 199-D5-19 & A4576 & G & GG, SG & & & & & $\begin{array}{l}\text { Bden, Por, } \\
\text { SpG }\end{array}$ & & & $\mathrm{Y}$ & $\begin{array}{l}\text { DOE 1993a; } \\
\text { Peterson et al. } \\
1996 \\
\end{array}$ & Groundwater monitoring \\
\hline 142 & $100-D / D R$ & 199-D5-2 & A5557 & & & & & & & & & & $\mathrm{Y}$ & DOE 1993a & Abandoned \\
\hline 143 & $3100-\mathrm{D} / \mathrm{DR}$ & 199-D5-20 & A4577 & D, G, As-built & GG, SG & & & & & & & & $Y$ & HWIS & Groundwater monitoring \\
\hline 144 & $4100-\mathrm{D} / \mathrm{DR}$ & 199-D5-21 & A5567 & D, G & GG, SG & & & & & & & & & HWIS & \\
\hline 145 & |100-D/DR & 199-D5-22 & A5568 & G & GG, SG & & & & & & & & $Y$ & HWIS & Abandoned; B8778 \\
\hline 146 & |100-D/DR & 199-D5-23 & A5569 & G & GG, SG & & & & & & & & $\mathrm{Y}$ & HWIS & Abandoned; B8779 \\
\hline 147 & $7100-D / D R$ & 199-D5-24 & A5570 & D, G & GG & & & & & & & & $\mathrm{Y}$ & HWIS & Abandoned \\
\hline 148 & 100-D/DR & 199-D5-25 & A5571 & $\mathrm{D}, \mathrm{G}$ & GG, SG & & & & & & & & $Y$ & HWIS & Abandoned \\
\hline 149 & 100-D/DR & 199-D5-26 & A5572 & D, G & GG & & & & & & & & $\mathrm{Y}$ & HWIS & Abandoned \\
\hline 150 & $100-D / D R$ & 199-D5-27 & A5573 & D, G, As-built & GG & & & & & & & & $Y$ & HWIS & Abandoned \\
\hline 151 & $100-\mathrm{D} / \mathrm{DR}$ & 199-D5-28 & A5574 & D, G, As-built & GG, SG & & & & & & & & $\mathrm{Y}$ & HWIS & Abandoned \\
\hline 152 & 100-D/DR & 199-D5-29 & A5575 & $\mathrm{D}, \mathrm{G}$ & & & & & & & & & $Y$ & HWIS & Abandoned \\
\hline 153 & $3100-D / D R$ & 199-D5-30 & A5576 & D, G & SG & $\mathrm{Y}$ & & & & & & & $\mathrm{Y}$ & $\begin{array}{l}\text { See R. Khaleel } \\
\text { or G. Freeman } \\
\text { for sieve data }\end{array}$ & \\
\hline 154 & 100-D/DR & 199-D5-32 & C4185 & G & & $Y$ & & & & & & & & Martinez 2004 & 100-HR-3 \\
\hline 155 & $100-D / D R$ & 199-D5-33 & C4186 & G & & $Y$ & & & & & & & & Martinez 2004 & 100-HR-3 \\
\hline 156 & 100-D/DR & 199-D5-34 & C4187 & G & & Y & & & & & & & & Martinez 2004 & 100-HR-3 \\
\hline 157 & $100-\mathrm{D} / \mathrm{DR}$ & 199-D5-36 & B8744 & G & & $Y$ & & & & & & & & Lee 1999 & Groundwater monitoring \\
\hline 158 & 100-D/DR & 199-D5-37 & B8745 & G & & $Y$ & & & & & & & & Lee 1999 & Groundwater monitoring \\
\hline 159 & 100-D/DR & 199-D5-38 & B8747 & G & & $Y$ & & & & & & & & Lee 1999 & Groundwater monitoring \\
\hline 160 & 100-D/DR & 199-D5-39 & B8748 & G & & $Y$ & & & & & & & & Lee 1999 & Groundwater monitoring \\
\hline 161 & 100-D/DR & 199-D5-40 & B8749 & G & & $\mathrm{Y}$ & & & & & & & & Lee 1999 & Groundwater monitoring \\
\hline
\end{tabular}




\begin{tabular}{|c|c|c|c|c|c|c|c|c|c|c|c|c|c|c|c|}
\hline Sortindex & Area & Well Name & WellID & Log Type & GPX Log Runs & Sieved & $\mathrm{CaCO} 3$ & Moisture & $\begin{array}{c}\text { Chemical } \\
\text { Properties }\end{array}$ & $\begin{array}{l}\text { Physical } \\
\text { Properties }\end{array}$ & $\begin{array}{c}\text { Min } \\
\text { Properties } \\
\end{array}$ & $\begin{array}{l}\text { Geochron } \\
\text { Properties }\end{array}$ & Archived & Source & Comments \\
\hline 162 & $100-\mathrm{D} / \mathrm{DR}$ & 199-D5-41 & B8751 & G & & $Y$ & & & & & & & & Lee 1999 & Groundwater monitoring \\
\hline 163 & $100-\mathrm{D} / \mathrm{DR}$ & 199-D5-42 & B8752 & G & & $Y$ & & & & & & & & Lee 1999 & Groundwater monitoring \\
\hline 164 & $100-D / D R$ & 199-D5-43 & B8753 & G & & $Y$ & & & & & & & & Lee 1999 & Groundwater monitoring \\
\hline 165 & $100-\mathrm{D} / \mathrm{DR}$ & 199-D5-44 & B8754 & G & & Y & & & & & & & & Lee 1999 & Groundwater monitoring \\
\hline 166 & $100-\mathrm{D} / \mathrm{DR}$ & 199-D8-2 & A5580 & $\mathrm{D}$ & GG, SG, TP & & & & & & & & $Y$ & DOE 1993a & Decommissioned 3/11/98 \\
\hline 167 & $100-\mathrm{D} / \mathrm{DR}$ & 199-D8-3 & A4578 & D & DN, GG, NT, SG, TP & & & & & & & & $\mathrm{Y}$ & DOE 1993a & Decommissioned 9/2/98 \\
\hline 168 & $100-D / D R$ & 199-D8-4 & A4579 & G & GG, SG & & Y & $Y$ & & & & & $Y$ & HWIS & Groundwater monitoring \\
\hline 169 & 100-D/DR & 199-D8-5 & A4580 & G & GG, SG & & $Y$ & $Y$ & & & & & Y & DOE 1993a & Groundwater monitoring \\
\hline 170 & $100-D / D R$ & 199-D8-53 & A4581 & $\mathrm{D}, \mathrm{G}$ & & $Y$ & & & & & & & $\mathrm{Y}$ & $\begin{array}{l}\text { DOE 1993a; } \\
\text { Khaleel 1999; } \\
\text { Peterson et al. } \\
1996\end{array}$ & ISRM extraction \\
\hline 171 & $100-D / D R$ & 199-D8-54A & A4582 & $D, G$ & & $Y$ & & & & & & & $Y$ & $\begin{array}{l}\text { DOE 1993a; } \\
\text { Khaleel 1999 }\end{array}$ & ISRM extraction \\
\hline 172 & $100-D / D R$ & 199-D8-54B & A4583 & D, G, Well summary & GG, SG & $Y$ & & & & $\begin{array}{l}\text { Bden. Por, } \\
\text { SpG }\end{array}$ & & & $Y$ & $\begin{array}{l}\text { DOE 1993a; } \\
\text { Khaleel 1999; } \\
\text { Peterson et al. } \\
1996\end{array}$ & ISRM performance \\
\hline 173 & 100-D/DR & 199-D8-55 & A4584 & D, G, As-built & GG, SG & $Y$ & & & & Bden, SpG & & & $\mathrm{Y}$ & $\begin{array}{l}\text { DOE 1993a; } \\
\text { Khaleel 1999; } \\
\text { Peterson et al. } \\
1996\end{array}$ & Groundwater monitoring \\
\hline 174 & 100-D/DR & 199-D8-59 & A5630 & $\mathrm{D}, \mathrm{G}$ & GG, SG & & & & & & & & $\mathrm{Y}$ & HWIS & Abandoned \\
\hline 175 & $100-D / D R$ & 199-D8-6 & A4585 & G & GG, MG, SG & $Y$ & $Y$ & $Y$ & & & & & Y & DOE 1993a & Groundwater monitoring \\
\hline 176 & 100-D/DR & 199-D8-60 & A5631 & G & GG & & & & & & & & $Y$ & HWIS & Abandoned \\
\hline 177 & $100-D / D R$ & 199-D8-61 & A5632 & $\mathrm{D}, \mathrm{G}$ & GG & & & & & & & & $Y$ & HWIS & Abandoned \\
\hline 178 & $100-\mathrm{D} / \mathrm{DR}$ & 199-D8-62 & A5633 & D, G & GG & & & & & & & & $Y$ & HWIS & Abandoned \\
\hline 179 & 100-D/DR & 199-D8-63 & A5634 & D, G & GG, SG & & & & & & & & $Y$ & HWIS & Abandoned \\
\hline 180 & $100-\mathrm{D} / \mathrm{DR}$ & 199-D8-64 & A5635 & G & GG & & & & & & & & $Y$ & HWIS & Abandoned \\
\hline 181 & 100-D/DR & 199-D8-65 & A5636 & G & GG & & & & & & & & $Y$ & HWIS & Abandoned \\
\hline 182 & $100-\mathrm{D} / \mathrm{DR}$ & 199-D8-66 & A5637 & G & & & & & & & & & $Y$ & HWIS & Abandoned \\
\hline 183 & $100-D / D R$ & 199-D8-68 & B2772 & G, Well summary & & $Y$ & & & & & & & & HWIS & ISRM compliance \\
\hline 184 & $100-D / D R$ & 199-D8-69 & B2773 & G, Well summary & & $Y$ & & & & & & & & HWIS & ISRM compliance \\
\hline 185 & 100-D/DR & 199-D8-70 & B2774 & G, Well summary & & $Y$ & & & & & & & & HWIS & ISRM compliance \\
\hline 186 & $100-D / D R$ & 199-D8-71 & B2775 & G, Well summary & & Y & & & & & & & & HWIS & ISRM performance \\
\hline 187 & $100-\mathrm{D} / \mathrm{DR}$ & 199-D8-72 & C3829 & G & & $Y$ & & & & & & & & Sump 2002a & \\
\hline 188 & $100-\mathrm{F}$ & $199-\mathrm{F} 1-2$ & A4586 & G & SG & & & & & & & & Y & HWIS & \\
\hline 189 & $100-\mathrm{F}$ & 199-F2-1 & A5640 & $\mathrm{D}$ & GG, NT, SG & & & & & & & & & HWIS & \\
\hline 190 & $100-\mathrm{F}$ & 199-F2-2 & A5641 & As-built & GG, NT & & & & & & & & & HWIS & \\
\hline
\end{tabular}




\begin{tabular}{|c|c|c|c|c|c|c|c|c|c|c|c|c|c|c|c|}
\hline SortIndex & Area & Well Name & WellID & Log Type & GPX Log Runs & Sieved & $\mathrm{CaCO} 3$ & Moisture & $\begin{array}{l}\text { Chemical } \\
\text { Properties }\end{array}$ & $\begin{array}{l}\text { Physical } \\
\text { Properties }\end{array}$ & $\begin{array}{c}\text { Min } \\
\text { Properties }\end{array}$ & $\begin{array}{l}\text { Geochron } \\
\text { Properties }\end{array}$ & Archived & Source & Comments \\
\hline 191 & $100-\mathrm{F}$ & $199-\mathrm{F} 2-3$ & A5642 & D & & & & & & & & & & HWIS & \\
\hline 192 & $100-\mathrm{F}$ & $199-F 4-2$ & A5645 & $\mathrm{D}, \mathrm{G}$ & SG & & & & & & & & & HWIS & \\
\hline 193 & $100-\mathrm{F}$ & $199-F 4-45$ & & G & & & & & & & & & $\mathrm{Y}$ & & \\
\hline 194 & $100-\mathrm{F}$ & 199-F5-1 & A4587 & D & DN, GG, NT, SG, TP & & & & & & & & $\mathrm{Y}$ & HWIS & \\
\hline 195 & $100-\mathrm{F}$ & $199-F 5-2$ & A4588 & $\mathrm{D}$ & & & & & & & & & $\mathrm{Y}$ & HWIS & \\
\hline 196 & $100-\mathrm{F}$ & $199-F 5-3$ & A4589 & D & GG, SG, TP & & & & & & & & $\mathrm{Y}$ & HWIS & \\
\hline 197 & $100-\mathrm{F}$ & $199-F 5-4$ & A4590 & D & GG, TP & & & & & & & & $\mathrm{Y}$ & HWIS & \\
\hline 198 & $100-\mathrm{F}$ & 199-F5-42 & A4591 & G & GG, SG & & & & & & & & & HWIS & \\
\hline 199 & $100-\mathrm{F}$ & 199-F5-43A & A4592 & G & GG, SG & & & & & & & & $\mathrm{Y}$ & HWIS & \\
\hline 200 & $100-\mathrm{F}$ & 199-F5-43B & A4593 & G & GG, SG & $Y$ & & & & & & & $\mathrm{Y}$ & See R. Khaleel or & G. Freeman for sieve data \\
\hline 201 & $100-\mathrm{F}$ & $199-F 5-44$ & A4594 & G & GG, SG & & & & & & & & $\mathrm{Y}$ & HWIS & \\
\hline 202 & $100-\mathrm{F}$ & $199-F 5-45$ & A4595 & G, As-built & GG, SG & & & & & & & & & HWIS & \\
\hline 203 & $100-\mathrm{F}$ & 199-F5-46 & A4596 & G & GG & & & & & & & & $\mathrm{Y}$ & HWIS & \\
\hline 204 & $100-\mathrm{F}$ & 199-F5-47 & A4597 & G & SG & & & & & & & & $\mathrm{Y}$ & HWIS & \\
\hline 205 & $100-\mathrm{F}$ & 199-F5-48 & A4598 & G & GG, SG & $Y$ & & & & & & & $\mathrm{Y}$ & See R. Khaleel or & G. Freeman for sieve data \\
\hline 206 & $100-\mathrm{F}$ & 199-F5-49 & A5680 & D, G & SG & & & & & & & & & HWIS & \\
\hline 207 & $100-\mathrm{F}$ & $199-F 5-5$ & A4599 & D & & & & & & & & & $\mathrm{Y}$ & HWIS & \\
\hline 208 & $100-\mathrm{F}$ & 199-F5-50 & A5681 & $D, G$ & SG & & & & & & & & & HWIS & \\
\hline 209 & $100-\mathrm{F}$ & 199-F5-51 & A5682 & $\mathrm{D}, \mathrm{G}$ & SG & & & & & & & & & HWIS & \\
\hline 210 & $100-\mathrm{F}$ & $199-F 5-6$ & A4600 & D & DN, GG, NT, SN, TP & & & & & & & & $\mathrm{Y}$ & HWIS & \\
\hline 211 & $100-\mathrm{F}$ & $199-F 5-7$ & A4601 & & GG, SG, TP & & & & & & & & & & \\
\hline 212 & $100-\mathrm{F}$ & 199-F6-1 & A4602 & G & GG, SG & & & & & & & & $\mathrm{Y}$ & HWIS & \\
\hline 213 & $100-\mathrm{F}$ & 199-F6-46 & & & SG & & & & & & & & & & \\
\hline 214 & $100-\mathrm{F}$ & 199-F7-1 & A4603 & D & TP & & & & & & & & $\mathrm{Y}$ & HWIS & \\
\hline 215 & $100-\mathrm{F}$ & $199-\mathrm{F} 7-2$ & A4604 & G & & & & & & & & & $\mathrm{Y}$ & HWIS & \\
\hline 216 & $100-\mathrm{F}$ & $199-\mathrm{F} 7-3$ & A4605 & G & SG & & & & & & & & $\mathrm{Y}$ & HWIS & \\
\hline 217 & $100-\mathrm{F}$ & 199-F8-1 & A4606 & D & GG, TP & & & & & & & & $\mathrm{Y}$ & HWIS & \\
\hline 218 & $100-\mathrm{F}$ & $199-\mathrm{F} 8-2$ & A4607 & D & & & & & & & & & $\mathrm{Y}$ & HWIS & \\
\hline 219 & $100-\mathrm{F}$ & $199-\mathrm{F} 8-3$ & A4608 & $G$ & SG & $Y$ & & & & & & & $\mathrm{Y}$ & See R. Khaleel or & o. Freeman for sieve data \\
\hline 220 & $100-\mathrm{F}$ & $199-\mathrm{F} 8-4$ & A4609 & G & GG, SG & & & & & & & & $\mathrm{Y}$ & HWIS & \\
\hline 221 & $100-\mathrm{F}$ & $199-\mathrm{F} 8-5$ & A5683 & $D, G$ & SG & & & & & & & & & HWIS & \\
\hline 222 & $100-\mathrm{F}$ & $199-\mathrm{F} 8-6$ & A5684 & $\mathrm{D}, \mathrm{G}$ & SG & & & & & & & & & HWIS & \\
\hline 223 & $100-\mathrm{F}$ & \multicolumn{2}{|c|}{ 108-F soil samples } & & & & & & \multicolumn{3}{|c|}{ VOC, SVOC, An, Metals, Lab Rad } & & & DOE 1993b & \\
\hline 224 & $100-\mathrm{F}$ & \multicolumn{2}{|l|}{$116-F-1 A$ test pit } & & & & & & \multicolumn{3}{|c|}{ VOC, SVOC, An, Metals, Lab Rad } & & & DOE 1993b & \\
\hline 225 & $100-\mathrm{F}$ & \multirow{2}{*}{\multicolumn{2}{|c|}{ 116-F-1A shallow borehole }} & & & & & & \multicolumn{3}{|c|}{ VOC, SVOC, An, Metals, Lab Rad } & & & DOE 1993b & \\
\hline 226 & $100-\mathrm{F}$ & & & & & & & & \multicolumn{3}{|c|}{ VOC, SVOC, An, Metals, Lab Rad } & & & DOE 1993b & \\
\hline 227 & $100-\mathrm{F}$ & \multicolumn{2}{|c|}{$116-\mathrm{F}-2$ shallow borehole } & & & & & & \multicolumn{3}{|c|}{ VOC, SVOC, An, Metals, Lab Rad } & & & DOE 1993b & \\
\hline 228 & $100-\mathrm{F}$ & 116-F-3 test pit & & & & & & & \multirow{2}{*}{\multicolumn{3}{|c|}{ VOC SVOC An Metals I Lab Rau }} & & & DOE 1993b & \\
\hline 229 & $100-\mathrm{F}$ & \multicolumn{2}{|c|}{ 116-F-4 shallow borehole } & & & & & & & & & & & DOE 1993b & \\
\hline 230 & $100-\mathrm{F}$ & \multicolumn{2}{|c|}{ 116-F-6 shallow borehole } & & & & & & \multicolumn{3}{|c|}{ VOC, SVOC, An, Metals, Lab Rad } & & & DOE 1993b & \\
\hline 231 & $100-\mathrm{F}$ & \multicolumn{2}{|c|}{ 116-F9 shallow borehole } & & & & & & \multicolumn{3}{|c|}{ VOC, SVOC, An, Metals, Lab Rad } & & & DOE 1993b & \\
\hline 232 & $100-\mathrm{F}$ & 116-F9D test pit & & & & & & & \multicolumn{3}{|c|}{ VOC, SVOC, An, Metals, Lab Rad } & & & DOE 1993b & \\
\hline 233 & $100-\mathrm{F}$ & 116-F14 shallow & borehole & & & & & & VOC, SVOC, & An, Metals, Le & b $\mathrm{Rad}$ & & & DOE 1993b & \\
\hline 234 & $100-\mathrm{H}$ & $199-\mathrm{H} 3-1$ & A4610 & D & GG, NT, SG, TP & & & & & & & & Y & DOE 1993b & Decommissioned \\
\hline 235 & $100-\mathrm{H}$ & 199-H3-2A & A4611 & $\mathrm{D}, \mathrm{G}$ & DN, GG, NT & & & $Y$ & & & & & $\mathrm{Y}$ & $\begin{array}{l}\text { Liikala et al. } \\
1988\end{array}$ & \\
\hline 236 & $100-\mathrm{H}$ & 199-H3-2B & A4612 & D, G & DN, GG, NT & & & $Y$ & & & & & $\mathrm{Y}$ & $\begin{array}{l}\text { Liikala et al. } \\
1988\end{array}$ & \\
\hline 237 & $100-\mathrm{H}$ & $199-\mathrm{H} 3-2 \mathrm{C}$ & A4613 & $\mathrm{D}, \mathrm{G}$ & CP, DN, GG, NT & & & $Y$ & & Bden & & & Y & $\begin{array}{l}\text { Liikala et al. } \\
1988\end{array}$ & \\
\hline 238 & $100-\mathrm{H}$ & 199-H3-3 & B2778 & G & & $Y$ & & & & & & & & $\begin{array}{l}\text { Liikala et al. } \\
1988\end{array}$ & \\
\hline 239 & $100-\mathrm{H}$ & $199-\mathrm{H} 3-4$ & B2779 & G & & $Y$ & & & & & & & & Myers et al. 1996 & \\
\hline
\end{tabular}




\begin{tabular}{|c|c|c|c|c|c|c|c|c|c|c|c|c|c|c|c|}
\hline Sortindex & Area & Well Name & WellID & Log Type & GPX Log Runs & Sieved & $\mathrm{CaCO} 3$ & Moisture & $\begin{array}{l}\text { Chemical } \\
\text { Properties }\end{array}$ & $\begin{array}{c}\text { Physical } \\
\text { Properties }\end{array}$ & $\begin{array}{c}\text { Min } \\
\text { Properties } \\
\end{array}$ & $\begin{array}{l}\text { Geochron } \\
\text { Properties }\end{array}$ & Archived & Source & Comments \\
\hline & $100-\mathrm{H}$ & 199-H3-5 & B2780 & G & & $Y$ & & & & & & & & Myers et al. 1996 & \\
\hline 241 & $100-\mathrm{H}$ & 199-H4-1 & A5685 & D & GG, TP & & & & & & & & $\mathrm{Y}$ & HWIS & Abandoned \\
\hline 242 & $100-\mathrm{H}$ & $199-\mathrm{H} 4-10$ & A4614 & D, G & DN, GG, NT & & & $Y$ & & & & & $Y$ & $\begin{array}{l}\text { Liikala et al. } \\
1988\end{array}$ & \\
\hline 243 & $100-\mathrm{H}$ & 199-H4-11 & A4615 & D, G & DN, GG, NT, SG & & & $Y$ & & & & & $Y$ & $\begin{array}{l}\text { Liikala et al. } \\
\text { 1988; DOE } \\
\text { 1993b }\end{array}$ & \\
\hline 244 & $100-\mathrm{H}$ & 199-H4-12A & A4616 & D, G & DN, GG, NT & & & $Y$ & & & & & $Y$ & $\begin{array}{l}\text { Liikala et al. } \\
1988\end{array}$ & \\
\hline 245 & $100-\mathrm{H}$ & 199-H4-12B & A4617 & D, G & DN, GG, NT & & & $Y$ & & & & & $Y$ & $\begin{array}{l}\text { Liikala et al. } \\
1988\end{array}$ & \\
\hline 246 & $100-H$ & $199-\mathrm{H} 4-12 \mathrm{C}$ & A4618 & D, G & CP, DN, GG, NT & & & $Y$ & & Bden & & & $Y$ & $\begin{array}{l}\text { Liikala et al. } \\
1988\end{array}$ & \\
\hline 247 & $100-\mathrm{H}$ & 199-H4-13 & A4619 & D, G & $\mathrm{DN}, \mathrm{GG}, \mathrm{NT}, \mathrm{SG}$ & & & $Y$ & & & & & $Y$ & $\begin{array}{l}\text { Liikala et al. } \\
\text { 1988; DOE } \\
\text { 1993b }\end{array}$ & \\
\hline 248 & $100-\mathrm{H}$ & 199-H4-14 & A4620 & D, G & DN, GG, NT & & & $Y$ & & & & & $Y$ & \begin{tabular}{|l|} 
Likkala et al. \\
1988
\end{tabular} & \\
\hline 249 & $100-\mathrm{H}$ & 199-H4-15A & A4621 & D, G & DN, GG, NT & & & $Y$ & & & & & $Y$ & $\begin{array}{l}\text { Liikala et al. } \\
1988\end{array}$ & \\
\hline 250 & $100-\mathrm{H}$ & 199-H4-15B & A4622 & D, G & DN, GG, NT & & & $Y$ & & & & & $Y$ & $\begin{array}{l}\text { Liikala et al. } \\
1988\end{array}$ & \\
\hline 251 & $100-\mathrm{H}$ & $199-\mathrm{H} 4-15 \mathrm{C}$ & A5689 & D, G & CP, DN, GG, NT, TP & & & $Y$ & XRF (basalt) & Bden & & & $Y$ & $\begin{array}{l}\text { Liikala et al. } \\
1988\end{array}$ & Top of basalt@314' \\
\hline 252 & $100-\mathrm{H}$ & $199-\mathrm{H} 4-16$ & A4626 & D, G & $\mathrm{DN}, \mathrm{GG}, \mathrm{NT}, \mathrm{SG}$ & & & $Y$ & & & & & $Y$ & $\begin{array}{l}\text { Liikala et al. } \\
\text { 1988; DOE } \\
\text { 1993b }\end{array}$ & \\
\hline 253 & $100-\mathrm{H}$ & 199-H4-17 & A4627 & $\mathrm{D}, \mathrm{G}$ & $\mathrm{DN}, \mathrm{GG}, \mathrm{NT}$ & & & $Y$ & & & & & $Y$ & \begin{tabular}{|l|} 
Likikala et al. \\
1988; DOE \\
1993b
\end{tabular} & \\
\hline 254 & $100-\mathrm{H}$ & $199-\mathrm{H} 4-18$ & A4628 & D, G & $\mathrm{DN}, \mathrm{GG}, \mathrm{NT}, \mathrm{SG}$ & & & $Y$ & & & & & $Y$ & $\begin{array}{l}\text { Liikala et al. } \\
1988\end{array}$ & \\
\hline 255 & $100-H$ & $199-\mathrm{H} 4-2$ & A5686 & D & $\begin{array}{l}\text { CP, DN, GG, MG, NT, } \\
\text { SG, SN, TP }\end{array}$ & $\begin{array}{l}\text { Virtual } \\
\text { Library }\end{array}$ & $\begin{array}{l}\text { Virtual } \\
\text { Library }\end{array}$ & & XRF (basalt) & & & & $Y$ & $\begin{array}{l}\text { Liikala et al. } \\
1988\end{array}$ & Top of basalt @381 ft \\
\hline 256 & $100-\mathrm{H}$ & $199-\mathrm{H} 4-3$ & A4629 & D & GG, NT, SG & & & & & & & & $Y$ & DOE 1993b & \\
\hline 257 & $100-\mathrm{H}$ & $199-\mathrm{H} 4-4$ & A4630 & $\mathrm{D}$ & GG, NT & & & & & & & & & HWIS & \\
\hline 258 & $100-\mathrm{H}$ & 199-H4-45 & A4631 & G & $G G, S G$ & $Y$ & & $Y$ & & & & & Y & $\begin{array}{l}\text { Peterson et al. } \\
\text { 1996; DOE } \\
\text { 1993b; Khaleel } \\
\text { 1999 }\end{array}$ & \\
\hline 259 & $100-\mathrm{H}$ & 199-H4-46 & A4632 & D, G & SG & $Y$ & & & & & & & $Y$ & $\begin{array}{l}\text { Peterson et al. } \\
\text { 1996; DOE } \\
\text { 1993b; Khaleel } \\
\text { 1999 }\end{array}$ & \\
\hline 260 & $100-\mathrm{H}$ & 199-H4-47 & A4633 & D, G & $\mathrm{GG}, \mathrm{SG}$ & & & & & & & & Y & $\begin{array}{l}\text { Peterson et al. } \\
\text { 1996; DOE } \\
\text { 1993b } \\
\end{array}$ & \\
\hline 261 & $100-\mathrm{H}$ & $199-\mathrm{H} 4-48$ & A4634 & $\mathrm{D}, \mathrm{G}$ & GG, SG & & & & & & & & $Y$ & $\begin{array}{l}\text { Peterson et al. } \\
\text { 1996; DOE } \\
\text { 1993b }\end{array}$ & \\
\hline 262 & $100-H$ & 199-H4-49 & A4635 & $\mathrm{D}, \mathrm{G}$ & GG, SG & & & & & & & & $Y$ & $\begin{array}{l}\text { Peterson et al. } \\
\text { 1996; DOE } \\
\text { 1993b } \\
\end{array}$ & \\
\hline
\end{tabular}




\begin{tabular}{|c|c|c|c|c|c|c|c|c|c|c|c|c|c|c|c|}
\hline Sortlndex & Area & Well Name & WelliD & Log Type & GPX Log Runs & Sieved & $\mathrm{CaCO} 3$ & Moisture & \begin{tabular}{|l|} 
Chemical \\
Properties \\
\end{tabular} & $\begin{array}{l}\text { Physical } \\
\text { Properties }\end{array}$ & $\begin{array}{c}\text { Min } \\
\text { Properties } \\
\end{array}$ & $\begin{array}{l}\text { Geochron } \\
\text { Properties }\end{array}$ & Archived & Source & Comments \\
\hline $\begin{array}{l}263 \\
264\end{array}$ & $\frac{100-H}{400-H}$ & $\frac{199-\text {-H4-5 }}{199-\text { - }}$ & $\frac{14636}{A 5723}$ & $\frac{D}{G}$ & GG, NT & & & & & & & & & $\begin{array}{l}\text { HWIS } \\
\text { HWIS }\end{array}$ & \\
\hline 265 & $100-\mathrm{H}$ & 199-H4-58 & A5724 & G & SG & & & & & & & & Y & HWIS & Vadose-zone hole \\
\hline 266 & $100-\mathrm{H}$ & 199-H4-59 & A5725 & $\mathrm{D}, \mathrm{G}$, Well summary & SG & & & & & & & & YY & HWIS & Vadose-zone hole \\
\hline 267 & $100-\mathrm{H}$ & 199-H4-6 & A4637 & D & GG, NT & & & & & & & & & HWIS & \\
\hline 268 & $100-\mathrm{H}$ & $199-\mathrm{H} 4-60$ & A5726 & G & SG & & & & & & & & YY & HWIS & Vadose-zone hole \\
\hline 269 & $100-H$ & 199-H4-61 & A5727 & G & $\mathrm{GG}, \mathrm{SG}$ & & & & & & & & Y & HWIS & Vadose-zone hole \\
\hline 270 & $100-\mathrm{H}$ & 199-H4-62 & A5728 & D, G, As-built & GG & & & & & & & & Y & HWIS & Vadose-zone hole \\
\hline 271 & $100-H$ & 199-H4-63 & B2776 & G & & $\bar{Y}$ & & & & & & & & Myers et al. 1996 & \\
\hline 272 & $100-H$ & 199-H4-64 & B2777 & G & & $\mathrm{Y}$ & & & & & & & & Myers et al. 1996 & \\
\hline 273 & $100-\mathrm{H}$ & $199-\mathrm{H} 4-65$ & B8759 & G & & $\bar{Y}$ & & & & & & & & HWIS & \\
\hline 274 & $100-\mathrm{H}$ & 199-H4-7 & A4638 & $\mathrm{D}, \mathrm{G}$ & $\mathrm{DN}, \mathrm{GG}, \mathrm{NT}$ & & & Y & & & & & $Y$ & \begin{tabular}{|l|} 
Liikala et al. \\
1988
\end{tabular} & \\
\hline 275 & $100-\mathrm{H}$ & 199-H4-8 & A4639 & $\mathrm{D}, \mathrm{G}$ & $\mathrm{DN}, \mathrm{GG}, \mathrm{NT}$ & & & Y & & & & & $Y$ & Liikala et al. & \\
\hline 276 & $100-H$ & 199-H4-9 & A4640 & $\mathrm{D}, \mathrm{G}$ & $\mathrm{DN}, \mathrm{GG}, \mathrm{NT}$ & & & $\mathrm{Y}$ & & & & & Y & $\begin{array}{l}\text { Likikala et al. } \\
\text { tikaga }\end{array}$ & \\
\hline 277 & $100-\mathrm{H}$ & 199-H5-10 & A9982 & As-built & & Y & & & & & & & & $\begin{array}{l}\text { Fruchter et al. } \\
\text { 1996; HWIS }\end{array}$ & \\
\hline 278 & $100-\mathrm{H}$ & 199-H5-11 & B2426 & G & & $\bar{Y}$ & & Y & & $\begin{array}{l}\text { Pden, Bden, } \\
\text { Por }\end{array}$ & & & & $\begin{array}{l}\text { Vermeul et al. } \\
\text { 1995; Fruchter et } \\
\text { al. } 1996\end{array}$ & Microbiology \\
\hline 279 & $100-H$ & $199-\mathrm{H} 5-12$ & B2427 & As-built & & Y & & & & & & & & $\begin{array}{l}\text { Fruchter et al. } \\
\text { 1996; HWIS }\end{array}$ & \\
\hline 280 & $100-\mathrm{H}$ & 199-H5-13 & B2433 & As-built & & Y & & & & & & & & $\begin{array}{l}\text { Fruchter et al. } \\
\text { 1996; HWIS }\end{array}$ & \\
\hline 281 & $100-\mathrm{H}$ & 199-H5-14 & B2434 & As-built & & $\mathrm{Y}$ & & & & & & & & $\begin{array}{l}\text { Fruchter et al. } \\
\text { 1996; HWIS }\end{array}$ & \\
\hline 282 & $100-H$ & 199-H5-15 & B2629 & As-built & & Y & & & & & & & & $\begin{array}{l}\text { Fruchter et al. } \\
\text { 1996; HWIS }\end{array}$ & \\
\hline 283 & $3100-\mathrm{H}$ & 199-H5-1A & A4641 & G & & & & $\mathrm{Y}$ & TFe/Fe2 & & & & Y & $\begin{array}{l}\text { Peterson et al. } \\
\text { 1996; DOE } \\
\text { 1993b }\end{array}$ & \\
\hline 284 & $100-\mathrm{H}$ & 199-H5-1B & A9809 & Well Summary & GG & & & Y & & & & & & $\begin{array}{l}\text { Peterson et al. } \\
\text { 1996; DOE } \\
\text { 1993b; HWIS }\end{array}$ & \\
\hline
\end{tabular}




\begin{tabular}{|c|c|c|c|c|c|c|c|c|c|c|c|c|c|c|c|}
\hline Sortindex & Area & Well Name & WellID & Log Type & GPX Log Runs & Sieved & $\mathrm{CaCO} 3$ & Moisture & $\begin{array}{l}\text { Chemical } \\
\text { Properties }\end{array}$ & $\begin{array}{c}\text { Physical } \\
\text { Properties }\end{array}$ & \begin{tabular}{|c|} 
Min \\
Properties \\
\end{tabular} & $\begin{array}{l}\text { Geochron } \\
\text { Properties }\end{array}$ & Archived & Source & Comments \\
\hline 285 & 5 $100-\mathrm{H}$ & 199-H5-2 & A9892 & G & & Y & & $Y$ & & $\begin{array}{l}\text { Pden, Bden, } \\
\text { Por }\end{array}$ & & & & $\begin{array}{l}\text { Vermeul et al. } \\
\text { 1995; Fruchter et } \\
\text { al. 1996 }\end{array}$ & $\begin{array}{l}\text { ISRM injection well; } \\
\text { Microbiology }\end{array}$ \\
\hline 286 & $100-\mathrm{H}$ & $199-\mathrm{H} 5-3$ & & G & & $Y$ & & $Y$ & & $\begin{array}{l}\text { Pden, Bden, } \\
\text { Por, Hydrom }\end{array}$ & & & & $\begin{array}{l}\text { Vermeul et al. } \\
\text { 1995; Fruchter et } \\
\text { al. } 1996\end{array}$ & Piezometer well; Microbiology \\
\hline 287 & 7 & $199-\mathrm{H} 5-4$ & & G & & Y & & $\mathrm{Y}$ & & Pden & & & & \begin{tabular}{|l|} 
Vermeul et al. \\
1995; Fruchter et \\
al. 1996
\end{tabular} & Piezometer well; Microbiology \\
\hline 288 & 3 & $199-\mathrm{H} 5-5$ & & As-built & & Y & & Y & & $\begin{array}{l}\text { Pden, Bden, } \\
\text { Por }\end{array}$ & & & & $\begin{array}{l}\text { Vermeul et al. } \\
\text { 1995; Fruchter et } \\
\text { al. 1996; HWIS }\end{array}$ & Piezometer well; Microbiology \\
\hline 289 & $100-\mathrm{H}$ & $199-\mathrm{H} 5-6$ & A9979 & As-built & & $Y$ & & & & & & & & $\begin{array}{l}\text { Fruchter et al. } \\
\text { 1996; HWIS }\end{array}$ & \\
\hline 290 & $100-\mathrm{H}$ & $199-\mathrm{H} 5-7$ & A9980 & As-built & & $Y$ & & & & & & & & $\begin{array}{l}\text { Fruchter et al. } \\
\text { 1996; HWIS }\end{array}$ & \\
\hline 291 & $100-\mathrm{H}$ & 199-H5-8 & B2425 & G & & Y & & $Y$ & & $\begin{array}{l}\text { Pden, Bden, } \\
\text { Por }\end{array}$ & & & & $\begin{array}{l}\text { Vermeul et al. } \\
\text { 1995; Fruchter et } \\
\text { al. } 1996\end{array}$ & Microbiology \\
\hline 292 & $100-\mathrm{H}$ & $199-\mathrm{H} 5-9$ & A9981 & As-built & & Y & & & & & & & & $\begin{array}{l}\text { Fruchter et al. } \\
\text { 1996; HWIS }\end{array}$ & \\
\hline 293 & $100-\mathrm{H}$ & $199-\mathrm{H6}-1$ & A4642 & G & GG, SG & Y & & & & & & & Y & $\begin{array}{l}\text { Peterson et al. } \\
\text { 1996; DOE } \\
\text { 1993b }\end{array}$ & \\
\hline 294 & $100-\mathrm{H}$ & C3048 & C3048 & G & & & & $Y$ & & & & & & Kious 2001 & \\
\hline 295 & $100-\mathrm{H}$ & Redox-core1 & & & & & & & & & & & & & \\
\hline 296 & $100-\mathrm{H}$ & Redox-core2 & & & & & & & & & & & & & \\
\hline 297 & $7100-\mathrm{H}$ & Redox-core3 & & & & & & & & & & & & & \\
\hline 298 & $100-\mathrm{H}$ & Redox-core4 & & & & & & & & & & & & & \\
\hline 299 & $100-\mathrm{H}$ & Redox-core5 & & & & & & & & & & & & & \\
\hline 300 & $100-\mathrm{H}$ & Redox-core6 & & & & & & & & & & & & & \\
\hline 301 & $100-\mathrm{H}$ & Redox-core7 & & & & & & & & & & & & $\begin{array}{l}\text { Fruchter et al. } \\
1996\end{array}$ & Abandoned corehole \\
\hline 302 & $100-\mathrm{H}$ & Redox-core8 & & & & & & & & & & & & & \\
\hline 303 & $3100-\mathrm{H}$ & 199-HR-46 & & & GG & & & & & & & & & & \\
\hline 304 & $4100-\mathrm{H}$ & 199-H5-1 & & & GG, SG & & & & & & & & & & \\
\hline 305 & $100-K$ & 199-K-1 & A5729 & $\mathrm{D}$ & & & & & & & & & & & \\
\hline 306 & | $100-K$ & $199-K-10$ & A5738 & D, As-built & & & & & & & & & & HWIS & \\
\hline 307 & $700-K$ & 199-K-106A & A9842 & D, G, Well summary & SG & $Y$ & Y & $Y$ & $\begin{array}{l}\text { Field Rad, } \\
\text { Field VOA }\end{array}$ & $\begin{array}{l}\text { Pden, Bden, } \\
\text { Por }\end{array}$ & XRD & & & HWIS & Aquifer Slug Test \\
\hline 308 & $100-K$ & 199-K-107A & A9843 & D, G, Well summary & GG, SG & $\mathrm{Y}$ & Y & $\mathrm{Y}$ & $\begin{array}{l}\text { Field Rad, } \\
\text { Field VOA }\end{array}$ & $\begin{array}{l}\text { Pden, Bden, } \\
\text { Por }\end{array}$ & XRD & & & HWIS & Aquifer Slug Test \\
\hline 309 & $900-K$ & $199-K-108 \mathrm{~A}$ & A9844 & Well summary & SG & & & & & & & & & HWIS & \\
\hline 310 & $100-K$ & 199-K-109A & A9828 & D, G, Well summary & GG, SG & $\mathrm{Y}$ & Y & $Y$ & $\begin{array}{l}\text { Field Rad, } \\
\text { Field VOA }\end{array}$ & $\begin{array}{l}\text { Pden, Bden, } \\
\text { Por }\end{array}$ & XRD & & & HWIS & Aquifer Slug Test \\
\hline 311 & $1100-K$ & $199-\mathrm{K}-11$ & A4643 & D, As-built & GG, OT, TP & & & & & & & & & HWIS & \\
\hline
\end{tabular}




\begin{tabular}{|c|c|c|c|c|c|c|c|c|c|c|c|c|c|c|c|}
\hline Sortindex & Area & Well Name & WellID & Log Type & GPX Log Runs & Sieved & $\mathrm{CaCO} 3$ & Moisture & $\begin{array}{l}\text { Chemical } \\
\text { Properties }\end{array}$ & $\begin{array}{c}\text { Physical } \\
\text { Properties }\end{array}$ & \begin{tabular}{|c|} 
Min \\
Properties \\
\end{tabular} & $\begin{array}{l}\text { Geochron } \\
\text { Properties }\end{array}$ & Archived & Source & Comments \\
\hline 312 & $100-K$ & 199-K-110A & A9829 & D, G, Well summary & GG, SG & $\mathrm{Y}$ & Y & Y & $\begin{array}{l}\text { Field Rad, } \\
\text { Field VOA }\end{array}$ & $\begin{array}{l}\text { Pden, Bden, } \\
\text { Por }\end{array}$ & \begin{tabular}{|l|} 
XRD \\
\end{tabular} & & & HWIS & Aquifer Slug Test \\
\hline 313 & $100-K$ & 199-K-111A & A9830 & D, G, Well summary & GG, SG & Y & $\mathrm{Y}$ & $\mathrm{Y}$ & $\begin{array}{l}\text { Field Rad, } \\
\text { Field VOA }\end{array}$ & $\begin{array}{l}\text { Pden, Bden, } \\
\text { Por } \\
\end{array}$ & XRD & & & HWIS & \\
\hline 314 & $100-K$ & 199-K-112A & B2799 & G, Well summary & & $\mathrm{Y}$ & & & & & & & & HWIS & CH2 Library \\
\hline 315 & $100-K$ & 199-K-113A & B2800 & G, Well summary & & $\mathrm{Y}$ & & & & & & & & HWIS & \\
\hline 316 & $100-K$ & 199-K-114A & B2801 & G, Well summary & & $\mathrm{Y}$ & & & & & & & & HWIS & \\
\hline 317 & $100-K$ & 199-K-115A & B2802 & G, Well summary & & $\mathrm{Y}$ & & & & & & & & HWIS & \\
\hline 318 & $100-K$ & 199-K-116A & B2803 & G, Well summary & & $Y$ & & & & & & & & HWIS & \\
\hline 319 & $100-K$ & 199-K-117A & B2804 & G, Well summary & & $Y$ & & & & & & & & HWIS & \\
\hline 320 & $100-K$ & 199-K-118A & B2805 & G, Well summary & & $\mathrm{Y}$ & & & & & & & & HWIS & \\
\hline 321 & $100-K$ & 199-K-119A & B2806 & G, Well summary & & $\mathrm{Y}$ & & & & & & & & HWIS & \\
\hline 322 & $100-K$ & $199-K-12$ & A5739 & D, As-built & OT, TP & & & & & & & & & HWIS & \\
\hline 323 & $100-K$ & $199-\mathrm{K}-120 \mathrm{~A}$ & B2807 & G, Well summary & & $Y$ & & & & & & & & HWIS & \\
\hline 324 & $100-K$ & 199-K-121A & B2808 & G, Well summary & & $\mathrm{Y}$ & & & & & & & & HWIS & \\
\hline 325 & $100-K$ & 199-K-122A & B2809 & G, Well summary & & $\mathrm{Y}$ & & & & & & & & HWIS & \\
\hline 326 & $100-K$ & 199-K-123A & B2810 & G, Well summary & & $Y$ & & & & & & & & HWIS & \\
\hline 327 & $100-K$ & $199-\mathrm{K}-124 \mathrm{~A}$ & B2811 & G, Well summary & & $Y$ & & & & & & & & HWIS & \\
\hline 328 & $100-K$ & 199-K-125A & B8559 & Well summary & & & & & & & & & & HWIS & \\
\hline 329 & $100-K$ & 199-K-126A & & Well summary & & & & & & & & & & HWIS & \\
\hline 330 & $100-K$ & $199-K-127$ & C3662 & G & & $Y$ & & & & & & & & Sump 2002b & \\
\hline 331 & $100-K$ & $199-K-128$ & C3663 & G & & $Y$ & & & & & & & & Sump 2002b & \\
\hline 332 & $100-K$ & 199-K-129 & C4117 & G & & & & & & & & & & HWIS & Extraction well \\
\hline 333 & $100-K$ & $199-K-13$ & A4644 & D, As-built & OT, TP & & & & & & & & & HWIS & \\
\hline 334 & $100-K$ & $199-K-130$ & C4120 & G & & & & & & & & & & HWIS & Extraction well \\
\hline 335 & $100-K$ & 199-K-14 & A5740 & D, As-built & & & & & & & & & & HWIS & aka 105-KW-1 \\
\hline 336 & $100-K$ & 199-K-15 & A4645 & D, As-built & & & & & & & & & & HWIS & \\
\hline 337 & $100-K$ & $199-\mathrm{K}-16$ & A4646 & D, As-built & & & & & & & & & & HWIS & \\
\hline 338 & $100-K$ & 199-K-17 & A5741 & D, As-built & & & & & & & & & & HWIS & \\
\hline 339 & $100-K$ & $199-K-18$ & A4647 & D, As-built & $\mathrm{DN}, \mathrm{GG}, \mathrm{NT}, \mathrm{OT}, \mathrm{TP}$ & & & & & & & & & HWIS & \\
\hline 340 & $100-K$ & $199-\mathrm{K}-19$ & A4648 & D, As-built & $\mathrm{GG}, \mathrm{OT}, \mathrm{TP}$ & & & & & & & & & HWIS & \\
\hline 341 & $100-K$ & $199-K-2$ & A5730 & D & & & & & & & & & & & \\
\hline 342 & $100-K$ & 199-K-20 & A4649 & D, As-built & OT, TP & & & & & & & & & HWIS & \\
\hline 343 & $100-K$ & $199-K-21$ & A4650 & D, As-built & OT, TP & & & & & & & & & HWIS & \\
\hline 344 & $100-K$ & $199-\mathrm{K}-22$ & A4651 & D, As-built & OT, TP & & & & & & & & & HWIS & \\
\hline 345 & $100-K$ & $199-\mathrm{K}-23$ & A4652 & D, As-built & OT, TP & & & & & & & & & HWIS & \\
\hline 346 & $100-K$ & $199-K-24$ & A5742 & D, As-built & OT, TP & & & & & & & & & HWIS & \\
\hline 347 & $100-K$ & $199-K-25$ & A5743 & D, As-built & GG, MO, OT, TP & & & & & & & & & HWIS & \\
\hline 348 & $100-K$ & $199-K-26$ & A5744 & D & & & & & & & & & & & \\
\hline 349 & $100-K$ & $199-\mathrm{K}-27$ & A4653 & $\mathrm{D}$, As-built & $\mathrm{DN}, \mathrm{GG}, \mathrm{NT}, \mathrm{OT}, \mathrm{TP}$ & & & & & & & & & HWIS & \\
\hline 350 & $100-K$ & $199-\mathrm{K}-28$ & A4654 & D, As-built & DN, GG, NT, OT, TP & & & & & & & & & HWIS & \\
\hline 351 & $100-\mathrm{K}$ & $199-K-29$ & A5480 & D, As-built & $\mathrm{DN}, \mathrm{GG}, \mathrm{NT}, \mathrm{OT}, \mathrm{TP}$ & & & & & & & & & HWIS & \\
\hline 352 & $100-K$ & $199-K-3$ & A5731 & D & & & & & & & & & & & \\
\hline 353 & $100-K$ & $199-\mathrm{K}-30$ & A4655 & D, As-built & DN, GG, NT, OT, TP & & & & & & & & & HWIS & \\
\hline 354 & $100-K$ & $199-\mathrm{K}-31$ & A4656 & D, As-built & & & & & & & & & & HWIS & \\
\hline 355 & $100-K$ & 199-K-32A & A4657 & Well summary & GG, SG & & & & & & & & & HWIS & Aquifer test (slug test) \\
\hline 356 & $100-K$ & 199-K-32B & A4658 & Well summary & GG, SG & & & & & & & & & HWIS & \\
\hline 357 & $100-K$ & $199-K-33$ & A4659 & Well summary & GG, SG & Y & & & & & & & & $\begin{array}{l}\text { see R. Khaleel or } \\
\text { G. Freeman for } \\
\text { sieve data }\end{array}$ & Aquifer test (slug test) \\
\hline
\end{tabular}




\begin{tabular}{|c|c|c|c|c|c|c|c|c|c|c|c|c|c|c|c|}
\hline SortIndex & Area & Well Name & WellID & Log Type & GPX Log Runs & Sieved & $\mathrm{CaCO} 3$ & Moisture & $\begin{array}{c}\text { Chemical } \\
\text { Properties }\end{array}$ & $\begin{array}{c}\text { Physical } \\
\text { Properties }\end{array}$ & $\begin{array}{c}\text { Min } \\
\text { Properties } \\
\end{array}$ & $\begin{array}{l}\text { Geochron } \\
\text { Properties }\end{array}$ & Archived & Source & Comments \\
\hline & $100-K$ & 199-K-34 & A4660 & D, G, Well summary & GG, SG & & & & & & & & & & Aquifer test (slug test) \\
\hline 359 & $100-K$ & $199-K-35$ & A4661 & $\mathrm{D}, \mathrm{G}$, Well summary & GG, SG & $Y$ & & & & & & & & $\begin{array}{l}\text { see R. Khaleel or } \\
\text { G. Freeman for } \\
\text { sieve data }\end{array}$ & Aquifer test (slug test) \\
\hline 360 & $100-K$ & $199-K-36$ & A4662 & D, G, Well summary & GG, SG & & & & & & & & & & Aquifer test (slug test) \\
\hline 361 & $100-K$ & $199-K-37$ & A4663 & $\mathrm{D}, \mathrm{G}$, Well summary & GG, SG & $Y$ & & & & & & & & $\begin{array}{l}\text { see R. Khaleel or } \\
\text { G. Freeman for } \\
\text { sieve data }\end{array}$ & Aquifer test (slug test) \\
\hline 362 & $100-K$ & $199-K-38$ & A5745 & D, G, Well summary & SG & & & & & & & & & & \\
\hline 363 & $100-K$ & $199-\mathrm{K}-39$ & A5746 & $\mathrm{D}, \mathrm{G}$, Well summary & SG & & & & & & & & & & \\
\hline 364 & $100-K$ & $199-\mathrm{K}-4$ & A5732 & $\mathrm{D}$ & & & & & & & & & & & \\
\hline 365 & $100-K$ & $199-\mathrm{K}-40$ & A5747 & D, G, Well summary & GG & & & & & & & & & & \\
\hline 366 & $100-K$ & $199-K-41$ & A5748 & D, G, Well summary & SG & & & & & & & & & & \\
\hline 367 & $100-K$ & $199-\mathrm{K}-5$ & A5733 & D & & & & & & & & & & & \\
\hline 368 & $100-K$ & $199-\mathrm{K}-6$ & A5734 & D & & & & & & & & & & & \\
\hline 369 & $100-K$ & $199-\mathrm{K}-7$ & A5735 & D & & & & & & & & & & & \\
\hline 370 & $100-K$ & 199-K-8 & A5736 & D & & & & & & & & & & & \\
\hline 371 & $100-K$ & 199-K-9 & A5737 & D & & & & & & & & & & & \\
\hline 372 & $100-\mathrm{N}$ & $199-\mathrm{N}-1$ & A5813 & $\mathrm{D}$ & TP & & & & & & & & $\mathrm{Y}$ & HWIS & \\
\hline 373 & $100-\mathrm{N}$ & $199-\mathrm{N}-10$ & A5823 & D, G, Well summary & GG, NT, TP & & & & & & & & & HWIS & \\
\hline 374 & $100-\mathrm{N}$ & 199-N-103A & A9988 & G & SG & $Y$ & & & & & & & & HWIS & \\
\hline 375 & $100-\mathrm{N}$ & $199-\mathrm{N}-104$ & & G & & $\mathrm{Y}$ & & & & & & & & & \\
\hline 376 & $100-\mathrm{N}$ & 199-N-104A & A9989 & D, G, As-built & SG & & & & & & & & & HWIS & \\
\hline 377 & $100-\mathrm{N}$ & $199-\mathrm{N}-105$ & & G & & $Y$ & & & & & & & & & \\
\hline 378 & $100-\mathrm{N}$ & 199-N-105A & B2408 & D, G, As-built & SG & & & & & & & & & HWIS & \\
\hline 379 & $100-\mathrm{N}$ & $199-\mathrm{N}-106 \mathrm{~A}$ & B2538 & G, As-built & SG & & & & & & & & & HWIS & \\
\hline 380 & $100-\mathrm{N}$ & $199-\mathrm{N}-119$ & C4471 & G, As-built & SG & & & & & & & & & HWIS & \\
\hline 381 & $100-\mathrm{N}$ & $199-\mathrm{N}-12$ & A5824 & D & TP & & & & & & & & $\mathrm{Y}$ & HWIS & \\
\hline 382 & $100-\mathrm{N}$ & $199-\mathrm{N}-120$ & C4472 & G, As-built & SG & & & & & & & & & HWIS & \\
\hline 383 & $100-\mathrm{N}$ & $199-\mathrm{N}-121$ & C4473 & G, As-built & SG & & & & & & & & & HWIS & \\
\hline 384 & $100-\mathrm{N}$ & $199-\mathrm{N}-13$ & A5825 & & GG, NT, TP & & & & & & & & & & \\
\hline 385 & $100-\mathrm{N}$ & $199-\mathrm{N}-14$ & A4664 & D & SG, TP & & & & & & & & $Y$ & HWIS & \\
\hline 386 & $100-\mathrm{N}$ & $199-\mathrm{N}-15$ & A5826 & D & TP & & & & & & & & $\mathrm{Y}$ & HWIS & \\
\hline 387 & $100-\mathrm{N}$ & $199-\mathrm{N}-16$ & A4665 & D & GG, SG & & & & & & & & Y & HWIS & Drilled per spec $\mathrm{H}-517-\mathrm{C} 1$ \\
\hline 388 & $100-\mathrm{N}$ & $199-\mathrm{N}-17$ & A4666 & $\mathrm{D}$ & GG, SG & & & & & & & & $Y$ & HWIS & Drilled per spec $\mathrm{H}-517-\mathrm{C} 1$ \\
\hline 389 & $100-\mathrm{N}$ & $199-\mathrm{N}-18$ & A4667 & D & GG, SG & & & & & & & & $Y$ & HWIS & Drilled per spec $\mathrm{H}-517-\mathrm{C} 1$ \\
\hline 390 & $100-\mathrm{N}$ & $199-\mathrm{N}-19$ & A4668 & D & GG, SG & & & & & & & & $Y$ & HWIS & Drilled per spec $\mathrm{H}-517-\mathrm{C} 1$ \\
\hline
\end{tabular}




\begin{tabular}{|c|c|c|c|c|c|c|c|c|c|c|c|c|c|c|c|}
\hline SortIndex & Area & Well Name & WellID & Log Type & GPX Log Runs & Sieved & $\mathrm{CaCO} 3$ & Moisture & $\begin{array}{l}\text { Chemical } \\
\text { Properties }\end{array}$ & $\begin{array}{l}\text { Physical } \\
\text { Properties }\end{array}$ & \begin{tabular}{|c|} 
Min \\
Properties
\end{tabular} & $\begin{array}{l}\text { Geochron } \\
\text { Properties }\end{array}$ & Archived & Source & Comments \\
\hline 391 & $100-\mathrm{N}$ & $199-\mathrm{N}-2$ & A4669 & $\mathrm{D}$ & TP & & & & & & & & $\mathrm{Y}$ & HWIS & \\
\hline 392 & $100-\mathrm{N}$ & $199-\mathrm{N}-20$ & A4670 & D & GG, SG & & & & & & & & $Y$ & HWIS & Drilled per spec $\mathrm{H}-517-\mathrm{C} 1$ \\
\hline 393 & $100-\mathrm{N}$ & $199-\mathrm{N}-21$ & A4671 & D, As-built & GG, SG & & & & & & & & & HWIS & \\
\hline 394 & $100-\mathrm{N}$ & $199-\mathrm{N}-21 \mathrm{~A}$ & & D & & & & & & & & & $\mathrm{Y}$ & & Drilled per spec $\mathrm{H}-517-\mathrm{C} 1$ \\
\hline 395 & $100-N$ & $199-\mathrm{N}-22$ & A5827 & D & GG, SG & & & & & & & & $\mathrm{Y}$ & HWIS & Drilled per spec $\mathrm{H}-517-\mathrm{C} 1$ \\
\hline 396 & $100-\mathrm{N}$ & $199-\mathrm{N}-23$ & A4672 & D & & & & & & & & & $Y$ & HWIS & Drilled per spec $\mathrm{H}-517-\mathrm{C} 1$ \\
\hline 397 & $100-\mathrm{N}$ & $199-\mathrm{N}-24$ & A4673 & D & & & & & & & & & Y & HWIS & Drilled per spec $\mathrm{H}-517-\mathrm{C} 1$ \\
\hline 398 & $100-\mathrm{N}$ & $199-\mathrm{N}-25$ & A4674 & D & GG, SG & & & & & & & & $\mathrm{Y}$ & HWIS & Drilled per spec $\mathrm{H}-517-\mathrm{C} 1$ \\
\hline 399 & $100-\mathrm{N}$ & $199-\mathrm{N}-26$ & A4675 & D & GG, SG & & & & & & & & $\mathrm{Y}$ & HWIS & Drilled per spec $\mathrm{H}-517-\mathrm{C} 1$ \\
\hline 400 & $100-\mathrm{N}$ & $199-\mathrm{N}-27$ & A4676 & D & DN, GG, NT, SG, TP & & & & & & & & $\mathrm{Y}$ & Prater 1984 & \\
\hline 401 & $100-\mathrm{N}$ & $199-\mathrm{N}-28$ & A4677 & D & DN, GG, NT, SG, TP & & & & & & & & $Y$ & Prater 1984 & \\
\hline 402 & $100-\mathrm{N}$ & $199-\mathrm{N}-29$ & A4678 & D & DN, GG, NT, SG, TP & & & & & & & & $\mathrm{Y}$ & Prater 1984 & \\
\hline 403 & $100-\mathrm{N}$ & $199-\mathrm{N}-3$ & A4679 & D & TP & & & & & & & & $\mathrm{Y}$ & HWIS & \\
\hline 404 & $100-\mathrm{N}$ & $199-\mathrm{N}-30$ & A5828 & D & DN, GG, NT, TP & & & & & & & & $\mathrm{Y}$ & Prater 1984 & \\
\hline 405 & $100-\mathrm{N}$ & $199-\mathrm{N}-31$ & A4680 & D & DN, GG, NT, TP & & & & & & & & $Y$ & Prater 1984 & \\
\hline 406 & $100-\mathrm{N}$ & $199-\mathrm{N}-32$ & A4681 & $\mathrm{D}$ & DN, GG, NT, TP & & & & & & & & $\mathrm{Y}$ & Prater 1984 & \\
\hline 407 & $100-\mathrm{N}$ & $199-\mathrm{N}-33$ & A4682 & D & DN, GG, NT, TP & & & & & & & & $Y$ & Prater 1984 & \\
\hline 408 & $100-\mathrm{N}$ & $199-\mathrm{N}-34$ & A4683 & D & DN, GG, NT, TP & & & & & & & & $Y$ & Prater 1984 & \\
\hline 409 & $100-\mathrm{N}$ & $199-\mathrm{N}-35$ & A5829 & D & DN, GG, NT, SG & & & & & & & & $\mathrm{Y}$ & HWIS & \\
\hline 410 & $100-\mathrm{N}$ & $199-\mathrm{N}-36$ & A4684 & D, G & DN, GG, NT & & & & & & & & $\mathrm{Y}$ & HWIS & \\
\hline 411 & $100-\mathrm{N}$ & $199-\mathrm{N}-37$ & A4685 & $\mathrm{D}, \mathrm{G}$ & DN, GG, NT & & & & & & & & $Y$ & HWIS & \\
\hline 412 & $100-\mathrm{N}$ & $199-\mathrm{N}-38$ & A5830 & D, G & DN, GG, NT & & & & & & & & $Y$ & HWIS & \\
\hline 413 & $100-\mathrm{N}$ & $199-\mathrm{N}-39$ & A4686 & D, G & DN, GG, NT, SG & & & & & & & & $\mathrm{Y}$ & HWIS & \\
\hline 414 & $100-\mathrm{N}$ & $199-\mathrm{N}-4$ & A4687 & D & TP & & & & & & & & $Y$ & HWIS & \\
\hline 415 & $100-\mathrm{N}$ & $199-\mathrm{N}-40$ & A4688 & D, G & DN, GG, NT & & & & & & & & $\mathrm{Y}$ & HWIS & \\
\hline 416 & $100-N$ & $199-\mathrm{N}-41$ & A4689 & $\mathrm{D}, \mathrm{G}$ & DN, GG, NT & & & & & & & & $\mathrm{Y}$ & $\begin{array}{l}\text { Hartman and } \\
\text { Lindsey } 1993\end{array}$ & \\
\hline 417 & $100-\mathrm{N}$ & $199-\mathrm{N}-42$ & A4690 & $D, G$ & DN, GG, NT & & & & & & & & $Y$ & & \\
\hline 418 & $100-N$ & $199-\mathrm{N}-43$ & A5831 & $\mathrm{D}, \mathrm{G}$ & DN, GG, NT & & & & & & & & $Y$ & \begin{tabular}{l|} 
Hartman and \\
Lindsey 1993
\end{tabular} & \\
\hline 419 & $100-\mathrm{N}$ & $199-\mathrm{N}-44$ & A4691 & $D, G$ & DN, GG, NT, SG & & & & & & & & $Y$ & HWIS & \\
\hline 420 & $100-\mathrm{N}$ & $199-\mathrm{N}-45$ & A5832 & $\mathrm{D}, \mathrm{G}$ & $\mathrm{DN}, \mathrm{GG}, \mathrm{NT}, \mathrm{SG}$ & & & & & & & & $\mathrm{Y}$ & HWIS & \\
\hline 421 & $100-\mathrm{N}$ & $199-\mathrm{N}-47$ & A5834 & D & & & & & & & & & $Y$ & HWIS & \\
\hline 422 & $100-\mathrm{N}$ & $199-\mathrm{N}-48$ & A5835 & $\mathrm{D}$ & & & & & & & & & $Y$ & HWIS & \\
\hline 423 & $100-\mathrm{N}$ & $199-\mathrm{N}-49$ & A4692 & D & GG, SG & & & & & & & & $Y$ & HWIS & \\
\hline 424 & $100-\mathrm{N}$ & $199-\mathrm{N}-5$ & A5814 & $\mathrm{D}$ & DN, GG, NT, TP & & & & & & & & $\mathrm{Y}$ & HWIS & \\
\hline 425 & $100-\mathrm{N}$ & $199-\mathrm{N}-50$ & A4693 & D & GG, SG & & & & & & & & $\mathrm{Y}$ & HWIS & \\
\hline 426 & $100-\mathrm{N}$ & $199-\mathrm{N}-51$ & A4694 & $\mathrm{D}$ & GG, SG & & & & & & & & $Y$ & HWIS & \\
\hline 427 & $100-\mathrm{N}$ & $199-\mathrm{N}-52$ & A4695 & D & GG, SG & & & & & & & & $Y$ & HWIS & \\
\hline 428 & $100-\mathrm{N}$ & $199-\mathrm{N}-53$ & A4696 & D & GG, SG & & & & & & & & $\mathrm{Y}$ & HWIS & \\
\hline
\end{tabular}




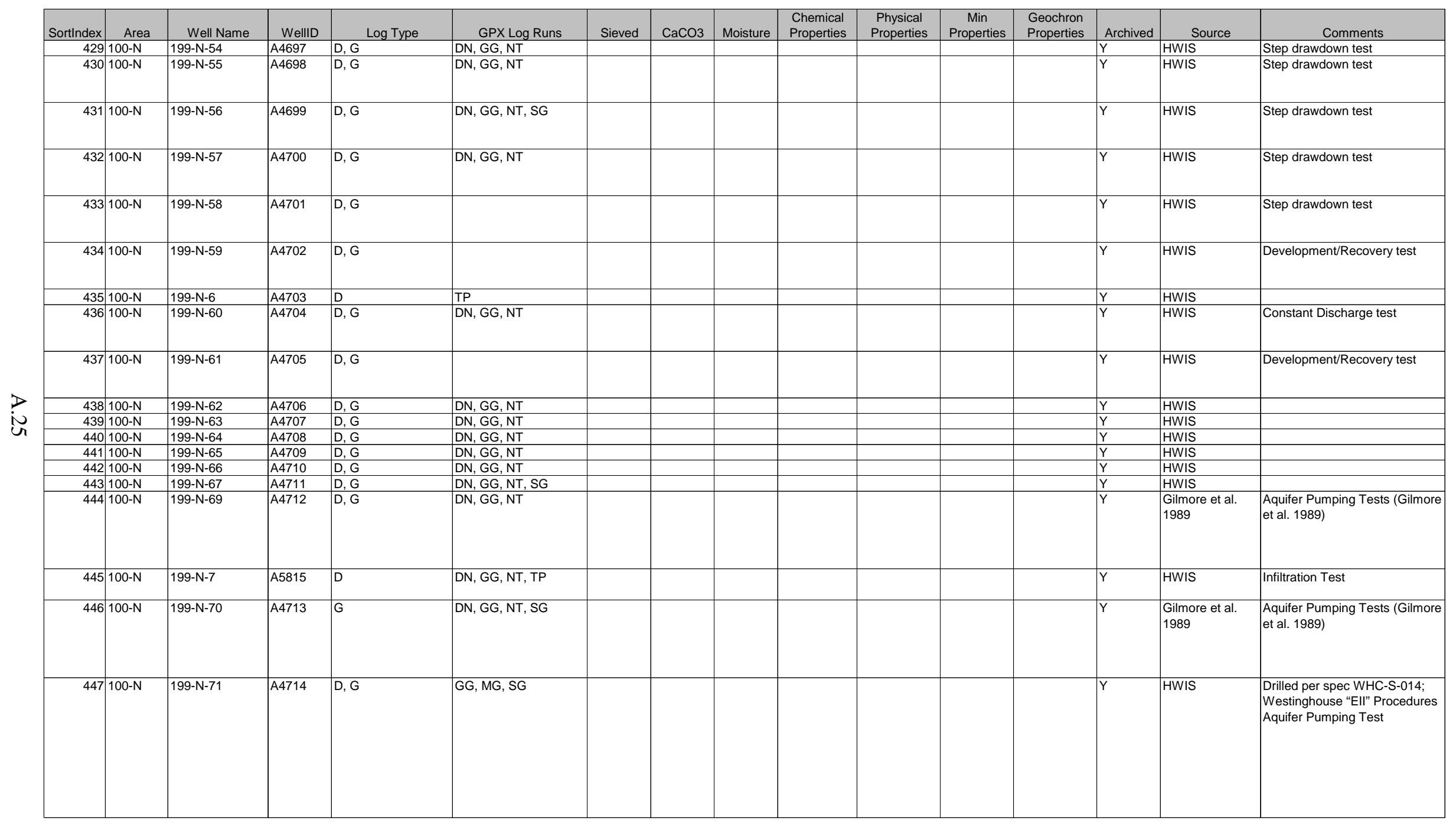




\begin{tabular}{|c|c|c|c|c|c|c|c|c|c|c|c|c|c|c|c|}
\hline Sortindex & Area & Well Name & WellID & Log Type & GPX Log Runs & Sieved & $\mathrm{CaCO} 3$ & Moisture & $\begin{array}{l}\text { Chemical } \\
\text { Properties }\end{array}$ & $\begin{array}{c}\text { Physical } \\
\text { Properties }\end{array}$ & \begin{tabular}{|c|} 
Min \\
Properties
\end{tabular} & $\begin{array}{l}\text { Geochron } \\
\text { Properties }\end{array}$ & Archived & Source & Comments \\
\hline & $100-\mathrm{N}$ & 199-N-72 & A4715 & $D, G$ & GG, SG & & & & & & & & $Y$ & HWIS & $\begin{array}{l}\text { Drilled per spec WHC-S-014; } \\
\text { Westinghouse "EII" Procedures } \\
\text { Aquifer Pumping Test }\end{array}$ \\
\hline 449 & $100-\mathrm{N}$ & 199-N-73 & A4716 & $D, G$ & GG, SG & & & & & & & & $Y$ & HWIS & $\begin{array}{l}\text { Drilled per spec WHC-S-014; } \\
\text { Westinghouse "EII" Procedures } \\
\text { Aquifer Pumping Test }\end{array}$ \\
\hline 450 & $100-\mathrm{N}$ & $199-\mathrm{N}-74$ & A4717 & $D, G$ & GG, SG & & & & & & & & $Y$ & HWIS & $\begin{array}{l}\text { Drilled per spec WHC-S-014; } \\
\text { Westinghouse "Ell" Procedures } \\
\text { Aquifer Pumping Test }\end{array}$ \\
\hline 451 & $100-N$ & $199-\mathrm{N}-75$ & A4718 & $\mathrm{D}, \mathrm{G}$ & GG, SG & & & & & & & & $\mathrm{Y}$ & HWIS & $\begin{array}{l}\text { Drilled per spec WHC-S-014; } \\
\text { Westinghouse "Ell" Procedures } \\
\text { Aquifer Pumping Test }\end{array}$ \\
\hline 452 & $100-\mathrm{N}$ & $199-\mathrm{N}-76$ & A4719 & $\mathrm{D}, \mathrm{G}$ & GG, SG & & & & & & & & $Y$ & HWIS & \\
\hline 453 & $100-\mathrm{N}$ & 199-N-77 & A5442 & $D, G$ & GG, SG & & & & & & & & $Y$ & HWIS & \\
\hline 454 & $100-\mathrm{N}$ & $199-\mathrm{N}-80$ & A4720 & D, G & GG, SG & $Y$ & & & & & & & $Y$ & $\begin{array}{l}\text { see R. Khaleel or } \\
\text { G. Freeman for } \\
\text { sieve data }\end{array}$ & \\
\hline 455 & $100-\mathrm{N}$ & $199-\mathrm{N}-81$ & A5443 & $\mathrm{D}, \mathrm{G}$ & & $Y$ & & & & & & & $Y$ & HWIS & \\
\hline 456 & $100-\mathrm{N}$ & $199-\mathrm{N}-83$ & A5838 & D, G & & $Y$ & & & & & & & $Y$ & HWIS & \\
\hline 457 & $100-\mathrm{N}$ & $199-\mathrm{N}-84$ & A5839 & & SG & & & & & & & & & & \\
\hline 458 & $100-\mathrm{N}$ & $199-\mathrm{N}-86$ & A5841 & & SG & & & & & & & & & & \\
\hline 459 & $100-\mathrm{N}$ & $199-\mathrm{N}-87$ & A5842 & & SG & & & & & & & & & & \\
\hline 460 & $100-\mathrm{N}$ & $199-\mathrm{N}-88$ & A5843 & & SG & & & & & & & & & & \\
\hline 461 & $100-\mathrm{N}$ & $199-\mathrm{N}-89$ & A5844 & & SG & & & & & & & & & & \\
\hline 462 & $100-\mathrm{N}$ & 199-N-8P & A5816 & D & TP & & & & & & & & $Y$ & HWIS & \\
\hline 463 & $100-\mathrm{N}$ & $199-\mathrm{N}-8 \mathrm{Q}$ & A5817 & $\mathrm{D}$ & TP & & & & & & & & $Y$ & HWIS & \\
\hline 464 & $100-\mathrm{N}$ & $199-\mathrm{N}-8 \mathrm{R}$ & A5818 & D & & & & & & & & & $\mathrm{Y}$ & HWIS & \\
\hline 465 & $100-\mathrm{N}$ & $199-\mathrm{N}-8 \mathrm{~S}$ & A4721 & D & & & & & & & & & $Y$ & HWIS & \\
\hline 466 & $100-\mathrm{N}$ & $199-\mathrm{N}-8 \mathrm{~T}$ & A5819 & D & & & & & & & & & $Y$ & HWIS & \\
\hline 467 & $100-\mathrm{N}$ & $199-\mathrm{N}-8 \mathrm{U}$ & A5820 & D & & & & & & & & & $Y$ & HWIS & \\
\hline 468 & $100-\mathrm{N}$ & $199-\mathrm{N}-8 \mathrm{~V}$ & A5821 & D & & & & & & & & & $Y$ & HWIS & \\
\hline 469 & $100-\mathrm{N}$ & $199-\mathrm{N}-9$ & A5822 & D & GG, NT & & & & & & & & $Y$ & HWIS & \\
\hline 470 & $100-\mathrm{N}$ & $199-\mathrm{N}-90$ & A5845 & & SG & & & & & & & & & & \\
\hline 471 & $100-\mathrm{N}$ & $199-\mathrm{N}-91 \mathrm{~A}$ & A9877 & G & & $Y$ & & & $\begin{array}{l}\mathrm{H}-3, \mathrm{Sr}-90, \\
\text { GEA }\end{array}$ & & & & & $\begin{array}{l}\text { Johnson et al. } \\
1995\end{array}$ & N-Springs Barrier \\
\hline
\end{tabular}




\begin{tabular}{|c|c|c|c|c|c|c|c|c|c|c|c|c|c|c|c|}
\hline SortIndex & Area & Well Name & WellID & Log Type & GPX Log Runs & Sieved & $\mathrm{CaCO} 3$ & Moisture & $\begin{array}{l}\text { Chemical } \\
\text { Properties }\end{array}$ & $\begin{array}{l}\text { Physical } \\
\text { Properties }\end{array}$ & \begin{tabular}{|c|} 
Min \\
Properties
\end{tabular} & $\begin{array}{l}\text { Geochron } \\
\text { Properties }\end{array}$ & Archived & Source & Comments \\
\hline 472 & $100-\mathrm{N}$ & 199-N-92A & A9878 & G & & Y & & & $\begin{array}{l}\mathrm{H}-3, \mathrm{Sr}-90, \\
\text { GEA }\end{array}$ & & & & & $\begin{array}{l}\text { Johnson et al. } \\
1995\end{array}$ & $\mathrm{~N}$-Springs Barrier \\
\hline 473 & $100-\mathrm{N}$ & 199-N-93A & A9879 & G & & $Y$ & & & $\begin{array}{l}\mathrm{H}-3, \mathrm{Sr}-90, \\
\text { GEA }\end{array}$ & & & & & $\begin{array}{l}\text { Johnson et al. } \\
1995\end{array}$ & N-Springs Barrier \\
\hline 474 & $100-\mathrm{N}$ & 199-N-94A & A9880 & G & & $Y$ & & & $\begin{array}{l}\mathrm{H}-3, \mathrm{Sr}-90, \\
\text { GEA }\end{array}$ & & & & & $\begin{array}{l}\text { Johnson et al. } \\
1995\end{array}$ & N-Springs Barrier \\
\hline 475 & $100-\mathrm{N}$ & 199-N-95A & A9881 & G & & Y & & & $\begin{array}{l}\mathrm{H}-3, \mathrm{Sr}-90 \\
\text { GEA }\end{array}$ & & & & & $\begin{array}{l}\text { Johnson et al. } \\
1995\end{array}$ & N-Springs Barrier \\
\hline 476 & $100-N$ & 199-N-96A & A9882 & G & & $\mathrm{Y}$ & & & $\begin{array}{l}\text { H-3, Sr-90, } \\
\text { GEA }\end{array}$ & & & & & $\begin{array}{l}\text { Johnson et al. } \\
1995\end{array}$ & $\mathrm{~N}$-Springs Barrier \\
\hline 477 & $100-\mathrm{N}$ & 199-N-97A & A9883 & G & & $Y$ & & & $\begin{array}{l}\mathrm{H}-3, \mathrm{Sr}-90, \\
\text { GEA }\end{array}$ & & & & & $\begin{array}{l}\text { Johnson et al. } \\
1995\end{array}$ & N-Springs Barrier \\
\hline 478 & $100-\mathrm{N}$ & 199-N-99A & A9910 & G & & & & & & & & & & $\begin{array}{l}\text { Johnson et al. } \\
1995\end{array}$ & $\mathrm{~N}$-Springs Barrier \\
\hline 479 & $100-N$ & $\begin{array}{l}\text { 100N Backwash } \\
\text { Lake }\end{array}$ & & & & $Y$ & & & $\mathrm{pH}$ & Cond & & & & Opitz 1982 & \\
\hline 480 & $200-E$ & 299-E13-1 & A5849 & D & $\begin{array}{l}\text { DN, GG, MO, NT, SG, } \\
\text { TP }\end{array}$ & \begin{tabular}{|l} 
Virtual \\
Library
\end{tabular} & \begin{tabular}{|l} 
Virtual \\
Library
\end{tabular} & & $\mathrm{pH}$ & $\begin{array}{l}\% \text { GSSC, } 15- \\
\text { ATM, CEC }\end{array}$ & & & Y & McHenry 1957 & \\
\hline 481 & $200-E$ & 299-E13-10 & A4724 & D & DN, GG, NT, SG, TP & $\begin{array}{l}\text { Virtual } \\
\text { Library }\end{array}$ & $\begin{array}{l}\text { Virtual } \\
\text { Library }\end{array}$ & & & Por & & & $Y$ & \begin{tabular}{|l|} 
Bierschenk 1959 \\
\end{tabular} & \\
\hline 482 & $200-E$ & 299-E13-11 & A5858 & D & DN, GG, NT, SG & $\begin{array}{l}\text { Virtual } \\
\text { Library }\end{array}$ & $\begin{array}{l}\text { Virtual } \\
\text { Library }\end{array}$ & & & & & & $Y$ & HWIS & \\
\hline 483 & $200-E$ & 299-E13-12 & A4725 & D & $\begin{array}{l}\text { DN, GG, MO, NT, SG, } \\
\text { TP }\end{array}$ & $\begin{array}{l}\text { Virtual } \\
\text { Library }\end{array}$ & $\begin{array}{l}\text { Virtual } \\
\text { Library }\end{array}$ & & & & & & $Y$ & HWIS & \\
\hline 484 & $200-E$ & 299-E13-13 & A5859 & D & GG, NT & $\begin{array}{l}\text { Virtual } \\
\text { Library }\end{array}$ & $\begin{array}{l}\text { Virtual } \\
\text { Library }\end{array}$ & & & & & & Y & HWIS & \\
\hline 485 & $200-E$ & 299-E13-14 & A4726 & D & DN, GG, NT, SG, TP & $\begin{array}{l}\text { Virtual } \\
\text { Library }\end{array}$ & \begin{tabular}{|l|l|} 
Virtual \\
Library
\end{tabular} & & Lab Rad & & & & $Y$ & Haney 1967 & \\
\hline 486 & $200-E$ & 299-E13-15 & A5860 & D & DN, GG, NT, SG & \begin{tabular}{|l|} 
Virtual \\
Library
\end{tabular} & \begin{tabular}{|l|} 
Virtual \\
Library
\end{tabular} & & & & & & $Y$ & HWIS & \\
\hline 487 & $200-E$ & 299-E13-16 & A5861 & D & DN, GG, NT, SG & $\begin{array}{l}\text { Virtual } \\
\text { Library }\end{array}$ & $\begin{array}{l}\text { Virtual } \\
\text { Library }\end{array}$ & & & & & & $Y$ & HWIS & \\
\hline 488 & $200-E$ & 299-E13-17 & A5862 & D & DN, GG, NT, SG, TP & \begin{tabular}{|l} 
Virtual \\
Library
\end{tabular} & \begin{tabular}{|l} 
Virtual \\
Library
\end{tabular} & & & & & & $Y$ & HWIS & \\
\hline 489 & $200-E$ & 299-E13-18 & A5863 & D & DN, GG, NT, SG & $\begin{array}{l}\text { Virtual } \\
\text { Library }\end{array}$ & $\begin{array}{l}\text { Virtual } \\
\text { Library }\end{array}$ & & & & & & $Y$ & HWIS & \\
\hline 490 & $200-E$ & 299-E13-19 & A5864 & D & DN, GG, NT, SG, TP & \begin{tabular}{|l} 
Virtual \\
Library
\end{tabular} & \begin{tabular}{|l} 
Virtual \\
Library
\end{tabular} & & & & & & $Y$ & HWIS & \\
\hline 491 & $200-E$ & 299-E13-2 & A5850 & D & DN, GG, MO, NT, SG & $\begin{array}{l}\text { Virtual } \\
\text { Library }\end{array}$ & $\begin{array}{l}\text { Virtual } \\
\text { Library }\end{array}$ & & & & & & Y & HWIS & \\
\hline 492 & $200-E$ & 299-E13-20 & A5865 & D & GG, TP & & & & Field Rad & & & & & HWIS & \\
\hline 493 & $200-E$ & 299-E13-21 & A5866 & $\mathrm{D}$ & $\mathrm{DN}, \mathrm{GG}, \mathrm{MO}, \mathrm{NT}, \mathrm{SG}$ & & & & Field Rad & & & & & HWIS & \\
\hline 494 & $200-E$ & 299-E13-3 & A5851 & D & $\begin{array}{l}\text { DN, GG, MO, NT, SG, } \\
\text { TP }\end{array}$ & \begin{tabular}{|l|} 
Virtual \\
Library
\end{tabular} & \begin{tabular}{|l|} 
Virtual \\
Library
\end{tabular} & & Lab Rad & & & & $Y$ & Haney 1967 & \\
\hline 495 & $200-E$ & 299-E13-4 & A5852 & D & $\mathrm{DN}, \mathrm{GG}, \mathrm{MO}, \mathrm{NT}, \mathrm{SG}$ & $\begin{array}{l}\text { Virtual } \\
\text { Library }\end{array}$ & $\begin{array}{l}\text { Virtual } \\
\text { Library }\end{array}$ & & & & & & $\mathrm{Y}$ & HWIS & \\
\hline 496 & $200-E$ & 299-E13-5 & A5853 & D & $\mathrm{DN}, \mathrm{GG}, \mathrm{MO}, \mathrm{NT}, \mathrm{SG}$ & $\begin{array}{l}\text { Virtual } \\
\text { Library }\end{array}$ & $\begin{array}{l}\text { Virtual } \\
\text { Library }\end{array}$ & & $\mathrm{pH}$ & $\begin{array}{l}\text { \%GSSC, 15- } \\
\text { ATM, CEC }\end{array}$ & & & $Y$ & McHenry 1957 & \\
\hline 497 & $200-E$ & 299-E13-51 & A5867 & D & GG, NT & & & & Field Rad & & & & & HWIS & \\
\hline 498 & $200-E$ & 299-E13-52 & A5868 & D & GG, MO, NT, SG & & & & Field Rad & & & & & HWIS & \\
\hline 499 & $200-E$ & 299-E13-54 & A5869 & D & GG, MO, SG & & & & Field Rad & & & & $Y$ & HWIS & \\
\hline 500 & $200-E$ & 299-E13-55 & A5870 & D & GG, MO, SG & & & & & & & & $\mathrm{Y}$ & HWIS & \\
\hline 501 & $200-E$ & 299-E13-56 & A5871 & D & GG, MO, SG & & & & Field Rad & & & & $Y$ & HWIS & \\
\hline 502 & $200-E$ & 299-E13-57 & A5872 & $\mathrm{D}$ & $\mathrm{GG}, \mathrm{MO}, \mathrm{SG}$ & & & & Field Rad & & & & $Y$ & HWIS & \\
\hline 503 & $200-E$ & 299-E13-58 & A5873 & $\mathrm{D}$ & GG, MO, SG & & & & Field Rad & & & & $\mathrm{Y}$ & HWIS & \\
\hline
\end{tabular}




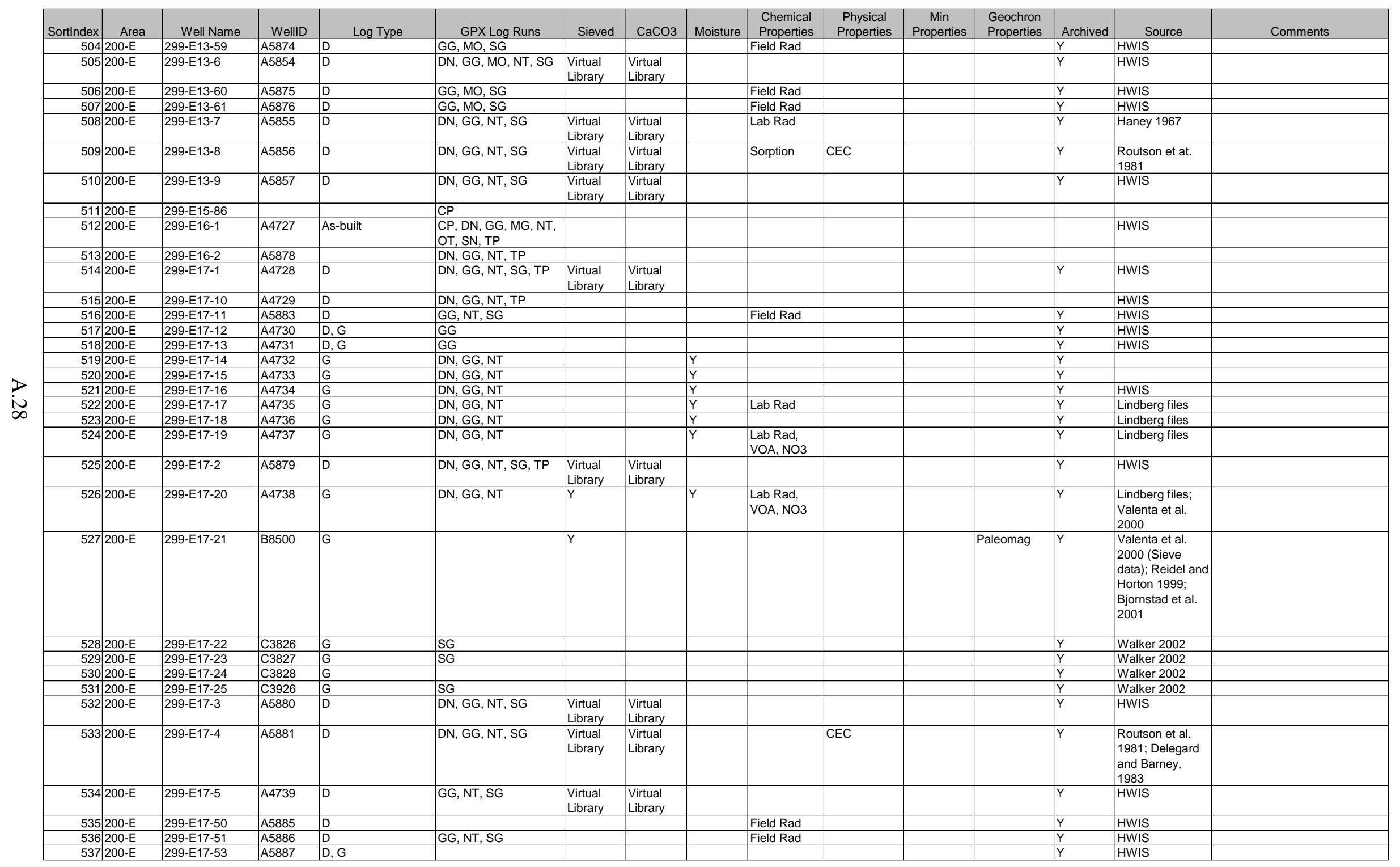




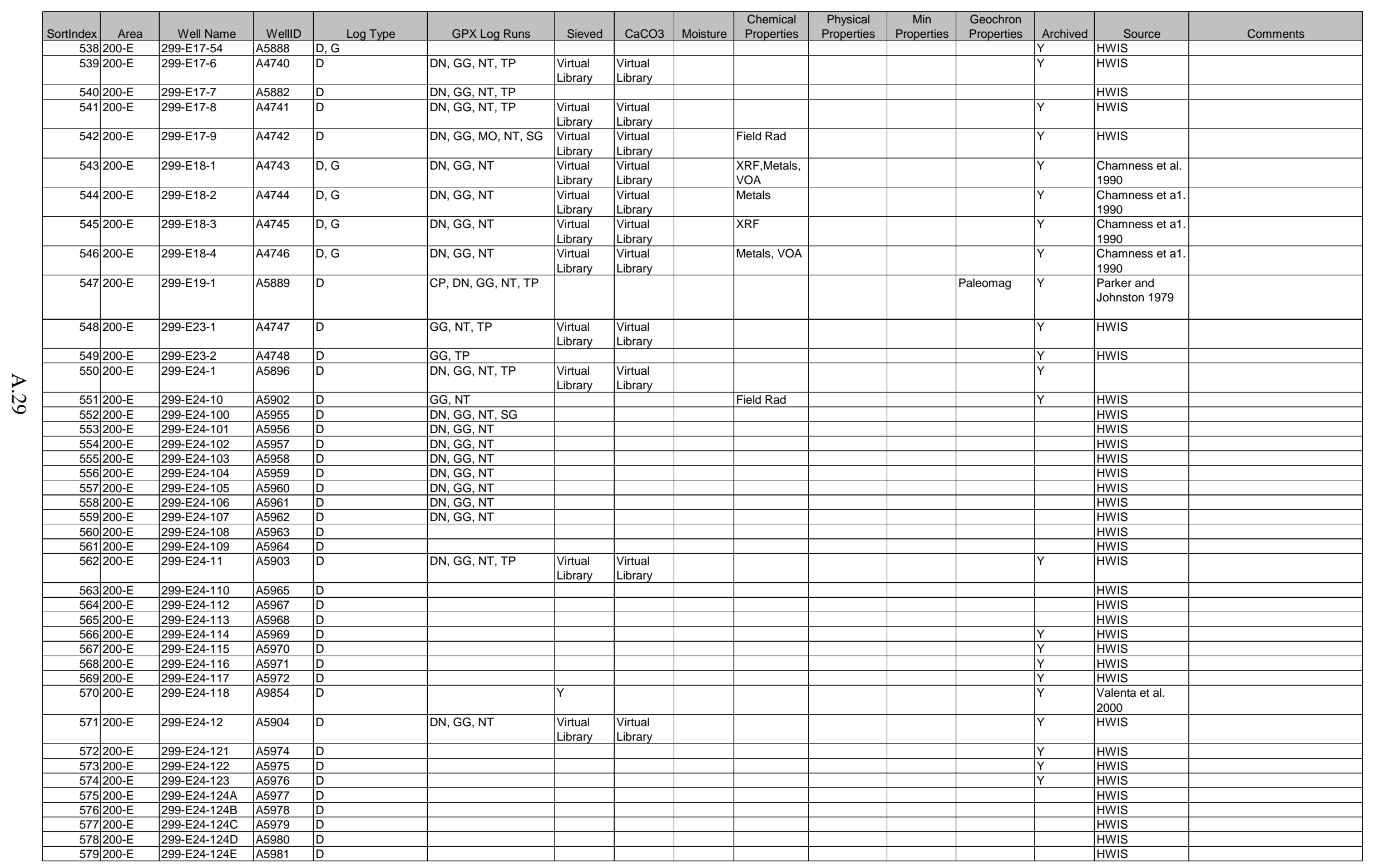




\begin{tabular}{|c|c|c|c|c|c|c|c|c|c|c|c|c|c|c|c|}
\hline SortIndex & Area & Well Name & WelllD & Log Type & GPX Log Runs & Sieved & $\mathrm{CaCO} 3$ & Moisture & $\begin{array}{c}\text { Chemical } \\
\text { Properties } \\
\end{array}$ & $\begin{array}{l}\text { Physical } \\
\text { Properties }\end{array}$ & \begin{tabular}{|c|} 
Min \\
Properties \\
\end{tabular} & $\begin{array}{l}\text { Geochron } \\
\text { Properties } \\
\end{array}$ & Archived & Source & Comments \\
\hline 580 & $200-E$ & 299-E24-124F & A5982 & D & & & & & & & & & & HWIS & \\
\hline 581 & $200-E$ & 299-E24-124G & A5983 & D & & & & & & & & & & HWIS & \\
\hline 582 & $200-E$ & 299-E24-124H & A5984 & D & & & & & & & & & 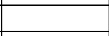 & HWIS & \\
\hline 583 & $200-E$ & 299-E24-124I & A5985 & D & & & & & & & & & & HWIS & \\
\hline 584 & $200-E$ & 299-E24-124J & A5986 & D & & & & & & & & & & HWIS & \\
\hline 585 & $200-\mathrm{E}$ & 299-E24-13 & A4749 & D & & $\begin{array}{l}\text { Virtual } \\
\text { Library }\end{array}$ & $\begin{array}{l}\text { Virtual } \\
\text { Library }\end{array}$ & & & & & & $Y$ & HWIS & \\
\hline 586 & $200-\mathrm{E}$ & \begin{tabular}{|l|} 
299-E24-14 \\
\end{tabular} & A4750 & D & & $\begin{array}{l}\text { Virtual } \\
\text { Library }\end{array}$ & $\begin{array}{l}\text { Virtual } \\
\text { Library }\end{array}$ & & & & & & $Y$ & HWIS & \\
\hline 587 & $200-E$ & 299-E24-15 & A5905 & $\mathrm{D}, \mathrm{G}$ & GG, NT & & & & Field Rad & & & & $\mathrm{Y}$ & HWIS & \\
\hline 588 & $200-E$ & 299-E24-155 & A6016 & D & & & & & & & & & $\mathrm{Y}$ & HWIS & \\
\hline 589 & $200-\mathrm{E}$ & 299-E24-156 & A6017 & D & & $\begin{array}{l}\text { Virtual } \\
\text { Library }\end{array}$ & $\begin{array}{l}\text { Virtual } \\
\text { Library }\end{array}$ & & & & & & $Y$ & HWIS & \\
\hline 590 & $200-\mathrm{E}$ & 299-E24-157 & A6018 & D & & $\begin{array}{l}\text { Virtual } \\
\text { Library }\end{array}$ & $\begin{array}{l}\text { Virtual } \\
\text { Library }\end{array}$ & & & & & & $Y$ & HWIS & \\
\hline 591 & $200-\mathrm{E}$ & 299-E24-158 & A6019 & D & & $\begin{array}{l}\text { Virtual } \\
\text { Library }\end{array}$ & $\begin{array}{l}\text { Virtual } \\
\text { Library }\end{array}$ & & & & & & $Y$ & & \\
\hline 592 & $200-\mathrm{E}$ & 299-E24-159 & A6020 & D & & $\begin{array}{l}\text { Virtual } \\
\text { Library }\end{array}$ & $\begin{array}{l}\text { Virtual } \\
\text { Library }\end{array}$ & & & & & & Y & HWIS & \\
\hline 593 & $200-\mathrm{E}$ & \begin{tabular}{|l|} 
299-E24-16 \\
\end{tabular} & A4751 & G & DN, GG, NT & & & Y & $\begin{array}{l}\text { Lab Rad, } \\
\text { VOA, TOC }\end{array}$ & & & & Y & Lindberg files & \\
\hline 594 & $200-E$ & 299-E24-160 & A6021 & D, G & GG, SG & & & & & & & & $\mathrm{Y}$ & HWIS & \\
\hline 595 & $200-\mathrm{E}$ & 299-E24-17 & A4752 & G & DN, GG, NT & & & $\mathrm{Y}$ & \begin{tabular}{|l|} 
Lab Rad, \\
VOA, NO3
\end{tabular} & & & & $Y$ & Lindberg files & \\
\hline 596 & $200-E$ & 299-E24-18 & A4753 & G & DN, GG, NT & & & $Y$ & & & & & $\mathrm{Y}$ & & \\
\hline 597 & $200-E$ & 299-E24-19 & A4754 & G & GG & $\begin{array}{l}\text { Virtual } \\
\text { Library }\end{array}$ & & $Y$ & $Y$ & & & & $Y$ & $\begin{array}{l}\text { Pearson 1990; } \\
\text { Valenta et al. } \\
2000 \text { (sieve } \\
\text { data) }\end{array}$ & \\
\hline 598 & $200-E$ & 299-E24-1A & & & & & & & Lab Rad & & & & & Haney 1967 & \\
\hline 599 & $200-E$ & 299-E24-2 & A4755 & D & DN, GG, NT, SG, TP & $\begin{array}{l}\text { Virtual } \\
\text { Library }\end{array}$ & $\begin{array}{l}\text { Virtual } \\
\text { Library }\end{array}$ & & & & & & $\mathrm{Y}$ & HWIS & \\
\hline 600 & $200-E$ & 299-E24-20 & A4756 & G & GG, SG & & & & & & & & $\mathrm{Y}$ & HWIS & \\
\hline 601 & $200-E$ & 299-E24-21 & C3177 & G & SG & $\mathrm{Y}$ & $Y$ & $Y$ & \begin{tabular}{|l|} 
TC, TOC, \\
XRF, Iron \\
Extractions, \\
$1: 1$
\end{tabular} & CEC & XRF & Paleomag & $Y$ & $\begin{array}{l}\text { Walker 2001; } \\
\text { Horton et al. } \\
\text { 2003a; Pluhar } \\
\text { 2002 }\end{array}$ & \\
\hline 602 & $200-\mathrm{E}$ & 299-E24-22 & C4123 & G & SG & $Y$ & & & & & & & $Y$ & $\begin{array}{l}\text { Martinez 2003; } \\
\text { Williams and } \\
\text { Narbutovskih } \\
2003\end{array}$ & \\
\hline 603 & $200-E$ & 299-E24-3 & A5897 & D & DN, GG, NT & \begin{tabular}{|l|}
$\begin{array}{l}\text { Virtual } \\
\text { Library }\end{array}$ \\
\end{tabular} & $\begin{array}{l}\text { Virtual } \\
\text { Library }\end{array}$ & & & & & & $Y$ & HWIS & \\
\hline 604 & $200-E$ & 299-E24-33 & C4257 & G, Well summary & NT, SG & & & & & & & & & HWIS & \\
\hline 605 & $200-E$ & 299-E24-4 & A5898 & D & $\mathrm{DN}, \mathrm{GG}, \mathrm{NT}, \mathrm{TP}$ & $\begin{array}{l}\text { Virtual } \\
\text { Library }\end{array}$ & \begin{tabular}{|l|} 
Virtual \\
Library
\end{tabular} & & & & & & $\mathrm{Y}$ & HWIS & \\
\hline 606 & $200-\mathrm{E}$ & 299-E24-5 & A5899 & D & DN, GG, NT, TP & \begin{tabular}{|l|} 
Virtual \\
Library
\end{tabular} & \begin{tabular}{|l|} 
Virtual \\
Library
\end{tabular} & & & & & & $Y$ & HWIS & \\
\hline 607 & $200-E$ & 299-E24-51 & A5908 & D & & & & & & & & & & HWIS & \\
\hline 608 & $200-E$ & 299-E24-52 & A5909 & D & & & & & & & & & & HWIS & \\
\hline 609 & $200-\mathrm{E}$ & 299-E24-53 & A5910 & D & DN, GG, MO, NT, SG & $\begin{array}{l}\text { Virtual } \\
\text { Library }\end{array}$ & \begin{tabular}{|l|} 
Virtual \\
Library
\end{tabular} & & & & & & $Y$ & HWIS & \\
\hline 610 & 200-E & 299-E24-54 & A5911 & D & GG, MO, NT, SG & $\begin{array}{l}\text { Virtual } \\
\text { Library }\end{array}$ & $\begin{array}{l}\text { Virtual } \\
\text { Library }\end{array}$ & & & & & & $\mathrm{Y}$ & HWIS & \\
\hline
\end{tabular}




\begin{tabular}{|c|c|c|c|c|c|c|c|c|c|c|c|c|c|c|c|}
\hline SortIndex & Area & Well Name & WellID & Log Type & GPX Log Runs & Sieved & $\mathrm{CaCO} 3$ & Moisture & $\begin{array}{l}\text { Chemical } \\
\text { Properties }\end{array}$ & $\begin{array}{c}\text { Physical } \\
\text { Properties } \\
\end{array}$ & \begin{tabular}{|c|} 
Min \\
Properties \\
\end{tabular} & $\begin{array}{l}\text { Geochron } \\
\text { Properties } \\
\end{array}$ & Archived & Source & Comments \\
\hline 611 & $200-E$ & 299-E24-55 & A5912 & D & & & & & & & & & $\mathrm{Y}$ & HWIS & \\
\hline 612 & $200-\mathrm{E}$ & 299-E24-56 & A5913 & D & DN, GG, NT & \begin{tabular}{|l} 
Virtual \\
Library
\end{tabular} & \begin{tabular}{|l} 
Virtual \\
Library
\end{tabular} & & & & & & Y & HWIS & \\
\hline 613 & $200-\mathrm{E}$ & \begin{tabular}{|l|} 
299-E24-57 \\
\end{tabular} & A5914 & D & DN, GG, NT & $\begin{array}{l}\text { Virtual } \\
\text { Library }\end{array}$ & $\begin{array}{l}\text { Virtual } \\
\text { Library }\end{array}$ & & & & & & $Y$ & HWIS & \\
\hline 614 & 200-E & 299-E24-58 & A5915 & D & DN, GG, NT & \begin{tabular}{|l} 
Virtual \\
Library
\end{tabular} & $\begin{array}{l}\text { Virtual } \\
\text { Library }\end{array}$ & & & & & & $Y$ & HWIS & \\
\hline 615 & $200-\mathrm{E}$ & \begin{tabular}{|l|} 
299-E24-59 \\
\end{tabular} & A5916 & D & GG, NT, SG & $\begin{array}{l}\text { Virtual } \\
\text { Library }\end{array}$ & $\begin{array}{l}\text { Virtual } \\
\text { Library }\end{array}$ & & & & & & $Y$ & HWIS & \\
\hline 616 & $200-E$ & 299-E24-6 & A5900 & D & GG, NT & & & & & & & & $Y$ & & \\
\hline 617 & $200-E$ & 299-E24-60 & A5917 & D & GG, NT, SG & \begin{tabular}{|l|}
$\begin{array}{l}\text { Virtual } \\
\text { Library }\end{array}$ \\
\end{tabular} & \begin{tabular}{|l|l|} 
Virtual \\
Library
\end{tabular} & & & & & & $Y$ & HWIS & \\
\hline 618 & $200-E$ & 299-E24-63 & A5918 & D & $\mathrm{DN}, \mathrm{GG}, \mathrm{NT}$ & & & & & & & & & HWIS & \\
\hline 619 & $200-E$ & 299-E24-64 & A5919 & D & & & & & & & & & & HWIS & \\
\hline 620 & $200-E$ & 299-E24-65 & A5920 & & GG & & & & & & & & & & \\
\hline 621 & $200-E$ & 299-E24-66 & A5921 & $\mathrm{D}$ & & & & & & & & & $\mathrm{Y}$ & HWIS & \\
\hline 622 & $200-E$ & 299-E24-67 & A5922 & D & & & & & & & & & $\mathrm{Y}$ & & \\
\hline 623 & $200-E$ & 299-E24-68 & A5923 & D & & & & & & & & & $Y$ & HWIS & \\
\hline 624 & $200-E$ & 299-E24-69 & A5924 & D & & & & & & & & & & HWIS & \\
\hline 625 & $200-\mathrm{E}$ & 299-E24-7 & A4757 & D & GG, NT, TP & $\begin{array}{l}\text { Virtual } \\
\text { Library }\end{array}$ & $\begin{array}{l}\text { Virtual } \\
\text { Library }\end{array}$ & & & & & & Y & HWIS & \\
\hline 626 & $200-E$ & 299-E24-70 & A5925 & D & & & & & & & & & $Y$ & HWIS & \\
\hline 627 & $200-E$ & 299-E24-71 & A5926 & D & & & & & & & & & $Y$ & HWIS & \\
\hline 628 & $200-E$ & 299-E24-72 & A5927 & D & & & & & & & & & $Y$ & HWIS & \\
\hline 629 & $200-E$ & 299-E24-73 & A5928 & D & & & & & & & & & & HWIS & \\
\hline 630 & $200-E$ & 299-E24-75 & A5930 & D & & & & & & & & & $Y$ & HWIS & \\
\hline 631 & $200-\mathrm{E}$ & 299-E24-76 & A5931 & D & DN, GG, NT & & & & & & & & & HWIS & \\
\hline 632 & $200-E$ & 299-E24-77 & A5932 & $\mathrm{D}$ & $\mathrm{DN}, \mathrm{GG}, \mathrm{NT}$ & & & & & & & & & HWIS & \\
\hline 633 & $200-E$ & 299-E24-78 & A5933 & D & DN, GG, MO, NT & & & & & & & & $Y$ & HWIS & \\
\hline 634 & $200-E$ & \begin{tabular}{|l|} 
299-E24-79 \\
\end{tabular} & A5934 & D & $\mathrm{DN}, \mathrm{GG}, \mathrm{NT}, \mathrm{OT}$ & \begin{tabular}{|l|} 
Virtual \\
Library
\end{tabular} & $\begin{array}{l}\text { Virtual } \\
\text { Library }\end{array}$ & & & & & & Y & HWIS & \\
\hline 635 & $200-\mathrm{E}$ & 299-E24-8 & A4758 & D & DN, GG, NT & $\begin{array}{l}\text { Virtual } \\
\text { Library }\end{array}$ & $\begin{array}{l}\text { Virtual } \\
\text { Library }\end{array}$ & & & & & & $Y$ & HWIS & \\
\hline 636 & $200-E$ & 299-E24-80 & A5935 & D & DN, GG, NT & & & & & & & & & HWIS & \\
\hline 637 & $200-E$ & 299-E24-81 & A5936 & D & DN, GG, NT & & & & & & & & & HWIS & \\
\hline 638 & $200-E$ & 299-E24-82 & A5937 & $\mathrm{D}$ & DN, GG, NT & & & & & & & & & HWIS & \\
\hline 639 & $200-E$ & 299-E24-83 & A5938 & D & DN, GG, NT & & & & & & & & & HWIS & \\
\hline 640 & $200-E$ & 299-E24-84 & A5939 & $\mathrm{D}$ & DN, GG, NT, SG & & & & & & & & & HWIS & \\
\hline 641 & $200-E$ & 299-E24-85 & A5940 & D & DN, GG, NT & & & & & & & & & HWIS & \\
\hline 642 & $200-E$ & 299-E24-86 & A5941 & $\mathrm{D}$ & DN, GG, NT & & & & & & & & & HWIS & \\
\hline 643 & $200-E$ & 299-E24-87 & A5942 & $\mathrm{D}$ & DN, GG, NT & & & & & & & & & HWIS & \\
\hline 644 & $200-E$ & 299-E24-88 & A5943 & $\mathrm{D}$ & DN, GG, NT & & & & & & & & & HWIS & \\
\hline 645 & $200-E$ & 299-E24-89 & A5944 & $\mathrm{D}$ & DN, GG, NT & & & & & & & & & HWIS & \\
\hline 646 & $200-E$ & 299-E24-9 & A5901 & D & $\mathrm{DN}, \mathrm{GG}, \mathrm{NT}, \mathrm{TP}$ & $\begin{array}{l}\text { Virtual } \\
\text { Library }\end{array}$ & $\begin{array}{l}\text { Virtual } \\
\text { Library }\end{array}$ & & & & & & $Y$ & HWIS & \\
\hline 647 & $200-E$ & 299-E24-90 & A5945 & $\mathrm{D}$ & DN, GG, NT & & & & & & & & & HWIS & \\
\hline 648 & $200-\mathrm{E}$ & 299-E24-91 & A5946 & D & DN, GG, NT & & & & & & & & & HWIS & \\
\hline 649 & $200-\mathrm{E}$ & 299-E24-92 & A5947 & D & $\mathrm{DN}, \mathrm{GG}, \mathrm{NT}, \mathrm{OT}$ & \begin{tabular}{|l|} 
Virtual \\
Library
\end{tabular} & $\begin{array}{l}\text { Virtual } \\
\text { Library }\end{array}$ & & & & & & $Y$ & HWIS & \\
\hline 650 & $200-E$ & 299-E24-93 & A5948 & D & DN, GG, NT & & & & & & & & $Y$ & HWIS & \\
\hline 651 & $200-E$ & 299-E24-94 & A5949 & D & DN, GG, NT & & & & & & & & Y & HWIS & \\
\hline 652 & $200-\mathrm{E}$ & \begin{tabular}{|l|} 
299-E24-95 \\
\end{tabular} & A5950 & D & $\mathrm{DN}, \mathrm{GG}, \mathrm{NT}, \mathrm{OT}$ & \begin{tabular}{|l|} 
Virtual \\
Library
\end{tabular} & $\begin{array}{l}\text { Virtual } \\
\text { Library }\end{array}$ & & & & & & $Y$ & HWIS & \\
\hline 653 & $200-E$ & 299-E24-96 & A5951 & D & DN, GG, NT & & & & & & & & & HWIS & \\
\hline 654 & $200-\mathrm{E}$ & 299-E24-97 & A5952 & $\mathrm{D}$ & DN, GG, NT & & & & & & & & & HWIS & \\
\hline 655 & $200-E$ & 299-E24-98 & A5953 & D & DN, GG, NT & & & & & & & & & HWIS & \\
\hline
\end{tabular}




\begin{tabular}{|c|c|c|c|c|c|c|c|c|c|c|c|c|c|c|c|}
\hline SortIndex & Area & Well Name & WelllD & Log Type & GPX Log Runs & Sieved & $\mathrm{CaCO} 3$ & Moisture & $\begin{array}{l}\text { Chemical } \\
\text { Properties }\end{array}$ & $\begin{array}{l}\text { Physical } \\
\text { Properties }\end{array}$ & \begin{tabular}{|c|} 
Min \\
Properties \\
\end{tabular} & $\begin{array}{l}\text { Geochron } \\
\text { Properties } \\
\end{array}$ & Archived & Source & Comments \\
\hline 656 & $200-E$ & 299-E24-99 & A5954 & $\mathrm{D}$ & DN, GG, NT & & & & & & & & & & \\
\hline 657 & $200-\mathrm{E}$ & 299-E25-1 & A4759 & D & GG & $\begin{array}{l}\text { Virtual } \\
\text { Library }\end{array}$ & $\begin{array}{l}\text { Virtual } \\
\text { Library }\end{array}$ & & Field Rad & $\% G S S C$ & & & $Y$ & Brown 1963 & \\
\hline 658 & $200-E$ & 299-E25-10 & A4760 & D & GG, MO, NT, SG, TP & $\begin{array}{l}\text { Virtual } \\
\text { Library }\end{array}$ & $\begin{array}{l}\text { Virtual } \\
\text { Library }\end{array}$ & & & & & & $\mathrm{Y}$ & HWIS & \\
\hline 659 & $200-\mathrm{E}$ & 299-E25-100 & A6535 & D & & \begin{tabular}{|l} 
Virtual \\
Library
\end{tabular} & $\begin{array}{l}\text { Virtual } \\
\text { Library }\end{array}$ & & & & & & $Y$ & HWIS & \\
\hline 660 & 200-E & 299-E25-1000 & A6536 & G & GG, MO, SG & & $Y$ & $Y$ & $\begin{array}{l}\text { Field Rad } \\
\text { (Alpha, } \\
\text { Beta/Gamma } \\
\text { ) }\end{array}$ & & & & $Y$ & Swanson 1994 & \\
\hline 661 & $200-\mathrm{E}$ & 299-E25-101 & A6537 & D & & \begin{tabular}{|l} 
Virtual \\
Library
\end{tabular} & $\begin{array}{l}\text { Virtual } \\
\text { Library }\end{array}$ & & & & & & $\mathrm{Y}$ & HWIS & \\
\hline 662 & $200-\mathrm{E}$ & 299-E25-102 & A6538 & D & & \begin{tabular}{|l} 
Virtual \\
Library
\end{tabular} & \begin{tabular}{|l} 
Virtual \\
Library
\end{tabular} & & & & & & $Y$ & HWIS & \\
\hline 663 & $200-\mathrm{E}$ & 299-E25-103 & A6539 & D & & \begin{tabular}{|l} 
Virtual \\
Library
\end{tabular} & $\begin{array}{l}\text { Virtual } \\
\text { Library }\end{array}$ & & & & & & $Y$ & HWIS & \\
\hline 664 & $200-\mathrm{E}$ & 299-E25-104 & A6540 & D & & $\begin{array}{l}\text { Virtual } \\
\text { Library }\end{array}$ & $\begin{array}{l}\text { Virtual } \\
\text { Library }\end{array}$ & & & & & & $Y$ & HWIS & \\
\hline 665 & $200-\mathrm{E}$ & 299-E25-105 & A6541 & D & & $\begin{array}{l}\text { Virtual } \\
\text { Library }\end{array}$ & $\begin{array}{l}\text { Virtual } \\
\text { Library }\end{array}$ & & & & & & $Y$ & HWIS & \\
\hline 666 & $200-E$ & 299-E25-106 & A6542 & D & & \begin{tabular}{|l} 
Virtual \\
Library
\end{tabular} & $\begin{array}{l}\text { Virtual } \\
\text { Library }\end{array}$ & & & & & & $Y$ & HWIS & \\
\hline 667 & $200-\mathrm{E}$ & 299-E25-107 & A6543 & D & & $\begin{array}{l}\text { Virtual } \\
\text { Library }\end{array}$ & $\begin{array}{l}\text { Virtual } \\
\text { Library }\end{array}$ & & & & & & $Y$ & HWIS & \\
\hline 668 & $200-E$ & 299-E25-108 & A6544 & D & & \begin{tabular}{|l|} 
Virtual \\
Library
\end{tabular} & \begin{tabular}{|l|} 
Virtual \\
Library
\end{tabular} & & & & & & $\mathrm{Y}$ & HWIS & \\
\hline 669 & $200-\mathrm{E}$ & 299-E25-109 & A6545 & D & & \begin{tabular}{|l} 
Virtual \\
Library
\end{tabular} & $\begin{array}{l}\text { Virtual } \\
\text { Library }\end{array}$ & & & & & & $Y$ & HWIS & \\
\hline 670 & $200-\mathrm{E}$ & 299-E25-11 & A4761 & D & DN, GG, NT, TP & $\begin{array}{l}\text { Virtual } \\
\text { Library }\end{array}$ & $\begin{array}{l}\text { Virtual } \\
\text { Library }\end{array}$ & & & & & & $\mathrm{Y}$ & HWIS & \\
\hline 671 & $200-\mathrm{E}$ & 299-E25-110 & A6546 & D & & $\begin{array}{l}\text { Virtual } \\
\text { Library }\end{array}$ & $\begin{array}{l}\text { Virtual } \\
\text { Library }\end{array}$ & & & & & & $Y$ & HWIS & \\
\hline 672 & $200-\mathrm{E}$ & 299-E25-111 & A6547 & D & & $\begin{array}{l}\text { Virtual } \\
\text { Library }\end{array}$ & $\begin{array}{l}\text { Virtual } \\
\text { Library }\end{array}$ & & & & & & $\mathrm{Y}$ & HWIS & \\
\hline 673 & $200-\mathrm{E}$ & 299-E25-112 & A6548 & D & & $\begin{array}{l}\text { Virtual } \\
\text { Library }\end{array}$ & $\begin{array}{l}\text { Virtual } \\
\text { Library }\end{array}$ & & & & & & $Y$ & HWIS & \\
\hline 674 & $200-\mathrm{E}$ & 299-E25-113 & A6549 & D & & \begin{tabular}{|l|} 
Virtual \\
Library
\end{tabular} & \begin{tabular}{|l|} 
Virtual \\
Library
\end{tabular} & & Field Rad & & & & $\mathrm{Y}$ & HWIS & \\
\hline 675 & $200-E$ & 299-E25-114 & A6550 & D & & & & & & & & & $\mathrm{Y}$ & HWIS & \\
\hline 676 & $200-\mathrm{E}$ & 299-E25-115 & A6551 & D & & $\begin{array}{l}\text { Virtual } \\
\text { Library } \\
\end{array}$ & $\begin{array}{l}\text { Virtual } \\
\text { Library }\end{array}$ & & & & & & $Y$ & HWIS & \\
\hline 677 & $200-\mathrm{E}$ & 299-E25-116 & A6552 & D & & $\begin{array}{l}\text { Virtual } \\
\text { Library }\end{array}$ & $\begin{array}{l}\text { Virtual } \\
\text { Library }\end{array}$ & & Field Rad & & & & $\mathrm{Y}$ & HWIS & \\
\hline 678 & $200-\mathrm{E}$ & 299-E25-117 & A6553 & D & & $\begin{array}{l}\text { Virtual } \\
\text { Library }\end{array}$ & $\begin{array}{l}\text { Virtual } \\
\text { Library }\end{array}$ & & & & & & $\mathrm{Y}$ & HWIS & \\
\hline 679 & $200-\mathrm{E}$ & 299-E25-118 & A6554 & D & & $\begin{array}{l}\text { Virtual } \\
\text { Library }\end{array}$ & $\begin{array}{l}\text { Virtual } \\
\text { Library }\end{array}$ & & & & & & & HWIS & \\
\hline 680 & $200-\mathrm{E}$ & 299-E25-119 & A6555 & D & & \begin{tabular}{|l} 
Virtual \\
Library
\end{tabular} & \begin{tabular}{|l|} 
Virtual \\
Library
\end{tabular} & & Field Rad & & & & $Y$ & HWIS & \\
\hline 681 & $200-\mathrm{E}$ & 299-E25-12 & A6028 & D & DN, GG, NT, TP & $\begin{array}{l}\text { Virtual } \\
\text { Library }\end{array}$ & $\begin{array}{l}\text { Virtual } \\
\text { Library }\end{array}$ & & & & & & $\mathrm{Y}$ & HWIS & \\
\hline 682 & $200-\mathrm{E}$ & 299-E25-120 & A6556 & D & & \begin{tabular}{|l} 
Virtual \\
Library
\end{tabular} & $\begin{array}{l}\text { Virtual } \\
\text { Library }\end{array}$ & & & & & & $Y$ & HWIS & \\
\hline 683 & $200-E$ & 299-E25-121 & A6557 & D & & \begin{tabular}{|l} 
Virtual \\
Library
\end{tabular} & \begin{tabular}{|l|} 
Virtual \\
Library
\end{tabular} & & & & & & $\mathrm{Y}$ & HWIS & 1-ft sample interval \\
\hline
\end{tabular}




\begin{tabular}{|c|c|c|c|c|c|c|c|c|c|c|c|c|c|c|c|}
\hline SortIndex & Area & Well Name & WellID & Log Type & GPX Log Runs & Sieved & $\mathrm{CaCO} 3$ & Moisture & $\begin{array}{c}\text { Chemical } \\
\text { Properties } \\
\end{array}$ & $\begin{array}{c}\text { Physical } \\
\text { Properties } \\
\end{array}$ & \begin{tabular}{|c|} 
Min \\
Properties \\
\end{tabular} & $\begin{array}{l}\text { Geochron } \\
\text { Properties } \\
\end{array}$ & Archived & Source & Comments \\
\hline 684 & $200-E$ & 299-E25-122 & A6558 & D & & \begin{tabular}{|l|} 
Virtual \\
Library
\end{tabular} & \begin{tabular}{|l|} 
Virtual \\
Library
\end{tabular} & & & & & & $\mathrm{Y}$ & HWIS & \\
\hline 685 & 5 & 299-E25-123 & A6559 & D & & $\begin{array}{l}\text { Virtual } \\
\text { Library }\end{array}$ & $\begin{array}{l}\text { Virtual } \\
\text { Library }\end{array}$ & & & & & & $Y$ & HWIS & \\
\hline 686 & $200-E$ & 299-E25-124 & A6560 & D & & $\begin{array}{l}\text { Virtual } \\
\text { Library }\end{array}$ & $\begin{array}{l}\text { Virtual } \\
\text { Library }\end{array}$ & & Field Rad & & & & $Y$ & HWIS & \\
\hline 687 & $200-E$ & 299-E25-127 & A6561 & D & & & & & & & & & $Y$ & HWIS & \\
\hline 688 & $200-E$ & 299-E25-128 & A6562 & D & & & & & Field Rad & & & & & HWIS & \\
\hline 689 & $200-E$ & 299-E25-13 & A4762 & D & & \begin{tabular}{|l|} 
Virtual \\
Library
\end{tabular} & $\begin{array}{l}\text { Virtual } \\
\text { Library }\end{array}$ & & & & & & $Y$ & HWIS & \\
\hline 690 & $200-E$ & 299-E25-132 & A6563 & D & & & & & & & & & $Y$ & HWIS & \\
\hline 691 & $200-E$ & 299-E25-14 & A6029 & D & DN, GG, NT & & & & Field Rad & $\%$ GSSC & & & & Brown 1963 & \\
\hline 692 & $200-E$ & 299-E25-146 & A6564 & D & & \begin{tabular}{|l|} 
Virtual \\
Library
\end{tabular} & $\begin{array}{l}\text { Virtual } \\
\text { Library }\end{array}$ & & & & & & $Y$ & HWIS & \\
\hline 693 & $200-E$ & 299-E25-147 & A6565 & D & & & & & & & & & $Y$ & & \\
\hline 694 & $200-E$ & 299-E25-148 & A6566 & D & & & & & Field Rad & & & & & HWIS & \\
\hline 695 & $200-E$ & 299-E25-149 & A6567 & D & & & & & Field Rad & & & & & HWIS & \\
\hline 696 & $200-E$ & 299-E25-15 & A4763 & D & & \begin{tabular}{|l|} 
Virtual \\
Library
\end{tabular} & $\begin{array}{l}\text { Virtual } \\
\text { Library }\end{array}$ & & & & XRD, EM & & $Y$ & Ames 1976 & \\
\hline 697 & $200-E$ & 299-E25-152 & A6568 & D & & & & & & & & & & HWIS & \\
\hline 698 & $200-E$ & 299-E25-153 & A6569 & D & & & & & & & & & $Y$ & HWIS & \\
\hline 699 & $200-E$ & 299-E25-154 & A6570 & D & & & & & & & & & $Y$ & HWIS & \\
\hline 700 & $200-E$ & 299-E25-155 & A6571 & D & & & & & & & & & $Y$ & HWIS & \\
\hline 701 & $200-E$ & 299-E25-156 & A6572 & D & & & & & & & & & & HWIS & \\
\hline 702 & $200-E$ & 299-E25-157 & A6573 & D & & & & & & & & & $Y$ & HWIS & \\
\hline 703 & $200-E$ & 299-E25-158 & A6574 & D & & & & & & & & & $Y$ & HWIS & \\
\hline 704 & $200-E$ & 299-E25-159 & A6575 & D & & & & & & & & & $Y$ & HWIS & \\
\hline 705 & $200-E$ & 299-E25-16 & A6030 & D & & & & & & & & & & HWIS & \\
\hline 706 & $200-E$ & 299-E25-160 & A6576 & D & & & & & & & & & $Y$ & HWIS & \\
\hline 707 & $200-E$ & 299-E25-161 & A6577 & D & & & & & & & & & $Y$ & HWIS & \\
\hline 708 & $200-E$ & 299-E25-162 & A6578 & D & & & & & & & & & $Y$ & HWIS & \\
\hline 709 & $200-\mathrm{E}$ & 299-E25-163 & A6579 & D & & & & & & & & & $Y$ & HWIS & \\
\hline 710 & $200-E$ & 299-E25-164 & A6580 & D & & & & & & & & & $Y$ & HWIS & \\
\hline 711 & $200-E$ & 299-E25-165 & A9793 & D & & & & & & & & & $Y$ & HWIS & \\
\hline 712 & $200-E$ & 299-E25-166 & A6581 & D & & & & & & & & & $Y$ & HWIS & \\
\hline 713 & $200-E$ & 299-E25-167 & A6582 & $\mathrm{D}$ & & & & & & & & & $Y$ & HWIS & \\
\hline 714 & $200-E$ & 299-E25-168 & A6583 & $\mathrm{D}$ & & & & & & & & & $Y$ & HWIS & \\
\hline 715 & $200-E$ & 299-E25-169 & A6584 & D & GG, NT & & & & Field Rad & & & & & HWIS & (Same as well 299-E25-98) \\
\hline 716 & 200-E & 299-E25-17 & A6031 & D & GG, NT, SG & $\begin{array}{l}\text { Virtual } \\
\text { Library }\end{array}$ & $\begin{array}{l}\text { Virtual } \\
\text { Library }\end{array}$ & & & & & & $Y$ & HWIS & \\
\hline 717 & $200-E$ & 299-E25-170 & A6585 & D & & & & & & & & & & HWIS & \\
\hline 718 & $200-E$ & 299-E25-171 & A6586 & D & & & & & & & & & & HWIS & \\
\hline 719 & $200-E$ & 299-E25-172 & A6587 & $\mathrm{D}$ & & & & & Field Rad & & & & & HWIS & \\
\hline 720 & $200-E$ & 299-E25-173 & A6588 & $\mathrm{D}$ & & & & & Field Rad & & & & & HWIS & \\
\hline 721 & $200-E$ & 299-E25-174 & A6589 & D & & & & & & & & & & HWIS & \\
\hline 722 & $200-E$ & 299-E25-179 & A6590 & D & & & & & & & & & & HWIS & \\
\hline 723 & $200-E$ & 299-E25-18 & A4764 & D & GG, NT, SG & $\begin{array}{l}\text { Virtual } \\
\text { Library }\end{array}$ & $\begin{array}{l}\text { Virtual } \\
\text { Library }\end{array}$ & & & & & & $Y$ & HWIS & \\
\hline 724 & $200-E$ & 299-E25-181 & A6591 & D & & & & & & & & & & HWIS & \\
\hline 725 & $200-E$ & 299-E25-182 & A6592 & D & & & & & & & & & & HWIS & \\
\hline 726 & $200-E$ & 299-E25-183 & A6593 & D & & & & & & & & & & HWIS & \\
\hline 727 & $200-E$ & 299-E25-184 & A6594 & D & & & & & & & & & $Y$ & HWIS & \\
\hline 728 & $200-E$ & 299-E25-185 & A6595 & $\mathrm{D}$ & & & & & & & & & & & \\
\hline 729 & 200-E & 299-E25-189 & & D & & & & & & & & & & & \\
\hline
\end{tabular}




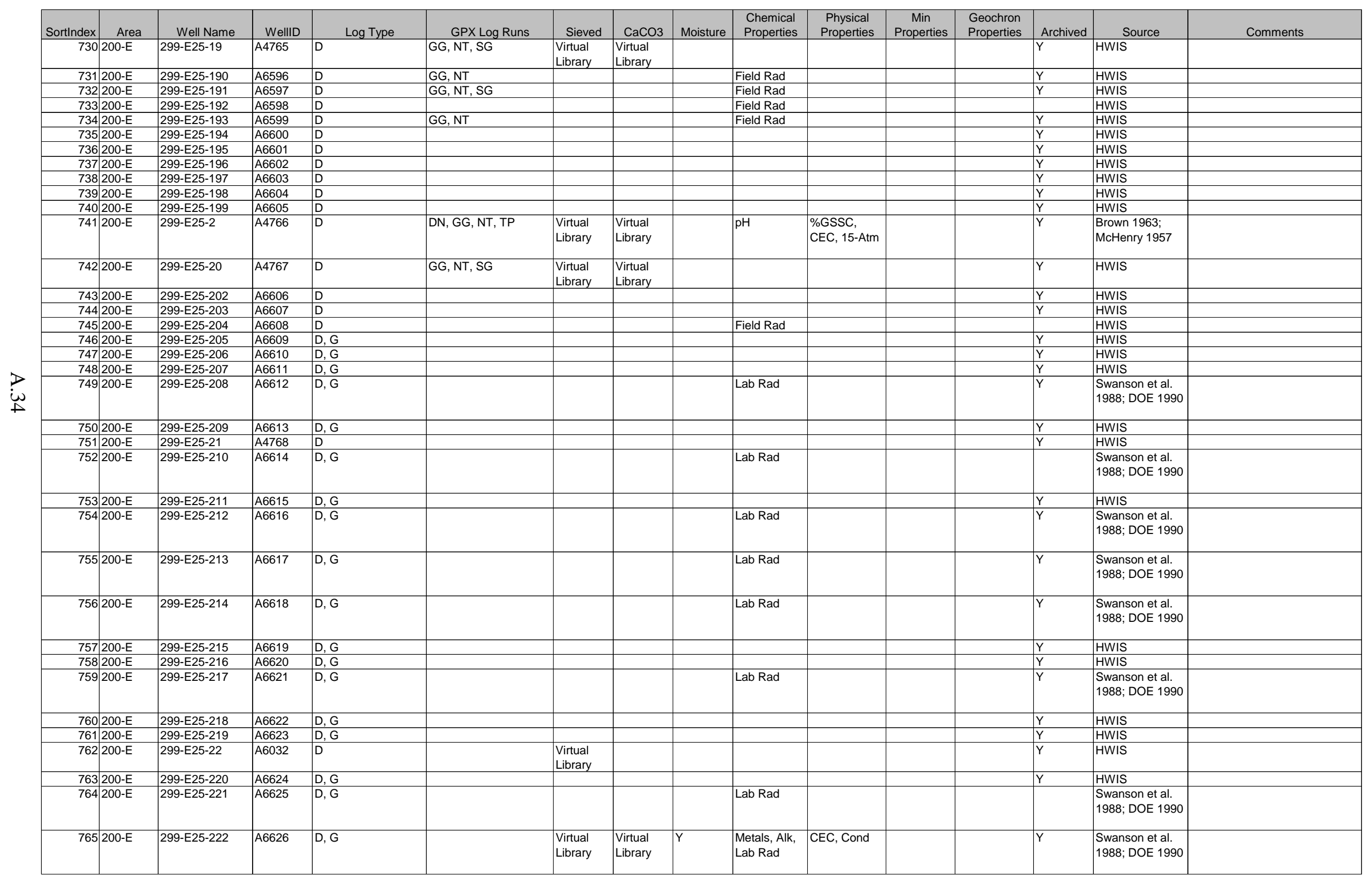




\begin{tabular}{|c|c|c|c|c|c|c|c|c|c|c|c|c|c|c|c|}
\hline Sortindex & $x \quad$ Area & Well Name & WellID & Log Type & GPX Log Runs & Sieved & $\mathrm{CaCO} 3$ & Moisture & $\begin{array}{l}\text { Chemical } \\
\text { Properties }\end{array}$ & $\begin{array}{l}\text { Physical } \\
\text { Properties }\end{array}$ & $\begin{array}{c}\text { Min } \\
\text { Properties }\end{array}$ & $\begin{array}{l}\text { Geochron } \\
\text { Properties }\end{array}$ & Archived & Source & Comments \\
\hline \begin{tabular}{r|}
766 \\
\end{tabular} & \begin{tabular}{|l|l|}
6 & $200-E$ \\
\end{tabular} & 299-E25-223 & A6627 & D, G & & $\begin{array}{l}\text { Virtual } \\
\text { Library }\end{array}$ & & Y & $\begin{array}{l}\text { Metals, Alk, } \\
\text { Lab Rad }\end{array}$ & CEC, Cond & & & $\mathrm{Y}$ & $\begin{array}{l}\text { Swanson et al. } \\
\text { 1988; DOE 1990 }\end{array}$ & \\
\hline 767 & $7200-E$ & 299-E25-224 & A6628 & D, G & & & & Y & $\begin{array}{l}\text { Metals, Alk, } \\
\text { Lab Rad }\end{array}$ & CEC, Cond & & & $Y$ & $\begin{array}{l}\text { Swanson et al. } \\
\text { 1988; DOE } 1990\end{array}$ & \\
\hline 768 & $8200-E$ & 299-E25-225 & A6629 & D, G & & \begin{tabular}{|l|} 
Virtual \\
Library
\end{tabular} & & Y & $\begin{array}{l}\text { Metals, Alk, } \\
\text { Lab Rad }\end{array}$ & CEC, Cond & & & Y & $\begin{array}{l}\text { Swanson et al. } \\
\text { 1988; DOE } 1990\end{array}$ & \\
\hline 769 & $9200-E$ & 299-E25-226 & A6630 & D, G & & \begin{tabular}{|l|} 
Virtual \\
Library
\end{tabular} & & Y & $\begin{array}{l}\text { Metals, Alk, } \\
\text { Lab Rad }\end{array}$ & CEC, Cond & & & $Y$ & \begin{tabular}{|l|} 
Swanson et al. \\
1988; DOE 1990
\end{tabular} & \\
\hline 770 & $0200-E$ & 299-E25-227 & A6631 & D, G & & \begin{tabular}{|l|} 
Virtual \\
Library
\end{tabular} & & Y & $\begin{array}{l}\text { Metals, Alk, } \\
\text { Lab Rad }\end{array}$ & CEC, Cond & & & $Y$ & \begin{tabular}{|l|} 
Swanson et al. \\
1988; DOE 1990
\end{tabular} & \\
\hline 771 & $200-E$ & 299-E25-228 & A6632 & D, G & & $\begin{array}{l}\text { Virtual } \\
\text { Library }\end{array}$ & & & & & & & $\mathrm{Y}$ & HWIS & \\
\hline 772 & $2200-E$ & 299-E25-229 & A6633 & D, G & & Y & & & & & & & Y & Serkowski 1986 & \\
\hline 773 & $3200-E$ & 299-E25-23 & A6033 & D & & & & & & & & & Y & HWIS & \\
\hline 774 & $4200-E$ & 299-E25-230 & A6634 & D, G & & $Y$ & & & & & & & $Y$ & Serkowski 1986 & \\
\hline 775 & $5200-E$ & 299-E25-231 & A6635 & $D, G$ & & \begin{tabular}{|l} 
Virtual \\
Library
\end{tabular} & Y & & & & & & $Y$ & Serkowski 1986 & \\
\hline 776 & $\begin{array}{l}6200-E \\
\end{array}$ & 299-E25-232 & A6636 & D, G & & $\begin{array}{l}\text { Virtual } \\
\text { Library }\end{array}$ & $Y$ & & & & & & $Y$ & Serkowski 1986 & \\
\hline 777 & $2200-E$ & 299-E25-233 & A6637 & D, G & & & & & & & & & $\mathrm{Y}$ & HWIS & \\
\hline 778 & $8200-E$ & 299-E25-234 & A6638 & D, G & & \begin{tabular}{|l|} 
Virtual \\
Library
\end{tabular} & & Y & & Bden & & & $\mathrm{Y}$ & $\begin{array}{l}\text { DOE 1990; } \\
\text { Swanson et al. } \\
\text { 1988; Smoot } \\
\text { et al. } 1989\end{array}$ & \\
\hline 779 & $9200-E$ & 299-E25-235 & A6639 & D, G & & \begin{tabular}{|l|l|} 
Virtual \\
Library
\end{tabular} & \begin{tabular}{|l|} 
Virtual \\
Library
\end{tabular} & Y & & & & & $\mathrm{Y}$ & DOE 1990 & \\
\hline 780 & $200-E$ & 299-E25-24 & A4769 & D & & & & & & & & & $Y$ & HWIS & \\
\hline 781 & $200-E$ & 299-E25-25 & A4770 & D, G & DN, GG, NT & \begin{tabular}{|l|} 
Virtual \\
Library
\end{tabular} & & Y & $\begin{array}{l}\text { Metals, Alk, } \\
\text { Lab Rad }\end{array}$ & CEC, Cond & & & $\mathrm{Y}$ & \begin{tabular}{|l|} 
Swanson et al. \\
1988; DOE 1990
\end{tabular} & \\
\hline 782 & $200-E$ & 299-E25-26 & A4771 & $D, G$ & DN, GG, NT & \begin{tabular}{|l|} 
Virtual \\
Library
\end{tabular} & $\begin{array}{l}\text { Virtual } \\
\text { Library }\end{array}$ & Y & $\begin{array}{l}\text { Metals, Alk, } \\
\text { Lab Rad }\end{array}$ & CEC, Cond & & & $\mathrm{Y}$ & $\begin{array}{l}\text { Swanson et al. } \\
\text { 1988; DOE } 1990\end{array}$ & \\
\hline 783 & $200-E$ & 299-E25-27 & A4772 & D, G & DN, GG, MO, NT & & & Y & $\begin{array}{l}\text { Metals, Alk, } \\
\text { Lab Rad }\end{array}$ & CEC, Cond & & & $Y$ & $\begin{array}{l}\text { Swanson et al. } \\
\text { 1988; DOE } 1990\end{array}$ & \\
\hline 784 & $200-E$ & 299-E25-28 & A4773 & $D, G$ & DN, GG, NT & \begin{tabular}{|l|l|} 
Virtual \\
Library
\end{tabular} & & Y & & & & & $\mathrm{Y}$ & DOE 1990 & \\
\hline 785 & 200-E & \begin{tabular}{|l|}
$299-E 25-29$ \\
\end{tabular} & A6034 & D, G & GG, MO, NT & $\begin{array}{l}\text { Virtual } \\
\text { Library }\end{array}$ & $\begin{array}{l}\text { Virtual } \\
\text { Library }\end{array}$ & Y & & & & & $\mathrm{Y}$ & DOE 1990 & \\
\hline 786 & |200-E & 299-E25-3 & A6024 & D & DN, GG, NT, TP & $\begin{array}{l}\text { Virtual } \\
\text { Library }\end{array}$ & $\begin{array}{l}\text { Virtual } \\
\text { Library }\end{array}$ & & & & & & $\mathrm{Y}$ & HWIS & \\
\hline 787 & $7200-E$ & \begin{tabular}{|l|} 
299-E25-30 \\
\end{tabular} & A6035 & D, G & & $\begin{array}{l}\text { Virtual } \\
\text { Library }\end{array}$ & $\begin{array}{l}\text { Virtual } \\
\text { Library }\end{array}$ & Y & & & & & $\mathrm{Y}$ & DOE 1990 & \\
\hline 788 & $200-E$ & 299-E25-30Q & A4777 & & GG, MO & & & & & & & & & & \\
\hline 789 & $200-E$ & 299-E25-31 & A4778 & D, G & GG, MO, SG & \begin{tabular}{|l|} 
Virtual \\
Library
\end{tabular} & $\begin{array}{l}\text { Virtual } \\
\text { Library }\end{array}$ & & & & & & $\mathrm{Y}$ & HWIS & \\
\hline 790 & $200-E$ & \begin{tabular}{|l|} 
299-E25-32 \\
\end{tabular} & A6036 & D, G & DN, GG, NT & $\begin{array}{l}\text { Virtual } \\
\text { Library }\end{array}$ & $\begin{array}{l}\text { Virtual } \\
\text { Library }\end{array}$ & Y & & & & & $Y$ & DOE 1990 & \\
\hline
\end{tabular}




\begin{tabular}{|c|c|c|c|c|c|c|c|c|c|c|c|c|c|c|c|}
\hline SortIndex & Area & Well Name & WelliD & Log Type & GPX Log Runs & Sieved & $\mathrm{CaCO} 3$ & Moisture & $\begin{array}{l}\text { Chemical } \\
\text { Properties }\end{array}$ & $\begin{array}{c}\text { Physical } \\
\text { Properties } \\
\end{array}$ & $\begin{array}{c}\text { Min } \\
\text { Properties } \\
\end{array}$ & $\begin{array}{l}\text { Geochron } \\
\text { Properties } \\
\end{array}$ & Archived & Source & Comments \\
\hline 791 & $200-E$ & 299-E25-32P & A4779 & & $\mathrm{MO}$ & & & & & & & & & & \\
\hline 792 & $200-E$ & 299-E25-32Q & A4780 & & $\mathrm{MO}$ & & & & & & & & & & \\
\hline 793 & $200-E$ & 299-E25-33 & A4781 & $\mathrm{D}, \mathrm{G}$ & DN, GG, NT & & & $\mathrm{Y}$ & & & & & $Y$ & DOE 1990 & \\
\hline 794 & $200-E$ & 299-E25-34 & A4782 & G & & & & $Y$ & & & & & $Y$ & & \\
\hline 795 & $200-\mathrm{E}$ & 299-E25-35 & A4783 & G & DN, GG, NT & & & $Y$ & & & & & $Y$ & & \\
\hline 796 & $200-E$ & 299-E25-36 & A4784 & G & DN, GG, NT & & & & & & & & $Y$ & & \\
\hline 797 & $200-E$ & 299-E25-37 & A4785 & G & GG, MO, SG & \begin{tabular}{|l|} 
Virtual \\
Library
\end{tabular} & & $Y$ & $Y$ & & & & $Y$ & Teel 1990 & \\
\hline 798 & $200-\mathrm{E}$ & 299-E25-38 & A4786 & G & GG, MO, SG & $\begin{array}{l}\text { Virtual } \\
\text { Library }\end{array}$ & & $Y$ & $Y$ & & & & $Y$ & Teel 1990 & \\
\hline 799 & $200-\mathrm{E}$ & 299-E25-39 & A4787 & G & $\mathrm{GG}, \mathrm{MO}, \mathrm{SG}$ & & $Y$ & $Y$ & & & & & $Y$ & Swanson 1992 & \\
\hline 800 & $200-E$ & 299-E25-4 & A4788 & D & DN, GG, NT, SG, TP & \begin{tabular}{|l|} 
Virtual \\
Library
\end{tabular} & \begin{tabular}{|l|} 
Virtual \\
Library
\end{tabular} & & & & & & $Y$ & HWIS & \\
\hline 801 & $200-\mathrm{E}$ & 299-E25-40 & A4789 & G & GG & $\begin{array}{l}\text { Virtual } \\
\text { Library }\end{array}$ & & $Y$ & $Y$ & & & & $Y$ & Pearson 1990 & \\
\hline 802 & $200-\mathrm{E}$ & 299-E25-41 & A4790 & G & GG & $\begin{array}{l}\text { Virtual } \\
\text { Library }\end{array}$ & & $Y$ & $Y$ & & & & $Y$ & Pearson 1990 & \\
\hline 803 & $200-E$ & 299-E25-42 & A4791 & G & GG, SG & $Y$ & $Y$ & $Y$ & & & & & $Y$ & Kasza 1992 & \\
\hline 804 & $200-E$ & 299-E25-43 & A4792 & G & $\mathrm{GG}, \mathrm{SG}$ & $Y$ & $Y$ & $Y$ & & & & & $Y$ & Kasza 1992 & \\
\hline 805 & $200-\mathrm{E}$ & 299-E25-44 & A5448 & G & GG, MO, SG & & $Y$ & $Y$ & & & & & $Y$ & Swanson 1993 & \\
\hline 806 & $200-E$ & \begin{tabular}{|l|}
$299-E 25-45$ \\
\end{tabular} & A5449 & G & GG, MO, SG & & $Y$ & $Y$ & & & & & $Y$ & Swanson 1993 & \\
\hline 807 & $200-E$ & 299-E25-46 & A4793 & G & GG & $Y$ & $\mathrm{Y}$ & Y & & & & & $Y$ & Caggiano 1993 & \\
\hline 808 & $200-E$ & 299-E25-47 & A4794 & G & GG & & $Y$ & $Y$ & & & & & Y & Kasza 1993 & \\
\hline 809 & $200-E$ & 299-E25-48 & A4795 & G & GG, MG, SG & & $Y$ & $Y$ & & & & & $Y$ & Kasza 1993 & \\
\hline 810 & $200-E$ & 299-E25-49 & A6038 & G & $\mathrm{GG}, \mathrm{MO}, \mathrm{SG}$ & & $Y$ & $Y$ & \begin{tabular}{|l|} 
Field Rad \\
(Alpha, \\
Beta/Gamma \\
)
\end{tabular} & & & & $Y$ & Swanson 1994 & \\
\hline 811 & $200-\mathrm{E}$ & 299-E25-5 & A6025 & D & $\mathrm{DN}, \mathrm{GG}, \mathrm{NT}, \mathrm{SG}$ & \begin{tabular}{|l|} 
Virtual \\
Library
\end{tabular} & $\begin{array}{l}\text { Virtual } \\
\text { Library }\end{array}$ & & \begin{tabular}{|l|} 
MiscMetals \\
\end{tabular} & & & & $Y$ & Haney 1967 & \\
\hline 812 & $200-\mathrm{E}$ & 299-E25-50 & A6039 & G & GG, MO, SG & & Y & $Y$ & \begin{tabular}{|l|} 
Field Rad \\
(Alpha, \\
Beta/Gamma \\
\end{tabular} & & & & $Y$ & Swanson 1994 & \\
\hline 813 & $200-E$ & 299-E25-51 & A6040 & D & & \begin{tabular}{|l|} 
Virtual \\
Library
\end{tabular} & \begin{tabular}{|l|} 
Virtual \\
Library
\end{tabular} & & & & & & $Y$ & HWIS & \\
\hline 814 & $200-\mathrm{E}$ & 299-E25-53 & A6042 & D & GG & $\begin{array}{l}\text { Virtual } \\
\text { Library }\end{array}$ & \begin{tabular}{|l|} 
Virtual \\
Library
\end{tabular} & & & & & & $Y$ & HWIS & \\
\hline 815 & $200-E$ & 299-E25-54 & A6043 & $\mathrm{D}$ & GG, SG & & & & & & & & $Y$ & HWIS & \\
\hline 816 & $200-\mathrm{E}$ & 299-E25-55 & A6044 & D & GG & $\begin{array}{l}\text { Virtual } \\
\text { Library }\end{array}$ & \begin{tabular}{|l|} 
Virtual \\
Library
\end{tabular} & & & & & & $Y$ & HWIS & \\
\hline 817 & $200-E$ & 299-E25-56 & A6045 & $\mathrm{D}$ & GG & & & & & & & & $\mathrm{Y}$ & HWIS & \\
\hline 818 & $200-E$ & 299-E25-57 & A6046 & D & GG & $\begin{array}{l}\text { Virtual } \\
\text { Library }\end{array}$ & \begin{tabular}{|l|} 
Virtual \\
Library
\end{tabular} & & & & & & Y & HWIS & \\
\hline 819 & $200-\mathrm{E}$ & 299-E25-58 & A6047 & D & GG & $\begin{array}{l}\text { Virtual } \\
\text { Library }\end{array}$ & $\begin{array}{l}\text { Virtual } \\
\text { Library }\end{array}$ & & & & & & $Y$ & HWIS & \\
\hline 820 & $200-\mathrm{E}$ & 299-E25-59 & A6048 & & GG & $\begin{array}{l}\text { Virtual } \\
\text { Library }\end{array}$ & $\begin{array}{l}\text { Virtual } \\
\text { Library }\end{array}$ & & & & & & & & \\
\hline 821 & $200-\mathrm{E}$ & 299-E25-6 & A4796 & D & $\mathrm{DN}, \mathrm{GG}, \mathrm{NT}, \mathrm{SG}$ & $\begin{array}{l}\text { Virtual } \\
\text { Library }\end{array}$ & \begin{tabular}{|l|} 
Virtual \\
Library
\end{tabular} & & MiscMetals & & & & $Y$ & Haney 1967 & \\
\hline 822 & $200-E$ & 299-E25-60 & A6049 & & GG & $\begin{array}{l}\text { Virtual } \\
\text { Library }\end{array}$ & $\begin{array}{l}\text { Virtual } \\
\text { Library }\end{array}$ & & & & & & $Y$ & & \\
\hline
\end{tabular}




\begin{tabular}{|c|c|c|c|c|c|c|c|c|c|c|c|c|c|c|c|}
\hline Sortindex & Area & Well Name & WelllD & Log Type & GPX Log Runs & Sieved & $\mathrm{CaCO} 3$ & Moisture & $\begin{array}{l}\text { Chemical } \\
\text { Properties }\end{array}$ & $\begin{array}{c}\text { Physical } \\
\text { Properties } \\
\end{array}$ & $\begin{array}{c}\text { Min } \\
\text { Properties } \\
\end{array}$ & $\begin{array}{l}\text { Geochron } \\
\text { Properties } \\
\end{array}$ & Archived & Source & Comments \\
\hline 823 & $\begin{array}{ll}3200-E \\
\end{array}$ & 299-E25-61 & A6500 & D & GG & \begin{tabular}{|l|} 
Virtual \\
Library
\end{tabular} & $\begin{array}{l}\text { Virtual } \\
\text { Library }\end{array}$ & & & & & & $\mathrm{Y}$ & HWIS & \\
\hline 824 & $4200-E$ & 299-E25-62 & A6501 & D & GG & & & & & & & & & HWIS & \\
\hline 825 & 5 & 299-E25-63 & A6502 & D & GG & $\begin{array}{l}\text { Virtual } \\
\text { Library }\end{array}$ & $\begin{array}{l}\text { Virtual } \\
\text { Library }\end{array}$ & & & & & & $Y$ & HWIS & \\
\hline 826 & \begin{tabular}{|l|l|}
6 & $200-E$ \\
\end{tabular} & 299-E25-64 & A6503 & & GG & & & & & & & & & & \\
\hline 827 & $200-E$ & 299-E25-65 & A6504 & D & GG & $\begin{array}{l}\text { Virtual } \\
\text { Library }\end{array}$ & \begin{tabular}{|l|} 
Virtual \\
Library
\end{tabular} & & & & & & $\mathrm{Y}$ & HWIS & \\
\hline 828 & \begin{tabular}{|l|l|}
$3200-E$ \\
\end{tabular} & 299-E25-66 & A6505 & D & GG & & & & & & & & $\mathrm{Y}$ & HWIS & \\
\hline 829 & $200-E$ & 299-E25-67 & A6506 & D & GG & $\begin{array}{l}\text { Virtual } \\
\text { Library }\end{array}$ & $\begin{array}{l}\text { Virtual } \\
\text { Library }\end{array}$ & & & & & & $\mathrm{Y}$ & & \\
\hline 830 & $200-E$ & 299-E25-68 & A6507 & D & GG & & & & Field Rad & & & & $\mathrm{Y}$ & HWIS & \\
\hline 831 & $1200-E$ & 299-E25-69 & A6508 & D & GG & $\begin{array}{l}\text { Virtual } \\
\text { Library }\end{array}$ & $\begin{array}{l}\text { Virtual } \\
\text { Library }\end{array}$ & & & & & & Y & HWIS & \\
\hline 832 & $200-E$ & 299-E25-7 & A6026 & D & DN, GG, MO, NT, SG & $\begin{array}{l}\text { Virtual } \\
\text { Library }\end{array}$ & $\begin{array}{l}\text { Virtual } \\
\text { Library }\end{array}$ & & & & & & $\mathrm{Y}$ & HWIS & \\
\hline 833 & \begin{tabular}{|l|l|}
$200-E$ \\
\end{tabular} & 299-E25-70 & A6509 & D & GG & & & & & & & & $\mathrm{Y}$ & HWIS & \\
\hline 834 & $4200-E$ & 299-E25-71 & A6510 & D & GG & & & & & & & & $Y$ & HWIS & \\
\hline 835 & $200-\mathrm{E}$ & 299-E25-72 & A6511 & D & GG & & & & Field Rad & & & & $\mathrm{Y}$ & HWIS & \\
\hline 836 & $6200-E$ & 299-E25-73 & A6512 & D & GG & & & & & & & & $\mathrm{Y}$ & HWIS & \\
\hline 837 & $200-E$ & 299-E25-74 & A6513 & D & GG & \begin{tabular}{|l|} 
Virtual \\
Library
\end{tabular} & $\begin{array}{l}\text { Virtual } \\
\text { Library }\end{array}$ & & Field Rad & & & & $Y$ & HWIS & \\
\hline 838 & \begin{tabular}{|l|l|}
3 & $200-E$ \\
\end{tabular} & 299-E25-75 & A6514 & D & GG & $\begin{array}{l}\text { Virtual } \\
\text { Library }\end{array}$ & $\begin{array}{l}\text { Virtual } \\
\text { Library }\end{array}$ & & & & & & $Y$ & HWIS & \\
\hline 839 & $9200-E$ & 299-E25-76 & A6515 & D & GG & & & & & & & & & HWIS & \\
\hline 840 & $200-E$ & 299-E25-77 & A6516 & D & GG & $\begin{array}{l}\text { Virtual } \\
\text { Library }\end{array}$ & $\begin{array}{l}\text { Virtual } \\
\text { Library }\end{array}$ & & & & & & $\mathrm{Y}$ & HWIS & \\
\hline 841 & $200-E$ & 299-E25-78 & A6517 & D & GG & & & & & & & & $Y$ & HWIS & \\
\hline 842 & $200-E$ & 299-E25-79 & A6518 & D & GG & $\begin{array}{l}\text { Virtual } \\
\text { Library }\end{array}$ & $\begin{array}{l}\text { Virtual } \\
\text { Library }\end{array}$ & & & & & & $\mathrm{Y}$ & HWIS & \\
\hline 843 & $3200-E$ & 299-E25-8 & A6027 & D & DN, GG, NT, SG & $\begin{array}{l}\text { Virtual } \\
\text { Library }\end{array}$ & $\begin{array}{l}\text { Virtual } \\
\text { Library }\end{array}$ & & & & & & $\mathrm{Y}$ & HWIS & \\
\hline 844 & (200-E & 299-E25-80 & A6519 & D & GG & $\begin{array}{l}\text { Virtual } \\
\text { Library }\end{array}$ & $\begin{array}{l}\text { Virtual } \\
\text { Library }\end{array}$ & & Field Rad & & & & $\mathrm{Y}$ & HWIS & \\
\hline 845 & 200-E & 299-E25-81 & A6520 & D & GG & $\begin{array}{l}\text { Virtual } \\
\text { Library }\end{array}$ & $\begin{array}{l}\text { Virtual } \\
\text { Library }\end{array}$ & & & & & & Y & HWIS & \\
\hline 846 & $6200-E$ & 299-E25-82 & A6521 & D & GG & & & & & & & & $\mathrm{Y}$ & HWIS & \\
\hline 847 & $200-E$ & 299-E25-83 & A6522 & $\mathrm{D}$ & GG & & & & & & & & $Y$ & HWIS & \\
\hline 848 & $200-\mathrm{E}$ & 299-E25-84 & A6523 & D & GG & & & & & & & & $\mathrm{Y}$ & HWIS & \\
\hline 849 & $200-E$ & 299-E25-85 & A6524 & D & GG & \begin{tabular}{|l|} 
Virtual \\
Library
\end{tabular} & $\begin{array}{l}\text { Virtual } \\
\text { Library }\end{array}$ & & & & & & $\mathrm{Y}$ & HWIS & \\
\hline 850 & $200-E$ & 299-E25-86 & A6525 & D & GG & $\begin{array}{l}\text { Virtual } \\
\text { Library }\end{array}$ & $\begin{array}{l}\text { Virtual } \\
\text { Library }\end{array}$ & & & & & & $\mathrm{Y}$ & HWIS & \\
\hline 851 & 1 $200-\mathrm{E}$ & 299-E25-87 & A6526 & D & GG & & & & & & & & $Y$ & HWIS & \\
\hline 852 & $200-E$ & 299-E25-88 & A6527 & D & GG & & & & & & & & $\mathrm{Y}$ & HWIS & \\
\hline 853 & $200-E$ & 299-E25-89 & A6528 & D & GG & \begin{tabular}{|l|} 
Virtual \\
Library
\end{tabular} & $\begin{array}{l}\text { Virtual } \\
\text { Library }\end{array}$ & & & & & & $Y$ & HWIS & \\
\hline 854 & $200-E$ & 299-E25-9 & A4797 & D & DN, GG, NT, SG, TP & $\begin{array}{l}\text { Virtual } \\
\text { Library }\end{array}$ & $\begin{array}{l}\text { Virtual } \\
\text { Library }\end{array}$ & & & & & & $\mathrm{Y}$ & HWIS & \\
\hline 855 & $200-E$ & 299-E25-90 & A6529 & D & GG & & & & & & & & $\mathrm{Y}$ & HWIS & \\
\hline 856 & $200-E$ & 299-E25-91 & A6530 & D & GG & $\begin{array}{l}\text { Virtual } \\
\text { Library }\end{array}$ & \begin{tabular}{|l|} 
Virtual \\
Library
\end{tabular} & & & & & & $\mathrm{Y}$ & HWIS & \\
\hline 857 & $7200-E$ & 299-E25-92 & A6531 & D & GG & & & & & & & & $Y$ & HWIS & \\
\hline
\end{tabular}




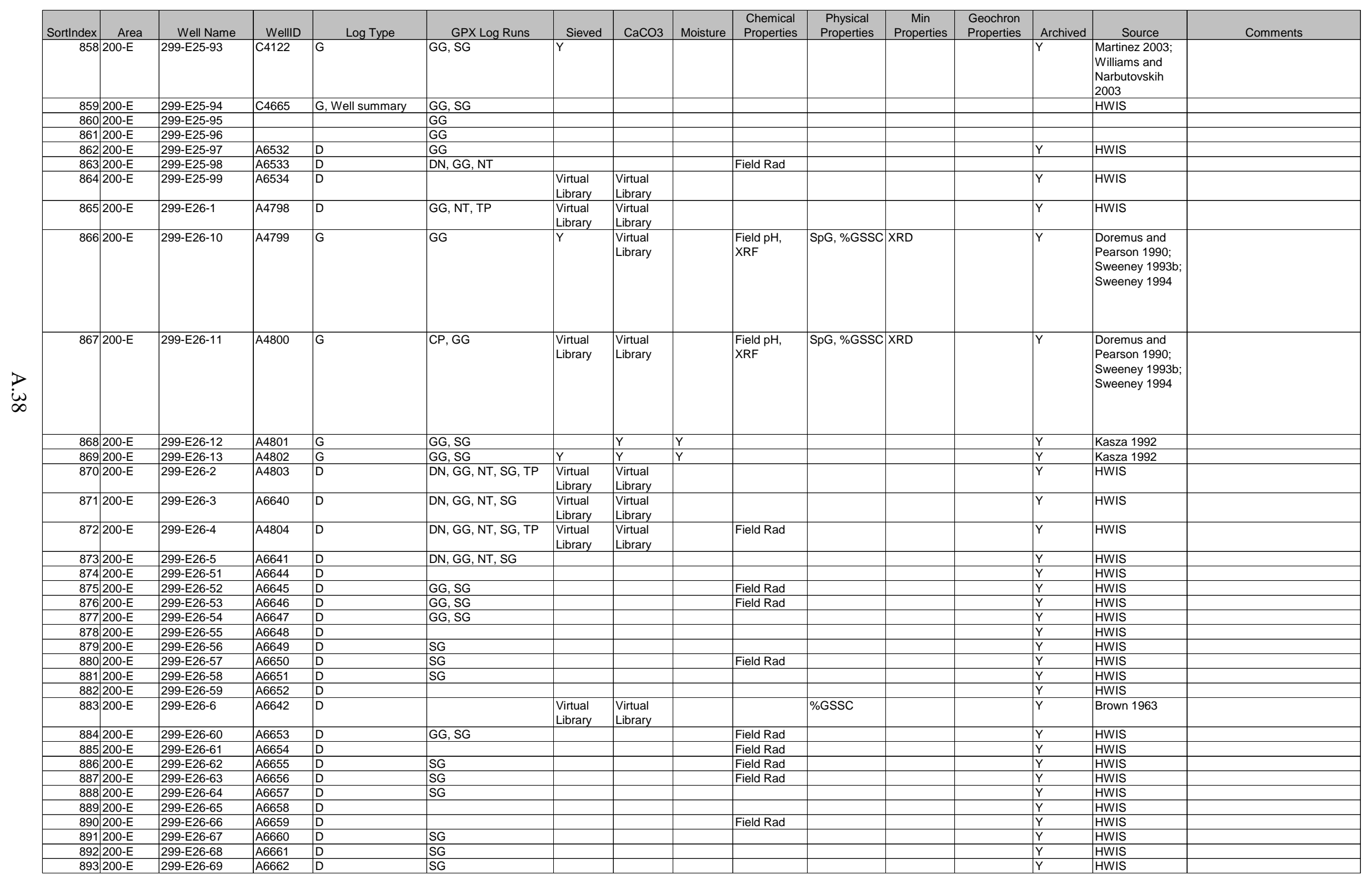




\begin{tabular}{|c|c|c|c|c|c|c|c|c|c|c|c|c|c|c|c|}
\hline Sortindex & Area & Well Name & WellID & Log Type & GPX Log Runs & Sieved & $\mathrm{CaCO} 3$ & Moisture & $\begin{array}{l}\text { Chemical } \\
\text { Properties }\end{array}$ & $\begin{array}{c}\text { Physical } \\
\text { Properties }\end{array}$ & \begin{tabular}{|c|} 
Min \\
Properties
\end{tabular} & $\begin{array}{l}\text { Geochron } \\
\text { Properties }\end{array}$ & Archived & Source & Comments \\
\hline 894 & $200-E$ & 299-E26-7 & A6643 & D & DN, GG, NT, SG & & & & & & & & & HWIS & \\
\hline 895 & $200-E$ & 299-E26-70 & A6663 & $\mathrm{D}$ & & & & & & & & & $Y$ & HWIS & \\
\hline 896 & $200-E$ & 299-E26-71 & A6664 & D & SG & & & & Field Rad & & & & $Y$ & HWIS & \\
\hline 897 & $200-E$ & 299-E26-72 & A6665 & D & & & & & & & & & $Y$ & HWIS & \\
\hline 898 & $200-E$ & 299-E26-73 & A6666 & $\mathrm{D}$ & SG & & & & & & & & $\mathrm{Y}$ & HWIS & \\
\hline 899 & $200-E$ & 299-E26-74 & A6667 & D & SG & & & & Field Rad & & & & $Y$ & HWIS & \\
\hline 900 & $200-E$ & 299-E26-75 & A6668 & D & SG & & & & Field Rad & & & & $Y$ & HWIS & \\
\hline 901 & $200-E$ & 299-E26-76 & A6669 & $\mathrm{D}$ & GG, SG & & & & Field Rad & & & & $Y$ & HWIS & \\
\hline 902 & $200-E$ & 299-E26-8 & A4805 & D & $\begin{array}{l}\text { CP, DN, GG, MG, NT, } \\
\text { OT, SN, TP }\end{array}$ & & & & & & & & Y & HWIS & \\
\hline 903 & $200-E$ & 299-E26-9 & A4806 & G & GG & $Y$ & \begin{tabular}{|l} 
Virtual \\
Library
\end{tabular} & & $\begin{array}{l}\text { Field pH, } \\
\text { XRF }\end{array}$ & $|\mathrm{SpG}, \% \mathrm{GSSC}|$ & XRD & & $Y$ & $\begin{array}{l}\text { Doremus and } \\
\text { Pearson 1990; } \\
\text { Sweeney 1993b; } \\
\text { Sweeney } 1994\end{array}$ & \\
\hline 904 & $200-E$ & 299-E27-1 & A4807 & D & GG, MO, NT, SG, TP & \begin{tabular}{|l|} 
Virtual \\
Library
\end{tabular} & \begin{tabular}{|l|} 
Virtual \\
Library
\end{tabular} & & $\mathrm{pH}$ & \begin{tabular}{|l|} 
CEC, \\
$\%$ \%SSC, 15- \\
Atm
\end{tabular} & & & $Y$ & McHenry 1957 & \\
\hline 905 & $200-E$ & 299-E27-10 & A4808 & G & DN, GG, NT & \begin{tabular}{|l|} 
Virtual \\
Library
\end{tabular} & & & & & & & Y & HWIS & \\
\hline 906 & $200-E$ & 299-E27-100 & A6725 & D & & $\begin{array}{l}\text { Virtual } \\
\text { Library }\end{array}$ & $\begin{array}{l}\text { Virtual } \\
\text { Library }\end{array}$ & & & & & & $Y$ & HWIS & \\
\hline 907 & $200-E$ & 299-E27-101 & A6726 & D & & $\begin{array}{l}\text { Virtual } \\
\text { Library }\end{array}$ & $\begin{array}{l}\text { Virtual } \\
\text { Library }\end{array}$ & & & & & & $Y$ & HWIS & \\
\hline 908 & $200-E$ & 299-E27-102 & A6727 & D & & \begin{tabular}{|l|} 
Virtual \\
Library
\end{tabular} & \begin{tabular}{|l|l|} 
Virtual \\
Library
\end{tabular} & & & & & & $Y$ & HWIS & \\
\hline 909 & $200-E$ & 299-E27-103 & A6728 & $\mathrm{D}$ & & & & & & & & & $\mathrm{Y}$ & HWIS & \\
\hline 910 & $200-E$ & 299-E27-104 & A6729 & D & & & & & & & & & $Y$ & HWIS & \\
\hline 911 & $200-E$ & 299-E27-105 & A6730 & $\mathrm{D}$ & & & & & & & & & $Y$ & HWIS & \\
\hline 912 & $200-E$ & 299-E27-106 & A6731 & D & & & & & & & & & $\mathrm{Y}$ & HWIS & \\
\hline 913 & $200-E$ & 299-E27-107 & A6732 & D & & & & & & & & & $\mathrm{Y}$ & HWIS & \\
\hline 914 & $200-E$ & 299-E27-108 & A6733 & D & & & & & & & & & $Y$ & HWIS & \\
\hline 915 & $200-E$ & 299-E27-109 & A6734 & D & & & & & & & & & $Y$ & HWIS & \\
\hline 916 & $200-E$ & 299-E27-11 & A4809 & G & GG & & & $\mathrm{Y}$ & \begin{tabular}{|l|} 
An, TOC, \\
VOA, Field \\
Rad
\end{tabular} & & & & $Y$ & $\begin{array}{l}\text { Goodwin and } \\
\text { Bjornstad } 1990\end{array}$ & Rich Mercer \\
\hline 917 & $200-E$ & 299-E27-115 & A6735 & D & & $\begin{array}{l}\text { Virtual } \\
\text { Library }\end{array}$ & \begin{tabular}{|l|} 
Virtual \\
Library
\end{tabular} & & & & & & $Y$ & HWIS & \\
\hline 918 & $200-E$ & 299-E27-116 & A6736 & D & & $\begin{array}{l}\text { Virtual } \\
\text { Library }\end{array}$ & $\begin{array}{l}\text { Virtual } \\
\text { Library }\end{array}$ & & Field Rad & & & & $Y$ & HWIS & \\
\hline \begin{tabular}{c|}
919 \\
\end{tabular} & $200-E$ & 299-E27-117 & A6737 & D & & \begin{tabular}{|l|} 
Virtual \\
Library
\end{tabular} & \begin{tabular}{|l|} 
Virtual \\
Library
\end{tabular} & & Field Rad & & & & $Y$ & HWIS & \\
\hline 920 & $200-E$ & 299-E27-118 & A6738 & D & & $\begin{array}{l}\text { Virtual } \\
\text { Library }\end{array}$ & \begin{tabular}{|l|} 
Virtual \\
Library
\end{tabular} & & Field Rad & & & & $Y$ & HWIS & \\
\hline 921 & $200-E$ & 299-E27-119 & A6739 & D & & $\begin{array}{l}\text { Virtual } \\
\text { Library }\end{array}$ & \begin{tabular}{|l|} 
Virtual \\
Library
\end{tabular} & & & & & & $Y$ & HWIS & \\
\hline 922 & $200-E$ & 299-E27-12 & A4810 & G & GG & & & & & & & & $Y$ & HWIS & \\
\hline 923 & $200-E$ & 299-E27-120 & A6740 & D & & & & & & & & & $Y$ & HWIS & \\
\hline 924 & $200-E$ & 299-E27-121 & A6741 & D & & & & & & & & & $Y$ & HWIS & \\
\hline 925 & $200-E$ & 299-E27-122 & A6742 & D & & & & & & & & & $Y$ & & \\
\hline 926 & $200-E$ & 299-E27-124 & A6744 & D & & & & & & & & & $Y$ & HWIS & \\
\hline 927 & $200-E$ & 299-E27-125 & A6745 & D & & & & & & & & & $Y$ & HWIS & \\
\hline 928 & $200-E$ & 299-E27-126 & A6746 & D & & & & & Field Rad & & & & $Y$ & HWIS & \\
\hline 929 & $200-E$ & 299-E27-127 & A6747 & D & & $\begin{array}{l}\text { Virtual } \\
\text { Library }\end{array}$ & \begin{tabular}{|l|} 
Virtual \\
Library
\end{tabular} & & Field Rad & & & & $Y$ & HWIS & \\
\hline
\end{tabular}




\begin{tabular}{|c|c|c|c|c|c|c|c|c|c|c|c|c|c|c|c|}
\hline SortIndex & Area & Well Name & WelllD & Log Type & GPX Log Runs & Sieved & $\mathrm{CaCO} 3$ & Moisture & $\begin{array}{l}\text { Chemical } \\
\text { Properties } \\
\end{array}$ & $\begin{array}{c}\text { Physical } \\
\text { Properties } \\
\end{array}$ & \begin{tabular}{|c|} 
Min \\
Properties \\
\end{tabular} & $\begin{array}{l}\text { Geochron } \\
\text { Properties } \\
\end{array}$ & Archived & Source & Comments \\
\hline 930 & $200-E$ & 299-E27-128 & A6748 & $\mathrm{D}$ & & & & & Field Rad & & & & $Y$ & HWIS & \\
\hline 931 & $200-E$ & 299-E27-129 & A6749 & D & & & & & Field Rad & & & & $\mathrm{Y}$ & HWIS & \\
\hline 932 & $200-\mathrm{E}$ & 299-E27-13 & A4811 & G & GG & \begin{tabular}{|l} 
Virtual \\
Library
\end{tabular} & & $Y$ & $Y$ & & & & $\mathrm{Y}$ & Pearson 1990 & \\
\hline 933 & $200-E$ & 299-E27-130 & A6750 & D & & & & & Field Rad & & & & $Y$ & HWIS & \\
\hline 934 & $200-E$ & 299-E27-131 & A6751 & D & & & & & Field Rad & & & & $Y$ & HWIS & \\
\hline 935 & $200-E$ & 299-E27-132 & A6752 & D & & & & & Field Rad & & & & $\mathrm{Y}$ & HWIS & \\
\hline 936 & $200-E$ & 299-E27-133 & A6753 & D & GG & & & & Field Rad & & & & $Y$ & HWIS & \\
\hline 937 & $200-E$ & 299-E27-135 & A6754 & D & & & & & & & & & $Y$ & HWIS & \\
\hline 938 & $200-E$ & 299-E27-137 & A6766 & $\mathrm{D}, \mathrm{G}$ & & & & & & & & & $Y$ & HWIS & \\
\hline 939 & $200-E$ & 299-E27-138 & A6767 & D, G & & & & & Field Rad & & & & & HWIS & \\
\hline 940 & $200-E$ & 299-E27-139 & A6768 & $\mathrm{D}, \mathrm{G}$ & & & & & & & & & $\mathrm{Y}$ & HWIS & \\
\hline 941 & $200-\mathrm{E}$ & 299-E27-14 & A4812 & G & GG & & & $Y$ & $\mathrm{Y}$ & & & & $Y$ & \begin{tabular}{|l|l} 
Pearson 1990 \\
\end{tabular} & \\
\hline 942 & $200-\mathrm{E}$ & 299-E27-140 & A6769 & $D, G$ & & & & & Field Rad & & & & $\mathrm{Y}$ & HWIS & \\
\hline 943 & $200-E$ & 299-E27-141 & A6770 & D, G & & & & & & & & & $Y$ & HWIS & \\
\hline 944 & $200-E$ & 299-E27-142 & A6771 & D & & & & & & & & & $\mathrm{Y}$ & HWIS & \\
\hline 945 & $200-E$ & 299-E27-143 & A6772 & D & & & & & & & & & $Y$ & HWIS & \\
\hline 946 & $200-\mathrm{E}$ & 299-E27-144 & A6773 & D & & & & & & & & & $Y$ & HWIS & \\
\hline 947 & $200-E$ & 299-E27-145 & A6774 & D & & & & & & & & & $\mathrm{Y}$ & HWIS & \\
\hline 948 & $200-E$ & 299-E27-146 & A6775 & D & & & & & & & & & $Y$ & HWIS & \\
\hline 949 & $200-\mathrm{E}$ & 299-E27-147 & A6776 & D & & \begin{tabular}{|l} 
Virtual \\
Library
\end{tabular} & $\begin{array}{l}\text { Virtual } \\
\text { Library }\end{array}$ & & & & & & $Y$ & HWIS & \\
\hline 950 & $200-E$ & 299-E27-148 & A6777 & D, G & & & & & Lab Rad & & & & $Y$ & $\begin{array}{l}\text { Subrahmanyam } \\
1986\end{array}$ & \\
\hline 951 & $200-E$ & 299-E27-149 & A6778 & D, G & & & & & & & & & $Y$ & HWIS & \\
\hline 952 & $200-\mathrm{E}$ & 299-E27-15 & A4813 & G & GG & & & $Y$ & $Y$ & & & & $\mathrm{Y}$ & Pearson 1990 & \\
\hline 953 & $200-\mathrm{E}$ & 299-E27-150 & A6779 & $D, G$ & & & & & & & & & Y & HWIS & \\
\hline 954 & $200-E$ & 299-E27-151 & A6780 & D, G & & & & & & & & & $Y$ & HWIS & \\
\hline 955 & $200-E$ & 299-E27-152 & A6781 & D & & & & & Lab Rad & & & & $Y$ & $\begin{array}{l}\text { Subrahmanyam } \\
1986\end{array}$ & \\
\hline 956 & $200-\mathrm{E}$ & 299-E27-153 & A6782 & D, G & & & & & Lab Rad & & & & $Y$ & $\begin{array}{l}\text { Subrahmanyam } \\
1986\end{array}$ & \\
\hline 957 & $200-E$ & 299-E27-154 & A6783 & D, G & & & & & & & & & & HWIS & \\
\hline 958 & $200-E$ & 299-E27-155 & & $D, G$ & & & & & & & & & $Y$ & & \\
\hline 959 & $200-\mathrm{E}$ & 299-E27-16 & A4814 & G & GG & & & & & & & & $Y$ & & \\
\hline 960 & $200-\mathrm{E}$ & 299-E27-17 & A4815 & G & GG, SG & & $Y$ & $Y$ & Field Rad & & & & $Y$ & Mercer 1993b & \\
\hline 961 & $200-\mathrm{E}$ & 299-E27-18 & A6674 & G & $G G, S G$ & & $\mathrm{Y}$ & $Y$ & & & & & $Y$ & Bjornstad 1993 & \\
\hline 962 & $200-E$ & 299-E27-19 & A6675 & G & GG, SG & & $Y$ & Y & & & & & $\mathrm{Y}$ & Bjornstad 1993 & \\
\hline 963 & 200-E & 299-E27-2 & A6670 & $\mathrm{D}, \mathrm{G}$ & & & & & & & & & $Y$ & HWIS & \\
\hline 964 & $200-E$ & 299-E27-20 & C4126 & G & & Y & & & & & & & Y & Martinez 2003 & \\
\hline 965 & $200-\mathrm{E}$ & 299-E27-21 & C4127 & G & SG & $Y$ & & & & & & & $Y$ & $\begin{array}{l}\text { Martinez 2003; } \\
\text { Williams and } \\
\text { Narbutovskih } \\
2004\end{array}$ & \\
\hline 966 & $200-E$ & 299-E27-22 & C4124 & $G$ & SG & Y & & & & & & & $\mathrm{Y}$ & $\begin{array}{l}\text { Martinez 2003; } \\
\text { Williams and } \\
\text { Narbutovskih } \\
2004\end{array}$ & \\
\hline
\end{tabular}




\begin{tabular}{|c|c|c|c|c|c|c|c|c|c|c|c|c|c|c|c|}
\hline SortIndex & Area & Well Name & WelllD & Log Type & GPX Log Runs & Sieved & $\mathrm{CaCO} 3$ & Moisture & $\begin{array}{c}\text { Chemical } \\
\text { Properties } \\
\end{array}$ & $\begin{array}{c}\text { Physical } \\
\text { Properties } \\
\end{array}$ & \begin{tabular}{|c|} 
Min \\
Properties \\
\end{tabular} & $\begin{array}{l}\text { Geochron } \\
\text { Properties } \\
\end{array}$ & Archived & Source & Comments \\
\hline 967 & $200-E$ & 299-E27-23 & C4190 & G & SG & $Y$ & & & & & & & Y & $\begin{array}{l}\text { Martinez 2003; } \\
\text { Williams and } \\
\text { Narbutovskih }\end{array}$ & \\
\hline 968 & $200-E$ & 299-E27-3 & A6671 & D & GG, NT, TP & $\begin{array}{l}\text { Virtual } \\
\text { Library }\end{array}$ & $\begin{array}{l}\text { Virtual } \\
\text { Library }\end{array}$ & & & & & & $Y$ & HWIS & \\
\hline 969 & $200-E$ & 299-E27-4 & C4125 & G & SG & $\mathrm{Y}$ & & & & & & & Y & $\begin{array}{l}\text { Martinez 2003; } \\
\text { Williams and } \\
\text { Narbutovskih } \\
2004\end{array}$ & \\
\hline 970 & $200-E$ & 299-E27-5 & A6672 & D & DN, GG, NT, SG, TP & \begin{tabular}{|l|} 
Virtual \\
Library
\end{tabular} & \begin{tabular}{|l|l|} 
Virtual \\
Library
\end{tabular} & & & & & & $Y$ & HWIS & \\
\hline 971 & $200-E$ & 299-E27-51 & A6676 & D & GG & & & & & & & & & HWIS & \\
\hline 972 & $200-E$ & 299-E27-52 & A6677 & D & GG & & & & & & & & $Y$ & HWIS & \\
\hline 973 & $200-E$ & 299-E27-53 & A6678 & $\mathrm{D}$ & & & & & & & & & & HWIS & \\
\hline 974 & $200-E$ & 299-E27-54 & A6679 & $\mathrm{D}$ & GG & & & & & & & & & HWIS & \\
\hline 975 & $200-E$ & 299-E27-55 & A6680 & D & GG & & & & & & & & & & \\
\hline 976 & $200-E$ & 299-E27-56 & A6681 & D & GG & & & & & & & & & HWIS & \\
\hline 977 & $200-E$ & 299-E27-57 & A6682 & D & GG & & & & & & & & . & HWIS & \\
\hline 978 & $200-E$ & 299-E27-58 & A6683 & D & & & & & & & & & & HWIS & \\
\hline 979 & $200-E$ & 299-E27-59 & A6684 & D & & & & & & & & & & HWIS & \\
\hline 980 & $200-E$ & 299-E27-6 & A6673 & D & & $\begin{array}{l}\text { Virtual } \\
\text { Library }\end{array}$ & $\begin{array}{l}\text { Virtual } \\
\text { Library }\end{array}$ & & & & & & $\mathrm{Y}$ & HWIS & \\
\hline 981 & $200-E$ & 299-E27-60 & A6685 & D & & & & & Field Rad & & & & $Y$ & HWIS & \\
\hline 982 & $200-E$ & 299-E27-61 & A6686 & $\mathrm{D}$ & & & & & & & & & & HWIS & \\
\hline 983 & $200-E$ & 299-E27-62 & A6687 & $\mathrm{D}$ & & & & & & & & & & HWIS & \\
\hline 984 & $200-E$ & 299-E27-63 & A6688 & D & & & & & Field Rad & & & & 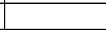 & HWIS & \\
\hline 985 & $200-E$ & 299-E27-64 & A6689 & $\mathrm{D}$ & & & & & & & & & & HWIS & \\
\hline 986 & $200-E$ & 299-E27-65 & A6690 & D & & \begin{tabular}{|l|} 
Virtual \\
Library
\end{tabular} & \begin{tabular}{|l|}
$\begin{array}{l}\text { Virtual } \\
\text { Library }\end{array}$ \\
\end{tabular} & & & & & & $Y$ & HWIS & \\
\hline 987 & $200-E$ & 299-E27-66 & A6691 & D & & \begin{tabular}{|l|} 
Virtual \\
Library
\end{tabular} & \begin{tabular}{|l|} 
Virtual \\
Library
\end{tabular} & & & & & & $Y$ & HWIS & \\
\hline 988 & $200-E$ & 299-E27-67 & A6692 & D & & $\begin{array}{l}\text { Virtual } \\
\text { Library }\end{array}$ & \begin{tabular}{|l|} 
Virtual \\
Library
\end{tabular} & & & & & & $Y$ & HWIS & \\
\hline 989 & $200-E$ & 299-E27-68 & A6693 & D & & & & & & & & & $Y$ & HWIS & \\
\hline 990 & $200-E$ & 299-E27-69 & A6694 & D & & & & & & & & & $Y$ & HWIS & \\
\hline 991 & $200-E$ & 299-E27-7 & A4816 & D & & & & & & & & & $Y$ & HWIS & \\
\hline 992 & $200-E$ & 299-E27-70 & A6695 & D & & \begin{tabular}{|l|} 
Virtual \\
Library
\end{tabular} & \begin{tabular}{|l|}
$\begin{array}{l}\text { Virtual } \\
\text { Library }\end{array}$ \\
\end{tabular} & & & & & & $Y$ & HWIS & \\
\hline 993 & $200-\mathrm{E}$ & 299-E27-71 & A6696 & D & & & & & & & & & $Y$ & HWIS & \\
\hline 994 & $200-E$ & 299-E27-72 & A6697 & D & & $\begin{array}{l}\text { Virtual } \\
\text { Library }\end{array}$ & \begin{tabular}{|l|} 
Virtual \\
Library
\end{tabular} & & & & & & $Y$ & HWIS & \\
\hline 995 & $200-E$ & 299-E27-73 & A6698 & D & & & & & & & & & $Y$ & HWIS & \\
\hline 996 & $200-E$ & 299-E27-74 & A6699 & D & & $\begin{array}{l}\text { Virtual } \\
\text { Library }\end{array}$ & \begin{tabular}{|l|} 
Virtual \\
Library
\end{tabular} & & & & & & $Y$ & HWIS & \\
\hline 997 & $200-E$ & 299-E27-75 & A6700 & D & & \begin{tabular}{|l|} 
Virtual \\
Library
\end{tabular} & \begin{tabular}{|l|} 
Virtual \\
Library
\end{tabular} & & & & & & $Y$ & HWIS & \\
\hline 998 & $200-E$ & \begin{tabular}{|l|} 
299-E27-76 \\
\end{tabular} & A6701 & D & & $\begin{array}{l}\text { Virtual } \\
\text { Library }\end{array}$ & $\begin{array}{l}\text { Virtual } \\
\text { Library }\end{array}$ & & & & & & $Y$ & HWIS & \\
\hline 999 & $200-E$ & 299-E27-77 & A6702 & D & & & & & & & & & $Y$ & HWIS & \\
\hline 1000 & $200-E$ & 299-E27-78 & A6703 & D & & $\begin{array}{l}\text { Virtual } \\
\text { Library }\end{array}$ & \begin{tabular}{|l|} 
Virtual \\
Library
\end{tabular} & & & & & & $\mathrm{Y}$ & HWIS & \\
\hline 1001 & $200-E$ & 299-E27-79 & A6704 & D & & $\begin{array}{l}\text { Virtual } \\
\text { Library }\end{array}$ & \begin{tabular}{|l|} 
Virtual \\
Library
\end{tabular} & & & & & & Y & HWIS & \\
\hline 1002 & $200-E$ & 299-E27-8 & A4817 & D, G & DN, GG, NT & $\begin{array}{l}\text { Virtual } \\
\text { Library }\end{array}$ & & $Y$ & & & & & $Y$ & Last et al. 1989 & \\
\hline
\end{tabular}




\begin{tabular}{|c|c|c|c|c|c|c|c|c|c|c|c|c|c|c|c|}
\hline SortIndex & Area & Well Name & WelllI & Log Type & GPX Log Runs & Sieved & $\mathrm{CaCO} 3$ & Moisture & $\begin{array}{l}\text { Chemical } \\
\text { Properties }\end{array}$ & $\begin{array}{c}\text { Physical } \\
\text { Properties }\end{array}$ & $\begin{array}{c}\text { Min } \\
\text { Properties }\end{array}$ & $\begin{array}{l}\text { Geochron } \\
\text { Properties }\end{array}$ & Archived & Source & Comments \\
\hline 1003 & $200-E$ & 299-E27-80 & A6705 & D & & & & & & & & & Y & HWIS & \\
\hline 1004 & $200-E$ & 299-E27-81 & A6706 & $\mathrm{D}$ & & & & & & & & & $\bar{Y}$ & HWIS & \\
\hline 1005 & $200-E$ & \begin{tabular}{|l|} 
299-E27-82 \\
\end{tabular} & A6707 & D & & \begin{tabular}{|l|} 
Virtual \\
Library
\end{tabular} & \begin{tabular}{|l|} 
Virtual \\
Library
\end{tabular} & & & & & & Y & HWIS & \\
\hline 1006 & $200-E$ & \begin{tabular}{|l|} 
299-E27-83 \\
\end{tabular} & A6708 & $\mathrm{D}$ & & $\begin{array}{l}\text { Virtual } \\
\text { Library }\end{array}$ & $\begin{array}{l}\text { Virtual } \\
\text { Library }\end{array}$ & & & & & & $\mathrm{Y}$ & HWIS & \\
\hline 1007 & $200-E$ & 299-E27-84 & A6709 & D & & $\begin{array}{l}\text { Virtual } \\
\text { Library }\end{array}$ & $\begin{array}{l}\text { Virtual } \\
\text { Library }\end{array}$ & & & & & & Y & HWIS & \\
\hline 1008 & $200-E$ & \begin{tabular}{|l|} 
299-E27-85 \\
\end{tabular} & A6710 & D & & $\begin{array}{l}\text { Virtual } \\
\text { Library }\end{array}$ & $\begin{array}{l}\text { Virtual } \\
\text { Library }\end{array}$ & & & & & & Y & HWIS & \\
\hline 1009 & 200-E & 299-E27-86 & A6711 & D & & $\begin{array}{l}\text { Virtual } \\
\text { Library }\end{array}$ & $\begin{array}{l}\text { Virtual } \\
\text { Library }\end{array}$ & & & & & & Y & & \\
\hline 1010 & $200-E$ & 299-E27-87 & A6712 & D & & & & & & & & & $Y$ & HWIS & \\
\hline 1011 & 200-E & 299-E27-88 & A6713 & $\mathrm{D}$ & & & & & & & & & $Y$ & HWIS & \\
\hline 1012 & $200-E$ & 299-E27-89 & A6714 & D & & & & & & & & & $Y$ & HWIS & \\
\hline 1013 & $200-E$ & 299-E27-9 & A4818 & D, G & DN, GG, NT & $\begin{array}{l}\text { Virtual } \\
\text { Library }\end{array}$ & & & & & & & $Y$ & HWIS & \\
\hline 1014 & $200-E$ & 299-E27-90 & A6715 & D & & & & & & & & & $Y$ & HWIS & \\
\hline 1015 & $200-E$ & 299-E27-91 & A6716 & D & & \begin{tabular}{|l|l|} 
Virtual \\
Library
\end{tabular} & $\begin{array}{l}\text { Virtual } \\
\text { Library }\end{array}$ & & & & & & $Y$ & HWIS & \\
\hline 1016 & $200-E$ & 299-E27-92 & A6717 & D & & $\begin{array}{l}\text { Virtual } \\
\text { Library }\end{array}$ & $\begin{array}{l}\text { Virtual } \\
\text { Library }\end{array}$ & & & & & & $\mathrm{Y}$ & HWIS & \\
\hline 1017 & 200-E & 299-E27-93 & A6718 & D & & $\begin{array}{l}\text { Virtual } \\
\text { Library }\end{array}$ & $\begin{array}{l}\text { Virtual } \\
\text { Library }\end{array}$ & & & & & & $Y$ & HWIS & \\
\hline 1018 & $200-E$ & \begin{tabular}{|l|} 
299-E27-94 \\
\end{tabular} & A6719 & D & & $\begin{array}{l}\text { Virtual } \\
\text { Library }\end{array}$ & $\begin{array}{l}\text { Virtual } \\
\text { Library }\end{array}$ & & & & & & Y & HWIS & \\
\hline 1019 & 200-E & 299-E27-95 & A6720 & D & & & & & & & & & $\mathrm{Y}$ & HWIS & \\
\hline 1020 & 200-E & 299-E27-96 & A6721 & D & & $\begin{array}{l}\text { Virtual } \\
\text { Library }\end{array}$ & $\begin{array}{l}\text { Virtual } \\
\text { Library }\end{array}$ & & & & & & $Y$ & HWIS & \\
\hline 1021 & $200-E$ & \begin{tabular}{|l|} 
299-E27-97 \\
\end{tabular} & A6722 & D & & $\begin{array}{l}\text { Virtual } \\
\text { Library }\end{array}$ & $\begin{array}{l}\text { Virtual } \\
\text { Library }\end{array}$ & & & & & & $Y$ & HWIS & \\
\hline 1022 & $200-\mathrm{E}$ & \begin{tabular}{|l|} 
299-E27-98 \\
\end{tabular} & A6723 & D & & & & & & & & & $Y$ & HWIS & \\
\hline 1023 & $200-E$ & \begin{tabular}{|l|} 
299-E27-99 \\
\end{tabular} & A6724 & D & & $\begin{array}{l}\text { Virtual } \\
\text { Library }\end{array}$ & $\begin{array}{l}\text { Virtual } \\
\text { Library }\end{array}$ & & & & & & $Y$ & HWIS & \\
\hline 1024 & $200-E$ & 299-E28-1 & A6784 & D & DN, GG, NT, SG & $\begin{array}{l}\text { Virtual } \\
\text { Library }\end{array}$ & $\begin{array}{l}\text { Virtual } \\
\text { Library }\end{array}$ & & & & & & $Y$ & HWIS & \\
\hline 1025 & $200-E$ & 299-E28-10 & A6789 & D & GG, NT, SG & & & & & & & & & HWIS & \\
\hline 1026 & $200-E$ & 299-E28-11 & A6790 & D & & & & & & & & & & HWIS & \\
\hline 1027 & $200-E$ & 299-E28-12 & A4819 & D & DN, GG, NT, TP & & & & & & & & & HWIS & \\
\hline 1028 & $200-E$ & 299-E28-13 & A6791 & D & $\mathrm{DN}, \mathrm{GG}, \mathrm{NT}, \mathrm{TP}$ & $\begin{array}{l}\text { Virtual } \\
\text { Library }\end{array}$ & $\begin{array}{l}\text { Virtual } \\
\text { Library }\end{array}$ & & & & & & $Y$ & HWIS & \\
\hline 1029 & $200-E$ & 299-E28-14 & A6792 & As-built & DN, GG, NT, SG & $\begin{array}{l}\text { Virtual } \\
\text { Library }\end{array}$ & $\begin{array}{l}\text { Virtual } \\
\text { Library }\end{array}$ & & & & & & $\mathrm{Y}$ & HWIS & \\
\hline 1030 & 200-E & \begin{tabular}{|l|}
$299-E 28-15$ \\
\end{tabular} & A6793 & D & GG, TP & $\begin{array}{l}\text { Virtual } \\
\text { Library }\end{array}$ & $\begin{array}{l}\text { Virtual } \\
\text { Library }\end{array}$ & & & & & & $Y$ & HWIS & \\
\hline 1031 & $200-E$ & 299-E28-16 & A6794 & D & $\mathrm{DN}, \mathrm{GG}, \mathrm{NT}, \mathrm{SG}$ & $\begin{array}{l}\text { Virtual } \\
\text { Library }\end{array}$ & $\begin{array}{l}\text { Virtual } \\
\text { Library }\end{array}$ & & Field Rad & & & & $\mathrm{Y}$ & HWIS & \\
\hline 1032 & $200-E$ & 299-E28-17 & A4820 & D & DN, GG, NT & $\begin{array}{l}\text { Virtual } \\
\text { Library }\end{array}$ & $\begin{array}{l}\text { Virtual } \\
\text { Library }\end{array}$ & & & & & & $\mathrm{Y}$ & HWIS & \\
\hline 1033 & $200-E$ & \begin{tabular}{|l|} 
299-E28-18 \\
\end{tabular} & A4821 & D & DN, GG, NT, SG, TP & $\begin{array}{l}\text { Virtual } \\
\text { Library }\end{array}$ & $\begin{array}{l}\text { Virtual } \\
\text { Library }\end{array}$ & & & & & & $\mathrm{Y}$ & HWIS & \\
\hline 1034 & $200-E$ & 299-E28-19 & A6795 & D & $\mathrm{DN}, \mathrm{GG}, \mathrm{NT}, \mathrm{TP}$ & $\begin{array}{l}\text { Virtual } \\
\text { Library }\end{array}$ & $\begin{array}{l}\text { Virtual } \\
\text { Library }\end{array}$ & & & & & & $\mathrm{Y}$ & HWIS & \\
\hline 1035 & $200-E$ & 299-E28-2 & A6785 & D & GG, NT, SG, TP & $\begin{array}{l}\text { Virtual } \\
\text { Library }\end{array}$ & $\begin{array}{l}\text { Virtual } \\
\text { Library }\end{array}$ & & & & & & $Y$ & HWIS & \\
\hline 1036 & $200-E$ & 299-E28-20 & A6796 & D & $\mathrm{DN}, \mathrm{GG}, \mathrm{NT}, \mathrm{TP}$ & $\begin{array}{l}\text { Virtual } \\
\text { Library }\end{array}$ & $\begin{array}{l}\text { Virtual } \\
\text { Library }\end{array}$ & & & & & & $\mathrm{Y}$ & HWIS & \\
\hline
\end{tabular}




\begin{tabular}{|c|c|c|c|c|c|c|c|c|c|c|c|c|c|c|c|}
\hline SortIndex & Area & Well Name & WellID & Log Type & GPX Log Runs & Sieved & $\mathrm{CaCO} 3$ & Moisture & $\begin{array}{l}\text { Chemical } \\
\text { Properties }\end{array}$ & $\begin{array}{c}\text { Physical } \\
\text { Properties }\end{array}$ & \begin{tabular}{|c|} 
Min \\
Properties
\end{tabular} & $\begin{array}{l}\text { Geochron } \\
\text { Properties }\end{array}$ & Archived & Source & Comments \\
\hline 1037 & $200-E$ & 299-E28-21 & A6797 & D & DN, GG, NT, TP & & & & & & & & & HWIS & \\
\hline 1038 & $200-E$ & 299-E28-22 & A6798 & D & $\mathrm{DN}, \mathrm{GG}, \mathrm{NT}, \mathrm{TP}$ & $\begin{array}{l}\text { Virtual } \\
\text { Library }\end{array}$ & $\begin{array}{l}\text { Virtual } \\
\text { Library }\end{array}$ & & & & & & $Y$ & HWIS & \\
\hline 1039 & $200-E$ & 299-E28-23 & A6799 & D & SG & $\begin{array}{l}\text { Virtual } \\
\text { Library }\end{array}$ & $\begin{array}{l}\text { Virtual } \\
\text { Library }\end{array}$ & & Lab Rad & & & & $\mathrm{Y}$ & Smith 1980 & \\
\hline 1040 & $200-E$ & 299-E28-24 & A6800 & D & GG, SG & $\begin{array}{l}\text { Virtual } \\
\text { Library }\end{array}$ & $\begin{array}{l}\text { Virtual } \\
\text { Library }\end{array}$ & & Lab Rad & & & & $Y$ & Smith 1980 & \\
\hline 1041 & $200-E$ & 299-E28-25 & A6801 & D & GG, SG & $\begin{array}{l}\text { Virtual } \\
\text { Library }\end{array}$ & $\begin{array}{l}\text { Virtual } \\
\text { Library }\end{array}$ & & Lab Rad & & & & $Y$ & Smith 1980 & \\
\hline 1042 & $200-E$ & 299-E28-26 & A4822 & D, G & DN, GG, NT & $\begin{array}{l}\text { Virtual } \\
\text { Library }\end{array}$ & $\begin{array}{l}\text { Virtual } \\
\text { Library }\end{array}$ & & $\begin{array}{l}\text { XRF, TC, IC, } \\
\text { TOC }\end{array}$ & $\begin{array}{l}\% G S S C, \\
\text { CEC }\end{array}$ & XRD & & $Y$ & $\begin{array}{l}\text { Schramke 1988; } \\
\text { Ames and Serne } \\
1991\end{array}$ & \\
\hline 1043 & $200-E$ & 299-E28-27 & A4823 & $\mathrm{D}, \mathrm{G}$ & DN, GG, NT & \begin{tabular}{|l|} 
Virtual \\
Library
\end{tabular} & \begin{tabular}{|l|} 
Virtual \\
Library
\end{tabular} & & & & & & $\mathrm{Y}$ & HWIS & \\
\hline 1044 & $200-E$ & \begin{tabular}{|l|} 
299-E28-28 \\
\end{tabular} & A4824 & D, G & GG & & $\begin{array}{l}\text { Virtual } \\
\text { Library }\end{array}$ & & \begin{tabular}{|l|} 
An,Rad,VOA, \\
TOC
\end{tabular} & & & & $Y$ & Barton 1990 & Rich Mercer \\
\hline 1045 & $200-E$ & 299-E28-3 & A6786 & D & GG, NT, SG & $\begin{array}{l}\text { Virtual } \\
\text { Library }\end{array}$ & $\begin{array}{l}\text { Virtual } \\
\text { Library }\end{array}$ & & & & & & $Y$ & HWIS & \\
\hline 1046 & $200-E$ & 299-E28-4 & A4825 & $\mathrm{D}$ & GG, NT, TP & $\begin{array}{l}\text { Virtual } \\
\text { Library }\end{array}$ & $\begin{array}{l}\text { Virtual } \\
\text { Library }\end{array}$ & & & & & & $Y$ & HWIS & \\
\hline 1047 & $200-E$ & 299-E28-5 & \begin{tabular}{|l|} 
A6787 \\
\end{tabular} & D & DN, GG, NT, SG, TP & $\begin{array}{l}\text { Virtual } \\
\text { Library }\end{array}$ & $\begin{array}{l}\text { Virtual } \\
\text { Library }\end{array}$ & & & & & & $Y$ & HWIS & \\
\hline 1048 & $200-E$ & \begin{tabular}{|l|}
$299-E 28-50$ \\
\end{tabular} & C3659 & & SG & & & & & & & & & & \\
\hline 1049 & $200-E$ & 299-E28-51 & A6802 & D & & & & & & & & & & HWIS & \\
\hline 1050 & $200-E$ & \begin{tabular}{|l|} 
299-E28-52 \\
\end{tabular} & A6803 & D & & $\begin{array}{l}\text { Virtual } \\
\text { Library }\end{array}$ & $\begin{array}{l}\text { Virtual } \\
\text { Library }\end{array}$ & & & & & & $Y$ & HWIS & \\
\hline 1051 & $200-E$ & 299-E28-53 & A6804 & D & GG, SG & $\begin{array}{l}\text { Virtual } \\
\text { Library }\end{array}$ & $\begin{array}{l}\text { Virtual } \\
\text { Library }\end{array}$ & & & & & & $Y$ & HWIS & \\
\hline 1052 & $200-E$ & \begin{tabular}{|l|} 
299-E28-54 \\
\end{tabular} & A6805 & D & $\mathrm{DN}, \mathrm{GG}, \mathrm{NT}, \mathrm{SG}$ & $\begin{array}{l}\text { Virtual } \\
\text { Library }\end{array}$ & $\begin{array}{l}\text { Virtual } \\
\text { Library }\end{array}$ & & & & & & $Y$ & HWIS & \\
\hline 1053 & $200-E$ & 299-E28-55 & A6806 & D & GG, SG & $\begin{array}{l}\text { Virtual } \\
\text { Library }\end{array}$ & $\begin{array}{l}\text { Virtual } \\
\text { Library }\end{array}$ & & & & & & $Y$ & HWIS & \\
\hline 1054 & $200-E$ & \begin{tabular}{|l|} 
299-E28-56 \\
\end{tabular} & A6807 & D & $\mathrm{DN}, \mathrm{GG}, \mathrm{NT}, \mathrm{SG}$ & $\begin{array}{l}\text { Virtual } \\
\text { Library }\end{array}$ & $\begin{array}{l}\text { Virtual } \\
\text { Library }\end{array}$ & & & & & & $Y$ & HWIS & \\
\hline 1055 & $200-E$ & \begin{tabular}{|l|} 
299-E28-57 \\
\end{tabular} & A6808 & D & $\mathrm{DN}, \mathrm{GG}, \mathrm{NT}, \mathrm{SG}$ & $\begin{array}{l}\text { Virtual } \\
\text { Library }\end{array}$ & $\begin{array}{l}\text { Virtual } \\
\text { Library }\end{array}$ & & & & & & $Y$ & HWIS & \\
\hline 1056 & $200-E$ & \begin{tabular}{|l|} 
299-E28-58 \\
\end{tabular} & A6809 & D & GG, SG & $\begin{array}{l}\text { Virtual } \\
\text { Library }\end{array}$ & $\begin{array}{l}\text { Virtual } \\
\text { Library }\end{array}$ & & & & & & $\mathrm{Y}$ & HWIS & \\
\hline 1057 & $200-E$ & \begin{tabular}{|l|} 
299-E28-59 \\
\end{tabular} & A6810 & D & GG, SG & $\begin{array}{l}\text { Virtual } \\
\text { Library }\end{array}$ & $\begin{array}{l}\text { Virtual } \\
\text { Library }\end{array}$ & & & & & & $Y$ & HWIS & \\
\hline 1058 & $200-E$ & 299-E28-6 & A4826 & D & GG, NT, TP & $\begin{array}{l}\text { Virtual } \\
\text { Library }\end{array}$ & $\begin{array}{l}\text { Virtual } \\
\text { Library }\end{array}$ & & & & & & $Y$ & HWIS & \\
\hline 1059 & $200-E$ & \begin{tabular}{|l|} 
299-E28-60 \\
\end{tabular} & A6811 & D & $\mathrm{DN}, \mathrm{GG}, \mathrm{NT}, \mathrm{SG}$ & $\begin{array}{l}\text { Virtual } \\
\text { Library }\end{array}$ & $\begin{array}{l}\text { Virtual } \\
\text { Library }\end{array}$ & & & & & & $Y$ & HWIS & \\
\hline 1060 & $200-E$ & 299-E28-61 & A6812 & $\mathrm{D}$ & $\mathrm{DN}, \mathrm{GG}, \mathrm{NT}, \mathrm{SG}$ & $\begin{array}{l}\text { Virtual } \\
\text { Library }\end{array}$ & $\begin{array}{l}\text { Virtual } \\
\text { Library }\end{array}$ & & & & & & $Y$ & HWIS & \\
\hline 1061 & $200-E$ & 299-E28-62 & A6813 & $\mathrm{D}$ & GG, SG & & & & & & & & & HWIS & \\
\hline 1062 & $200-E$ & 299-E28-63 & A6814 & D & & & & & & & & & & HWIS & \\
\hline 1063 & $200-E$ & 299-E28-64 & A6815 & D & GG & $\begin{array}{l}\text { Virtual } \\
\text { Library }\end{array}$ & $\begin{array}{l}\text { Virtual } \\
\text { Library }\end{array}$ & & Field Rad & & & & $Y$ & HWIS & \\
\hline 1064 & $200-E$ & 299-E28-65 & A6816 & D & GG, NT, SG & $\begin{array}{l}\text { Virtual } \\
\text { Library }\end{array}$ & $\begin{array}{l}\text { Virtual } \\
\text { Library }\end{array}$ & & & & & & $Y$ & HWIS & \\
\hline 1065 & $200-E$ & 299-E28-66 & A6817 & D & DN, GG, NT, SG & $\begin{array}{l}\text { Virtual } \\
\text { Library }\end{array}$ & $\begin{array}{l}\text { Virtual } \\
\text { Library }\end{array}$ & & & & & & $Y$ & HWIS & \\
\hline 1066 & $200-E$ & \begin{tabular}{|l|}
$299-E 28-67$ \\
\end{tabular} & A6818 & & SG & & & & & & & & & & \\
\hline 1067 & $200-E$ & 299-E28-68 & A6819 & & SG & & & & & & & & & & \\
\hline
\end{tabular}




\begin{tabular}{|c|c|c|c|c|c|c|c|c|c|c|c|c|c|c|c|}
\hline Sortindex & Area & Well Name & WellID & Log Type & GPX Log Runs & Sieved & $\mathrm{CaCO} 3$ & Moisture & $\begin{array}{l}\text { Chemical } \\
\text { Properties }\end{array}$ & $\begin{array}{c}\text { Physical } \\
\text { Properties }\end{array}$ & $\begin{array}{c}\text { Min } \\
\text { Properties }\end{array}$ & $\begin{array}{l}\text { Geochron } \\
\text { Properties }\end{array}$ & Archived & Source & Comments \\
\hline $\begin{array}{ll}1068 \\
\end{array}$ & $200-E$ & 299-E28-69 & A6820 & D & GG, NT & & & & Field Rad & & & & & HWIS & \\
\hline 1069 & $200-E$ & 299-E28-7 & A4827 & D & DN, GG, NT, SG, TP & $\begin{array}{l}\text { Virtual } \\
\text { Library }\end{array}$ & $\begin{array}{l}\text { Virtual } \\
\text { Library }\end{array}$ & & Lab Rad & & & & $Y$ & Smith 1980 & \\
\hline 1070 & $200-E$ & 299-E28-70 & A6821 & D & GG, NT & & & & Field Rad & & & & & HWIS & \\
\hline 1071 & $200-E$ & 299-E28-71 & A6822 & $\mathrm{D}$ & $\mathrm{GG}, \mathrm{NT}$ & & & & Field Rad & & & & & HWIS & \\
\hline 1072 & $200-E$ & 299-E28-73 & A6824 & D & GG, SG & $\begin{array}{l}\text { Virtual } \\
\text { Library }\end{array}$ & $\begin{array}{l}\text { Virtual } \\
\text { Library }\end{array}$ & & Lab Rad & & & & $Y$ & Smith 1980 & \\
\hline 1073 & $200-E$ & 299-E28-74 & A6825 & D & GG, SG & $\begin{array}{l}\text { Virtual } \\
\text { Library }\end{array}$ & $\begin{array}{l}\text { Virtual } \\
\text { Library }\end{array}$ & & Lab Rad & & & & $Y$ & Smith 1980 & \\
\hline 1074 & $200-E$ & 299-E28-75 & A6826 & D & SG & & & & Field Rad & & & & $\mathrm{Y}$ & HWIS & \\
\hline 1075 & $200-E$ & 299-E28-76 & A6827 & D & GG & & & & Field Rad & & & & $Y$ & HWIS & \\
\hline 1076 & $200-E$ & 299-E28-77 & A6828 & D & & & & & & & & & $Y$ & HWIS & Backfilled \\
\hline 1077 & $200-E$ & 299-E28-78 & A6829 & D & & & & & & & & & $Y$ & HWIS & Backfilled \\
\hline 1078 & $200-E$ & \begin{tabular}{|l|}
$299-E 28-8$ \\
\end{tabular} & A6788 & $\mathrm{D}$ & GG, NT, SG, TP & $\begin{array}{l}\text { Virtual } \\
\text { Library }\end{array}$ & $\begin{array}{l}\text { Virtual } \\
\text { Library }\end{array}$ & & & & & & Y & HWIS & \\
\hline 1079 & $200-\mathrm{E}$ & 299-E28-84 & A6835 & D & SG & & & & Field Rad & & & & $Y$ & HWIS & \\
\hline 1080 & $200-E$ & 299-E28-85 & A6836 & $\mathrm{D}$ & SG & & & & Field Rad & & & & $Y$ & HWIS & \\
\hline 1081 & $200-E$ & 299-E28-86 & A6837 & D & SG & & & & Field Rad & & & & $Y$ & HWIS & \\
\hline 1082 & $200-E$ & 299-E28-87 & A6838 & D & SG & & & & Field Rad & & & & $Y$ & HWIS & \\
\hline 1083 & $200-E$ & 299-E28-88 & A6839 & $\mathrm{D}$ & SG & & & & Field Rad & & & & $Y$ & HWIS & \\
\hline 1084 & $200-\mathrm{E}$ & 299-E28-89 & A6840 & D & SG & & & & Field Rad & & & & $Y$ & HWIS & \\
\hline 1085 & $200-E$ & 299-E28-9 & A4828 & D & $\mathrm{DN}, \mathrm{GG}, \mathrm{NT}, \mathrm{TP}$ & $\begin{array}{l}\text { Virtual } \\
\text { Library }\end{array}$ & $\begin{array}{l}\text { Virtual } \\
\text { Library }\end{array}$ & & & & & & Y & HWIS & \\
\hline 1086 & $200-E$ & 299-E28-90 & A6841 & D, G & & & & & $\begin{array}{l}\text { Field Rad, } \\
\text { Lab Rad }\end{array}$ & & & & Y & PNNL files & Can't find \\
\hline 1087 & $200-E$ & 299-E28-91 & A6842 & D & SG & & & & Field Rad & & & & $Y$ & HWIS & \\
\hline 1088 & $200-E$ & 299-E28-93 & A6844 & $D$ & & & & & & & & & Y & HWIS & \\
\hline 1089 & $200-E$ & 299-E28-94 & A6845 & G, Well summary & SG & & & & & & & & & HWIS & \\
\hline $\begin{array}{l}1090 \\
1090\end{array}$ & $200-E$ & 299-다-E28-95 & $\mathrm{A} 6846$ & $\mathrm{D}, \mathrm{G}$, Well summary & SG & & & & & & & & & HWIS & \\
\hline 1091 & $200-E$ & 299-E29-1 & \begin{tabular}{|l|}
$A 6847$ \\
\end{tabular} & $\mathrm{D}$ & & & & & & & & & & HWIS & \\
\hline 1092 & $200-E$ & 299-E29-2 & & D, G & & $\begin{array}{l}\text { Virtual } \\
\text { Library }\end{array}$ & & & & & & & $\mathrm{Y}$ & & \\
\hline 1093 & $200-E$ & 299-E29-3 & & D, G & & $\begin{array}{l}\text { Virtual } \\
\text { Library }\end{array}$ & & & & & & & $\mathrm{Y}$ & & \\
\hline 1094 & $200-E$ & 299-E29-4 & & D, G & & $\begin{array}{l}\text { Virtual } \\
\text { Library }\end{array}$ & & & & & & & $\mathrm{Y}$ & & \\
\hline 1095 & $200-E$ & 299-E29-5 & & D, G & & $\begin{array}{l}\text { Virtual } \\
\text { Library }\end{array}$ & $\begin{array}{l}\text { Virtual } \\
\text { Library }\end{array}$ & $Y$ & \begin{tabular}{|l|} 
An, Rad, \\
TOC, VOA
\end{tabular} & & & & $\mathrm{Y}$ & $\begin{array}{l}\text { Goodwin and } \\
\text { Bjornstad 1990; } \\
\text { Barton } 1990\end{array}$ & \\
\hline 1096 & $200-E$ & 299-E32-1 & A4829 & D, As-built & GG, NT, TP & & & & & & & & & HWIS & \\
\hline 1097 & $200-E$ & 299-E32-10 & A5432 & G & GG, SG & & $Y$ & $Y$ & Field Rad & & & & $Y$ & Mercer 1993a & \\
\hline 1098 & $200-E$ & 299-E32-2 & A4830 & D, As-built & DN, GG, NT & & & & & & & & & HWIS & \\
\hline 1099 & $200-E$ & 299-E32-3 & A4831 & D, As-built & DN, GG, NT & & & & & & & & & HWIS & \\
\hline 1100 & $200-E$ & 299-E32-4 & A4832 & $\mathrm{D}, \mathrm{G}$ & DN, GG, NT & $Y$ & & & & & & & & $\begin{array}{l}\text { see R. Khaleel or } \\
\text { G. Freeman for } \\
\text { sieve data }\end{array}$ & \\
\hline 1101 & $200-E$ & 299-E32-5 & A4833 & As-built & GG & & & & & & & & & HWIS & \\
\hline 1102 & $200-E$ & 299-E32-6 & A4834 & G & GG, SG & & $Y$ & $Y$ & Field Rad & & & & $\mathrm{Y}$ & Mercer 1993b & \\
\hline 1103 & $200-E$ & 299-E32-7 & A4835 & G & GG, SG & & $Y$ & $Y$ & Field Rad & & & & $Y$ & Mercer 1993b & \\
\hline 1104 & $200-E$ & 299-E32-8 & A4836 & G & GG, SG & & Y & $\mathrm{Y}$ & Field Rad & & & & Y & Mercer 1993b & \\
\hline
\end{tabular}




\begin{tabular}{|c|c|c|c|c|c|c|c|c|c|c|c|c|c|c|c|}
\hline SortIndex & Area & Well Name & WellID & Log Type & GPX Log Runs & Sieved & $\mathrm{CaCO} 3$ & Moisture & $\begin{array}{l}\text { Chemical } \\
\text { Properties }\end{array}$ & $\begin{array}{c}\text { Physical } \\
\text { Properties }\end{array}$ & \begin{tabular}{|c|} 
Min \\
Properties
\end{tabular} & $\begin{array}{l}\text { Geochron } \\
\text { Properties }\end{array}$ & Archived & Source & Comments \\
\hline \begin{tabular}{|l|}
1105 \\
\end{tabular} & $200-E$ & 299-E32-9 & A4837 & G & GG, SG & & $Y$ & Y & Field Rad & & & & $Y$ & Mercer 1993b & \\
\hline 1106 & $200-E$ & 299-E33-10 & A6853 & $\mathrm{D}$ & $\mathrm{GG}, \mathrm{MO}, \mathrm{NT}, \mathrm{SG}$ & & & & Field Rad & & & & $Y$ & HWIS & \\
\hline 1107 & $200-E$ & 299-E33-100 & A6908 & D & & & & & & & & & $Y$ & HWIS & \\
\hline 1108 & $200-E$ & 299-E33-101 & A6909 & D & & & & & Field Rad & & & & & HWIS & \\
\hline 1109 & $200-E$ & 299-E33-102 & A6910 & D & & & & & Field Rad & & & & & HWIS & \\
\hline 1110 & $200-E$ & 299-E33-103 & A6911 & D & & & & & Field Rad & & & & & HWIS & \\
\hline 1111 & $200-E$ & 299-E33-104 & A6912 & D & & & & & Field Rad & & & & & HWIS & \\
\hline 1112 & $200-E$ & 299-E33-105 & A6913 & D & & & & & Field Rad & & & & & HWIS & \\
\hline 1113 & $200-E$ & 299-E33-106 & A6914 & D & & & & & Field Rad & & & & & HWIS & \\
\hline 1114 & $200-E$ & 299-E33-107 & A6915 & D & & & & & Field Rad & & & & & HWIS & \\
\hline 1115 & $200-E$ & 299-E33-108 & A6916 & D & & & & & Field Rad & & & & $Y$ & HWIS & \\
\hline 1116 & $200-E$ & 299-E33-109 & A6917 & D & & & & & Field Rad & & & & & HWIS & \\
\hline 1117 & $200-E$ & 299-E33-11 & A6854 & D & DN, GG, NT, SG & \begin{tabular}{|l|} 
Virtual \\
Library
\end{tabular} & \begin{tabular}{|l|}
$\begin{array}{l}\text { Virtual } \\
\text { Library }\end{array}$ \\
\end{tabular} & & & & & & $Y$ & HWIS & \\
\hline 1118 & $200-E$ & 299-E33-110 & A6918 & D & & & & & Field Rad & & & & & HWIS & \\
\hline 1119 & $200-E$ & 299-E33-111 & A6919 & D & & & & & Field Rad & & & & & HWIS & \\
\hline 1120 & $200-E$ & 299-E33-112 & A6920 & D & & & & & Field Rad & & & & & HWIS & \\
\hline 1121 & $200-E$ & 299-E33-113 & A6921 & D & & & & & Field Rad & & & & & HWIS & \\
\hline 1122 & $200-E$ & 299-E33-114 & A6922 & D & & & & & Field Rad & & & & & HWIS & \\
\hline 1123 & $200-E$ & 299-E33-115 & A6923 & D & & & & & Field Rad & & & & & HWIS & \\
\hline 1124 & $200-E$ & 299-E33-116 & A6924 & D & & & & & Field Rad & & & & & HWIS & \\
\hline 1125 & 200-E & 299-E33-117 & A6925 & D & & & & & Field Rad & & & & & HWIS & \\
\hline 1126 & $200-E$ & 299-E33-118 & A6926 & D & & & & & Field Rad & & & & & HWIS & \\
\hline 1127 & $200-E$ & 299-E33-119 & A6927 & D & & & & & Field Rad & & & & & HWIS & \\
\hline 1128 & $200-E$ & 299-E33-12 & A4839 & D & DN, GG, NT, SG, TP & \begin{tabular}{|l|} 
Virtual \\
Library
\end{tabular} & \begin{tabular}{|l|} 
Virtual \\
Library
\end{tabular} & & $\mathrm{pH}$ & $\begin{array}{l}\text { CEC, } \\
\% \text { GSSC, 15- } \\
\text { Atm }\end{array}$ & & & Y & McHenry 1957 & \\
\hline 1129 & $200-E$ & 299-E33-120 & A6928 & D & & & & & Field Rad & & & & & HWIS & \\
\hline 1130 & $200-E$ & 299-E33-121 & A6929 & D & & & & & Field Rad & & & & & HWIS & \\
\hline 1131 & $200-E$ & 299-E33-122 & A6930 & $\mathrm{D}$ & & & & & Field Rad & & & & & HWIS & \\
\hline 1132 & $200-E$ & 299-E33-123 & A6931 & D & & & & & Field Rad & & & & & HWIS & \\
\hline 1133 & $200-E$ & 299-E33-124 & A6932 & D & & & & & Field Rad & & & & & HWIS & \\
\hline 1134 & $200-E$ & 299-E33-125 & A6933 & D & & & & & Field Rad & & & & & HWIS & \\
\hline 1135 & $200-E$ & 299-E33-126 & A6934 & D & & & & & Field Rad & & & & & HWIS & \\
\hline 1136 & $200-E$ & 299-E33-127 & A6935 & D & & & & & Field Rad & & & & & HWIS & \\
\hline 1137 & $200-E$ & 299-E33-128 & A6936 & D & & & & & Field Rad & & & & & HWIS & \\
\hline 1138 & $200-E$ & 299-E33-129 & A6937 & D & & & & & & & & & & HWIS & \\
\hline 1139 & 200-E & 299-E33-13 & A4840 & D & DN, GG, NT, SG, TP & $\begin{array}{l}\text { Virtual } \\
\text { Library }\end{array}$ & \begin{tabular}{|l} 
Virtual \\
Library
\end{tabular} & & $\mathrm{pH}$ & $\begin{array}{l}\text { CEC, } \\
\% \text { \%SSC, 15- } \\
\text { Atm }\end{array}$ & & & $Y$ & McHenry 1957 & \\
\hline 1140 & $200-E$ & 299-E33-130 & A6938 & D & & & & & & & & & & HWIS & \\
\hline 1141 & $200-E$ & 299-E33-131 & A6939 & $\mathrm{D}$ & & & & & & & & & & HWIS & \\
\hline 1142 & $200-E$ & 299-E33-132 & A6940 & D & GG, NT & & & & Field Rad & & & & & HWIS & \\
\hline 1143 & $200-E$ & 299-E33-133 & A6941 & D & GG, NT & & & & & & & & & HWIS & \\
\hline 1144 & $200-E$ & 299-E33-134 & A6942 & D & GG, NT & & & & & & & & & HWIS & \\
\hline 1145 & $200-E$ & 299-E33-135 & A6943 & $\mathrm{D}$ & GG, NT & & & & & & & & & HWIS & \\
\hline 1146 & $200-\mathrm{E}$ & 299-E33-136 & A6944 & D & GG, NT & & & & Field Rad & & & & & HWIS & \\
\hline 1147 & $200-E$ & 299-E33-137 & A6945 & D & GG, NT & & & & Field Rad & & & & & HWIS & \\
\hline 1148 & $200-E$ & 299-E33-138 & A6946 & D & & & & & Field Rad & & & & & HWIS & \\
\hline 1149 & $200-E$ & 299-E33-139 & A6947 & $\mathrm{D}$ & & & & & Field Rad & & & & & HWIS & \\
\hline 1150 & 200-E & \begin{tabular}{|l|} 
299-E33-14 \\
\end{tabular} & A4841 & D & DN, GG, NT, SG, TP & $\begin{array}{l}\text { Virtual } \\
\text { Library }\end{array}$ & $\begin{array}{l}\text { Virtual } \\
\text { Library }\end{array}$ & & & & & & $Y$ & HWIS & \\
\hline 1151 & $200-E$ & 299-E33-140 & A6948 & D & & & & & Field Rad & & & & & HWIS & \\
\hline 1152 & $200-E$ & 299-E33-141 & A6949 & $\mathrm{D}$ & GG, NT & & & & Field Rad & & & & & HWIS & \\
\hline 1153 & $200-E$ & 299-E33-142 & A6950 & D & GG, NT & & & & Field Rad & & & & & HWIS & \\
\hline 1154 & $200-E$ & 299-E33-143 & A6951 & $\mathrm{D}$ & GG, NT & & & & & & & & & HWIS & \\
\hline 1155 & $200-E$ & 299-E33-144 & A6952 & D & & & & & & & & & & HWIS & \\
\hline
\end{tabular}




\begin{tabular}{|c|c|c|c|c|c|c|c|c|c|c|c|c|c|c|c|}
\hline Sortlndex & Area & Well Name & WelliD & Log Type & GPX Log Runs & Sieved & $\mathrm{CaCO} 3$ & Moisture & $\begin{array}{c}\text { Chemical } \\
\text { Properties } \\
\end{array}$ & $\begin{array}{c}\text { Physical } \\
\text { Properties } \\
\end{array}$ & \begin{tabular}{|c|} 
Min \\
Properties \\
\end{tabular} & $\begin{array}{l}\text { Geochron } \\
\text { Properties } \\
\end{array}$ & Archived & Source & Comments \\
\hline 1156 & $200-E$ & 299-E33-145 & A6953 & $\mathrm{D}$ & GG, NT & & & & & & & & & HWIS & \\
\hline 1157 & $200-E$ & 299-E33-146 & A6954 & D & GG, NT & & & & Field Rad & & & & & HWIS & \\
\hline 1158 & $200-E$ & 299-E33-147 & A6955 & D & & & & & & & & & & HWIS & \\
\hline 1159 & $200-E$ & 299-E33-148 & A6956 & D & & \begin{tabular}{|l|} 
Virtual \\
Library
\end{tabular} & $\begin{array}{l}\text { Virtual } \\
\text { Library }\end{array}$ & & & & & & Y & HWIS & \\
\hline 1160 & $200-E$ & 299-E33-149 & A6957 & D & & & & & & & & & & HWIS & \\
\hline 1161 & $200-E$ & 299-E33-15 & A4842 & D & DN, GG, NT, SG & \begin{tabular}{|l|} 
Virtual \\
Library
\end{tabular} & $\begin{array}{l}\text { Virtual } \\
\text { Library }\end{array}$ & & & & & & $Y$ & HWIS & \\
\hline 1162 & $200-E$ & 299-E33-150 & A6958 & D & & $\begin{array}{l}\text { Virtual } \\
\text { Library }\end{array}$ & $\begin{array}{l}\text { Virtual } \\
\text { Library }\end{array}$ & & & & & & $Y$ & HWIS & \\
\hline 1163 & $200-E$ & 299-E33-151 & A6959 & D & & \begin{tabular}{|l|l|} 
Virtual \\
Library
\end{tabular} & \begin{tabular}{|l|l|} 
Virtual \\
Library
\end{tabular} & & & & & & $Y$ & HWIS & \\
\hline 1164 & $200-E$ & 299-E33-152 & A6960 & $\mathrm{D}$ & & & & & & & & & $\mathrm{Y}$ & HWIS & \\
\hline 1165 & $200-E$ & 299-E33-153 & A6961 & D & & \begin{tabular}{|l|} 
Virtual \\
Library
\end{tabular} & $\begin{array}{l}\text { Virtual } \\
\text { Library }\end{array}$ & & & & & & Y & HWIS & \\
\hline 1166 & $200-E$ & 299-E33-154 & A6962 & D & & $\begin{array}{l}\text { Virtual } \\
\text { Library }\end{array}$ & $\begin{array}{l}\text { Virtual } \\
\text { Library }\end{array}$ & & & & & & $Y$ & HWIS & \\
\hline 1167 & $200-E$ & 299-E33-155 & A6963 & D & & $\begin{array}{l}\text { Virtual } \\
\text { Library }\end{array}$ & $\begin{array}{l}\text { Virtual } \\
\text { Library }\end{array}$ & & & & & & Y & HWIS & \\
\hline 1168 & $200-E$ & 299-E33-156 & A6964 & D & & & & & & & & & $Y$ & HWIS & \\
\hline 1169 & $200-E$ & 299-E33-157 & A6965 & D & & & & & & & & & Y & HWIS & \\
\hline 1170 & $200-E$ & 299-E33-158 & A6966 & D & & & & & & & & & $Y$ & HWIS & \\
\hline 1171 & $200-E$ & 299-E33-159 & A6967 & D & & \begin{tabular}{|l|} 
Virtual \\
Library
\end{tabular} & \begin{tabular}{|l|} 
Virtual \\
Library
\end{tabular} & & & & & & $Y$ & HWIS & \\
\hline 1172 & $200-E$ & 299-E33-16 & A6855 & D & $\mathrm{DN}, \mathrm{GG}, \mathrm{NT}, \mathrm{SG}$ & $\begin{array}{l}\text { Virtual } \\
\text { Library }\end{array}$ & $\begin{array}{l}\text { Virtual } \\
\text { Library }\end{array}$ & & & & & & $Y$ & HWIS & \\
\hline 1173 & $200-E$ & 299-E33-160 & A6968 & D & & $\begin{array}{l}\text { Virtual } \\
\text { Library }\end{array}$ & $\begin{array}{l}\text { Virtual } \\
\text { Library }\end{array}$ & & & & & & $\mathrm{Y}$ & HWIS & \\
\hline 1174 & $200-E$ & 299-E33-161 & A6969 & D & & $\begin{array}{l}\text { Virtual } \\
\text { Library }\end{array}$ & $\begin{array}{l}\text { Virtual } \\
\text { Library } \\
\end{array}$ & & & & & & & HWIS & \\
\hline 1175 & $200-E$ & 299-E33-162 & A6970 & $\mathrm{D}$ & & & & & & & & & $Y$ & HWIS & \\
\hline 1176 & $200-E$ & 299-E33-163 & A6971 & D & & $\begin{array}{l}\text { Virtual } \\
\text { Library }\end{array}$ & $\begin{array}{l}\text { Virtual } \\
\text { Library }\end{array}$ & & & & & & $Y$ & HWIS & \\
\hline 1177 & $200-E$ & 299-E33-164 & A6972 & D & & $\begin{array}{l}\text { Virtual } \\
\text { Library }\end{array}$ & $\begin{array}{l}\text { Virtual } \\
\text { Library }\end{array}$ & & & & & & $Y$ & HWIS & \\
\hline 1178 & $200-E$ & 299-E33-165 & A6973 & D & & $\begin{array}{l}\text { Virtual } \\
\text { Library }\end{array}$ & $\begin{array}{l}\text { Virtual } \\
\text { Library }\end{array}$ & & & & & & $\mathrm{Y}$ & HWIS & \\
\hline 1179 & $200-E$ & 299-E33-166 & A6974 & D & & & & & & & & & $\mathrm{Y}$ & HWIS & \\
\hline 1180 & $200-E$ & 299-E33-167 & A6975 & D & & & & & & & & & & HWIS & \\
\hline 1181 & $200-E$ & 299-E33-168 & A6976 & D & & & & & & & & & & HWIS & \\
\hline 1182 & $200-E$ & 299-E33-169 & A6977 & $\mathrm{D}$ & & & & & & & & & & HWIS & \\
\hline 1183 & $200-E$ & 299-E33-17 & A4843 & D & DN, GG, NT, SG, TP & $\begin{array}{l}\text { Virtual } \\
\text { Library }\end{array}$ & $\begin{array}{l}\text { Virtual } \\
\text { Library }\end{array}$ & & $\mathrm{pH}$ & \begin{tabular}{|l|} 
CEC, \\
\%GSSC, 15- \\
Atm
\end{tabular} & & & $\mathrm{Y}$ & McHenry 1957 & \\
\hline 1184 & $200-E$ & 299-E33-170 & A6978 & $\mathrm{D}$ & & & & & & & & & & HWIS & \\
\hline 1185 & $200-E$ & 299-E33-171 & A6979 & D & & & & & & & & & & HWIS & \\
\hline 1186 & $200-E$ & 299-E33-172 & A6980 & D & & & & & & & & & & HWIS & \\
\hline 1187 & $200-E$ & 299-E33-173 & A6981 & D & & & & & & & & & & HWIS & \\
\hline 1188 & $200-E$ & 299-E33-174 & A6982 & $\mathrm{D}$ & & & & & & & & & & HWIS & \\
\hline 1189 & $200-\mathrm{E}$ & 299-E33-175 & A6983 & D & & & & & & & & & & HWIS & \\
\hline 1190 & $200-E$ & 299-E33-176 & A6984 & D & & \begin{tabular}{|l|} 
Virtual \\
Library
\end{tabular} & $\begin{array}{l}\text { Virtual } \\
\text { Library }\end{array}$ & & & & & & $Y$ & HWIS & \\
\hline 1191 & $200-E$ & 299-E33-177 & A6985 & D & & $\begin{array}{l}\text { Virtual } \\
\text { Library }\end{array}$ & $\begin{array}{l}\text { Virtual } \\
\text { Library }\end{array}$ & & & & & & Y & HWIS & \\
\hline 1192 & $200-E$ & 299-E33-178 & A6986 & D & & $\begin{array}{l}\text { Virtual } \\
\text { Library }\end{array}$ & $\begin{array}{l}\text { Virtual } \\
\text { Library }\end{array}$ & & & & & & $\mathrm{Y}$ & HWIS & \\
\hline
\end{tabular}




\begin{tabular}{|c|c|c|c|c|c|c|c|c|c|c|c|c|c|c|c|}
\hline SortIndex & Area & Well Name & WelllD & Log Type & GPX Log Runs & Sieved & $\mathrm{CaCO} 3$ & Moisture & $\begin{array}{l}\text { Chemical } \\
\text { Properties }\end{array}$ & $\begin{array}{c}\text { Physical } \\
\text { Properties } \\
\end{array}$ & \begin{tabular}{|c|} 
Min \\
Properties \\
\end{tabular} & $\begin{array}{l}\text { Geochron } \\
\text { Properties } \\
\end{array}$ & Archived & Source & Comments \\
\hline 1193 & $200-E$ & 299-E33-179 & A6987 & D & & \begin{tabular}{|l|} 
Virtual \\
Library
\end{tabular} & $\begin{array}{l}\text { Virtual } \\
\text { Library }\end{array}$ & & & & & & $\mathrm{Y}$ & HWIS & \\
\hline 1194 & $200-E$ & 299-E33-18 & A4844 & D & DN, GG, NT, SG, TP & $\begin{array}{l}\text { Virtual } \\
\text { Library }\end{array}$ & $\begin{array}{l}\text { Virtual } \\
\text { Library }\end{array}$ & & & & & & $Y$ & HWIS & \\
\hline 1195 & $200-E$ & 299-E33-180 & A6988 & D & & $\begin{array}{l}\text { Virtual } \\
\text { Library }\end{array}$ & \begin{tabular}{|l|}
$\begin{array}{l}\text { Virtual } \\
\text { Library }\end{array}$ \\
\end{tabular} & & & & & & $Y$ & HWIS & \\
\hline 1196 & $200-E$ & 299-E33-181 & A6989 & D & & & & & & & & & $Y$ & HWIS & \\
\hline 1197 & $200-E$ & 299-E33-182 & A6990 & D & & $\begin{array}{l}\text { Virtual } \\
\text { Library }\end{array}$ & $\begin{array}{l}\text { Virtual } \\
\text { Library }\end{array}$ & & & & & & Y & HWIS & \\
\hline 1198 & $200-E$ & 299-E33-183 & A6991 & $\mathrm{D}$ & & & & & & & & & $Y$ & HWIS & \\
\hline 1199 & $200-E$ & 299-E33-184 & A6992 & D & & $\begin{array}{l}\text { Virtual } \\
\text { Library }\end{array}$ & $\begin{array}{l}\text { Virtual } \\
\text { Library }\end{array}$ & & & & & & Y & HWIS & \\
\hline 1200 & $200-E$ & 299-E33-185 & A6993 & D & & $\begin{array}{l}\text { Virtual } \\
\text { Library }\end{array}$ & $\begin{array}{l}\text { Virtual } \\
\text { Library }\end{array}$ & & & & & & $\mathrm{Y}$ & HWIS & \\
\hline 1201 & $200-E$ & 299-E33-186 & A6994 & D & & $\begin{array}{l}\text { Virtual } \\
\text { Library }\end{array}$ & $\begin{array}{l}\text { Virtual } \\
\text { Library }\end{array}$ & & & & & & $Y$ & HWIS & \\
\hline 1202 & $200-E$ & 299-E33-187 & A6995 & D & & & & & & & & & $\mathrm{Y}$ & HWIS & \\
\hline 1203 & $200-E$ & 299-E33-188 & A6996 & D & & & & & & & & & $Y$ & HWIS & \\
\hline 1204 & $200-E$ & 299-E33-189 & A6997 & D & & \begin{tabular}{|l|} 
Virtual \\
Library
\end{tabular} & \begin{tabular}{|l} 
Virtual \\
Library
\end{tabular} & & & & & & $Y$ & HWIS & \\
\hline 1205 & $200-E$ & \begin{tabular}{|l|} 
299-E33-19 \\
\end{tabular} & A4845 & D & $\mathrm{DN}, \mathrm{GG}, \mathrm{NT}, \mathrm{SG}$ & $\begin{array}{l}\text { Virtual } \\
\text { Library }\end{array}$ & $\begin{array}{l}\text { Virtual } \\
\text { Library }\end{array}$ & & & & & & $Y$ & HWIS & \\
\hline 1206 & $200-E$ & 299-E33-190 & A6998 & D & & $\begin{array}{l}\text { Virtual } \\
\text { Library }\end{array}$ & $\begin{array}{l}\text { Virtual } \\
\text { Library }\end{array}$ & & & & & & $\mathrm{Y}$ & HWIS & \\
\hline 1207 & $200-E$ & 299-E33-191 & A6999 & D & & $\begin{array}{l}\text { Virtual } \\
\text { Library }\end{array}$ & $\begin{array}{l}\text { Virtual } \\
\text { Library }\end{array}$ & & & & & & $\mathrm{Y}$ & HWIS & \\
\hline 1208 & $200-E$ & 299-E33-192 & A7000 & D & & $\begin{array}{l}\text { Virtual } \\
\text { Library }\end{array}$ & $\begin{array}{l}\text { Virtual } \\
\text { Library }\end{array}$ & & & & & & & HWIS & \\
\hline 1209 & $200-E$ & 299-E33-193 & A7001 & D & & $\begin{array}{l}\text { Virtual } \\
\text { Library } \\
\end{array}$ & $\begin{array}{l}\text { Virtual } \\
\text { Library }\end{array}$ & & & & & & Y & HWIS & \\
\hline 1210 & $200-E$ & 299-E33-194 & A7002 & $\mathrm{D}$ & & & & & & & & & $Y$ & HWIS & \\
\hline 1211 & $200-E$ & 299-E33-195 & A7003 & D & & $\begin{array}{l}\text { Virtual } \\
\text { Library }\end{array}$ & \begin{tabular}{|l|} 
Virtual \\
Library
\end{tabular} & & & & & & $Y$ & HWIS & \\
\hline 1212 & $200-E$ & 299-E33-196 & A7004 & D & & $\begin{array}{l}\text { Virtual } \\
\text { Library }\end{array}$ & $\begin{array}{l}\text { Virtual } \\
\text { Library }\end{array}$ & & & & & & $Y$ & HWIS & \\
\hline 1213 & $200-E$ & 299-E33-197 & A7005 & D & & & & & & & & & $Y$ & HWIS & \\
\hline 1214 & $200-E$ & 299-E33-198 & A7006 & $\mathrm{D}$ & & & & & & & & & $Y$ & HWIS & \\
\hline 1215 & $200-E$ & 299-E33-199 & A7007 & $\mathrm{D}$ & & & & & & & & & $\mathrm{Y}$ & HWIS & \\
\hline 1216 & $200-E$ & 299-E33-1A & A4838 & D & DN, GG, NT, SG & \begin{tabular}{|l|} 
Virtual \\
Library
\end{tabular} & \begin{tabular}{|l} 
Virtual \\
Library
\end{tabular} & & Lab Rad & & & & $Y$ & Haney 1967 & \\
\hline 1217 & $200-E$ & 299-E33-1B & A6851 & D & & & & & Field Rad & & & & $\mathrm{Y}$ & HWIS & \\
\hline 1218 & $200-E$ & 299-E33-2 & A4846 & D & $\mathrm{DN}, \mathrm{GG}, \mathrm{NT}, \mathrm{SG}$ & $\begin{array}{l}\text { Virtual } \\
\text { Library }\end{array}$ & \begin{tabular}{|l|} 
Virtual \\
Library
\end{tabular} & & & & & & $\mathrm{Y}$ & HWIS & \\
\hline 1219 & $200-E$ & 299-E33-20 & A4847 & D & $\mathrm{DN}, \mathrm{GG}, \mathrm{MO}, \mathrm{NT}, \mathrm{SG}$ & $\begin{array}{l}\text { Virtual } \\
\text { Library }\end{array}$ & $\begin{array}{l}\text { Virtual } \\
\text { Library }\end{array}$ & & & & & & $\mathrm{Y}$ & HWIS & \\
\hline 1220 & $200-E$ & 299-E33-200 & A7008 & $\mathrm{D}$ & & & & & & & & & $\mathrm{Y}$ & HWIS & \\
\hline 1221 & $200-E$ & 299-E33-201 & A7009 & D & & $\begin{array}{l}\text { Virtual } \\
\text { Library }\end{array}$ & \begin{tabular}{|l|} 
Virtual \\
Library
\end{tabular} & & & & XRD, EM & & $\mathrm{Y}$ & Ames 1976 & \\
\hline 1222 & $200-E$ & 299-E33-202 & A7010 & D & & $\begin{array}{l}\text { Virtual } \\
\text { Library }\end{array}$ & $\begin{array}{l}\text { Virtual } \\
\text { Library }\end{array}$ & & & & & & $Y$ & HWIS & \\
\hline 1223 & $200-E$ & 299-E33-203 & A7011 & D & & $\begin{array}{l}\text { Virtual } \\
\text { Library }\end{array}$ & $\begin{array}{l}\text { Virtual } \\
\text { Library }\end{array}$ & & & & & & $\mathrm{Y}$ & HWIS & \\
\hline 1224 & $200-E$ & 299-E33-204 & A7012 & D & & $\begin{array}{l}\text { Virtual } \\
\text { Library }\end{array}$ & \begin{tabular}{|l|l|} 
Virtual \\
Library
\end{tabular} & & & & XRD, EM & & $Y$ & Ames 1976 & \\
\hline 1225 & $200-E$ & 299-E33-206 & A7013 & $\mathrm{D}$ & & & & & & & & & $\mathrm{Y}$ & HWIS & \\
\hline 1226 & $200-E$ & 299-E33-207 & A7014 & D & & & & & & & & & $Y$ & HWIS & \\
\hline
\end{tabular}




\begin{tabular}{|c|c|c|c|c|c|c|c|c|c|c|c|c|c|c|c|}
\hline SortIndex & Area & Well Name & WellID & Log Type & GPX Log Runs & Sieved & $\mathrm{CaCO} 3$ & Moisture & $\begin{array}{l}\text { Chemical } \\
\text { Properties }\end{array}$ & $\begin{array}{l}\text { Physical } \\
\text { Properties }\end{array}$ & \begin{tabular}{|c|} 
Min \\
Properties
\end{tabular} & $\begin{array}{l}\text { Geochron } \\
\text { Properties }\end{array}$ & Archived & Source & Comments \\
\hline 1227 & $200-E$ & 299-E33-208 & A7015 & D & & $\begin{array}{l}\text { Virtual } \\
\text { Library }\end{array}$ & $\begin{array}{l}\text { Virtual } \\
\text { Library }\end{array}$ & & & & & & Y & & \\
\hline 1228 & $200-E$ & 299-E33-209 & A7016 & D & & $\begin{array}{l}\text { Virtual } \\
\text { Library }\end{array}$ & \begin{tabular}{|l|l}
$\begin{array}{l}\text { Virtual } \\
\text { Library }\end{array}$ \\
\end{tabular} & & & & & & $Y$ & HWIS & \\
\hline 1229 & $200-E$ & 299-E33-21 & A4848 & D & DN, GG, NT, SG, TP & \begin{tabular}{|l|}
$\begin{array}{l}\text { Virtual } \\
\text { Library }\end{array}$ \\
\end{tabular} & \begin{tabular}{|l|} 
Virtual \\
Library
\end{tabular} & & Field Rad & & & & $Y$ & HWIS & \\
\hline 1230 & $200-E$ & 299-E33-210 & A7017 & $\mathrm{D}$ & & & & & Field Rad & & & & & HWIS & \\
\hline 1231 & $200-E$ & 299-E33-211 & A7018 & D & & & & & & & & & & HWIS & \\
\hline 1232 & $200-E$ & 299-E33-212 & A7019 & D & & \begin{tabular}{|l|} 
Virtual \\
Library
\end{tabular} & \begin{tabular}{|l|} 
Virtual \\
Library
\end{tabular} & & Field Rad & & & & $\mathrm{Y}$ & HWIS & \\
\hline 1233 & $200-E$ & 299-E33-213 & A7020 & D & & & & & & & & & $Y$ & HWIS & \\
\hline 1234 & $200-E$ & 299-E33-214 & A7021 & D & & \begin{tabular}{|l|} 
Virtual \\
Library
\end{tabular} & \begin{tabular}{|l|}
$\begin{array}{l}\text { Virtual } \\
\text { Library }\end{array}$ \\
\end{tabular} & & Field Rad & & & & $\mathrm{Y}$ & HWIS & \\
\hline 1235 & $200-E$ & 299-E33-215 & A7022 & D & & \begin{tabular}{|l|}
$\begin{array}{l}\text { Virtual } \\
\text { Library }\end{array}$ \\
\end{tabular} & \begin{tabular}{|l|} 
Virtual \\
Library
\end{tabular} & & Field Rad & & & & $\mathrm{Y}$ & HWIS & \\
\hline 1236 & $200-E$ & 299-E33-216 & A7023 & D & & \begin{tabular}{|l|} 
Virtual \\
Library
\end{tabular} & \begin{tabular}{|l|} 
Virtual \\
Library
\end{tabular} & & Field Rad & & & & $\mathrm{Y}$ & HWIS & \\
\hline 1237 & $200-E$ & 299-E33-217 & A7024 & D & & \begin{tabular}{|l|} 
Virtual \\
Library
\end{tabular} & \begin{tabular}{|l|} 
Virtual \\
Library
\end{tabular} & & & & & & $\mathrm{Y}$ & HWIS & \\
\hline 1238 & $200-E$ & 299-E33-218 & A7025 & D & & $\begin{array}{l}\text { Virtual } \\
\text { Library }\end{array}$ & $\begin{array}{l}\text { Virtual } \\
\text { Library }\end{array}$ & & Field Rad & & & & $Y$ & HWIS & \\
\hline 1239 & $200-E$ & 299-E33-219 & A7026 & D & & $\begin{array}{l}\text { Virtual } \\
\text { Library }\end{array}$ & $\begin{array}{l}\text { Virtual } \\
\text { Library }\end{array}$ & & & & & & $Y$ & HWIS & \\
\hline 1240 & $200-E$ & 299-E33-22 & A6856 & D & $\mathrm{DN}, \mathrm{GG}, \mathrm{NT}, \mathrm{SG}$ & \begin{tabular}{|l} 
Virtual \\
Library
\end{tabular} & \begin{tabular}{|l} 
Virtual \\
Library
\end{tabular} & & $\begin{array}{l}\text { Field Rad; } \\
\text { Sorption; }\end{array}$ & CEC & XRD & & $Y$ & \begin{tabular}{|l|} 
Routson et al. \\
1981; Delegard \\
and Barney 1983
\end{tabular} & \\
\hline 1241 & $200-E$ & 299-E33-220 & A7027 & D & & \begin{tabular}{|l|} 
Virtual \\
Library
\end{tabular} & \begin{tabular}{|l|} 
Virtual \\
Library
\end{tabular} & & & & & & $Y$ & HWIS & \\
\hline 1242 & $200-E$ & 299-E33-221 & A7028 & D & & \begin{tabular}{|l|} 
Virtual \\
Library
\end{tabular} & \begin{tabular}{|l|} 
Virtual \\
Library
\end{tabular} & & & & & & $\mathrm{Y}$ & HWIS & \\
\hline 1243 & $200-E$ & 299-E33-222 & A7029 & D & & $\begin{array}{l}\text { Virtual } \\
\text { Library }\end{array}$ & $\begin{array}{l}\text { Virtual } \\
\text { Library }\end{array}$ & & & & & & $Y$ & HWIS & \\
\hline 1244 & $200-E$ & 299-E33-223 & A7030 & D & & \begin{tabular}{|l|} 
Virtual \\
Library
\end{tabular} & \begin{tabular}{|l|} 
Virtual \\
Library
\end{tabular} & & Field Rad & & & & $\mathrm{Y}$ & HWIS & \\
\hline 1245 & $200-E$ & 299-E33-224 & A7031 & D & & $\begin{array}{l}\text { Virtual } \\
\text { Library }\end{array}$ & \begin{tabular}{|l|} 
Virtual \\
Library
\end{tabular} & & & & & & $\mathrm{Y}$ & HWIS & \\
\hline 1246 & $200-E$ & 299-E33-225 & A7032 & D & & $\begin{array}{l}\text { Virtual } \\
\text { Library }\end{array}$ & \begin{tabular}{|l|} 
Virtual \\
Library
\end{tabular} & & & & & & $\mathrm{Y}$ & HWIS & \\
\hline 1247 & $200-E$ & 299-E33-226 & A7033 & D & & \begin{tabular}{|l|} 
Virtual \\
Library
\end{tabular} & \begin{tabular}{|l|} 
Virtual \\
Library
\end{tabular} & & & & & & $Y$ & HWIS & \\
\hline 1248 & $200-E$ & 299-E33-227 & A7034 & D & & $\begin{array}{l}\text { Virtual } \\
\text { Library }\end{array}$ & \begin{tabular}{|l|} 
Virtual \\
Library
\end{tabular} & & & & & & $Y$ & HWIS & \\
\hline 1249 & $200-E$ & 299-E33-228 & A7035 & D & & \begin{tabular}{|l} 
Virtual \\
Library
\end{tabular} & $\begin{array}{l}\text { Virtual } \\
\text { Library }\end{array}$ & & & & & & $Y$ & HWIS & \\
\hline 1250 & $200-E$ & 299-E33-229 & A7036 & D & & $\begin{array}{l}\text { Virtual } \\
\text { Library }\end{array}$ & \begin{tabular}{|l|} 
Virtual \\
Library
\end{tabular} & & Field Rad & & & & $Y$ & HWIS & \\
\hline 1251 & $200-E$ & 299-E33-23 & A6857 & D & GG, NT, SG & & & & Field Rad & & & & & HWIS & \\
\hline 1252 & $200-E$ & 299-E33-230 & A7037 & D & & & & & & & & & $Y$ & HWIS & \\
\hline 1253 & $200-E$ & 299-E33-231 & A7038 & D & & $\begin{array}{l}\text { Virtual } \\
\text { Library }\end{array}$ & \begin{tabular}{|l|} 
Virtual \\
Library
\end{tabular} & & & & & & $Y$ & HWIS & \\
\hline 1254 & $200-E$ & 299-E33-232 & A7039 & D & & \begin{tabular}{|l|} 
Virtual \\
Library
\end{tabular} & \begin{tabular}{|l|} 
Virtual \\
Library
\end{tabular} & & & & & & $Y$ & HWIS & \\
\hline 1255 & $200-E$ & 299-E33-233 & A7040 & D & & \begin{tabular}{|l} 
Virtual \\
Library
\end{tabular} & $\begin{array}{l}\text { Virtual } \\
\text { Library }\end{array}$ & & & & & & Y & HWIS & \\
\hline 1256 & $200-E$ & 299-E33-234 & A7041 & D & & \begin{tabular}{|l|} 
Virtual \\
Library
\end{tabular} & \begin{tabular}{|l|} 
Virtual \\
Library
\end{tabular} & & & & & & Y & HWIS & \\
\hline
\end{tabular}




\begin{tabular}{|c|c|c|c|c|c|c|c|c|c|c|c|c|c|c|c|}
\hline SortIndex & Area & Well Name & WellID & Log Type & GPX Log Runs & Sieved & $\mathrm{CaCO} 3$ & Moisture & $\begin{array}{l}\text { Chemical } \\
\text { Properties }\end{array}$ & $\begin{array}{c}\text { Physical } \\
\text { Properties }\end{array}$ & \begin{tabular}{|c|} 
Min \\
Properties
\end{tabular} & $\begin{array}{l}\text { Geochron } \\
\text { Properties }\end{array}$ & Archived & Source & Comments \\
\hline \begin{tabular}{|l|}
1257 \\
\end{tabular} & $200-E$ & 299-E33-235 & A7042 & D & & \begin{tabular}{|l|} 
Virtual \\
Library
\end{tabular} & $\begin{array}{l}\text { Virtual } \\
\text { Library }\end{array}$ & & & & & & $Y$ & HWIS & \\
\hline 1258 & $200-E$ & 299-E33-236 & A7043 & D & & & & & & & & & $\mathrm{Y}$ & HWIS & \\
\hline 1259 & $200-E$ & 299-E33-237 & A7044 & D & & $\begin{array}{l}\text { Virtual } \\
\text { Library }\end{array}$ & $\begin{array}{l}\text { Virtual } \\
\text { Library }\end{array}$ & & Field Rad & & & & Y & HWIS & \\
\hline 1260 & $200-E$ & 299-E33-238 & A7045 & D & & & & & & & & & & HWIS & \\
\hline 1261 & $200-E$ & 299-E33-239 & A7046 & $\mathrm{D}$ & & & & & & & & & $\mathrm{Y}$ & HWIS & \\
\hline 1262 & $200-E$ & 299-E33-24 & A4849 & D & DN, GG, NT, SG & & & & & & & & & HWIS & \\
\hline 1263 & $200-E$ & 299-E33-240 & A7047 & D & & $\begin{array}{l}\text { Virtual } \\
\text { Library }\end{array}$ & $\begin{array}{l}\text { Virtual } \\
\text { Library }\end{array}$ & & & & & & $Y$ & HWIS & \\
\hline 1264 & $200-E$ & 299-E33-241 & A7048 & D & & $\begin{array}{l}\text { Virtual } \\
\text { Library }\end{array}$ & $\begin{array}{l}\text { Virtual } \\
\text { Library }\end{array}$ & & & & & & Y & HWIS & \\
\hline 1265 & $200-E$ & 299-E33-242 & A7049 & D & & $\begin{array}{l}\text { Virtual } \\
\text { Library }\end{array}$ & $\begin{array}{l}\text { Virtual } \\
\text { Library }\end{array}$ & & & & & & Y & HWIS & \\
\hline 1266 & $200-E$ & 299-E33-243 & A7050 & D & & $\begin{array}{l}\text { Virtual } \\
\text { Library }\end{array}$ & $\begin{array}{l}\text { Virtual } \\
\text { Library }\end{array}$ & & & & & & $\mathrm{Y}$ & HWIS & \\
\hline 1267 & $200-E$ & 299-E33-244 & A7051 & D & & $\begin{array}{l}\text { Virtual } \\
\text { Library }\end{array}$ & $\begin{array}{l}\text { Virtual } \\
\text { Library }\end{array}$ & & & & & & $\mathrm{Y}$ & & \\
\hline 1268 & $200-E$ & 299-E33-245 & A7052 & D & & $\begin{array}{l}\text { Virtual } \\
\text { Library }\end{array}$ & $\begin{array}{l}\text { Virtual } \\
\text { Library }\end{array}$ & & & & & & $\mathrm{Y}$ & HWIS & \\
\hline 1269 & $200-E$ & 299-E33-246 & A7053 & D & & $\begin{array}{l}\text { Virtual } \\
\text { Library }\end{array}$ & $\begin{array}{l}\text { Virtual } \\
\text { Library }\end{array}$ & & & & & & $Y$ & HWIS & \\
\hline 1270 & $200-E$ & 299-E33-247 & A7054 & D & & & & & & & & & & HWIS & \\
\hline 1271 & $200-E$ & 299-E33-248 & A7055 & D & & $\begin{array}{l}\text { Virtual } \\
\text { Library }\end{array}$ & $\begin{array}{l}\text { Virtual } \\
\text { Library }\end{array}$ & & & & & & $Y$ & HWIS & \\
\hline 1272 & $200-E$ & 299-E33-249 & A7056 & D & & $\begin{array}{l}\text { Virtual } \\
\text { Library }\end{array}$ & $\begin{array}{l}\text { Virtual } \\
\text { Library }\end{array}$ & & & & & & $Y$ & HWIS & \\
\hline 1273 & $200-E$ & 299-E33-25 & A6858 & D & DN, GG, NT, SG & $\begin{array}{l}\text { Virtual } \\
\text { Library }\end{array}$ & $\begin{array}{l}\text { Virtual } \\
\text { Library }\end{array}$ & & & & & & $\mathrm{Y}$ & HWIS & \\
\hline 1274 & $200-E$ & 299-E33-250 & A7057 & D & & $\begin{array}{l}\text { Virtual } \\
\text { Library }\end{array}$ & $\begin{array}{l}\text { Virtual } \\
\text { Library }\end{array}$ & & & & & & $\mathrm{Y}$ & HWIS & \\
\hline 1275 & $200-E$ & 299-E33-251 & A7058 & D & & $\begin{array}{l}\text { Virtual } \\
\text { Library }\end{array}$ & $\begin{array}{l}\text { Virtual } \\
\text { Library }\end{array}$ & & & & & & $Y$ & HWIS & \\
\hline 1276 & $200-E$ & 299-E33-252 & A7059 & D & & $\begin{array}{l}\text { Virtual } \\
\text { Library }\end{array}$ & $\begin{array}{l}\text { Virtual } \\
\text { Library }\end{array}$ & & & & & & $\mathrm{Y}$ & HWIS & \\
\hline 1277 & $200-E$ & 299-E33-253 & A7060 & D & & $\begin{array}{l}\text { Virtual } \\
\text { Library }\end{array}$ & $\begin{array}{l}\text { Virtual } \\
\text { Library }\end{array}$ & & & & & & $\mathrm{Y}$ & HWIS & \\
\hline 1278 & $200-E$ & 299-E33-254 & A7061 & D & & $\begin{array}{l}\text { Virtual } \\
\text { Library }\end{array}$ & $\begin{array}{l}\text { Virtual } \\
\text { Library }\end{array}$ & & & & & & $Y$ & HWIS & \\
\hline 1279 & $200-E$ & 299-E33-255 & A7062 & D & & $\begin{array}{l}\text { Virtual } \\
\text { Library }\end{array}$ & $\begin{array}{l}\text { Virtual } \\
\text { Library }\end{array}$ & & & & & & $\mathrm{Y}$ & HWIS & \\
\hline 1280 & $200-E$ & 299-E33-256 & A7063 & D & & $\begin{array}{l}\text { Virtual } \\
\text { Library }\end{array}$ & $\begin{array}{l}\text { Virtual } \\
\text { Library }\end{array}$ & & & & & & $\mathrm{Y}$ & HWIS & \\
\hline 1281 & $200-E$ & 299-E33-257 & A7064 & D & & $\begin{array}{l}\text { Virtual } \\
\text { Library }\end{array}$ & $\begin{array}{l}\text { Virtual } \\
\text { Library }\end{array}$ & & & & & & $\mathrm{Y}$ & HWIS & \\
\hline 1282 & $200-E$ & 299-E33-258 & A7065 & $\mathrm{D}$ & & $\begin{array}{l}\text { Virtual } \\
\text { Library }\end{array}$ & $\begin{array}{l}\text { Virtual } \\
\text { Library }\end{array}$ & & & & & & $Y$ & HWIS & \\
\hline 1283 & $200-E$ & 299-E33-259 & A7066 & D & & & & & & & & & $\mathrm{Y}$ & HWIS & \\
\hline 1284 & $200-E$ & 299-E33-26 & A4850 & D & DN, GG, NT, SG, TP & $\begin{array}{l}\text { Virtual } \\
\text { Library }\end{array}$ & $\begin{array}{l}\text { Virtual } \\
\text { Library }\end{array}$ & & & & & & $\mathrm{Y}$ & HWIS & \\
\hline 1285 & $200-E$ & 299-E33-260 & A7067 & D & & & & & Field Rad & & & & $\mathrm{Y}$ & HWIS & \\
\hline 1286 & $200-E$ & 299-E33-261 & A7068 & D & & & & & & & & & $\mathrm{Y}$ & HWIS & \\
\hline 1287 & $200-\mathrm{E}$ & 299-E33-262 & A7069 & D & & & & & & & & & $\mathrm{Y}$ & HWIS & \\
\hline 1288 & $200-E$ & 299-E33-263 & A7070 & D & & & & & & & & & $Y$ & HWIS & \\
\hline 1289 & $200-E$ & 299-E33-264 & A7071 & $\mathrm{D}$ & & & & & & & & & $Y$ & HWIS & \\
\hline 1290 & $200-E$ & 299-E33-27 & A4851 & D & GG, NT & & & & Field Rad & & & & & HWIS & \\
\hline 1291 & $200-E$ & 299-E33-273 & A7073 & D & & & & & Field Rad & & & & & HWIS & \\
\hline
\end{tabular}




\begin{tabular}{|c|c|c|c|c|c|c|c|c|c|c|c|c|c|c|c|}
\hline SortIndex & Area & Well Name & WelliD & Log Type & GPX Log Runs & Sieved & $\mathrm{CaCO} 3$ & Moisture & $\begin{array}{l}\text { Chemical } \\
\text { Properties }\end{array}$ & $\begin{array}{l}\text { Physical } \\
\text { Properties }\end{array}$ & $\begin{array}{c}\text { Min } \\
\text { Properties }\end{array}$ & $\begin{array}{l}\text { Geochron } \\
\text { Properties }\end{array}$ & Archived & Source & Comments \\
\hline 1292 & $200-E$ & 299-E33-274 & A7074 & $\mathrm{D}$ & & & & & & & & & Y & HWIS & \\
\hline 1293 & $200-E$ & 299-E33-277 & A7076 & $\mathrm{D}$ & & & & & & & & & $Y$ & HWIS & \\
\hline 1294 & $200-E$ & 299-E33-28 & A4852 & $\mathrm{D}, \mathrm{G}$ & DN, GG, NT & \begin{tabular}{|l|} 
Virtual \\
Library
\end{tabular} & & & & & & & $Y$ & HWIS & \\
\hline 1295 & $200-E$ & 299-E33-286 & A7082 & D & GG, MO, SG & & & & Field Rad & & & & $Y$ & HWIS & \\
\hline 1296 & $200-E$ & 299-E33-287 & A7083 & D & GG, MO, SG & & & & Field Rad & & & & $Y$ & HWIS & \\
\hline 1297 & $200-E$ & 299-E33-288 & A7084 & $\mathrm{D}$ & GG, MO, SG & & & & Field Rad & & & & $\mathrm{Y}$ & HWIS & \\
\hline 1298 & $200-E$ & 299-E33-289 & A7085 & $\mathrm{D}$ & GG, MO, SG & & & & Field Rad & & & & $\mathrm{Y}$ & HWIS & \\
\hline 1299 & $200-E$ & 299-E33-29 & A4853 & $\mathrm{D}, \mathrm{G}$ & DN, GG, NT & \begin{tabular}{|l} 
Virtual \\
Library
\end{tabular} & & & & & & & Y & & \\
\hline 1300 & $200-E$ & 299-E33-290 & A7086 & $\mathrm{D}$ & GG, MO, SG & & & & Field Rad & & & & $Y$ & HWIS & \\
\hline 1301 & $200-E$ & 299-E33-291 & A7087 & D & & & & & & & & & & HWIS & \\
\hline 1302 & $200-E$ & 299-E33-292 & A7088 & D & & & & & & & & & & HWIS & \\
\hline 1303 & $200-E$ & 299-E33-293 & A7089 & $\mathrm{D}$ & & & & & & & & & & HWIS & \\
\hline 1304 & $200-E$ & 299-E33-294 & A7090 & D & & & & & & & & & & HWIS & \\
\hline 1305 & $200-E$ & 299-E33-295 & A7091 & D & & & & & & & & & & HWIS & \\
\hline 1306 & $200-E$ & 299-E33-296 & A7092 & G & SG & $Y$ & $Y$ & $Y$ & & $\begin{array}{l}\text { SpG, Por, } \\
\text { Bden }\end{array}$ & & & Y & $\begin{array}{l}\text { Hoffman 1992; } \\
\text { HWIS }\end{array}$ & 200-BP-1 \\
\hline 1307 & $200-E$ & 299-E33-297 & A7093 & G & SG & & & & & & & & & HWIS & 200-BP-1 \\
\hline 1308 & $200-E$ & 299-E33-298 & A7094 & G & SG & & & & & & & & & HWIS & 200-BP-1 \\
\hline 1309 & $200-E$ & 299-E33-299 & A7095 & G & SG & & & & & & & & & HWIS & 200-BP-1 \\
\hline 1310 & 200-E & 299-E33-2A & & & & & & & Lab Rad & & & & & Haney 1967 & \\
\hline 1311 & $200-E$ & 299-E33-3 & A4854 & D & $\mathrm{DN}, \mathrm{GG}, \mathrm{NT}, \mathrm{SG}$ & & & & & & & & & HWIS & \\
\hline 1312 & $200-E$ & 299-E33-30 & A4855 & D, G & $\mathrm{DN}, \mathrm{GG}, \mathrm{NT}$ & $\begin{array}{l}\text { Virtual } \\
\text { Library }\end{array}$ & & & TC, IC, TOC & $\% G S S C, X R F$ & & & $Y$ & $\begin{array}{l}\text { Schramke 1988; } \\
\text { Ames and Serne } \\
1991\end{array}$ & \\
\hline 1313 & $200-E$ & 299-E33-300 & A7096 & G & SG & & & & & & & & & HWIS & 200-BP-1 \\
\hline 1314 & $200-\mathrm{E}$ & 299-E33-301 & A7097 & G & SG & & & & & & & & & HWIS & 200-BP-1 \\
\hline 1315 & $200-E$ & 299-E33-302 & A7098 & G & SG & & & & & & & & & HWIS & 200-BP-1 \\
\hline 1316 & $200-E$ & 299-E33-303 & A7099 & G & SG & & & & & & & & & HWIS & 200-BP-1 \\
\hline 1317 & 200-E & 299-E33-304 & A7100 & G & SG & Y & Y & Y & & $\begin{array}{l}\text { SpG, Por, } \\
\text { Bden }\end{array}$ & & & $Y$ & $\begin{array}{l}\text { Hoffman 1992; } \\
\text { HWIS }\end{array}$ & 200-BP-1 \\
\hline 1318 & $200-E$ & 299-E33-305 & A7101 & G & SG & & & & & & & & & HWIS & 200-BP-1 \\
\hline 1319 & $200-E$ & 299-E33-306 & A7102 & G & SG & & & & & & & & & HWIS & 200-BP-1 \\
\hline 1320 & $200-E$ & 299-E33-307 & A7103 & G & SG & $\mathrm{Y}$ & $Y$ & $Y$ & & $\begin{array}{l}\text { SpG, Por, } \\
\text { Bden }\end{array}$ & & & & $\begin{array}{l}\text { Hoffman 1992; } \\
\text { HWIS }\end{array}$ & 200-BP-1 \\
\hline 1321 & $200-E$ & 299-E33-308 & A7104 & D, G, Well summary & SG & & & & & & & & & HWIS & \\
\hline 1322 & $200-E$ & 299-E33-309 & A7105 & D, G, Well summary & SG & & & & & & & & & HWIS & \\
\hline 1323 & $200-E$ & 299-E33-31 & A4856 & G & GG, SG & $\begin{array}{l}\text { Virtual } \\
\text { Library }\end{array}$ & & $Y$ & Rad,TOC,An & & & & $Y$ & Pearson 1990 & \\
\hline 1324 & $200-E$ & 299-E33-310 & A7106 & G & SG & & & & & & & & & HWIS & 200-BP-1 \\
\hline 1325 & $200-E$ & 299-E33-311 & A7107 & G & SG & & & & & & & & & HWIS & 200-BP-1 \\
\hline 1326 & $200-E$ & 299-E33-312 & A7108 & G & SG & & & & & & & & & HWIS & 200-BP-1 \\
\hline 1327 & $200-E$ & 299-E33-313 & A7109 & G & & & & & & & & & & HWIS & 200-BP-1 \\
\hline 1328 & $200-E$ & 299-E33-314 & A7110 & G & & & & & & & & & & HWIS & 200-BP-1 \\
\hline 1329 & $200-E$ & 299-E33-315 & A7111 & G & & & & & & & & & & HWIS & 200-BP-1 \\
\hline 1330 & $200-E$ & 299-E33-316 & A7112 & G & & & & & & & & & & HWIS & 200-BP-1 \\
\hline 1331 & $200-E$ & 299-E33-317 & A7113 & G & & & & & & & & & & HWIS & 200-BP-1 \\
\hline 1332 & $200-E$ & 299-E33-318 & A7114 & G & & & & & & & & & & HWIS & 200-BP-1 \\
\hline 1333 & $200-E$ & 299-E33-32 & A4857 & G & GG, SG & \begin{tabular}{|l|} 
Virtual \\
Library
\end{tabular} & & $Y$ & Field Rad & & & & Y & Pearson 1990 & \\
\hline 1334 & $200-E$ & 299-E33-320 & A7116 & G & & & & & & & & & & HWIS & 200-BP-1 \\
\hline 1335 & $200-E$ & 299-E33-321 & A7117 & G & & & & & & & & & & HWIS & 200-BP-1 \\
\hline 1336 & $200-E$ & 299-E33-322 & A7118 & G & & & & & & & & & & HWIS & 200-BP-1 \\
\hline
\end{tabular}




\begin{tabular}{|c|c|c|c|c|c|c|c|c|c|c|c|c|c|c|c|}
\hline SortIndex & Area & Well Name & WelllD & Log Type & GPX Log Runs & Sieved & $\mathrm{CaCO} 3$ & Moisture & $\begin{array}{l}\text { Chemical } \\
\text { Properties }\end{array}$ & $\begin{array}{c}\text { Physical } \\
\text { Properties }\end{array}$ & $\begin{array}{c}\text { Min } \\
\text { Properties }\end{array}$ & $\begin{array}{l}\text { Geochron } \\
\text { Properties }\end{array}$ & Archived & Source & Comments \\
\hline 1337 & $200-E$ & 299-E33-323 & A7119 & G & & & & & & & & & & HWIS & 200-BP-1 \\
\hline 1338 & $200-E$ & 299-E33-324 & A7120 & & GG, SG & & & & & & & & & & \\
\hline 1339 & $200-E$ & 299-E33-325 & A7121 & & GG, SG & & & & & & & & & & \\
\hline 1340 & $200-E$ & 299-E33-326 & A7122 & & SG & & & & & & & & & & \\
\hline 1341 & $200-E$ & 299-E33-327 & A7123 & & GG, SG & & & & & & & & & & \\
\hline 1342 & $200-E$ & 299-E33-328 & A7124 & & SG & & & & & & & & & & \\
\hline 1343 & $200-E$ & 299-E33-329 & A9942 & & GG & & & & & & & & & & \\
\hline 1344 & $200-E$ & $\frac{\mid 599-E 33-33}{299}$ & A4858 & G & GG & $\begin{array}{l}\text { Virtual } \\
\text { Library }\end{array}$ & & $Y$ & \begin{tabular}{|l} 
Field Rad, \\
PID
\end{tabular} & & & & $Y$ & Pearson 1990 & \\
\hline 1345 & $200-E$ & 299-E33-331 & A9944 & & SG & & & & & & & & & & \\
\hline 1346 & $200-E$ & 299-E33-332 & A9945 & & GG, SG & & & & & & & & & & \\
\hline 1347 & $200-E$ & 299-E33-333 & B8079 & G & & $Y$ & $Y$ & $Y$ & $\begin{array}{l}\text { Field Rad, } \\
\text { Metals, An, } \\
\text { VOA, SVOA, } \\
\text { Lab Rad. }\end{array}$ & $\begin{array}{l}\text { Bden, SpG, } \\
\text { CEC, Pden }\end{array}$ & & & & $\begin{array}{l}\text { Rohay and } \\
\text { Weekes } 1998\end{array}$ & \\
\hline 1348 & $200-E$ & 299-E33-334 & B8810 & G & NT, SG & & & Y & & & & & $Y$ & Horton 2000 & \\
\hline 1349 & $200-E$ & 299-E33-335 & B8811 & G & NT & & & & & & & Paleomag & $Y$ & $\begin{array}{l}\text { Horton 2000a; } \\
\text { Bjornstad et al. } \\
2001\end{array}$ & \\
\hline 1350 & $200-E$ & 299-E33-336 & B8908 & & SG & & & & & & & & & & \\
\hline 1351 & $200-E$ & 299-E33-337 & C3390 & G & NT, SG & Y & & & & & & & $Y$ & Horton 2002d & \\
\hline 1352 & $200-E$ & 299-E33-338 & C3391 & G & NT, SG & $\mathrm{Y}$ & & & & & & & $\mathrm{Y}$ & Horton 2002d & \\
\hline 1353 & $200-\mathrm{E}$ & 299-E33-339 & C3392 & G & NT, SG & $Y$ & & & & & & & $Y$ & Horton 2002d & \\
\hline 1354 & $200-E$ & 299-E33-34 & A4859 & G & GG & & & $Y$ & \begin{tabular}{|l|} 
An, Rad, \\
TOC, VOA
\end{tabular} & & & & $Y$ & Barton 1990 & \\
\hline 1355 & |200-E & 299-E33-35 & A4860 & G & GG & & & $Y$ & $\begin{array}{l}\text { An,Rad,TOC, } \\
\text { VOA }\end{array}$ & & & & $Y$ & Barton 1990 & \\
\hline 1356 & $200-E$ & 299-E33-36 & A4861 & G & GG & & & & & & & & $Y$ & & \\
\hline 1357 & $200-E$ & 299-E33-37 & A4862 & G & GG & & & & & & & & $Y$ & & \\
\hline 1358 & $200-E$ & 299-E33-38 & A4863 & G & GG, NT, SG & $Y$ & $Y$ & $Y$ & & $\begin{array}{l}\text { SpG, Bden, } \\
\text { Por }\end{array}$ & & & $Y$ & Hoffman 1992 & 200-BP-1 \\
\hline 1359 & $200-E$ & 299-E33-39 & A4864 & G & GG, SG & $Y$ & $Y$ & $Y$ & & $\begin{array}{l}\text { SpG, Bden, } \\
\text { Por }\end{array}$ & & & $Y$ & Hoffman 1992 & 200-BP-1 \\
\hline 1360 & $200-E$ & 299-E33-3A & & & & & & & Lab Rad & & & & & Haney 1967 & \\
\hline 1361 & $200-E$ & 299-E33-4 & A4865 & D & DN, GG, NT, SG & \begin{tabular}{|l} 
Virtual \\
Library
\end{tabular} & \begin{tabular}{|l|} 
Virtual \\
Library
\end{tabular} & & $\mathrm{pH}$ & $\begin{array}{l}\text { CEC, } \\
\% G S S C, 15- \\
\text { Atm }\end{array}$ & & & $Y$ & McHenry 1957 & \\
\hline 1362 & $200-E$ & 299-E33-40 & A4866 & G & GG, SG & $Y$ & Y & $Y$ & & $\begin{array}{l}\text { SpG, Bden, } \\
\text { Por }\end{array}$ & & & $Y$ & Hoffman 1992 & 200-BP-1 \\
\hline 1363 & $200-E$ & 299-E33-41 & A4867 & G & GG, SG & & & & & & & & $Y$ & & \\
\hline 1364 & $200-E$ & 299-E33-42 & A4868 & G & GG, SG & $Y$ & $\mathrm{Y}$ & $Y$ & Field Rad & & & & $Y$ & Caggiano 1993 & \\
\hline 1365 & $200-E$ & 299-E33-43 & A4869 & G & GG, SG & $Y$ & $Y$ & $Y$ & Field Rad & & & & Y & Caggiano 1993 & \\
\hline 1366 & $200-E$ & 299-E33-44 & B8554 & G & MO, SG & $Y$ & & Y & 1:01 & CEC, Cond & & & $Y$ & $\begin{array}{l}\text { Horton and } \\
\text { Narbutovskih } \\
1999\end{array}$ & \\
\hline 1367 & $200-E$ & 299-E33-45 & C3269 & G & GG, SG & & & & & & & & $\mathrm{Y}^{*}$ & $\begin{array}{l}\text { Lindsey et al. } \\
2001\end{array}$ & Backfilled \\
\hline 1368 & 200-E & 299-E33-46 & C3360 & G & $\mathrm{MO}, \mathrm{SG}$ & & & & & & & & $\mathrm{Y}^{*}$ & $\begin{array}{l}\text { Lindsey et al. } \\
2001\end{array}$ & \\
\hline 1369 & $200-E$ & 299-E33-47 & C4259 & G, Well summary & SG & & & & & & & & & HWIS & \\
\hline$\frac{1370}{1370}$ & $200-E$ & 299-E33-48 & C4260 & G, Well summary & SG & & & & & & & & & HWIS & \\
\hline 1371 & $200-\mathrm{E}$ & 299-E33-49 & C4261 & G, Well summary & NT, SG & & & & & & & & & HWIS & \\
\hline 1372 & $200-E$ & 299-E33-4A & & & & & & & Lab Rad & & & & & Haney 1967 & \\
\hline
\end{tabular}




\begin{tabular}{|c|c|c|c|c|c|c|c|c|c|c|c|c|c|c|c|}
\hline SortIndex & Area & Well Name & WelliD & Log Type & GPX Log Runs & Sieved & $\mathrm{CaCO} 3$ & Moisture & $\begin{array}{l}\text { Chemical } \\
\text { Properties }\end{array}$ & $\begin{array}{l}\text { Physical } \\
\text { Properties }\end{array}$ & $\begin{array}{c}\text { Min } \\
\text { Properties }\end{array}$ & $\begin{array}{l}\text { Geochron } \\
\text { Properties }\end{array}$ & Archived & Source & Comments \\
\hline 1373 & $200-E$ & 299-E33-5 & A4870 & D & DN, GG, MO, NT, SG & \begin{tabular}{|l|} 
Virtual \\
Library
\end{tabular} & $\begin{array}{l}\text { Virtual } \\
\text { Library }\end{array}$ & & & & & & $\mathrm{Y}$ & HWIS & \\
\hline 1374 & $200-E$ & 299-E33-51 & A6859 & G & GG & & & & & & & & & HWIS & \\
\hline 1375 & $200-E$ & 299-E33-52 & A6860 & D & GG & & & & & & & & & HWIS & \\
\hline 1376 & $200-E$ & 299-E33-53 & A6861 & D & GG & & & & & & & & & HWIS & \\
\hline 1377 & $200-E$ & 299-E33-54 & A6862 & D & GG & & & & & & & & & HWIS & \\
\hline 1378 & $200-E$ & 299-E33-55 & A6863 & D & GG & & & & & & & & & HWIS & \\
\hline 1379 & $200-E$ & 299-E33-56 & A6864 & $\mathrm{D}$ & GG & & & & & & & & & HWIS & \\
\hline 1380 & $200-E$ & 299-E33-57 & A6865 & D & GG & & & & & & & & & & \\
\hline 1381 & $200-E$ & 299-E33-58 & A6866 & D & DN, GG, NT, SG & $\begin{array}{l}\text { Virtual } \\
\text { Library }\end{array}$ & $\begin{array}{l}\text { Virtual } \\
\text { Library }\end{array}$ & & & & & & $Y$ & HWIS & $\begin{array}{l}1 \mathrm{ft} \mathrm{Sieve/CaCO3} \text { interval 20-50 } \\
\mathrm{ft}\end{array}$ \\
\hline 1382 & $200-E$ & 299-E33-59 & A6867 & D & DN, GG, NT, SG & \begin{tabular}{|l} 
Virtual \\
Library
\end{tabular} & \begin{tabular}{|l} 
Virtual \\
Library
\end{tabular} & & & & & & $Y$ & HWIS & $\begin{array}{l}1 \mathrm{ft} \text { Sieve/CaCO3 interval 20-50 } \\
\mathrm{ft}\end{array}$ \\
\hline 1383 & $200-E$ & 299-E33-6 & A6852 & D & DN, GG, NT, SG & \begin{tabular}{|l|} 
Virtual \\
Library
\end{tabular} & \begin{tabular}{|l|} 
Virtual \\
Library
\end{tabular} & & & & & & Y & HWIS & \\
\hline 1384 & $200-E$ & 299-E33-60 & A6868 & $\mathrm{D}$ & $\mathrm{DN}, \mathrm{GG}, \mathrm{NT}, \mathrm{SG}$ & $\begin{array}{l}\text { Virtual } \\
\text { Library }\end{array}$ & $\begin{array}{l}\text { Virtual } \\
\text { Library }\end{array}$ & & & & & & Y & HWIS & $\begin{array}{l}1 \mathrm{ft} \text { Sieve/CaCO3 interval 20-50 } \\
\mathrm{ft}\end{array}$ \\
\hline 1385 & 200-E & 299-E33-61 & A6869 & D & GG & Virtual & Virtual & & & & & & Y & HWIS & \\
\hline 1386 & $200-E$ & 299-E33-62 & A6870 & D & GG & $\begin{array}{l}\text { Litrary } \\
\text { Virtual } \\
\text { Library }\end{array}$ & \begin{tabular}{|l} 
Litbrary \\
Virtual \\
Library
\end{tabular} & & & & & & $\mathrm{Y}$ & HWIS & \\
\hline 1387 & $200-E$ & 299-E33-63 & A6871 & D & GG & $\begin{array}{l}\text { Virtual } \\
\text { Library }\end{array}$ & $\begin{array}{l}\text { Virtual } \\
\text { Library }\end{array}$ & & & & & & $Y$ & HWIS & \\
\hline 1388 & $200-E$ & 299-E33-64 & A6872 & D & GG & $\begin{array}{l}\text { Virtual } \\
\text { Library }\end{array}$ & $\begin{array}{l}\text { Virtual } \\
\text { Library }\end{array}$ & & & & & & $\mathrm{Y}$ & HWIS & \\
\hline 1389 & $200-E$ & 299-E33-65 & A6873 & D & GG & $\begin{array}{l}\text { Virtual } \\
\text { Library }\end{array}$ & $\begin{array}{l}\text { Virtual } \\
\text { Library }\end{array}$ & & & & & & $\mathrm{Y}$ & HWIS & \\
\hline 1390 & $200-E$ & 299-E33-66 & A6874 & D & GG, SG & $\begin{array}{l}\text { Virtual } \\
\text { Library }\end{array}$ & $\begin{array}{l}\text { Virtual } \\
\text { Library }\end{array}$ & & & & & & $\mathrm{Y}$ & HWIS & \\
\hline 1391 & $200-E$ & 299-E33-67 & A6875 & D & GG, SG & $\begin{array}{l}\text { Virtual } \\
\text { Library }\end{array}$ & $\begin{array}{l}\text { Virtual } \\
\text { Library }\end{array}$ & & & & & & $Y$ & HWIS & \\
\hline 1392 & $200-E$ & 299-E33-68 & A6876 & D & GG, SG & $\begin{array}{l}\text { Virtual } \\
\text { Library }\end{array}$ & $\begin{array}{l}\text { Virtual } \\
\text { Library }\end{array}$ & & & & & & $\mathrm{Y}$ & HWIS & \\
\hline 1393 & $200-E$ & 299-E33-69 & A6877 & D & $\mathrm{DN}, \mathrm{GG}, \mathrm{NT}, \mathrm{SG}$ & $\begin{array}{l}\text { Virtual } \\
\text { Library }\end{array}$ & $\begin{array}{l}\text { Virtual } \\
\text { Library }\end{array}$ & & & & & & $\mathrm{Y}$ & HWIS & \\
\hline 1394 & $200-E$ & 299-E33-7 & A4871 & D & DN, GG, NT, SG & $\begin{array}{l}\text { Virtual } \\
\text { Library }\end{array}$ & $\begin{array}{l}\text { Virtual } \\
\text { Library }\end{array}$ & & & & & & $\mathrm{Y}$ & HWIS & \\
\hline 1395 & $200-E$ & 299-E33-70 & A6878 & D & GG, SG & $\begin{array}{l}\text { Virtual } \\
\text { Library }\end{array}$ & $\begin{array}{l}\text { Virtual } \\
\text { Library }\end{array}$ & & & & & & $Y$ & HWIS & \\
\hline 1396 & $200-E$ & 299-E33-71 & A6879 & D & $\mathrm{DN}, \mathrm{GG}, \mathrm{NT}, \mathrm{SG}$ & $\begin{array}{l}\text { Virtual } \\
\text { Library }\end{array}$ & $\begin{array}{l}\text { Virtual } \\
\text { Library }\end{array}$ & & & & & & $Y$ & HWIS & \\
\hline 1397 & $200-E$ & 299-E33-72 & A6880 & D & DN, GG, NT, SG & $\begin{array}{l}\text { Virtual } \\
\text { Library }\end{array}$ & $\begin{array}{l}\text { Virtual } \\
\text { Library }\end{array}$ & & & & & & $\mathrm{Y}$ & HWIS & \\
\hline 1398 & $200-E$ & 299-E33-73 & A6881 & D & $\mathrm{DN}, \mathrm{GG}, \mathrm{NT}, \mathrm{SG}$ & $\begin{array}{l}\text { Virtual } \\
\text { Library }\end{array}$ & $\begin{array}{l}\text { Virtual } \\
\text { Library }\end{array}$ & & & & & & $Y$ & HWIS & \\
\hline 1399 & $200-E$ & 299-E33-74 & A6882 & D & $\mathrm{DN}, \mathrm{GG}, \mathrm{NT}, \mathrm{SG}$ & $\begin{array}{l}\text { Virtual } \\
\text { Library }\end{array}$ & $\begin{array}{l}\text { Virtual } \\
\text { Library }\end{array}$ & & & & & & & HWIS & \\
\hline 1400 & $200-E$ & 299-E33-75 & A6883 & D & DN, GG, NT, SG & $\begin{array}{l}\text { Virtual } \\
\text { Library }\end{array}$ & $\begin{array}{l}\text { Virtual } \\
\text { Library }\end{array}$ & & & & & & $Y$ & HWIS & \\
\hline 1401 & $200-E$ & 299-E33-76 & A6884 & D & $\mathrm{DN}, \mathrm{GG}, \mathrm{NT}, \mathrm{SG}$ & $\begin{array}{l}\text { Virtual } \\
\text { Library }\end{array}$ & $\begin{array}{l}\text { Virtual } \\
\text { Library }\end{array}$ & & & & & & $Y$ & HWIS & \\
\hline
\end{tabular}




\begin{tabular}{|c|c|c|c|c|c|c|c|c|c|c|c|c|c|c|c|}
\hline Sortindex & Area & Well Name & WellID & Log Type & GPX Log Runs & Sieved & $\mathrm{CaCO} 3$ & Moisture & $\begin{array}{l}\text { Chemical } \\
\text { Properties }\end{array}$ & $\begin{array}{l}\text { Physical } \\
\text { Properties }\end{array}$ & \begin{tabular}{|c|} 
Min \\
Properties
\end{tabular} & $\begin{array}{l}\text { Geochron } \\
\text { Properties }\end{array}$ & Archived & Source & Comments \\
\hline 1402 & $200-E$ & 299-E33-77 & A6885 & D & GG & \begin{tabular}{|l|} 
Virtual \\
Library
\end{tabular} & $\begin{array}{l}\text { Virtual } \\
\text { Library }\end{array}$ & & & & & & $\mathrm{Y}$ & HWIS & \\
\hline 1403 & $200-E$ & \begin{tabular}{|l|} 
299-E33-78 \\
\end{tabular} & A6886 & D & GG & $\begin{array}{l}\text { Virtual } \\
\text { Library }\end{array}$ & $\begin{array}{l}\text { Virtual } \\
\text { Library }\end{array}$ & & & & & & $Y$ & HWIS & \\
\hline 1404 & $200-E$ & 299-E33-79 & A6887 & $\mathrm{D}$ & SG & & & & & & & & & HWIS & \\
\hline 1405 & 200-E & 299-E33-8 & A4872 & D & $\begin{array}{l}\text { DN, GG, MO, NT, SG, } \\
\text { TP }\end{array}$ & $\begin{array}{l}\text { Virtual } \\
\text { Library }\end{array}$ & $\begin{array}{l}\text { Virtual } \\
\text { Library }\end{array}$ & & & & & & $Y$ & HWIS & \\
\hline 1406 & $200-E$ & 299-E33-80 & A6888 & $\mathrm{D}$ & & & & & & & & & & HWIS & \\
\hline 1407 & $200-E$ & 299-E33-81 & A6889 & D & SG & & & & & & & & . & HWIS & \\
\hline 1408 & $200-E$ & 299-E33-82 & A6890 & D & SG & & & & & & & & & HWIS & \\
\hline 1409 & 200-E & 299-E33-83 & A6891 & D & & & & & & & & & $Y$ & HWIS & \\
\hline 1410 & $200-E$ & 299-E33-84 & A6892 & D & GG & $\begin{array}{l}\text { Virtual } \\
\text { Library }\end{array}$ & \begin{tabular}{|l|} 
Virtual \\
Library
\end{tabular} & & & & & & $Y$ & HWIS & \\
\hline 1411 & $200-E$ & \begin{tabular}{|l|} 
299-E33-85 \\
\end{tabular} & A6893 & $\mathrm{D}$ & GG & $\begin{array}{l}\text { Virtual } \\
\text { Library }\end{array}$ & $\begin{array}{l}\text { Virtual } \\
\text { Library }\end{array}$ & & & & & & $Y$ & HWIS & \\
\hline 1412 & $200-E$ & 299-E33-86 & A6894 & D & GG & $\begin{array}{l}\text { Virtual } \\
\text { Library }\end{array}$ & \begin{tabular}{|l|} 
Virtual \\
Library
\end{tabular} & & & & & & & HWIS & \\
\hline 1413 & $200-E$ & 299-E33-87 & A6895 & D & GG & $\begin{array}{l}\text { Virtual } \\
\text { Library }\end{array}$ & \begin{tabular}{|l|} 
Virtual \\
Library
\end{tabular} & & & & & & $Y$ & HWIS & \\
\hline 1414 & $200-E$ & 299-E33-88 & A6896 & D & GG & $\begin{array}{l}\text { Virtual } \\
\text { Library }\end{array}$ & $\begin{array}{l}\text { Virtual } \\
\text { Library }\end{array}$ & & & & & & $Y$ & HWIS & \\
\hline 1415 & $200-E$ & 299-E33-89 & A6897 & D & GG & $\begin{array}{l}\text { Virtual } \\
\text { Library }\end{array}$ & \begin{tabular}{|l|} 
Virtual \\
Library
\end{tabular} & & & & & & $Y$ & HWIS & \\
\hline 1416 & $200-E$ & 299-E33-9 & A4873 & D & GG & $\begin{array}{l}\text { Virtual } \\
\text { Library }\end{array}$ & $\begin{array}{l}\text { Virtual } \\
\text { Library }\end{array}$ & & $\mathrm{pH}$ & $\begin{array}{l}\text { CEC, } \\
\% \text { GSSC, 15- } \\
\text { Atm }\end{array}$ & & & $Y$ & McHenry 1957 & \\
\hline 1417 & $200-E$ & 299-E33-90 & A6898 & D & DN, GG, NT & & & & Field Rad & & & & & HWIS & \\
\hline 1418 & $200-E$ & 299-E33-91 & A6899 & D & GG & & & & Field Rad & & & & & HWIS & \\
\hline 1419 & $200-E$ & 299-E33-92 & A6900 & D & & & & & Field Rad & & & & . & HWIS & \\
\hline 1420 & $200-E$ & 299-E33-93 & A6901 & D & & & & & Field Rad & & & & & HWIS & \\
\hline 1421 & $200-E$ & 299-E33-94 & A6902 & $\mathrm{D}$ & & & & & Field Rad & & & & $Y$ & HWIS & \\
\hline 1422 & 200-E & 299-E33-95 & A6903 & D & & $\begin{array}{l}\text { Virtual } \\
\text { Library }\end{array}$ & \begin{tabular}{|l|} 
Virtual \\
Library
\end{tabular} & & & & & & $Y$ & HWIS & \\
\hline 1423 & $200-E$ & 299-E33-96 & A6904 & D & & & & & & & & & $Y$ & HWIS & \\
\hline 1424 & 200-E & 299-E33-97 & A6905 & D & & $\begin{array}{l}\text { Virtual } \\
\text { Library }\end{array}$ & \begin{tabular}{|l|l|} 
Virtual \\
Library
\end{tabular} & & & & & & $Y$ & HWIS & \\
\hline 1425 & $200-E$ & \begin{tabular}{|l} 
299-E33-98 \\
\end{tabular} & A6906 & D & & & & & & & & & $Y$ & HWIS & \\
\hline 1426 & $200-E$ & 299-E33-99 & A6907 & D & & $\begin{array}{l}\text { Virtual } \\
\text { Library }\end{array}$ & $\begin{array}{l}\text { Virtual } \\
\text { Library }\end{array}$ & & & & & & $Y$ & HWIS & \\
\hline 1427 & $200-E$ & 299-E34-1 & A4874 & D & GG, NT, TP & $\begin{array}{l}\text { Virtual } \\
\text { Library }\end{array}$ & $\begin{array}{l}\text { Virtual } \\
\text { Library }\end{array}$ & & & & & & $Y$ & & \\
\hline 1428 & $200-E$ & 299-E34-10 & A4875 & G & GG, SG & & $\mathrm{Y}$ & $Y$ & Field Rad & & & & $Y$ & Mercer 1993b & \\
\hline 1429 & $200-E$ & \begin{tabular}{|l|} 
299-E34-11 \\
\end{tabular} & A4876 & G & $\mathrm{GG}, \mathrm{SG}$ & & Y & $Y$ & Field Rad & & & & $Y$ & Mercer 1993a & \\
\hline 1430 & $200-E$ & 299-E34-12 & A5433 & G & GG, SG & & $Y$ & $Y$ & Field Rad & & & & $\mathrm{Y}$ & Mercer 1993a & \\
\hline 1431 & $200-E$ & 299-E34-2 & A4877 & D, G & DN, GG, NT & \begin{tabular}{|l} 
Virtual \\
Library
\end{tabular} & & & $\begin{array}{l}\text { XRF,TC, IC, } \\
\text { TOC }\end{array}$ & $\begin{array}{l}\% G S S C, \\
\text { CEC }\end{array}$ & XRD & & $\mathrm{Y}$ & $\begin{array}{l}\text { PNNL files; Last } \\
\text { et al. 1989; } \\
\text { Schramke 1988; } \\
\text { Ames and Serne } \\
1991\end{array}$ & \\
\hline
\end{tabular}




\begin{tabular}{|c|c|c|c|c|c|c|c|c|c|c|c|c|c|c|c|}
\hline SortIndex & Area & Well Name & WellID & Log Type & GPX Log Runs & Sieved & $\mathrm{CaCO} 3$ & Moisture & $\begin{array}{r}\text { Chemical } \\
\text { Properties } \\
\end{array}$ & \begin{tabular}{|c|} 
Physical \\
Properties \\
\end{tabular} & \begin{tabular}{|c|} 
Min \\
Properties
\end{tabular} & $\begin{array}{l}\text { Geochron } \\
\text { Properties }\end{array}$ & Archived & Source & Comments \\
\hline 1432 & $200-E$ & 299-E34-3 & A4878 & G & DN, GG, NT & & & & TC, IC, TOC & $\begin{array}{l}\% \text { GSSC, } \\
\text { CEC }\end{array}$ & & & Y & $\begin{array}{l}\text { Schramke 1988; } \\
\text { Ames and Serne } \\
1991\end{array}$ & \\
\hline 1433 & $3200-E$ & 299-E34-4 & A4879 & $\mathrm{D}, \mathrm{G}$ & $\mathrm{DN}, \mathrm{GG}, \mathrm{NT}$ & \begin{tabular}{|l|l|} 
Virtual \\
Library
\end{tabular} & & & & & & & $Y$ & HWIS & \\
\hline 1434 & 4200-E & 299-E34-5 & A4880 & D, G & DN, GG, NT & \begin{tabular}{|l|} 
Virtual \\
Library
\end{tabular} & & & & & & & $\mathrm{Y}$ & HWIS & \\
\hline 1435 & $200-E$ & 299-E34-51A & A7125 & D & & & & & & & & & & HWIS & \\
\hline 1436 & $200-E$ & 299-E34-51B & A7126 & D & & & & & & & & & & HWIS & \\
\hline 1437 & $7200-E$ & 299-E34-51C & A7127 & $\mathrm{D}$ & & & & & & & & & & HWIS & \\
\hline 1438 & $3200-E$ & 299-E34-51D & A7128 & D & & & & & & & & & & HWIS & \\
\hline 1439 & $200-E$ & 299-E34-51E & A7129 & D & & & & & & & & & & HWIS & \\
\hline 1440 & $200-E$ & 299-E34-6 & A4881 & D, G & $\mathrm{DN}, \mathrm{GG}, \mathrm{NT}$ & \begin{tabular}{|l|} 
Virtual \\
Library
\end{tabular} & & $Y$ & & & & & $Y$ & Last et al. 1989 & \\
\hline 1441 & $200-E$ & 299-E34-7 & A4882 & G & GG & & & & $\begin{array}{l}\text { An, Rad, TOC, } \\
\text { VOA }\end{array}$ & & & & $Y$ & \begin{tabular}{|l|} 
Goodwin and \\
Bjornstad 1990
\end{tabular} & \\
\hline 1442 & $200-E$ & 299-E34-8 & A4883 & G & GG & & & & & & & & $Y$ & & \\
\hline 1443 & 3200-E & 299-E34-9 & A4884 & G & GG, SG & & $Y$ & $Y$ & Field Rad & & & & $Y$ & Mercer 1993b & \\
\hline 1444 & $4200-E$ & 299-E35-1 & A4885 & G & GG & $\begin{array}{l}\text { Virtual } \\
\text { Library }\end{array}$ & \begin{tabular}{|l} 
Virtual \\
Library
\end{tabular} & Y & $\begin{array}{l}\text { An, Rad, TOC, } \\
\text { VOA }\end{array}$ & & & & $Y$ & $\begin{array}{l}\text { Goodwin and } \\
\text { Bornstad } 1990\end{array}$ & \\
\hline 1445 & $200-E$ & 299-E35-2 & A4886 & G & GG & $\begin{array}{l}\text { Virtual } \\
\text { Library }\end{array}$ & $\begin{array}{l}\text { Virtual } \\
\text { Library }\end{array}$ & Y & Field $\mathrm{pH}, \mathrm{XRF}$ & SpG, \%GSSC & XRD & & $Y$ & $\begin{array}{l}\text { Doremus and } \\
\text { Pearson 1990; } \\
\text { Sweeney 1993b; } \\
\text { Sweeney } 1994\end{array}$ & \\
\hline 1446 & 5200-E & 399-E33-319 & & G & & & & & & & & & & HWIS & 200-BP-1 \\
\hline 1447 & $7200-E$ & B2469 & B2469 & & GG, MO, SG & & & & & & & & & & \\
\hline 1448 & $200-E$ & B2470 & B2470 & & $\mathrm{MO}, \mathrm{SG}$ & & & & & & & & & & \\
\hline 1449 & $200-E$ & B8501 & B8501 & As-built & $\mathrm{MO}, \mathrm{SG}$ & & & & & & & & & HWIS & \\
\hline 1450 & $200-E$ & B8502 & B8502 & As-built & MO, SG & & & & & & & & & HWIS & \\
\hline 1451 & $1200-E$ & B8826 & B8826 & G & NT, SG & & & $\mathrm{Y}$ & $\begin{array}{l}\text { An, Metals, } \\
\text { LabRad }\end{array}$ & $\begin{array}{l}\text { Bden, } \\
\% G S S C\end{array}$ & & & & DOE, 2004b & 216-A-29 \\
\hline 1452 & $200-E$ & B8827 & B8827 & G & NT, SG & & & Y & $\begin{array}{l}\text { An, Metals, } \\
\text { LabRad }\end{array}$ & $\begin{array}{l}\text { Bden, } \\
\% \text { GSSC }\end{array}$ & & & & DOE, 2004b & 216-A-29 \\
\hline 1453 & $3200-E$ & C3103 & C3103 & G & NT, SG & & & & & Bden & & & & $\begin{array}{l}\text { Todd and Trice } \\
\text { 2002; HWIS }\end{array}$ & \\
\hline 1454 & $4200-E$ & C3104 & C3104 & G & & & & & & Bden & & & & $\begin{array}{l}\text { Todd and Trice } \\
\text { 2002; HWIS }\end{array}$ & \\
\hline 1455 & $200-E$ & c3245 & C3245 & G & NT, SG & $Y$ & & Y & $\begin{array}{l}\text { metals, } \\
\text { anions, VOA, } \\
\text { SVOA, } \\
\text { numerous } \\
\text { radionuclides }\end{array}$ & & & & & $\begin{array}{l}\text { Hulstrom 2004; } \\
\text { DOE 2004a }\end{array}$ & \\
\hline
\end{tabular}




\begin{tabular}{|c|c|c|c|c|c|c|c|c|c|c|c|c|c|c|c|}
\hline SortIndex & Area & Well Name & WelllD & Log Type & GPX Log Runs & Sieved & $\mathrm{CaCO} 3$ & Moisture & $\begin{array}{c}\text { Chemical } \\
\text { Properties }\end{array}$ & $\begin{array}{c}\text { Physical } \\
\text { Properties }\end{array}$ & $\begin{array}{c}\text { Min } \\
\text { Properties }\end{array}$ & $\begin{array}{l}\text { Geochron } \\
\text { Properties }\end{array}$ & Archived & Source & Comments \\
\hline 1456 & 200-E & C3246 & C3246 & G & NT, SG & Y & & Y & $\begin{array}{l}\text { metals, } \\
\text { anions, VOA, } \\
\text { SVOA, } \\
\text { numerous } \\
\text { radionuclides }\end{array}$ & & & & & $\begin{array}{l}\text { Hulstrom 2004; } \\
\text { DOE 2004a }\end{array}$ & \\
\hline 1457 & $7200-E$ & C3247 & C3247 & G & & $Y$ & & $\mathrm{Y}$ & $\begin{array}{l}\text { metals, } \\
\text { anions, VOA, } \\
\text { SVOA, } \\
\text { numerous } \\
\text { radionuclides }\end{array}$ & & & & & $\begin{array}{l}\text { Hulstrom 2004; } \\
\text { DOE 2004a }\end{array}$ & \\
\hline 1458 & 3200-E & C3248 & C3248 & G & & 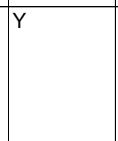 & & $Y$ & $\begin{array}{l}\text { metals, } \\
\text { anions, VOA, } \\
\text { SVOA, } \\
\text { numerous } \\
\text { radionuclides }\end{array}$ & & & & & $\begin{array}{l}\text { Hulstrom 2004; } \\
\text { DOE 2004a }\end{array}$ & \\
\hline 1459 & $200-E$ & C3340 & C3340 & Well Summary & & & & & & & & & & $\begin{array}{l}\text { Todd and Trice } \\
\text { 2002; HWIS }\end{array}$ & \\
\hline 1460 & $200-E$ & C3341 & C3341 & Well Summary & & & & & & & & & & $\begin{array}{l}\text { Todd and Trice } \\
\text { 2002; HWIS }\end{array}$ & \\
\hline 1461 & 200-E & C3342 & C3342 & Well Summary & & & & & & & & & & $\begin{array}{l}\text { Todd and Trice } \\
\text { 2002; HWIS }\end{array}$ & \\
\hline 1462 & $200-E$ & C3344 & C3344 & Well Summary & & & & & & & & & & $\begin{array}{l}\text { Todd and Trice } \\
\text { 2002; HWIS }\end{array}$ & \\
\hline 1463 & B200-E & C4069 & C4069 & & SG & & & & & & & & & & \\
\hline 1464 & $4200-E$ & C4070 & C4070 & & SG & & & & & & & & & & \\
\hline 1465 & $200-E$ & C4071 & C4071 & & SG & & & & & & & & & & \\
\hline 1466 & $200-E$ & C4106 & C4106 & G, As-built & NT, SG & & & & & & & & & HWIS & \\
\hline 1467 & $7200-E$ & C4160 & C4160 & G & NT, SG & & & & & & & & & HWIS & $216-A-36 B$ \\
\hline 1468 & $200-E$ & C4174 & C4174 & G, As-built & NT, SG & & & & & & & & & HWIS & \\
\hline 1469 & $200-E$ & C4191 & C4191 & G, As-built & NT, SG & & & & & & & & & HWIS & \\
\hline 1470 & $200-E$ & C4192 & C4192 & As-built & NT, SG & & & & & & & & & HWIS & \\
\hline 1471 & $200-E$ & C4193 & C4193 & As-built & NT, SG & & & & & & & & & HWIS & \\
\hline 1472 & $200-E$ & C4194 & C4194 & As-built & NT, SG & & & & & & & & & HWIS & \\
\hline 1473 & $200-E$ & C4195 & C4195 & As-built & NT, SG & & & & & & & & & HWIS & \\
\hline 1474 & $4200-E$ & C4196 & C4196 & As-built & NT, SG & & & & & & & & & HWIS & \\
\hline 1475 & $200-E$ & C4197 & C4197 & As-built & NT, SG & & & & & & & & & HWIS & \\
\hline 1476 & $200-E$ & C4297 & & & NT, SG & & & & & & & & & & \\
\hline 1477 & $200-E$ & C4671 & C4671 & & NT, SG & & & & & & & & & & \\
\hline 1478 & $3200-E$ & $\mathrm{~S}-1$ & & $G$ & & & & $Y$ & 1:1 anions & & & & & \begin{tabular}{|l|} 
Last and \\
Caldwell 2001
\end{tabular} & Sisson and Lu site \\
\hline 1479 & 200-E & $\mathrm{S}-2$ & & G & & & & $Y$ & 1:1 anions & & & & & \begin{tabular}{|l} 
Last and \\
Caldwell 2001
\end{tabular} & Sisson and Lu site \\
\hline 1480 & $200-E$ & $S-3$ & & G & & & & $\mathrm{Y}$ & $1: 1$ anions & & & & & \begin{tabular}{|l|} 
Last and \\
Caldwell 2001
\end{tabular} & Sisson and Lu site \\
\hline 1481 & 200-E & WL-10 & & G & & $\begin{array}{l}\%<0.053 \\
\mathrm{~mm}\end{array}$ & & $Y$ & 1:1 anions & & & & & Last et al. 2001 & Sisson and Lu site \\
\hline
\end{tabular}




\begin{tabular}{|c|c|c|c|c|c|c|c|c|c|c|c|c|c|c|c|}
\hline Sortlndex & Area & Well Name & WellID & Log Type & GPX Log Runs & Sieved & $\mathrm{CaCO} 3$ & Moisture & $\begin{array}{c}\text { Chemical } \\
\text { Properties }\end{array}$ & $\begin{array}{c}\text { Physical } \\
\text { Properties }\end{array}$ & $\begin{array}{c}\text { Min } \\
\text { Properties } \\
\end{array}$ & $\begin{array}{l}\text { Geochron } \\
\text { Properties }\end{array}$ & Archived & Source & Comments \\
\hline & $200-\mathrm{E}$ & WL-11 & & G & & $\mid \begin{array}{l}\%<0.053 \\
\mathrm{~mm}\end{array}$ & & Y & 1:1 anions & & & & & Last et al. 2001 & Sisson and Lu site \\
\hline 1483 & $200-E$ & WL-12 & & G & & $\begin{array}{l}\%<0.053 \\
\mathrm{~mm}\end{array}$ & & $Y$ & 1:1 anions & & & & & Last et al. 2001 & Sisson and Lu site \\
\hline 1484 & $200-\mathrm{E}$ & WL-3A & & G & & $\begin{array}{l}\%<0.053 \\
\mathrm{~mm}\end{array}$ & & $Y$ & 1:1 anions & & & & & Last et al. 2001 & Sisson and Lu site \\
\hline 1485 & $200-\mathrm{E}$ & WL-4 & & G & & $\begin{array}{l}\%<0.053 \\
\mathrm{~mm}\end{array}$ & & $Y$ & 1:1 anions & & & & & Last et al. 2001 & Sisson and Lu site \\
\hline 1486 & $200-E$ & WL-5 & & G & & $\begin{array}{l}\%<0.053 \\
\mathrm{~mm}\end{array}$ & & $Y$ & 1:1 anions & & & & & Last et al. 2001 & Sisson and Lu site \\
\hline 1487 & $200-E$ & WL-6 & & G & & $\begin{array}{l}\%<0.053 \\
\mathrm{~mm}\end{array}$ & & $Y$ & 1:1 anions & & & & & Last et al. 2001 & Sisson and Lu site \\
\hline 1488 & $200-\mathrm{E}$ & WL-7 & & G & & $\begin{array}{l}\%<0.053 \\
\mathrm{~mm}\end{array}$ & & $Y$ & 1:1 anions & & & & & Last et al. 2001 & Sisson and Lu site \\
\hline 1489 & $200-E$ & WL-8 & & G & & $\begin{array}{l}\%<0.053 \\
\mathrm{~mm}\end{array}$ & & $Y$ & 1:1 anions & & & & & Last et al. 2001 & Sisson and Lu site \\
\hline 1490 & $200-\mathrm{E}$ & WL-9 & & G & & $\begin{array}{l}\%<0.053 \\
\mathrm{~mm}\end{array}$ & & $Y$ & 1:1 anions & & & & & Last et al. 2001 & Sisson and Lu site \\
\hline 1491 & $200-W$ & 299-W10-1 & A7136 & D & DN, GG, NT, TP & \begin{tabular}{|l} 
Virtual \\
Library
\end{tabular} & $\begin{array}{l}\text { Virtual } \\
\text { Library }\end{array}$ & & Rad Info. & & & & $Y$ & PNNL files & \\
\hline 1492 & $200-W$ & 299-W10-10 & A4887 & D & & $\begin{array}{l}\text { Virtual } \\
\text { Library }\end{array}$ & & & & & & & $\mathrm{Y}$ & HWIS & \\
\hline 1493 & $200-W$ & 299-W10-101 & A7191 & D & & \begin{tabular}{|l|} 
Virtual \\
Library
\end{tabular} & $\begin{array}{l}\text { Virtual } \\
\text { Library }\end{array}$ & & & & & & $Y$ & HWIS & \\
\hline 1494 & $200-W$ & 299-W10-102 & A7192 & D & GG & & & & Field Rad & & & & $Y$ & HWIS & \\
\hline \begin{tabular}{|l|}
1495 \\
1496
\end{tabular} & $200-W$ & $\frac{299-W 10-103}{299-W 10-104}$ & \begin{tabular}{|l|} 
A7193 \\
A7194 \\
\end{tabular} & \begin{tabular}{|l}
$\mathrm{D}$ \\
$\mathrm{D}$
\end{tabular} & & Virtual & Virtual & & Field Rad & & & & $Y$ & \begin{tabular}{|l} 
HWIS \\
HWIS
\end{tabular} & \\
\hline 1497 & $200-W$ & 299-W10-105 & A7195 & D & GG & \begin{tabular}{|l|} 
Library \\
Virtual \\
Library
\end{tabular} & $\begin{array}{l}\text { LLbrary } \\
\text { Virtual } \\
\text { Library }\end{array}$ & & & & & & Y & HWIS & \\
\hline 1498 & $200-W$ & 299-W10-106 & A7196 & D & & & & & $\begin{array}{l}\text { Field Rad, Lab } \\
\text { Rad }\end{array}$ & & & & $\mathrm{Y}$ & ARH-2874 & \\
\hline 1499 & $200-W$ & 299-W10-107 & A7197 & D & & & & & $\begin{array}{l}\text { Field Rad, Lab } \\
\text { Rad }\end{array}$ & & & & $\mathrm{Y}$ & ARH-2874 & \\
\hline 1500 & $200-W$ & 299-W10-108 & A7198 & D & & & & & $\begin{array}{l}\text { Field Rad, Lab } \\
\text { Rad }\end{array}$ & & & & $Y$ & ARH-2874 & \\
\hline 1501 & $200-W$ & 299-W10-109 & A7199 & D & & $\begin{array}{l}\text { Virtual } \\
\text { Library }\end{array}$ & & & $\begin{array}{l}\text { Field Rad, Lab } \\
\text { Rad }\end{array}$ & & & & $\mathrm{Y}$ & ARH-2874 & \\
\hline 1502 & $200-W$ & 299-W10-11 & A4888 & D & & $\begin{array}{l}\text { Virtual } \\
\text { Library }\end{array}$ & & & & & & & $Y$ & HWIS & \\
\hline 1503 & $200-W$ & 299-W10-110 & A7200 & D & GG & & & & $\begin{array}{l}\text { Field Rad, Lab } \\
\text { Rad }\end{array}$ & & & & & ARH-2874 & \\
\hline 1504 & $200-W$ & 299-W10-111 & A7201 & D & GG & & & & $\begin{array}{l}\text { Field Rad, Lab } \\
\text { Rad }\end{array}$ & & & & & ARH-2874 & \\
\hline 1505 & $200-W$ & 299-W10-112 & A7202 & $\mathrm{D}$ & & \begin{tabular}{|l|l|} 
Virtual \\
Library
\end{tabular} & \begin{tabular}{|l|} 
Virtual \\
Library
\end{tabular} & & & & & & $Y$ & HWIS & \\
\hline 1506 & $200-W$ & 299-W10-113 & A7203 & D & & $\begin{array}{l}\text { Virtual } \\
\text { Library }\end{array}$ & $\begin{array}{l}\text { Virtual } \\
\text { Library }\end{array}$ & & & & & & $Y$ & HWIS & \\
\hline
\end{tabular}




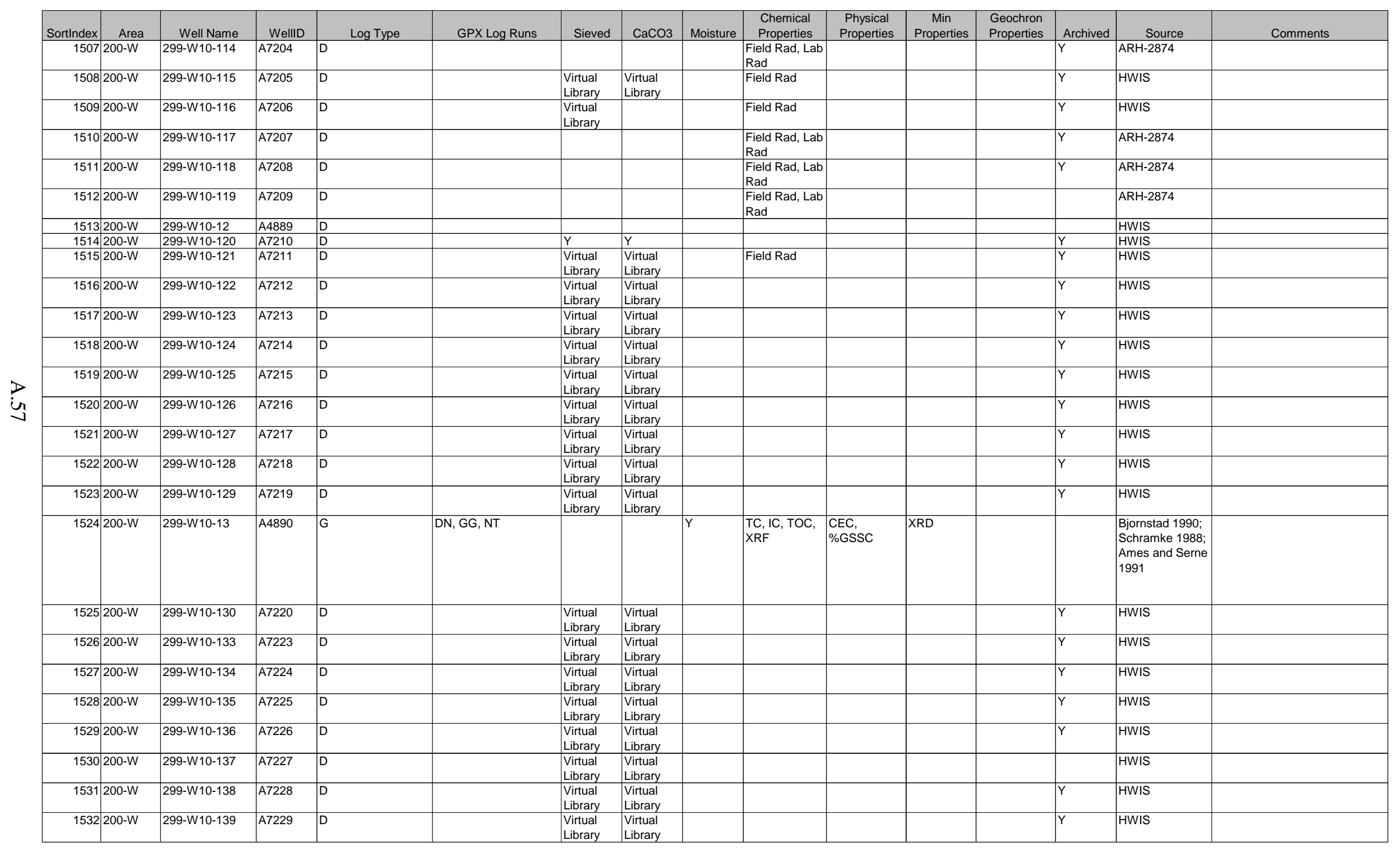




\begin{tabular}{|c|c|c|c|c|c|c|c|c|c|c|c|c|c|c|c|}
\hline SortIndex & Area & Well Name & WellID & Log Type & GPX Log Runs & Sieved & $\mathrm{CaCO} 3$ & Moisture & $\begin{array}{l}\text { Chemical } \\
\text { Properties }\end{array}$ & \begin{tabular}{|c|} 
Physical \\
Properties
\end{tabular} & $\begin{array}{c}\text { Min } \\
\text { Properties }\end{array}$ & $\begin{array}{l}\text { Geochron } \\
\text { Properties }\end{array}$ & Archived & Source & Comments \\
\hline 1533 & $200-W$ & 299-W10-14 & A4891 & G & $\mathrm{DN}, \mathrm{GG}, \mathrm{NT}$ & & & Y & $\begin{array}{l}\text { TC, IC, TOC, } \\
\text { XRF }\end{array}$ & & & & & $\begin{array}{l}\text { Bjornstad 1990; } \\
\text { Schramke 1988; } \\
\text { Ames and Serne } \\
1991\end{array}$ & \\
\hline 1534 & $200-W$ & 299-W10-140 & A7230 & D & & \begin{tabular}{|l|} 
Virtual \\
Library
\end{tabular} & $\begin{array}{l}\text { Virtual } \\
\text { Library }\end{array}$ & & & & & & $Y$ & HWIS & \\
\hline 1535 & $200-W$ & 299-W10-141 & A7231 & D & & \begin{tabular}{|l} 
Virtual \\
Library
\end{tabular} & $\begin{array}{l}\text { Virtual } \\
\text { Library }\end{array}$ & & & & & & $Y$ & HWIS & \\
\hline 1536 & $200-W$ & 299-W10-142 & A7232 & D & & \begin{tabular}{|l|} 
Virtual \\
Library
\end{tabular} & $\begin{array}{l}\text { Virtual } \\
\text { Library }\end{array}$ & & & & & & Y & HWIS & \\
\hline 1537 & $200-W$ & 299-W10-143 & A7233 & D & & \begin{tabular}{|l|} 
Virtual \\
Library
\end{tabular} & $\begin{array}{l}\text { Virtual } \\
\text { Library }\end{array}$ & & & & & & $\mathrm{Y}$ & HWIS & \\
\hline 1538 & $200-W$ & 299-W10-144 & A7234 & D & & $\begin{array}{l}\text { Virtual } \\
\text { Library }\end{array}$ & $\begin{array}{l}\text { Virtual } \\
\text { Library }\end{array}$ & & & & & & Y & HWIS & \\
\hline 1539 & $200-W$ & 299-W10-145 & A7235 & D & & \begin{tabular}{|l|l|} 
Virtual \\
Library
\end{tabular} & $\begin{array}{l}\text { Virtual } \\
\text { Library }\end{array}$ & & Field Rad & & & & $Y$ & HWIS & \\
\hline 1540 & $200-W$ & 299-W10-146 & A7236 & $\mathrm{D}$ & & & & & & & & & $\mathrm{Y}$ & HWIS & \\
\hline 1541 & $200-W$ & 299-W10-147 & A7237 & D & & $\begin{array}{l}\text { Virtual } \\
\text { Library }\end{array}$ & $\begin{array}{l}\begin{array}{l}\text { Virtual } \\
\text { Library }\end{array} \\
\end{array}$ & & & & & & $\mathrm{Y}$ & HWIS & \\
\hline 1542 & $200-W$ & 299-W10-148 & A7238 & D & & \begin{tabular}{|l} 
Virtual \\
Library
\end{tabular} & \begin{tabular}{|l|} 
Virtual \\
Library
\end{tabular} & & & & $\begin{array}{l}\text { XRD, SEM, } \\
\text { EM }\end{array}$ & & Y & $\begin{array}{l}\text { Routson et al. } \\
\text { 1979; Ames } \\
1976\end{array}$ & \\
\hline 1543 & $200-W$ & 299-W10-149 & A7239 & D & & $\begin{array}{l}\text { Virtual } \\
\text { Library }\end{array}$ & $\begin{array}{l}\text { Virtual } \\
\text { Library }\end{array}$ & & Field Rad & & & & $\mathrm{Y}$ & HWIS & \\
\hline 1544 & $200-W$ & 299-W10-15 & A4892 & G & GG & Y & & $Y$ & $\mathrm{YL}$ & & & & $Y$ & Pearson 1990 & \\
\hline 1545 & $200-W$ & 299-W10-150 & A7240 & D & & & & & Field Rad & & & & Y & HWIS & \\
\hline 1546 & $200-W$ & 299-W10-151 & A7241 & D & & & & & & & & & $Y$ & HWIS & \\
\hline 1547 & $200-W$ & 299-W10-152 & A7242 & $\mathrm{D}$ & & & & & & & & & $Y$ & HWIS & \\
\hline 1548 & $200-W$ & 299-W10-153 & A7243 & D & & & & & & & & & $Y$ & HWIS & \\
\hline 1549 & $200-W$ & 299-W10-154 & A7244 & D & & & & & & & & & $\mathrm{Y}$ & HWIS & \\
\hline 1550 & $200-W$ & 299-W10-16 & A4893 & G & GG & $Y$ & & $Y$ & $\mathrm{YL}$ & & & & Y & Pearson 1990 & \\
\hline 1551 & $200-W$ & 299-W10-161 & A7245 & D & & & & & & & & & & HWIS & \\
\hline 1552 & $200-W$ & 299-W10-162 & A7246 & D & & \begin{tabular}{|l} 
Virtual \\
Library
\end{tabular} & $\begin{array}{l}\text { Virtual } \\
\text { Library }\end{array}$ & & & & & & $\mathrm{Y}$ & HWIS & \\
\hline 1553 & $200-W$ & 299-W10-164 & A7247 & D, G & & & & $Y$ & Field Rad & & & & Y & HWIS & Moisture data in geologic logs \\
\hline 1554 & $200-\mathrm{W}$ & 299-W10-165 & A7248 & D, G & & & & $\mathrm{Y}$ & Field Rad & & & & $\mathrm{Y}$ & HWIS & Moisture data in geologic logs \\
\hline 1555 & $200-W$ & 299-W10-166 & A7249 & D & & $\begin{array}{l}\text { Virtual } \\
\text { Library }\end{array}$ & \begin{tabular}{|l|} 
Virtual \\
Library
\end{tabular} & & Field Rad & & & & Y & HWIS & \\
\hline 1556 & $200-W$ & 299-W10-167 & A7250 & D & GG & \begin{tabular}{|l|} 
Virtual \\
Library
\end{tabular} & $\begin{array}{l}\begin{array}{l}\text { Virtual } \\
\text { Library }\end{array} \\
\end{array}$ & & Field Rad & & & & $\mathrm{Y}$ & HWIS & \\
\hline 1557 & $200-W$ & 299-W10-168 & A7251 & D & & & & & Field Rad & & & & Y & HWIS & \\
\hline 1558 & $200-W$ & 299-W10-17 & A4894 & G & GG & Y & $Y$ & $Y$ & & & & & Y & Caggiano 1992 & \\
\hline 1559 & $200-W$ & 299-W10-176 & A7255 & D & & & & & Field Rad & & & & $\mathrm{Y}$ & HWIS & \\
\hline 1560 & $200-w$ & 299-W10-177 & A7256 & $\mathrm{D}$ & & & & & Field Rad & & & & $Y$ & HWIS & \\
\hline 1561 & $200-W$ & 299-W10-178 & A7257 & $\mathrm{D}$ & & & & & Field Rad & & & & $\mathrm{Y}$ & HWIS & \\
\hline 1562 & $200-\mathrm{W}$ & 299-W10-179 & A7258 & $\mathrm{D}$ & & & & & Field Rad & & & & & HWIS & \\
\hline 1563 & $200-W$ & 299-W10-18 & A4895 & G & GG & Y & $\mathrm{Y}$ & $Y$ & & & & & Y & Caggiano 1992 & \\
\hline
\end{tabular}




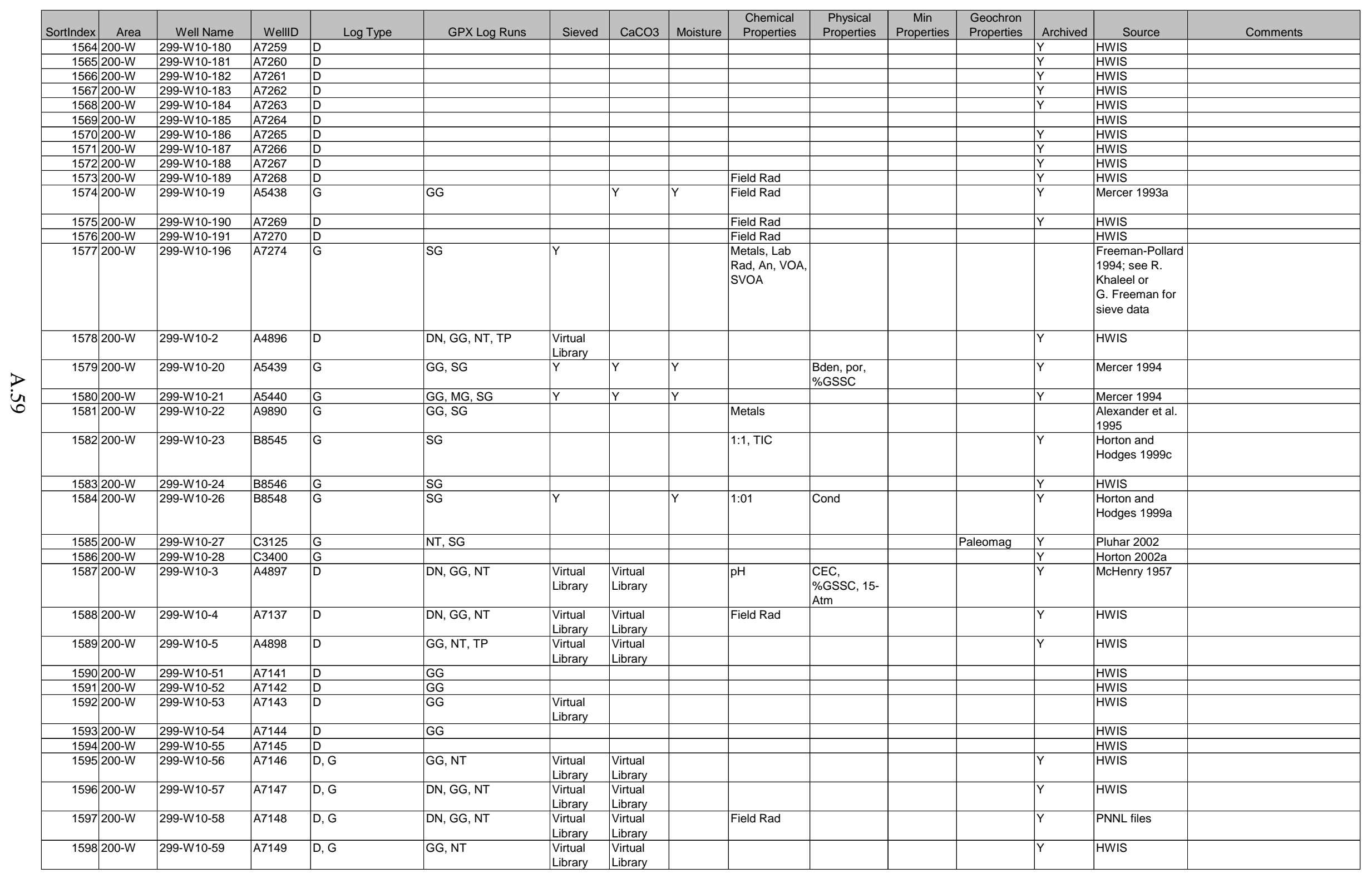




\begin{tabular}{|c|c|c|c|c|c|c|c|c|c|c|c|c|c|c|c|}
\hline SortIndex & Area & Well Name & WellID & Log Type & GPX Log Runs & Sieved & $\mathrm{CaCO} 3$ & Moisture & $\begin{array}{l}\text { Chemical } \\
\text { Properties }\end{array}$ & $\begin{array}{c}\text { Physical } \\
\text { Properties }\end{array}$ & $\begin{array}{c}\text { Min } \\
\text { Properties }\end{array}$ & $\begin{array}{l}\text { Geochron } \\
\text { Properties }\end{array}$ & Archived & Source & Comments \\
\hline 1599 & $200-W$ & 299-W10-6 & A7138 & $\begin{array}{l}\text { As-built, Well } \\
\text { Summary }\end{array}$ & & & & & & & & & & HWIS & \begin{tabular}{|l|} 
Abandoned? \\
?
\end{tabular} \\
\hline 1600 & $200-W$ & 299-W10-60 & A7150 & D, G & GG & \begin{tabular}{|l|} 
Virtual \\
Library
\end{tabular} & $\begin{array}{l}\text { Virtual } \\
\text { Library }\end{array}$ & & & & & & $Y$ & HWIS & \\
\hline 1601 & $200-W$ & 299-W10-61 & A7151 & D, G & GG & $\begin{array}{l}\text { Virtual } \\
\text { Library }\end{array}$ & $\begin{array}{l}\text { Virtual } \\
\text { Library }\end{array}$ & & & & & & $Y$ & HWIS & \\
\hline 1602 & $200-W$ & 299-W10-62 & \begin{tabular}{|l} 
A7152 \\
\end{tabular} & D, G & DN, GG, NT & $\begin{array}{l}\text { Virtual } \\
\text { Library }\end{array}$ & $\begin{array}{l}\text { Virtual } \\
\text { Library }\end{array}$ & & & & & & $Y$ & HWIS & \\
\hline 1603 & $200-W$ & 299-W10-63 & A7153 & $D, G$ & GG, NT & & & & & & & & $Y$ & HWIS & \\
\hline 1604 & $200-W$ & 299-W10-64 & A7154 & $D, G$ & GG, NT & & & & Field Rad & & & & $Y$ & PNNL files & \\
\hline 1605 & $200-W$ & 299-W10-65 & A7155 & $D, G$ & $\mathrm{GG}, \mathrm{NT}$ & & & & & & & & $Y$ & HWIS & \\
\hline 1606 & $200-W$ & 299-W10-66 & A7156 & $\mathrm{D}, \mathrm{G}$ & GG, NT & \begin{tabular}{|l|} 
Virtual \\
Library
\end{tabular} & \begin{tabular}{|l|} 
Virtual \\
Library
\end{tabular} & & & & & & $Y$ & HWIS & \\
\hline 1607 & $200-W$ & 299-W10-67 & A7157 & D, G & DN, GG & $\begin{array}{l}\text { Virtual } \\
\text { Library }\end{array}$ & $\begin{array}{l}\text { Virtual } \\
\text { Library }\end{array}$ & & & & & & $Y$ & HWIS & \\
\hline 1608 & $200-W$ & 299-W10-68 & A7158 & D, G & GG & $\begin{array}{l}\text { Virtual } \\
\text { Library }\end{array}$ & $\begin{array}{l}\text { Virtual } \\
\text { Library }\end{array}$ & & & & & & $Y$ & HWIS & \\
\hline 1609 & $200-W$ & 299-W10-69 & A7159 & D, G & DN, GG, NT & $\begin{array}{l}\text { Virtual } \\
\text { Library }\end{array}$ & $\begin{array}{l}\text { Virtual } \\
\text { Library }\end{array}$ & & & & & & $Y$ & HWIS & \\
\hline 1610 & $200-W$ & 299-W10-7 & A7139 & As-built & & & & & & & & & & HWIS & Abandoned? \\
\hline 1611 & $200-W$ & 299-W10-70 & A7160 & D, G & DN, GG, NT & \begin{tabular}{|l|} 
Virtual \\
Library
\end{tabular} & \begin{tabular}{|l|} 
Virtual \\
Library
\end{tabular} & & & & & & $Y$ & HWIS & \\
\hline 1612 & $200-W$ & 299-W10-71 & A7161 & D, G & $\mathrm{DN}, \mathrm{GG}, \mathrm{NT}$ & \begin{tabular}{|l|} 
Virtual \\
Library
\end{tabular} & \begin{tabular}{|l|} 
Virtual \\
Library
\end{tabular} & & & & & & $Y$ & HWIS & \\
\hline 1613 & $200-W$ & 299-W10-72 & \begin{tabular}{|l|} 
A7162 \\
\end{tabular} & D, G & GG, NT & $\begin{array}{l}\text { Virtual } \\
\text { Library }\end{array}$ & $\begin{array}{l}\text { Virtual } \\
\text { Library }\end{array}$ & & & & & & $Y$ & HWIS & \\
\hline 1614 & $200-W$ & 299-W10-73 & A7163 & D, G & GG & $\begin{array}{l}\text { Virtual } \\
\text { Library }\end{array}$ & $\begin{array}{l}\text { Virtual } \\
\text { Library }\end{array}$ & & & & & & $Y$ & HWIS & \\
\hline 1615 & $200-W$ & 299-W10-74 & A7164 & $\mathrm{D}, \mathrm{G}$ & GG, NT & $\begin{array}{l}\text { Virtual } \\
\text { Library }\end{array}$ & $\begin{array}{l}\text { Virtual } \\
\text { Library }\end{array}$ & & & & & & $Y$ & HWIS & \\
\hline 1616 & $200-W$ & 299-W10-75 & A7165 & $D, G$ & DN, GG, NT & & & & & & & & $Y$ & HWIS & \\
\hline 1617 & $200-W$ & 299-W10-76 & A7166 & D, G & GG & \begin{tabular}{|l|} 
Virtual \\
Library
\end{tabular} & $\begin{array}{l}\text { Virtual } \\
\text { Library }\end{array}$ & & & & & & $Y$ & HWIS & \\
\hline 1618 & $200-W$ & 299-W10-77 & A7167 & & GG & & & & & & & & & & \\
\hline 1619 & $200-W$ & 299-W10-78 & A7168 & & GG & & & & & & & & & & \\
\hline 1620 & $200-W$ & 299-W10-79 & A7169 & & GG & & & & & & & & & & \\
\hline 1621 & $200-w$ & 299-W10-8 & A4899 & D & & \begin{tabular}{|l|} 
Virtual \\
Library
\end{tabular} & $\begin{array}{l}\text { Virtual } \\
\text { Library }\end{array}$ & & & & & & $Y$ & HWIS & \\
\hline 1622 & $200-W$ & 299-W10-80 & A7170 & D & GG & $\begin{array}{l}\text { Virtual } \\
\text { Library }\end{array}$ & $\begin{array}{l}\text { Virtual } \\
\text { Library }\end{array}$ & & & & & & $Y$ & HWIS & \\
\hline 1623 & $200-W$ & 299-W10-81 & A7171 & & $\mathrm{GG}, \mathrm{NT}$ & & & & & & & & & & \\
\hline 1624 & $200-\mathrm{W}$ & $299-W 10-82$ & A7172 & $\mathrm{D}$ & GG & & & & & & & & $Y$ & HWIS & \\
\hline 1625 & $200-W$ & $299-W 10-83$ & A7173 & D & GG & & & & & & & & $Y$ & HWIS & \\
\hline 1626 & $200-W$ & 299-W10-88 & A7178 & $\mathrm{D}$ & & & & & & & & & $Y$ & HWIS & \\
\hline 1627 & $200-w$ & 299-W10-89 & A7179 & $\mathrm{D}$ & & & & & & & & & $Y$ & HWIS & \\
\hline 1628 & $200-W$ & 299-W10-9 & A4900 & D & & \begin{tabular}{|l|} 
Virtual \\
Library
\end{tabular} & & & & & & & $Y$ & HWIS & \\
\hline 1629 & $200-W$ & 299-W10-90 & A7180 & $\mathrm{D}$ & & & & & & & & & $Y$ & HWIS & \\
\hline 1630 & $200-W$ & 299-W10-91 & A7181 & $\mathrm{D}$ & & & & & & & & & $Y$ & HWIS & \\
\hline 1631 & $200-W$ & 299-W10-92 & A7182 & D & & & & & & & & & $Y$ & HWIS & \\
\hline 1632 & $200-W$ & 299-W10-93 & A7183 & $\mathrm{D}$ & & & & & & & & & $Y$ & HWIS & \\
\hline 1633 & $200-W$ & 299-W10-94 & A7184 & $\mathrm{D}$ & & & & & & & & & $Y$ & HWIS & \\
\hline 1634 & $200-W$ & $299-$ W10-95 & A7185 & $\mathrm{D}$ & & & & & & & & & $Y$ & HWIS & \\
\hline 1635 & $200-W$ & 299-W10-96 & A7186 & $\mathrm{D}$ & & & & & & & & & $Y$ & HWIS & \\
\hline 1636 & $200-W$ & 299-W10-97 & A7187 & $\mathrm{D}$ & & & & & & & & & Y & HWIS & \\
\hline 1637 & $200-W$ & 299-W10-98 & A7188 & D & & & & & & & & & $Y$ & HWIS & \\
\hline 1638 & $200-\mathrm{W}$ & 299-W10-99 & A7189 & D & & & & & & & & & $Y$ & HWIS & \\
\hline
\end{tabular}




\begin{tabular}{|c|c|c|c|c|c|c|c|c|c|c|c|c|c|c|c|}
\hline SortIndex & Area & Well Name & WellID & Log Type & GPX Log Runs & Sieved & $\mathrm{CaCO} 3$ & Moisture & $\begin{array}{l}\text { Chemical } \\
\text { Properties }\end{array}$ & $\begin{array}{c}\text { Physical } \\
\text { Properties }\end{array}$ & \begin{tabular}{c|c|} 
Min \\
Properties
\end{tabular} & $\begin{array}{l}\text { Geochron } \\
\text { Properties }\end{array}$ & Archived & Source & Comments \\
\hline $\begin{array}{l}1639 \\
\end{array}$ & $200-W$ & 299-W11-1 & A7275 & $D, G$ & $\mathrm{DN}, \mathrm{GG}, \mathrm{NT}, \mathrm{SG}$ & \begin{tabular}{|l|} 
Virtual \\
Library
\end{tabular} & $\begin{array}{l}\text { Virtual } \\
\text { Library }\end{array}$ & & & & & & $\mathrm{Y}$ & HWIS & \\
\hline 1640 & $200-W$ & 299-W11-10 & A4901 & D & GG, NT, TP & \begin{tabular}{|l|} 
Virtual \\
Library
\end{tabular} & $\begin{array}{l}\text { Virtual } \\
\text { Library }\end{array}$ & & & & & & $\mathrm{Y}$ & HWIS & \\
\hline 1641 & $200-W$ & 299-W11-11 & A7280 & D & $\mathrm{DN}, \mathrm{GG}, \mathrm{NT}, \mathrm{SG}$ & \begin{tabular}{|l|} 
Virtual \\
Library
\end{tabular} & \begin{tabular}{|l|} 
Virtual \\
Library
\end{tabular} & & & & & & $Y$ & HWIS & \\
\hline 1642 & $200-W$ & 299-W11-12 & A4902 & D & GG, NT, TP & $\begin{array}{l}\text { Virtual } \\
\text { Library }\end{array}$ & $\begin{array}{l}\text { Virtual } \\
\text { Library }\end{array}$ & & & & & & $\mathrm{Y}$ & HWIS & \\
\hline 1643 & $200-W$ & 299-W11-13 & A5465 & D & GG, TP & & & & & & & & $\mathrm{Y}$ & HWIS & \\
\hline 1644 & $200-W$ & 299-W11-14 & A4903 & D & $\mathrm{DN}, \mathrm{GG}, \mathrm{NT}$ & \begin{tabular}{|l|}
$\begin{array}{l}\text { Virtual } \\
\text { Library }\end{array}$ \\
\end{tabular} & \begin{tabular}{|l|} 
Virtual \\
Library
\end{tabular} & & & & & & $\mathrm{Y}$ & HWIS & \\
\hline 1645 & $200-W$ & 299-W11-15 & A7281 & D & DN, GG, NT & & & & & & & & $\mathrm{Y}$ & HWIS & \\
\hline 1646 & $200-W$ & 299-W11-16 & A7282 & D & DN, GG, NT & \begin{tabular}{|l|} 
Virtual \\
Library
\end{tabular} & $\begin{array}{l}\text { Virtual } \\
\text { Library }\end{array}$ & & & & & & $\mathrm{Y}$ & HWIS & \\
\hline 1647 & $200-W$ & 299-W11-17 & A7283 & D & DN, GG, NT & \begin{tabular}{|l|} 
Virtual \\
Library
\end{tabular} & $\begin{array}{l}\text { Virtual } \\
\text { Library }\end{array}$ & & & & & & $\mathrm{Y}$ & HWIS & \\
\hline 1648 & $200-W$ & 299-W11-18 & A7284 & D & $\mathrm{DN}, \mathrm{GG}, \mathrm{NT}, \mathrm{SG}$ & \begin{tabular}{|l|} 
Virtual \\
Library
\end{tabular} & $\begin{array}{l}\text { Virtual } \\
\text { Library }\end{array}$ & & & & & & $Y$ & HWIS & \\
\hline 1649 & $200-W$ & 299-W11-19 & A4904 & D & $\mathrm{DN}, \mathrm{GG}, \mathrm{NT}, \mathrm{TP}$ & \begin{tabular}{|l} 
Virtual \\
Library
\end{tabular} & $\begin{array}{l}\text { Virtual } \\
\text { Library }\end{array}$ & & & & & & $\mathrm{Y}$ & HWIS & \\
\hline 1650 & $200-W$ & 299-W11-2 & A7276 & $D, G$ & GG, TP & \begin{tabular}{|l} 
Virtual \\
Library
\end{tabular} & $\begin{array}{l}\text { Virtual } \\
\text { Library }\end{array}$ & & $\mathrm{pH}$ & $\begin{array}{l}\text { CEC, } \\
\% \text { \%SSC, 15- } \\
\text { Atm }\end{array}$ & & & $\mathrm{Y}$ & McHenry 1957 & \\
\hline 1651 & $200-W$ & 299-W11-20 & A7285 & D & DN, GG, NT & \begin{tabular}{|l|} 
Virtual \\
Library
\end{tabular} & $\begin{array}{l}\text { Virtual } \\
\text { Library }\end{array}$ & & & & & & $Y$ & HWIS & \\
\hline 1652 & $200-W$ & 299-W11-21 & A7286 & D & DN, GG, NT & \begin{tabular}{|l} 
Virtual \\
Library
\end{tabular} & $\begin{array}{l}\text { Virtual } \\
\text { Library }\end{array}$ & & & & & & $\mathrm{Y}$ & HWIS & \\
\hline 1653 & $200-W$ & 299-W11-23 & A4905 & D & & \begin{tabular}{|l} 
Virtual \\
Library
\end{tabular} & $\begin{array}{l}\text { Virtual } \\
\text { Library }\end{array}$ & & & & & & $\mathrm{Y}$ & HWIS & \\
\hline 1654 & $200-W$ & 299-W11-24 & A4906 & D & & & & & & & & & $Y$ & HWIS & \\
\hline 1655 & $200-W$ & 299-W11-25B & C4669 & & SG & & & & & & & & & & \\
\hline 1656 & $200-W$ & 299-W11-26 & A7287 & $D, G$ & DN, GG, NT, TP & \begin{tabular}{|l|} 
Virtual \\
Library
\end{tabular} & \begin{tabular}{|l} 
Virtual \\
Library
\end{tabular} & $Y$ & & $\begin{array}{l}\text { Bden, } \\
\% \text { GSSC }\end{array}$ & SEM, XRD & Paleomag & $Y$ & \begin{tabular}{|l|} 
Virtual Library \\
2000 (PPI only, \\
includes \\
description); \\
Routson et al. \\
1979; Bjornstad \\
1984; Parker and \\
Johnston 1979; \\
Summers and \\
Hanson 1977
\end{tabular} & DH-6 \\
\hline 1657 & $200-W$ & 299-W11-27 & A4907 & G & GG, SG & Y & $\mathrm{Y}$ & $\mathrm{Y}$ & Field Rad & & & & $Y$ & Caggiano 1993 & \\
\hline 1658 & $200-W$ & 299-W11-28 & A4908 & G & $\mathrm{CP}, \mathrm{GG}, \mathrm{SG}$ & $Y$ & $Y$ & $Y$ & Field Rad & & & & $Y$ & Caggiano 1993 & \\
\hline 1659 & $200-W$ & 299-W11-29 & A7288 & G & GG & & & & VOA & & & & & $\begin{array}{l}\text { Weekes and } \\
\text { Glaman 1995; } \\
\text { Newcomer et al. } \\
1995\end{array}$ & \\
\hline 1660 & $200-W$ & 299-W11-3 & A5473 & $D, G$ & GG, NT & \begin{tabular}{|l|} 
Virtual \\
Library
\end{tabular} & $\begin{array}{l}\text { Virtual } \\
\text { Library }\end{array}$ & & & & & & $Y$ & HWIS & \\
\hline
\end{tabular}




\begin{tabular}{|c|c|c|c|c|c|c|c|c|c|c|c|c|c|c|c|}
\hline SortIndex & Area & Well Name & WellID & Log Type & GPX Log Runs & Sieved & $\mathrm{CaCO} 3$ & Moisture & $\begin{array}{l}\text { Chemical } \\
\text { Properties }\end{array}$ & $\begin{array}{c}\text { Physical } \\
\text { Properties }\end{array}$ & $\begin{array}{c}\text { Min } \\
\text { Properties }\end{array}$ & $\begin{array}{l}\text { Geochron } \\
\text { Properties }\end{array}$ & Archived & Source & Comments \\
\hline 1661 & $200-W$ & 299-W11-30 & A7289 & G & GG, SG & & & & $\begin{array}{l}\text { TC, TOC, IC, } \\
\text { VOA }\end{array}$ & & & & & $\begin{array}{l}\text { Weekes and } \\
\text { Glaman 1995; } \\
\text { Newcomer et al. } \\
1995\end{array}$ & \\
\hline 1662 & $200-W$ & 299-W11-31 & A5472 & G & GG, SG & & $Y$ & $Y$ & Field Rad & & & & $Y$ & Mercer 1993a & \\
\hline 1663 & $200-W$ & 299-W11-32 & A7290 & G & GG, SG & $\mathrm{Y}$ & & & $\begin{array}{l}\text { TC, TOC, IC, } \\
\text { VOA, N, P, } \\
\text { TFe/Fe2, } \\
\text { ASio2 }\end{array}$ & $\begin{array}{l}\text { \%GSSC, } \\
\text { Pden, Bden, } \\
\text { Por }\end{array}$ & XRD & & & $\begin{array}{l}\text { Weekes and } \\
\text { Glaman 1995; } \\
\text { Newcomer et al. } \\
1995\end{array}$ & \\
\hline 1664 & $200-W$ & 299-W11-33 & A9827 & G & & Y & & & & Pden, Cond & & & & $\begin{array}{l}\text { Weekes and } \\
\text { Glaman } 1995\end{array}$ & \\
\hline 1665 & $200-W$ & 299-W11-34 & A9826 & G & & Y & & & & Pden, Cond & & & & $\begin{array}{l}\text { Weekes and } \\
\text { Glaman } 1995\end{array}$ & \\
\hline 1666 & $200-W$ & 299-W11-35 & A9924 & G & & Y & & & & Pden, Cond & & & & $\begin{array}{l}\text { Weekes and } \\
\text { Glaman } 1995\end{array}$ & \\
\hline 1667 & $200-W$ & 299-W11-37 & A9889 & Well Summary & SG & & & & Metals & & & & & $\begin{array}{l}\text { Sweeney et al } \\
1995 ; \text { HWIS }\end{array}$ & \\
\hline 1668 & $200-W$ & 299-W11-38 & C3116 & G & NT, SG & & & & & & & & $\mathrm{Y}$ & $\begin{array}{l}\text { Horton and } \\
\text { Hodges 2001a }\end{array}$ & \\
\hline 1669 & $200-W$ & 299-W11-39 & C3117 & G & & $\mathrm{Y}$ & & & & & & Paleomag & $\mathrm{Y}$ & $\begin{array}{l}\text { Horton and } \\
\text { Hodges 2001a; } \\
\text { Pluhar 2002 }\end{array}$ & \\
\hline 1670 & $200-W$ & 299-W11-4 & A7277 & D & GG, NT & \begin{tabular}{|l|} 
Virtual \\
Library
\end{tabular} & \begin{tabular}{|l|} 
Virtual \\
Library
\end{tabular} & & & & & & $\mathrm{Y}$ & HWIS & \\
\hline 1671 & $200-W$ & 299-W11-40 & C3118 & G & & $Y$ & & & & & & & $Y$ & $\begin{array}{l}\text { Horton and } \\
\text { Hodges 2001a }\end{array}$ & \\
\hline 1672 & $200-W$ & 299-W11-41 & C3119 & G & NT, SG & Y & & & & & & & $\mathrm{Y}$ & $\begin{array}{l}\text { Horton and } \\
\text { Hodges 2001a }\end{array}$ & \\
\hline 1673 & $200-W$ & 299-W11-42 & C3242 & G & & $\mathrm{Y}$ & & & & & & & $Y$ & $\begin{array}{l}\text { Horton and } \\
\text { Hodges 2001a }\end{array}$ & \\
\hline 1674 & $200-W$ & 299-W11-5 & A7278 & D & GG, NT & \begin{tabular}{|l|}
$\begin{array}{l}\text { Virtual } \\
\text { Library }\end{array}$ \\
\end{tabular} & \begin{tabular}{|l} 
Virtual \\
Library \\
\end{tabular} & & & & & & $\mathrm{Y}$ & HWIS & \\
\hline 1675 & $200-W$ & 299-W11-51 & A7291 & $\mathrm{D}$ & GG & & & & & & & & & HWIS & \\
\hline 1676 & $200-W$ & 299-W11-52 & A7293 & D & & & & & & & & & & HWIS & \\
\hline 1677 & $200-W$ & 299-W11-53 & A7294 & D & GG & & & & & & & & & HWIS & \\
\hline 1678 & $200-W$ & 299-W11-54 & A7296 & $D, G$ & GG, NT, SG & $\begin{array}{l}\text { Virtual } \\
\text { Library }\end{array}$ & $\begin{array}{l}\text { Virtual } \\
\text { Library }\end{array}$ & & Field Rad & & & & $\mathrm{Y}$ & PNNL files & \\
\hline 1679 & $200-W$ & 299-W11-55 & A7297 & $D, G$ & DN, GG, NT, SG & \begin{tabular}{|l} 
Virtual \\
Library
\end{tabular} & $\begin{array}{l}\text { Virtual } \\
\text { Library }\end{array}$ & & Field Rad & & & & $Y$ & PNNL files & \\
\hline 1680 & $200-W$ & 299-W11-56 & A7298 & $D, G$ & DN, GG, NT, SG & \begin{tabular}{|l} 
Virtual \\
Library
\end{tabular} & $\begin{array}{l}\text { Virtual } \\
\text { Library }\end{array}$ & & Field Rad & & & & $\mathrm{Y}$ & PNNL files & \\
\hline 1681 & $200-W$ & 299-W11-57 & A7299 & $D, G$ & GG, SG & \begin{tabular}{|l} 
Virtual \\
Library
\end{tabular} & $\begin{array}{l}\text { Virtual } \\
\text { Library }\end{array}$ & & Field Rad & & & & $\mathrm{Y}$ & PNNL files & \\
\hline 1682 & $200-W$ & 299-W11-58 & A7300 & $D, G$ & DN, GG, NT, SG & \begin{tabular}{|l} 
Virtual \\
Library
\end{tabular} & \begin{tabular}{|l} 
Virtual \\
Library
\end{tabular} & & Field Rad & & & & $\mathrm{Y}$ & PNNL files & \\
\hline 1683 & $200-W$ & 299-W11-59 & A7301 & $D, G$ & GG, SG & & & & Field Rad & & & & & PNNL files & \\
\hline 1684 & $200-W$ & 299-W11-6 & A4909 & D & GG, NT & \begin{tabular}{|l} 
Virtual \\
Library
\end{tabular} & $\begin{array}{l}\text { Virtual } \\
\text { Library }\end{array}$ & & & & & & $\mathrm{Y}$ & HWIS & \\
\hline
\end{tabular}




\begin{tabular}{|c|c|c|c|c|c|c|c|c|c|c|c|c|c|c|c|}
\hline SortIndex & Area & Well Name & WellID & Log Type & GPX Log Runs & Sieved & $\mathrm{CaCO} 3$ & Moisture & $\begin{array}{l}\text { Chemical } \\
\text { Properties }\end{array}$ & $\begin{array}{c}\text { Physical } \\
\text { Properties }\end{array}$ & $\begin{array}{c}\text { Min } \\
\text { Properties }\end{array}$ & $\begin{array}{l}\text { Geochron } \\
\text { Properties }\end{array}$ & Archived & Source & Comments \\
\hline 1685 & $200-W$ & 299-W11-60 & A7302 & $D, G$ & DN, GG, NT, SG & \begin{tabular}{|l|} 
Virtual \\
Library
\end{tabular} & $\begin{array}{l}\text { Virtual } \\
\text { Library }\end{array}$ & & & & & & $\mathrm{Y}$ & \begin{tabular}{|l|} 
PNNL files \\
\end{tabular} & \\
\hline 1686 & $200-W$ & 299-W11-61 & A7303 & D, G & GG, SG & $\begin{array}{l}\text { Virtual } \\
\text { Library }\end{array}$ & $\begin{array}{l}\text { Virtual } \\
\text { Library }\end{array}$ & & Field Rad & & & & $Y$ & PNNL files & \\
\hline 1687 & $200-W$ & 299-W11-62 & A7304 & D, G & GG, SG & \begin{tabular}{|l} 
Virtual \\
Library
\end{tabular} & $\begin{array}{l}\text { Virtual } \\
\text { Library }\end{array}$ & & Field Rad & & & & $Y$ & PNNL files & \\
\hline 1688 & $200-W$ & 299-W11-63 & A7305 & D, G & DN, GG, NT, SG & $\begin{array}{l}\text { Virtual } \\
\text { Library }\end{array}$ & $\begin{array}{l}\text { Virtual } \\
\text { Library }\end{array}$ & & & & & & $Y$ & HWIS & \\
\hline 1689 & $200-W$ & 299-W11-64 & A7306 & D, G & GG, SG & \begin{tabular}{|l|} 
Virtual \\
Library
\end{tabular} & \begin{tabular}{|l} 
Virtual \\
Library
\end{tabular} & & & & & & Y & HWIS & \\
\hline 1690 & $200-W$ & 299-W11-65 & A7307 & $D, G$ & GG, SG & \begin{tabular}{|l|} 
Virtual \\
Library
\end{tabular} & \begin{tabular}{|l|} 
Virtual \\
Library
\end{tabular} & & & & & & $\mathrm{Y}$ & HWIS & \\
\hline 1691 & $1200-W$ & 299-W11-66 & A7308 & D & DN, GG, NT, SG & $\begin{array}{l}\text { Virtual } \\
\text { Library }\end{array}$ & \begin{tabular}{|l} 
Virtual \\
Library
\end{tabular} & & & & & & $\mathrm{Y}$ & HWIS & \\
\hline 1692 & $200-W$ & 299-W11-67 & A7309 & D & GG, SG & \begin{tabular}{|l|l|} 
Virtual \\
Library
\end{tabular} & \begin{tabular}{|l|} 
Virtual \\
Library
\end{tabular} & & & & & & $Y$ & HWIS & \\
\hline 1693 & $3200-W$ & 299-W11-68 & A7310 & D & DN, GG, NT & \begin{tabular}{|l|} 
Virtual \\
Library
\end{tabular} & \begin{tabular}{|l|} 
Virtual \\
Library
\end{tabular} & & & & & & $\mathrm{Y}$ & HWIS & \\
\hline 1694 & $4200-W$ & 299-W11-69 & A7311 & D & DN, GG, NT & & & & & & & & $Y$ & HWIS & \\
\hline 1695 & $5200-W$ & 299-W11-7 & A4910 & D & DN, GG, NT, SG, TP & \begin{tabular}{|l|}
$\begin{array}{l}\text { Virtual } \\
\text { Library }\end{array}$ \\
\end{tabular} & \begin{tabular}{|l} 
Virtual \\
Library
\end{tabular} & & & & & & Y & HWIS & \\
\hline 1696 & $200-W$ & 299-W11-70 & A7312 & D & DN, GG, NT, SG & \begin{tabular}{|l|}
$\begin{array}{l}\text { Virtual } \\
\text { Library }\end{array}$ \\
\end{tabular} & \begin{tabular}{|l|} 
Virtual \\
Library \\
\end{tabular} & & & & & & $\mathrm{Y}$ & HWIS & \\
\hline 1697 & $7200-\mathrm{W}$ & 299-W11-79 & A7321 & D & GG, SG & & & & Field Rad & & & & $Y$ & HWIS & \\
\hline 1698 & $200-W$ & 299-W11-8 & A7279 & D & GG, NT, TP & \begin{tabular}{|l|} 
Virtual \\
Library
\end{tabular} & \begin{tabular}{|l} 
Virtual \\
Library
\end{tabular} & & & & & & $Y$ & HWIS & \\
\hline 1699 & $200-W$ & 299-W11-80 & A7322 & D & GG & & & & & & & & $Y$ & HWIS & \\
\hline 1700 & $200-W$ & 299-W11-81 & A7323 & $\mathrm{D}$ & GG, SG & & & & Field Rad & & & & $Y$ & HWIS & \\
\hline 1701 & $1200-W$ & 299-W11-82 & A7324 & $\mathrm{D}$ & GG, SG & & & & Field Rad & & & & $Y$ & HWIS & \\
\hline 1702 & $200-W$ & 299-W11-9 & A4911 & D & GG, NT, TP & \begin{tabular}{|l} 
Virtual \\
Library
\end{tabular} & $\begin{array}{l}\text { Virtual } \\
\text { Library }\end{array}$ & & & & & & $Y$ & HWIS & \\
\hline 1703 & $200-W$ & 299-W12-1 & A4912 & D, As-built & GG, NT, TP & & & & & & & & & HWIS & \\
\hline 1704 & $4200-W$ & 299-W13-1 & C4238 & G & GG & & & & & & & & & Martinez 2004 & 200-ZP-1 \\
\hline 1705 & $200-W$ & 299-W14-1 & A4913 & D & DN, GG, NT, SG, TP & $\begin{array}{l}\text { Virtual } \\
\text { Library }\end{array}$ & \begin{tabular}{|l|} 
Virtual \\
Library
\end{tabular} & & & & & & $Y$ & HWIS & \\
\hline 1706 & $200-W$ & 299-W14-10 & A7334 & $\mathrm{D}$ & DN, GG, NT, TP & & & & & & & & $\mathrm{Y}$ & HWIS & \\
\hline 1707 & $200-W$ & 299-W14-11 & C4668 & & & & & & & & & & & & \\
\hline 1708 & $3200-W$ & 299-W14-12 & \begin{tabular}{|l|} 
A4914 \\
\end{tabular} & G & GG, SG & Y & $\mathrm{Y}$ & $Y$ & & & & & Y & Caggiano 1993 & \\
\hline 1709 & $200-W$ & 299-W14-13 & B8549 & G & SG & & & $Y$ & 1:01 & Cond & & & $Y$ & $\begin{array}{l}\text { Horton and } \\
\text { Hodges 1999a }\end{array}$ & \\
\hline 1710 & $200-W$ & 299-W14-14 & B8547 & G & SG & & & & & & & & $Y$ & HWIS & \\
\hline 1711 & $1200-W$ & 299-W14-15 & C3114 & G & & $\mathrm{Y}$ & & & & & & & Y & $\begin{array}{l}\text { Horton and } \\
\text { Hodges 2001b }\end{array}$ & \\
\hline 1712 & $200-W$ & 299-W14-16 & C3120 & G & & $y$ & & & & & & & Y & $\begin{array}{l}\text { Horton and } \\
\text { Hodges 2001b }\end{array}$ & \\
\hline 1713 & $200-W$ & 299-W14-17 & C3121 & D & NT, SG & & Y & $Y$ & Stlso & $\begin{array}{l}\text { Bden, } \\
\% G S S C\end{array}$ & & & & $\begin{array}{l}\text { Horton and } \\
\text { Hodges 2001b; } \\
\text { Virtual Library } \\
2000\end{array}$ & \\
\hline 1714 & $200-W$ & 299-W14-18 & C3396 & G & & & & & & & & & $Y$ & Horton 2002b & \\
\hline 1715 & $200-W$ & 299-W14-19 & C3957 & G & SG & $\mathrm{Y}$ & & & & & & & $Y$ & Horton 2003b & \\
\hline
\end{tabular}




\begin{tabular}{|c|c|c|c|c|c|c|c|c|c|c|c|c|c|c|c|}
\hline SortIndex & Area & Well Name & WellID & Log Type & GPX Log Runs & Sieved & $\mathrm{CaCO} 3$ & Moisture & $\begin{array}{l}\text { Chemical } \\
\text { Properties }\end{array}$ & $\begin{array}{c}\text { Physical } \\
\text { Properties }\end{array}$ & \begin{tabular}{|c|} 
Min \\
Properties \\
\end{tabular} & $\begin{array}{l}\text { Geochron } \\
\text { Properties }\end{array}$ & Archived & Source & Comments \\
\hline 1716 & $200-W$ & 299-W14-2 & A7328 & D & DN, GG, NT, SG & \begin{tabular}{|l|} 
Virtual \\
Library
\end{tabular} & $\begin{array}{l}\text { Virtual } \\
\text { Library }\end{array}$ & & & & & & Y & HWIS & \\
\hline 1717 & $200-W$ & 299-W14-3 & A7329 & $\mathrm{D}$ & $\mathrm{DN}, \mathrm{GG}, \mathrm{NT}, \mathrm{SG}$ & & & & & & & & & HWIS & \\
\hline 1718 & $200-W$ & 299-W14-4 & A7330 & D & $\mathrm{DN}, \mathrm{GG}, \mathrm{NT}, \mathrm{SG}$ & & & & & & & & & HWIS & \\
\hline 1719 & $200-W$ & 299-W14-5 & A5475 & D & & & $\begin{array}{l}\text { Virtual } \\
\text { Library }\end{array}$ & & & & & & $\mathrm{Y}$ & HWIS & \\
\hline 1720 & $200-W$ & 299-W14-51 & A7335 & D & GG, NT & & & & & & & & $Y$ & HWIS & \\
\hline 1721 & $200-W$ & 299-W14-52 & A7336 & & GG, NT & & & & & & & & & & \\
\hline 1722 & $200-W$ & 299-W14-53 & A7337 & D & $\mathrm{DN}, \mathrm{GG}, \mathrm{NT}, \mathrm{SG}$ & \begin{tabular}{|l|} 
Virtual \\
Library
\end{tabular} & \begin{tabular}{|l|} 
Virtual \\
Library
\end{tabular} & & & & & & $\mathrm{Y}$ & HWIS & \\
\hline 1723 & $200-W$ & 299-W14-54 & A7338 & D & & & & & & & & & $Y$ & HWIS & \\
\hline 1724 & $200-W$ & 299-W14-55 & A7339 & D & DN & & & & & & & & $Y$ & HWIS & \\
\hline 1725 & $200-W$ & 299-W14-56 & A7340 & D & & & & & & & & & & HWIS & \\
\hline 1726 & $200-W$ & 299-W14-57 & A7341 & D & & & & & & & & & & HWIS & \\
\hline 1727 & $200-W$ & 299-W14-58 & A7342 & D & & & & & & & & & & HWIS & \\
\hline 1728 & $200-W$ & 299-W14-59 & A7343 & D & & & & & & & & & & HWIS & \\
\hline 1729 & $200-W$ & \begin{tabular}{|l|}
$299-W 14-6$ \\
\end{tabular} & A7331 & $\mathrm{D}$ & & \begin{tabular}{|l|} 
Virtual \\
Library
\end{tabular} & $\begin{array}{l}\text { Virtual } \\
\text { Library }\end{array}$ & & & & & & $\mathrm{Y}$ & HWIS & \\
\hline 1730 & $200-W$ & 299-W14-60 & A7344 & D & & & & & & & & & & HWIS & \\
\hline 1731 & $200-W$ & 299-W14-61 & A7345 & D & & & & & & & & & & HWIS & \\
\hline 1732 & $200-W$ & 299-W14-62 & A7346 & D & GG, SG & & & & Field Rad & & & & $Y$ & HWIS & \\
\hline 1733 & $200-W$ & 299-W14-63 & A7347 & D & & \begin{tabular}{|l|} 
Virtual \\
Library
\end{tabular} & $\begin{array}{l}\text { Virtual } \\
\text { Library }\end{array}$ & & & & & & $\mathrm{Y}$ & HWIS & \\
\hline 1734 & $200-W$ & 299-W14-7 & A5467 & $D, G$ & & \begin{tabular}{|l|} 
Virtual \\
Library
\end{tabular} & $\begin{array}{l}\text { Virtual } \\
\text { Library }\end{array}$ & & & & & \begin{tabular}{|l|} 
Date, \\
Paleomag
\end{tabular} & $\mathrm{Y}$ & \begin{tabular}{|l|} 
Baker et al. \\
1991; Bjornstad \\
1984
\end{tabular} & $\begin{array}{l}\text { Open Hole 105-531', Filled } \\
\text { w/Drilling Mud, }\end{array}$ \\
\hline 1735 & $200-W$ & 299-W14-8 & & $D, G$ & $\mathrm{DN}, \mathrm{GG}, \mathrm{NT}, \mathrm{TP}$ & \begin{tabular}{|l} 
Virtual \\
Library
\end{tabular} & \begin{tabular}{|l|} 
Virtual \\
Library
\end{tabular} & & & & & Paleomag & Y & Webster 1977 & Parker and Johnston 1979 \\
\hline 1736 & $200-W$ & 299-W14-8A & A7332 & D & & & & & & & & & $\mathrm{Y}$ & HWIS & \\
\hline 1737 & $200-W$ & 299-W14-9 & A4915 & D & DN, GG, NT, SG, TP & & & & & & & & & HWIS & \\
\hline 1738 & $200-w$ & 299-W15-1 & A7348 & D, G & GG, NT, TP & \begin{tabular}{|l} 
Virtual \\
Library
\end{tabular} & $\begin{array}{l}\text { Virtual } \\
\text { Library }\end{array}$ & & & & & & $Y$ & HWIS & \\
\hline 1739 & $200-W$ & 299-W15-10 & A4916 & D & DN, GG, NT, OT, SG & \begin{tabular}{|l} 
Virtual \\
Library
\end{tabular} & & & & & & & $Y$ & HWIS & \\
\hline 1740 & $200-W$ & 299-W15-100 & A7399 & D & & & & & & & & & & HWIS & \\
\hline 1741 & $200-W$ & 299-W15-101 & A7400 & D & GG, NT & & & & & & & & & HWIS & \\
\hline 1742 & $200-W$ & 299-W15-102 & A7401 & D & GG, NT & & & & & & & & $Y$ & HWIS & \\
\hline 1743 & $200-W$ & 299-W15-109 & A7408 & D & & & & & & & & & & HWIS & \\
\hline 1744 & $200-W$ & 299-W15-11 & A5474 & D & $\mathrm{DN}, \mathrm{GG}, \mathrm{NT}, \mathrm{TP}$ & \begin{tabular}{|l|} 
Virtual \\
Library
\end{tabular} & \begin{tabular}{|l|} 
Virtual \\
Library
\end{tabular} & & & & & & $Y$ & HWIS & \\
\hline 1745 & $200-W$ & 299-W15-110 & A7409 & D & & & & & & & & & & HWIS & \\
\hline 1746 & $200-W$ & 299-W15-111 & A7410 & D & & & & & & & & & & HWIS & \\
\hline 1747 & $200-W$ & 299-W15-112 & A7411 & D & & & & & & & & & & HWIS & \\
\hline 1748 & $200-W$ & 299-W15-113 & A7412 & D & & & & & & & & & & HWIS & \\
\hline 1749 & $200-W$ & 299-W15-114 & A7413 & D & & & & & & & & & & HWIS & \\
\hline 1750 & $200-W$ & 299-W15-115 & A7414 & D & & & & & & & & & & HWIS & \\
\hline 1751 & $200-W$ & 299-W15-116 & A7415 & D & & & & & & & & & & HWIS & \\
\hline 1752 & $200-W$ & 299-W15-117 & A7416 & D & & & & & & & & & & HWIS & \\
\hline 1753 & $200-W$ & 299-W15-118 & A7417 & D & & & & & & & & & & HWIS & \\
\hline 1754 & $200-W$ & 299-W15-119 & A7418 & $\mathrm{D}$ & & & & & & & & & & HWIS & \\
\hline 1755 & $200-W$ & 299-W15-12 & A4917 & $\mathrm{D}$ & TP & \begin{tabular}{|l|} 
Virtual \\
Library
\end{tabular} & $\begin{array}{l}\text { Virtual } \\
\text { Library }\end{array}$ & & & & & & $\mathrm{Y}$ & HWIS & \\
\hline 1756 & $200-W$ & 299-W15-120 & A7419 & D & & & & & & & & & & HWIS & \\
\hline 1757 & $200-W$ & 299-W15-121 & A7420 & D & & & & & & & & & & HWIS & \\
\hline
\end{tabular}




\begin{tabular}{|c|c|c|c|c|c|c|c|c|c|c|c|c|c|c|c|}
\hline Sortlndex & Area & Well Name & WellID & Log Type & GPX Log Runs & Sieved & $\mathrm{CaCO} 3$ & Moisture & $\begin{array}{l}\text { Chemical } \\
\text { Properties }\end{array}$ & $\begin{array}{c}\text { Physical } \\
\text { Properties }\end{array}$ & $\begin{array}{c}\text { Min } \\
\text { Properties }\end{array}$ & $\begin{array}{l}\text { Geochron } \\
\text { Properties }\end{array}$ & Archived & Source & Comments \\
\hline 1758 & $200-W$ & 299-W15-122 & A7421 & D & & & & & & & & & & HWIS & \\
\hline 1759 & $200-W$ & 299-W15-123 & A7422 & D & & & & & Field Rad & & & & & HWIS & \\
\hline 1760 & $200-W$ & 299-W15-124 & A7423 & D & & & & & & & & & & HWIS & \\
\hline 1761 & $200-W$ & 299-W15-125 & A7424 & D & & & & & & & & & & HWIS & \\
\hline 1762 & $200-W$ & 299-W15-126 & A7425 & D & & & & & & & & & & HWIS & \\
\hline 1763 & $200-W$ & 299-W15-127 & A7426 & D & & $\begin{array}{l}\text { Virtual } \\
\text { Library }\end{array}$ & $\begin{array}{l}\text { Virtual } \\
\text { Library }\end{array}$ & & & & & & $Y$ & HWIS & \\
\hline 1764 & $200-W$ & 299-W15-128 & A7427 & D & & $\begin{array}{l}\begin{array}{l}\text { Virtual } \\
\text { Library }\end{array} \\
\end{array}$ & $\begin{array}{l}\text { Virtual } \\
\text { Library }\end{array}$ & & & & & & $Y$ & HWIS & \\
\hline 1765 & $200-W$ & 299-W15-129 & A7428 & D, G & & \begin{tabular}{|l|} 
Virtual \\
Library
\end{tabular} & \begin{tabular}{|l|} 
Virtual \\
Library
\end{tabular} & & & & & & $Y$ & HWIS & \\
\hline 1766 & $200-W$ & 299-W15-13 & A4918 & D & & \begin{tabular}{|l|} 
Virtual \\
Library
\end{tabular} & $\begin{array}{l}\text { Virtual } \\
\text { Library }\end{array}$ & & & & & & $Y$ & HWIS & \\
\hline 1767 & $200-W$ & 299-W15-130 & A7429 & D & & \begin{tabular}{|l|}
$\begin{array}{l}\text { Virtual } \\
\text { Library }\end{array}$ \\
\end{tabular} & \begin{tabular}{|l|} 
Virtual \\
Library
\end{tabular} & & & & & & $Y$ & HWIS & \\
\hline 1768 & $200-W$ & 299-W15-131 & A7430 & D & & \begin{tabular}{|l|} 
Virtual \\
Library
\end{tabular} & $\begin{array}{l}\text { Virtual } \\
\text { Library }\end{array}$ & & & & & & $Y$ & HWIS & \\
\hline 1769 & $200-W$ & 299-W15-132 & A7431 & D & & \begin{tabular}{|l|}
$\begin{array}{l}\text { Virtual } \\
\text { Library }\end{array}$ \\
\end{tabular} & \begin{tabular}{|l|} 
Virtual \\
Library
\end{tabular} & & & & & & $Y$ & HWIS & \\
\hline 1770 & $200-W$ & 299-W15-133 & \begin{tabular}{|l|} 
A7432 \\
\end{tabular} & D & & $\begin{array}{l}\text { Virtual } \\
\text { Library }\end{array}$ & $\begin{array}{l}\text { Virtual } \\
\text { Library }\end{array}$ & & & & & & $Y$ & HWIS & \\
\hline 1771 & $200-W$ & 299-W15-134 & A7433 & D & & \begin{tabular}{|l|} 
Virtual \\
Library
\end{tabular} & \begin{tabular}{|l|} 
Virtual \\
Library \\
\end{tabular} & & & & & & $Y$ & HWIS & \\
\hline 1772 & $200-W$ & 299-W15-135 & \begin{tabular}{|l|l} 
A7434 \\
\end{tabular} & D & & & & & & & & & $Y$ & HWIS & \\
\hline 1773 & $200-W$ & 299-W15-136 & A7435 & D & & $\begin{array}{l}\text { Virtual } \\
\text { Library }\end{array}$ & $\begin{array}{l}\text { Virtual } \\
\text { Library }\end{array}$ & & & & & & $Y$ & HWIS & \\
\hline 1774 & $200-W$ & 299-W15-137 & A7436 & D & & \begin{tabular}{|l|} 
Virtual \\
Library
\end{tabular} & \begin{tabular}{|l|l|} 
Virtual \\
Library
\end{tabular} & & & & & & $Y$ & HWIS & \\
\hline 1775 & $200-W$ & 299-W15-138 & A7437 & D & & & & & & & & & & HWIS & \\
\hline 1776 & $200-W$ & 299-W15-139 & A7438 & D & & & & & & & & & & HWIS & \\
\hline$\left.1777\right|^{2}$ & $200-W$ & 299-W15-14 & A7350 & D & TP & \begin{tabular}{|l} 
Virtual \\
Library
\end{tabular} & $\begin{array}{l}\text { Virtual } \\
\text { Library }\end{array}$ & $\mathrm{Y}$ & Stlso & $\begin{array}{l}\text { Bden, } \\
\% \text { GSSC }\end{array}$ & & \begin{tabular}{|l} 
Date, \\
Paleomag
\end{tabular} & & \begin{tabular}{|l|} 
Virtual Library \\
2000 (PPI only, \\
includes \\
description); \\
Baker et al. \\
1991; Parker and \\
Johnston 1979
\end{tabular} & \\
\hline 1778 & $200-W$ & 299-W15-140 & A7439 & D & & & & & & & & & & HWIS & \\
\hline 1779 & $200-W$ & 299-W15-141 & A7440 & D & & & & & & & & & & HWIS & \\
\hline 1780 & $200-W$ & 299-W15-142 & A7441 & D & & & & & & & & & & HWIS & \\
\hline 1781 & $200-W$ & 299-W15-143 & A7442 & D & & & & & & & & & & HWIS & \\
\hline 1782 & $200-W$ & 299-W15-144 & A7443 & D & & & & & & & & & & HWIS & \\
\hline 1783 & $200-W$ & 299-W15-145 & A7444 & D & & & & & & & & & & HWIS & \\
\hline 1784 & $200-W$ & 299-W15-146 & A7445 & D & & & & & & & & & & HWIS & \\
\hline 1785 & $200-W$ & 299-W15-147 & A7446 & D & & & & & & & & & & HWIS & \\
\hline 1786 & $200-W$ & 299-W15-148 & A7447 & D & & & & & & & & & & HWIS & \\
\hline 1787 & $200-W$ & 299-W15-149 & A7448 & D & & & & & & & & & & HWIS & \\
\hline 1788 & $200-W$ & 299-W15-15 & A4919 & $\mathrm{D}, \mathrm{G}$ & $\mathrm{DN}, \mathrm{GG}, \mathrm{NT}$ & $\begin{array}{l}\text { Virtual } \\
\text { Library }\end{array}$ & $\begin{array}{l}\text { Virtual } \\
\text { Library }\end{array}$ & Y & & & & & $Y$ & Last et al. 1989 & \\
\hline 1789 & $200-W$ & 299-W15-153 & A7451 & $\mathrm{D}$ & & & & & & & & & $Y$ & HWIS & \\
\hline 1790 & $200-w$ & 299-W15-154 & A7452 & $\mathrm{D}$ & & & & & & & & & $Y$ & HWIS & \\
\hline 1791 & $200-W$ & 299-W15-155 & A7453 & D & & \begin{tabular}{|l|} 
Virtual \\
Library
\end{tabular} & & & & & & & $Y$ & HWIS & \\
\hline 1792 & $200-W$ & 299-W15-156 & A7454 & D & & & & & & & & & $Y$ & HWIS & \\
\hline 1793 & $200-W$ & 299-W15-158 & A7456 & D & & & & & & & & & $Y$ & HWIS & \\
\hline
\end{tabular}




\begin{tabular}{|c|c|c|c|c|c|c|c|c|c|c|c|c|c|c|c|}
\hline SortIndex & Area & Well Name & WellID & Log Type & GPX Log Runs & Sieved & $\mathrm{CaCO} 3$ & Moisture & $\begin{array}{l}\text { Chemical } \\
\text { Properties }\end{array}$ & $\begin{array}{c}\text { Physical } \\
\text { Properties }\end{array}$ & $\begin{array}{c}\text { Min } \\
\text { Properties } \\
\end{array}$ & $\begin{array}{l}\text { Geochron } \\
\text { Properties }\end{array}$ & Archived & Source & Comments \\
\hline \begin{tabular}{|l|}
1794 \\
\end{tabular} & $200-W$ & 299-W15-159 & A7457 & D & & \begin{tabular}{|l|} 
Virtual \\
Library
\end{tabular} & & & & & & & $\mathrm{Y}$ & HWIS & \\
\hline 1795 & $200-W$ & 299-W15-16 & A4920 & D, G & DN, GG, NT & \begin{tabular}{|l} 
Virtual \\
Library
\end{tabular} & \begin{tabular}{|l} 
Virtual \\
Library
\end{tabular} & $Y$ & $\begin{array}{l}\text { TC, IC, TOC, } \\
\text { XRF }\end{array}$ & $\begin{array}{l}\text { CEC, } \\
\% G S S C\end{array}$ & XRD & & $Y$ & $\begin{array}{l}\text { Last et al. 1989; } \\
\text { Schramke 1988; } \\
\text { Bjornstad et al. } \\
\text { no date; ;mes } \\
\text { and Serne } 1991\end{array}$ & \\
\hline 1796 & $200-W$ & 299-W15-160 & A7458 & D & & \begin{tabular}{|l|} 
Virtual \\
Library
\end{tabular} & & & & & & & $Y$ & HWIS & \\
\hline 1797 & $200-W$ & 299-W15-161 & A7459 & D & & \begin{tabular}{|l|} 
Virtual \\
Library
\end{tabular} & & & & & & & $Y$ & HWIS & \\
\hline 1798 & $200-W$ & 299-W15-162 & A7460 & D & & \begin{tabular}{|l|} 
Virtual \\
Library
\end{tabular} & & & & & & & $Y$ & HWIS & \\
\hline 1799 & $200-W$ & 299-W15-163 & A7461 & D & & \begin{tabular}{|l|} 
Virtual \\
Library
\end{tabular} & & & & & & & $Y$ & HWIS & \\
\hline 1800 & $200-W$ & 299-W15-164 & A7462 & D & & \begin{tabular}{|l} 
Virtual \\
Library
\end{tabular} & & & & & & & $Y$ & HWIS & \\
\hline 1801 & $200-W$ & 299-W15-165 & A7463 & D & & $\begin{array}{l}\text { Virtual } \\
\text { Library }\end{array}$ & & & & & & & & HWIS & \\
\hline 1802 & $200-W$ & 299-W15-166 & A7464 & D & & \begin{tabular}{|l|} 
Virtual \\
Library
\end{tabular} & \begin{tabular}{|l|} 
Virtual \\
Library
\end{tabular} & & & & & & $Y$ & HWIS & \\
\hline 1803 & $200-W$ & 299-W15-167 & A7465 & D & & \begin{tabular}{|l} 
Virtual \\
Library
\end{tabular} & & & & & & & $Y$ & HWIS & \\
\hline 1804 & $200-W$ & 299-W15-168 & A7466 & D & & \begin{tabular}{|l} 
Virtual \\
Library
\end{tabular} & \begin{tabular}{|l|}
$\begin{array}{l}\text { Virtual } \\
\text { Library }\end{array}$ \\
\end{tabular} & & & & & & $Y$ & HWIS & \\
\hline 1805 & $200-W$ & 299-W15-169 & A7467 & D & & \begin{tabular}{|l|} 
Virtual \\
Library
\end{tabular} & \begin{tabular}{|l|} 
Virtual \\
Library
\end{tabular} & & & & & & $Y$ & HWIS & \\
\hline 1806 & $200-W$ & 299-W15-17 & A4921 & $D, G$ & DN, GG, NT & $\begin{array}{l}\text { Virtual } \\
\text { Library }\end{array}$ & $\begin{array}{l}\text { Virtual } \\
\text { Library }\end{array}$ & & $\begin{array}{l}\text { TC, IC, TOC, } \\
\text { XRF }\end{array}$ & CEC & XRD & & $Y$ & $\begin{array}{l}\text { Last et al. 1989; } \\
\text { Schramke 1988; } \\
\text { Bjornstad et al. } \\
\text { no date; Ames } \\
\text { and Serne } 1991\end{array}$ & \\
\hline 1807 & $200-W$ & 299-W15-170 & A7468 & D & & \begin{tabular}{|l} 
Virtual \\
Library
\end{tabular} & $\begin{array}{l}\text { Virtual } \\
\text { Library }\end{array}$ & & & & & & $Y$ & HWIS & \\
\hline 1808 & $200-W$ & 299-W15-171 & A7469 & D & & \begin{tabular}{|l|} 
Virtual \\
Library
\end{tabular} & \begin{tabular}{|l|} 
Virtual \\
Library
\end{tabular} & & & & & & $Y$ & HWIS & \\
\hline 1809 & $200-W$ & 299-W15-172 & A7470 & D & & \begin{tabular}{|l} 
Virtual \\
Library
\end{tabular} & $\begin{array}{l}\text { Virtual } \\
\text { Library }\end{array}$ & & & & & & $Y$ & HWIS & \\
\hline 1810 & $200-W$ & 299-W15-173 & A7471 & D & & \begin{tabular}{|l} 
Virtual \\
Library
\end{tabular} & \begin{tabular}{|l|} 
Virtual \\
Library
\end{tabular} & & & & & & $Y$ & HWIS & \\
\hline 1811 & $200-W$ & 299-W15-174 & A7472 & D & & \begin{tabular}{|l|} 
Virtual \\
Library
\end{tabular} & & & & & & & $Y$ & HWIS & \\
\hline 1812 & $200-W$ & 299-W15-175 & A7473 & D & & $\begin{array}{l}\text { Virtual } \\
\text { Library }\end{array}$ & $\begin{array}{l}\text { Virtual } \\
\text { Library }\end{array}$ & & & & & & $Y$ & HWIS & \\
\hline 1813 & $200-W$ & 299-W15-176 & A7474 & D & & \begin{tabular}{|l|} 
Virtual \\
Library
\end{tabular} & $\begin{array}{l}\text { Virtual } \\
\text { Library }\end{array}$ & & & & & & $Y$ & HWIS & \\
\hline 1814 & $200-W$ & 299-W15-177 & A7475 & D & & $\begin{array}{l}\text { Virtual } \\
\text { Library }\end{array}$ & $\begin{array}{l}\text { Virtual } \\
\text { Library }\end{array}$ & & & & & & $Y$ & HWIS & \\
\hline 1815 & $200-W$ & 299-W15-178 & A7476 & D & & \begin{tabular}{|l|} 
Virtual \\
Library
\end{tabular} & & & & & & & $Y$ & HWIS & \\
\hline
\end{tabular}




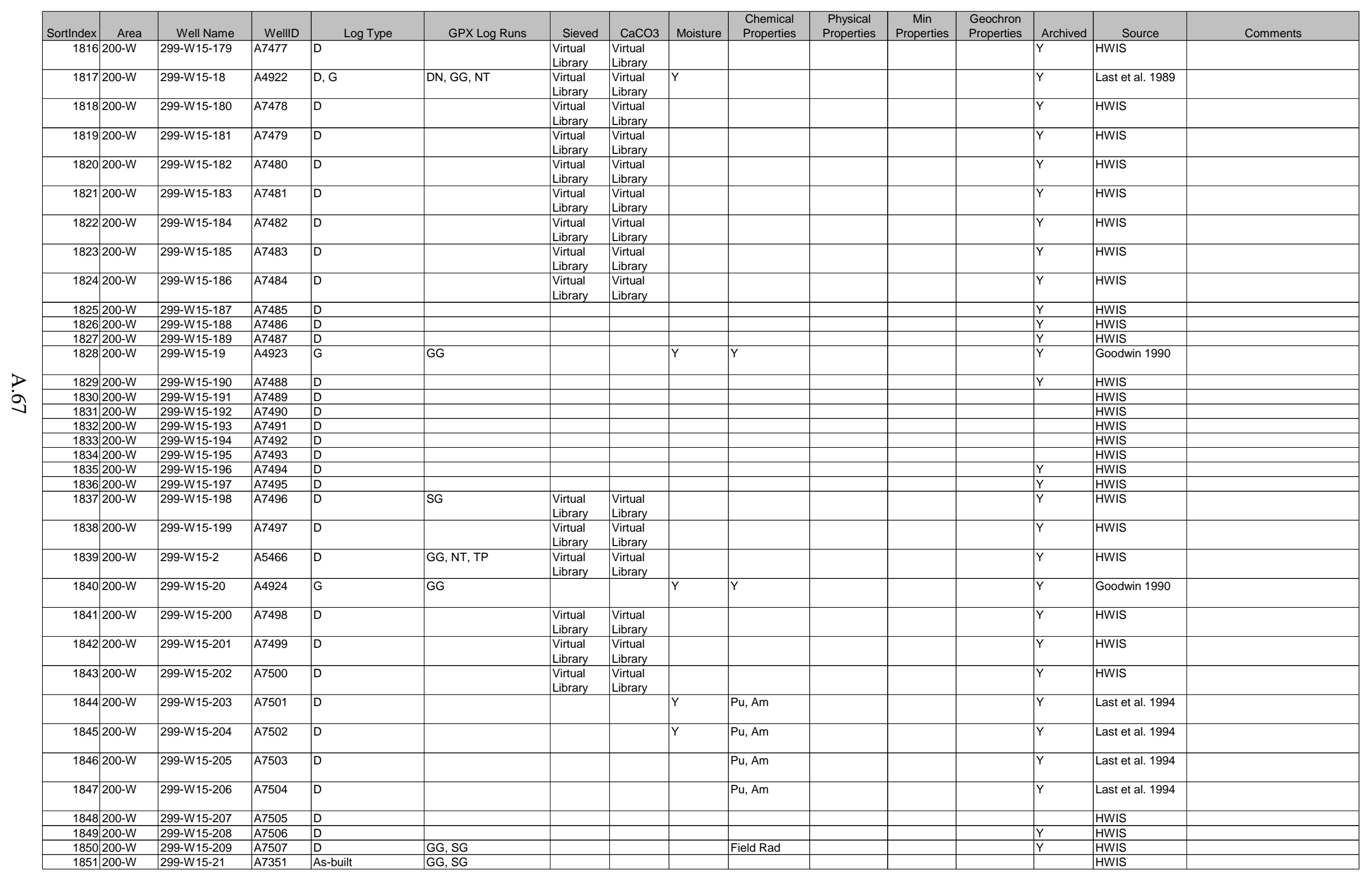




\begin{tabular}{|c|c|c|c|c|c|c|c|c|c|c|c|c|c|c|c|}
\hline Sortindex & Area & Well Name & WellID & Log Type & GPX Log Runs & Sieved & $\mathrm{CaCO} 3$ & Moisture & $\begin{array}{l}\text { Chemical } \\
\text { Properties }\end{array}$ & $\begin{array}{c}\text { Physical } \\
\text { Properties }\end{array}$ & $\begin{array}{c}\text { Min } \\
\text { Properties } \\
\end{array}$ & $\begin{array}{l}\text { Geochron } \\
\text { Properties }\end{array}$ & Archived & Source & Comments \\
\hline 1852 & $200-W$ & 299-W15-210 & A7508 & D & $\mathrm{GG}, \mathrm{SG}$ & & & ivivioture & Field Rad & & & & Y & HWIS & Covitinerits \\
\hline 1853 & $200-W$ & 299-W15-211 & A7509 & D & GG, SG & & & & Field Rad & & & & $Y$ & HWIS & \\
\hline 1854 & $200-W$ & 299-W15-212 & A7510 & D & GG, SG & & & & & & & & $Y$ & HWIS & \\
\hline 1855 & $200-W$ & 299-W15-213 & A7511 & D & SG & & & & & & & & & HWIS & \\
\hline 1856 & $200-W$ & 299-W15-214 & A7512 & & SG & & & & & & & & & & \\
\hline 1857 & $200-W$ & 299-W15-216 & A7514 & G & GG, SG & $Y$ & & $Y$ & $\begin{array}{l}\text { Metals, VOA, } \\
\text { SVOA, An, } \\
\text { Lab Rad }\end{array}$ & $\begin{array}{l}\text { Bden, } \\
\% G S S C\end{array}$ & & & & \begin{tabular}{|l|} 
Rohay et al. \\
1994; Last and \\
Rohay 1993; see \\
R. Khaleel or G. \\
Freemen for \\
sieve data
\end{tabular} & \\
\hline 1858 & $200-W$ & 299-W15-217 & A7515 & G & GG, SG & $Y$ & & $Y$ & $\begin{array}{l}\text { Metals, VOA, } \\
\text { SVOA, An, } \\
\text { Lab Rad }\end{array}$ & $\begin{array}{l}\text { Bden, } \\
\% G S S C\end{array}$ & & & & \begin{tabular}{|l|} 
Rohay et al. \\
1994; Last and \\
Rohay 1993; see \\
R. Khaleel or G. \\
Freemen for \\
sieve data
\end{tabular} & \\
\hline 1859 & $200-W$ & 299-W15-218 & A7516 & G & GG, SG & Y & Y & $Y$ & $\begin{array}{l}\text { VOA, SVOA, } \\
\text { Metals, An, } \\
\text { Rad }\end{array}$ & & & & & $\begin{array}{l}\text { Rohay et al. } \\
\text { 1994; Rohay et } \\
\text { al. 1993; see R. } \\
\text { Khaleel or G. } \\
\text { Freeman for } \\
\text { sieve data }\end{array}$ & \\
\hline 1860 & $200-W$ & 299-W15-219 & A7517 & G & GG & $Y$ & Y & $Y$ & $\begin{array}{l}\text { VOA, SVOA, } \\
\text { Metals, An, } \\
\text { Rad }\end{array}$ & & & & & \begin{tabular}{|l|} 
Rohay et al. \\
1994; Rohay et \\
al. 1993 ; see R. \\
Khaleel or G. \\
Freeman for \\
sieve data
\end{tabular} & \\
\hline 1861 & $200-W$ & 299-W15-22 & A4925 & G & GG & Y & \begin{tabular}{|l} 
Virtual \\
Library
\end{tabular} & $Y$ & & & & & $\mathrm{Y}$ & Caggiano 1992 & \\
\hline 1862 & $200-W$ & 299-W15-220 & A7518 & G & GG, SG & $Y$ & $\mathrm{Y}$ & $Y$ & VOA & & & & & $\begin{array}{l}\text { Rohay et al. } \\
\text { 1994; see } \\
\text { R. Khaleel or G. } \\
\text { Freeman for } \\
\text { sieve data }\end{array}$ & \\
\hline 1863 & $200-W$ & 299-W15-223 & A7521 & G & GG, SG & $Y$ & $Y$ & $Y$ & $\begin{array}{l}\text { VOA, SVOA, } \\
\text { An, CN, Hg, } \\
\text { Pb, Metals }\end{array}$ & Bden & & & & \begin{tabular}{|l|} 
Rohay et al. \\
1994; Rohay \\
1995
\end{tabular} & \\
\hline 1864 & $200-W$ & 299-W15-23 & A4926 & G & GG & & & $Y$ & & & & & $Y$ & Barton 1990 & \\
\hline 1865 & $200-W$ & 299-W15-24 & A4927 & G & GG & & & & & & & & Y & Barton 1990 & \\
\hline 1866 & $200-W$ & $299-W 15-25$ & A9831 & G & DN, GG, OT & $Y$ & $\mathrm{Y}$ & $Y$ & VOA & & & & & $\begin{array}{l}\text { Weekes and } \\
\text { Glaman } 1995\end{array}$ & \\
\hline 1867 & $200-W$ & 299-W15-252 & & & & $Y$ & & & & & & & & $\begin{array}{l}\text { Rohay et al. } \\
1993\end{array}$ & \\
\hline 1868 & $200-W$ & 299-W15-29 & B2409 & G & & Y & & & & & & & & $\begin{array}{l}\text { CH2M HILL files } \\
\end{array}$ & \\
\hline 1869 & $200-W$ & 299-W15-3 & A4928 & D & GG & \begin{tabular}{|l|} 
Virtual \\
Library
\end{tabular} & & & & & & & $\mathrm{Y}$ & HWIS & \\
\hline 1870 & $200-W$ & 299-W15-30 & B2410 & G & & & & & & & & & & CH2M HILL files & \\
\hline 1871 & $200-W$ & 299-W15-31 & B2411 & G & SG & & & & & & & & & HWIS & \\
\hline
\end{tabular}




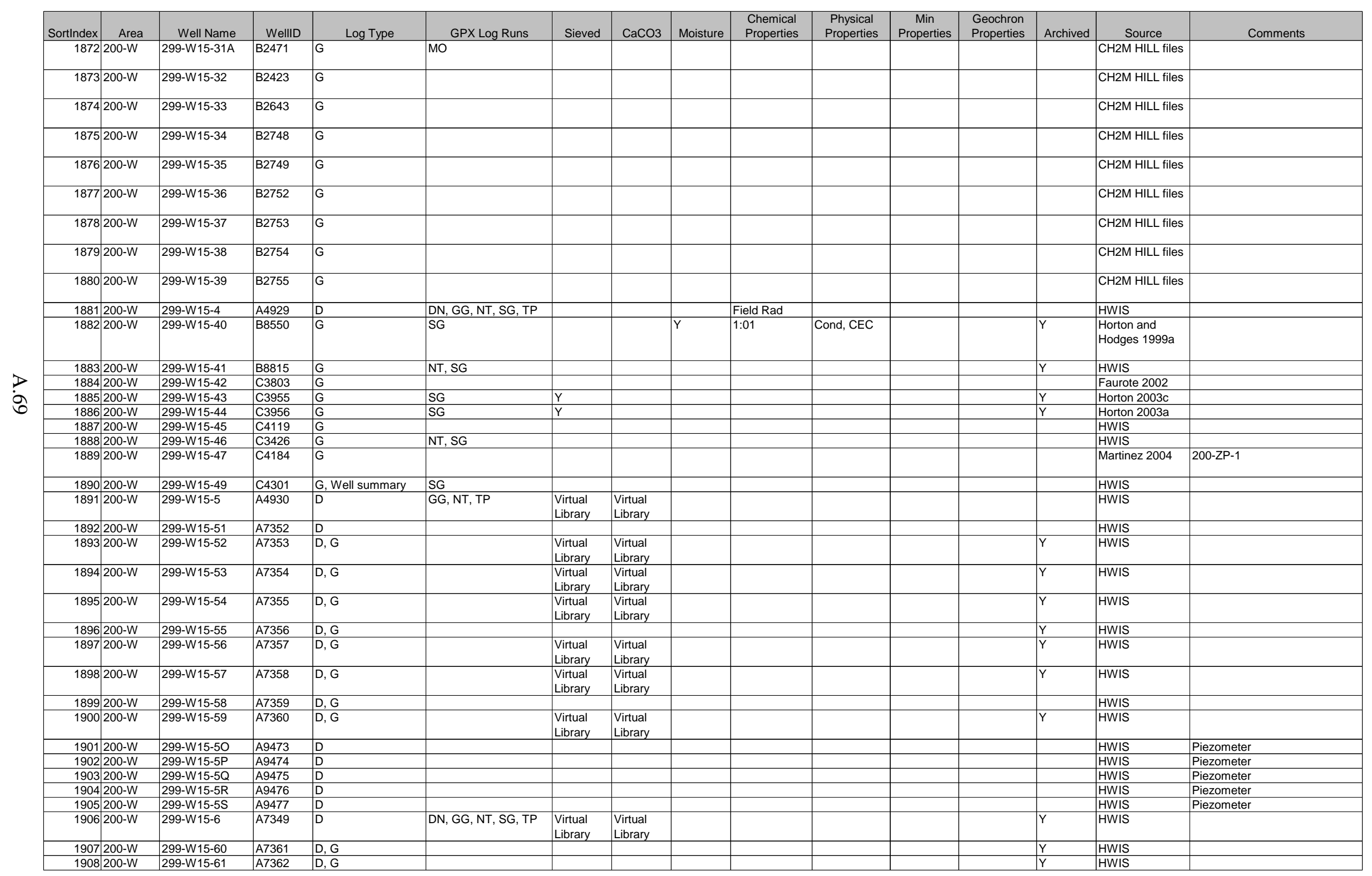




\begin{tabular}{|c|c|c|c|c|c|c|c|c|c|c|c|c|c|c|c|}
\hline SortIndex & Area & Well Name & WellID & Log Type & GPX Log Runs & Sieved & $\mathrm{CaCO} 3$ & Moisture & $\begin{array}{c}\text { Chemical } \\
\text { Properties }\end{array}$ & $\begin{array}{c}\text { Physical } \\
\text { Properties }\end{array}$ & $\begin{array}{c}\text { Min } \\
\text { Properties } \\
\end{array}$ & $\begin{array}{l}\text { Geochron } \\
\text { Properties }\end{array}$ & Archived & Source & Comments \\
\hline \begin{tabular}{|l|}
1909 \\
\end{tabular} & $200-W$ & 299-W15-62 & A7363 & D & DN, GG, NT, SG & & & & & & & & $\mathrm{Y}$ & HWIS & \\
\hline 1910 & $200-W$ & 299-W15-63 & A7364 & As-built & DN, GG, NT, SG & & & & & & & & $Y$ & HWIS & \\
\hline 1911 & $200-W$ & 299-W15-64 & A7365 & $\mathrm{D}$ & $\mathrm{DN}, \mathrm{GG}, \mathrm{NT}, \mathrm{SG}$ & & & & & & & & $Y$ & HWIS & \\
\hline 1912 & $200-W$ & 299-W15-65 & A7366 & & DN, GG, NT & & & & & & & & & & \\
\hline 1913 & $200-W$ & 299-W15-66 & A7367 & D & DN, GG, NT & & & & & & & & $\mathrm{Y}$ & HWIS & \\
\hline 1914 & $4200-W$ & 299-W15-67 & A7368 & D, G & GG & \begin{tabular}{|l|} 
Virtual \\
Library
\end{tabular} & $\begin{array}{l}\text { Virtual } \\
\text { Library }\end{array}$ & & & & & & $\mathrm{Y}$ & HWIS & \\
\hline 1915 & $200-W$ & 299-W15-68 & A7369 & D, G & GG & $\begin{array}{l}\text { Virtual } \\
\text { Library }\end{array}$ & $\begin{array}{l}\text { Virtual } \\
\text { Library }\end{array}$ & & & & & & $Y$ & HWIS & \\
\hline 1916 & $200-W$ & 299-W15-69 & A7370 & D, G & GG & $\begin{array}{l}\text { Virtual } \\
\text { Library }\end{array}$ & $\begin{array}{l}\text { Virtual } \\
\text { Library }\end{array}$ & & & & & & $\mathrm{Y}$ & HWIS & \\
\hline 1917 & $200-W$ & 299-W15-7 & A5476 & D & DN, GG, NT, SG, TP & \begin{tabular}{|l|} 
Virtual \\
Library
\end{tabular} & $\begin{array}{l}\text { Virtual } \\
\text { Library }\end{array}$ & & & & & & $Y$ & HWIS & \\
\hline 1918 & $200-W$ & 299-W15-70 & A7371 & $D, G$ & & \begin{tabular}{|l|} 
Virtual \\
Library
\end{tabular} & $\begin{array}{l}\text { Virtual } \\
\text { Library }\end{array}$ & & & & & & $Y$ & HWIS & \\
\hline 1919 & $200-W$ & 299-W15-71 & A7372 & $D, G$ & & \begin{tabular}{|l} 
Virtual \\
Library
\end{tabular} & $\begin{array}{l}\text { Virtual } \\
\text { Library }\end{array}$ & & & & & & $Y$ & HWIS & \\
\hline 1920 & $200-W$ & 299-W15-72 & A7373 & D, G & GG & \begin{tabular}{|l|} 
Virtual \\
Library
\end{tabular} & $\begin{array}{l}\text { Virtual } \\
\text { Library }\end{array}$ & & & & & & Y & HWIS & \\
\hline 1921 & 1 200-W & 299-W15-73 & A7374 & $D, G$ & GG & $\begin{array}{l}\text { Virtual } \\
\text { Library }\end{array}$ & $\begin{array}{l}\text { Virtual } \\
\text { Library }\end{array}$ & & & & & & $\mathrm{Y}$ & HWIS & \\
\hline 1922 & $200-W$ & 299-W15-74 & A7375 & $D, G$ & GG & \begin{tabular}{|l|} 
Virtual \\
Library
\end{tabular} & $\begin{array}{l}\text { Virtual } \\
\text { Library }\end{array}$ & & & & & & $\mathrm{Y}$ & HWIS & \\
\hline 1923 & $200-W$ & 299-W15-75 & A7376 & $D, G$ & GG & \begin{tabular}{|l|} 
Virtual \\
Library
\end{tabular} & $\begin{array}{l}\text { Virtual } \\
\text { Library }\end{array}$ & & & & & & $Y$ & HWIS & \\
\hline 1924 & $4200-W$ & 299-W15-76 & A7377 & $D, G$ & DN, GG, MO, NT, SG & $\begin{array}{l}\text { Virtual } \\
\text { Library }\end{array}$ & & & & & & & $\mathrm{Y}$ & HWIS & \\
\hline 1925 & $200-W$ & 299-W15-763 & C3339 & G & & $y$ & & & & & & & $\mathrm{Y}$ & $\begin{array}{l}\text { Horton and } \\
\text { Hodges 2001b }\end{array}$ & \\
\hline 1926 & $200-W$ & 299-W15-764 & C3494 & G & & & & & & & & & & Faurote 2002 & \\
\hline 1927 & $7200-W$ & 299-W15-765 & C3397 & G & NT, SG & & & & & & & & $Y$ & Horton $2002 b$ & \\
\hline 1928 & $200-W$ & 299-W15-77 & A7378 & D & DN, GG, MO, NT, SG & \begin{tabular}{|l} 
Virtual \\
Library
\end{tabular} & & & & & & & $\mathrm{Y}$ & HWIS & \\
\hline 1929 & $200-W$ & 299-W15-78 & A7379 & D & $\mathrm{DN}, \mathrm{GG}, \mathrm{MO}, \mathrm{NT}, \mathrm{SG}$ & & & & & & & & $\mathrm{Y}$ & HWIS & \\
\hline 1930 & $200-W$ & 299-W15-79 & A7380 & D & $G G$ & \begin{tabular}{|l} 
Virtual \\
Library
\end{tabular} & & & & & & & $Y$ & HWIS & \\
\hline 1931 & $1200-W$ & 299-W15-8 & A5468 & D & CP, DN, GG, NT, SG & \begin{tabular}{|l} 
Virtual \\
Library
\end{tabular} & $\begin{array}{l}\text { Virtual } \\
\text { Library }\end{array}$ & & & & & & $Y$ & HWIS & \\
\hline 1932 & $200-W$ & 299-W15-80 & A7381 & D & GG, SG & & & & & & & & $\mathrm{Y}$ & HWIS & \\
\hline 1933 & $200-W$ & 299-W15-81 & A7382 & $\mathrm{D}$ & GG, SG & & & & & & & & $Y$ & HWIS & \\
\hline 1934 & $200-W$ & 299-W15-82 & A7383 & D & DN, GG, NT, SG & \begin{tabular}{|l|} 
Virtual \\
Library
\end{tabular} & $\begin{array}{l}\text { Virtual } \\
\text { Library }\end{array}$ & & & & & & $\mathrm{Y}$ & HWIS & \\
\hline 1935 & $200-W$ & 299-W15-83 & C4683 & & GG & & & & & & & & & & \\
\hline 1936 & $200-W$ & 299-W15-84 & A7384 & D, G & $\mathrm{CP}, \mathrm{DN}, \mathrm{GG}, \mathrm{NT}, \mathrm{SG}$ & & & & & & & & Steve & $\begin{array}{l}\text { CH2M HILL } \\
2001\end{array}$ & \\
\hline 1937 & $200-W$ & 299-W15-85 & A7385 & $D$ & $\mathrm{DN}, \mathrm{GG}, \mathrm{NT}, \mathrm{SG}$ & & & & & & & & & HWIS & \\
\hline 1938 & $200-W$ & 299-W15-86 & A7386 & D & $\mathrm{CP}, \mathrm{GG}, \mathrm{MG}, \mathrm{NT}, \mathrm{SG}$ & \begin{tabular}{|l} 
Virtual \\
Library
\end{tabular} & $\begin{array}{l}\text { Virtual } \\
\text { Library }\end{array}$ & & & & & & $Y$ & HWIS & \\
\hline 1939 & $200-W$ & 299-W15-9 & A5477 & D & CP, DN, GG, NT, SG & & & & & & & & & HWIS & \\
\hline 1940 & $200-W$ & 299-W15-92 & A7392 & D & GG, NT & & & & & & & & & HWIS & \\
\hline 1941 & $200-W$ & 299-W15-94 & C4684 & D & GG & \begin{tabular}{|l|l|} 
Virtual \\
Library
\end{tabular} & \begin{tabular}{|l|} 
Virtual \\
Library
\end{tabular} & & & & & & $\mathrm{Y}$ & HWIS & \\
\hline 1942 & $200-W$ & 299-W15-95 & A7394 & $D, G$ & DN, GG, NT, SG & & & & & & & & & $\begin{array}{l}\text { CH2M HILL } \\
2001\end{array}$ & \\
\hline 1943 & $3200-W$ & 299-W15-96 & A7395 & D & GG & Y & & & & & & & & $\begin{array}{l}\text { Rohay et al. } \\
1993\end{array}$ & \\
\hline
\end{tabular}




\begin{tabular}{|c|c|c|c|c|c|c|c|c|c|c|c|c|c|c|c|}
\hline Sortindex & Area & Well Name & WellID & Log Type & GPX Log Runs & Sieved & $\mathrm{CaCO} 3$ & Moisture & $\begin{array}{l}\text { Chemical } \\
\text { Properties }\end{array}$ & $\begin{array}{c}\text { Physical } \\
\text { Properties }\end{array}$ & \begin{tabular}{|c|} 
Min \\
Properties \\
\end{tabular} & $\begin{array}{l}\text { Geochron } \\
\text { Properties } \\
\end{array}$ & Archived & Source & Comments \\
\hline 1944 & $200-W$ & 299-W15-97 & A7396 & D & & & & & & & & & & HWIS & \\
\hline 1945 & $200-W$ & 299-W15-98 & A7397 & D & & & & & & & & & & HWIS & \\
\hline 1946 & $200-W$ & 299-W15-99 & A7398 & D & & & & & & & & & & HWIS & \\
\hline 1947 & $200-W$ & 299-W17-1 & C4237 & G & & & & & & & & & & Martinez 2004 & 200-ZP-1 \\
\hline 1948 & $200-W$ & 299-W18-1 & A5481 & D & GG, NT, TP & \begin{tabular}{|l|} 
Virtual \\
Library
\end{tabular} & $\begin{array}{l}\text { Virtual } \\
\text { Library }\end{array}$ & & & & & & Y & HWIS & \\
\hline 1949 & $200-W$ & 299-W18-10 & A4931 & D & $\begin{array}{l}\text { CP, DN, GG, NT, SG, } \\
\text { TP }\end{array}$ & $\begin{array}{l}\text { Virtual } \\
\text { Library }\end{array}$ & $\begin{array}{l}\text { Virtual } \\
\text { Library }\end{array}$ & & & & & & Y & HWIS & \\
\hline 1950 & $200-W$ & 299-W18-100 & A7583 & D & & \begin{tabular}{|l} 
Virtual \\
Library
\end{tabular} & $\begin{array}{l}\text { Virtual } \\
\text { Library }\end{array}$ & & & & & & Y & HWIS & \\
\hline 1951 & $1200-W$ & 299-W18-101 & A7584 & D & & & & & & & & & Y & HWIS & \\
\hline 1952 & $200-W$ & 299-W18-102 & A7585 & D & & \begin{tabular}{|l|} 
Virtual \\
Library
\end{tabular} & $\begin{array}{l}\text { Virtual } \\
\text { Library }\end{array}$ & & & & & & $\mathrm{Y}$ & HWIS & \\
\hline 1953 & $200-W$ & 299-W18-103 & A7586 & D & & $\begin{array}{l}\text { Virtual } \\
\text { Library }\end{array}$ & $\begin{array}{l}\text { Virtual } \\
\text { Library }\end{array}$ & & & & & & $\mathrm{Y}$ & HWIS & \\
\hline 1954 & $4200-W$ & 299-W18-104 & A7587 & D & & $\begin{array}{l}\text { Virtual } \\
\text { Library }\end{array}$ & $\begin{array}{l}\text { Virtual } \\
\text { Library }\end{array}$ & & & & & & $\mathrm{Y}$ & HWIS & \\
\hline 1955 & $200-W$ & 299-W18-105 & A7588 & D & & \begin{tabular}{|l} 
Virtual \\
Library
\end{tabular} & $\begin{array}{l}\text { Virtual } \\
\text { Library }\end{array}$ & & & & & & $\mathrm{Y}$ & HWIS & \\
\hline 1956 & $200-W$ & 299-W18-107 & A7590 & D & & & & & & & & & $Y$ & HWIS & \\
\hline 1957 & $7200-W$ & 299-W18-109 & A7592 & D & & \begin{tabular}{|l|} 
Virtual \\
Library
\end{tabular} & \begin{tabular}{|l|l|} 
Virtual \\
Library
\end{tabular} & & & & & & $\mathrm{Y}$ & HWIS & \\
\hline 1958 & $200-W$ & 299-W18-11 & A7527 & $\mathrm{D}$ & $\mathrm{CP}, \mathrm{DN}, \mathrm{GG}, \mathrm{NT}, \mathrm{SG}$ & & & & & & & & $\mathrm{Y}$ & HWIS & \\
\hline 1959 & $200-W$ & 299-W18-110 & A7593 & D & & \begin{tabular}{|l} 
Virtual \\
Library
\end{tabular} & $\begin{array}{l}\text { Virtual } \\
\text { Library }\end{array}$ & & & & & & $Y$ & HWIS & \\
\hline 1960 & $200-W$ & 299-W18-111 & A7594 & D & & & & & & & & & & HWIS & \\
\hline 1961 & $200-W$ & 299-W18-113 & A7596 & D & & \begin{tabular}{|l} 
Virtual \\
Library
\end{tabular} & $\begin{array}{l}\text { Virtual } \\
\text { Library }\end{array}$ & & \begin{tabular}{|l|} 
Field Rad in \\
logs
\end{tabular} & & & & $\mathrm{Y}$ & PNNL files & \\
\hline 1962 & $200-W$ & 299-W18-114 & A7597 & D & & & & & & & & & $Y$ & HWIS & \\
\hline 1963 & $200-W$ & 299-W18-115 & A7598 & D & & \begin{tabular}{|l|} 
Virtual \\
Library
\end{tabular} & $\begin{array}{l}\text { Virtual } \\
\text { Library }\end{array}$ & & & & & & $\mathrm{Y}$ & HWIS & \\
\hline 1964 & $200-W$ & 299-W18-116 & A7599 & D & & & & & $\begin{array}{l}\text { Field Rad in } \\
\text { logs }\end{array}$ & & & & $\mathrm{Y}$ & PNNL files & \\
\hline 1965 & $200-W$ & 299-W18-117 & A7600 & D & & \begin{tabular}{|l|} 
Virtual \\
Library
\end{tabular} & $\begin{array}{l}\text { Virtual } \\
\text { Library }\end{array}$ & & & & & & $Y$ & HWIS & \\
\hline 1966 & $200-W$ & 299-W18-118 & A7601 & D & & & & & & & & & $Y$ & HWIS & \\
\hline 1967 & $200-W$ & 299-W18-119 & A7602 & D & & \begin{tabular}{|l} 
Virtual \\
Library
\end{tabular} & \begin{tabular}{|l|} 
Virtual \\
Library
\end{tabular} & & & & & & $Y$ & HWIS & \\
\hline 1968 & $200-W$ & 299-W18-12 & A7528 & D, As-built & DN, GG, NT & & & & & & & & $Y$ & HWIS & \\
\hline 1969 & $200-W$ & $299-W 18-120$ & A7603 & $\mathrm{D}$ & & & & & & & & & $Y$ & HWIS & \\
\hline 1970 & $200-W$ & 299-W18-121 & A7604 & $\mathrm{D}$ & & & & & & & & & $Y$ & HWIS & \\
\hline 1971 & $200-W$ & 299-W18-122 & A7605 & D & & & & & & & & & $Y$ & HWIS & \\
\hline 1972 & $200-W$ & 299-W18-123 & A7606 & D & & \begin{tabular}{|l} 
Virtual \\
Library
\end{tabular} & $\begin{array}{l}\text { Virtual } \\
\text { Library }\end{array}$ & & & & & & $Y$ & HWIS & \\
\hline 1973 & $200-W$ & 299-W18-124 & A7607 & D & & \begin{tabular}{|l|} 
Virtual \\
Library
\end{tabular} & $\begin{array}{l}\text { Virtual } \\
\text { Library }\end{array}$ & & & & & & Y & HWIS & \\
\hline 1974 & $200-W$ & 299-W18-125 & A7608 & D & & $\begin{array}{l}\text { Virtual } \\
\text { Library }\end{array}$ & $\begin{array}{l}\text { Virtual } \\
\text { Library }\end{array}$ & & & & & & $Y$ & HWIS & \\
\hline 1975 & $200-W$ & 299-W18-126 & A7609 & D & & $\begin{array}{l}\text { Virtual } \\
\text { Library }\end{array}$ & $\begin{array}{l}\text { Virtual } \\
\text { Library }\end{array}$ & & & & & & $Y$ & HWIS & \\
\hline 1976 & $200-W$ & 299-W18-127 & A7610 & D & & $\begin{array}{l}\text { Virtual } \\
\text { Library }\end{array}$ & $\begin{array}{l}\text { Virtual } \\
\text { Library }\end{array}$ & & & & & & Y & HWIS & \\
\hline 1977 & $200-W$ & 299-W18-128 & A7611 & D & & \begin{tabular}{|l|} 
Virtual \\
Library
\end{tabular} & $\begin{array}{l}\text { Virtual } \\
\text { Library }\end{array}$ & & & & & & $Y$ & HWIS & \\
\hline 1978 & $200-W$ & 299-W18-129 & A7612 & D & & $\begin{array}{l}\text { Virtual } \\
\text { Library }\end{array}$ & $\begin{array}{l}\text { Virtual } \\
\text { Library }\end{array}$ & & & & & & $Y$ & HWIS & \\
\hline
\end{tabular}




\begin{tabular}{|c|c|c|c|c|c|c|c|c|c|c|c|c|c|c|c|}
\hline SortIndex & Area & Well Name & WellID & Log Type & GPX Log Runs & Sieved & $\mathrm{CaCO} 3$ & Moisture & $\begin{array}{l}\text { Chemical } \\
\text { Properties }\end{array}$ & $\begin{array}{c}\text { Physical } \\
\text { Properties }\end{array}$ & \begin{tabular}{|c|} 
Min \\
Properties
\end{tabular} & $\begin{array}{l}\text { Geochron } \\
\text { Properties }\end{array}$ & Archived & Source & Comments \\
\hline 1979 & $200-W$ & 299-W18-13 & A7529 & D & & & & & & & & & & HWIS & \\
\hline 1980 & $200-W$ & 299-W18-130 & A7613 & D & & $\begin{array}{l}\text { Virtual } \\
\text { Library }\end{array}$ & $\begin{array}{l}\text { Virtual } \\
\text { Library }\end{array}$ & & & & & & $Y$ & HWIS & \\
\hline 1981 & $200-W$ & 299-W18-131 & A7614 & D & & \begin{tabular}{|l|} 
Virtual \\
Library
\end{tabular} & $\begin{array}{l}\text { Virtual } \\
\text { Library }\end{array}$ & & & & & & $Y$ & HWIS & \\
\hline 1982 & $200-W$ & 299-W18-132 & A7615 & D & & \begin{tabular}{|l|} 
Virtual \\
Library
\end{tabular} & $\begin{array}{l}\text { Virtual } \\
\text { Library }\end{array}$ & & & & & & $Y$ & HWIS & \\
\hline 1983 & $200-W$ & 299-W18-133 & A7616 & D & & $\begin{array}{l}\text { Virtual } \\
\text { Library }\end{array}$ & $\begin{array}{l}\text { Virtual } \\
\text { Library }\end{array}$ & & & & & & $Y$ & HWIS & \\
\hline 1984 & $200-W$ & 299-W18-134 & A7617 & D & & & & & & & & & $Y$ & HWIS & \\
\hline 1985 & $200-W$ & 299-W18-135 & A7618 & D & & $\begin{array}{l}\text { Virtual } \\
\text { Library }\end{array}$ & \begin{tabular}{|l|} 
Virtual \\
Library
\end{tabular} & & & & & & $Y$ & HWIS & \\
\hline 1986 & $200-W$ & 299-W18-137 & A7620 & D & & \begin{tabular}{|l|} 
Virtual \\
Library
\end{tabular} & $\begin{array}{l}\text { Virtual } \\
\text { Library }\end{array}$ & & & & & & $Y$ & HWIS & \\
\hline 1987 & $200-W$ & 299-W18-138 & A7621 & D & & \begin{tabular}{|l|} 
Virtual \\
Library
\end{tabular} & $\begin{array}{l}\text { Virtual } \\
\text { Library }\end{array}$ & & & & & & & HWIS & \\
\hline 1988 & $200-W$ & 299-W18-139 & A7622 & D & & $\begin{array}{l}\text { Virtual } \\
\text { Library }\end{array}$ & $\begin{array}{l}\text { Virtual } \\
\text { Library }\end{array}$ & & & & & & $Y$ & HWIS & \\
\hline 1989 & $200-W$ & 299-W18-14 & A7530 & D & & & & & & & & Paleomag & & Bjornstad 1984 & \\
\hline 1990 & $200-W$ & 299-W18-140 & A7623 & D & & & & & & & & & $\mathrm{Y}$ & HWIS & \\
\hline 1991 & $200-w$ & 299-W18-141 & A7624 & D & & \begin{tabular}{|l|l|} 
Virtual \\
Library
\end{tabular} & \begin{tabular}{|l|} 
Virtual \\
Library
\end{tabular} & & & & & & $Y$ & HWIS & \\
\hline 1992 & $200-W$ & 299-W18-142 & A7625 & D & & $\begin{array}{l}\text { Virtual } \\
\text { Library }\end{array}$ & $\begin{array}{l}\text { Virtual } \\
\text { Library }\end{array}$ & & & & & & $Y$ & HWIS & \\
\hline 1993 & $200-W$ & 299-W18-143 & A7626 & D & & $\begin{array}{l}\text { Virtual } \\
\text { Library }\end{array}$ & $\begin{array}{l}\text { Virtual } \\
\text { Library }\end{array}$ & & & & & & $Y$ & HWIS & \\
\hline 1994 & $200-W$ & 299-W18-144 & A7627 & D & & \begin{tabular}{|l|} 
Virtual \\
Library
\end{tabular} & $\begin{array}{l}\text { Virtual } \\
\text { Library }\end{array}$ & & & & & & $Y$ & HWIS & \\
\hline 1995 & $200-W$ & 299-W18-145 & A7628 & D & & $\begin{array}{l}\text { Virtual } \\
\text { Library }\end{array}$ & $\begin{array}{l}\text { Virtual } \\
\text { Library }\end{array}$ & & & & & & $Y$ & HWIS & \\
\hline 1996 & $200-W$ & 299-W18-146 & A7629 & D & & & & & & & & & $Y$ & HWIS & \\
\hline 1997 & $200-W$ & 299-W18-147 & A7630 & D & & \begin{tabular}{|l} 
Virtual \\
Library
\end{tabular} & \begin{tabular}{|l|} 
Virtual \\
Library \\
\end{tabular} & & & & & & $Y$ & HWIS & \\
\hline 1998 & $200-W$ & 299-W18-148 & A7631 & D & & \begin{tabular}{|l|} 
Virtual \\
Library
\end{tabular} & $\begin{array}{l}\text { Virtual } \\
\text { Library }\end{array}$ & & & & & & $Y$ & HWIS & \\
\hline 1999 & $200-W$ & 299-W18-149 & A7632 & G & SG & & & & $\mathrm{Pu}, \mathrm{Am}$ & & & & & Price et al. 1979 & \\
\hline 2000 & $200-W$ & 299-W18-15 & A4932 & D & GG, SG & $\begin{array}{l}\text { Virtual } \\
\text { Library }\end{array}$ & \begin{tabular}{|l|} 
Virtual \\
Library
\end{tabular} & $Y$ & $\mathrm{Cs}, \mathrm{Pu}, \mathrm{Am}$ & & & & $Y$ & Last et al. 1994 & \\
\hline 2001 & $200-W$ & 299-W18-150 & A7633 & G & GG & & & & $\mathrm{Pu}, \mathrm{Am}$ & & & & $Y$ & Price et al. 1979 & \\
\hline 2002 & $200-W$ & 299-W18-151 & A7634 & D & GG, SG & & & & & & & & & & \\
\hline 2003 & $200-W$ & 299-W18-152 & A7635 & D & $\mathrm{GG}, \mathrm{SG}$ & \begin{tabular}{|l|} 
Virtual \\
Library
\end{tabular} & $\begin{array}{l}\text { Virtual } \\
\text { Library }\end{array}$ & & $\mathrm{Pu}, \mathrm{Am}$ & & & & $Y$ & $\begin{array}{l}\text { Kasper 1981; } \\
\text { Kasper 1982 }\end{array}$ & \\
\hline 2004 & $200-W$ & 299-W18-153 & A7636 & D & GG, SG & $\begin{array}{l}\text { Virtual } \\
\text { Library }\end{array}$ & $\begin{array}{l}\text { Virtual } \\
\text { Library }\end{array}$ & & $\mathrm{Pu}, \mathrm{Am}$ & & & & $Y$ & $\begin{array}{l}\text { Kasper 1981; } \\
\text { Kasper 1982 }\end{array}$ & \\
\hline 2005 & $200-W$ & 299-W18-154 & A7637 & D & GG, SG & & & & $\mathrm{Pu}, \mathrm{Am}$ & & & & $Y$ & Kasper 1982 & \\
\hline 2006 & $200-W$ & 299-W18-155 & A7638 & D & $\mathrm{GG}, \mathrm{SG}$ & & & & & & & & $Y$ & HWIS & \\
\hline 2007 & $200-\mathrm{W}$ & 299-W18-156 & A7639 & D & SG & & & & & & & & $\mathrm{Y}$ & HWIS & \\
\hline 2008 & $200-w$ & 299-W18-157 & A7640 & D & GG, SG & \begin{tabular}{|l|} 
Virtual \\
Library
\end{tabular} & & & $\mathrm{Pu}, \mathrm{Am}$ & & & & $Y$ & Kasper 1982 & \\
\hline 2009 & $200-W$ & 299-W18-158 & A7641 & G & GG, SG & & & & $\mathrm{Pu}, \mathrm{Am}$ & & & & $Y$ & Price et al. 1979 & \\
\hline
\end{tabular}




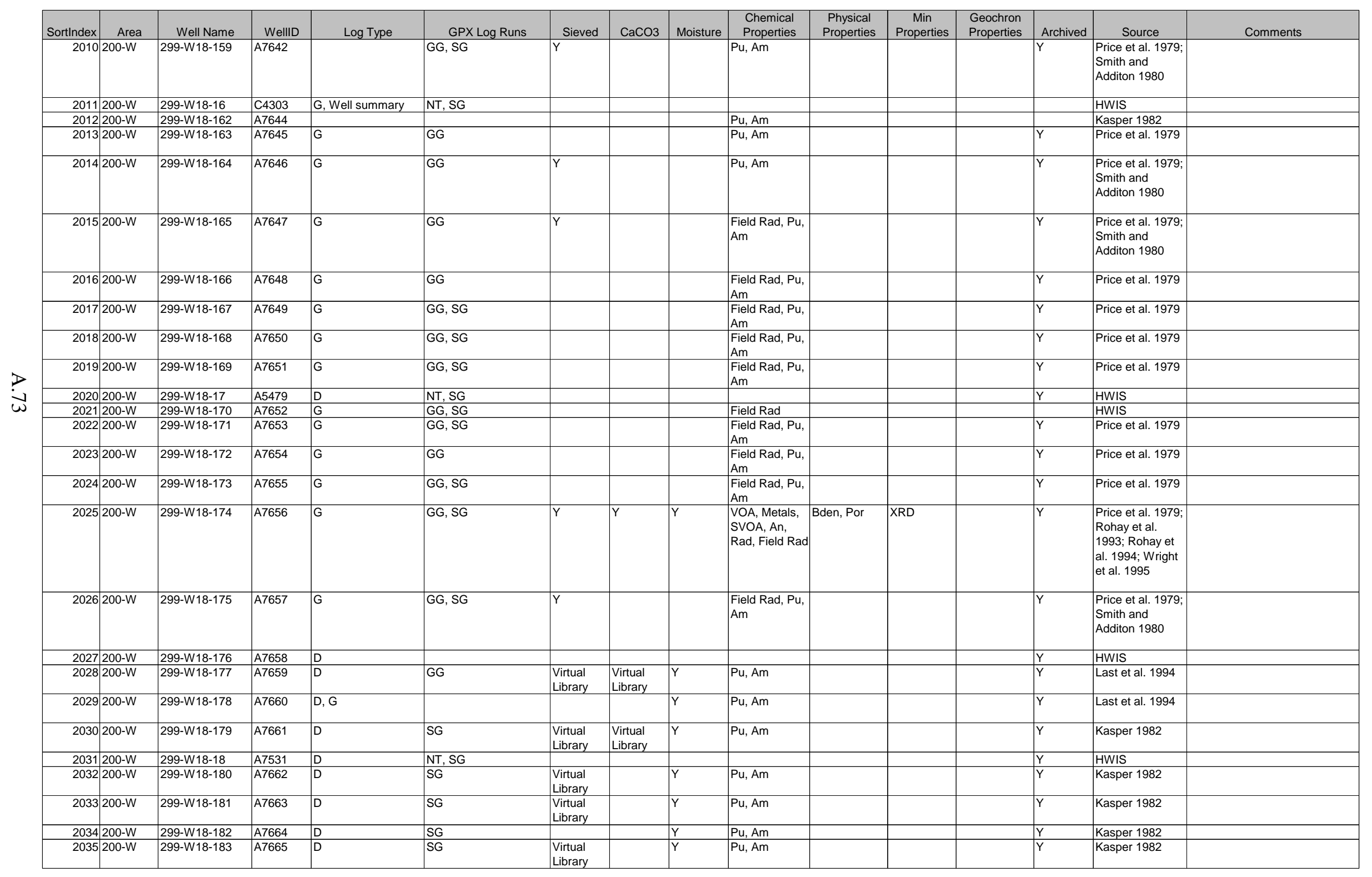




\begin{tabular}{|c|c|c|c|c|c|c|c|c|c|c|c|c|c|c|c|}
\hline SortIndex & Area & Well Name & WellID & Log Type & GPX Log Runs & Sieved & $\mathrm{CaCO} 3$ & Moisture & $\begin{array}{l}\text { Chemical } \\
\text { Properties }\end{array}$ & $\begin{array}{l}\text { Physical } \\
\text { Properties }\end{array}$ & \begin{tabular}{|c|} 
Min \\
Properties \\
\end{tabular} & $\begin{array}{l}\text { Geochron } \\
\text { Properties }\end{array}$ & Archived & Source & Comments \\
\hline 2036 & $200-W$ & 299-W18-184 & A7666 & D & SG & & & $\mathrm{Y}$ & $\mathrm{Pu}, \mathrm{Am}$ & & & & $\mathrm{Y}$ & Kasper 1982 & \\
\hline 2037 & $200-W$ & 299-W18-185 & A7667 & D & SG & & & $\mathrm{Y}$ & $\mathrm{Pu}, \mathrm{Am}$ & & & & $\mathrm{Y}$ & Kasper 1982 & \\
\hline 2038 & $200-W$ & 299-W18-186 & A7668 & $D, G$ & & & & $Y$ & $\mathrm{Pu}, \mathrm{Am}$ & & & & $\mathrm{Y}$ & \begin{tabular}{|l|} 
Last et al. 1994 \\
\end{tabular} & \\
\hline 2039 & $200-W$ & 299-W18-187 & A7669 & $D, G$ & & & & $Y$ & $\mathrm{Pu}, \mathrm{Am}$ & & & & & Last et al. 1994 & \\
\hline 2040 & $200-W$ & 299-W18-188 & A7670 & $D, G$ & & & & $Y$ & $\mathrm{Pu}, \mathrm{Am}$ & & & & $\mathrm{Y}$ & Last et al. 1994 & \\
\hline 2041 & $200-W$ & 299-W18-189 & A7671 & $D, G$ & & & & & $\mathrm{Pu}, \mathrm{Am}$ & & & & & Last et al. 1994 & \\
\hline 2042 & $200-W$ & 299-W18-19 & A7532 & $\mathrm{D}$ & NT, SG & & & & & & & & $\mathrm{Y}$ & HWIS & \\
\hline 2043 & $200-W$ & 299-W18-192 & A7672 & $D, G$ & & & & $Y$ & $\mathrm{Pu}, \mathrm{Am}$ & & & & $Y$ & Last et al. 1994 & \\
\hline 2044 & $200-W$ & 299-W18-193 & A7673 & $D, G$ & & & & $Y$ & $\mathrm{Pu}, \mathrm{Am}$ & & & & $Y$ & Last et al. 1994 & \\
\hline 2045 & $200-W$ & 299-W18-194 & A7674 & $D, G$ & & & & $\mathrm{Y}$ & $\mathrm{Pu}, \mathrm{Am}$ & & & & $\mathrm{Y}$ & Last et al. 1994 & \\
\hline 2046 & $200-W$ & 299-W18-195 & A7675 & $D, G$ & & & & & $\mathrm{Pu}, \mathrm{Am}$ & & & & & Last et al. 1994 & \\
\hline 2047 & $200-W$ & 299-W18-196 & A7676 & $D, G$ & & & & & $\mathrm{Pu}, \mathrm{Am}$ & & & & & Last et al. 1994 & \\
\hline 2048 & $200-W$ & 299-W18-197 & A7677 & $D, G$ & & & & & $\mathrm{Pu}, \mathrm{Am}$ & & & & $Y$ & Last et al. 1994 & \\
\hline 2049 & $200-W$ & 299-W18-198 & A7678 & $D, G$ & & & & & $\mathrm{Pu}, \mathrm{Am}$ & & & & $Y$ & Last et al. 1994 & \\
\hline 2050 & $200-W$ & 299-W18-198 & A7678 & $D, G$ & & & & & & & & & $\mathrm{Y}$ & HWIS & \\
\hline 2051 & $200-\mathrm{W}$ & 299-W18-199 & A7679 & $D, G$ & & & & & & & & & Y & Last et al. 1994 & \\
\hline 2052 & $200-W$ & 299-W18-2 & A5478 & D & DN, GG, NT & \begin{tabular}{|l|}
$\begin{array}{l}\text { Virtual } \\
\text { Library }\end{array}$ \\
\end{tabular} & \begin{tabular}{|l|} 
Virtual \\
Library
\end{tabular} & $\mathrm{Y}$ & & Por & & & $\mathrm{Y}$ & \begin{tabular}{|l|l|} 
Bierschenk 1959 \\
\end{tabular} & \\
\hline 2053 & $200-\mathrm{W}$ & 299-W18-20 & A5471 & D & NT, SG & & & & & & & & $Y$ & HWIS & \\
\hline 2054 & $200-W$ & 299-W18-200 & A7680 & $D, G$ & & & & & $\mathrm{Pu}, \mathrm{Am}$ & & & & $\mathrm{Y}$ & Last et al. 1994 & \\
\hline 2055 & $200-W$ & 299-W18-201 & A7681 & $\mathrm{D}, \mathrm{G}$ & & & & & & & & & & HWIS & \\
\hline 2056 & $200-W$ & 299-W18-202 & A7682 & $\mathrm{D}, \mathrm{G}$ & & & & & & & & & & HWIS & \\
\hline 2057 & $200-w$ & 299-W18-203 & A7683 & D, G & & & & & & & & & $Y$ & HWIS & \\
\hline 2058 & $200-W$ & 299-W18-204 & A7684 & $D, G$ & & & & & & & & & $Y$ & HWIS & \\
\hline 2059 & $200-W$ & 299-W18-205 & A7685 & D & & & & & & & & & $Y$ & HWIS & \\
\hline 2060 & $200-W$ & 299-W18-206 & A7686 & D & & & & & & & & & $\mathrm{Y}$ & HWIS & \\
\hline 2061 & $200-W$ & 299-W18-207 & A76887 & $\mathrm{D}$ & & & & & & & & & $\mathrm{Y}$ & HWIS & \\
\hline 2062 & $200-W$ & 299-W18-208 & A7688 & $D, G$ & & & & & & & & & $Y$ & HWIS & \\
\hline 2063 & $200-W$ & 299-W18-209 & A7689 & $\mathrm{D}$ & & & & & & & & & $Y$ & HWIS & \\
\hline 2064 & $200-W$ & 299-W18-21 & A4933 & G & DN, GG, NT & \begin{tabular}{|l} 
Virtual \\
Library
\end{tabular} & $\begin{array}{l}\text { Virtual } \\
\text { Library }\end{array}$ & $Y$ & $\begin{array}{l}\text { XRF, TC, IC, } \\
\text { TOC }\end{array}$ & CEC & & C-14 & $\mathrm{Y}$ & $\begin{array}{l}\text { Last et al. 1989; } \\
\text { Schramke 1988; } \\
\text { Bjornstad et al. } \\
\text { no date; Ames } \\
\text { and Serne } 1991\end{array}$ & \\
\hline 2065 & $200-W$ & 299-W18-210 & A7690 & $D, G$ & & & & & & & & & $Y$ & HWIS & \\
\hline 2066 & $200-W$ & 299-W18-211 & A7691 & D & & & & & & & & & $Y$ & HWIS & \\
\hline 2067 & $200-w$ & $299-W 18-212$ & A7692 & $\mathrm{D}$ & & & & & & & & & $Y$ & HWIS & \\
\hline 2068 & $200-W$ & 299-W18-213 & A7693 & $D, G$ & & & & & & & & & $Y$ & HWIS & \\
\hline 2069 & $200-W$ & $299-W 18-214$ & A7694 & D & & & & & & & & & $Y$ & HWIS & \\
\hline 2070 & $200-W$ & 299-W18-215 & A7695 & D & & & & & & & & & $Y$ & HWIS & \\
\hline
\end{tabular}




\begin{tabular}{|c|c|c|c|c|c|c|c|c|c|c|c|c|c|c|c|}
\hline SortIndex & Area & Well Name & WellID & Log Type & GPX Log Runs & Sieved & $\mathrm{CaCO} 3$ & Moisture & $\begin{array}{l}\text { Chemical } \\
\text { Properties }\end{array}$ & $\begin{array}{c}\text { Physical } \\
\text { Properties }\end{array}$ & $\begin{array}{c}\text { Min } \\
\text { Properties }\end{array}$ & $\begin{array}{l}\text { Geochron } \\
\text { Properties }\end{array}$ & Archived & Source & Comments \\
\hline 2071 & $1200-W$ & 299-W18-216 & A7696 & D & & Y & & & & & & & $\mathrm{Y}$ & $\begin{array}{l}\text { Rohay et al. } \\
1993\end{array}$ & Hole backfilled \\
\hline 2072 & $200-W$ & 299-W18-217 & A7697 & $\mathrm{D}$ & & & & & & & & & $Y$ & HWIS & \\
\hline 2073 & $200-W$ & 299-W18-218 & A7698 & $D, G$ & & & & & & & & & $Y$ & HWIS & \\
\hline 2074 & $200-W$ & 299-W18-219 & A7699 & $\mathrm{D}$ & & & & & & & & & $Y$ & HWIS & \\
\hline 2075 & $200-W$ & 299-W18-22 & A4934 & $G$ & DN, GG, NT & $\begin{array}{l}\text { Virtual } \\
\text { Library }\end{array}$ & \begin{tabular}{|l|} 
Virtual \\
Library
\end{tabular} & & $\begin{array}{l}\text { XRF, TC, IC, } \\
\text { TOC }\end{array}$ & $\begin{array}{l}\text { \%GSSC, } \\
\text { CEC }\end{array}$ & XRD & & $Y$ & $\begin{array}{l}\text { Last et al. 1989; } \\
\text { Schramke 1988; } \\
\text { Bjornstad et al. } \\
\text { no date; Ames } \\
\text { and Serne } 1991\end{array}$ & \\
\hline 2076 & 5 200-W & 299-W18-220 & A7700 & $D, G$ & & Y & $\mathrm{Y}$ & $Y$ & $\begin{array}{l}\text { VOA, SVOA, } \\
\text { Metals, An, } \\
\text { Rad }\end{array}$ & & & & $Y$ & $\begin{array}{l}\text { Rohay et al. } \\
1992\end{array}$ & \\
\hline 2077 & $200-W$ & 299-W18-221 & A7701 & D & & & & & & & & & $Y$ & HWIS & \\
\hline 2078 & $200-W$ & 299-W18-222 & A7702 & D & & & & & & & & & $Y$ & HWIS & \\
\hline 2079 & $200-W$ & 299-W18-223 & A7703 & D & & & & & & & & & $Y$ & HWIS & \\
\hline 2080 & $200-W$ & 299-W18-224 & A7704 & D & & & & & & & & & $Y$ & HWIS & \\
\hline 2081 & $200-W$ & 299-W18-225 & A7705 & D & & & & & & & & & $Y$ & HWIS & \\
\hline 2082 & $200-W$ & $299-W 18-226$ & A7706 & D & & & & & & & & & $Y$ & HWIS & \\
\hline 2083 & $3200-W$ & 299-W18-227 & A7707 & $\mathrm{D}$ & & & & & & & & & $Y$ & HWIS & \\
\hline 2084 & $4200-W$ & 299-W18-228 & A7708 & D & & & & & & & & & $Y$ & HWIS & \\
\hline 2085 & $200-W$ & 299-W18-229 & A7709 & D & & & & & & & & & $\mathrm{Y}$ & HWIS & \\
\hline 2086 & $200-W$ & 299-W18-23 & A4935 & G & DN, GG, NT & \begin{tabular}{|l} 
Virtual \\
Library
\end{tabular} & $\begin{array}{l}\text { Virtual } \\
\text { Library }\end{array}$ & $Y$ & & & & & $Y$ & Last et al. 1989 & \\
\hline 2087 & $200-W$ & 299-W18-230 & A7710 & D & & & & & & & & & $Y$ & HWIS & \\
\hline 2088 & $200-W$ & 299-W18-231 & A7711 & D & & & & & & & & & $Y$ & HWIS & \\
\hline 2089 & $200-W$ & 299-W18-232 & A7712 & D & & & & & & & & & $Y$ & HWIS & \\
\hline 2090 & $200-W$ & 299-W18-233 & A7713 & D & & & & & & & & & $Y$ & HWIS & \\
\hline 2091 & $200-W$ & 299-W18-234 & A7714 & D & & & & & & & & & & HWIS & \\
\hline 2092 & $200-W$ & 299-W18-235 & A7715 & D & & & & & & & & & & & \\
\hline 2093 & $200-W$ & 299-W18-236 & A7716 & D & & & & & & & & & $Y$ & HWIS & \\
\hline 2094 & $200-W$ & 299-W18-237 & A7717 & D & & & & & & & & & $Y$ & HWIS & \\
\hline 2095 & $200-W$ & 299-W18-238 & A7718 & D & & & & & & & & & $Y$ & HWIS & \\
\hline 2096 & $200-W$ & 299-W18-239 & A7719 & D & & & & & & & & & $\mathrm{Y}$ & HWIS & \\
\hline 2097 & $200-W$ & 299-W18-24 & A4936 & G & $\mathrm{CP}, \mathrm{DN}, \mathrm{GG}, \mathrm{NT}$ & \begin{tabular}{|l|l|} 
Virtual \\
Library
\end{tabular} & \begin{tabular}{|l|l|} 
Virtual \\
Library
\end{tabular} & Y & & & & & $\mathrm{Y}$ & Last et al. 1989 & \\
\hline 2098 & $200-W$ & 299-W18-240 & A7720 & D & & & & & & & & & $Y$ & HWIS & \\
\hline 2099 & $200-W$ & 299-W18-241 & A7721 & $\mathrm{D}$ & & & & & & & & & $Y$ & HWIS & \\
\hline 2100 & $200-W$ & 299-W18-242 & A7722 & D & & & & & & & & & & HWIS & \\
\hline 2101 & $1200-W$ & 299-W18-243 & A7723 & D & & & & & & & & & $Y$ & HWIS & \\
\hline 2102 & $200-W$ & 299-W18-244 & A7724 & D & & & & & & & & & $\mathrm{Y}$ & HWIS & \\
\hline 2103 & $200-W$ & 299-W18-245 & A7725 & D & & & & & & & & & $\mathrm{Y}$ & HWIS & \\
\hline 2104 & $200-W$ & 299-W18-246 & A7726 & $G$ & GG, SG & $\mathrm{Y}$ & & $Y$ & $\begin{array}{l}\text { Metals, VOA, } \\
\text { SVOA, An, } \\
\text { Lab Rad }\end{array}$ & $\begin{array}{l}\text { Bden, } \\
\% \text { GSSC }\end{array}$ & & & & $\begin{array}{l}\text { Rohay et al. } \\
\text { 1994; Last and } \\
\text { Rohay 1993; } \\
\text { Rohay et al. } \\
\text { 1992 }\end{array}$ & \\
\hline 2105 & $200-W$ & 299-W18-247 & A7727 & G & GG, SG & & & Y & $\begin{array}{l}\text { Metals, VOA, } \\
\text { SVOA, An, } \\
\text { Lab Rad }\end{array}$ & $\begin{array}{l}\text { Bden, } \\
\% \text { GSSC }\end{array}$ & & & & $\begin{array}{l}\text { Rohay et al. } \\
\text { 1994; Last and } \\
\text { Rohay } 1993\end{array}$ & \\
\hline
\end{tabular}




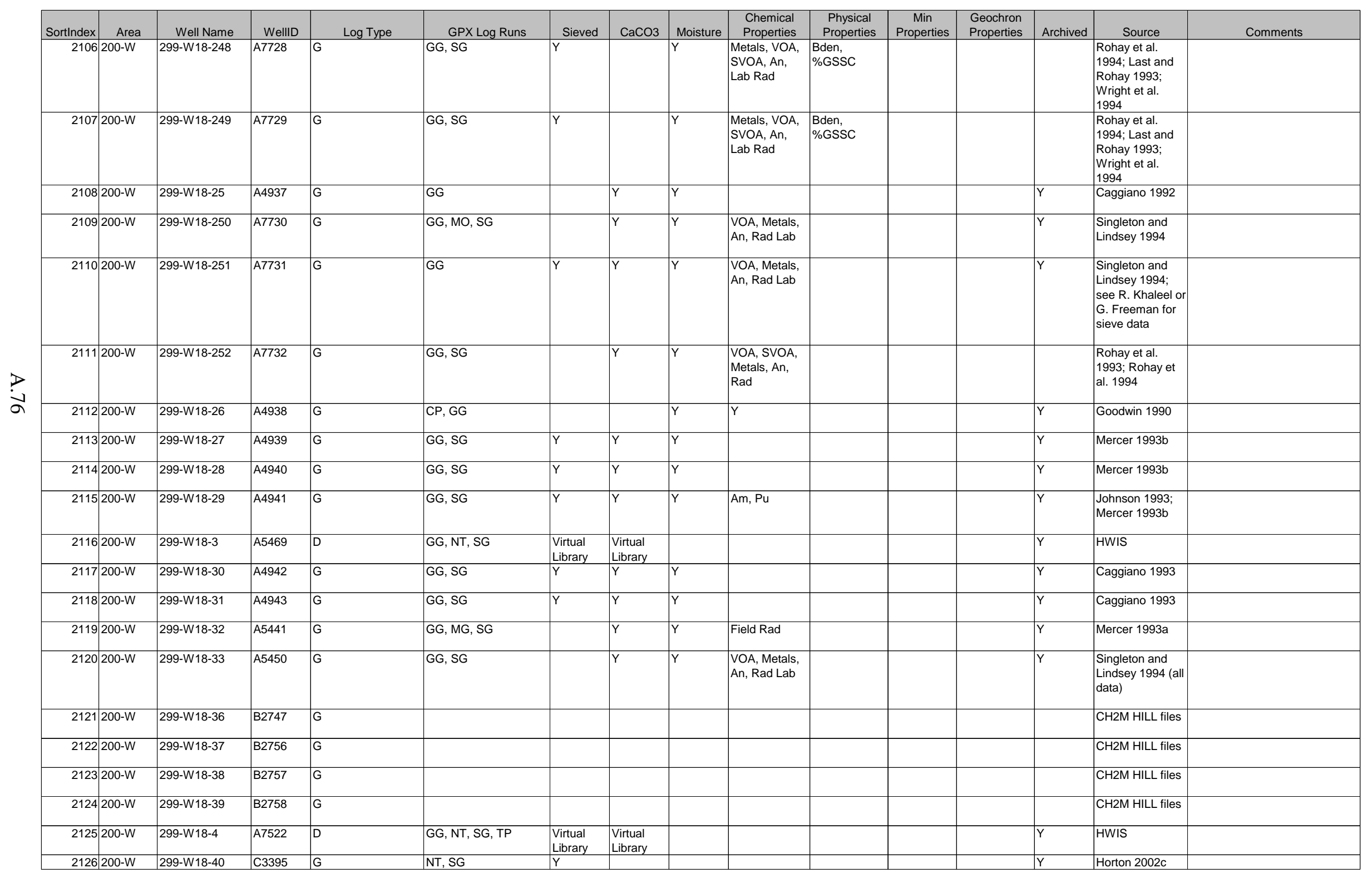




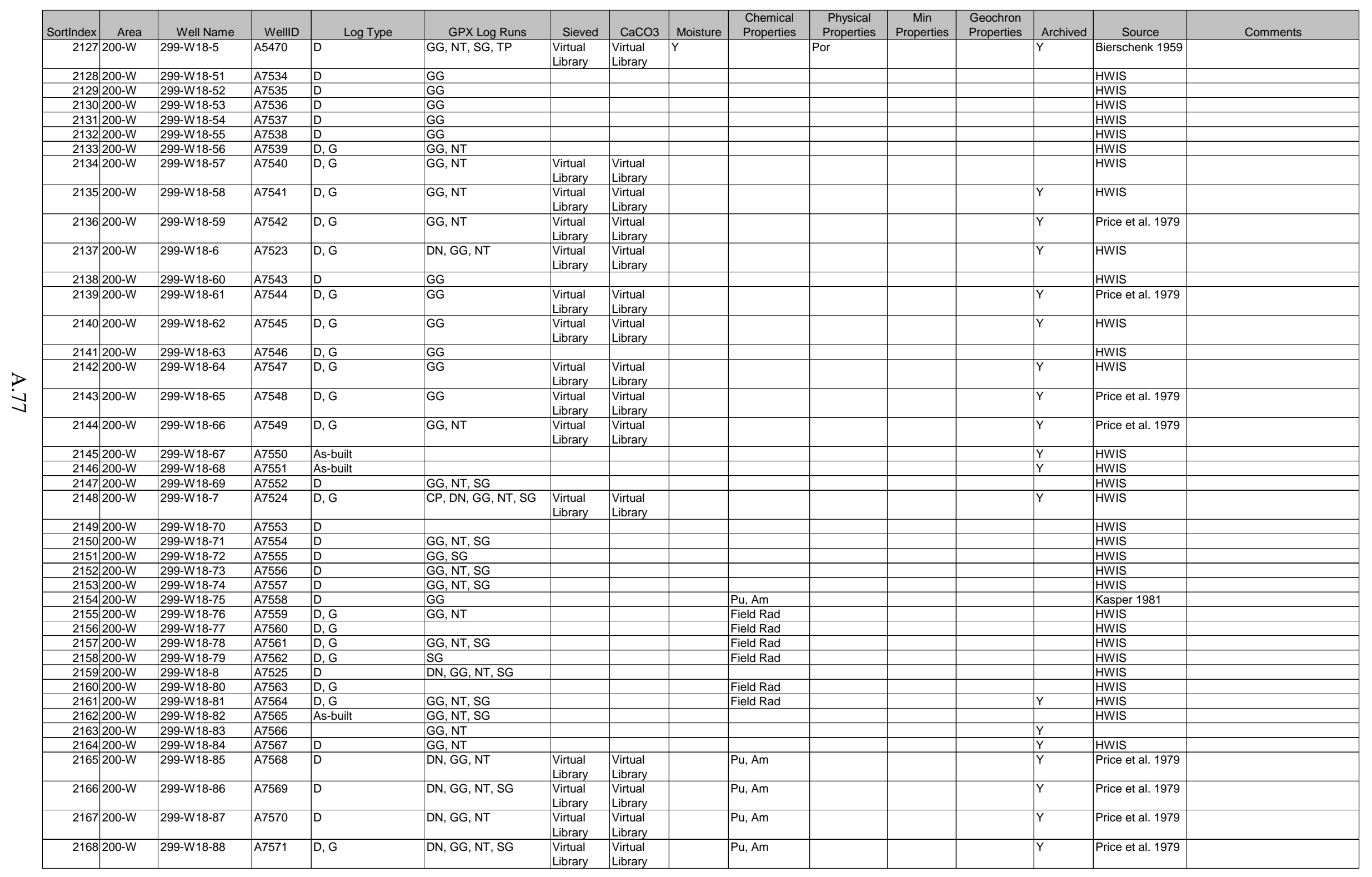




\begin{tabular}{|c|c|c|c|c|c|c|c|c|c|c|c|c|c|c|c|}
\hline Sortindex & Area & Well Name & WelllD & Log Type & GPX Log Runs & Sieved & $\mathrm{CaCO} 3$ & Moisture & $\begin{array}{l}\text { Chemical } \\
\text { Properties }\end{array}$ & $\begin{array}{c}\text { Physical } \\
\text { Properties }\end{array}$ & $\begin{array}{c}\text { Min } \\
\text { Properties } \\
\end{array}$ & $\begin{array}{l}\text { Geochron } \\
\text { Properties }\end{array}$ & Archived & Source & Comments \\
\hline 2169 & $200-W$ & 299-W18-89 & A7572 & D & DN, GG, NT & \begin{tabular}{|l|} 
Virtual \\
Library
\end{tabular} & $\begin{array}{l}\text { Virtual } \\
\text { Library }\end{array}$ & & & & & & $\mathrm{Y}$ & Price et al. 1979 & \\
\hline 2170 & $200-W$ & 299-W18-9 & A7526 & D & $\mathrm{CP}, \mathrm{DN}, \mathrm{GG}, \mathrm{NT}, \mathrm{SG}$ & \begin{tabular}{|l|} 
Virtual \\
Library
\end{tabular} & \begin{tabular}{|l|l|} 
Virtual \\
Library
\end{tabular} & & & & & & $\mathrm{Y}$ & HWIS & \\
\hline 2171 & $200-W$ & 299-W18-90 & A7573 & D & & & & & & & & & & HWIS & \\
\hline 2172 & $200-W$ & 299-W18-91 & A7574 & D & & & & & & & & & & HWIS & \\
\hline 2173 & $200-W$ & 299-W18-92 & A7575 & D & & & & & & & & & & HWIS & \\
\hline 2174 & $200-\mathrm{W}$ & 299-W18-93 & A7576 & D & $\mathrm{DN}, \mathrm{GG}, \mathrm{NT}$ & & & & & & & & & HWIS & \\
\hline 2175 & $200-W$ & 299-W18-94 & A7577 & D & CP, DN, GG, NT, SG & & & & & & & & & HWIS & \\
\hline 2176 & $200-W$ & 299-W18-95 & A7578 & D & DN, GG, NT, SG & & & & & & & & & HWIS & \\
\hline 2177 & $200-W$ & 299-W18-96 & A7579 & $D, G$ & $\begin{array}{l}\text { CP, DN, GG, MG, NT, } \\
\text { SG }\end{array}$ & $\mathrm{Y}$ & $Y$ & $\mathrm{Y}$ & $\begin{array}{l}\text { VOA, Metals, } \\
\text { An, Rad, } \\
\text { SVOA }\end{array}$ & Bden, Por & & & $\mathrm{Y}$ & $\begin{array}{l}\text { Rohay et al. } \\
\text { 1993; Rohay et } \\
\text { al. } 1994\end{array}$ & \\
\hline 2178 & $200-W$ & 299-W18-97 & A7580 & $D, G$ & DN, GG, NT & & & & & & & & $\mathrm{Y}$ & HWIS & \\
\hline 2179 & $200-W$ & 299-W18-98 & A7581 & $D, G$ & $\mathrm{CP}, \mathrm{DN}, \mathrm{GG}, \mathrm{NT}, \mathrm{SG}$ & & & & & & & & $Y$ & HWIS & \\
\hline 2180 & $200-W$ & 299-W18-99 & A7582 & $D, G$ & $\mathrm{DN}, \mathrm{GG}, \mathrm{NT}$ & & & & & & & & $Y$ & HWIS & \\
\hline 2181 & $200-W$ & 299-W19-1 & A4944 & $\mathrm{D}$ & $\mathrm{DN}, \mathrm{GG}, \mathrm{NT}, \mathrm{SG}, \mathrm{TP}$ & \begin{tabular}{|l|} 
Virtual \\
Library
\end{tabular} & \begin{tabular}{|l} 
Virtual \\
Library
\end{tabular} & & & & & & $\mathrm{Y}$ & HWIS & \\
\hline 2182 & $200-W$ & 299-W19-10 & A7738 & $\mathrm{D}, \mathrm{G}$ & DN, NT, TP & & $Y$ & $Y$ & & $\begin{array}{l}\text { Bden, } \\
\text { \%GSSC }\end{array}$ & EM, XRD & Paleomag & & $\begin{array}{l}\text { Virtual Library } \\
2000 \text { (PPI only, } \\
\text { includes } \\
\text { descriptions); } \\
\text { Ames 1976; } \\
\text { Bjornstad 1984; } \\
\text { Parker and } \\
\text { Johnston 1979; } \\
\text { Summers and } \\
\text { Hanson 1977 }\end{array}$ & DH-7 \\
\hline 2183 & $200-W$ & 299-W19-11 & A7739 & D & GG, NT, SG & \begin{tabular}{|l} 
Virtual \\
Library
\end{tabular} & \begin{tabular}{|l|}
$\begin{array}{l}\text { Virtual } \\
\text { Library }\end{array}$ \\
\end{tabular} & & & & & & $\mathrm{Y}$ & HWIS & \\
\hline 2184 & $200-W$ & 299-W19-12 & A4945 & D & & & & & & & & & $\mathrm{Y}$ & HWIS & \\
\hline 2185 & $200-W$ & 299-W19-13 & A7740 & D & GG, NT & \begin{tabular}{|l} 
Virtual \\
Library
\end{tabular} & $\begin{array}{l}\text { Virtual } \\
\text { Library }\end{array}$ & & & & & & $\mathrm{Y}$ & HWIS & \\
\hline 2186 & $200-W$ & 299-W19-14 & A4946 & D & GG, NT & \begin{tabular}{|l} 
Virtual \\
Library
\end{tabular} & $\begin{array}{l}\begin{array}{l}\text { Virtual } \\
\text { Library }\end{array} \\
\end{array}$ & & & & & & $Y$ & HWIS & \\
\hline 2187 & $200-W$ & 299-W19-15 & A4947 & $D, G$ & $\mathrm{DN}, \mathrm{GG}, \mathrm{NT}, \mathrm{SG}$ & \begin{tabular}{|l|} 
Virtual \\
Library
\end{tabular} & $\begin{array}{l}\text { Virtual } \\
\text { Library }\end{array}$ & $Y$ & GEA & & & & $\mathrm{Y}$ & PNNL files & \\
\hline 2188 & $200-W$ & 299-W19-16 & A7741 & $D, G$ & $\mathrm{DN}, \mathrm{GG}, \mathrm{NT}$ & \begin{tabular}{|l|} 
Virtual \\
Library
\end{tabular} & & $\mathrm{Y}$ & GEA & & & & $\mathrm{Y}$ & PNNL files & \\
\hline 2189 & $200-W$ & 299-W19-17 & A7742 & $D, G$ & GG & \begin{tabular}{|l} 
Virtual \\
Library
\end{tabular} & & & & & & & $\mathrm{Y}$ & HWIS & \\
\hline 2190 & 200-W & 299-W19-18 & A7743 & $D, G$ & GG & & & & & & & & $Y$ & HWIS & \\
\hline 2191 & $200-W$ & 299-W19-19 & A7744 & $D, G$ & $\mathrm{DN}, \mathrm{GG}, \mathrm{NT}, \mathrm{SG}$ & \begin{tabular}{|l|} 
Virtual \\
Library
\end{tabular} & \begin{tabular}{|l|} 
Virtual \\
Library
\end{tabular} & $Y$ & & & & & $\mathrm{Y}$ & HWIS & \\
\hline 2192 & $200-W$ & 299-W19-2 & A4948 & D & DN, GG, NT, SG, TP & \begin{tabular}{|l} 
Virtual \\
Library
\end{tabular} & \begin{tabular}{|l}
$\begin{array}{l}\text { Virtual } \\
\text { Library }\end{array}$ \\
\end{tabular} & & & & & & $Y$ & HWIS & \\
\hline 2193 & $200-W$ & 299-W19-20 & A4949 & D, G & GG, NT, SG & \begin{tabular}{|l} 
Virtual \\
Library
\end{tabular} & $\begin{array}{l}\text { Virtual } \\
\text { Library }\end{array}$ & & & & & & $\mathrm{Y}$ & HWIS & \\
\hline 2194 & $200-W$ & 299-W19-21 & A4950 & $D, G$ & GG, NT, SG & $\begin{array}{l}\text { Virtual } \\
\text { Library }\end{array}$ & $\begin{array}{l}\text { Virtual } \\
\text { Library }\end{array}$ & & MiscMetals & & & & $\mathrm{Y}$ & Horton files & \\
\hline 2195 & $200-W$ & 299-W19-22 & A4951 & D, G & GG, NT, SG & \begin{tabular}{|l|} 
Virtual \\
Library
\end{tabular} & \begin{tabular}{|l|} 
Virtual \\
Library
\end{tabular} & $\mathrm{Y}$ & & & & & $\mathrm{Y}$ & HWIS & \\
\hline
\end{tabular}




\begin{tabular}{|c|c|c|c|c|c|c|c|c|c|c|c|c|c|c|c|}
\hline SortIndex & Area & Well Name & WellID & Log Type & GPX Log Runs & Sieved & $\mathrm{CaCO} 3$ & Moisture & $\begin{array}{c}\text { Chemical } \\
\text { Properties }\end{array}$ & $\begin{array}{c}\text { Physical } \\
\text { Properties }\end{array}$ & \begin{tabular}{|c|} 
Min \\
Properties \\
\end{tabular} & $\begin{array}{l}\text { Geochron } \\
\text { Properties }\end{array}$ & Archived & Source & Comments \\
\hline \begin{tabular}{|l|}
2196 \\
\end{tabular} & $200-W$ & 299-W19-23 & A7745 & $D, G$ & DN, GG, NT & \begin{tabular}{|l|} 
Virtual \\
Library
\end{tabular} & $\begin{array}{l}\text { Virtual } \\
\text { Library }\end{array}$ & & & & & & $\mathrm{Y}$ & HWIS & \\
\hline 2197 & $200-W$ & 299-W19-24 & A4952 & $D, G$ & $\mathrm{DN}, \mathrm{GG}, \mathrm{NT}$ & \begin{tabular}{|l|} 
Virtual \\
Library
\end{tabular} & $\begin{array}{l}\text { Virtual } \\
\text { Library }\end{array}$ & & & & & & $\mathrm{Y}$ & HWIS & \\
\hline 2198 & $200-W$ & 299-W19-25 & A7746 & $D, G$ & GG, SG & \begin{tabular}{|l|} 
Virtual \\
Library
\end{tabular} & \begin{tabular}{|l|} 
Virtual \\
Library
\end{tabular} & & & & & & $\mathrm{Y}$ & HWIS & \\
\hline 2199 & $200-W$ & 299-W19-26 & A7747 & D, G & DN, GG, NT, SG & \begin{tabular}{|l} 
Virtual \\
Library
\end{tabular} & \begin{tabular}{|l} 
Virtual \\
Library
\end{tabular} & & & & & & $\mathrm{Y}$ & HWIS & \\
\hline 2200 & $200-W$ & 299-W19-27 & A4953 & $D, G$ & GG, SG & \begin{tabular}{|l} 
Virtual \\
Library
\end{tabular} & \begin{tabular}{|l} 
Virtual \\
Library
\end{tabular} & $\mathrm{Y}$ & MiscMetals & & & & $Y$ & Horton files & \\
\hline 2201 & $1200-W$ & 299-W19-28 & A4954 & $D, G$ & GG, SG & & & & & & & & $\mathrm{Y}$ & & \\
\hline 2202 & $200-W$ & 299-W19-29 & A4955 & $D, G$ & $\mathrm{CP}, \mathrm{GG}$ & & & & & & & & $\mathrm{Y}$ & & \\
\hline 2203 & $3200-W$ & 299-W19-3 & A7733 & D & DN, GG, NT, SG, TP & \begin{tabular}{|l|} 
Virtual \\
Library
\end{tabular} & $\begin{array}{l}\text { Virtual } \\
\text { Library }\end{array}$ & & & & & & $Y$ & HWIS & \\
\hline 2204 & $4200-W$ & 299-W19-30 & A7748 & $D, G$ & $G G$ & & & & & & & & $\mathrm{Y}$ & & \\
\hline 2205 & 5200-W & 299-W19-31 & A4956 & G & GG & $Y$ & $\mathrm{Y}$ & $\mathrm{Y}$ & & & & & $\mathrm{Y}$ & Caggiano 1992 & \\
\hline 2206 & $200-W$ & 299-W19-32 & A4957 & G & GG, SG & Y & $Y$ & $Y$ & & & & & Y & Caggiano 1992 & \\
\hline 2207 & $200-W$ & 299-W19-34 & & & SG & & & & & & & & & & \\
\hline 2208 & $200-W$ & 299-W19-34B & A9513 & D, G, Well summary & $G G, S G$ & & & & & & & & & HWIS & \\
\hline 2209 & $200-W$ & 299-W19-35 & A9515 & D, G, Well summary & SG & & & & & & & & & HWIS & \\
\hline 2210 & $200-W$ & 299-W19-36 & B2461 & G & & Y & & & & & & & & Darrach 1995 & \\
\hline 2211 & $1200-W$ & 299-W19-37 & B2465 & G & & $Y$ & & & & & & & & Darrach 1995 & \\
\hline 2212 & $200-W$ & 299-W19-38 & B2463 & G & & $Y$ & & & & & & & & Darrach 1995 & \\
\hline 2213 & $200-W$ & 299-W19-39 & B2460 & G & & $\mathrm{Y}$ & & & & & & & & Darrach 1995 & \\
\hline 2214 & $200-W$ & 299-W19-4 & A4958 & D & GG, NT, TP & & & & & & & & & HWIS & \\
\hline 2215 & $200-W$ & 299-W19-40 & B2464 & G & & $Y$ & & & & & & & & Darrach 1995 & \\
\hline 2216 & \begin{tabular}{|l|l|}
5 & $200-W$ \\
\end{tabular} & 299-W19-41 & B8551 & G & & Y & & & & & & & $\mathrm{Y}$ & $\begin{array}{l}\text { Horton and } \\
\text { Hodges 1999b }\end{array}$ & \\
\hline 2217 & $200-W$ & 299-W19-42 & B8553 & G & SG & Y & & Y & 1:1, Alk & Cond & & & $\mathrm{Y}$ & $\begin{array}{l}\text { Horton and } \\
\text { Hodges 1999b }\end{array}$ & \\
\hline 2218 & $200-W$ & 299-W19-43 & C3381 & G & & & & & & & & Paleomag & & $\begin{array}{l}\text { Faurote and } \\
\text { Wright 2001; } \\
\text { Pluhar 2002 }\end{array}$ & \\
\hline 2219 & $200-W$ & 299-W19-44 & C3393 & $G$ & & $\mathrm{Y}$ & & & & & & & $\mathrm{Y}$ & Horton 2002c & \\
\hline 2220 & $200-W$ & 299-W19-45 & C3394 & G & NT, SG & $Y$ & & & & & & & $\mathrm{Y}$ & Horton 2002c & \\
\hline 2221 & $200-W$ & 299-W19-46 & C3958 & $\mathrm{G}$ & SG & $Y$ & & & & & & & $\mathrm{Y}$ & HWIS & \\
\hline 2222 & $200-W$ & 299-W19-47 & C4258 & G, Well summary & SG & & & & & & & & & HWIS & \\
\hline 2223 & $200-W$ & 299-W19-48 & C4300 & G, Well summary & SG & & & & & & & & & HWIS & \\
\hline 2224 & $4200-W$ & 299-W19-5 & A7734 & D & DN, GG, NT, SG & & & & & & & & $\mathrm{Y}$ & HWIS & \\
\hline 2225 & $200-W$ & 299-W19-53 & A7751 & D & GG & & & & & & & & & HWIS & \\
\hline 2226 & $200-W$ & 299-W19-54 & A7753 & $\mathrm{D}$ & GG & & & & & & & & & HWIS & \\
\hline 2227 & $200-\mathrm{W}$ & 299-W19-55 & A7755 & D & & & & & & & & & & HWIS & \\
\hline 2228 & $200-W$ & 299-W19-6 & A4959 & D, As-built & DN, GG, NT, TP & & & & & & & & $\mathrm{Y}$ & HWIS & Only remediation logs available \\
\hline 2229 & $200-W$ & 299-W19-69 & A7769 & D & & \begin{tabular}{|l|} 
Virtual \\
Library
\end{tabular} & & & & & & & $\mathrm{Y}$ & HWIS & \\
\hline 2230 & $200-W$ & 299-W19-7 & A7735 & D & DN, GG, NT, SG & \begin{tabular}{|l|} 
Virtual \\
Library
\end{tabular} & $\begin{array}{l}\text { Virtual } \\
\text { Library }\end{array}$ & & & & & & $\mathrm{Y}$ & HWIS & \\
\hline
\end{tabular}




\begin{tabular}{|c|c|c|c|c|c|c|c|c|c|c|c|c|c|c|c|}
\hline SortIndex & Area & Well Name & WellID & Log Type & GPX Log Runs & Sieved & $\mathrm{CaCO} 3$ & Moisture & $\begin{array}{l}\text { Chemical } \\
\text { Properties }\end{array}$ & $\begin{array}{c}\text { Physical } \\
\text { Properties }\end{array}$ & $\begin{array}{c}\text { Min } \\
\text { Properties } \\
\end{array}$ & $\begin{array}{l}\text { Geochron } \\
\text { Properties }\end{array}$ & Archived & Source & Comments \\
\hline 2231 & $200-W$ & 299-W19-70 & A7770 & D & GG, SG & \begin{tabular}{|l|} 
Virtual \\
Library
\end{tabular} & $\begin{array}{l}\text { Virtual } \\
\text { Library }\end{array}$ & & & & & & $\mathrm{Y}$ & HWIS & \\
\hline 2232 & $200-W$ & 299-W19-71 & A7771 & D & GG, SG & $\begin{array}{l}\text { Virtual } \\
\text { Library }\end{array}$ & $\begin{array}{l}\text { Virtual } \\
\text { Library }\end{array}$ & & & & & & $\mathrm{Y}$ & HWIS & \\
\hline 2233 & $200-W$ & 299-W19-72 & A7772 & D & & \begin{tabular}{|l} 
Virtual \\
Library
\end{tabular} & & & & & & & $\mathrm{Y}$ & HWIS & \\
\hline 2234 & $200-W$ & 299-W19-73 & A7773 & D & & & & & & & & & $\mathrm{Y}$ & HWIS & \\
\hline 2235 & $200-W$ & 299-W19-74 & A7774 & D & & $\begin{array}{l}\text { Virtual } \\
\text { Library }\end{array}$ & \begin{tabular}{|l} 
Virtual \\
Library
\end{tabular} & & & & & & $\mathrm{Y}$ & HWIS & \\
\hline 2236 & $200-W$ & 299-W19-75 & A7775 & $\mathrm{D}$ & & & & & & & & & $\mathrm{Y}$ & HWIS & \\
\hline 2237 & $200-W$ & 299-W19-76 & A7776 & $\mathrm{D}$ & & \begin{tabular}{|l|} 
Virtual \\
Library
\end{tabular} & $\begin{array}{l}\text { Virtual } \\
\text { Library }\end{array}$ & & & & & & $\mathrm{Y}$ & HWIS & \\
\hline 2238 & $200-W$ & 299-W19-77 & A7777 & G & & & & & & & & & & Webster 1977 & \\
\hline 2239 & $200-W$ & 299-W19-8 & A7736 & D & GG, NT & \begin{tabular}{|l|l|} 
Virtual \\
Library
\end{tabular} & \begin{tabular}{|l|}
$\begin{array}{l}\text { Virtual } \\
\text { Library }\end{array}$ \\
\end{tabular} & & & & & & Y & HWIS & \\
\hline 2240 & $200-W$ & 299-W19-89 & A7789 & $D, G$ & GG, SG & \begin{tabular}{|l|} 
Virtual \\
Library
\end{tabular} & & $Y$ & & & & & $Y$ & HWIS & \\
\hline 2241 & $200-W$ & 299-W19-9 & A7737 & D & $\mathrm{CP}, \mathrm{GG}, \mathrm{NT}, \mathrm{SG}$ & & & & & & & & & HWIS & \\
\hline 2242 & $200-W$ & 299-W19-90 & A7790 & $D, G$ & GG, SG & \begin{tabular}{|l} 
Virtual \\
Library
\end{tabular} & $\begin{array}{l}\text { Virtual } \\
\text { Library }\end{array}$ & Y & & & & & $Y$ & HWIS & \\
\hline 2243 & $200-W$ & 299-W19-91 & A4960 & $D, G$ & $\mathrm{DN}, \mathrm{GG}, \mathrm{NT}, \mathrm{SG}$ & $\begin{array}{l}\text { Virtual } \\
\text { Library }\end{array}$ & $\begin{array}{l}\text { Virtual } \\
\text { Library }\end{array}$ & $Y$ & MiscMetals & & & & $\mathrm{Y}$ & \begin{tabular}{|l|} 
Routson $1987 ;$ \\
D. G. Horton files
\end{tabular} & \\
\hline 2244 & $200-W$ & 299-W19-92 & A4961 & $D, G$ & $\mathrm{DN}, \mathrm{GG}, \mathrm{NT}, \mathrm{SG}$ & $\begin{array}{l}\text { Virtual } \\
\text { Library }\end{array}$ & $\begin{array}{l}\text { Virtual } \\
\text { Library }\end{array}$ & $Y$ & MiscMetals & & & & $\mathrm{Y}$ & \begin{tabular}{|l|} 
Routson 1987;D. \\
G. Horton files
\end{tabular} & \\
\hline 2245 & $200-W$ & 299-W19-93 & A4962 & $D, G$ & $\mathrm{DN}, \mathrm{GG}, \mathrm{NT}, \mathrm{SG}$ & $\begin{array}{l}\text { Virtual } \\
\text { Library }\end{array}$ & \begin{tabular}{|l} 
Virtual \\
Library
\end{tabular} & $Y$ & MiscMetals & & & & $\mathrm{Y}$ & $\begin{array}{l}\text { Routson } 1987 ; \\
\text { D. G. Horton files }\end{array}$ & \\
\hline 2246 & $200-W$ & 299-W19-94 & A9796 & D, G, Well summary & $S G$ & & & & & & & & & HWIS & \\
\hline 2247 & $200-W$ & 299-W19-95 & A7791 & Well Summary & SG & Y & & & & & & & & $\begin{array}{l}\text { see R. Khaleel or } \\
\text { G. Freeman for } \\
\text { sieve data }\end{array}$ & \\
\hline 2248 & $200-W$ & 299-W19-96 & A9797 & D, G, Well summary & $S G$ & & & & & & & & & HWIS & \\
\hline 2249 & $200-W$ & 299-W19-97 & A7792 & D, G, Well summary & $S G$ & & & & & & & & & HWIS & \\
\hline 2250 & $200-W$ & 299-W19-98 & A9798 & D, G, Well summary & SG & & & & & & & & & HWIS & \\
\hline 2251 & $200-W$ & 299-W21-1 & A4963 & D, As-built & GG, SG, TP & & & & & & & & & HWIS & \\
\hline 2252 & $200-\mathrm{W}$ & 299-W21-2 & C4639 & G, Well summary & GG & & & & & & & & & HWIS & \\
\hline 2253 & $200-W$ & 299-W21-51 & A7793 & D & & \begin{tabular}{|l|l} 
Virtual \\
Library
\end{tabular} & & & & & & & & HWIS & \\
\hline 2254 & $200-W$ & 299-W21-52 & A7794 & D & & \begin{tabular}{|l|} 
Virtual \\
Library
\end{tabular} & & & & & & & $\mathrm{Y}$ & HWIS & \\
\hline 2255 & $200-W$ & 299-W21-53 & A7795 & D & & $\begin{array}{l}\text { Virtual } \\
\text { Library }\end{array}$ & & & & & & & $Y$ & HWIS & \\
\hline 2256 & $200-W$ & 299-W21-54 & A7796 & D & & \begin{tabular}{|l|} 
Virtual \\
Library
\end{tabular} & & & & & & & $\mathrm{Y}$ & HWIS & \\
\hline 2257 & $200-W$ & 299-W21-55 & A7797 & D & & $\begin{array}{l}\text { Virtual } \\
\text { Library }\end{array}$ & & & & & & & $\mathrm{Y}$ & HWIS & \\
\hline 2258 & $200-W$ & 299-W21-56 & A7798 & D & & \begin{tabular}{|l} 
Virtual \\
Library
\end{tabular} & & & & & & & $Y$ & HWIS & \\
\hline
\end{tabular}




\begin{tabular}{|c|c|c|c|c|c|c|c|c|c|c|c|c|c|c|c|}
\hline SortIndex & Area & Well Name & WellID & Log Type & GPX Log Runs & Sieved & $\mathrm{CaCO} 3$ & Moisture & $\begin{array}{l}\text { Chemical } \\
\text { Properties }\end{array}$ & $\begin{array}{c}\text { Physical } \\
\text { Properties }\end{array}$ & $\begin{array}{c}\text { Min } \\
\text { Properties } \\
\end{array}$ & $\begin{array}{l}\text { Geochron } \\
\text { Properties }\end{array}$ & Archived & Source & Comments \\
\hline $\begin{array}{r}2259 \\
\end{array}$ & $200-W$ & 299-W21-57 & A7799 & D & & \begin{tabular}{|l|} 
Virtual \\
Library
\end{tabular} & & & & & & & $\mathrm{Y}$ & HWIS & \\
\hline 2260 & $200-W$ & 299-W21-58 & A7800 & D & & $\begin{array}{l}\text { Virtual } \\
\text { Library }\end{array}$ & & & & & & & $Y$ & HWIS & \\
\hline 2261 & $200-W$ & 299-W21-59 & A7801 & D & & \begin{tabular}{|l|} 
Virtual \\
Library
\end{tabular} & & & & & & & $Y$ & HWIS & \\
\hline 2262 & $200-W$ & 299-W21-60 & A7802 & D & & $\begin{array}{l}\text { Virtual } \\
\text { Library }\end{array}$ & & & & & & & $Y$ & HWIS & \\
\hline 2263 & $200-W$ & 299-W21-61 & A7803 & D & & \begin{tabular}{|l|} 
Virtual \\
Library
\end{tabular} & & & & & & & $Y$ & HWIS & \\
\hline 2264 & $200-W$ & 299-W21-62 & A7804 & D & & $\begin{array}{l}\text { Virtual } \\
\text { Library }\end{array}$ & & & & & & & $Y$ & HWIS & \\
\hline 2265 & $200-W$ & 299-W21-63 & A7805 & D & & \begin{tabular}{|l|} 
Virtual \\
Library
\end{tabular} & & & & & & & $Y$ & HWIS & \\
\hline 2266 & $200-W$ & 299-W21-64 & A7806 & D & & \begin{tabular}{|l|} 
Virtual \\
Library
\end{tabular} & & & & & & & $Y$ & HWIS & \\
\hline 2267 & $200-W$ & 299-W21-65 & A7807 & D & & \begin{tabular}{|l|} 
Virtual \\
Library
\end{tabular} & & & & & & & $Y$ & HWIS & \\
\hline 2268 & $200-W$ & 299-W21-66 & A7808 & D & & \begin{tabular}{|l} 
Virtual \\
Library
\end{tabular} & & & & & & & $Y$ & HWIS & \\
\hline 2269 & $200-W$ & 299-W21-67 & A7809 & D & & $\begin{array}{l}\text { Virtual } \\
\text { Library }\end{array}$ & & & & & & & $Y$ & HWIS & \\
\hline 2270 & $200-W$ & 299-W21-68 & A7810 & D & & \begin{tabular}{|l|} 
Virtual \\
Library
\end{tabular} & & & & & & & $Y$ & HWIS & \\
\hline 2271 & $1200-W$ & 299-W21-69 & A7811 & D & & \begin{tabular}{|l} 
Virtual \\
Library
\end{tabular} & & & & & & & $Y$ & HWIS & \\
\hline 2272 & $200-W$ & 299-W21-70 & A7812 & D & & \begin{tabular}{|l} 
Virtual \\
Library
\end{tabular} & & & & & & & $Y$ & HWIS & \\
\hline 2273 & $3200-W$ & 299-W21-71 & A7813 & D & & \begin{tabular}{|l|} 
Virtual \\
Library
\end{tabular} & & & & & & & $Y$ & HWIS & \\
\hline 2274 & $4200-W$ & 299-W21-72 & A7814 & D & & $\begin{array}{l}\text { Virtual } \\
\text { Library }\end{array}$ & & & & & & & $Y$ & HWIS & \\
\hline 2275 & $200-W$ & 299-W21-73 & A7815 & D & & $\begin{array}{l}\text { Virtual } \\
\text { Library }\end{array}$ & & & & & & & $Y$ & HWIS & \\
\hline 2276 & $200-W$ & 299-W21-74 & A7816 & D & & \begin{tabular}{|l|} 
Virtual \\
Library
\end{tabular} & & & & & & & $Y$ & HWIS & \\
\hline 2277 & $200-W$ & 299-W21-75 & A7817 & D & & \begin{tabular}{|l|} 
Virtual \\
Library
\end{tabular} & & & & & & & $Y$ & HWIS & \\
\hline 2278 & $3200-W$ & 299-W21-76 & A7818 & D & & \begin{tabular}{|l|}
$\begin{array}{l}\text { Virtual } \\
\text { Library }\end{array}$ \\
\end{tabular} & & & & & & & $Y$ & HWIS & \\
\hline 2279 & $200-W$ & 299-W21-77 & A7819 & $\mathrm{D}$ & & & & & & & & & $Y$ & HWIS & \\
\hline 2280 & $200-W$ & 299-W21-78 & A7820 & D & & & & & & & & & $Y$ & HWIS & \\
\hline 2281 & $200-W$ & 299-W21-79 & A7821 & D & & & & & & & & & $Y$ & HWIS & \\
\hline 2282 & $200-W$ & 299-W21-80 & A7822 & D & & & & & & & & & $Y$ & HWIS & \\
\hline 2283 & $200-W$ & 299-W21-81 & A7823 & D & & & & & & & & & $Y$ & HWIS & \\
\hline 2284 & $4200-W$ & 299-W21-82 & A7824 & D & & & & & & & & & $Y$ & HWIS & \\
\hline 2285 & $200-W$ & 299-W21-83 & A7825 & D & & & & & & & & & $\mathrm{Y}$ & HWIS & \\
\hline 2286 & $200-W$ & 299-W22-1 & A7827 & D & DN, GG, NT & \begin{tabular}{|l} 
Virtual \\
Library
\end{tabular} & \begin{tabular}{|l} 
Virtual \\
Library
\end{tabular} & & \begin{tabular}{|l|} 
Field \\
Rad/Temp
\end{tabular} & & & & $Y$ & HWIS & \\
\hline 2287 & $200-W$ & 299-W22-10 & A7835 & D & $\mathrm{DN}, \mathrm{GG}, \mathrm{NT}, \mathrm{TP}$ & \begin{tabular}{|l|} 
Virtual \\
Library
\end{tabular} & $\begin{array}{l}\text { Virtual } \\
\text { Library }\end{array}$ & & \begin{tabular}{|l|} 
Field \\
Rad/Temp
\end{tabular} & & & & $Y$ & HWIS & \\
\hline 2288 & $200-W$ & 299-W22-11 & A7836 & D & GG, TP & $\begin{array}{l}\text { Virtual } \\
\text { Library }\end{array}$ & $\begin{array}{l}\text { Virtual } \\
\text { Library }\end{array}$ & & $\begin{array}{l}\text { Sr, Beta, } \\
\text { Gamma }\end{array}$ & & & & $\mathrm{Y}$ & $\begin{array}{l}\text { Haney and } \\
\text { Linderoth } 1959\end{array}$ & \\
\hline 2289 & $200-W$ & 299-W22-12 & A7837 & D & GG, NT, SG & \begin{tabular}{|l|} 
Virtual \\
Library
\end{tabular} & $\begin{array}{l}\text { Virtual } \\
\text { Library }\end{array}$ & & & & & & $\mathrm{Y}$ & HWIS & \\
\hline
\end{tabular}




\begin{tabular}{|c|c|c|c|c|c|c|c|c|c|c|c|c|c|c|c|}
\hline Sortlndex & Area & Well Name & WellID & Log Type & GPX Log Runs & Sieved & $\mathrm{CaCO} 3$ & Moisture & $\begin{array}{l}\text { Chemical } \\
\text { Properties }\end{array}$ & $\begin{array}{c}\text { Physical } \\
\text { Properties }\end{array}$ & $\begin{array}{c}\text { Min } \\
\text { Properties }\end{array}$ & $\begin{array}{l}\text { Geochron } \\
\text { Properties }\end{array}$ & Archived & Source & Comments \\
\hline 2290 & $200-W$ & 299-W22-13 & A7838 & D & DN, GG, NT, SG & \begin{tabular}{|l|} 
Virtual \\
Library
\end{tabular} & $\begin{array}{l}\text { Virtual } \\
\text { Library }\end{array}$ & & & & & & $\mathrm{Y}$ & HWIS & \\
\hline 2291 & $200-W$ & 299-W22-14 & A7839 & D & GG, SG, TP & $\begin{array}{l}\text { Virtual } \\
\text { Library }\end{array}$ & $\begin{array}{l}\text { Virtual } \\
\text { Library }\end{array}$ & & Field Rad & & & & $Y$ & HWIS & \\
\hline 2292 & $200-W$ & 299-W22-15 & A7840 & D & DN, GG, NT, TP & & & & $\begin{array}{l}\text { Field Rad, Sr, } \\
\text { Beta, Gamma }\end{array}$ & & & & & \begin{tabular}{l|} 
Haney and \\
Linderoth 1959
\end{tabular} & \\
\hline 2293 & $200-W$ & 299-W22-16 & A7841 & D & DN, GG, NT, TP & & & & $\begin{array}{l}\text { Sr, Beta, } \\
\text { Gamma }\end{array}$ & & & & & $\begin{array}{l}\text { Haney and } \\
\text { Linderoth } 1959\end{array}$ & \\
\hline 2294 & $200-W$ & 299-W22-17 & A4964 & $\mathrm{D}$ & DN, GG, NT, TP & & & & Field Rad & & & & & HWIS & \\
\hline 2295 & $200-W$ & 299-W22-18 & A7842 & D & $\mathrm{DN}, \mathrm{GG}, \mathrm{NT}$ & $\begin{array}{l}\text { Virtual } \\
\text { Library }\end{array}$ & \begin{tabular}{|l|} 
Virtual \\
Library
\end{tabular} & & $\begin{array}{l}\text { Sr, Beta, } \\
\text { Gamma }\end{array}$ & & & & $\mathrm{Y}$ & $\begin{array}{l}\text { Haney and } \\
\text { Linderoth } 1959\end{array}$ & \\
\hline 2296 & $200-W$ & 299-W22-19 & A4965 & D & DN, GG, NT, SG, TP & $\begin{array}{l}\text { Virtual } \\
\text { Library }\end{array}$ & \begin{tabular}{|l|}
$\begin{array}{l}\text { Virtual } \\
\text { Library }\end{array}$ \\
\end{tabular} & & & & & & Y & HWIS & \\
\hline 2297 & $200-W$ & 299-W22-2 & \begin{tabular}{|l|} 
A7828 \\
\end{tabular} & D & DN, GG, NT & \begin{tabular}{|l|} 
Virtual \\
Library
\end{tabular} & \begin{tabular}{|l|} 
Virtual \\
Library
\end{tabular} & & \begin{tabular}{|l|} 
Field \\
Rad/Temp, Sr, \\
Beta, Gamma
\end{tabular} & & & & $\mathrm{Y}$ & $\begin{array}{l}\text { Haney and } \\
\text { Linderoth } 1959\end{array}$ & \\
\hline 2298 & $200-W$ & 299-W22-20 & A7843 & D & DN, GG, NT, SG, TP & \begin{tabular}{|l|} 
Virtual \\
Library
\end{tabular} & $\begin{array}{l}\text { Virtual } \\
\text { Library }\end{array}$ & & & & & & $Y$ & HWIS & \\
\hline 2299 & $200-W$ & 299-W22-21 & A4966 & D & DN, GG, NT, SG, TP & \begin{tabular}{|l|} 
Virtual \\
Library
\end{tabular} & \begin{tabular}{|l|} 
Virtual \\
Library
\end{tabular} & & & & & & $Y$ & HWIS & \\
\hline 2300 & $200-W$ & 299-W22-22 & A4967 & D & $\mathrm{DN}, \mathrm{GG}, \mathrm{NT}, \mathrm{SG}$ & $\begin{array}{l}\text { Virtual } \\
\text { Library }\end{array}$ & $\begin{array}{l}\text { Virtual } \\
\text { Library }\end{array}$ & & & & & & $Y$ & HWIS & \\
\hline 2301 & $200-W$ & 299-W22-23 & A7844 & D & $\mathrm{DN}, \mathrm{GG}, \mathrm{NT}, \mathrm{SG}$ & $\begin{array}{l}\text { Virtual } \\
\text { Library }\end{array}$ & $\begin{array}{l}\text { Virtual } \\
\text { Library }\end{array}$ & & & & & & $Y$ & HWIS & \\
\hline 2302 & $200-W$ & 299-W22-24 & A7845 & $\mathrm{D}$ & TP & $\begin{array}{l}\text { Virtual } \\
\text { Library }\end{array}$ & $\begin{array}{l}\text { Virtual } \\
\text { Library }\end{array}$ & & & & & & $Y$ & HWIS & \\
\hline 2303 & $200-W$ & 299-W22-25 & A7846 & D & DN, GG, NT, TP & $\begin{array}{l}\text { Virtual } \\
\text { Library }\end{array}$ & $\begin{array}{l}\text { Virtual } \\
\text { Library }\end{array}$ & & & & & & $Y$ & HWIS & \\
\hline 2304 & $200-W$ & 299-W22-26 & A4968 & D & DN, GG, NT & $\begin{array}{l}\text { Virtual } \\
\text { Library }\end{array}$ & $\begin{array}{l}\text { Virtual } \\
\text { Library }\end{array}$ & & & & & & $Y$ & HWIS & \\
\hline 2305 & $200-W$ & 299-W22-27 & A7847 & D & GG, TP & $\begin{array}{l}\text { Virtual } \\
\text { Library }\end{array}$ & $\begin{array}{l}\begin{array}{l}\text { Virtual } \\
\text { Library }\end{array} \\
\end{array}$ & & & & & & $\mathrm{Y}$ & HWIS & \\
\hline 2306 & $200-W$ & 299-W22-27O & A9573 & D & & & & & & & & & $Y$ & HWIS & \\
\hline 2307 & $200-\mathrm{W}$ & 299-W22-27P & A9574 & $\mathrm{D}$ & & & & & & & & & Y & HWIS & \\
\hline 2308 & $200-W$ & 299-W22-27Q & A9575 & $\mathrm{D}$ & & & & & & & & & $Y$ & HWIS & \\
\hline 2309 & $200-W$ & 299-W22-27R & A9576 & D & & & & & & & & & $Y$ & HWIS & \\
\hline 2310 & $200-W$ & $299-W 22-27 S$ & A9577 & $\mathrm{D}$ & & & & & & & & & $Y$ & HWIS & \\
\hline 2311 & $200-w$ & $299-W 22-28$ & A4969 & $\mathrm{D}$ & GG, NT, SG, TP & \begin{tabular}{|l|} 
Virtual \\
Library
\end{tabular} & & & & & & & $Y$ & HWIS & \\
\hline 2312 & $200-W$ & 299-W22-29 & A7848 & D & $\mathrm{DN}, \mathrm{GG}, \mathrm{NT}$ & & & & & & & & & HWIS & \\
\hline 2313 & $200-W$ & 299-W22-3 & A7829 & D & & $\begin{array}{l}\text { Virtual } \\
\text { Library }\end{array}$ & $\begin{array}{l}\text { Virtual } \\
\text { Library }\end{array}$ & & & & & & $Y$ & HWIS & \\
\hline 2314 & $200-W$ & 299-W22-30 & A7849 & $\mathrm{D}$ & $\mathrm{DN}, \mathrm{GG}, \mathrm{NT}$ & & & & & & & & & HWIS & \\
\hline 2315 & $200-W$ & 299-W22-31 & A7850 & D & DN, GG, NT & & & & & & & & $Y$ & HWIS & \\
\hline 2316 & $200-W$ & 299-W22-32 & A7851 & $\mathrm{D}$ & DN, GG, NT, SG & & & & & & & & & & \\
\hline 2317 & $200-\mathrm{W}$ & 299-W22-33 & A7852 & D & DN, GG, NT, SG & & & & & & & & & HWIS & \\
\hline 2318 & $200-W$ & 299-W22-34 & A7853 & $\mathrm{D}$ & DN, GG, NT & & & & & & & & & HWIS & \\
\hline 2319 & $200-W$ & 299-W22-35 & A7854 & $\mathrm{D}$ & $\mathrm{DN}, \mathrm{GG}, \mathrm{NT}$ & & & & & & & & & HWIS & \\
\hline 2320 & $200-W$ & 299-W22-36 & A7855 & $\mathrm{D}$ & DN, GG, NT & & & & & & & & & HWIS & \\
\hline 2321 & $200-w$ & $299-W 22-37$ & A7856 & $\mathrm{D}$ & $\mathrm{DN}, \mathrm{GG}, \mathrm{NT}, \mathrm{SG}$ & \begin{tabular}{|l|} 
Virtual \\
Library
\end{tabular} & \begin{tabular}{|l|} 
Virtual \\
Library
\end{tabular} & & & & & & $Y$ & HWIS & \\
\hline 2322 & $200-W$ & 299-W22-38 & A7857 & D & $\mathrm{DN}, \mathrm{GG}, \mathrm{NT}, \mathrm{SG}$ & $\begin{array}{l}\text { Virtual } \\
\text { Library }\end{array}$ & \begin{tabular}{|l|} 
Virtual \\
Library
\end{tabular} & & & & & & $Y$ & HWIS & \\
\hline
\end{tabular}




\begin{tabular}{|c|c|c|c|c|c|c|c|c|c|c|c|c|c|c|c|}
\hline SortIndex & Area & Well Name & WellID & Log Type & GPX Log Runs & Sieved & $\mathrm{CaCO} 3$ & Moisture & $\begin{array}{l}\text { Chemical } \\
\text { Properties }\end{array}$ & $\begin{array}{c}\text { Physical } \\
\text { Properties }\end{array}$ & $\begin{array}{c}\text { Min } \\
\text { Properties }\end{array}$ & $\begin{array}{l}\text { Geochron } \\
\text { Properties }\end{array}$ & Archived & Source & Comments \\
\hline 2323 & $200-W$ & 299-W22-39 & A4970 & G & GG, SG & & $Y$ & $\mathrm{Y}$ & & & & & $\mathrm{Y}$ & Caggiano 1992 & \\
\hline 2324 & $200-W$ & 299-W22-4 & A7830 & D & GG, NT, SG, TP & & & & & & & & Y & HWIS & \\
\hline 2325 & $200-W$ & 299-W22-40 & A4971 & $D, G$ & GG & $\mathrm{Y}$ & $Y$ & $Y$ & & & & & Y & Goodwin 1990 & \\
\hline 2326 & $200-W$ & 299-W22-41 & A4972 & $D, G$ & GG & $\mathrm{Y}$ & $Y$ & $Y$ & & & & & Y & Goodwin 1990 & \\
\hline 2327 & $200-W$ & 299-W22-42 & A4973 & $D, G$ & GG & $\mathrm{Y}$ & $\mathrm{Y}$ & $Y$ & $Y$ & & & & Y & Goodwin 1990 & \\
\hline 2328 & 200-W & 299-W22-43 & A4974 & $D, G$ & GG & $\mathrm{Y}$ & $\mathrm{Y}$ & $\mathrm{Y}$ & $Y$ & & & & Y & Goodwin 1990 & \\
\hline 2329 & $200-W$ & 299-W22-44 & A4975 & G & GG, SG & $\mathrm{Y}$ & $\mathrm{Y}$ & $Y$ & \begin{tabular}{|l|} 
Field Rad \\
\end{tabular} & & & & Y & Caggiano 1993 & \\
\hline 2330 & $200-W$ & 299-W22-45 & A4976 & G & GG, SG & & $\mathrm{Y}$ & $\mathrm{Y}$ & & & & & $Y$ & Caggiano 1993 & \\
\hline 2331 & $200-W$ & 299-W22-46 & A4977 & G & GG, SG & & $\mathrm{Y}$ & $Y$ & & & & & $Y$ & Caggiano 1993 & \\
\hline 2332 & $200-W$ & 299-W22-48 & B8812 & G & NT, SG & $Y$ & $\mathrm{Y}$ & $\mathrm{Y}$ & $\begin{array}{l}\text { 1:1, Pwater, } \\
\text { Aex, TOC, } \\
\text { XRF }\end{array}$ & \begin{tabular}{|l} 
Cond, \\
$\%$ GSSC, \\
Bden, CEC
\end{tabular} & XRD, TEM & Paleomag & $Y$ & $\begin{array}{l}\text { Bjornstad et al. } \\
\text { 2001; Horton } \\
\text { and Johnson } \\
\text { 2000; Serne et } \\
\text { al. 2001a }\end{array}$ & \\
\hline 2333 & $200-W$ & 299-W22-49 & B8813 & G & $\mathrm{NT}, \mathrm{SG}$ & & & & & & & & $\mathrm{Y}$ & \begin{tabular}{|l|} 
Horton and \\
Johnson 2000
\end{tabular} & \\
\hline 2334 & $200-W$ & 299-W22-5 & A7831 & D & DN, GG, NT & \begin{tabular}{|l|l|} 
Virtual \\
Library
\end{tabular} & $\begin{array}{l}\text { Virtual } \\
\text { Library }\end{array}$ & & Field Rad & & & & $\mathrm{Y}$ & HWIS & \\
\hline 2335 & $200-W$ & 299-W22-50 & B8814 & G & & & & $\mathrm{Y}$ & $\begin{array}{l}\text { 1:1, Pwater, } \\
\text { Aex, TOC, } \\
\text { XRF }\end{array}$ & $\begin{array}{l}\text { Cond, } \\
\% \text { GSSC, } \\
\text { Bden, CEC }\end{array}$ & XRD & Paleomag & $\mathrm{Y}$ & $\begin{array}{l}\text { Bjornstad et al. } \\
\text { 2001; Horton } \\
\text { and Johnson } \\
\text { 2000, Serne et } \\
\text { al. 2001a }\end{array}$ & \\
\hline 2336 & $200-W$ & 299-W22-51 & A7858 & $D, G$ & & & & & & & & & $Y$ & HWIS & \\
\hline 2337 & $200-\mathrm{W}$ & 299-W22-52 & A7859 & $D, G$ & & & & & & & & & $Y$ & HWIS & \\
\hline 2338 & $200-W$ & 299-W22-53 & A7860 & $D, G$ & & & & & & & & & $\mathrm{Y}$ & HWIS & \\
\hline 2339 & $200-W$ & $299-W 22-54$ & A7861 & $D, G$ & & & & & & & & & $Y$ & HWIS & \\
\hline 2340 & $200-\mathrm{W}$ & 299-W22-55 & A7862 & $D, G$ & & & & & & & & & $\mathrm{Y}$ & HWIS & \\
\hline 2341 & $200-W$ & 299-W22-56 & A7863 & $D, G$ & & & & & & & & & & HWIS & \\
\hline 2342 & $200-\mathrm{W}$ & 299-W22-57 & A7864 & $D, G$ & & & & & & & & & $Y$ & HWIS & \\
\hline 2343 & $200-W$ & $299-W 22-58$ & A7865 & $D, G$ & & & & & & & & & $Y$ & HWIS & \\
\hline 2344 & $200-\mathrm{W}$ & 299-W22-59 & \begin{tabular}{|l|l|} 
A7866 \\
\end{tabular} & $D, G$ & & & & & & & & & $Y$ & HWIS & \\
\hline 2345 & $200-W$ & $299-W 22-6$ & A7832 & D & DN, GG, NT & \begin{tabular}{|l|} 
Virtual \\
Library
\end{tabular} & $\begin{array}{l}\text { Virtual } \\
\text { Library }\end{array}$ & & & & & & $Y$ & HWIS & \\
\hline 2346 & $200-W$ & 299-W22-60 & A7867 & $\mathrm{D}$ & GG & & & & & & & & $Y$ & HWIS & \\
\hline 2347 & $200-\mathrm{W}$ & 299-W22-61 & A7868 & $D, G$ & SG & & & & & & & & Y & HWIS & \\
\hline 2348 & $200-w$ & $299-W 22-62$ & A7869 & D & & & & & & & & & & HWIS & \\
\hline 2349 & $200-w$ & $299-W 22-63$ & A7870 & D & SG & & & & & & & & $Y$ & HWIS & \\
\hline 2350 & $200-W$ & $299-W 22-65$ & A7872 & D & & & & & & & & & $Y$ & HWIS & \\
\hline 2351 & $200-\mathrm{W}$ & 299-W22-66 & A7873 & D & & & & & & & & & $\mathrm{Y}$ & HWIS & \\
\hline 2352 & $200-W$ & 299-W22-67 & A7874 & D & DN, GG, NT & $\begin{array}{l}\text { Virtual } \\
\text { Library }\end{array}$ & $\begin{array}{l}\text { Virtual } \\
\text { Library }\end{array}$ & & & & & & $\mathrm{Y}$ & HWIS & \\
\hline 2353 & $200-W$ & 299-W22-7 & A4978 & D & GG, NT, TP & $\begin{array}{l}\text { Virtual } \\
\text { Library }\end{array}$ & $\begin{array}{l}\text { Virtual } \\
\text { Library }\end{array}$ & & & & & & $Y$ & HWIS & \\
\hline 2354 & $200-W$ & 299-W22-70 & A7876 & D & & & & & & & & & & HWIS & \\
\hline 2355 & $200-W$ & $299-W 22-73$ & A7877 & $D, G$ & GG, NT & \begin{tabular}{|l|l} 
Virtual \\
Library
\end{tabular} & \begin{tabular}{|l} 
Virtual \\
Library
\end{tabular} & & $\begin{array}{l}\begin{array}{l}\text { Field Rad in } \\
\text { logs }\end{array} \\
\end{array}$ & & & & $Y$ & PNNL files & \\
\hline
\end{tabular}




\begin{tabular}{|c|c|c|c|c|c|c|c|c|c|c|c|c|c|c|c|}
\hline Sortindex & Area & Well Name & WellID & Log Type & GPX Log Runs & Sieved & $\mathrm{CaCO} 3$ & Moisture & $\begin{array}{l}\text { Chemical } \\
\text { Properties }\end{array}$ & $\begin{array}{c}\text { Physical } \\
\text { Properties }\end{array}$ & $\begin{array}{c}\text { Min } \\
\text { Properties }\end{array}$ & $\begin{array}{l}\text { Geochron } \\
\text { Properties }\end{array}$ & Archived & Source & Comments \\
\hline 2356 & $200-W$ & 299-W22-74 & A7878 & D & GG, SG & & & & & & & & $Y$ & HWIS & \\
\hline 2357 & $200-\mathrm{W}$ & 299-W22-75 & A7879 & $D, G$ & GG, NT, SG & $\begin{array}{l}\text { Virtual } \\
\text { Library }\end{array}$ & $\begin{array}{l}\text { Virtual } \\
\text { Library }\end{array}$ & & $\begin{array}{l}\text { Field Rad in } \\
\text { logs }\end{array}$ & & & & $\mathrm{Y}$ & PNNL files & \\
\hline 2358 & $200-W$ & 299-W22-76 & A7880 & $D, G$ & & & & & Field Rad & & & & $Y$ & HWIS & \\
\hline 2359 & $200-W$ & 299-W22-77 & A7881 & $D, G$ & & & & & Field Rad & & & & $Y$ & HWIS & \\
\hline 2360 & $200-W$ & 299-W22-78 & A7882 & D, G, Well summary & SG & & & & & & & & & HWIS & \\
\hline 2361 & $200-W$ & 299-W22-79 & B8552 & G & & & & & & & & & $Y$ & & \\
\hline 2362 & $200-W$ & 299-W22-8 & A7833 & D & GG, NT, SG, TP & & & & & & & & $\mathrm{Y}$ & HWIS & \\
\hline 2363 & $200-W$ & 299-W22-80 & C3115 & G & & Y & & & & & & & $\mathrm{Y}$ & \begin{tabular}{|l|} 
Horton and \\
Johnson 2001
\end{tabular} & \\
\hline 2364 & 200-W & 299-W22-81 & C3123 & G & NT, SG & Y & & & & & & & Y & $\begin{array}{l}\text { Horton and } \\
\text { Johnson } 2001\end{array}$ & \\
\hline 2365 & $200-W$ & 299-W22-82 & C3124 & G & NT, SG & Y & & & & & & & Y & $\begin{array}{l}\text { Horton and } \\
\text { Johnson } 2001\end{array}$ & \\
\hline 2366 & $200-W$ & 299-W22-83 & C3126 & G & NT, SG & $Y$ & & & & & & & Y & \begin{tabular}{|l|} 
Horton and \\
Johnson 2001
\end{tabular} & \\
\hline 2367 & $200-W$ & 299-W22-84 & C3398 & G & & $\mathrm{Y}$ & & & & & & & $\mathrm{Y}$ & Horton 2002e & \\
\hline 2368 & $200-W$ & 299-W22-85 & C3399 & G & NT, SG & $\mathrm{Y}$ & & & & & & & $\mathrm{Y}$ & Horton $2002 \mathrm{e}$ & \\
\hline 2369 & $200-W$ & 299-W22-9 & A7834 & D & GG, NT, SG & \begin{tabular}{|l} 
Virtual \\
Library
\end{tabular} & \begin{tabular}{|l}
$\begin{array}{l}\text { Virtual } \\
\text { Library }\end{array}$ \\
\end{tabular} & & & & & & $Y$ & HWIS & \\
\hline 2370 & $200-W$ & 299-W23-1 & A4979 & $D, G$ & GG & $\begin{array}{l}\text { Virtual } \\
\text { Library }\end{array}$ & $\begin{array}{l}\text { Virtual } \\
\text { Library }\end{array}$ & & $\mathrm{pH}$ & $\begin{array}{l}\text { CEC, } \\
\% \text { GSSC, 15- } \\
\text { Atm }\end{array}$ & & & $\mathrm{Y}$ & McHenry 1957 & \\
\hline 2371 & $1200-W$ & 299-W23-10 & A7884 & D & DN, GG, NT, TP & \begin{tabular}{|l|} 
Virtual \\
Library
\end{tabular} & $\begin{array}{l}\text { Virtual } \\
\text { Library }\end{array}$ & & & & & & $\mathrm{Y}$ & HWIS & \\
\hline 2372 & $200-W$ & 299-W23-100 & A7936 & D & GG & $\begin{array}{l}\text { Virtual } \\
\text { Library }\end{array}$ & $\begin{array}{l}\text { Virtual } \\
\text { Library }\end{array}$ & & & & & & Y & HWIS & \\
\hline 2373 & $200-W$ & 299-W23-101 & A7937 & D & GG & & & & & & & & & HWIS & \\
\hline 2374 & $200-W$ & 299-W23-102 & A7938 & D & GG & & & & & & & & 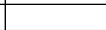 & HWIS & \\
\hline 2375 & $200-W$ & 299-W23-103 & A7939 & D & GG & & & & & & & & & HWIS & \\
\hline 2376 & $200-W$ & 299-W23-104 & A7940 & D & GG & & & & & & & & & HWIS & \\
\hline 2377 & $200-W$ & 299-W23-105 & A7941 & $\mathrm{D}$ & GG & \begin{tabular}{|l|l|} 
Virtual \\
Library
\end{tabular} & \begin{tabular}{|l|} 
Virtual \\
Library
\end{tabular} & & & & & & $\mathrm{Y}$ & HWIS & \\
\hline 2378 & $200-W$ & 299-W23-106 & A7942 & D & GG & $\begin{array}{l}\text { Virtual } \\
\text { Library }\end{array}$ & $\begin{array}{l}\text { Virtual } \\
\text { Library }\end{array}$ & & & & & & Y & HWIS & \\
\hline 2379 & $200-W$ & 299-W23-107 & A7943 & D & GG & \begin{tabular}{|l|} 
Virtual \\
Library
\end{tabular} & $\begin{array}{l}\text { Virtual } \\
\text { Library }\end{array}$ & & & & & & $\mathrm{Y}$ & HWIS & \\
\hline 2380 & $200-W$ & 299-W23-108 & A7944 & D & GG & $\begin{array}{l}\text { Virtual } \\
\text { Library }\end{array}$ & $\begin{array}{l}\text { Virtual } \\
\text { Library }\end{array}$ & & & & EM, XRD & & $\mathrm{Y}$ & $\begin{array}{l}\text { Ames 1976; } \\
\text { Tallman et al. } \\
1979\end{array}$ & \\
\hline 2381 & $200-W$ & 299-W23-109 & A7945 & D & GG & \begin{tabular}{|l|l|} 
Virtual \\
Library
\end{tabular} & $\begin{array}{l}\text { Virtual } \\
\text { Library }\end{array}$ & & & & & & $\mathrm{Y}$ & & \\
\hline 2382 & $200-W$ & 299-W23-11 & A4980 & D & DN, GG, NT, TP & $\begin{array}{l}\text { Virtual } \\
\text { Library }\end{array}$ & $\begin{array}{l}\text { Virtual } \\
\text { Library }\end{array}$ & & & & & & Y & HWIS & \\
\hline 2383 & $200-W$ & 299-W23-110 & A7946 & D & GG & $\begin{array}{l}\text { Virtual } \\
\text { Library }\end{array}$ & $\begin{array}{l}\text { Virtual } \\
\text { Library }\end{array}$ & & & & & & & HWIS & \\
\hline 2384 & 200-W & 299-W23-111 & A7947 & D & GG & $\begin{array}{l}\text { Virtual } \\
\text { Library }\end{array}$ & $\begin{array}{l}\text { Virtual } \\
\text { Library }\end{array}$ & & & & & & Y & HWIS & \\
\hline 2385 & $200-W$ & 299-W23-112 & A7948 & D & GG & \begin{tabular}{|l|} 
Virtual \\
Library
\end{tabular} & $\begin{array}{l}\text { Virtual } \\
\text { Library }\end{array}$ & & & & & & $\mathrm{Y}$ & HWIS & \\
\hline 2386 & $200-W$ & 299-W23-113 & A7949 & D & GG & $\begin{array}{l}\text { Virtual } \\
\text { Library }\end{array}$ & $\begin{array}{l}\text { Virtual } \\
\text { Library }\end{array}$ & & & & & & $Y$ & HWIS & \\
\hline
\end{tabular}




\begin{tabular}{|c|c|c|c|c|c|c|c|c|c|c|c|c|c|c|c|}
\hline SortIndex & Area & Well Name & WellID & Log Type & GPX Log Runs & Sieved & $\mathrm{CaCO} 3$ & Moisture & $\begin{array}{l}\text { Chemical } \\
\text { Properties }\end{array}$ & $\begin{array}{c}\text { Physical } \\
\text { Properties }\end{array}$ & \begin{tabular}{|c|} 
Min \\
Properties
\end{tabular} & $\begin{array}{l}\text { Geochron } \\
\text { Properties }\end{array}$ & Archived & Source & Comments \\
\hline 2387 & $200-w$ & 299-W23-114 & A7950 & D & GG & \begin{tabular}{|l|} 
Virtual \\
Library
\end{tabular} & $\begin{array}{l}\text { Virtual } \\
\text { Library }\end{array}$ & & & & & & $Y$ & HWIS & \\
\hline 2388 & 200-W & 299-W23-115 & A7951 & D & GG & & & & & & & & & HWIS & \\
\hline 2389 & 200-W & 299-W23-116 & A7952 & D & GG & \begin{tabular}{|l} 
Virtual \\
Library
\end{tabular} & \begin{tabular}{|l|} 
Virtual \\
Library
\end{tabular} & & & & & & $Y$ & HWIS & \\
\hline 2390 & 200-W & 299-W23-117 & A7953 & D & $G G$ & \begin{tabular}{|l} 
Virtual \\
Library
\end{tabular} & $\begin{array}{l}\text { Virtual } \\
\text { Library }\end{array}$ & & & & & & $Y$ & HWIS & \\
\hline 2391 & $1200-W$ & 299-W23-118 & A7954 & D & GG & & & & & & & & & HWIS & \\
\hline 2392 & $200-W$ & 299-W23-119 & A7955 & D & GG & \begin{tabular}{|l} 
Virtual \\
Library
\end{tabular} & $\begin{array}{l}\text { Virtual } \\
\text { Library }\end{array}$ & & & & & & $Y$ & HWIS & \\
\hline 2393 & $200-W$ & 299-W23-12 & A4981 & $\mathrm{D}$ & & & & & & & & & & HWIS & \\
\hline 2394 & $4200-w$ & $299-W 23-120$ & A7956 & D & GG & \begin{tabular}{|l|l|} 
Virtual \\
Library
\end{tabular} & $\begin{array}{l}\begin{array}{l}\text { Virtual } \\
\text { Library }\end{array} \\
\end{array}$ & & & & & & $Y$ & HWIS & \\
\hline 2395 & $5200-W$ & 299-W23-121 & A7957 & D & GG & \begin{tabular}{|l|} 
Virtual \\
Library
\end{tabular} & \begin{tabular}{|l|}
$\begin{array}{l}\text { Virtual } \\
\text { Library }\end{array}$ \\
\end{tabular} & & & & & & $Y$ & HWIS & \\
\hline 2396 & $200-W$ & 299-W23-122 & A7958 & D & & & & & & & & & & HWIS & \\
\hline 2397 & $7200-W$ & 299-W23-123 & A7959 & D & & & & & & & & & & HWIS & \\
\hline 2398 & $200-w$ & 299-W23-124 & A7960 & D & & \begin{tabular}{|l} 
Virtual \\
Library
\end{tabular} & \begin{tabular}{|l} 
Virtual \\
Library
\end{tabular} & & & & & & $Y$ & HWIS & \\
\hline 2399 & $200-W$ & 299-W23-125 & A7961 & D & & $\begin{array}{l}\text { Virtual } \\
\text { Library }\end{array}$ & $\begin{array}{l}\text { Virtual } \\
\text { Library }\end{array}$ & & & & & & & HWIS & \\
\hline 2400 & $200-W$ & 299-W23-126 & A7962 & D & & & & & & & & & Y & HWIS & \\
\hline 2401 & $1200-W$ & 299-W23-127 & A7963 & $\mathrm{D}$ & & & & & & & & & Y & HWIS & \\
\hline 2402 & $200-\mathrm{W}$ & 299-W23-128 & A7964 & D & & & & & & & & & $Y$ & HWIS & \\
\hline 2403 & $3200-W$ & 299-W23-129 & A7965 & D & & & & & & & & & & HWIS & \\
\hline 2404 & $4200-\mathrm{W}$ & 299-W23-13 & A4982 & G & GG & Y & $Y$ & $Y$ & & & & & $Y$ & Caggiano 1992 & \\
\hline 2405 & $200-w$ & 299-W23-130 & A7966 & $\mathrm{D}$ & & & & & & & & & $\mathrm{Y}$ & HWIS & \\
\hline 2406 & $5200-W$ & 299-W23-131 & A7967 & D & & & & & & & & & $\mathrm{Y}$ & HWIS & \\
\hline 2407 & $200-W$ & 299-W23-132 & A7968 & D & & \begin{tabular}{|l} 
Virtual \\
Library
\end{tabular} & $\begin{array}{l}\text { Virtual } \\
\text { Library }\end{array}$ & & Field Rad & & & & $Y$ & HWIS & \\
\hline 2408 & 200-W & 299-W23-133 & A7969 & D & & $\begin{array}{l}\text { Virtual } \\
\text { Library }\end{array}$ & $\begin{array}{l}\text { Virtual } \\
\text { Library }\end{array}$ & & Field Rad & & & & $\mathrm{Y}$ & HWIS & \\
\hline 2409 & 200-W & 299-W23-134 & A7970 & D & & $\begin{array}{l}\text { Virtual } \\
\text { Library }\end{array}$ & $\begin{array}{l}\text { Virtual } \\
\text { Library }\end{array}$ & & & & & & $Y$ & HWIS & \\
\hline 2410 & 200-W & 299-W23-135 & A7971 & D & & $\begin{array}{l}\text { Virtual } \\
\text { Library }\end{array}$ & $\begin{array}{l}\text { Virtual } \\
\text { Library }\end{array}$ & & Field Rad & & & & $\mathrm{Y}$ & HWIS & \\
\hline 2411 & $1200-W$ & 299-W23-136 & A7972 & D & & \begin{tabular}{|l} 
Virtual \\
Library
\end{tabular} & $\begin{array}{l}\text { Virtual } \\
\text { Library }\end{array}$ & & & & & & & HWIS & \\
\hline 2412 & $200-W$ & 299-W23-137 & A7973 & D & & $\begin{array}{l}\text { Virtual } \\
\text { Library }\end{array}$ & $\begin{array}{l}\text { Virtual } \\
\text { Library }\end{array}$ & & & & & & Y & HWIS & \\
\hline 2413 & $3200-W$ & 299-W23-138 & A7974 & D & & $\begin{array}{l}\text { Virtual } \\
\text { Library }\end{array}$ & $\begin{array}{l}\text { Virtual } \\
\text { Library }\end{array}$ & & & & & & $Y$ & HWIS & \\
\hline 2414 & $4200-W$ & 299-W23-139 & A7975 & D & & $\begin{array}{l}\text { Virtual } \\
\text { Library }\end{array}$ & $\begin{array}{l}\text { Virtual } \\
\text { Library }\end{array}$ & & & & & & Y & HWIS & \\
\hline 2415 & $5200-W$ & 299-W23-14 & A4983 & G & GG, SG & $\mathrm{Y}$ & $Y$ & Y & & & & & $Y$ & Caggiano 1992 & \\
\hline 2416 & 200-W & 299-W23-140 & A7976 & D & & $\begin{array}{l}\text { Virtual } \\
\text { Library }\end{array}$ & $\begin{array}{l}\text { Virtual } \\
\text { Library }\end{array}$ & & & & & & Y & HWIS & \\
\hline 2417 & 200-W & 299-W23-141 & A7977 & D & & $\begin{array}{l}\text { Virtual } \\
\text { Library }\end{array}$ & $\begin{array}{l}\text { Virtual } \\
\text { Library }\end{array}$ & & & & & & & HWIS & \\
\hline 2418 & 200-W & 299-W23-142 & A7978 & D & & $\begin{array}{l}\text { Virtual } \\
\text { Library }\end{array}$ & $\begin{array}{l}\text { Virtual } \\
\text { Library }\end{array}$ & & & & & & Y & HWIS & \\
\hline 2419 & $200-W$ & 299-W23-143 & A7979 & D & & \begin{tabular}{|l} 
Virtual \\
Library
\end{tabular} & $\begin{array}{l}\text { Virtual } \\
\text { Library }\end{array}$ & & & & & & $Y$ & HWIS & \\
\hline 2420 & 200-W & 299-W23-144 & A7980 & D & & $\begin{array}{l}\text { Virtual } \\
\text { Library }\end{array}$ & $\begin{array}{l}\text { Virtual } \\
\text { Library }\end{array}$ & & & & & & Y & HWIS & \\
\hline
\end{tabular}




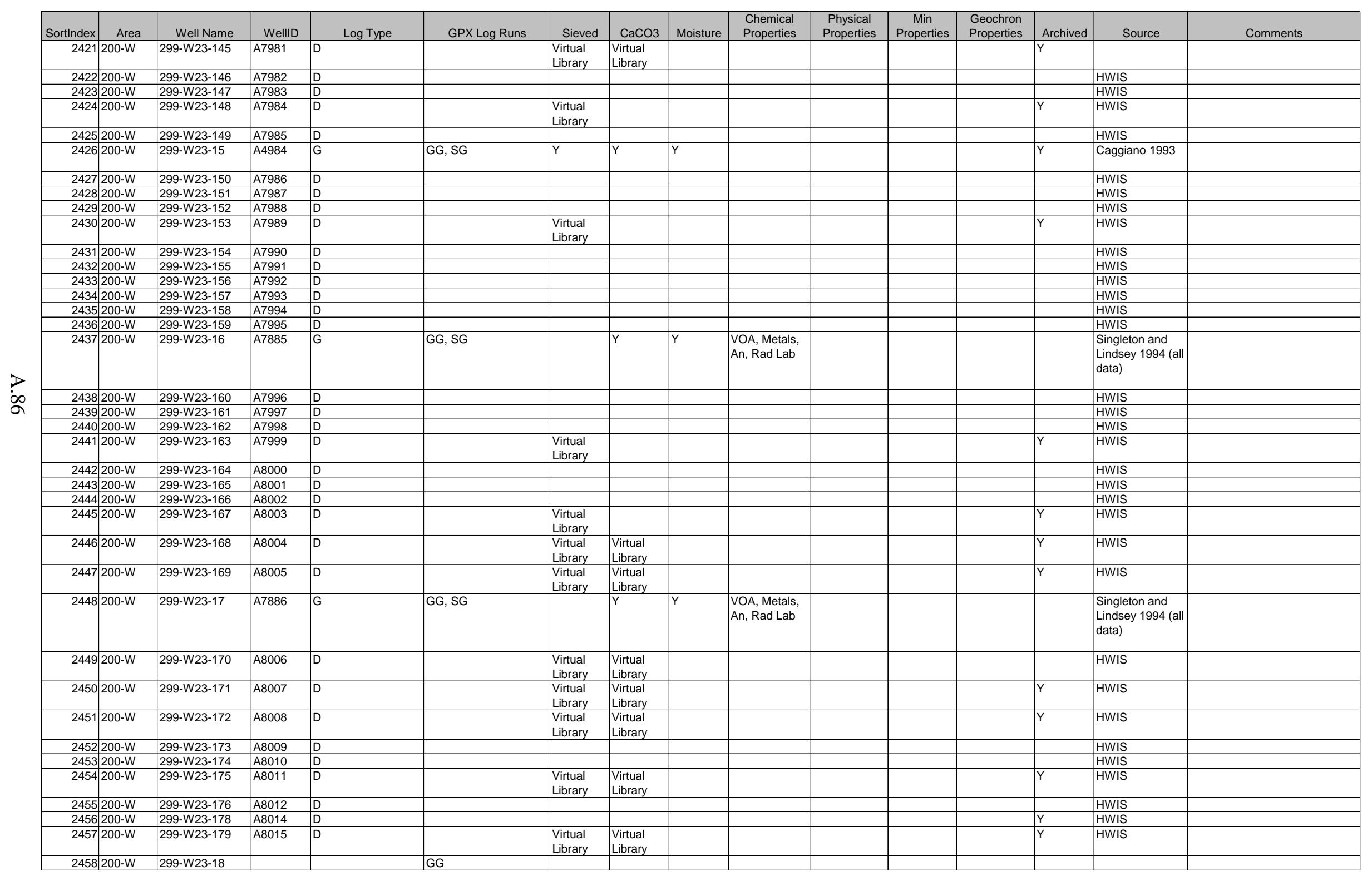




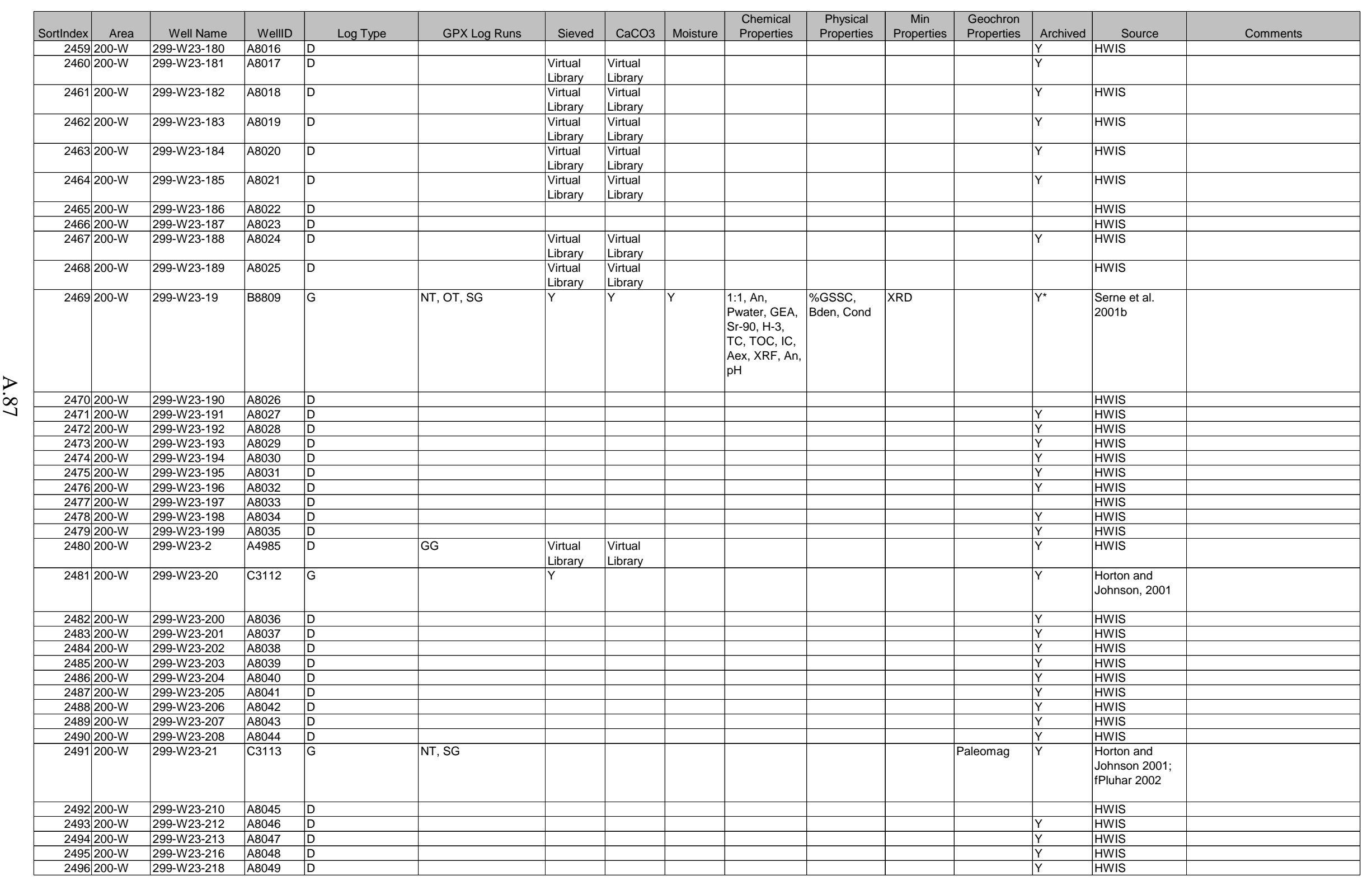




\begin{tabular}{|c|c|c|c|c|c|c|c|c|c|c|c|c|c|c|c|}
\hline Sortlndex & Area & Well Name & WellID & Log Type & GPX Log Runs & Sieved & $\mathrm{CaCO} 3$ & Moisture & $\begin{array}{l}\text { Chemical } \\
\text { Properties }\end{array}$ & $\begin{array}{c}\text { Physical } \\
\text { Properties }\end{array}$ & \begin{tabular}{|c|} 
Min \\
Properties \\
\end{tabular} & $\begin{array}{l}\text { Geochron } \\
\text { Properties }\end{array}$ & Archived & Source & Comments \\
\hline 2497 & $200-W$ & 299-W23-220 & A8050 & D & & & & & & & & & & HWIS & \\
\hline 2498 & $200-W$ & 299-W23-223 & A8051 & D & & & & & & & & & $Y$ & HWIS & \\
\hline 2499 & $200-W$ & 299-W23-225 & A8052 & D & & & & & & & & & $Y$ & HWIS & \\
\hline 2500 & $200-W$ & 299-W23-226 & A8053 & D & & & & & & & & & $Y$ & HWIS & \\
\hline 2501 & $200-W$ & 299-W23-227 & A8054 & $\mathrm{D}$ & & & & & & & & & $Y$ & & \\
\hline 2502 & $200-W$ & 299-W23-228 & A9874 & G & & & & $Y$ & $\mathrm{Pu}, \mathrm{Cs}, \mathrm{Sr}$ & & & & & Last et al. 1994 & \\
\hline 2503 & $200-W$ & 299-W23-229 & A8055 & $\mathrm{D}$ & & & & & & & & & & HWIS & \\
\hline 2504 & $200-W$ & 299-W23-231 & A9518 & D, G, Well summary & SG & & & & & & & & & HWIS & \\
\hline 2505 & $200-W$ & 299-W23-234 & \begin{tabular}{|l|} 
B2828 \\
\end{tabular} & G & & $Y$ & Y & $\mathrm{Y}$ & $\begin{array}{l}\text { 1:1, metals, } \\
\text { An, pH, } \\
\text { Pwater, GEA, } \\
\text { TC-99, Sr-90, } \\
\text { TC, IC, Aex, } \\
\text { XRF }\end{array}$ & $\begin{array}{l}\text { \%GSSC, } \\
\text { CEC, Cond }\end{array}$ & TEM, XRD & & $\mathrm{Y}^{*}$ & $\begin{array}{l}\text { Serne et al. } \\
2001 c\end{array}$ & \\
\hline 2506 & $200-W$ & 299-W23-3 & A4986 & D & GG & $\begin{array}{l}\text { Virtual } \\
\text { Library }\end{array}$ & $\begin{array}{l}\text { Virtual } \\
\text { Library }\end{array}$ & & & & & & Y & HWIS & \\
\hline 2507 & $200-W$ & 299-W23-4 & A4987 & D & $\begin{array}{l}\text { DN, GG, MO, NT, SG, } \\
\text { TP }\end{array}$ & $\begin{array}{l}\text { Virtual } \\
\text { Library }\end{array}$ & $\begin{array}{l}\text { Virtual } \\
\text { Library }\end{array}$ & & & & & & $\mathrm{Y}$ & HWIS & \\
\hline 2508 & $200-W$ & 299-W23-5 & A4988 & D & GG & $\begin{array}{l}\text { Virtual } \\
\text { Library }\end{array}$ & $\begin{array}{l}\text { Virtual } \\
\text { Library }\end{array}$ & & & & & & $\mathrm{Y}$ & HWIS & \\
\hline 2509 & $200-W$ & 299-W23-51 & A7887 & D, G & GG & & $\mathrm{Y}$ & & & & & & $Y$ & HWIS & \\
\hline 2510 & $200-W$ & 299-W23-52 & A7888 & D, G & GG & $\begin{array}{l}\text { Virtual } \\
\text { Library }\end{array}$ & $\begin{array}{l}\text { Virtual } \\
\text { Library }\end{array}$ & & & & XRD, EM & & $\mathrm{Y}$ & \begin{tabular}{|l} 
Ames 1976; \\
Tallman et al. \\
1979
\end{tabular} & \\
\hline 2511 & $200-W$ & 299-W23-53 & A7889 & D, G & GG & \begin{tabular}{|l|} 
Virtual \\
Library
\end{tabular} & $\begin{array}{l}\text { Virtual } \\
\text { Library }\end{array}$ & & & & & & $\mathrm{Y}$ & HWIS & \\
\hline 2512 & $200-W$ & 299-W23-54 & A7890 & D, G & GG & $\begin{array}{l}\text { Virtual } \\
\text { Library }\end{array}$ & $\begin{array}{l}\text { Virtual } \\
\text { Library }\end{array}$ & & & & & & $\mathrm{Y}$ & HWIS & \\
\hline 2513 & $200-W$ & 299-W23-55 & A7891 & D, G & GG & \begin{tabular}{|l|} 
Virtual \\
Library
\end{tabular} & $\begin{array}{l}\text { Virtual } \\
\text { Library }\end{array}$ & & & & & & $\mathrm{Y}$ & HWIS & \\
\hline 2514 & $200-W$ & 299-W23-56 & A7892 & D & GG & \begin{tabular}{|l|} 
Virtual \\
Library
\end{tabular} & \begin{tabular}{|l|} 
Virtual \\
Library
\end{tabular} & & & & & & $\mathrm{Y}$ & HWIS & \\
\hline 2515 & $200-W$ & 299-W23-57 & A7893 & D & GG & $\begin{array}{l}\text { Virtual } \\
\text { Library }\end{array}$ & \begin{tabular}{|l} 
Virtual \\
Library
\end{tabular} & & & & & & $\mathrm{Y}$ & HWIS & \\
\hline 2516 & $200-W$ & 299-W23-58 & A7894 & D & GG & $\begin{array}{l}\text { Virtual } \\
\text { Library }\end{array}$ & $\begin{array}{l}\text { Virtual } \\
\text { Library }\end{array}$ & & & & & & $\mathrm{Y}$ & HWIS & \\
\hline 2517 & $200-W$ & 299-W23-59 & A7895 & $\mathrm{D}$ & GG & & & & & & & & & HWIS & \\
\hline 2518 & $200-W$ & 299-W23-6 & A4989 & D & GG, TP & $\begin{array}{l}\text { Virtual } \\
\text { Library }\end{array}$ & $\begin{array}{l}\text { Virtual } \\
\text { Library }\end{array}$ & & & & & & $\mathrm{Y}$ & HWIS & \\
\hline 2519 & $200-W$ & 299-W23-60 & A7896 & D & GG & & & & & & & & & HWIS & \\
\hline 2520 & $200-W$ & 299-W23-61 & A7897 & D & GG & & & & & & & & & HWIS & \\
\hline 2521 & $200-W$ & 299-W23-62 & A7898 & D & GG & $\begin{array}{l}\text { Virtual } \\
\text { Library }\end{array}$ & $\begin{array}{l}\text { Virtual } \\
\text { Library }\end{array}$ & & & & & & $\mathrm{Y}$ & HWIS & \\
\hline 2522 & $200-W$ & 299-W23-64 & A7900 & D & GG & \begin{tabular}{|l|} 
Virtual \\
Library
\end{tabular} & $\begin{array}{l}\text { Virtual } \\
\text { Library }\end{array}$ & & & & & & $\mathrm{Y}$ & HWIS & \\
\hline 2523 & $200-W$ & 299-W23-65 & \begin{tabular}{|l|} 
A7901 \\
\end{tabular} & D & GG & $\begin{array}{l}\text { Virtual } \\
\text { Library }\end{array}$ & $\begin{array}{l}\text { Virtual } \\
\text { Library }\end{array}$ & & & & & & $\mathrm{Y}$ & HWIS & \\
\hline 2524 & $200-W$ & 299-W23-66 & A7902 & D & GG & \begin{tabular}{|l|} 
Virtual \\
Library
\end{tabular} & $\begin{array}{l}\text { Virtual } \\
\text { Library }\end{array}$ & & & & & & $\mathrm{Y}$ & HWIS & \\
\hline 2525 & $200-W$ & 299-W23-67 & A7903 & D & GG & \begin{tabular}{|l|} 
Virtual \\
Library
\end{tabular} & $\begin{array}{l}\text { Virtual } \\
\text { Library }\end{array}$ & & & & & & Y & HWIS & \\
\hline 2526 & $200-W$ & 299-W23-68 & A7904 & D & GG & $\begin{array}{l}\text { Virtual } \\
\text { Library }\end{array}$ & $\begin{array}{l}\text { Virtual } \\
\text { Library }\end{array}$ & & & & & & $\mathrm{Y}$ & HWIS & \\
\hline
\end{tabular}




\begin{tabular}{|c|c|c|c|c|c|c|c|c|c|c|c|c|c|c|c|}
\hline SortIndex & Area & Well Name & WellID & Log Type & GPX Log Runs & Sieved & $\mathrm{CaCO} 3$ & Moisture & $\begin{array}{c}\text { Chemical } \\
\text { Properties }\end{array}$ & $\begin{array}{c}\text { Physical } \\
\text { Properties }\end{array}$ & \begin{tabular}{|c|} 
Min \\
Properties \\
\end{tabular} & $\begin{array}{l}\text { Geochron } \\
\text { Properties }\end{array}$ & Archived & Source & Comments \\
\hline \begin{tabular}{|l|}
2527 \\
\end{tabular} & $200-W$ & 299-W23-69 & A7905 & D & GG & \begin{tabular}{|l|} 
Virtual \\
Library
\end{tabular} & $\begin{array}{l}\text { Virtual } \\
\text { Library }\end{array}$ & & & & & & $\mathrm{Y}$ & HWIS & \\
\hline 2528 & $200-W$ & 299-W23-7 & A4990 & D & GG & $\begin{array}{l}\text { Virtual } \\
\text { Library }\end{array}$ & $\begin{array}{l}\text { Virtual } \\
\text { Library }\end{array}$ & & & & & & $\mathrm{Y}$ & HWIS & \\
\hline 2529 & $200-W$ & 299-W23-70 & A7906 & D & GG & $\begin{array}{l}\text { Virtual } \\
\text { Library }\end{array}$ & $\begin{array}{l}\text { Virtual } \\
\text { Library }\end{array}$ & & & & & & $\mathrm{Y}$ & HWIS & \\
\hline 2530 & $200-W$ & 299-W23-71 & A7907 & D & & & & & & & & & & HWIS & \\
\hline 2531 & $200-W$ & 299-W23-72 & A7908 & D & GG & \begin{tabular}{|l} 
Virtual \\
Library
\end{tabular} & \begin{tabular}{|l|} 
Virtual \\
Library
\end{tabular} & & & & XRD, EM & & $\mathrm{Y}$ & $\begin{array}{l}\text { Ames 1976; } \\
\text { Tallman et al. } \\
1979\end{array}$ & \\
\hline 2532 & $200-W$ & 299-W23-73 & A7909 & D & GG & \begin{tabular}{|l|} 
Virtual \\
Library
\end{tabular} & $\begin{array}{l}\text { Virtual } \\
\text { Library }\end{array}$ & & & & & & $Y$ & & \\
\hline 2533 & $200-W$ & 299-W23-74 & A7910 & D & GG & \begin{tabular}{|l|} 
Virtual \\
Library
\end{tabular} & $\begin{array}{l}\text { Virtual } \\
\text { Library }\end{array}$ & & & & & & $Y$ & HWIS & \\
\hline 2534 & $200-W$ & 299-W23-75 & A7911 & D & GG & \begin{tabular}{|l|} 
Virtual \\
Library
\end{tabular} & $\begin{array}{l}\text { Virtual } \\
\text { Library }\end{array}$ & & & & & & $\mathrm{Y}$ & HWIS & \\
\hline 2535 & $200-W$ & 299-W23-76 & A7912 & D & GG & \begin{tabular}{|l|} 
Virtual \\
Library
\end{tabular} & $\begin{array}{l}\text { Virtual } \\
\text { Library }\end{array}$ & & & & & & $Y$ & HWIS & \\
\hline 2536 & $200-W$ & 299-W23-77 & A7913 & D & GG & \begin{tabular}{|l} 
Virtual \\
Library
\end{tabular} & $\begin{array}{l}\text { Virtual } \\
\text { Library }\end{array}$ & & & & & & $\mathrm{Y}$ & HWIS & \\
\hline 2537 & $200-W$ & 299-W23-78 & A7914 & D & GG & $\begin{array}{l}\text { Virtual } \\
\text { Library }\end{array}$ & $\begin{array}{l}\text { Virtual } \\
\text { Library }\end{array}$ & & & & & & $\mathrm{Y}$ & HWIS & \\
\hline 2538 & $200-W$ & 299-W23-79 & A7915 & D & GG & \begin{tabular}{|l|} 
Virtual \\
Library
\end{tabular} & \begin{tabular}{|l|} 
Virtual \\
Library
\end{tabular} & & & & & & $Y$ & HWIS & \\
\hline 2539 & $200-W$ & 299-W23-8 & A4991 & D & DN, GG, NT, SG & \begin{tabular}{|l} 
Virtual \\
Library
\end{tabular} & $\begin{array}{l}\text { Virtual } \\
\text { Library }\end{array}$ & & & & & & $Y$ & HWIS & \\
\hline 2540 & $200-W$ & 299-W23-80 & A7916 & D & GG & \begin{tabular}{|l} 
Virtual \\
Library
\end{tabular} & \begin{tabular}{|l}
$\begin{array}{l}\text { Virtual } \\
\text { Library }\end{array}$ \\
\end{tabular} & & & & & & $Y$ & HWIS & \\
\hline 2541 & $200-W$ & 299-W23-81 & A7917 & D & GG & & & & & & & & & HWIS & \\
\hline 2542 & $200-W$ & 299-W23-82 & A7918 & D & GG & \begin{tabular}{|l} 
Virtual \\
Library
\end{tabular} & $\begin{array}{l}\begin{array}{l}\text { Virtual } \\
\text { Library }\end{array} \\
\end{array}$ & & & & & & $\mathrm{Y}$ & HWIS & \\
\hline 2543 & $200-W$ & 299-W23-83 & A7919 & D & GG & \begin{tabular}{|l|} 
Virtual \\
Library
\end{tabular} & $\begin{array}{l}\text { Virtual } \\
\text { Library }\end{array}$ & & & & & & $Y$ & HWIS & \\
\hline 2544 & $200-W$ & 299-W23-84 & A7920 & D & GG & \begin{tabular}{|l|} 
Virtual \\
Library
\end{tabular} & $\begin{array}{l}\text { Virtual } \\
\text { Library }\end{array}$ & & & & & & $Y$ & HWIS & \\
\hline 2545 & $200-W$ & 299-W23-85 & A7921 & D & GG & \begin{tabular}{|l|} 
Virtual \\
Library
\end{tabular} & $\begin{array}{l}\text { Virtual } \\
\text { Library }\end{array}$ & & & & & & $Y$ & HWIS & \\
\hline 2546 & $200-W$ & 299-W23-86 & A7922 & D & GG & \begin{tabular}{|l} 
Virtual \\
Library
\end{tabular} & $\begin{array}{l}\text { Virtual } \\
\text { Library }\end{array}$ & & & & & & $Y$ & HWIS & \\
\hline 2547 & $200-W$ & 299-W23-87 & A7923 & $\mathrm{D}$ & GG & & & & & & & & & HWIS & \\
\hline 2548 & $200-W$ & $299-W 23-88$ & A7924 & $\mathrm{D}$ & GG & & & & & & & & & HWIS & \\
\hline 2549 & $200-W$ & 299-W23-89 & A7925 & D & GG & & & & & & & & & HWIS & \\
\hline 2550 & $200-w$ & 299-W23-9 & A7883 & $\mathrm{D}$ & $\mathrm{DN}, \mathrm{GG}, \mathrm{NT}, \mathrm{TP}$ & \begin{tabular}{|l|} 
Virtual \\
Library
\end{tabular} & \begin{tabular}{|l|} 
Virtual \\
Library
\end{tabular} & & & & & & Y & HWIS & \\
\hline 2551 & $200-W$ & 299-W23-90 & A7926 & D & GG & $\begin{array}{l}\text { Virtual } \\
\text { Library }\end{array}$ & $\begin{array}{l}\text { Virtual } \\
\text { Library }\end{array}$ & & & & & & $\mathrm{Y}$ & HWIS & \\
\hline 2552 & $200-W$ & 299-W23-91 & A7927 & D & GG & \begin{tabular}{|l|} 
Virtual \\
Library
\end{tabular} & $\begin{array}{l}\text { Virtual } \\
\text { Library }\end{array}$ & & & & & & $\mathrm{Y}$ & HWIS & \\
\hline 2553 & $200-W$ & 299-W23-92 & A7928 & D & GG & $\begin{array}{l}\text { Virtual } \\
\text { Library }\end{array}$ & $\begin{array}{l}\text { Virtual } \\
\text { Library }\end{array}$ & & & & & & $Y$ & HWIS & \\
\hline 2554 & $200-W$ & 299-W23-93 & A7929 & D & GG & \begin{tabular}{|l|} 
Virtual \\
Library
\end{tabular} & $\begin{array}{l}\text { Virtual } \\
\text { Library }\end{array}$ & & & & & & $\mathrm{Y}$ & HWIS & \\
\hline 2555 & $200-W$ & 299-W23-94 & A7930 & D & GG & $\begin{array}{l}\text { Virtual } \\
\text { Library }\end{array}$ & $\begin{array}{l}\text { Virtual } \\
\text { Library }\end{array}$ & & & & & & $\mathrm{Y}$ & HWIS & \\
\hline 2556 & $200-W$ & 299-W23-95 & A7931 & D & GG & \begin{tabular}{|l|} 
Virtual \\
Library
\end{tabular} & $\begin{array}{l}\text { Virtual } \\
\text { Library }\end{array}$ & & & & & & & HWIS & \\
\hline
\end{tabular}




\begin{tabular}{|c|c|c|c|c|c|c|c|c|c|c|c|c|c|c|c|}
\hline Sortindex & Area & Well Name & WellID & Log Type & GPX Log Runs & Sieved & $\mathrm{CaCO} 3$ & Moisture & $\begin{array}{l}\text { Chemical } \\
\text { Properties }\end{array}$ & $\begin{array}{l}\text { Physical } \\
\text { Properties }\end{array}$ & $\begin{array}{c}\text { Min } \\
\text { Properties }\end{array}$ & $\begin{array}{l}\text { Geochron } \\
\text { Properties }\end{array}$ & Archived & Source & Comments \\
\hline 2557 & $200-W$ & 299-W23-96 & A7932 & D & GG & $\begin{array}{l}\text { Virtual } \\
\text { Library }\end{array}$ & \begin{tabular}{|l|} 
Virtual \\
Library
\end{tabular} & & & & & & Y & HWIS & \\
\hline 2558 & $200-W$ & 299-W23-97 & A7933 & D & GG & $\begin{array}{l}\text { Virtual } \\
\text { Library }\end{array}$ & $\begin{array}{l}\text { Virtual } \\
\text { Library }\end{array}$ & & & & & & $\mathrm{Y}$ & HWIS & \\
\hline 2559 & $200-W$ & 299-W23-98 & A7934 & D & GG & $\begin{array}{l}\text { Virtual } \\
\text { Library }\end{array}$ & $\begin{array}{l}\text { Virtual } \\
\text { Library }\end{array}$ & & & & & & $\mathrm{Y}$ & HWIS & \\
\hline 2560 & 200-W & 299-W23-99 & A7935 & D & GG & \begin{tabular}{|l} 
Virtual \\
Library
\end{tabular} & \begin{tabular}{|l} 
Virtual \\
Library
\end{tabular} & & & & & & $Y$ & HWIS & \\
\hline 2561 & $200-W$ & 299-W26-1 & A8057 & D & $\mathrm{DN}, \mathrm{GG}, \mathrm{NT}, \mathrm{SG}$ & & & & & & & & & HWIS & \\
\hline 2562 & $200-W$ & 299-W26-10 & A4992 & G & GG, SG & & $\mathrm{Y}$ & $\mathrm{Y}$ & & & & & Y & Williams 1992 & \\
\hline 2563 & $200-W$ & 299-W26-11 & A4993 & $\mathrm{D}, \mathrm{G}$ & GG & \begin{tabular}{|l|} 
Virtual \\
Library
\end{tabular} & & $Y$ & \begin{tabular}{|l|} 
An, VOA, \\
TOC, Lab Rad
\end{tabular} & & & & $Y$ & \begin{tabular}{|l|} 
Airhart et al. \\
1990
\end{tabular} & \\
\hline 2564 & $200-W$ & 299-W26-12 & A5409 & G & GG, SG & & $\mathrm{Y}$ & $\mathrm{Y}$ & & & & & $\mathrm{Y}$ & Williams 1992 & \begin{tabular}{|l|}
$\begin{array}{l}\text { (CaCO3, moisture plots only, no } \\
\text { tabulated data) }\end{array}$ \\
\end{tabular} \\
\hline 2565 & $200-W$ & 299-W26-13 & B8817 & G & NT, SG & & & Y & $\begin{array}{l}\text { Lab Rad, } \\
\text { Metals, An, } \\
\text { SVOA, VOA }\end{array}$ & $\begin{array}{l}\text { Bden, } \\
\% G S S C\end{array}$ & & & $Y$ & DOE 2004b & 216-S-10 pond \\
\hline 2566 & $200-W$ & 299-W26-14 & B8828 & G & & & & $\mathrm{Y}$ & \begin{tabular}{|l|} 
An, VOA, \\
TOC, Lab \\
Rad, Metals
\end{tabular} & $\begin{array}{l}\text { Bden, } \\
\% G S S C\end{array}$ & & & & $\begin{array}{l}\text { HWIS, DOE } \\
2004 \mathrm{~b}\end{array}$ & 216-S-10 ditch \\
\hline 2567 & $200-W$ & 299-W26-2 & A8058 & D & DN, GG, NT, SG & $\begin{array}{l}\text { Virtual } \\
\text { Library }\end{array}$ & \begin{tabular}{|l|} 
Virtual \\
Library
\end{tabular} & & & & & & $\mathrm{Y}$ & HWIS & $1 \mathrm{ft}$ sieve $/ \mathrm{CaCO} 3,5-100 \mathrm{ft}$ \\
\hline 2568 & $200-W$ & 299-W26-3 & A5444 & $\mathrm{D}$ & $\mathrm{DN}, \mathrm{GG}, \mathrm{NT}, \mathrm{TP}$ & \begin{tabular}{|l|}
$\begin{array}{l}\text { Virtual } \\
\text { Library }\end{array}$ \\
Libre
\end{tabular} & \begin{tabular}{|l|} 
Virtual \\
Library
\end{tabular} & & & & & & 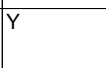 & HWIS & $1 \mathrm{ft}$ sieve/CaCO3, 5-90 ft \\
\hline 2569 & $200-W$ & 299-W26-4 & A8059 & D & DN, GG, NT & \begin{tabular}{|l|} 
Virtual \\
Library
\end{tabular} & \begin{tabular}{|l|} 
Virtual \\
Library
\end{tabular} & & & & & & $Y$ & HWIS & \\
\hline 2570 & $200-W$ & 299-W26-5 & A8060 & D & DN, GG, NT & $\begin{array}{l}\text { Virtual } \\
\text { Library }\end{array}$ & $\begin{array}{l}\text { Virtual } \\
\text { Library }\end{array}$ & & & & & & $\mathrm{Y}$ & HWIS & \\
\hline$\frac{2571}{2572}$ & $\frac{200-W}{200-W}$ & \begin{tabular}{|l|} 
299-W26-51 \\
$299-W 26-6$
\end{tabular} & \begin{tabular}{|l|} 
A8061 \\
A5445
\end{tabular} & $\frac{D}{D}$ & \begin{tabular}{|l} 
GG, SG \\
$S G$
\end{tabular} & & & & \begin{tabular}{|l} 
Field Rad \\
Field Rad
\end{tabular} & & & & \begin{tabular}{|l|l}
$Y$ \\
$Y$
\end{tabular} & HWIS & \\
\hline 2573 & $200-W$ & $299-W 26-7$ & A5446 & G & GG & \begin{tabular}{|l|} 
Virtual \\
Library
\end{tabular} & \begin{tabular}{|l|} 
Virtual \\
Library
\end{tabular} & $\mathrm{Y}$ & & & & & Y & Williams 1992 & \\
\hline 2574 & $200-W$ & 299-W26-8 & A4994 & $\mathrm{D}, \mathrm{G}$ & GG & \begin{tabular}{|l} 
Virtual \\
Library
\end{tabular} & & $\mathrm{Y}$ & \begin{tabular}{|l|} 
An, VOA, \\
TOC, Lab Rad
\end{tabular} & & & & $\mathrm{Y}$ & $\begin{array}{l}\text { Airhart et al. } \\
1990\end{array}$ & \\
\hline 2575 & $200-W$ & 299-W26-9 & A4995 & D, G & GG & \begin{tabular}{|l} 
Virtual \\
Library
\end{tabular} & & $\mathrm{Y}$ & \begin{tabular}{|l|} 
An, VOA, \\
TOC, Lab Rad
\end{tabular} & & & & $\mathrm{Y}$ & $\begin{array}{l}\text { Airhart et al. } \\
1990\end{array}$ & \\
\hline 2576 & $200-W$ & 299-W27-1 & A8062 & D & & & & & & & & & Y & HWIS & \\
\hline 2577 & $200-W$ & $\mid 299-W 27-2$ & A5410 & G & GG, SG & & $Y$ & $Y$ & & & & & $Y$ & $\begin{array}{l}\text { Williams and } \\
\text { Barnett } 1993\end{array}$ & \\
\hline 2578 & $200-W$ & 299-W6-1 & A4996 & D & GG, NT, TP & \begin{tabular}{|l} 
Virtual \\
Library
\end{tabular} & \begin{tabular}{|l} 
Virtual \\
Library
\end{tabular} & & & & & & $Y$ & HWIS & \\
\hline 2579 & $200-W$ & 299-W6-10 & A5435 & G & GG, MG, SG & & Y & $\mathrm{Y}$ & Field Rad & & & & $\mathrm{Y}$ & Mercer 1993a & \\
\hline 2580 & $200-W$ & 299-W6-11 & A5436 & G & GG, SG & & $Y$ & Y & Field Rad & & & & Y & Mercer 1993a & \\
\hline 2581 & $200-W$ & 299-W6-12 & A5437 & G & GG, SG & & $Y$ & Y & Field Rad & & & & $\mathrm{Y}$ & Mercer 1993a & \\
\hline
\end{tabular}




\begin{tabular}{|c|c|c|c|c|c|c|c|c|c|c|c|c|c|c|c|}
\hline Sortindex & Area & Well Name & WellID & Log Type & GPX Log Runs & Sieved & $\mathrm{CaCO} 3$ & Moisture & $\begin{array}{l}\text { Chemical } \\
\text { Properties }\end{array}$ & $\begin{array}{c}\text { Physical } \\
\text { Properties }\end{array}$ & $\begin{array}{c}\text { Min } \\
\text { Properties } \\
\end{array}$ & $\begin{array}{l}\text { Geochron } \\
\text { Properties } \\
\end{array}$ & Archived & Source & Comments \\
\hline 2582 & $200-W$ & 299-W6-2 & A4997 & G & $\mathrm{DN}, \mathrm{GG}, \mathrm{NT}$ & \begin{tabular}{|l|} 
Virtual \\
Library
\end{tabular} & $\begin{array}{l}\text { Virtual } \\
\text { Library }\end{array}$ & $Y$ & & & & & $\mathrm{Y}$ & Last et al. 1989 & \\
\hline 2583 & $200-W$ & 299-W6-3 & A4998 & G & GG, MG, SG & $Y$ & $Y$ & $Y$ & & & & & $\mathrm{Y}$ & & \\
\hline 2584 & $200-W$ & 299-W6-4 & A4999 & G & GG, MG, SG & $\mathrm{Y}$ & $Y$ & $\mathrm{Y}$ & & & & & $\mathrm{Y}$ & & \\
\hline 2585 & $200-W$ & 299-W6-5 & A5000 & $G$ & GG, SG & $Y$ & $Y$ & $\mathrm{Y}$ & & & & & $\mathrm{Y}$ & & \\
\hline 2586 & $200-W$ & 299-W6-6 & A5001 & G & GG, MG, SG & $\mathrm{Y}$ & $Y$ & $Y$ & & & & & $\mathrm{Y}$ & & \\
\hline 2587 & $200-W$ & 299-W6-7 & A5002 & G & GG, SG & $\mathrm{Y}$ & $Y$ & $Y$ & Rad Release & & & & $\mathrm{Y}$ & & \\
\hline 2588 & $200-W$ & 299-W6-8 & A5003 & G & CP, GG, SG & Y & $Y$ & $\mathrm{Y}$ & Rad Release & & & & $Y$ & & \\
\hline 2589 & $200-W$ & 299-W6-9 & A5434 & G & GG, SG & & $\mathrm{Y}$ & $Y$ & Field Rad & & & & $\mathrm{Y}$ & Mercer 1993a & \\
\hline 2590 & $200-W$ & 299-W7-1 & A5004 & G & GG, NT & \begin{tabular}{|l|} 
Virtual \\
Library
\end{tabular} & $\begin{array}{l}\text { Virtual } \\
\text { Library }\end{array}$ & & & & & & & Bjornstad 1990 & \\
\hline 2591 & $200-W$ & 299-W7-10 & A5005 & G & GG & & & $Y$ & & & & & $\mathrm{Y}$ & Barton 1990 & \\
\hline 2592 & $200-W$ & 299-W7-11 & A5006 & G & GG, SG & $Y$ & $\mathrm{Y}$ & $\mathrm{Y}$ & Field Rad & & & & $\mathrm{Y}$ & Mercer 1993b & \\
\hline 2593 & $200-W$ & 299-W7-12 & A5007 & G & GG, SG & $\mathrm{Y}$ & $Y$ & $Y$ & Field Rad & & & & $\mathrm{Y}$ & Mercer 1993b & \\
\hline 2594 & $200-W$ & 299-W7-2 & A5008 & G & DN, GG, NT & \begin{tabular}{|l} 
Virtual \\
Library
\end{tabular} & \begin{tabular}{|l} 
Virtual \\
Library
\end{tabular} & Y & $\begin{array}{l}\text { XRF, TC, IC, } \\
\text { TOC }\end{array}$ & $\begin{array}{l}\text { CEC, } \\
\% G S S C\end{array}$ & XRD & & & $\begin{array}{l}\text { Ames and Serne } \\
\text { 1991; Bjornstad } \\
\text { 1990; Last et al. } \\
\text { 1989; Schramke } \\
1988\end{array}$ & \\
\hline 2595 & $200-W$ & 299-W7-3 & A5009 & G & DN, GG, NT & \begin{tabular}{|l|} 
Virtual \\
Library
\end{tabular} & $\begin{array}{l}\text { Virtual } \\
\text { Library }\end{array}$ & $Y$ & $\begin{array}{l}\text { XRF, TC, IC, } \\
\text { TOC }\end{array}$ & $\begin{array}{l}\text { CEC, } \\
\% G S S C\end{array}$ & XRD & & $Y$ & \begin{tabular}{|l|} 
Ames and Serne \\
1991; Bjornstad \\
1990; Last et al. \\
1989; Schramke \\
1988
\end{tabular} & \\
\hline 2596 & $200-W$ & 299-W7-4 & A5010 & G & DN, GG, NT & \begin{tabular}{|l} 
Virtual \\
Library
\end{tabular} & $\begin{array}{l}\text { Virtual } \\
\text { Library }\end{array}$ & & & & & & $Y$ & Last et al. 1989 & \\
\hline 2597 & $200-W$ & 299-W7-5 & A5011 & G & DN, GG, NT & $\begin{array}{l}\text { Virtual } \\
\text { Library }\end{array}$ & $\begin{array}{l}\text { Virtual } \\
\text { Library }\end{array}$ & & $\begin{array}{l}\text { XRF, TC, IC, } \\
\text { TOC }\end{array}$ & $\begin{array}{l}\text { CEC, } \\
\% G S S C\end{array}$ & XRD & & $Y$ & \begin{tabular}{|l|} 
Ames and Serne \\
1991; Bjornstad \\
1990; Last et al. \\
no date; \\
Schramke 1988
\end{tabular} & \\
\hline 2598 & $200-W$ & 299-W7-51 & A7133 & D & & & & & & & & & & HWIS & \\
\hline 2599 & $200-W$ & 299-W7-52 & A7134 & D & & & & & & & & & & HWIS & \\
\hline 2600 & $200-W$ & 299-W7-53 & A7135 & D & & & & & & & & & & HWIS & \\
\hline 2601 & $200-W$ & 299-W7-6 & A5012 & G & DN, GG, NT & \begin{tabular}{|l} 
Virtual \\
Library
\end{tabular} & $\begin{array}{l}\text { Virtual } \\
\text { Library }\end{array}$ & $Y$ & & & & & $Y$ & PNNL files & \\
\hline 2602 & $200-W$ & 299-W7-7 & A5013 & G & GG & & & Y & Y & & & & Y & Goodwin 1990 & \\
\hline 2603 & $200-W$ & 299-W7-8 & A5014 & G & GG & $\mathrm{Y}$ & $Y$ & $Y$ & $Y$ & & & & $Y$ & Goodwin 1990 & \\
\hline 2604 & $200-W$ & 299-W7-9 & A5015 & $G$ & GG & $Y$ & $Y$ & $Y$ & & & & & $\mathrm{Y}$ & Barton 1990 & \\
\hline 2605 & $200-W$ & 299-W8-1 & A5016 & D, As-built & $\mathrm{DN}, \mathrm{GG}, \mathrm{NT}$ & & & & & & & & & HWIS & \\
\hline 2606 & $200-W$ & 299-W9-1 & A5017 & D, G & DN, GG, NT & \begin{tabular}{|l|} 
Virtual \\
Library
\end{tabular} & \begin{tabular}{|l|} 
Virtual \\
Library
\end{tabular} & $Y$ & & & & & $Y$ & $\begin{array}{l}\text { Bjornstad 1990; } \\
\text { Last et al. 1989 }\end{array}$ & \\
\hline
\end{tabular}




\begin{tabular}{|c|c|c|c|c|c|c|c|c|c|c|c|c|c|c|c|}
\hline SortIndex & Area & Well Name & WelllD & Log Type & GPX Log Runs & Sieved & $\mathrm{CaCO} 3$ & Moisture & $\begin{array}{l}\text { Chemical } \\
\text { Properties }\end{array}$ & $\begin{array}{c}\text { Physical } \\
\text { Properties }\end{array}$ & $\begin{array}{c}\text { Min } \\
\text { Properties } \\
\end{array}$ & $\begin{array}{l}\text { Geochron } \\
\text { Properties }\end{array}$ & Archived & Source & Comments \\
\hline 2607 & $200-W$ & C3082 & C3082 & G & & $Y$ & & Y & $\begin{array}{l}\text { 1:1, Alk, } \\
\text { Gamma, Sr- } \\
\text { 90, H-3, TC- } \\
\text { 99, U-238, TC, } \\
\text { IC }\end{array}$ & $\begin{array}{l}\text { Cond, } \\
\% \text { GSSC, } \\
\text { XRD }\end{array}$ & & & $Y^{*}$ & \begin{tabular}{|l|} 
Gardner and \\
Reynolds 2000; \\
Serne et al. 2002
\end{tabular} & \\
\hline 2608 & $200-W$ & C3102 & C3102 & G & NT, SG & & & & & Bden & & & & $\begin{array}{l}\text { Todd and Kahler- } \\
\text { Royer 2002; } \\
\text { HWIS }\end{array}$ & \\
\hline 2609 & $200-W$ & C3808 & C3808 & G & NT, SG & $Y$ & & $Y$ & $\begin{array}{l}\text { Metals, An, } \\
\text { VOA, SVOA, } \\
\text { lab rad }\end{array}$ & & & & & $\begin{array}{l}\text { Bauer and } \\
\text { Jacques 2002; } \\
\text { DOE/RL-2003- } \\
11\end{array}$ & 216-Z-11 \\
\hline 2610 & $200-W$ & C3830 & C3830 & G & & $Y$ & Y & $Y$ & $\begin{array}{l}\text { 1:1, Aex, Alk, } \\
\text { TC, TOC, } \\
\text { GEA, alpha, } \\
\text { beta }\end{array}$ & & & & & $\begin{array}{l}\text { Reynolds 2003c, } \\
\text { Serne et al } \\
\text { 2004b }\end{array}$ & TX-105 \\
\hline 2611 & $200-W$ & C3831 & C3831 & G & & $Y$ & Y & $Y$ & $\begin{array}{l}\text { 1:1, Aex, Alk, } \\
\text { TC, TOC, } \\
\text { GEA, alpha, } \\
\text { beta }\end{array}$ & & & & & $\begin{array}{l}\text { Gardner, et al } \\
\text { 2003a; Serne et } \\
\text { al. 2004b }\end{array}$ & TX-107 \\
\hline 2612 & $200-W$ & C3832 & C3832 & G & & $Y$ & $Y$ & $Y$ & $\begin{array}{l}\text { 1:1, Aex, Alk, } \\
\text { TC, TOC, } \\
\text { GEA, alpha, } \\
\text { beta }\end{array}$ & & & & & $\begin{array}{l}\text { Gardner et al. } \\
\text { 2003b, Serne et } \\
\text { al 2004b }\end{array}$ & TX-104 \\
\hline 2613 & $200-W$ & C4104 & C4104 & G & NT, SG & $Y$ & $\mathrm{Y}$ & $Y$ & $\begin{array}{l}\text { 1:1, Aex, Alk, } \\
\text { TC, TOC, } \\
\text { GEA, alpha, } \\
\text { beta }\end{array}$ & & & & & $\begin{array}{l}\text { Reynold 2003a, } \\
\text { Serne et al. } \\
\text { 2004a }\end{array}$ & T-106 \\
\hline 2614 & $200-W$ & C4105 & C4105 & $G$ & NT, SG & $Y$ & Y & $Y$ & $\begin{array}{l}\text { 1:1, Aex, Alk, } \\
\text { TC, TOC, } \\
\text { GEA, alpha, } \\
\text { beta }\end{array}$ & & & & & $\begin{array}{l}\text { Reynolds 2003b, } \\
\text { Serne et al. } \\
2004 a\end{array}$ & T-106 \\
\hline 2615 & $200-W$ & C4175 & C4175 & G, As-built & SG & & & & & & & & & HWIS & \\
\hline 2616 & $200-W$ & C4178 & C4178 & & NT, SG & & & & & & & & & & \\
\hline 2617 & $200-W$ & C4179 & C4179 & & NT, SG & & & & & & & & & & \\
\hline 2618 & $200-W$ & C4180 & C4180 & & NT, SG & & & & & & & & & & \\
\hline 2619 & $200-W$ & C4181 & C4181 & & NT, SG & & & & & & & & & & \\
\hline 2620 & $200-W$ & C4183 & C4183 & & NT, SG & & & & & & & & & & \\
\hline 2621 & $200-W$ & C4201 & C4201 & Well summary & SG & & & & & & & & & HWIS & \\
\hline 2622 & $200-W$ & C4202 & C4202 & Well summary & SG & & & & & & & & & HWIS & \\
\hline 2623 & $200-W$ & C4203 & C4203 & Well summary & SG & & & & & & & & & HWIS & \\
\hline 2624 & $200-W$ & C4204 & C4204 & Well summary & SG & & & & & & & & & HWIS & \\
\hline 2625 & $200-W$ & C4205 & C4205 & Well summary & SG & & & & & & & & & HWIS & \\
\hline 2626 & $200-W$ & C4206 & C4206 & Well summary & SG & & & & & & & & & HWIS & \\
\hline 2627 & $200-\mathrm{W}$ & C4207 & C4207 & Well summary & SG & & & & & & & & & HWIS & \\
\hline 2628 & $200-W$ & C4209 & C4209 & Well summary & SG & & & & & & & & & HWIS & \\
\hline 2629 & $200-W$ & C4210 & C4210 & Well summary & SG & & & & & & & & & HWIS & \\
\hline 2630 & $200-W$ & C4211 & C4211 & & SG & & & & & & & & & & \\
\hline 2631 & $200-\mathrm{W}$ & C4212 & C4212 & Well summary & SG & & & & & & & & & HWIS & \\
\hline 2632 & $200-W$ & C4213 & C4213 & Well summary & SG & & & & & & & & & HWIS & \\
\hline 2633 & $200-W$ & C4215 & C4215 & Well summary & SG & & & & & & & & & HWIS & \\
\hline 2634 & $200-W$ & C4217 & C4217 & Well summary & SG & & & & & & & & & HWIS & \\
\hline 2635 & $200-W$ & C4218 & C4218 & Well summary & SG & & & & & & & & & HWIS & \\
\hline 2636 & $200-W$ & C4219 & C4219 & Well summary & SG & & & & & & & & & HWIS & \\
\hline 2637 & $200-W$ & C4220 & C4220 & Well summary & SG & & & & & & & & & HWIS & \\
\hline 2638 & $200-\mathrm{W}$ & C4221 & C4221 & Well summary & SG & & & & & & & & & HWIS & \\
\hline
\end{tabular}




\begin{tabular}{|c|c|c|c|c|c|c|c|c|c|c|c|c|c|c|c|}
\hline SortIndex & Area & Well Name & WellID & Log Type & GPX Log Runs & Sieved & $\mathrm{CaCO} 3$ & Moisture & $\begin{array}{l}\text { Chemical } \\
\text { Properties }\end{array}$ & $\begin{array}{l}\text { Physical } \\
\text { Properties }\end{array}$ & \begin{tabular}{|c|} 
Min \\
Properties \\
\end{tabular} & $\begin{array}{l}\text { Geochron } \\
\text { Properties }\end{array}$ & Archived & Source & Comments \\
\hline 2639 & $200-W$ & C4222 & C4222 & Well summary & SG & & & & & & & & & HWIS & \\
\hline 2640 & $200-W$ & C4223 & C4223 & Well summary & SG & & & & & & & & & HWIS & \\
\hline 2641 & $200-W$ & C4224 & C4224 & Well summary & SG & & & & & & & & & HWIS & \\
\hline 2642 & $200-W$ & C4225 & C4225 & Well summary & SG & & & & & & & & & HWIS & \\
\hline 2643 & $200-W$ & C4226 & C4226 & Well summary & SG & & & & & & & & & HWIS & \\
\hline 2644 & $200-W$ & C4227 & C4227 & Well summary & SG & & & & & & & & & HWIS & \\
\hline 2645 & $200-W$ & C4228 & C4228 & Well summary & SG & & & & & & & & & HWIS & \\
\hline 2646 & $200-W$ & C4229 & C4229 & Well summary & SG & & & & & & & & & HWIS & \\
\hline 2647 & $200-W$ & $\mathrm{C} 4231$ & C4231 & Well summary & SG & & & & & & & & & HWIS & \\
\hline 2648 & $200-W$ & C4232 & C4232 & Well summary & SG & & & & & & & & & HWIS & \\
\hline 2649 & $200-W$ & C4548 & C4548 & Well summary & SG & & & & & & & & & HWIS & \\
\hline 2650 & $200-W$ & C4549 & C4549 & Well summary & NT, SG & & & & & & & & & HWIS & \\
\hline 2651 & $200-W$ & C4550 & C4550 & Well summary & SG & & & & & & & & & HWIS & \\
\hline 2652 & $200-W$ & C4551 & C4551 & Well summary & SG & & & & & & & & & HWIS & \\
\hline 2653 & $200-W$ & C4552 & C4552 & Well summary & SG & & & & & & & & & HWIS & \\
\hline 2654 & $200-W$ & C4553 & C4553 & Well summary & SG & & & & & & & & & HWIS & \\
\hline 2655 & $200-W$ & C4554 & C4554 & Well summary & SG & & & & & & & & & HWIS & \\
\hline 2656 & $200-W$ & C4555 & C4555 & Well summary & NT, SG & & & & & & & & & HWIS & \\
\hline 2657 & $200-W$ & C4556 & C4556 & Well summary & SG & & & & & & & & & HWIS & \\
\hline 2658 & $200-W$ & C4557 & C4557 & G, Well summary & SG & & & & & & & & & HWIS & \\
\hline 2659 & 300 & $399-1-10$ & & & DN, GG, NT & & & & & & & & & & \\
\hline 2660 & 300 & 399-1-10A & A5411 & As-built & SG & & & & & & & & & HWIS & \\
\hline 2661 & 300 & 399-1-10B & A8064 & Well summary & GG & & $\mathrm{Y}$ & $\mathrm{Y}$ & & $\%$ GSSC & & & & $\begin{array}{l}\text { Swanson 1992; } \\
\text { HWIS }\end{array}$ & \\
\hline 2662 & 300 & $399-1-11$ & A5020 & As-built & DN, GG, NT & & & & & \begin{tabular}{|l}
$\begin{array}{l}\text { Pden, Por, } \\
\text { Bden }\end{array}$ \\
\end{tabular} & & & & $\begin{array}{l}\text { Swanson 1992; } \\
\text { HWIS }\end{array}$ & \\
\hline 2663 & 300 & $399-1-12$ & A5021 & As-built & $\mathrm{DN}, \mathrm{GG}, \mathrm{NT}$ & & & & & & & & & HWIS & \\
\hline 2664 & 300 & $399-1-13$ & & & $\mathrm{DN}, \mathrm{GG}, \mathrm{NT}$ & & & & & & & & & & \\
\hline 2665 & 300 & $399-1-13 \mathrm{~A}$ & A5412 & As-built & SG & & & & & & & & & HWIS & \\
\hline 2666 & 300 & 399-1-13B & A8065 & Well summary & GG & & $Y$ & $Y$ & Field Rad & \begin{tabular}{|l} 
Pden, Por, \\
Bden, \\
$\%$ GSSC
\end{tabular} & & & & $\begin{array}{l}\text { Swanson 1992; } \\
\text { HWIS }\end{array}$ & \\
\hline 2667 & 300 & $399-1-14$ & & & DN, GG, NT & & & & & & & & & & \\
\hline 2668 & 300 & $399-1-14 \mathrm{~A}$ & A5413 & As-built & SG & & & & & & & & & HWIS & \\
\hline 2669 & 300 & $399-1-14 B$ & A8066 & Well summary & GG & & $\mathrm{Y}$ & Y & Field Rad & $\begin{array}{l}\text { Pden, Por, } \\
\text { Bden, } \\
\% G S S C\end{array}$ & & & & $\begin{array}{l}\text { Swanson 1992; } \\
\text { HWIS }\end{array}$ & \\
\hline 2670 & 300 & $399-1-15$ & A5024 & As-built & DN, GG, NT & & & & & & & & & HWIS & \\
\hline 2671 & 300 & $399-1-16 \mathrm{~A}$ & A5025 & As-built & DN, GG, NT & & & & & & & & & HWIS & \\
\hline 2672 & 300 & 399-1-16B & A5026 & As-built & DN, GG, NT & & & & & & & & & $\begin{array}{l}\text { Swanson 1992; } \\
\text { HWIS }\end{array}$ & \\
\hline 2673 & 300 & $399-1-16 C$ & A5027 & As-built & $\mathrm{CP}, \mathrm{DN}, \mathrm{GG}, \mathrm{NT}$ & & & & & & & C-14 & & $\begin{array}{l}\text { Swanson 1992; } \\
\text { HWIS }\end{array}$ & \\
\hline 2674 & 300 & 399-1-16D & A8067 & As-built & DN, GG, NT & & & & & & & & & HWIS & \\
\hline 2675 & 300 & 399-1-17A & A5028 & As-built & DN, GG, NT & & & & & & & & & HWIS & \\
\hline 2676 & 300 & 399-1-17B & A5029 & As-built & CP, DN, GG, NT & & & & & & & C-14 & & HWIS & \\
\hline 2677 & 300 & $399-1-17 \mathrm{C}$ & A5030 & As-built & DN, GG, NT & & & & & & & & & HWIS & \\
\hline 2678 & 300 & 399-1-18A & A5031 & As-built & DN, GG, NT & & & & & & & & & HWIS & \\
\hline 2679 & 300 & 399-1-18B & A5032 & As-built & DN, GG, NT & & & & & & & & & HWIS & \\
\hline 2680 & 300 & $399-1-18 \mathrm{C}$ & A5033 & As-built & $\mathrm{CP}, \mathrm{DN}, \mathrm{GG}, \mathrm{NT}$ & & & & & & & & & HWIS & \\
\hline 2681 & 300 & $399-1-19$ & A5034 & As-built & & & & & & & & & & HWIS & \\
\hline 2682 & 300 & $399-1-20$ & A8068 & As-built & DN, GG & & & & & & & & & HWIS & \\
\hline 2683 & 300 & $399-1-21 \mathrm{~A}$ & A5414 & Well summary & & & & & & & & & & HWIS & \\
\hline 2684 & 300 & $399-1-21 B$ & A5415 & Well summary & GG & & $Y$ & $Y$ & Field Rad & $\% G S S C$ & & & & $\begin{array}{l}\text { Swanson 1992; } \\
\text { HWIS }\end{array}$ & \\
\hline 2685 & 300 & $399-1-22$ & A8069 & Well summary & & & & & & & & & & HWIS & \\
\hline 2686 & 300 & $399-1-7$ & A5040 & D, As-built & DN, GG, NT, TP & & & & & & & & & HWIS & \\
\hline
\end{tabular}




\begin{tabular}{|c|c|c|c|c|c|c|c|c|c|c|c|c|c|c|c|}
\hline SortIndex & Area & Well Name & WellID & Log Type & GPX Log Runs & Sieved & $\mathrm{CaCO} 3$ & Moisture & $\begin{array}{l}\text { Chemical } \\
\text { Properties }\end{array}$ & $\begin{array}{l}\text { Physical } \\
\text { Properties }\end{array}$ & \begin{tabular}{|c|} 
Min \\
Properties \\
\end{tabular} & $\begin{array}{l}\text { Geochron } \\
\text { Properties }\end{array}$ & Archived & Source & Comments \\
\hline 2687 & 300 & $399-1-8$ & A5041 & As-built & DN, GG, NT, SN & & & & & & & & & HWIS & \\
\hline 2688 & 300 & $399-1-9$ & A5042 & As-built & DN, GG, NT & & & & & & & & & HWIS & \\
\hline 2689 & 300 & $399-2-1$ & A5043 & As-built & & & & & & & & & & HWIS & \\
\hline 2690 & 300 & $399-2-2$ & A5044 & As-built & & & & & & & & & & HWIS & \\
\hline 2691 & 300 & $399-2-3$ & A5045 & As-built & & & & & & & & & & HWIS & \\
\hline 2692 & 300 & $399-2-4$ & A8070 & Well summary & & & & & & & & & & HWIS & \\
\hline 2693 & & $399-3-1$ & A5046 & As-built & & & & & & & & & & HWIS & \\
\hline 2694 & 300 & $399-3-10$ & A5047 & As-built & DN, GG, NT, TP & & & & & & & & & HWIS & \\
\hline 2695 & 300 & $399-3-11$ & A8077 & As-built & & & & & & & & & & HWIS & \\
\hline 2696 & & $399-3-12$ & A5048 & As-built & DN, GG, NT, TP & & & & & & & & & HWIS & \\
\hline 2697 & 300 & $399-3-13$ & A8078 & Well summary & & & & & & & & & & HWIS & \\
\hline 2698 & 300 & $399-3-14$ & A8079 & Well summary & & & & & & & & & & HWIS & \\
\hline 2699 & 300 & $399-3-15$ & A8080 & Well summary & & & & & & & & & & HWIS & \\
\hline 2700 & & $399-3-16$ & A8081 & Well summary & & & & & & & & & & HWIS & \\
\hline 2701 & 300 & $399-3-17$ & A8082 & Well summary & & & & & & & & & & HWIS & \\
\hline 2702 & 300 & $399-3-2$ & A8071 & As-built & & & & & & & & & & HWIS & \\
\hline 2703 & 300 & $399-3-3$ & A8072 & As-built & NT & & & & & & & & & HWIS & \\
\hline 2704 & 300 & $399-3-4 B$ & A8074 & As-built & & & & & & & & & & HWIS & \\
\hline 2705 & 300 & $399-3-6$ & A5049 & As-built & & & & & & & & & & HWIS & \\
\hline 2706 & 300 & $399-3-7$ & A5050 & As-built & & & & & & & & & & HWIS & \\
\hline 2707 & 300 & $399-3-8$ & A8076 & As-built & & & & & & & & & & HWIS & \\
\hline 2708 & 300 & $399-3-9$ & A5051 & As-built & DN, GG, NT, TP & & & & & & & & & HWIS & \\
\hline 2709 & 300 & $399-4-1$ & A5052 & As-built & & & & & & & & & & HWIS & \\
\hline 2710 & 300 & $399-4-10$ & A5053 & As-built & DN, GG, NT, TP & & & & & & & & & HWIS & \\
\hline 2711 & 300 & $399-4-11$ & A5054 & As-built & $\mathrm{DN}, \mathrm{GG}, \mathrm{NT}$ & & & & & & & & & HWIS & \\
\hline 2712 & 300 & $399-4-12$ & A8089 & As-built & & & & & & & & & & HWIS & \\
\hline 2713 & 300 & $399-4-13$ & A8090 & As-built & & & & & & & & & & HWIS & \\
\hline 2714 & 300 & $399-4-5$ & A8086 & As-built & SG & & & & & & & & & HWIS & \\
\hline 2715 & 300 & $399-4-7$ & A5055 & As-built & NT & & & & & & & & & HWIS & \\
\hline 2716 & 300 & $399-4-8$ & A8088 & As-built & & & & & & & & & & HWIS & \\
\hline 2717 & 300 & $399-4-9$ & A5056 & As-built & DN, GG, NT, TP & & & & & & & & & HWIS & \\
\hline 2718 & 300 & $399-5-1$ & A5057 & As-built & & & & & & & & & & HWIS & \\
\hline 2719 & & $399-5-2$ & A8091 & As-built & $\mathrm{CP}, \mathrm{SG}$ & & & & & & & & & HWIS & \\
\hline 2720 & 300 & $399-5-3$ & A8092 & As-built & & & & & & & & & & HWIS & \\
\hline 2721 & 300 & $399-5-4 \mathrm{~A}$ & A8093 & Well summary & & & & & & & & & & HWIS & \\
\hline 2722 & 300 & $399-5-4 \mathrm{~B}$ & A8094 & Well summary & & & & & & & & & & HWIS & \\
\hline 2723 & 300 & $399-6-1$ & A5058 & As-built & & & & & & & & & & HWIS & \\
\hline 2724 & 300 & $399-6-2$ & A8095 & Well summary & & & & & & & & & & HWIS & \\
\hline 2725 & 300 & $399-8-1$ & A5059 & As-built & DN & & & & & & & & & HWIS & \\
\hline 2726 & 300 & $399-8-2$ & A5060 & As-built & & & & & & & & & & HWIS & \\
\hline 2727 & 300 & $399-8-3$ & A5061 & As-built & & & & & & & & & & HWIS & \\
\hline 2728 & 300 & $399-8-4$ & A8096 & As-built & & & & & & & & & & HWIS & \\
\hline 2729 & 300 & $399-8-5 A$ & A5416 & Well summary & & & & & & & & & & HWIS & \\
\hline 2730 & 300 & $399-8-5 B$ & A5417 & Well summary & & & & & & & & & & HWIS & \\
\hline 2731 & 300 & $399-8-5 \mathrm{C}$ & A5418 & Well summary & GG, SG & & Y & Y & Field Rad & $\%$ GSSC & & & & $\begin{array}{l}\text { Swanson 1992; } \\
\text { HWIS }\end{array}$ & \\
\hline 2732 & 300 & $399-8-5 \mathrm{D}$ & A8097 & Well summary & & & & & & & & & & HWIS & \\
\hline 2733 & 400 & 499-S1-8B & A8107 & As-built & SG & & & & & & & & & HWIS & \\
\hline 2734 & 400 & 499-S1-8L & A8116 & As-built & SG & & & & & & & & & HWIS & \\
\hline 2735 & 600 & 699-101-48A & A9101 & As-built & & & & & & & & & & HWIS & Groundwater monitoring \\
\hline 2736 & 600 & 699-101-48B & A5066 & As-built & TP & & & & & & & & & HWIS & Groundwater monitoring \\
\hline 2737 & 600 & $699-101-48 \mathrm{C}$ & A9102 & As-Built & & & & & & & & & & HWIS & Groundwater monitoring \\
\hline 2738 & 600 & 699-10-54A & A5063 & D, G, As-built & NT, TP & & & & & & & & & HWIS & \\
\hline 2739 & 600 & 699-10-54B & A5064 & D, As-built & $\mathrm{CP}, \mathrm{GG}, \mathrm{NT}, \mathrm{SN}$ & & & & & & & & & HWIS & \\
\hline
\end{tabular}




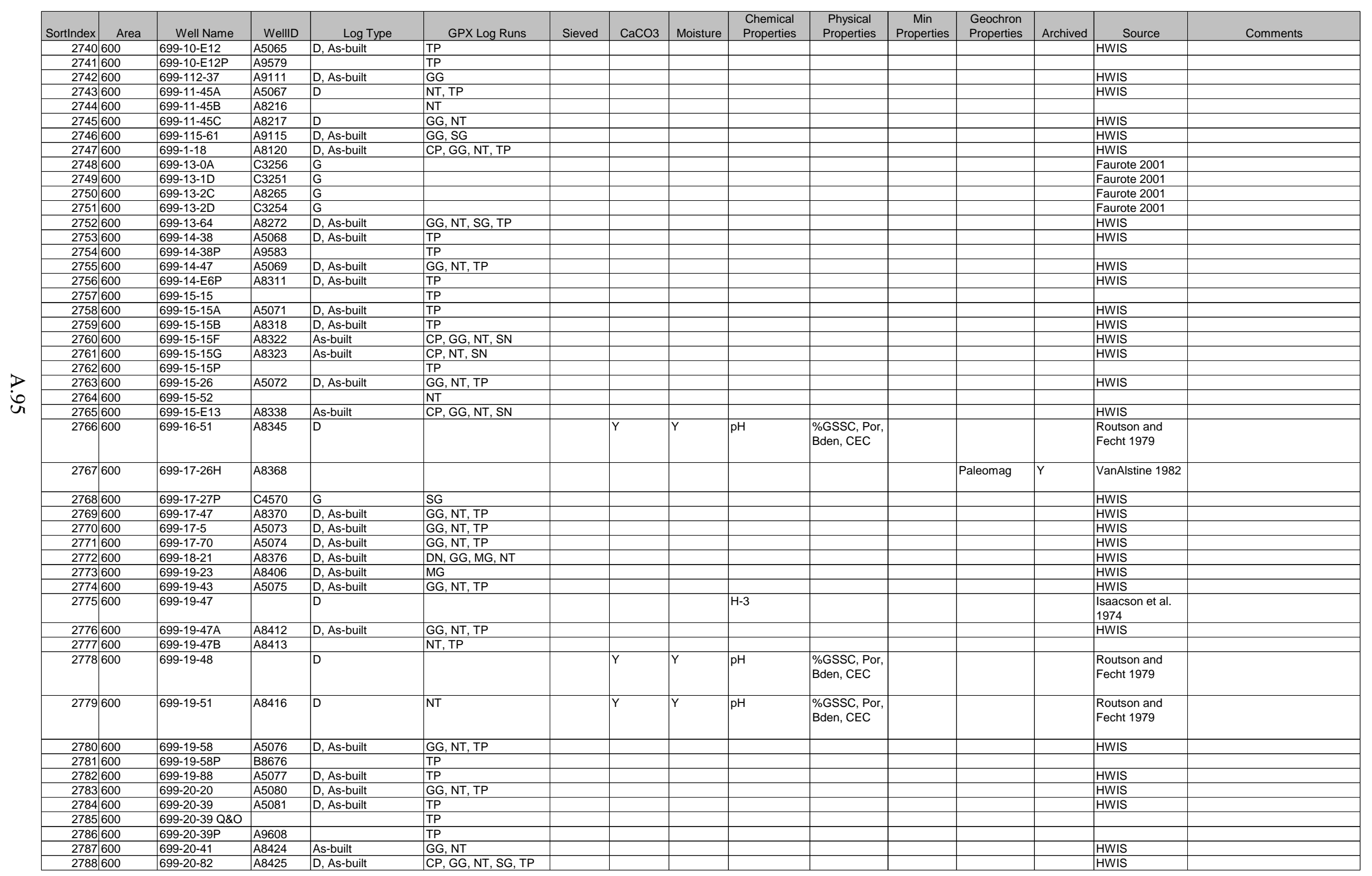




\begin{tabular}{|c|c|c|c|c|c|c|c|c|c|c|c|c|c|c|c|}
\hline Sortlndex & Area & Well Name & WellID & Log Type & GPX Log Runs & Sieved & $\mathrm{CaCO} 3$ & Moisture & $\begin{array}{l}\text { Chemical } \\
\text { Properties }\end{array}$ & $\begin{array}{l}\text { Physical } \\
\text { Properties }\end{array}$ & \begin{tabular}{|c|} 
Min \\
Properties
\end{tabular} & $\begin{array}{l}\text { Geochron } \\
\text { Properties }\end{array}$ & Archived & Source & Comments \\
\hline 2789 & 600 & 699-20-E12 & A5085 & D, As-built & TP & & & & & & & & & HWIS & \\
\hline 2790 & 600 & 699-20-E12P\&Q & & & TP & & & & & & & & & & \\
\hline 2791 & 600 & 699-20-E12Q & A9615 & & TP & & & & & & & & & & \\
\hline 2792 & 600 & 699-20-E12R & A9616 & & TP & & & & & & & & & & \\
\hline 2793 & & 699-20-E5A & A8428 & D, As-built & GG, NT & & & & & & & & & HWIS & \\
\hline 2794 & & 699-20-E5P & A8429 & D, As-built & TP & & & & & & & & & HWIS & \\
\hline 2795 & 600 & \begin{tabular}{|c|}
$699-21-17$ \\
\end{tabular} & A5086 & & GG, NT & & & & & & & & & & \\
\hline 2796 & 600 & 699-21-6 & A8438 & D, As-built & $\mathrm{GG}, \mathrm{NT}$ & & & & & & & & & HWIS & \\
\hline 2797 & 600 & 699-22-35 & A8443 & G, As-built & GG, SG & & & & & & & & & HWIS & \\
\hline 2798 & 600 & 699-22-55 & A8444 & D & NT & & $\mathrm{Y}$ & $Y$ & $\mathrm{pH}$ & $\begin{array}{l}\text { \%GSSC, Por, } \\
\text { Bden, CEC }\end{array}$ & & & & \begin{tabular}{|l|} 
Routson and \\
Fecht 1979
\end{tabular} & \\
\hline 2799 & 600 & $699-22-70$ & A8445 & D, As-built & $\mathrm{CP}, \mathrm{TP}$ & & & & & & & & & HWIS & \\
\hline 2800 & & 699-22-70P & A9480 & & TP & & & & & & & & & & \\
\hline 2801 & 600 & $699-2-3$ & A5078 & D, As-built & GG, NT, TP & & & & & & & & & HWIS & \\
\hline 2802 & 600 & $699-2-33$ & & & TP & & & & & & & & & & \\
\hline 2803 & & 699-23-33 & A8449 & & GG, NT & & & & & & & & & & \\
\hline 2804 & 600 & 699-23-34 & & G & GG, NT & $Y$ & & $Y$ & & & & & & $\begin{array}{l}\text { Fruland et al. } \\
1989\end{array}$ & \\
\hline 2805 & 600 & 699-23-34B & A8450 & G, As-built & GG, SG & & & & & & & & & HWIS & \\
\hline 2806 & & 699-2-33A & A5079 & D, As-built & GG, NT & & & & & & & & & HWIS & \\
\hline 2807 & 600 & 699-2-33P & & & TP & & & & & & & & & & \\
\hline 2808 & 600 & 699-24-1 P & & & TP & & & & & & & & & & \\
\hline 2809 & 600 & 699-24-33 & A5089 & D, G, As-built & TP & & $Y$ & & $\mathrm{pH}$ & $\begin{array}{l}\text { CEC, } \\
\% \text { \%SSC, 15- } \\
\text { Atm }\end{array}$ & & & & McHenry 1957 & \\
\hline 2810 & 600 & 699-24-34A & A5090 & G & GG, NT & $Y$ & & $\mathrm{Y}$ & & & & & & $\begin{array}{l}\text { Fruland et al. } \\
1989\end{array}$ & \\
\hline 2811 & 600 & 699-24-34B & A5091 & D, As-built & GG, NT & $Y$ & & $Y$ & & & & & & $\begin{array}{l}\text { HWIS; Fruland } \\
\text { et al. } 1989\end{array}$ & \\
\hline 2812 & 600 & $699-24-34 \mathrm{C}$ & A5092 & G & GG, NT & $Y$ & & Y & & & & & & $\begin{array}{l}\text { Fruland et al. } \\
1989\end{array}$ & \\
\hline 2813 & 600 & 699-24-35 & A5093 & G & GG, NT & $Y$ & & $Y$ & & & & & & $\begin{array}{l}\text { Fruland et al. } \\
1989\end{array}$ & \\
\hline 2814 & 600 & 699-24-46 & A8457 & D, As-built & GG, NT, TP & & & & & & & & & HWIS & \\
\hline 2815 & 600 & 699-24-E1 P & & & TP & & & & & & & & & & \\
\hline 2816 & 600 & 699-25-33A & A5094 & G & & $Y$ & $\mathrm{Y}$ & $\mathrm{Y}$ & & & & & & $\begin{array}{l}\text { Weekes et al. } \\
1987\end{array}$ & \\
\hline 2817 & 600 & 699-25-33B & A8462 & G & NT & $Y$ & $Y$ & $Y$ & & & & & & $\begin{array}{l}\text { Weekes et al. } \\
1987\end{array}$ & \\
\hline 2818 & 600 & 699-25-34A & A5095 & G & GG, NT & $Y$ & Y & $Y$ & & & & & & $\begin{array}{l}\text { Weekes et al. } \\
1987\end{array}$ & \\
\hline 2819 & 600 & 699-25-34B & A5096 & G & GG, NT & & & & & & & & & $\begin{array}{l}\text { Weekes et al. } \\
1987\end{array}$ & \\
\hline 2820 & 600 & $699-25-34 \mathrm{C}$ & A5097 & G & DN, GG, NT & $Y$ & & $Y$ & & & & & & $\begin{array}{l}\text { Fruland et al. } \\
1989\end{array}$ & \\
\hline 2821 & 600 & 699-25-34D & A5419 & G & GG, SG & & $Y$ & $Y$ & & & & & & Hodges 1993 & \\
\hline 2822 & 600 & 699-25-55 & A5098 & D & TP & & $Y$ & & $\mathrm{pH}$ & $\begin{array}{l}\text { CEC, } \\
\% \text { GSSC, 15- } \\
\text { Atm }\end{array}$ & & & & McHenry 1957 & \\
\hline 2823 & 600 & 699-25-70 & A5099 & D & GG, NT, TP & & $\mathrm{Y}$ & & $\mathrm{pH}$ & \begin{tabular}{|l|} 
CEC, \\
\%GSSC, 15- \\
Atm
\end{tabular} & & & & McHenry 1957 & \\
\hline 2824 & 600 & 699-25-70P & A9621 & & TP & & & & & & & & & & \\
\hline
\end{tabular}




\begin{tabular}{|c|c|c|c|c|c|c|c|c|c|c|c|c|c|c|c|}
\hline SortIndex & Area & Well Name & WellID & Log Type & GPX Log Runs & Sieved & $\mathrm{CaCO} 3$ & Moisture & $\begin{array}{l}\text { Chemical } \\
\text { Properties }\end{array}$ & $\begin{array}{c}\text { Physical } \\
\text { Properties }\end{array}$ & \begin{tabular}{|c|} 
Min \\
Properties \\
\end{tabular} & $\begin{array}{l}\text { Geochron } \\
\text { Properties }\end{array}$ & Archived & Source & Comments \\
\hline $\begin{array}{r}2825 \\
\end{array}$ & 600 & $699-25-80$ & A8465 & D, As-built & $\begin{array}{l}\text { CP, GG, NT, OT, SN, } \\
\text { TP }\end{array}$ & & & & & & & & & HWIS & \\
\hline 2826 & 600 & 699-26-15 & & & TP & & & & & & & & & & \\
\hline 2827 & 600 & \begin{tabular}{|l|l|}
$699-26-29$ \\
\end{tabular} & & & GG, NT & & & & & & & & & & \\
\hline 2828 & 600 & $699-26-33$ & A5101 & G & GG, NT & $Y$ & $\mathrm{Y}$ & $Y$ & & & & & & $\begin{array}{l}\text { Weekes et al. } \\
1987\end{array}$ & \\
\hline 2829 & 600 & 699-26-34 & & G & & $Y$ & $Y$ & $Y$ & & & & & & $\begin{array}{l}\text { Weekes et al. } \\
1987\end{array}$ & \\
\hline 2830 & 600 & 699-26-34A & A5102 & D, As-built & GG, NT & & & & & & & & & HWIS & \\
\hline 2831 & & 699-26-34B & A5420 & $G$ & GG & $Y$ & $Y$ & $Y$ & & & & & & Hodges 1993 & \\
\hline 2832 & & 699-26-34D & & & SG & & & & & & & & & & \\
\hline 2833 & 600 & 699-26-35A & A5103 & G & GG, NT & $\mathrm{Y}$ & Y & $\mathrm{Y}$ & & & & & & $\begin{array}{l}\text { Weekes et al. } \\
1987\end{array}$ & \\
\hline 2834 & 600 & 699-26-35B & A8471 & G & GG, NT & $Y$ & $Y$ & $Y$ & & & & & & $\begin{array}{l}\text { Weekes et al. } \\
1987\end{array}$ & \\
\hline 2835 & 600 & $699-26-35 C$ & A5104 & G & GG, NT & $Y$ & $\mathrm{Y}$ & $\mathrm{Y}$ & & & & & & $\begin{array}{l}\text { Weekes et al. } \\
1987\end{array}$ & \\
\hline 2836 & 600 & 699-26-35D & A8472 & G & GG, NT & & $Y$ & $Y$ & & & & & & $\begin{array}{l}\text { Weekes et al. } \\
1987\end{array}$ & \\
\hline 2837 & 600 & 699-26-47 & A8473 & D & NT & & $\mathrm{Y}$ & $\mathrm{Y}$ & $\mathrm{pH}$ & $\begin{array}{l}\text { \%GSSC, Por, } \\
\text { Bden, CEC }\end{array}$ & & & & \begin{tabular}{|l} 
Routson and \\
Fecht 1979
\end{tabular} & \\
\hline 2838 & 600 & 699-26-51 & A8474 & D & NT & & $Y$ & $Y$ & $\mathrm{pH}$ & $\begin{array}{l}\% \text { GSSC, Por, } \\
\text { Bden, CEC }\end{array}$ & & & & \begin{tabular}{|l} 
Routson and \\
Fecht 1979
\end{tabular} & \\
\hline 2839 & 600 & 699-26-83A & A8476 & As-built & GG, NT & & $\begin{array}{l}\text { Virtual } \\
\text { Library }\end{array}$ & $\mathrm{Y}$ & & $\begin{array}{l}\text { Bden, } \\
\% G S S C\end{array}$ & & Date & & \begin{tabular}{|l|} 
HWIS; Baker et \\
al. 1991; Virtual \\
Library 2000 (PPI \\
only, includes \\
descriptions)
\end{tabular} & \\
\hline 2840 & 600 & 699-26-83B & A8477 & As-built & NT & & & & & & & & & HWIS & \\
\hline 2841 & & 699-26-89 & A5108 & D, As-built & GG, NT, TP & & & & & & & & & HWIS & \\
\hline 2842 & 600 & $699-27-8$ & A5109 & D, As-built & GG, NT, TP & & & & & & & & & HWIS & \\
\hline 2843 & & $699-28-40$ & A5110 & D, As-built & TP & & & & & & & & & HWIS & \\
\hline 2844 & & 699-28-40P & A9628 & & TP & & & & & & & & & & \\
\hline 2845 & 600 & 699-28-49 & A8486 & D & NT & & $Y$ & $Y$ & $\mathrm{pH}$ & $\begin{array}{l}\text { \%GSSC, Por, } \\
\text { Bden, CEC }\end{array}$ & & & & $\begin{array}{l}\text { Routson and } \\
\text { Fecht } 1979\end{array}$ & \\
\hline 2846 & 600 & 699-28-52A & A5111 & D, As-built & NT, TP & & & & & & & & & HWIS & \\
\hline 2847 & 600 & 699-28-52B & A8487 & D, As-built & GG, NT & & & & & & & & & HWIS & \\
\hline 2848 & 600 & 699-28-55 & A8488 & D & GG, NT, TP & & $\mathrm{Y}$ & $Y$ & $\mathrm{pH}$ & $\begin{array}{l}\text { \%GSSC, Por, } \\
\text { Bden, CEC }\end{array}$ & & & & \begin{tabular}{|l|} 
Routson and \\
Fecht 1979
\end{tabular} & \\
\hline 2849 & 600 & 699-29-4 & A8490 & D, As-built & GG, NT & & & & & & & & & HWIS & \\
\hline 2850 & & \begin{tabular}{|l|l|}
$699-29-70 \mathrm{~A}$ \\
\end{tabular} & A8491 & As-built & $\mathrm{CP}, \mathrm{GG}, \mathrm{NT}, \mathrm{SN}$ & & & & & & & & & HWIS & \\
\hline 2851 & & 699-29-70C & A8492 & As-built & CP, GG, NT, SN & & & & & & & & & HWIS & \\
\hline 2852 & 600 & 699-29-70D & A8493 & As-built & $\mathrm{CP}, \mathrm{GG}, \mathrm{NT}, \mathrm{SN}$ & & & & & & & & & HWIS & \\
\hline 2853 & 600 & 699-29-78 & A5121 & D & GG, NT, TP & $\begin{array}{l}\text { Virtual } \\
\text { Library }\end{array}$ & $\begin{array}{l}\text { Virtual } \\
\text { Library }\end{array}$ & & & & & & $\mathrm{Y}$ & HWIS & \\
\hline 2854 & 600 & 699-29-83 & A8494 & As-built & CP, GG, NT & & & & & & & Paleomag & $\mathrm{Y}$ & $\begin{array}{l}\text { HWIS; WCC } \\
1982\end{array}$ & \\
\hline 2855 & 600 & 699-2-E14 & A8124 & As-built & $\mathrm{CP}, \mathrm{GG}, \mathrm{NT}, \mathrm{SN}, \mathrm{TP}$ & & & & & & & & & HWIS & \\
\hline 2856 & 600 & 699-30-16 & A8495 & & GG, NT & & & & & & & & & & \\
\hline 2857 & 600 & 699-30-25A & A8496 & & GG, NT & & & & & & & & & & \\
\hline
\end{tabular}




\begin{tabular}{|c|c|c|c|c|c|c|c|c|c|c|c|c|c|c|c|}
\hline Sortlndex & Area & Well Name & WellID & Log Type & GPX Log Runs & Sieved & $\mathrm{CaCO} 3$ & Moisture & $\begin{array}{l}\text { Chemical } \\
\text { Properties }\end{array}$ & $\begin{array}{c}\text { Physical } \\
\text { Properties }\end{array}$ & \begin{tabular}{|c|} 
Min \\
Properties \\
\end{tabular} & $\begin{array}{l}\text { Geochron } \\
\text { Properties }\end{array}$ & Archived & Source & Comments \\
\hline 2858 & 600 & 699-30-26C & & & & & & & & & & & & Goodwin 1993 & \\
\hline 2859 & & 699-30-47 & A8499 & D, As-built & NT & & Y & $\mathrm{Y}$ & $\mathrm{pH}$ & $\begin{array}{l}\text { \%GSSC, Por, } \\
\text { Bden, CEC }\end{array}$ & & & & $\begin{array}{l}\text { HWIS; Routson } \\
\text { and Fecht } 1979\end{array}$ & \\
\hline 2860 & 600 & 699-30-51 & A8500 & D & NT & & $\mathrm{Y}$ & $\mathrm{Y}$ & $\mathrm{pH}$ & $\begin{array}{l}\text { \%GSSC, Por, } \\
\text { Bden, CEC }\end{array}$ & & & & \begin{tabular}{|l|} 
Routson and \\
Fecht 1979
\end{tabular} & \\
\hline 2861 & 600 & 699-30-55 & A8501 & D & NT & & Y & $Y$ & $\mathrm{pH}$ & $\begin{array}{l}\text { \%GSSC, Por, } \\
\text { Bden, CEC }\end{array}$ & & & & \begin{tabular}{|l} 
Routson and \\
Fecht 1979
\end{tabular} & \\
\hline 2862 & 600 & 699-30-66 & C4298 & G, Well summary & SG & & & & & & & & & HWIS & \\
\hline 2863 & 600 & 699-31-11 & A8503 & D, As-built & GG, MG, NT & & & & & & & & & HWIS & \\
\hline 2864 & & 699-31-17 & A8504 & D, As-built & GG, MG, NT, SN & & & & & & & & & HWIS & \\
\hline 2865 & 600 & 699-31-30 & & & & & $\mathrm{Y}$ & & $\mathrm{pH}$ & $\begin{array}{l}\text { CEC, } \\
\text { \%GSSC, 15- } \\
\text { Atm }\end{array}$ & & & & McHenry 1957 & \\
\hline 2866 & 600 & 699-31-65 & A5124 & D, As-built & GG & & & & & & & & & HWIS & \\
\hline 2867 & & 699-31-8 & A8502 & & GG, MG, NT & & & & & & & & & & \\
\hline 2868 & 600 & 699-31-84A & A8509 & As-built & CP, GG, NT, SN & & & & & & & & & HWIS & \\
\hline 2869 & 600 & 699-31-84B & A5125 & As-built & $\mathrm{CP}, \mathrm{GG}, \mathrm{NT}$ & & & & & & & & & HWIS & \\
\hline 2870 & & $699-31-84 \mathrm{C}$ & A8510 & As-built & $\mathrm{CP}, \mathrm{GG}, \mathrm{NT}, \mathrm{SN}$ & & & & & & & & & HWIS & \\
\hline 2871 & 600 & 699-32-18 & A8511 & & GG, NT & & & & & & & & & & \\
\hline 2872 & 600 & 699-32-22 & & & $\mathrm{GG}, \mathrm{NT}$ & & & & & & & & & & \\
\hline 2873 & & 699-32-22B & A8512 & G & $\mathrm{CP}, \mathrm{GG}, \mathrm{SG}, \mathrm{TP}$ & & & & & & & & & $\begin{array}{l}\text { Chamness et al. } \\
1993\end{array}$ & Savage Island well \\
\hline 2874 & & 699-32-26 & A8513 & & GG, NT & & & & & & & & & & \\
\hline 2875 & 600 & 699-32-31 & A8514 & & GG, NT & & & & & & & & & & \\
\hline 2876 & 600 & 699-32-42 & A8516 & D, As-built & GG, NT & & & & & & & & & HWIS & \\
\hline 2877 & & 699-32-43 & A5127 & D, As-built & GG, NT & & & & & & & & & HWIS & \\
\hline 2878 & 600 & 699-32-49 & & & & & $Y$ & Y & $\mathrm{H}-3, \mathrm{pH}$ & $\begin{array}{l}\text { \%GSSC, Por, } \\
\text { Bden, CEC }\end{array}$ & & & & $\begin{array}{l}\text { Isaacson et al } \\
\text { 1974; Routson } \\
\text { and Fecht } 1979\end{array}$ & \\
\hline 2879 & 600 & 699-32-49A & A8517 & D, As-built & NT & & & & & & & & & HWIS & \\
\hline 2880 & & 699-32-49B & A8518 & D, As-built & NT & & & & & & & & & HWIS & \\
\hline 2881 & & 699-32-49D & A8520 & & NT & & & & & & & & & & \\
\hline 2882 & & 699-32-62 & A5128 & D, As-built & GG, SG & & & & & & & & & HWIS & \\
\hline 2883 & 600 & 699-32-70B & A5129 & D, As-built & GG, NT, SG & & & & & & & & & HWIS & \\
\hline 2884 & 600 & $699-32-72$ & & D & GG, NT, SG & \begin{tabular}{|l|l|} 
Virtual \\
Library
\end{tabular} & $\begin{array}{l}\text { Virtual } \\
\text { Library }\end{array}$ & & & & & & $Y$ & & \\
\hline 2885 & 600 & 699-32-72B & A9525 & G & GG, SG & Y & $Y$ & $Y$ & & $\begin{array}{l}\text { Sp. G, Bden, } \\
\text { Por }\end{array}$ & & & & $\begin{array}{l}\text { Weekes et al. } \\
1995\end{array}$ & \\
\hline 2886 & 600 & 699-32-77 & A5131 & D & GG, NT, SG & \begin{tabular}{|l|} 
Virtual \\
Library
\end{tabular} & \begin{tabular}{|l} 
Virtual \\
Library
\end{tabular} & & & & & & $Y$ & HWIS & \\
\hline 2887 & 600 & 699-33-14 & A8526 & & GG, NT & & & & & & & & & & \\
\hline 2888 & 600 & 699-33-42 & A5132 & D, As-built & GG, NT & & & & & & & & & HWIS & \\
\hline 2889 & & 699-33-6 & A8525 & & GG, NT, SN & & & & & & & & & & \\
\hline 2890 & & 699-34-39 & & & GG, NT & & & & & & & & & & \\
\hline 2891 & 600 & 699-34-39A & A5134 & D & & & & $\mathrm{Y}$ & $\mathrm{pH}$ & $\begin{array}{l}\text { CEC, } \\
\% \text { \%SSC, 15- } \\
\text { Atm }\end{array}$ & & & & McHenry 1957 & \\
\hline 2892 & & 699-34-41 & & & GG, NT & & & & & & & & & & \\
\hline 2893 & 600 & \begin{tabular}{|l}
$699-34-42$ \\
\end{tabular} & A5136 & D, As-built & GG, NT & & & & & & & & & HWIS & \\
\hline 2894 & 600 & $699-3-45$ & A5122 & D, As-built & GG, NT, SG, TP & & & & & & & & & HWIS & \\
\hline 2895 & 600 & 699-34-51 & A5137 & D, As-built & & & $Y$ & & $\mathrm{pH}$ & \begin{tabular}{|l|} 
CEC, \\
$\%$ \%GSC, 15- \\
Atm
\end{tabular} & & & & $\begin{array}{l}\text { HWIS; McHenry } \\
1957\end{array}$ & \\
\hline
\end{tabular}




\begin{tabular}{|c|c|c|c|c|c|c|c|c|c|c|c|c|c|c|c|}
\hline Sortlndex & Area & Well Name & WellID & Log Type & GPX Log Runs & Sieved & $\mathrm{CaCO} 3$ & Moisture & $\begin{array}{l}\text { Chemical } \\
\text { Properties }\end{array}$ & $\begin{array}{c}\text { Physical } \\
\text { Properties }\end{array}$ & \begin{tabular}{|c|} 
Min \\
Properties
\end{tabular} & $\begin{array}{l}\text { Geochron } \\
\text { Properties }\end{array}$ & Archived & Source & Comments \\
\hline $\begin{array}{r}2896 \\
\end{array}$ & 600 & 699-34-61 & A5463 & G & GG, SG & Y & Y & Y & & $\begin{array}{l}\text { Sp. G, Bden, } \\
\text { Por }\end{array}$ & & & & $\begin{array}{l}\text { Weekes et al. } \\
1995\end{array}$ & \\
\hline 2897 & 600 & 699-34-88 & A5138 & D, As-built & GG, NT & & & & & & & & & HWIS & \\
\hline 2898 & & 699-34-89 & & & $\mathrm{CP}, \mathrm{GG}, \mathrm{NT}$ & & $Y$ & & $\mathrm{pH}$ & $\begin{array}{l}\text { CEC, } \\
\% \text { \%SSC, 15- } \\
\text { Atm }\end{array}$ & & & & McHenry 1957 & \\
\hline 2899 & 600 & 699-34-89B & A8543 & As-built & $\mathrm{CP}, \mathrm{GG}, \mathrm{NT}, \mathrm{SN}$ & & & & & & & & & HWIS & \\
\hline 2900 & & 699-34-98 & A8544 & D, As-built & & & $Y$ & $Y$ & & $\begin{array}{l}\text { Bden, } \\
\% \text { GSSC }\end{array}$ & & & & $\begin{array}{l}\text { Virtual Library } \\
2000 \text { (PPI only, } \\
\text { includes } \\
\text { descriptions }\end{array}$ & \\
\hline 2901 & 600 & $699-35-16$ & A8549 & & GG, NT & & & & & & & & & & \\
\hline 2902 & & 699-35-19B & A8551 & & GG, NT & & & & & & & & & & \\
\hline 2903 & & 699-35-27 & A8554 & As-built & CP, NT & & & & & & & & & HWIS & \\
\hline 2904 & 600 & 699-35-28 & A8555 & & GG, NT & & & & & & & & & & \\
\hline 2905 & 600 & 699-35-58 & A8557 & As-built & & $Y$ & & & & & & & & $\begin{array}{l}\text { Bergeron et al. } \\
\text { 1987; HWIS }\end{array}$ & \\
\hline 2906 & 600 & 699-35-61A & A5456 & G & GG & Y & Y & $Y$ & & $\begin{array}{l}\text { Sp. G, Bden, } \\
\text { Por }\end{array}$ & & & & $\begin{array}{l}\text { Weekes et al. } \\
1995\end{array}$ & \\
\hline 2907 & 600 & 699-35-65A & A5454 & G & GG, SG & Y & $Y$ & $Y$ & & $\begin{array}{l}\text { Sp. G, Bden, } \\
\text { Por }\end{array}$ & & & & $\begin{array}{l}\text { Weekes et al. } \\
1995\end{array}$ & \\
\hline 2908 & 600 & 699-35-66 & & & GG, NT, SG & & & & & & & & & & \\
\hline 2909 & 600 & 699-35-66B & A5453 & G & GG, SG & $\mathrm{Y}$ & $Y$ & $Y$ & & $\begin{array}{l}\text { Sp. G, Bden, } \\
\text { Por }\end{array}$ & & & & $\begin{array}{l}\text { Weekes et al. } \\
1995\end{array}$ & \\
\hline 2910 & 600 & 699-35-68A & A5452 & G & SG & Y & $\mathrm{Y}$ & $\mathrm{Y}$ & & $\begin{array}{l}\text { Sp. G, Bden, } \\
\text { Por }\end{array}$ & & & & $\begin{array}{l}\text { Weekes et al. } \\
1995\end{array}$ & \\
\hline 2911 & 600 & 699-35-68B & A9823 & G & GG & & & & & & & & & CH2M HILL files & \\
\hline 2912 & 600 & 699-35-69A & A5451 & G & GG, SG & Y & $\mathrm{Y}$ & $Y$ & & $\begin{array}{l}\text { Sp. G, Bden, } \\
\text { Por }\end{array}$ & & & & $\begin{array}{l}\text { Weekes et al. } \\
1995\end{array}$ & \\
\hline 2913 & 600 & 699-35-69B & A9824 & G & & & & & & & & & & CH2M HILL files & \\
\hline 2914 & 600 & $699-35-70$ & A5140 & D & GG, NT, SG & & $\mathrm{Y}$ & & $\mathrm{pH}$ & $\begin{array}{l}\text { CEC, } \\
\% \text { GSSC, 15- } \\
\text { Atm }\end{array}$ & & & & McHenry 1957 & \\
\hline 2915 & 600 & 699-35-78A & A5141 & D & GG, NT & \begin{tabular}{|l} 
Virtual \\
Library
\end{tabular} & $\begin{array}{l}\text { Virtual } \\
\text { Library }\end{array}$ & & & & & & $Y$ & HWIS & \\
\hline 2916 & 600 & 699-35-78B & A8559 & As-built & $\mathrm{CP}, \mathrm{GG}, \mathrm{NT}, \mathrm{SN}$ & \begin{tabular}{|l|} 
Virtual \\
Library
\end{tabular} & $\begin{array}{l}\text { Virtual } \\
\text { Library }\end{array}$ & & & & & & $\mathrm{Y}$ & HWIS & \\
\hline 2917 & 600 & $699-35-9$ & A5142 & D, As-built & GG, NT & & & & & & & & & HWIS & \\
\hline 2918 & & 699-36-1 & A8561 & & GG, NT & & & & & & & & & & \\
\hline 2919 & & 699-36-17 & A8564 & & GG, NT & & & & & & & & & & \\
\hline 2920 & 600 & 699-36-2 & A8562 & & GG, NT, SN & & & & & & & & & & \\
\hline 2921 & 600 & 699-36-27 & A8566 & & GG, NT & & & & & & & & & & \\
\hline 2922 & 600 & 699-36-58A & A8571 & As-built & & $Y$ & & & & & & & & $\begin{array}{l}\text { Bergeron et al. } \\
\text { 1987; HWIS }\end{array}$ & \\
\hline 2923 & 600 & 699-36-58B & A8572 & As-built & & $Y$ & & & & & & & & $\begin{array}{l}\text { Bergeron et al. } \\
1987\end{array}$ & \\
\hline 2924 & 600 & 699-36-61 & & D & & Y & Y & & $\mathrm{pH}$ & $\begin{array}{l}\text { CEC, } \\
\% \text { \%SSC, 15- } \\
\text { Atm }\end{array}$ & & & & $\begin{array}{l}\text { Brown 1960; } \\
\text { McHenry 1957 }\end{array}$ & \\
\hline 2925 & 600 & 699-36-61A & A5144 & D, As-built & GG, NT, SG & & & & & & & & & HWIS & \\
\hline 2926 & 600 & 699-36-61B & A8573 & D & GG, NT, SG & Y & $\mathrm{Y}$ & & $\mathrm{pH}$ & CEC & & & & Brown 1960 & \\
\hline
\end{tabular}




\begin{tabular}{|c|c|c|c|c|c|c|c|c|c|c|c|c|c|c|c|}
\hline Sortindex & Area & Well Name & WelliD & Log Type & GPX Log Runs & Sieved & $\mathrm{CaCO} 3$ & Moisture & $\begin{array}{c}\text { Chemical } \\
\text { Properties }\end{array}$ & \begin{tabular}{|c|} 
Physical \\
Properties \\
\end{tabular} & $\begin{array}{c}\text { Min } \\
\text { Properties }\end{array}$ & $\begin{array}{l}\text { Geochron } \\
\text { Properties }\end{array}$ & Archived & Source & Comments \\
\hline 2927 & 600 & $699-36-63 A$ & A5455 & G & GG, SG & Y & Y & Y & & $\begin{array}{l}\text { Sp. G, Bden, } \\
\text { Por }\end{array}$ & & & & $\begin{array}{l}\text { Weekes et al. } \\
1995\end{array}$ & \\
\hline 2928 & 3600 & 699-36-63B & & G & & Y & Y & Y & & $\begin{array}{l}\text { Sp. G, Bden, } \\
\text { Por }\end{array}$ & & & & $\begin{array}{l}\text { Weekes et al. } \\
1995\end{array}$ & \\
\hline 2929 & 600 & 699-36-67 & B2733 & G & & & & & & & & & & CH2M HILL files & \\
\hline 2930 & 600 & 699-36-70A & A9901 & D, G, As-built & GG & & & & & & & & & HWIS & \\
\hline 2931 & 600 & 699-36-93 & A5145 & D, As-built & GG, NT & & & & & & & & & HWIS & \\
\hline 2932 & 600 & 699-36-E3 & A8574 & & $\mathrm{GG}, \mathrm{NT}, \mathrm{SN}$ & & & & & & & & & & \\
\hline 2933 & 600 & $699-37-22$ & A8577 & & & & & & & & & & & $\begin{array}{l}\text { Virtual Library } \\
2000 \text { (PPI only, } \\
\text { includes } \\
\text { descriptions }\end{array}$ & \\
\hline 2934 & 1600 & 699-37-43 & A5146 & D, As-built & GG, NT & & & & & & & & & HWIS & \\
\hline 2935 & 6000 & 699-37-47A & B2822 & G & GG & & & & & & XRD & & $\mathrm{Y}$ & $\begin{array}{l}\text { Lindberg et } \\
\text { al.1997 }\end{array}$ & \\
\hline 2936 & 600 & 699-37-68 & B2732 & G & & & & & & & & & & CH2M HILL files & \\
\hline 2937 & 600 & 699-37-82B & A8580 & D, As-built & NT & & & & & & & & & HWIS & \\
\hline 2938 & & 699-37-82D & A8582 & D, As-built & GG, NT, SN & & & & & & & & & HWIS & \\
\hline 2939 & & 699-37-83 & A8583 & As-built & $\mathrm{CP}$ & & & & & & & & & HWIS & \\
\hline 2940 & 600 & $699-37-84$ & A8584 & As-built & GG, NT & & $\mathrm{Y}$ & $\mathrm{Y}$ & & $\begin{array}{l}\text { Bden, } \\
\% \text { GSSC }\end{array}$ & & Date & & \begin{tabular}{|l|} 
HWIS; Baker et \\
al. 1991; Virtual \\
Library 2000 (PPI \\
only, includes \\
descriptions)
\end{tabular} & \\
\hline 2941 & 600 & 699-37-89 & A8585 & As-built & $\mathrm{CP}$ & & & & & & & & & HWIS & \\
\hline 2942 & 600 & 699-37-92 & A8586 & As-built & GG, NT & & & & XRF & & $\begin{array}{l}\mathrm{PC}, \mathrm{PbC}, \\
\text { XRD }\end{array}$ & & & \begin{tabular}{|l|} 
HWIS; Goodwin \\
1993; Virtual \\
Library 2000 (PPI \\
only, includes \\
descriptions); \\
Horton files
\end{tabular} & \\
\hline 2943 & 3600 & 699-37-E1 & A8587 & & GG, NT & & & & & & & & & & \\
\hline 2944 & & 699-38-15 & A8594 & $D, G$ & GG, NT & & & & & & & & & HWIS & \\
\hline 2945 & & $699-38-19$ & A8595 & & GG, NT & & & & & & & & & & \\
\hline 2946 & 600 & 699-38-34 & & & GG, NT & & & & & & & & & & \\
\hline 2947 & 600 & 699-38-43 & & & & & $\mathrm{Y}$ & & $\mathrm{pH}$ & $\begin{array}{l}\text { CEC, } \\
\% \text { \%SSC, 15- } \\
\text { Atm }\end{array}$ & & & & McHenry 1957 & \\
\hline 2948 & 3600 & 699-38-61 & A5464 & G & GG, SG & $Y$ & $Y$ & $Y$ & & $\begin{array}{l}\text { Sp. G, Bden, } \\
\text { Por }\end{array}$ & & & & $\begin{array}{l}\text { Weekes et al. } \\
1995\end{array}$ & \\
\hline 2949 & 600 & 699-38-65 & A5148 & D, As-built & GG, SG & & & & & & & & & HWIS & \\
\hline 2950 & 600 & 699-38-68A & A9516 & Well summary & GG, SG & $\mathrm{Y}$ & & & & & & & & $\begin{array}{l}\text { HWIS, see R. } \\
\text { Khaleel or G. } \\
\text { Freeman for } \\
\text { sieve data }\end{array}$ & \\
\hline 2951 & 600 & 699-38-70 & A5149 & D, As-built & GG, NT, SG & & & & & & & & & HWIS & \\
\hline 2952 & 600 & \begin{tabular}{|l|l|}
$699-38-70 B$ \\
\end{tabular} & C4236 & $G$ & SG & & & & & & & & & Martinez 2004 & 200-UP-1 \\
\hline 2953 & 3600 & $699-38-70 \mathrm{C}$ & C4256 & G & SG & & & & & & & & & Martinez 2004 & 200-UP-1 \\
\hline
\end{tabular}




\begin{tabular}{|c|c|c|c|c|c|c|c|c|c|c|c|c|c|c|c|}
\hline Sortindex & Area & Well Name & WellID & Log Type & GPX Log Runs & Sieved & $\mathrm{CaCO} 3$ & Moisture & $\begin{array}{l}\text { Chemical } \\
\text { Properties }\end{array}$ & $\begin{array}{c}\text { Physical } \\
\text { Properties }\end{array}$ & $\begin{array}{c}\text { Min } \\
\text { Properties } \\
\end{array}$ & $\begin{array}{l}\text { Geochron } \\
\text { Properties }\end{array}$ & Archived & Source & Comments \\
\hline 2954 & 600 & $699-38-9$ & A8592 & & GG, NT & & & & & & & & & & \\
\hline 2955 & 600 & 699-38-E0 & A8599 & & GG, NT & & & & & & & & & & \\
\hline 2956 & 600 & 699-39-0 & A8600 & D & GG, NT & & & & & & & & & HWIS & \\
\hline 2957 & & 699-39-103 & A8617 & As-built & $\mathrm{CP}, \mathrm{GG}, \mathrm{NT}$ & & & & & & & & & HWIS & \\
\hline 2958 & 600 & 699-39-23B & & & GG, NT & & & & & & & & & & \\
\hline 2959 & 600 & 699-39-39 & A5150 & D, As-built & GG, NT & & & & & & & & & HWIS & \\
\hline 2960 & & 699-39-79 & A5151 & D, As-built & GG, NT & & & & & & & & & HWIS & \\
\hline 2961 & 600 & 699-39-7A & A8603 & As-built & GG, MG, NT, SG, SN & & & & & & & & & HWIS & \\
\hline 2962 & 600 & 699-39-7B & A8604 & As-built & NT, SG & & & & & & & & & HWIS & \\
\hline 2963 & 600 & 699-39-7F & B2606 & As-built & $\mathrm{GG}$ & & & & & & & & & HWIS & \\
\hline 2964 & & 699-39-7H & B2608 & As-built & GG, NT, SG & & & & & & & & & HWIS & \\
\hline 2965 & 600 & 699-39-7L & B2612 & As-built & GG, NT & & & & & & & & & HWIS & \\
\hline 2966 & 600 & 699-39-7M & B2613 & As-built & GG, NT, SG & & & & & & & & & HWIS & \\
\hline 2967 & 600 & 699-39-7N & B2614 & As-built & $\mathrm{GG}, \mathrm{NT}$ & & & & & & & & & HWIS & \\
\hline 2968 & & 699-39-70 & B2615 & As-built & GG, NT & & & & & & & & & HWIS & \\
\hline 2969 & 600 & 699-39-82 & A8607 & As-built & GG, NT & & & & & & & & & HWIS & \\
\hline 2970 & 600 & 699-39-84A & A8608 & As-built & $\mathrm{CP}, \mathrm{GG}, \mathrm{NT}, \mathrm{OT}, \mathrm{SN}$ & & & & & & & & & HWIS & \\
\hline 2971 & 600 & 699-39-84B & A8609 & As-built & $\mathrm{CP}, \mathrm{GG}, \mathrm{NT}$ & & & & & & & & & HWIS & \\
\hline 2972 & 600 & 699-39-84C & A8610 & & $\mathrm{CP}, \mathrm{GG}, \mathrm{NT}, \mathrm{SN}$ & & & & & & & & & & \\
\hline 2973 & 600 & $699-40-1$ & A5152 & D, As-built & GG, NT & & & & & & & & & HWIS & \\
\hline 2974 & 600 & 699-40-12A & A8627 & D, As-built & GG & & & & & & & & & HWIS & \\
\hline 2975 & 600 & 699-40-12B & A8628 & & GG, NT & & & & & & & & & & \\
\hline 2976 & 600 & $699-40-12 \mathrm{C}$ & A8629 & & GG, NT & & & & & & & & & & \\
\hline 2977 & 600 & 699-40-13 & A8636 & & GG, NT & & & & & & $\mathrm{PC}, \mathrm{PbC}$ & & & Goodwin 1993 & \\
\hline 2978 & 600 & $699-40-20$ & A8637 & & GGNT & & & & & & & & & & \\
\hline 2979 & & $699-40-33$ & & D & & & Y & & $\mathrm{pH}$ & $\begin{array}{l}\text { CEC, } \\
\% \text { GSSC, 15- } \\
\text { Atm }\end{array}$ & & & & McHenry 1957 & \\
\hline 2980 & 600 & $699-40-33 C$ & A8642 & D, As-built & GG, NT, SN & & & & & & & & & HWIS & \\
\hline 2981 & 600 & $699-40-35$ & & G & & $Y$ & $\bar{Y}$ & Y & Field Rad & Por, SpG & & & $Y$ & Sweeney 1992 & \\
\hline 2982 & 600 & 699-40-36 & A5154 & G & $G G, S G$ & $Y$ & Y & Y & $\begin{array}{l}\text { Field Rad, } \\
\text { Metals, An, } \\
\text { VOA, SVOA, } \\
\text { CrIV, CN }\end{array}$ & Por, SpG & & & $Y$ & \begin{tabular}{|l|} 
Barnett 1993; \\
Sweeney 1992; \\
PNNL files
\end{tabular} & \\
\hline 2983 & 600 & 699-40-37 & & G & & & Y & & Field Rad & Por, SpG & & & $Y$ & Sweeney 1992 & \\
\hline 2984 & 600 & 699-40-39 & A5155 & G & GG & \begin{tabular}{|l|}
$\begin{array}{l}\text { Virtual } \\
\text { Library }\end{array}$ \\
\end{tabular} & & Y & $\begin{array}{l}\text { An, Rad, TOC, } \\
\text { XRF }\end{array}$ & & & & $Y$ & PNNL files & \\
\hline 2985 & 600 & 699-40-39B & & G & & & & & & & & & & CH2M HILL files & \\
\hline 2986 & 600 & 699-40-40A & A5156 & G & GG & & & & & & & & $Y$ & $\begin{array}{l}\text { Delaney 1992; } \\
\text { CH2M HILL files }\end{array}$ & \\
\hline 2987 & 600 & 699-40-40B & A5157 & G & GG, SG & & & & & & & & $\mathrm{Y}$ & Delaney 1992a & \\
\hline 2988 & 600 & 699-40-43 & & & GG & & & & & & & & & & \\
\hline 2989 & 600 & $699-40-6$ & A8621 & & $\mathrm{GG}, \mathrm{NT}$ & & & & & & & & & & \\
\hline 2990 & 600 & 699-40-62 & A5158 & D & $G G, N T$ & & $\mathrm{Y}$ & & $\mathrm{pH}$ & $\begin{array}{l}\text { CEC, } \\
\% \text { \%SSC, 15- } \\
\text { Atm }\end{array}$ & & & & McHenry 1957 & \\
\hline 2991 & 600 & 699-40-65 & C4235 & G & SG & & & & & & & & & Martinez 2004 & 200-UP-1 \\
\hline 2992 & 600 & 699-40-80 & A8643 & D & $\mathrm{CP}, \mathrm{GG}, \mathrm{NT}$ & & & & & & & & $Y$ & HWIS & \\
\hline
\end{tabular}




\begin{tabular}{|c|c|c|c|c|c|c|c|c|c|c|c|c|c|c|c|}
\hline Sortlndex & Area & Well Name & WellID & Log Type & GPX Log Runs & Sieved & $\mathrm{CaCO} 3$ & Moisture & $\begin{array}{l}\text { Chemical } \\
\text { Properties }\end{array}$ & $\begin{array}{c}\text { Physical } \\
\text { Properties }\end{array}$ & \begin{tabular}{|c|} 
Min \\
Properties \\
\end{tabular} & $\begin{array}{l}\text { Geochron } \\
\text { Properties }\end{array}$ & Archived & Source & Comments \\
\hline 2993 & 600 & 699-40-84 & A8644 & As-built & GG, NT & & $Y$ & $Y$ & XRF & $\begin{array}{l}\text { Bden, } \\
\% \text { GSSC }\end{array}$ & $\begin{array}{l}\text { XRD, PC, } \\
\text { PbC }\end{array}$ & Date & & $\begin{array}{l}\text { HWIS; Baker et } \\
\text { al. 1991; } \\
\text { Goodwin 1993; } \\
\text { Lindsey 1991; } \\
\text { Virtual Library } \\
2000 \text { (PPI only, } \\
\text { includes } \\
\text { descriptions); } \\
\text { Horton files }\end{array}$ & \\
\hline 2994 & 600 & $699-41-1$ & & & GG, NT & & & & & & & & & & \\
\hline 2995 & & $699-41-25$ & A8652 & & GG, NT & & & & & & & & & & \\
\hline 2996 & & 699-41-31 & A8653 & & GG, NT & & & & & & & & & & \\
\hline 2997 & 600 & 699-41-35 & A5160 & G & GG, SG & Y & $\mathrm{Y}$ & $Y$ & $\begin{array}{l}\text { Metals, An, } \\
\text { VOA, SVOA, } \\
\text { CrIV, CN }\end{array}$ & & & & & \begin{tabular}{|l|} 
Barnett 1993; \\
PNNL files, see \\
R. Khaleel or \\
G. Freeman for \\
sieve data
\end{tabular} & \\
\hline 2998 & 600 & 699-41-39 & & & & $\mathrm{Y}$ & & & & & & & & $\begin{array}{l}\text { see R. Khaleel or } \\
\text { G. Freeman for } \\
\text { sieve data }\end{array}$ & \\
\hline 2999 & 600 & 699-41-40 & A5161 & G & CP, GG, OT & \begin{tabular}{|l|} 
Virtual \\
Library
\end{tabular} & & $Y$ & $\begin{array}{l}\text { An, Rad, TOC, } \\
\text { XRF }\end{array}$ & & & & & PNNL files & \\
\hline 3000 & 600 & 699-41-41 & A8655 & G & GG & & & & & & & & $\mathrm{Y}$ & HWIS & \\
\hline 3001 & 600 & 699-41-42 & A5162 & G & GG, MG & & $Y$ & $Y$ & & & & & $Y$ & Delaney 1993 & \\
\hline 3002 & 600 & $699-41-5$ & A8648 & & $\begin{array}{l}\text { CP, GG, MG, NT, SG, } \\
\text { SN }\end{array}$ & & & & & & & & & & \\
\hline 3003 & 600 & 699-41-72 & & & MG, SG & & & & & & & & & & \\
\hline 3004 & 600 & 699-41-91 & A8659 & As-built & $\mathrm{CP}, \mathrm{GG}, \mathrm{NT}, \mathrm{OT}, \mathrm{SN}$ & & & & & & & & & HWIS & \\
\hline 3005 & 600 & 699-42-10 & A8662 & & GG, NT & & & & & & & & & & \\
\hline 3006 & & $699-42-12 B$ & A8663 & D, As-built & GG, NT & & & & & & & & & HWIS & \\
\hline 3007 & 600 & $699-42-2$ & A8660 & D, As-built & GG, NT & & & & & & & & & HWIS & \\
\hline 3008 & 600 & 699-42-21 & A8665 & & GG, NT & & & & & & & & & & \\
\hline 3009 & 600 & $699-42-30$ & A8668 & & & & & & & & $\mathrm{PC}, \mathrm{PbC}$ & & & Goodwin 1993 & \\
\hline 3010 & 600 & 699-42-37 & A5164 & G & GG, SG & $\mathrm{Y}$ & & $Y$ & $\begin{array}{l}\text { Metals, An, } \\
\text { VOA, SVOA, } \\
\text { CrIV, CN }\end{array}$ & $\begin{array}{l}\text { SpG, Bden, } \\
\text { Por }\end{array}$ & & & & $\begin{array}{l}\text { Barnett 1993; } \\
\text { PNNL files, see } \\
\text { R. Khaleel or } \\
\text { G. Freeman for } \\
\text { sieve data }\end{array}$ & \\
\hline 3011 & 600 & $699-42-39 A$ & A5165 & G & GG & & & & & & & & $Y$ & Delaney 1992a & \\
\hline 3012 & 600 & 699-42-39B & A5166 & G & GG & & & & & & & & $\mathrm{Y}$ & Delaney 1992a & \\
\hline 3013 & 600 & 699-42-40A & A5167 & $D, G$ & & $Y$ & $\mathrm{Y}$ & $Y$ & & SpG, Por & & & $\mathrm{Y}$ & HWIS & \\
\hline 3014 & 600 & $699-42-40 \mathrm{~B}$ & A5168 & D, G & & $\mathrm{Y}$ & $\mathrm{Y}$ & $\mathrm{Y}$ & & SpG, Por & & & $\mathrm{Y}$ & HWIS & \\
\hline 3015 & 600 & $699-42-40 \mathrm{C}$ & A5169 & D & $\mathrm{CP}, \mathrm{GG}, \mathrm{NT}, \mathrm{SN}$ & & & & & & & & $Y$ & HWIS & \\
\hline 3016 & 600 & 699-42-41 & A5170 & G & & $\mathrm{Y}$ & $\mathrm{Y}$ & $\mathrm{Y}$ & & SpG, Por & & & $\mathrm{Y}$ & Delaney 1992a & \\
\hline 3017 & & $699-42-41 \mathrm{~A}$ & A9914 & As-built & $\mathrm{GG}$ & & & & & & & & & HWIS & \\
\hline 3018 & 600 & $699-42-41 \mathrm{~B}$ & A8669 & $\mathrm{D}, \mathrm{G}, \mathrm{As}$-built & GG & & & & & & & & & HWIS & \\
\hline 3019 & 600 & $699-42-42 A$ & A8670 & D & GG, NT & & & & & & & & $Y$ & HWIS & \\
\hline
\end{tabular}




\begin{tabular}{|c|c|c|c|c|c|c|c|c|c|c|c|c|c|c|c|}
\hline Sortindex & Area & Well Name & WellID & Log Type & GPX Log Runs & Sieved & $\mathrm{CaCO} 3$ & Moisture & $\begin{array}{l}\text { Chemical } \\
\text { Properties }\end{array}$ & $\begin{array}{l}\text { Physical } \\
\text { Properties }\end{array}$ & $\begin{array}{c}\text { Min } \\
\text { Properties } \\
\end{array}$ & $\begin{array}{l}\text { Geochron } \\
\text { Properties }\end{array}$ & Archived & Source & Comments \\
\hline 3020 & 600 & 699-42-42B & A5171 & G & $\mathrm{DN}, \mathrm{GG}, \mathrm{NT}$ & Y & Y & Y & & & & & & \begin{tabular}{|l|} 
Delaney $1992 b ;$ \\
Luttrell et al. \\
1991
\end{tabular} & \\
\hline 3021 & 600 & \begin{tabular}{|l|}
$699-42-88$ \\
\end{tabular} & A8672 & G, As-built & $\mathrm{CP}, \mathrm{GG}, \mathrm{NT}, \mathrm{SN}$ & & & & & & & & & \begin{tabular}{|l|} 
HWIS \\
\end{tabular} & \\
\hline 3022 & 600 & \begin{tabular}{|l|}
$699-42-E 9 A$ \\
\end{tabular} & A8673 & $\mathrm{D}, \mathrm{G}$ & $\mathrm{CP}, \mathrm{GG}, \mathrm{SG}, \mathrm{TP}$ & & & & Wet Chem & & & & & \begin{tabular}{|l|} 
DOE 1993c; \\
Chamness et al. \\
1993
\end{tabular} & Savage Island well \\
\hline 3023 & 600 & 699-42-E9B & A8674 & G & $\mathrm{CP}, \mathrm{GG}, \mathrm{MG}, \mathrm{OT}, \mathrm{TP}$ & & & & & & & & & \begin{tabular}{|l|} 
Chamness et al. \\
1993
\end{tabular} & Savage Island well \\
\hline 3024 & 600 & 699-43-104 & A5172 & D, As-built & GG, NT, SG & & & & & & & & & HWIS & \\
\hline 3025 & & 699-43-18 & A8680 & & GG, NT & & & & & & & & & & \\
\hline 3026 & 600 & 699-43-23 & A8681 & & $\mathrm{GG}, \mathrm{NT}$ & & & & & & & & & & \\
\hline 3027 & 600 & 699-43-3 & A8677 & D, As-built & GG, NT & & & & & & & & & HWIS & \\
\hline 3028 & 600 & 699-43-40 & A5173 & G & $\mathrm{GG}, \mathrm{MG}, \mathrm{SG}$ & Y & $\mathrm{Y}$ & $\mathrm{Y}$ & & SpG, Por & & & $\mathrm{Y}$ & Delaney 1992a & \\
\hline 3029 & 600 & $699-43-41 \mathrm{~A}$ & A8682 & $D, G$ & & & & & & & & & $\bar{Y}$ & HWIS & \\
\hline 3030 & 600 & $699-43-41 \mathrm{~B}$ & 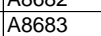 & $\frac{1,6}{D}$ & & & & & & & & & $\frac{T}{Y}$ & \begin{tabular}{|l} 
HWIS \\
\end{tabular} & \\
\hline 3031 & & $699-43-41 \mathrm{C}$ & A8684 & D & & & & & & & & & & HWIS & \\
\hline 3032 & 600 & 699-43-41D & A8685 & D & & & & & & & & & & HWIS & \\
\hline 3033 & 600 & \begin{tabular}{|l|}
$699-43-41 E$ \\
\end{tabular} & A5174 & G & GG & $\begin{array}{l}\text { Virtual } \\
\text { Library }\end{array}$ & & & $\begin{array}{l}\text { An, Rad, TOC, } \\
\text { XRF }\end{array}$ & & & & Y & PNNL files & \\
\hline 3034 & 600 & \begin{tabular}{|l|}
$699-43-41 \mathrm{~F}$ \\
\end{tabular} & A5175 & G & GG & $\begin{array}{l}\text { Virtual } \\
\text { Library }\end{array}$ & & Y & $\begin{array}{l}\text { An, Rad, TOC, } \\
\text { XRF }\end{array}$ & & & & $\mathrm{Y}$ & PNNL files & \\
\hline 3035 & 600 & 699-43-41G & A5176 & G & GG, SG & $\mathrm{Y}$ & $\mathrm{Y}$ & $\mathrm{Y}$ & & SpG, Por & & & $\mathrm{Y}$ & Delaney 1992a & \\
\hline 3036 & 600 & 699-43-41H & A8686 & D, G, Well summary & GG & & & & & & & & & HWIS & \\
\hline 3037 & 600 & $699-43-41 \mathrm{~K}$ & & G & & & & & & & & & $\bar{Y}$ & PNNL files & \\
\hline 3038 & & 699-43-42 & A5177 & D, As-built & GG, NT & & & & & & & & & HWIS & \\
\hline 3039 & & 699-43-42A & A8687 & $\mathrm{D}, \mathrm{G}$ & & & & & & & & & Y & HWIS & \\
\hline 3040 & 600 & 699-43-42B & A8688 & D & & & & & & & & & $\mathrm{Y}$ & HWIS & \\
\hline 3041 & 600 & $699-43-42 C$ & A8689 & D & & & & & & & & & $\begin{array}{r} \\
Y\end{array}$ & HWIS & \\
\hline 3042 & 600 & $699-43-42 \mathrm{D}$ & A8690 & D & & & & & & & & & Y & HWIS & \\
\hline 3043 & 600 & 699-43-42E & A8691 & D & & & & & & & & & & HWIS & \\
\hline 3044 & 600 & 699-43-42F & A8692 & G & & & & $Y$ & & & & & $\bar{Y}$ & Delaney 1992b & \\
\hline 3045 & 600 & $699-43-42 \mathrm{G}$ & A8693 & $\mathrm{D}$ & & & & & & & & & & HWIS & \\
\hline 3046 & 600 & $699-43-42 \mathrm{H}$ & A8694 & $\mathrm{D}$ & & & & & & & & & $\mathrm{Y}$ & HWIS & \\
\hline 3047 & 600 & \begin{tabular}{|l|}
$699-43-42 J$ \\
\end{tabular} & A5178 & G & DN, GG, NT & $Y$ & Y & $Y$ & & & & & $\mathrm{Y}$ & $\begin{array}{l}\text { Luttrell et al. } \\
1991\end{array}$ & \\
\hline 3048 & 600 & 699-43-42K & A8695 & D, G, As-built & $\mathrm{DN}, \mathrm{GG}, \mathrm{NT}$ & & & & & & & & & HWIS & \\
\hline 3049 & 600 & $\mid 699-43-43$ & A5179 & G & DN, GG, NT & \begin{tabular}{|l|} 
Virtual \\
Library
\end{tabular} & $\mathrm{Y}$ & $\mathrm{Y}$ & & & & & $\mathrm{Y}$ & $\begin{array}{l}\text { Delaney 1992b; } \\
\text { Luttrell et al. } \\
1991\end{array}$ & \\
\hline 3050 & 600 & 699-43-44 & B8758 & G & NT, SG & & & & $\begin{array}{l}\text { Metals, An, } \\
\text { SVOA, VOA, } \\
\text { Lab Rad }\end{array}$ & & & & & $\begin{array}{l}\text { Cearlock et al. } \\
2000\end{array}$ & \\
\hline 3051 & 600 & 699-43-45 & A5180 & G & GG & $\begin{array}{l}\text { Virtual } \\
\text { Library }\end{array}$ & & $Y$ & $\begin{array}{l}\text { An, Rad, TOC, } \\
\text { XRF }\end{array}$ & & & & $\mathrm{Y}$ & PNNL files & \\
\hline 3052 & 600 & 699-43-81 & A8696 & As-built & CP, GG, NT & & & & & & & & & HWIS & \\
\hline 3053 & 600 & \begin{tabular}{|l|}
$699-43-83$ \\
\end{tabular} & A8697 & D, As-built & GG, NT & & & & & & & & & HWIS & \\
\hline
\end{tabular}




\begin{tabular}{|c|c|c|c|c|c|c|c|c|c|c|c|c|c|c|c|}
\hline Sortlndex & Area & Well Name & WelliD & Log Type & GPX Log Runs & Sieved & $\mathrm{CaCO} 3$ & Moisture & $\begin{array}{l}\text { Chemical } \\
\text { Properties }\end{array}$ & $\begin{array}{c}\text { Physical } \\
\text { Properties }\end{array}$ & \begin{tabular}{|c|} 
Min \\
Properties \\
\end{tabular} & $\begin{array}{l}\text { Geochron } \\
\text { Properties }\end{array}$ & Archived & Source & Comments \\
\hline 3054 & 600 & 699-43-84 & A8698 & As-built & GG, NT, SN & & Y & Y & Stlso & \begin{tabular}{|l|} 
Bden, \\
$\% G S S C$
\end{tabular} & XRD & \begin{tabular}{|l|} 
Date, \\
Paleomag
\end{tabular} & & \begin{tabular}{|l|} 
HWIS; Baker et \\
al. 1991; Virtual \\
Library 2000 (PPI \\
only, includes \\
descriptions; \\
Horton files; \\
Bjornstad 1984; \\
WCC 1982
\end{tabular} & \\
\hline 3055 & 600 & 699-43-89 & A5181 & D, As-built & GG, NT, SN & & & & & & & & & HWIS & \\
\hline 3056 & & $699-43-9$ & A8679 & & GG, NT & & & & & & & & & & \\
\hline 3057 & & 699-43-91A & A8700 & As-built & $\mathrm{CP}, \mathrm{GG}, \mathrm{NT}, \mathrm{SN}$ & & & & & & & & & HWIS & \\
\hline 3058 & 600 & 699-43-91B & A8701 & As-built & $\mathrm{CP}, \mathrm{GG}, \mathrm{NT}, \mathrm{SN}$ & & & & & & & & & HWIS & \\
\hline 3059 & & 699-43-91D & A5184 & As-built & $\mathrm{CP}, \mathrm{GG}, \mathrm{NT}, \mathrm{SN}$ & & & & & & & & & HWIS & \\
\hline 3060 & & 699-44-16 & A8705 & & GG, NT & & & & & & & & & & \\
\hline 3061 & 600 & $699-44-28$ & A8707 & & GG & & & & & & & & & & \\
\hline 3062 & 600 & 699-44-39B & A5185 & G & GG, SG & & $\mathrm{Y}$ & $Y$ & & & & & $\mathrm{Y}$ & Delaney 1993 & \\
\hline 3063 & 600 & $699-44-4$ & A8703 & D & GG, NT & & & & & & & & & & \\
\hline 3064 & 600 & 699-44-42 & A5186 & G & GG & \begin{tabular}{|l} 
Virtual \\
Library
\end{tabular} & $\mathrm{Y}$ & $Y$ & & & & & $Y$ & $\begin{array}{l}\text { Delaney 1992b; } \\
\text { Luttrell et al. } \\
1991\end{array}$ & \\
\hline 3065 & 600 & 699-44-43A & A8710 & $D, G$ & & & & & & & & & $\mathrm{Y}$ & HWIS & \\
\hline 3066 & & 699-44-43B & A5187 & G & GG & $\mathrm{Y}$ & & $Y$ & $\begin{array}{l}\text { An, Rad, TOC, } \\
\text { XRF }\end{array}$ & & & & $\mathrm{Y}$ & PNNL files & \\
\hline 3067 & 600 & 699-44-64 & A5188 & D, As-built & GG, NT & & & & & & & & & HWIS & \\
\hline 3068 & 600 & 699-44-7 & A8704 & & $\begin{array}{l}\text { GG, MG, NT, OT, SG, } \\
\text { TP }\end{array}$ & & & & & & & & & & \\
\hline 3069 & 600 & 699-44-70 & A8712 & As-built & $\mathrm{CP}, \mathrm{GG}, \mathrm{NT}, \mathrm{SN}$ & & & & & & & & & HWIS & \\
\hline 3070 & 600 & 699-44-7S & & & MG & & & & & & & & & & \\
\hline 3071 & 600 & 699-44-91 & A8713 & As-built & CP, GG, NT, SN & & & & & & & & & HWIS & \\
\hline 3072 & & $699-45-2$ & A8715 & As-built & GG, NT & & & & & & & & & HWIS & \\
\hline 3073 & & 699-45-24 & A8719 & & GG, NT & & & & & & & & & & \\
\hline 3074 & 600 & 699-45-30 & A8721 & & GG, NT & & & & & & & & & & \\
\hline 3075 & & $699-45-4$ & A8716 & D & $\mathrm{GG}, \mathrm{NT}$ & & & & & & & & & HWIS & \\
\hline 3076 & 600 & 699-45-42 & A5195 & D, As-built & GG, NT & & $Y$ & & $\mathrm{pH}$ & $\begin{array}{l}\text { CEC, } \\
\% \text { GSSC, 15- } \\
\text { Atm }\end{array}$ & & & & $\begin{array}{l}\text { HWIS; McHenry } \\
1957\end{array}$ & \\
\hline 3077 & 600 & 699-45-69A & A5196 & D, As-built & GG, NT & & & & & & & & & HWIS & \\
\hline 3078 & & $699-45-78$ & A8723 & D & GG, NT, SN & & & & & & & & $Y$ & HWIS & \\
\hline 3079 & 600 & $699-46-21 B$ & A5197 & D, As-built & GG, NT & & & & & & & & & HWIS & \\
\hline 3080 & 600 & $699-46-3$ & A8725 & & GG, NT & & & & & & & & & & \\
\hline 3081 & & 699-46-31 & A8735 & & $\mathrm{GG}$ & & & & & & & & & & \\
\hline 3082 & & 699-46-32 & A8736 & & GG, NT & & & & & & & & & & \\
\hline 3083 & 600 & $699-46-33$ & A8737 & & GG, NT & & & & & & & & & & \\
\hline 3084 & 600 & $699-46-4$ & A8726 & D, As-built & $\mathrm{GG}, \mathrm{NT}$ & & & & & & & & & HWIS & \\
\hline 3085 & & 699-46-46 & A8738 & G & & \begin{tabular}{|l|l|} 
Virtual \\
Library
\end{tabular} & $\begin{array}{l}\text { Virtual } \\
\text { Library }\end{array}$ & & & & & & $Y$ & HWIS & \\
\hline 3086 & 600 & 699-46-5 & A8727 & As-built & $\begin{array}{l}\text { GG, MG, NT, OT, SG, } \\
\text { SN, TP }\end{array}$ & & & & & & & & & HWIS & \\
\hline 3087 & 600 & 699-46-5S & & & MG & & & & & & & & & & \\
\hline 3088 & 600 & \begin{tabular}{|l|}
$699-46-79$ \\
\end{tabular} & A8739 & $\mathrm{D}$ & $\mathrm{DN}, \mathrm{GG}$ & & & & & & & & & HWIS & \\
\hline
\end{tabular}




\begin{tabular}{|c|c|c|c|c|c|c|c|c|c|c|c|c|c|c|c|}
\hline Sortlndex & Area & Well Name & WellID & Log Type & GPX Log Runs & Sieved & $\mathrm{CaCO} 3$ & Moisture & $\begin{array}{l}\text { Chemical } \\
\text { Properties }\end{array}$ & $\begin{array}{c}\text { Physical } \\
\text { Properties }\end{array}$ & $\begin{array}{c}\text { Min } \\
\text { Properties } \\
\end{array}$ & $\begin{array}{l}\text { Geochron } \\
\text { Properties }\end{array}$ & Archived & Source & Comments \\
\hline 3089 & 500 & 699-46-85 & & & & & & & & & & Paleomag & & $\begin{array}{l}\text { Virtual Library } \\
2000 \text { (PPI only, } \\
\text { includes } \\
\text { descriptions); } \\
\text { WCC 1982 }\end{array}$ & \\
\hline 3090 & & 699-46-85A & A8741 & As-built & GG, NT & & & & & & & & & HWIS & \\
\hline 3091 & & 699-46-85B & \begin{tabular}{|l|} 
A8742 \\
A8747
\end{tabular} & As-built & CP, GG, NT & & & & & & & & & HWIS & \\
\hline & & $699-47-25$ & A8747 & & GG & & & & & & & & & & \\
\hline 3093 & & 699-47-35B & A5199 & D, As-built & GG, NT & & & & & & & & & HWIS & \\
\hline 3094 & & $699-47-42$ & A8749 & As-built & $\mathrm{CP}, \mathrm{GG}, \mathrm{NT}, \mathrm{SN}$ & & & & & & & & & HWIS & \\
\hline 3095 & & 699-47-46A & A5200 & $\mathrm{D}$ & GG, NT & & & & & & & & $\bar{Y}$ & HWIS & \\
\hline 3096 & & \begin{tabular}{|l|l|}
$699-47-5$ \\
\end{tabular} & A8744 & D, As-built & GG, NT & & & & & & & & & HWIS & \\
\hline 3097 & & 699-47-50 & A5201 & D, As-built & $\mathrm{CP}, \mathrm{GG}, \mathrm{NT}, \mathrm{SN}$ & & & & & & & & & HWIS & \\
\hline 3098 & & 699-47-51 & A8752 & D & GG, NT & & & & & & & & & HWIS & \\
\hline 3099 & & 699-47-60 & A5202 & D & GG, NT & & $\mathrm{Y}$ & & $\mathrm{pH}$ & $\begin{array}{l}\text { CEC, } \\
\% \text { \%SSC, 15- } \\
\text { Atm }\end{array}$ & & & & McHenry 1957 & \\
\hline 3100 & & 699-47-80A & A8753 & As-built & $\mathrm{CP}, \mathrm{GG}, \mathrm{NT}, \mathrm{SN}$ & & & & & & & & & HWIS & \\
\hline 3101 & & 699-47-80B & A8754 & As-built & CP, GG, NT, SN & & & & & & & & & HWIS & \\
\hline 3102 & 500 & $699-47-80 \mathrm{C}$ & A8755 & As-built & $\mathrm{CP}, \mathrm{GG}, \mathrm{NT}, \mathrm{OT}, \mathrm{SN}$ & & & & & & & & & HWIS & \\
\hline 3103 & & 699-47-80D & A8756 & As-built & $\mathrm{CP}, \mathrm{GG}, \mathrm{NT}, \mathrm{OT}, \mathrm{SN}$ & & & & & & & & & HWIS & \\
\hline 3104 & & $699-47-92$ & & & $\mathrm{CP}, \mathrm{GG}, \mathrm{NT}$ & & & & & & & & & & \\
\hline 3105 & & \begin{tabular}{|l|}
$699-48-18$ \\
\end{tabular} & A8764 & $\mathrm{D}$, As-built & GG, NT & & & & & & & & & HWIS & \\
\hline 3106 & & $699-48-35$ & A8767 & & GG, NT, SG & & & & & & & & & & \\
\hline 3107 & & 699-48-3S & & & GG & & & & & & & & & & \\
\hline 3108 & & \begin{tabular}{|l|}
$699-48-42$ \\
\end{tabular} & & & CP, DN, GG, NT, OT & & & & & & & & & & \\
\hline 3109 & & 699-48-48A & A8768 & D, G, As-built & $\begin{array}{l}\text { CP, DN, NT, OT, SN, } \\
\text { TP }\end{array}$ & & & & & & & & & HWIS & \\
\hline 3110 & & 699-48-48B & A8769 & D, G, As-built & $\mathrm{CP}, \mathrm{GG}, \mathrm{NT}, \mathrm{SN}$ & & & & & & & & & HWIS & \\
\hline 3111 & 500 & 699-48-50 & A5212 & G & GG & $\bar{Y}$ & $\mathrm{Y}$ & $\mathrm{Y}$ & & \begin{tabular}{|l|} 
SpG, Por, \\
Bden
\end{tabular} & & & $\bar{Y}$ & $\begin{array}{l}\text { Hoffman 1992; } \\
\text { HWIS }\end{array}$ & 200-BP-1 \\
\hline 3112 & 600 & 699-48-71 & A5214 & D, As-built & GG, NT & & & & & & & & & HWIS & \\
\hline 3113 & & 699-48-77 & & & $\mathrm{GG}, \mathrm{SG}$ & & & & & & & & & & \\
\hline 3114 & & 699-48-77A & A8772 & G & GG, SG & & & & $\begin{array}{l}\text { Metals, An, } \\
\text { Alpha, Beta, } \\
\text { pH }\end{array}$ & & & & & $\begin{array}{l}\text { CH2M HILL files; } \\
\text { Laurenz } 1993\end{array}$ & \\
\hline 3115 & 500 & 699-48-77B & A8773 & G & & & & & $\begin{array}{l}\text { Metals, An, } \\
\text { Alpha, Beta, } \\
\text { pH }\end{array}$ & & & & & $\begin{array}{l}\text { CH2M HILL files; } \\
\text { Laurenz } 1993\end{array}$ & \\
\hline 3116 & & $699-48-77 \mathrm{C}$ & A8774 & G, As-built & GG, SG & & & & & & & & & HWIS & \\
\hline 3117 & 500 & 699-48-77D & A8775 & G & GG, SG & & & & & & & & & CH2M HILL files & \\
\hline 3118 & 500 & 699-48-96 & A8776 & G & GG, SG & & & $\mathrm{Y}$ & $\begin{array}{l}\text { Wet Chem, IC. } \\
\text { TC, TOC }\end{array}$ & & & & & \begin{tabular}{|l|} 
DOE 1993c; \\
Fredrickson et al. \\
1993
\end{tabular} & \\
\hline 3119 & 500 & 699-49-100A & A8802 & G & $\mathrm{CP}, \mathrm{GG}, \mathrm{NT}, \mathrm{OT}$ & & & & & & & & & $\begin{array}{l}\text { Summers and } \\
\text { Hanson } 1977\end{array}$ & DH-9 \\
\hline 3120 & & 699-49-100B & A8803 & D, As-built & GG & & & & & & & & & HWIS & \\
\hline 3121 & & 699-49-111 & & & CP, GG, NT & & & & & & & & & & \\
\hline 3122 & 500 & 699-49-111A & A8805 & As-built & GG & & & & & & & & & HWIS & \\
\hline 3123 & & 699-49-12A & A8782 & As-built & GG, MO & & & & & & & & & HWIS & \\
\hline 3124 & 500 & 699-49-12B & A8783 & As-built & GG, MO & & & & & & & & & HWIS & \\
\hline 3125 & 500 & 699-49-21 & A8789 & & GG, NT & & & & & & & & & & \\
\hline 3126 & 500 & 699-49-28 & A5216 & D, As-built & GG, NT & & & & & & & & & HWIS & \\
\hline 3127 & & 699-49-31 & A8790 & & $\mathrm{GG}, \mathrm{NT}$ & & & & & & & & & & \\
\hline 3128 & 500 & 699-49-32A & A8791 & & GG, NT & & & & & & & & & & \\
\hline
\end{tabular}




\begin{tabular}{|c|c|c|c|c|c|c|c|c|c|c|c|c|c|c|c|}
\hline SortIndex & Area & Well Name & WellID & Log Type & GPX Log Runs & Sieved & $\mathrm{CaCO} 3$ & Moisture & $\begin{array}{c}\text { Chemical } \\
\text { Properties }\end{array}$ & $\begin{array}{c}\text { Physical } \\
\text { Properties }\end{array}$ & \begin{tabular}{|c|} 
Min \\
Properties \\
\end{tabular} & $\begin{array}{l}\text { Geochron } \\
\text { Properties }\end{array}$ & Archived & Source & Comments \\
\hline \begin{tabular}{|l|}
3129 \\
\end{tabular} & 600 & $699-49-32 B$ & A8792 & & GG, NT & & & & & & & & & & \\
\hline 3130 & 600 & 699-49-33 & A8793 & & CP, GG, NT & & & & & & & & & & \\
\hline 3131 & & 699-49-48 & & & NT & & & & & & & & & & \\
\hline 3132 & & 699-49-55A & A5217 & D, As-built & GG, NT & & & & & & & & & HWIS & \\
\hline 3133 & 600 & $699-49-55 B$ & A5218 & D, As-built & $\mathrm{CP}, \mathrm{GG}, \mathrm{NT}, \mathrm{SN}$ & & & & & & & & & HWIS & \\
\hline 3134 & & 699-49-57 & & & GG, NT & & & & & & & & & & \\
\hline 3135 & 600 & 699-49-57B & A5220 & G & GG & $Y$ & $\mathrm{Y}$ & $Y$ & & $\begin{array}{l}\text { SpG, Por, } \\
\text { Bden }\end{array}$ & & & $\mathrm{Y}$ & Hoffman 1992 & 200-BP-1 \\
\hline 3136 & 600 & 699-49-79 & A5221 & D, As-built & GG, NT & & & & & & & & & HWIS & \\
\hline 3137 & & $699-49-85 \mathrm{~A}$ & A8797 & G, As-built & $\mathrm{CP}, \mathrm{GG}, \mathrm{NT}, \mathrm{SN}$ & & & & & & & & & HWIS & \\
\hline 3138 & & 699-49-85B & A8798 & As-built & $\mathrm{CP}, \mathrm{GG}, \mathrm{NT}, \mathrm{SN}$ & & & & & & & & & HWIS & \\
\hline 3139 & 600 & 699-4-E6 & A8131 & D & GG, NT & & & & & & & & & HWIS & \\
\hline 3140 & 600 & 699-50-17C & & & & & & & & & $\mathrm{PC}, \mathrm{PbC}$ & & & Goodwin 1993 & \\
\hline 3141 & 600 & 699-50-28B & A5222 & D, As-built & GG, NT & & & & & & & & & HWIS & \\
\hline 3142 & 600 & 699-50-28D & B2535 & As-built & SG & & & & & & & & & HWIS & \\
\hline 3143 & 600 & 699-50-30 & A5223 & D, As-built & GG, NT & & & & & & & & & HWIS & \\
\hline 3144 & & 699-50-45 & A5225 & D, As-built & $\mathrm{CP}, \mathrm{GG}, \mathrm{NT}, \mathrm{SN}$ & & & & & & & & & HWIS & \\
\hline 3145 & 600 & 699-50-48A & A8812 & D, As-built & GG, NT & & & & & & & & & HWIS & \\
\hline 3146 & 600 & 699-50-48B & A5226 & D, As-built & $\mathrm{CP}, \mathrm{GG}, \mathrm{NT}, \mathrm{SN}$ & & & & & & & & & HWIS & \\
\hline 3147 & 600 & 699-50-53A & A5227 & D, As-built & GG, NT & & & & & & & & & HWIS & \\
\hline 3148 & 600 & 699-50-53B & A5228 & G & $\mathrm{CP}, \mathrm{GG}, \mathrm{NT}$ & $Y$ & $Y$ & $Y$ & & $\begin{array}{l}\text { SpG, Por, } \\
\text { Bden }\end{array}$ & & & & $\begin{array}{l}\text { Hoffman 1992; } \\
\text { HWIS }\end{array}$ & 200-BP-1 \\
\hline 3149 & 600 & 699-50-96 & A8814 & D, As-built & $\mathrm{CP}, \mathrm{GG}, \mathrm{NT}, \mathrm{SN}$ & $Y$ & & & & & & Paleomag & & $\begin{array}{l}\text { Virtual Library } \\
2000 \text { (PPI only, } \\
\text { includes } \\
\text { descriptions); } \\
\text { Bjornstad 1984; } \\
\text { ASC 1984 }\end{array}$ & \\
\hline 3150 & 600 & 699-50-99 & A8818 & As-built & CP, GG, NT & & & & & $\begin{array}{l}\text { Bden, } \\
\% \text { GSSC }\end{array}$ & & Paleomag & $\mathrm{Y}$ & $\begin{array}{l}\text { Lindsey 1991; } \\
\text { Virtual Library } \\
2000 \text { (PPI only, } \\
\text { includes } \\
\text { descriptions); } \\
\text { ASC 1984; } \\
\text { Bjornstad 1984 }\end{array}$ & \\
\hline 3151 & 600 & 699-51-36A & A8824 & D, As-built & $\mathrm{DN}, \mathrm{GG}, \mathrm{NT}, \mathrm{TP}$ & & & & & & & & & HWIS & \\
\hline 3152 & 600 & 699-51-36B & A8825 & & GG, NT & & & & & & & & & & \\
\hline 3153 & 600 & 699-51-36C & A8826 & & GG, NT & & & & & & & & & & \\
\hline 3154 & & 699-51-36D & A8827 & & CP, GG, NT & & & & & & & & & & \\
\hline 3155 & 600 & 699-51-46 & A5230 & D, As-built & $\mathrm{CP}, \mathrm{GG}, \mathrm{NT}, \mathrm{SN}$ & & & & & & & & & HWIS & \\
\hline 3156 & 600 & 699-51-7 & A8820 & As-built & GG, SG & & & & & & & & & HWIS & \\
\hline 3157 & 600 & \begin{tabular}{|l|l|}
$699-52-17$ \\
\end{tabular} & A8832 & & $\mathrm{CP}, \mathrm{GG}, \mathrm{NT}, \mathrm{SN}$ & & & & & & & & & & \\
\hline 3158 & & 699-52-18A & A8833 & As-built & GG & & & & & & & & & HWIS & \\
\hline 3159 & 600 & 699-52-18B & A8834 & & GG & & & & & & & & & & \\
\hline 3160 & 600 & 699-52-18C & A8835 & As-built & GG & & & & & & & & & HWIS & \\
\hline 3161 & 600 & 699-52-46A & A5234 & D, As-built & $\mathrm{CP}, \mathrm{GG}, \mathrm{NT}, \mathrm{SN}$ & & & & & & & & & HWIS & \\
\hline 3162 & 600 & 699-52-48 & A5235 & D, As-built & $\mathrm{CP}, \mathrm{GG}, \mathrm{NT}, \mathrm{SN}$ & & & & & & & & & HWIS & \\
\hline 3163 & 600 & 699-52-52 & A8842 & $\mathrm{D}, \mathrm{G}$ & & $\begin{array}{l}\text { Virtual } \\
\text { Library }\end{array}$ & \begin{tabular}{|l} 
Virtual \\
Library
\end{tabular} & & & & & & $\mathrm{Y}$ & $\begin{array}{l}\text { Fecht and Lillie } \\
1982\end{array}$ & \\
\hline 3164 & 600 & 699-52-54 & A5236 & G & & $Y$ & $\mathrm{Y}$ & Y & & $\begin{array}{l}\begin{array}{l}\text { SpG, Por, } \\
\text { Bden }\end{array} \\
\end{array}$ & & & $Y$ & $\begin{array}{l}\text { Hoffman 1992; } \\
\text { HWIS }\end{array}$ & 200-BP-1 \\
\hline
\end{tabular}




\begin{tabular}{|c|c|c|c|c|c|c|c|c|c|c|c|c|c|c|c|}
\hline SortIndex & Area & Well Name & WellID & Log Type & GPX Log Runs & Sieved & $\mathrm{CaCO} 3$ & Moisture & $\begin{array}{l}\text { Chemical } \\
\text { Properties }\end{array}$ & $\begin{array}{c}\text { Physical } \\
\text { Properties }\end{array}$ & \begin{tabular}{|c|} 
Min \\
Properties \\
\end{tabular} & $\begin{array}{l}\text { Geochron } \\
\text { Properties }\end{array}$ & Archived & Source & Comments \\
\hline \begin{tabular}{|l|l|}
3165 \\
\end{tabular} & 600 & $699-52-57$ & A5237 & G & GG & Y & Y & Y & & $\begin{array}{l}\text { SpG, Por, } \\
\text { Bden }\end{array}$ & & & Y & $\begin{array}{l}\text { Hoffman 1992: } \\
\text { HWIS }\end{array}$ & 200-BP-1 \\
\hline 3166 & 600 & 699-53-47 & & & NT & & & & & & & & & & \\
\hline 3167 & 600 & 699-53-47B & A5240 & D, As-built & DN, GG, MG, NT & & & & & & & & & HWIS & \\
\hline 3168 & 600 & 699-54-17 & & & & & & & & & XRD, EM & & & Ames 1976 & \\
\hline 3169 & & 699-54-17C & & G & & & & & & & & & & $\begin{array}{l}\text { Summers and } \\
\text { Hanson } 1977\end{array}$ & DH-9B \\
\hline 3170 & 600 & 699-54-18C & A8857 & D & & & & & & AirPerm & & Paleomag & $\mathrm{Y}$ & $\begin{array}{l}\text { Parker and } \\
\text { Johnston 1979; } \\
\text { Gaylord et al. } \\
1991\end{array}$ & \\
\hline 3171 & 600 & 699-54-18E & A8859 & As-built & GG & & & & & & & & & HWIS & \\
\hline 3172 & 600 & 699-54-37B & A8861 & D, As-built & GG & & & & & & & & & HWIS & \\
\hline 3173 & 600 & 699-54-42 & A5250 & D, As-built & & & $\mathrm{Y}$ & & $\mathrm{pH}$ & $\begin{array}{l}\text { CEC, } \\
\% \text { \%SSC, 15- } \\
\text { Atm }\end{array}$ & & & & $\begin{array}{l}\text { HWIS; McHenry } \\
1957\end{array}$ & \\
\hline 3174 & 600 & 699-54-45A & A5251 & D & & \begin{tabular}{|l|} 
Virtual \\
Library
\end{tabular} & $\begin{array}{l}\text { Virtual } \\
\text { Library }\end{array}$ & & & & & & $Y$ & HWIS & \\
\hline 3175 & 600 & 699-54-45B & A8862 & $\mathrm{D}$ & DN, GG, NT, SN, TP & & & & & & & & $\mathrm{Y}$ & HWIS & \\
\hline 3176 & 600 & 699-54-48 & A5252 & $\mathrm{D}$ & & & & & & & & & $\mathrm{Y}$ & HWIS & \\
\hline 3177 & 600 & 699-54-49 & A8863 & $\mathrm{D}$ & & & & & & & & & $\mathrm{Y}$ & HWIS & \\
\hline 3178 & 600 & $699-55-40$ & A5255 & D, As-built & GG, NT & & & & & & & & & HWIS & \\
\hline 3179 & & 699-55-44 & A5256 & D, As-built & GG, NT & & & & & & & & & HWIS & \\
\hline 3180 & & 699-55-50A & A8865 & D & & & $\mathrm{Y}$ & & $\mathrm{pH}$ & $\begin{array}{l}\text { CEC, } \\
\% \text { GSSC, 15- } \\
\text { Atm }\end{array}$ & & & $\mathrm{Y}$ & McHenry 1957 & \\
\hline 3181 & 600 & 699-55-50B & A8866 & D & & & & $\mathrm{Y}$ & & & & & $\mathrm{Y}$ & HWIS & \\
\hline 3182 & 600 & 699-55-50C & A5257 & D & & & Y & & $\mathrm{pH}$ & $\begin{array}{l}\text { CEC, } \\
\% \text { GSSC, 15- } \\
\text { Atm }\end{array}$ & & & $Y$ & McHenry 1957 & \\
\hline 3183 & 600 & 699-55-50D & A8867 & D & GG, NT & & & & & & & & $Y$ & HWIS & \\
\hline 3184 & 600 & 699-55-55 & A5258 & G & GG, SG & Y & $\mathrm{Y}$ & $Y$ & & $\begin{array}{l}\text { SpG, Por, } \\
\text { Bden }\end{array}$ & & & $\mathrm{Y}$ & $\begin{array}{l}\text { Hoffman 1992; } \\
\text { HWIS }\end{array}$ & 200-BP-1 \\
\hline 3185 & 600 & 699-55-57 & A5259 & D, As-built & GG, NT & & & & & & & & & HWIS & \\
\hline 3186 & & 699-55-60B & A8869 & As-built & $\mathrm{GG}, \mathrm{NT}$ & & & & & & & & & HWIS & \\
\hline 3187 & 600 & 699-55-76 & A5261 & D, As-built & GG, NT & & & & & & & & & HWIS & \\
\hline 3188 & & 699-55-89 & A5262 & D, As-built & GG, NT & & $Y$ & & $\mathrm{pH}$ & $\begin{array}{l}\text { CEC, } \\
\% \text { GSSC, 15- } \\
\text { Atm }\end{array}$ & & & & $\begin{array}{l}\text { HWIS; McHenry } \\
1957\end{array}$ & \\
\hline 3189 & 600 & 699-56-26B & A8880 & As-built & GG & & & & & & & & & HWIS & \\
\hline 3190 & & 699-56-40A & A8881 & & $\mathrm{CP}, \mathrm{GG}, \mathrm{NT}$ & & & & & & & & & & \\
\hline 3191 & & 699-56-40B & A8882 & & GG, NT & & & & & & & & & & \\
\hline 3192 & 600 & 699-56-40C & A8883 & & CP, GG, NT & & & & & & & & & & \\
\hline 3193 & 600 & 699-56-41 & A8884 & & $\mathrm{CP}, \mathrm{GG}, \mathrm{NT}$ & & & & & & & & & & \\
\hline 3194 & 600 & 699-56-42A & A8885 & & GG, NT & & & & & & & & & & \\
\hline 3195 & & 699-56-42B & A8886 & & GG, NT & & & & & & & & & & \\
\hline 3196 & 600 & $699-56-42 \mathrm{C}$ & A8887 & & CP, GG, NT & & & & & & & & & & \\
\hline 3197 & 600 & 699-56-42D & A8888 & & GG, NT & & & & & & & & & & \\
\hline 3198 & 600 & 699-56-42E & A8889 & & NT & & & & & & & & & & \\
\hline 3199 & 600 & $699-56-42 \mathrm{~F}$ & A8890 & & GG, NT & & & & & & & & & & \\
\hline 3200 & 600 & 699-56-43 & A5264 & D, As-built & GG, NT & & & & & & & & & HWIS & \\
\hline 3201 & 600 & 699-56-53 & A5265 & D & $\mathrm{CP}, \mathrm{GG}, \mathrm{NT}, \mathrm{SN}$ & & & & & & & & $Y$ & HWIS & \\
\hline 3202 & 600 & 699-56-S1 & & D & & & & & & & & & & & \\
\hline 3203 & 600 & 699-57-25B & A8894 & D, As-built & GG, NT & & & & & & & & & HWIS & \\
\hline 3204 & 600 & 699-57-29A & A5267 & D, As-built & NT & & & & & & & & & HWIS & \\
\hline
\end{tabular}




\begin{tabular}{|c|c|c|c|c|c|c|c|c|c|c|c|c|c|c|c|}
\hline SortIndex & Area & Well Name & WellID & Log Type & GPX Log Runs & Sieved & $\mathrm{CaCO} 3$ & Moisture & $\begin{array}{l}\text { Chemical } \\
\text { Properties }\end{array}$ & $\begin{array}{c}\text { Physical } \\
\text { Properties }\end{array}$ & \begin{tabular}{|c|} 
Min \\
Properties
\end{tabular} & $\begin{array}{l}\text { Geochron } \\
\text { Properties }\end{array}$ & Archived & Source & Comments \\
\hline 3205 & 500 & 699-57-29B & A5268 & D, As-built & GG, NT & & & & & & & & & HWIS & \\
\hline 3206 & & 699-57-41A & A8896 & & $\mathrm{CP}, \mathrm{GG}, \mathrm{NT}$ & & & & & & & & & & \\
\hline 3207 & & 699-57-41B & A8897 & & GG, NT & & & & & & & & & & \\
\hline 3208 & 500 & 699-57-41D & A8899 & & GG, NT & & & & & & & & & & \\
\hline 3209 & & 699-57-41E & A8900 & & $\mathrm{CP}, \mathrm{GG}, \mathrm{NT}$ & & & & & & & & & & \\
\hline 3210 & & $699-57-42$ & A8902 & & CP, GG, NT & & & & & & & & & & \\
\hline 3211 & & 699-57-59 & A5269 & G & GG, SG & & & & & & & & & HWIS & 200-BP-1 \\
\hline 3212 & 500 & 699-57-83A & A5270 & D, As-built & GG, NT & & & & & & & & & HWIS & \\
\hline 3213 & & 699-57-83B & A8903 & As-built & CP, GG, NT & & & & & & & & & HWIS & \\
\hline 3214 & & $699-57-83 \mathrm{C}$ & A5274 & As-built & CP, GG, NT & & & & & & & & & HWIS & \\
\hline 3215 & & $699-58-24$ & A5275 & D, As-built & GG & & & & & & & & & HWIS & \\
\hline 3216 & 500 & 699-58-40 & A8907 & & CP, GG, NT & & & & & & & & & & \\
\hline 3217 & & 699-58-41B & A8909 & & GG, NT & & & & & & & & & & \\
\hline 3218 & & 699-58-41C & A8910 & & GG, NT & & & & & & & & & & \\
\hline 3219 & & 699-58-41D & A8911 & & GG, NT & & & & & & & & & & \\
\hline 3220 & 500 & 699-58-41E & A8912 & & GG, NT & & & & & & & & & & \\
\hline 3221 & & 699-58-41F & A8913 & & GG, NT & & & & & & & & & & \\
\hline 3222 & & 699-59-101 & A8920 & D, As-built & $\mathrm{CP}, \mathrm{GG}, \mathrm{NT}$ & & & & & & & & & HWIS & \\
\hline 3223 & 600 & $699-59-32$ & A5276 & D, As-built & GG, NT & & & & & & & & & HWIS & \\
\hline 3224 & 600 & 699-59-55 & A8918 & & GG, NT & & & & & & & & $\mathrm{Y}$ & HWIS & \\
\hline 3225 & 500 & $\begin{array}{l}\text { 699-59-55 (DH- } \\
10)\end{array}$ & & & DN, GG, NT, TP & & & & & & & & & & \\
\hline 3226 & 500 & 699-59-58 & A5277 & D, As-built & GG, NT & & & & & & & & & HWIS & \\
\hline 3227 & 500 & 699-59-80B & A5278 & D, As-built & GG, NT & & & & & & & & & HWIS & \\
\hline 3228 & & $699-60-32$ & A5279 & D, As-built & GG, NT, TP & & & & & & & & & HWIS & \\
\hline 3229 & 500 & 699-60-57 & A5280 & D, As-built & GG, NT, TP & & & & & & & & & HWIS & \\
\hline 3230 & 500 & 699-60-59 & A5281 & As-built & $\mathrm{CP}, \mathrm{GG}, \mathrm{NT}, \mathrm{SN}$ & & & & & & & & & HWIS & \\
\hline 3231 & & $699-60-60$ & A5282 & D, As-built & TP & & $\mathrm{Y}$ & & $\mathrm{pH}$ & $\begin{array}{l}\text { CEC, } \\
\% \text { \%SSC, 15- } \\
\text { Atm }\end{array}$ & & & & $\begin{array}{l}\text { HWIS; McHenry } \\
1957\end{array}$ & \\
\hline 3232 & 500 & 699-61-102 & A8937 & As-built & $\mathrm{CP}$ & & & & & & & & & HWIS & \\
\hline 3233 & & 699-61-16A & A8927 & D, As-built & GG, SG & & & & & & & & & HWIS & \\
\hline 3234 & & 699-61-16B & A8928 & As-built & $\mathrm{GG}, \mathrm{SG}$ & & & & & & & & & HWIS & \\
\hline 3235 & & 699-61-24 & A8929 & & TP & & & & & & & & & & \\
\hline 3236 & & 699-61-37 & A5283 & D, As-built & NT, TP & & & & & & & & & HWIS & \\
\hline 3237 & & $699-61-41$ & A5284 & D, As-built & GG, NT, TP & & & & & & & & & HWIS & \\
\hline 3238 & 500 & 699-61-53 & A8932 & D, As-built & $\mathrm{CP}$ & & & & & & & & & HWIS & \\
\hline 3239 & 600 & 699-61-55 & & G & & & & & & & & & & $\begin{array}{l}\text { Summers and } \\
\text { Hanson } 1977\end{array}$ & DH-8 \\
\hline 3240 & 500 & 699-61-55B & A8934 & D, As-built & $\mathrm{CP}, \mathrm{GG}, \mathrm{NT}, \mathrm{SN}$ & & & & & & & & & HWIS & \\
\hline 3241 & 500 & 699-61-57 & A8935 & As-built & $\mathrm{GG}, \mathrm{NT}, \mathrm{SN}$ & & & & & & & & & HWIS & \\
\hline 3242 & 500 & 699-61-62 & A5285 & D, As-built & GG, NT, TP & & & & & & & & & HWIS & \\
\hline 3243 & & 699-61-66 & A5286 & D, As-built & GG, NT, TP & & & & & & & & & HWIS & \\
\hline 3244 & 500 & 699-62-31 & A5287 & D, As-built & TP & & & & & & & & & HWIS & \\
\hline 3245 & 500 & $699-62-32$ & A8939 & & TP & & & & & & & & & & \\
\hline 3246 & 500 & 699-62-43A & A5288 & D, As-built & GG, NT, TP & & & & & & & & & HWIS & \\
\hline 3247 & 500 & 699-62-43B & A8940 & D, As-built & GG & & & & & & & & & HWIS & \\
\hline 3248 & 500 & $699-62-43 C$ & A8941 & D, As-built & GG, NT, SN, TP & & & & & & & & & HWIS & \\
\hline 3249 & 500 & 699-62-43D & A8942 & D, As-built & TP & & & & & & & & & HWIS & \\
\hline 3250 & 500 & 699-62-43E & A8943 & D, As-built & GG, NT, SN & & & & & & & & & HWIS & \\
\hline 3251 & 500 & 699-62-43F & A8944 & D, As-built & TP & & & & & & & & & HWIS & \\
\hline 3252 & 000 & $699-62-43 \mathrm{H}$ & A8946 & D, As-built & GG, NT, SN & & & & & & & & & HWIS & \\
\hline 3253 & 500 & $699-63-25$ & & & TP & & & & & & & & & & \\
\hline 3254 & 500 & $699-63-25 A$ & A5289 & D, As-built & GG, NT, TP & & & & & & & & & HWIS & \\
\hline 3255 & 500 & $699-63-25 B$ & A8955 & D, As-built & NT & & & & & & & & & HWIS & \\
\hline 3256 & 600 & 699-63-51 & A5290 & D, As-built & TP & & & & & & & & & HWIS & \\
\hline
\end{tabular}




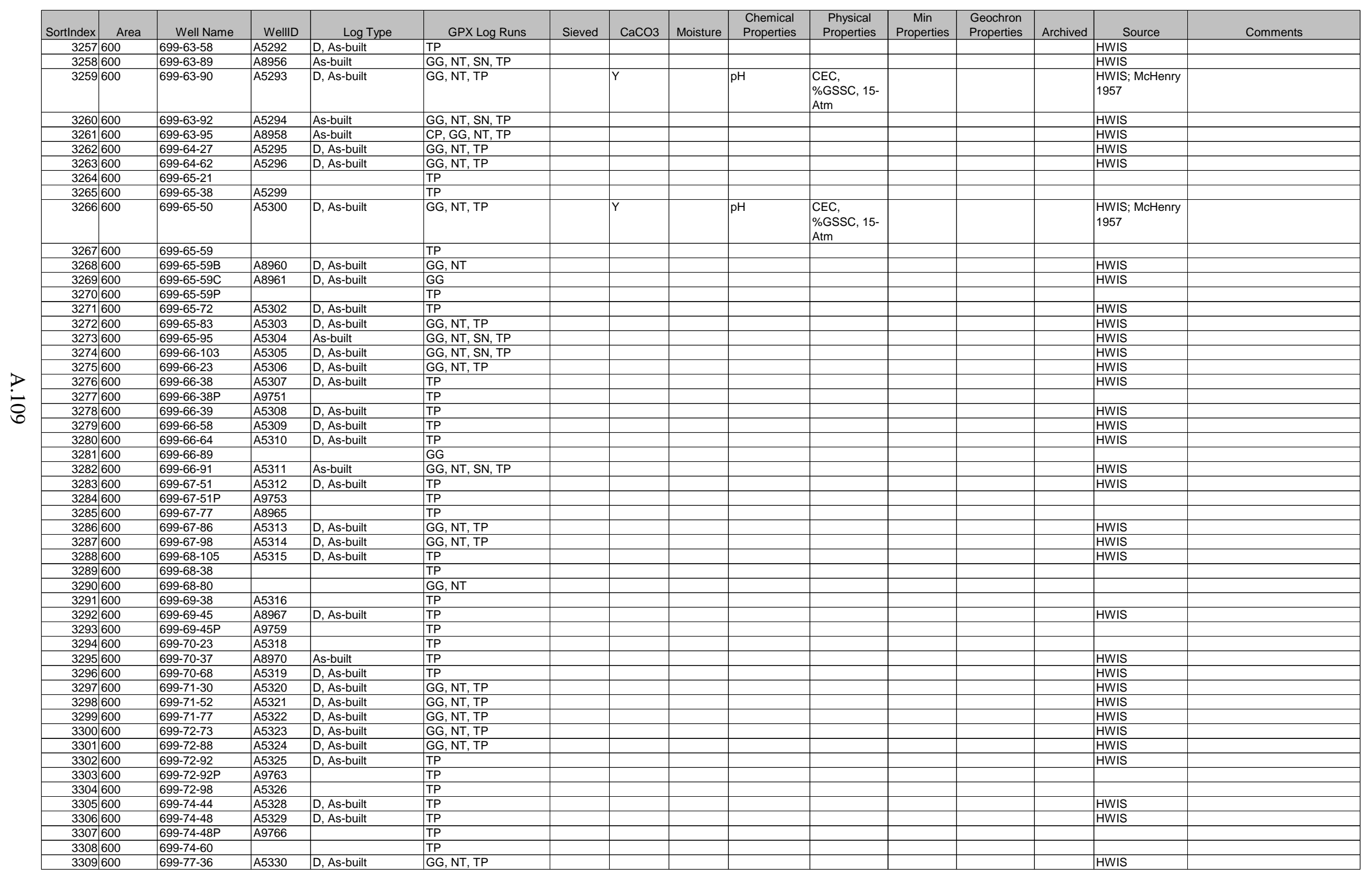




\begin{tabular}{|c|c|c|c|c|c|c|c|c|c|c|c|c|c|c|c|}
\hline Sortlndex & Area & Well Name & WellID & Log Type & GPX Log Runs & Sieved & $\mathrm{CaCO} 3$ & Moisture & $\begin{array}{c}\text { Chemical } \\
\text { Properties }\end{array}$ & $\begin{array}{c}\text { Physical } \\
\text { Properties }\end{array}$ & $\begin{array}{c}\text { Min } \\
\text { Properties } \\
\end{array}$ & $\begin{array}{l}\text { Geochron } \\
\text { Properties }\end{array}$ & Archived & Source & Comments \\
\hline 3310 & 600 & 699-77-54 & A5331 & D, As-built & GG, NT, TP & & & & & & & & & HWIS & \\
\hline 3311 & 600 & $699-78-62$ & A5332 & D, As-built & TP & & & & & & & & & HWIS & \\
\hline 3312 & & $699-79-104$ & A8987 & As-built & GG, SG & & & & & & & & & HWIS & \\
\hline 3313 & & $699-80-43$ & & & TP & & & & & & & & & & \\
\hline 3314 & 600 & 699-80-43P & A8993 & As-built & TP & & & & & & & & & HWIS & \\
\hline 3315 & 600 & $699-80-62$ & A8996 & As-built & GG & & & & & & & & & HWIS & \\
\hline 3316 & & 699-81-38 & A5337 & & TP & & & & & & & & & & \\
\hline 3317 & & 699-81-58 & A5338 & D, As-built & GG, NT, TP & & & & & & & & & HWIS & \\
\hline 3318 & 600 & 699-81-62 & A9000 & As-built & $\mathrm{CP}, \mathrm{GG}, \mathrm{NT}$ & & & & & & & & & HWIS & \\
\hline 3319 & 600 & 699-8-17 & A5333 & D, As-built & GG, NT, TP & & & & & & & & & HWIS & \\
\hline 3320 & & 699-82-45A & A5339 & & TP & & & & & & & & & & \\
\hline 3321 & 600 & $699-8-25$ & A5334 & D, As-built & GG, NT, TP & & & & & & & & & HWIS & \\
\hline 3322 & 600 & $699-8-32$ & A5335 & D, As-built & GG, NT, TP & & & & & & & & & HWIS & \\
\hline 3323 & & 699-83-32 & & & TP & & & & & & & & & & \\
\hline 3324 & & 699-83-36 & A5340 & As-built & TP & & & & & & & & & HWIS & \\
\hline 3325 & 600 & 699-83-47 & A5341 & D, As-built & TP & & & & & & & & & HWIS & \\
\hline 3326 & 600 & 699-83-47P & & & TP & & & & & & & & & & \\
\hline 3327 & & 699-84-33 & & & OT & & & & & & & & & & \\
\hline 3328 & 600 & 699-84-35 & & & TP & & & & & & & & & & \\
\hline 3329 & 600 & 699-84-35P & & & TP & & & & & & & & & & \\
\hline 3330 & 600 & 699-84-59 & A9031 & As-built & $\mathrm{CP}, \mathrm{GG}, \mathrm{NT}$ & & & & & & & & & HWIS & \\
\hline 3331 & & 699-85-40A & A5343 & & TP & & & & & & & & & & \\
\hline 3332 & 600 & 699-86-35 & & & TP & & & & & & & & & & \\
\hline 3333 & 600 & $699-86-42$ & A5344 & & TP & & & & & & & & & & \\
\hline 3334 & 600 & 699-86-60 & A9059 & D, As-built & $\mathrm{CP}, \mathrm{GG}, \mathrm{NT}, \mathrm{TP}$ & & & & & & & & & HWIS & \\
\hline 3335 & 600 & 699-86-95 & A9061 & D, As-built & GG, SG & & & & & & & & & HWIS & \\
\hline 3336 & 600 & $699-87-42$ & & & TP & & & & & & & & & & \\
\hline 3337 & 600 & 699-87-42A & A5345 & & TP & & & & & & & & & & \\
\hline 3338 & 600 & $699-87-43$ & & & TP & & & & & & & & & & \\
\hline 3339 & 600 & $699-87-55$ & A5346 & D, As-built & TP & & & & & & & & & HWIS & \\
\hline 3340 & 600 & 699-88-42 & A9072 & & TP & & & & & & & & & & \\
\hline 3341 & 600 & 699-89-35 & A5348 & D, As-built & GG, NT, TP & Y & $\mathrm{Y}$ & & & & & & & Virtual Library & \\
\hline 3342 & 600 & 699-90-34 & A5350 & G & & & & & & & & & & $\begin{array}{l}\text { Fruchter et al. } \\
1996\end{array}$ & Abandoned corehole \\
\hline 3343 & 600 & 699-90-37A & A9075 & D & & & & & & & & & & HWIS & \\
\hline 3344 & 600 & 699-90-38 & A5351 & D, As-built & TP & & & & & & & & & HWIS & \\
\hline 3345 & 600 & 699-90-45 & A5352 & As-built & TP & & & & & & & & & $\begin{array}{l}\text { Fruchter et al. } \\
1996\end{array}$ & Abandoned corehole \\
\hline 3346 & 600 & 699-91-37 & & $\mathrm{D}$ & TP & & & & & & & & & & \\
\hline 3347 & 600 & 699-91-38 & & & TP & & & & & & & & & & \\
\hline 3348 & 600 & $699-91-43$ & & & GG, SG & & & & & & & & & & \\
\hline 3349 & 600 & 699-91-46 & & As-built & & & & & & & & & & HWIS & \\
\hline 3350 & 600 & 699-91-46A & A5354 & As-built & & & & & & & & & $Y$ & HWIS & Abandoned corehole \\
\hline 3351 & 600 & 699-91-46B & A9810 & Well summary & GG & & & & & & & & & HWIS & \\
\hline 3352 & 600 & 699-91-48A & A9080 & vect surminary & TP & & & & & & & & & & \\
\hline 3353 & & 699-92-14 & A9082 & As-built & GG, SG & \begin{tabular}{|l} 
Virtual \\
Library
\end{tabular} & \begin{tabular}{|l} 
Virtual \\
Library
\end{tabular} & & & & & & $Y$ & HWIS & \\
\hline 3354 & 600 & 699-92-38 & & & TP & & & & & & & & & & \\
\hline 3355 & 600 & 699-92-48B & & & TP & & & & & & & & & & \\
\hline 3356 & & 699-92-49 & A5355 & D, Well Summary & TP & & & & & & & & & HWIS & \\
\hline 3357 & & 699-93-46 & & \begin{tabular}{|l|} 
As-built, Well \\
Summary
\end{tabular} & GG, SG & $Y$ & & & & & & & & $\begin{array}{l}\text { HWIS, see R. } \\
\text { Khaleel or G. } \\
\text { Freeman for } \\
\text { sieve data }\end{array}$ & \\
\hline
\end{tabular}




\begin{tabular}{|c|c|c|c|c|c|c|c|c|c|c|c|c|c|c|c|}
\hline Sortindex & Area & Well Name & WellID & Log Type & GPX Log Runs & Sieved & $\mathrm{CaCO} 3$ & Moisture & $\begin{array}{c}\text { Chemical } \\
\text { Properties } \\
\end{array}$ & $\begin{array}{c}\text { Physical } \\
\text { Properties }\end{array}$ & \begin{tabular}{|c|} 
Min \\
Properties \\
\end{tabular} & $\begin{array}{l}\text { Geochron } \\
\text { Properties } \\
\end{array}$ & Archived & Source & Comments \\
\hline 3358 & 600 & 699-93-48A & A5356 & As-built & GG & & & & & & & & $\mathrm{Y}$ & HWIS & Groundwater monitoring \\
\hline 3359 & & 699-93-48B & A9811 & & SG & & & & & & & & Y & & Decommissioned \\
\hline 3360 & 600 & 699-93-49B & & $\begin{array}{l}\text { As-built, Well } \\
\text { Summary }\end{array}$ & GG & & & & & & & & & HWIS & Groundwater monitoring \\
\hline 3361 & 600 & $699-93-50$ & A9086 & & TP & & & & & & & & & & \\
\hline 3362 & 600 & 699-93-93 & A9087 & As-built & GG & $\begin{array}{l}\text { Virtual } \\
\text { Library }\end{array}$ & $\begin{array}{l}\text { Virtual } \\
\text { Library }\end{array}$ & & & & & & $\mathrm{Y}$ & HWIS & \\
\hline 3363 & 600 & $699-94-47$ & A9088 & & TP & & & & & & & & & & \\
\hline 3364 & 600 & 699-96-43 & A5357 & $\begin{array}{l}\text { As-built, Well } \\
\text { Summary }\end{array}$ & & & & & & & & & $\mathrm{Y}$ & HWIS & Abandoned corehole \\
\hline 3365 & 600 & 699-96-44 & C4131 & G & & & & & & & & & $Y$ & HWIA & NABIR \\
\hline 3366 & 600 & $699-96-45$ & C4132 & G & & & & & & & & & $\mathrm{Y}$ & HWIS & NABIR \\
\hline 3367 & 600 & 699-96-49 & A5358 & As-built & TP & & & & & & & & & HWIS & Groundwater monitoring \\
\hline 3368 & 600 & 699-96-49P & A9775 & & TP & & & & & & & & & & \\
\hline 3369 & 600 & \begin{tabular}{|l|l|}
$699-96-52$ \\
\end{tabular} & A5359 & As-built & TP & & & & & & & & & HWIS & Abandoned \\
\hline 3370 & & 699-97-43 & A5360 & As-built & GG, NT, TP & \begin{tabular}{|l|} 
Virtual \\
Library
\end{tabular} & & & & & & & & $\begin{array}{l}\text { Fruchter et al. } \\
\text { 1996; HWIS }\end{array}$ & Abandoned corehole \\
\hline 3371 & 600 & 699-97-43P & A9777 & & TP & & & & & & & & & & \\
\hline 3372 & 600 & $699-97-47$ & A5361 & As-built & TP & & & & & & & & & HWIS & \\
\hline 3373 & 600 & $699-97-49$ & & & TP & & & & & & & & & & \\
\hline 3374 & 600 & 699-97-51A & A5362 & D, As-built & GG, NT, TP & & & & & & & & & HWIS & Groundwater monitoring \\
\hline 3375 & 600 & 699-97-51B & A9093 & As-built & TP & & & & & & & & & HWIS & Decommissioned 5/10/95 \\
\hline 3376 & 600 & 699-98-49 & & & TP & & & & & & & & & & \\
\hline 3377 & 600 & 699-98-49A & A5363 & As-built & & & & & & & & & & HWIS & Abandoned \\
\hline 3378 & 600 & 699-99-42 & A5364 & & TP & & & & & & & & & & \\
\hline 3379 & 600 & 699-9-E2 & A5349 & D, As-built & NT, TP & & & & & & & & & HWIS & \\
\hline 3380 & & 699-BP9-12 & & & SG & & & & & & & & & & \\
\hline 3381 & 600 & 699-BP9-13 & & & SG & & & & & & & & & & \\
\hline 3382 & 600 & $699-\mathrm{E}-9 \mathrm{~B}$ & & & CP, MG, SG, SN, TP & & & & & & & & & & \\
\hline 3383 & 600 & 699-S11-E12 & & & CP, GG, NT, TP & & & & & & & & & & \\
\hline 3384 & 600 & \begin{tabular}{|l|l|}
$699-S 12-29$ \\
\end{tabular} & A5365 & D, As-built & TP & & & & & & & & & HWIS & \\
\hline 3385 & 600 & 699-S12-3 & A5366 & D, Well Summary & $\mathrm{CP}, \mathrm{GG}, \mathrm{NT}, \mathrm{TP}$ & & & & & & & & & HWIS & \\
\hline 3386 & 600 & 699-S12-30 & & & TP & & & & & & & & & & \\
\hline 3387 & 600 & 699-S12-30P & & & TP & & & & & & & & & & \\
\hline 3388 & 600 & \begin{tabular}{|l|}
$699-S 14-20$ \\
\end{tabular} & & & TP & & & & & & & & & & \\
\hline 3389 & 600 & 699-S14-20A & A5367 & D, As-built & GG, NT & & & & & & & & & HWIS & \\
\hline 3390 & 600 & 699-S14-20P & & & TP & & & & & & & & & & \\
\hline 3391 & 600 & 699-S16-24 & A9189 & As-built & $\mathrm{CP}, \mathrm{NT}$ & & & & & & & & & HWIS & \\
\hline 3392 & 600 & 699-S16-E14 & A9190 & G, As-built & $\mathrm{CP}, \mathrm{GG}, \mathrm{NT}, \mathrm{SN}$ & & & & & & & & & HWIS & \\
\hline 3393 & 600 & 699-S18-E2A & A5368 & D, As-built & TP & & & & & & & & & HWIS & \\
\hline 3394 & 600 & 699-S18-E2B & A9199 & D, As-built & GG, NT & & & & & & & & & HWIS & \\
\hline 3395 & 600 & 699-S19-11 & A5369 & D, As-built & GG, NT, TP & & & & & & & & & HWIS & \\
\hline 3396 & 600 & 699-S19-E13 & A5370 & D, As-built & GG, NT, TP & & & & & & & & & HWIS & \\
\hline 3397 & 600 & 699-S19-E14 & A5421 & D, G, Well summary & GG & & & & & & & & & HWIS & \\
\hline 3398 & 600 & 699-S22-E9C & A5424 & D, G, Well summary & GG & & & & & & & & & HWIS & \\
\hline 3399 & 600 & 699-S23-26 & A9201 & As-built & TP & & & & & & & & & HWIS & \\
\hline 3400 & 600 & 699-S2-34B & B8101 & G & & & & & & & & & & HWIS & LIGO well \\
\hline 3401 & 600 & 699-S24-19 & A9202 & D, As-built & GG, NT & & & & & & & & & HWIS & \\
\hline 3402 & 600 & 699-S24-19A & & & $\mathrm{TP}$ & & & & & & & & & & \\
\hline
\end{tabular}




\begin{tabular}{|c|c|c|c|c|c|c|c|c|c|c|c|c|c|c|c|}
\hline SortIndex & Area & Well Name & WellID & Log Type & GPX Log Runs & Sieved & $\mathrm{CaCO} 3$ & Moisture & $\begin{array}{c}\text { Chemical } \\
\text { Properties }\end{array}$ & $\begin{array}{c}\text { Physical } \\
\text { Properties }\end{array}$ & $\begin{array}{c}\text { Min } \\
\text { Properties } \\
\end{array}$ & $\begin{array}{l}\text { Geochron } \\
\text { Properties }\end{array}$ & Archived & Source & Comments \\
\hline \begin{tabular}{|l|}
3403 \\
\end{tabular} & 600 & 699-S25-51 & A9203 & As-built & $\mathrm{CP}, \mathrm{GG}, \mathrm{NT}$ & & & & & & & & & HWIS & \\
\hline 3404 & 600 & 699-S27-E14 & A5371 & D, As-built & GG, NT, TP & & & & & & & & & HWIS & \\
\hline 3405 & 600 & 699-S27-E9B & A5426 & D, As-built & SG & & & & & & & & & HWIS & \\
\hline 3406 & 600 & 699-S27-E9C & A5427 & D, G, Well summary & GG, SG & & & & & & & & & HWIS & \\
\hline 3407 & 600 & 699-S28-E12 & A5428 & D, G, Well summary & GG & & & & & & & & & HWIS & \\
\hline 3408 & 600 & 699-S29-E12 & A5372 & D, As-built & GG, NT, TP & & & & & & & & & HWIS & \\
\hline 3409 & 600 & 699-S29-E16B & A5430 & D, Well summary & SG & & & & & & & & & HWIS & \\
\hline 3410 & 600 & 699-S29-E16C & A5431 & D, G, Well summary & GG, SG & & & & & & & & & HWIS & \\
\hline 3411 & 600 & 699-S30-E14 & A9209 & D, As-built & TP & & & & & & & & & HWIS & \\
\hline 3412 & 600 & 699-S30-E15A & A5377 & D, Well summary & TP & & & & & & & & & HWIS & \\
\hline 3413 & 600 & 699-S30-E15B & A9210 & As-built & GG, NT & & & & & & & & & HWIS & \\
\hline 3414 & & 699-S31-1 & A5378 & D, As-built & TP & & & & & & & & & HWIS & \\
\hline 3415 & 600 & 699-S31-1P & A9786 & & GG, NT, TP & & & & & & & & & & \\
\hline 3416 & 600 & 699-S3-25 & A5373 & D, As-built & GG, NT, TP & & & & & & & & & HWIS & \\
\hline 3417 & 600 & 699-S3-67 & A9144 & As-built & $\mathrm{CP}, \mathrm{GG}, \mathrm{NT}$ & & & & & & & & & HWIS & \\
\hline 3418 & 600 & 699-S37-E14 & A5394 & G & GG & & & & & & & & & $\begin{array}{l}\text { Bryce and } \\
\text { Goodwin } 1989\end{array}$ & \\
\hline 3419 & 600 & 699-S3-E12 & A5374 & D, As-built & GG, NT, TP & & & & & & & & & HWIS & \\
\hline 3420 & & 699-S40-E14 & A5398 & D, As-built & GG & & & & & & & & & HWIS & \\
\hline 3421 & 600 & 699-S40-E14A & & $\mathrm{G}$ & & & & & & & & & & $\begin{array}{l}\text { Bryce and } \\
\text { Goodwin } 1989\end{array}$ & \\
\hline 3422 & 600 & 699-S41-E13A & A5401 & G & GG & & & & & & & & & $\begin{array}{l}\text { Bryce and } \\
\text { Goodwin } 1989\end{array}$ & \\
\hline 3423 & 600 & 699-S41-E13B & A5402 & G & GG & & & & & & & & & \begin{tabular}{|l|} 
Bryce and \\
Goodwin 1989
\end{tabular} & \\
\hline 3424 & 600 & 699-S43-E12 & A5404 & G & GG & & & & & & & & & $\begin{array}{l}\text { Bryce and } \\
\text { Goodwin } 1989\end{array}$ & \\
\hline 3425 & 600 & 699-S6-E14 & & & TP & & & & & & & & & & \\
\hline 3426 & & 699-S6-E14A & A5405 & D & GG, NT & & & & & & & & & HWIS & \\
\hline 3427 & 600 & 699-S6-E14AP & A9494 & & TP & & & & & & & & & & \\
\hline 3428 & 600 & 699-S6-E14P & & & TP & & & & & & & & & & \\
\hline 3429 & & 699-S6-E4A & A9152 & D, As-built & GG, NT & & & & & & & & & HWIS & \\
\hline 3430 & & 699-S6-E4C & A9154 & D, As-built & $\mathrm{CP}, \mathrm{GG}, \mathrm{NT}, \mathrm{SG}, \mathrm{SN}$ & & & & & & & & & HWIS & \\
\hline 3431 & 600 & 699-S6-E4CP & A9788 & & TP & & & & & & & & & & \\
\hline 3432 & 600 & 699-S6-E4D & A5406 & D, As-built & TP & & & & & & & & & HWIS & \\
\hline 3433 & 600 & 699-S6-E4E & A9155 & D, As-built & GG, NT & & & & & & & & & HWIS & \\
\hline 3434 & & 699-S6-E4F & A9156 & D, As-built & GG, NT & & & & & & & & & HWIS & \\
\hline 3435 & 600 & 699-S6-E4G & A9157 & D, As-built & GG, NT & & & & & & & & & HWIS & \\
\hline 3436 & 600 & 699-S6-E4H & A9158 & D, As-built & GG, NT & & & & & & & & & HWIS & \\
\hline 3437 & 600 & 699-S6-E4J & A9159 & D, As-built & GG, NT & & & & & & & & & HWIS & \\
\hline 3438 & 600 & 699-S6-E4K & C4072 & $\mathrm{G}$ & SG & & & & & & & & $\mathrm{Y}$ & $\begin{array}{l}\text { Williams et al. } \\
2003\end{array}$ & $618-10$ \\
\hline 3439 & 600 & 699-S6-E4L & C4073 & G & NT, SG & & & & & & & & $\mathrm{Y}$ & $\begin{array}{l}\text { Williams et al. } \\
2003\end{array}$ & $618-10$ \\
\hline 3440 & 600 & 699-S7-34 & A5407 & D, As-built & TP & & & & & & & & & HWIS & \\
\hline 3441 & 600 & \begin{tabular}{|l|}
$699-S 7-34 P$ \\
\end{tabular} & A9791 & & TP & & & & & & & & & & \\
\hline \begin{tabular}{|c|}
34442 \\
\end{tabular} & 600 & $699-S 8-19$ & A5408 & D, As-built & GG, NT, TP & & & & & & & & & HWIS & \\
\hline 3443 & 600 & \begin{tabular}{|l|}
$699-S 9-65$ \\
\end{tabular} & A9177 & As-built & CP, GG, NT & & & & & & & & & HWIS & \\
\hline 3444 & 600 & C3252 & C3252 & G & & & & & & & & & & Faurote 2001 & \\
\hline
\end{tabular}




\begin{tabular}{|c|c|c|c|c|c|c|c|c|c|c|c|c|c|c|c|}
\hline SortIndex & Area & Well Name & WellID & Log Type & GPX Log Runs & Sieved & $\mathrm{CaCO} 3$ & Moisture & $\begin{array}{l}\text { Chemical } \\
\text { Properties }\end{array}$ & $\begin{array}{l}\text { Physical } \\
\text { Properties }\end{array}$ & $\begin{array}{c}\text { Min } \\
\text { Properties } \\
\end{array}$ & $\begin{array}{l}\text { Geochron } \\
\text { Properties }\end{array}$ & Archived & Source & Comments \\
\hline 3445 & 600 & C3255 & C3255 & G & & & & & & & & & & Faurote 2001 & \\
\hline 3446 & 5600 & C3264 & C3264 & G & & & & & & & & & $Y$ & HWIS & 618 tritium investigation \\
\hline 3447 & 7600 & C3265 & C3265 & G & & & & & & & & & $\mathrm{Y}$ & HWIS & 618 tritium investigation \\
\hline 3448 & 3600 & 108 & & G & & & & & & & & & & Golder 1982 & fault study \\
\hline 3449 & & 125 & & $G$ & & & & & & & & & & Golder 1982 & fault study \\
\hline 3450 & & WP-1 & & G & & & & & & & & & & Golder 1982 & fault study \\
\hline 3451 & 1600 & WP-10 & & G & & & & & & & & & & Golder 1982 & fault study \\
\hline 3452 & 2600 & WP-11 & & G & & & & & & & & & & Golder 1982 & fault study \\
\hline 3453 & 600 & WP-2 & & $\mathrm{G}$ & & & & & & & & & & Golder 1982 & fault study \\
\hline 3454 & 4600 & WP-3 & & $\mathrm{G}$ & & & & & & & & & & Golder 1982 & fault study \\
\hline 3455 & 600 & WP-4 & & G & & & & & & & & & & Golder 1982 & fault study \\
\hline 3456 & 600 & WP-5 & & G & & & & & & & & & & Golder 1982 & fault study \\
\hline 3457 & 7600 & WP-6 & & G & & & & & & & & & & Golder 1982 & fault study \\
\hline 3458 & 600 & WP-7 & & $\mathrm{G}$ & & & & & & & & & & Golder 1982 & fault study \\
\hline 3459 & 9600 & WP-8 & & $\mathrm{G}$ & & & & & & & & & & Golder 1982 & fault study \\
\hline 3460 & 600 & WP-9 & & G & & & & & & & & & & Golder 1982 & fault study \\
\hline 3461 & 1 MISC & 800 boreholes & & mixed & & & & & & & & & & $\begin{array}{l}\text { Fecht and Lillie } \\
1982\end{array}$ & \\
\hline 3462 & MISC & $\begin{array}{l}\text { AP tank farm - } \\
\text { six samples }\end{array}$ & & & & $Y$ & & & & Pip & & & & Last et al. 1995 & \\
\hline 3463 & 3 MISC & B5757 & & G & & & & & $\begin{array}{l}\text { Metals, An, } \\
\text { SVOA, VOA, } \\
\text { Lab Rad }\end{array}$ & & & & & $\begin{array}{l}\text { Cearlock et al. } \\
2000\end{array}$ & \\
\hline 3464 & 4 MISC & Bergmounds & & & & $Y$ & & & & & & & & Chamness 1993 & \\
\hline 3465 & MISC & $\mathrm{BH}-16$ & & & & & & & & & XRD & & & Horton files & \\
\hline 3466 & MISC & BH-17 & & & & & & & & & XRD & & & Horton files & \\
\hline 3467 & $7 \mathrm{MISC}$ & $\mathrm{DH}-33$ & & & & & & & & & $\mathrm{PC}, \mathrm{PbC}$ & & & Goodwin 1993 & \\
\hline 3468 & MISC & Outcrop & & & & Y & & & & & & & & Lindsey 1991 & \\
\hline 3460 & MISC & Outcrop & & & & $\mathrm{Y}$ & & & & Pip & & & & Bjornstad 1980 & \\
\hline 3470 & MISC & $\begin{array}{l}\text { Outcrops on } \\
\text { White Bluffs }\end{array}$ & & & & & & & & & $\mathrm{PC}, \mathrm{PbC}$ & Paleomag & & $\begin{array}{l}\text { Goodwin 1993; } \\
\text { WCC 1978; } \\
\text { Parker 1979; } \\
\text { Parker and } \\
\text { Johnston } 1979\end{array}$ & \\
\hline 3471 & MISC & PSPL-1 & & D & & $\mathrm{Y}$ & $\mathrm{Y}$ & $\mathrm{Y}$ & $\mathrm{pH}$ & $\begin{array}{l}\text { \%GSSC, } \\
\text { CEC Cond }\end{array}$ & & & & Heller et al. 1984 & \\
\hline 3472 & MISC & PSPL-2 & & D & & Y & Y & Y & $\mathrm{pH}$ & $\begin{array}{l}\% G S S C, \\
\text { CEC, Cond }\end{array}$ & & & & Heller et al. 1984 & \\
\hline 3473 & 3 MISC & PSPL-3 & & D & & $Y$ & $\mathrm{Y}$ & $\mathrm{Y}$ & $\mathrm{pH}$ & $\begin{array}{l}\% G S S C, \\
\text { CEC, Cond }\end{array}$ & & & & Heller et al. 1984 & \\
\hline 3474 & 4 MISC & PSPL-4 & & D & & Y & Y & Y & $\mathrm{pH}$ & $\begin{array}{l}\% G S S C, \\
\text { CEC, Cond }\end{array}$ & & & & Heller et al. 1984 & \\
\hline 3475 & MISC & PSPL-5 & & D & & $Y$ & Y & $\mathrm{Y}$ & $\mathrm{pH}$ & $\begin{array}{l}\% G S S C \\
\text { CEC, Cond }\end{array}$ & & & & Heller et al. 1984 & \\
\hline 3476 & MISC & $\begin{array}{l}\text { samples from } 3 \\
\text { boreholes at } \\
\text { Sisson and Lu } \\
\text { site }\end{array}$ & & & & & & Y & An & & & & & \begin{tabular}{|l|} 
Last and \\
Caldwell 2001
\end{tabular} & \\
\hline
\end{tabular}




\begin{tabular}{|c|c|c|c|c|c|c|c|c|c|c|c|c|c|c|c|}
\hline SortIndex & Area & Well Name & WelliD & Log Type & GPX Log Runs & Sieved & $\mathrm{CaCO} 3$ & Moisture & $\begin{array}{l}\text { Chemical } \\
\text { Properties }\end{array}$ & $\begin{array}{c}\text { Physical } \\
\text { Properties }\end{array}$ & $\begin{array}{c}\text { Min } \\
\text { Properties } \\
\end{array}$ & $\begin{array}{l}\text { Geochron } \\
\text { Properties } \\
\end{array}$ & Archived & Source & Comments \\
\hline 3477 & MISC & $\begin{array}{l}\text { Surface and near } \\
\text { surface soil }\end{array}$ & & & & & & & & & & & $Y$ & DOE 1996 & \\
\hline 3478 & MISC & $\begin{array}{l}\text { Surface soil at } \\
\text { former GTF }\end{array}$ & & & & & & & & Lab Rad & & & & $\begin{array}{l}\text { Swanson et al. } \\
1988\end{array}$ & \\
\hline 3479 & MISC & $\begin{array}{l}\text { Test pits at 216- } \\
\text { B-2-2 ditch }\end{array}$ & & G & & & & & $\begin{array}{l}\text { Metals, An, } \\
\text { SVOA, VOA, } \\
\text { Lab Rad }\end{array}$ & & & & & $\begin{array}{l}\text { Cearlock et al. } \\
2000\end{array}$ & \\
\hline 3480 & MISC & $\begin{array}{l}\text { Test pits at B- } \\
\text { pond and 216-B- } \\
\text { 3-3- ditch }\end{array}$ & & G & & & & & $\begin{array}{l}\text { Metals, An, } \\
\text { SVOA, VOA, } \\
\text { Lab Rad }\end{array}$ & & & & & $\begin{array}{l}\text { Cearlock et al. } \\
2000\end{array}$ & \\
\hline 3481 & MISC & $\begin{array}{l}\text { Test Pits at } \\
\text { Gable Mountain } \\
\text { Pond }\end{array}$ & & G & & & & & $\begin{array}{l}\text { Metals, An, } \\
\text { SVOA, VOA, } \\
\text { Lab Rad }\end{array}$ & & & & & $\begin{array}{l}\text { Cearlock et al. } \\
2000\end{array}$ & \\
\hline 3482 & MISC & $\begin{array}{l}\text { U. S. Ecology, } \\
\text { MW-10 }\end{array}$ & & & & $Y$ & & & & Por & & & & $\begin{array}{l}\text { Bergeron et al. } \\
1987\end{array}$ & \\
\hline 3483 & MISC & $\begin{array}{l}\text { U. S. Ecology, } \\
\text { MW-5 }\end{array}$ & & & & $Y$ & & & & Por & & & & $\begin{array}{l}\text { Bergeron et al. } \\
1987\end{array}$ & \\
\hline 3484 & MISC & $\begin{array}{l}\text { U. S. Ecology, } \\
\text { MW-8 }\end{array}$ & & & & $Y$ & & & & Por & & & & $\begin{array}{l}\text { Bergeron et al. } \\
1987\end{array}$ & \\
\hline 3485 & MISC & $\begin{array}{l}\text { Various shallow } \\
\text { boreholes done } \\
\text { for HWVP }\end{array}$ & & G & & $\mathrm{Y}$ & & $\mathrm{Y}$ & An, & Bden & & & & Horton files & \\
\hline 3486 & MISC & $\begin{array}{l}\text { Various wells at } \\
218-W-5 \text { burial } \\
\text { ground }\end{array}$ & & & & & & $\mathrm{Y}$ & Metals, & CEC & Min, XRD & & & Bjornstad 1990 & \\
\hline 3487 & MISC & $\begin{array}{l}104 \text { surface and } \\
\text { outcrop soil } \\
\text { samples }\end{array}$ & & & & & & & Metals, An & & & & & DOE 1993c & \\
\hline 3488 & MISC & $\begin{array}{l}11 \text { test pits at } \\
\text { FFTF }\end{array}$ & & & & $Y$ & & $\mathrm{Y}$ & & Bden, SpG & & & & $\begin{array}{l}\text { Shannon \& } \\
\text { Wilson } 1970 \\
\end{array}$ & \\
\hline 3489 & MISC & $\begin{array}{l}13 \text { samples of } \\
\text { topsoil by } \\
\text { ecosystem }\end{array}$ & & & & & & & Wet Chem & & & & & DOE 1993c & \\
\hline 3490 & MISC & $\begin{array}{l}14 \text { vadose zone } \\
\text { soil samples }\end{array}$ & & & & $Y$ & & & Wet Chem & & PC & & & DOE 1993c & \\
\hline 3491 & MISC & $\begin{array}{l}172 \text { surface soil } \\
\text { samples from } \\
\text { former GTF site }\end{array}$ & & & & & & & $\begin{array}{l}\text { An, Metals, } \\
\text { Lab Rad }\end{array}$ & & & & & \begin{tabular}{|l} 
Mitchell et al. \\
1998
\end{tabular} & \\
\hline 3492 & MISC & $\begin{array}{l}19 \text { borings at } \\
\text { WNP No. } 2\end{array}$ & & & & $Y$ & & $Y$ & & Bden, & & & & $\begin{array}{l}\text { Shannon and } \\
\text { Wilson } 1972\end{array}$ & \\
\hline 3493 & MISC & $\begin{array}{l}19 \text { samples from } \\
\text { the sub pit }\end{array}$ & & & & & & & $\begin{array}{l}\mathrm{pH}, \mathrm{Pb}, \mathrm{An}, \mathrm{IC} \text {, } \\
\mathrm{TC}, \mathrm{TOC}, \mathrm{Res}\end{array}$ & & & & & EBASCO 1992 & \\
\hline 3494 & MISC & $\begin{array}{l}23 \text { surface } \\
\text { samples }\end{array}$ & & & & & Y & & $\mathrm{pH}$ & $\begin{array}{l}\text { CEC, } \\
\% \text { GSSC, 15- } \\
\text { Atm }\end{array}$ & & & & McHenry 1957 & \\
\hline 3495 & MISC & $\begin{array}{l}\text { 241-AP } \\
\text { excavation }\end{array}$ & & & & $Y$ & $Y$ & & & & & & & Goldstrand 1984 & \\
\hline 3496 & MISC & $\begin{array}{l}4 \text { Hanford } \\
\text { standards }\end{array}$ & & & & $Y$ & $\mathrm{Y}$ & $\mathrm{Y}$ & $\begin{array}{l}\text { TOC, XRF, } \\
\text { 1:1, Pwater }\end{array}$ & $\begin{array}{l}\text { \%GSSC, } \\
\text { Bden, CEC }\end{array}$ & TEM, XRD & & & $\begin{array}{l}\text { Serne et al. } \\
2001 \mathrm{a}\end{array}$ & \\
\hline 3497 & MISC & $\begin{array}{l}4 \text { samples from } \\
\text { White Bluffs }\end{array}$ & & & & & & & & & XRD & & & $\begin{array}{l}\text { Moodie et al. } \\
1966\end{array}$ & \\
\hline
\end{tabular}




\begin{tabular}{|c|c|c|c|c|c|c|c|c|c|c|c|c|c|c|c|}
\hline Sortindex & Area & Well Name & WellID & Log Type & GPX Log Runs & Sieved & $\mathrm{CaCO} 3$ & Moisture & $\begin{array}{l}\text { Chemical } \\
\text { Properties }\end{array}$ & $\begin{array}{l}\text { Physical } \\
\text { Properties }\end{array}$ & \begin{tabular}{c|} 
Min \\
Properties
\end{tabular} & $\begin{array}{l}\text { Geochron } \\
\text { Properties }\end{array}$ & Archived & Source & Comments \\
\hline 3498 & MISC & $\begin{array}{l}4 \text { shallow } \\
\text { boreholes at } \\
\text { McGee Ranch }\end{array}$ & & & & & & Y & & & & & & Gee 1987 & \\
\hline 3499 & MISC & $\begin{array}{l}40 \text { boreholes at } \\
\text { McGee Ranch }\end{array}$ & & G & & $\mathrm{Y}$ & & $Y$ & & & & & & \begin{tabular}{|l|} 
Last et al. 1987 \\
\end{tabular} & \\
\hline 3500 & MISC & \begin{tabular}{|l|}
5 boreholes and \\
13 test pits in \\
LERF area
\end{tabular} & & D & & & & & & & & & & DOE 1991 & \\
\hline 3501 & MISC & \begin{tabular}{|l|}
5 un-numbered \\
boreholes at the \\
Wye Barricade \\
\end{tabular} & & D & & $\bar{Y}$ & $\mathrm{Y}$ & $\mathrm{Y}$ & $\mathrm{pH}$ & $\begin{array}{l}\text { \%GSSC, } \\
\text { Moist, CEC, } \\
\text { Cond }\end{array}$ & & & & Heller et al. 1985 & \\
\hline 3502 & MISC & $\begin{array}{l}5 \text { volcanic ash } \\
\text { samples }\end{array}$ & & & & & & & Wet Chem & & & & & DOE 1993c & \\
\hline 3503 & MISC & \begin{tabular}{|l|}
57 shallow \\
boreholes on the \\
McGee Ranch
\end{tabular} & & G & & & & & & & & & & Lindberg 1994 & \\
\hline 3504 & MISC & \begin{tabular}{|l|}
2 boreholes near \\
$618-4$ burial \\
ground (B8780, \\
B8781)
\end{tabular} & & G & & & & $\mathrm{Y}$ & & Bden & & & & & Resistivity data also \\
\hline 3505 & MISC & Soil Map & & & & & & & & & & & & Hajek 1966 & $\begin{array}{l}\text { Surface soils map of the } \\
\text { Hanford Site }\end{array}$ \\
\hline 3506 & MISC & 9 test pits & & & & & & & \begin{tabular}{|l} 
An, Metal, \\
LabRad, \\
VOS,SVOA \\
\end{tabular} & & & & & DOE 2004a & 216-A-29, 216-B-63, 216-S-10 \\
\hline 3507 & UNK & B8485 & B8485 & & SG & & & & & & & & & & \\
\hline 3508 & UNK & B8486 & B8486 & & SG & & & & & & & & & & \\
\hline 3509 & UNK & B8487 & B8487 & & NT, SG & & & & & & & & & & \\
\hline 3510 & UNK & B8488 & B8488 & & MO, SG & & & & & & & & & & \\
\hline$\frac{3511 \mid}{35121}$ & UNK & B8489 & B8489 & & NT, SG & & & & & & & & & & \\
\hline 3513 & UNK & BN 1-9 & & & MG & & & & & & & & & & \\
\hline 3514 & UNK & G-123 & & & GG, MG & & & & & & & & & & \\
\hline 3515 & UNK & LIGO WELL & & & SG & & & & & & & & & & \\
\hline 3516 & UNK & $\begin{array}{l}\text { PNL Research } \\
\text { Well } 1\end{array}$ & & & SG & & & & & & & & & & \\
\hline 3517 & UNK & RSH \#1 & & & DN, NT, OT, SN, TP & & & & & & & & & & \\
\hline 3518 & UNK & UN-200E-63 & & & SG & & & & & & & & & & \\
\hline 3519 & UNK & \begin{tabular}{|l} 
YAK. MIN. 2-33 \\
\end{tabular} & & & MG & & & & & & & & & & \\
\hline
\end{tabular}




\section{Appendix B}

Bibliography of Geologic Data for the Hanford Site 


\section{Appendix B}

\section{Bibliography of Geologic Data for the Hanford Site}

Airhart, S. P., A. W. Pearson, and J. V. Borghese. 1990. Borehole Completion Data Package for the 216-S-10 Ditch and Pond. WHC-MR-0206, Westinghouse Hanford Company, Richland, Washington.

Alexander, D. J., S. D. Evelo, V. G. Johnson, and M. D. Sweeney. 1995. Groundwater Impact Assessment Report for the 216-T-4-2 Ditch. WHC-EP-0815, Westinghouse Hanford Company, Richland, Washington. The report contains average results of analysis for metals in sediment from three test pits and one metal analyses of sediment in one groundwater well (split spoons).

Ames, L. L. 1976. Monthly Report: Sediment Mineralogy and Petrology. Unpublished data. This letter report contains the results of XRD and electron microprobe analyses of various size fractions of 17 samples of Ringold Formation sediment. Analyses were done by PNNL. See Duane G. Horton for data.

Ames, L. L. and R. J. Serne. 1991. Compilation of Data to Estimate Groundwater Migration Potential for Constituents in Active Liquid Discharges at the Hanford Site. PNL-7660, Pacific Northwest Laboratory, Richland, Washington.

ARH-2874. 1973. 241-T-106 Tank Leak Investigation. Atlantic Richfield Hanford Company, Richland, Washington. The report gives the results of laboratory radionuclide analysis of samples from 10 boreholes.

ASC. 1984. Paleomagnetic Analysis of Cores DH-27 and DH-28. Analytical Services Company, subcontractor report to Rockwell Hanford Operations, 3 volumes. Presents results of paleomagnetic analysis of Ringold Formation and younger suprabasalt sediments. A total of 16 and 59 separate stratigraphic horizons were sampled in coreholes 699-50-96 and 699-50-99 (DH-27 and DH-28), respectively.

Baker, V. R., B. N. Bjornstad, A. J. Busacca, K. R. Fecht, E. P. Kiver, U. L. Moody, J. G. Rigby, D. F. Stradling, and A. M. Tallman. 1991. Quaternary Geology of the Columbia Plateau. In. Morrison, R. B., ed. Quarternary Nonglacial Geology; Conterminous U.S.: The Geology of North America. Geol. Soc. of Am., Boulder, Colorado, v. K-2, pp 215-250. This paper contains radiometric and thermoluminesce age dates for Hanford formation sediment from a few boreholes and several outcrops. 
Barnett, D. B. 1993. Soil Chemistry Data Package for the Proposed W-049H Site. WHC-SD-EN-DP065, Westinghouse Hanford Company, Richland, Washington. Results of chemical analyses (metals, hexavalent chromium, anions, cyanide, and volatile and semi-volatile organics) and gross alpha, gross beta and gross gamma are reported for 20 sediment samples from three boreholes at the Treated Effluent Disposal Basin. Analyses were performed by EPA approved methods at the TW/NORCAL and Roy F. Weston laboratories.

Barton, K.R.O. 1990. Borehole Completion Data Package for Low-Level Burial Grounds - 1990. WHC-MR-0205, Westinghouse Hanford Company, Richland, Washington.

Bauer, R. G. and I. D. Jacques. 2002. Borehole Summary Report of Borehole C3808 in the 216-Z-11 Ditch, 200-CW-5 U-Pond/Z -Ditches Cooling Water Group Operable Unit. CP-12134, Fluor Hanford, Richland, Washington. The report contains the geologic log, the geophysical logs, and a minimal number of particle size distributions and moisture contents.

Bergeron, M. P., G. V. Last, and A. E. Reisenauer. 1987. Geohydrology of a Commercial Low-Level Radioactive Waste Disposal Facility Near Richland, Washington. PNWD-1127, Pacific Northwest Laboratory, Richland, Washington. The report presents results of 61 analyses of particle size distribution. Data are presented in graph form; minimal tabular data are given. Analyses were preformed by CH2M HILL and Benjamin J. Hajek Consulting Services.

Bierschenk, W. H. 1959. Techniques for Estimating the Specific Retention Properties of Hanford Soils. HW-61644, General Electric Company, Richland, Washington. The report gives some particle size distribution information (but no tabular data), porosity, and moisture content of samples from three boreholes in the 200 Areas. The text discusses the sampling and analysis procedures.

Bjornstad, B. N., K. A. Lindsey, and J. E. Amonette. no date. Mineralogical and Geochemical Characteristics of Suprabasalt Sedimentary Units: Hanford Site, South-Central Washington.

Unpublished data. This report contains XRD mineralogy and the mean, standard deviation, and range of major oxide and trace element analyses of Hanford, Ringold, and Plio-Pleistocene sediment. XRD analyses were done by PNNL; XRF analyses were done by WSU and PNNL. Much of the data is the same as that found in Bjornstad, 1990. See George V. Last for data.

Bjornstad, B. N. 1980. Sedimentology and Depositional Environment of the Touchet Beds, Walla Walla River Basin, Washington. RHO-BWI-SA-44, Rockwell Hanford Operations, Richland, Washington. The report presents results of grain size distribution in samples of Hanford formation Touchet bed material collected in and around the Pasco Basin.

Bjornstad, B. N. 1984. Suprabasalt Stratigraphy within and Adjacent to the Reference Repository Location. SD-BWI-DP-039, Rockwell Hanford Operations, Richland, Washington. 
Bjornstad, B. N. 1987. Late-Cenozoic Sediments Within a Subsiding Basin of the Yakima Fold Belt: Estimates on Age and Rates of Deformation. Unpublished data. This paper presents Ar-40/Ar-39 age dates for four samples of Ringold Formation tephra from four boreholes on the Hanford Site. See George V. Last for data.

Bjornstad, B. N. 1990. Geohydrology of the 218-W-5 Burial Ground, 200-West Area, Hanford Site. PNL-7336, Pacific Northwest Laboratory, Richland, Washington. This report gives the results of several analytical tests. Results include (1) 62 soil moisture measurements by ASTM 2216, (2) 11 CEC measurements (from Oregon State University using procedures in Schollenbeger and Limon, 1945), (3) average, minimum, maximum, and standard deviation of $55 \mathrm{XRF}$ analyses of major metals, (4) nine x-ray diffraction analysis of clays done by PNNL, (5) point count results from 33 samples done by Washington State University, and (6) hydrometer and wet sieve analysis of six samples by ASTM 854.

Bjornstad, B. N. 1993. Borehole Completion Data Package for the 216-B-63 Trench, CY 1992. WHC-SD-SN-DP-051, Westinghouse Hanford Company, Richland, Washington. The report gives moisture and calcium carbonate contents for samples from 5-ft intervals in two new RCRA wells.

Bjornstad, B. N., K. R. Fecht, and C. J. Pluhar. 2001. "Long History of Pre-Wisconsin, Ice Age Cataclysmic Floods: Evidence from Southeastern Washington State.” Journal of Geology 109: 695-713. Paleomagnetic results are presented for 57 sediment samples of Hanford formation from 4 coreholes (299-E33-335, 699-E17-21, 699-W22-48, and 699-W22-50), and Th/U age dates presented for 9 surface samples of caliche on flood deposits.

Brown, D. J. 1960. Evaluation of Earth Samples from Churn-Drilled Wells. HW-67415, General Electric Company, Richland, Washington. This report compares samples from two sets of wells sampled by (1) drive barrel and (2) bailer. The results of analyses for cation exchange capacity, calcium carbonate content, particle size distribution, and $\mathrm{pH}$. References to procedures are given. The well pairs are 699-36-61 and -61B and 699-37-82A and -82B.

Brown, D. J. 1963. Geology Underlying the 241-AX Tank Farm. HW-79805, General Electric Company, Richland, Washington. The report gives the results of percent gravel, sand, silt, and clay for a few samples from boreholes in the area of the 241-AX tank farm.

Bryce, R. W. and S. M. Goodwin. 1989. Borehole Summary Report for Five Monitoring wells Constructed in the 1100 Area. PNL-6824, Pacific Northwest Laboratory, Richland, Washington. The report contains geologic logs and geophysical logs for five wells constructed between the 1100 Area and the Richland City water supply wells.

Caggiano, J. A. 1992. Borehole Completion Data Package for CY90 Wells Installed at Single-Shell Tanks. WHC-SD-EN-DP-041, Westinghouse Hanford Company, Richland, Washington. Report contains calcium carbonate content and moisture content from samples at 5-ft intervals in 11 new wells. All analyses were done by Westinghouse Hanford Company laboratories. Geologist logs are included.

Caggiano, J. A. 1993. Borehole Data Completion Package for CY 1991 and CY 1992 Wells at SingleShell Tanks. WHC-SD-EN-DP-042, Westinghouse Hanford Company, Richland, Washington.

B. 3 
Report contains calcium carbonate content and moisture content from samples at 5-ft intervals in 12 new wells. Some particle size distribution (GEL-07) data is also given. All analyses were done by Westinghouse Hanford Company laboratories. Geologist logs are included.

Cearlock, C. S., K. M. Singleton, M. E. Todd, and D. B. Barnett. 2000. 200-CW-1 Operable Unit Borehole/Test Pit Summary Report. BHI-01367, Bechtel Hanford Inc., Richland, Washington. The report presents data for 210 samples from 29 test pits and 25 samples from 2 boreholes and 2 groundwater wells at Gable Mountain Pond, B-Pond, 216-B-2-2 ditch and 216-B-3-3 ditch. Data include results from 44 quality control samples. Physical properties analyses include bulk density (ASTM D2937), particle size distribution (ASTM D-422), and moisture content (ASTM D-2216) by Maxim Technologies laboratory. Radiological and chemical analyses (anions, $\mathrm{pH}$, selected metals, selected organics) were done at RECRA Environmental Inc. and ThermoRetec Nuclear Services laboratories with Quanterra laboratory as the split lab. Procedures for chemical analyses were EAP approved; procedures for radiological analyses were industry accepted. Geologist's logs for boreholes and pits are included in the report.

Chamness, M. A. 1993. An Investigation of Bergmounds as Analogs to Erosion Control Factors on Protective Barriers. PNL-8841, Pacific Northwest Laboratory, Richland, Washington. The report gives the results of particle size analysis, in graph form only, of several surface and near surface samples from two locations.

Chamness, M. A., S. P. Luttrell, D. J. Bates, and W. J. Martin. 1990. 2101-M Hydrogeologic Characterization Report. PNL-7468, Pacific Northwest Laboratory, Richland, Washington. The report presents physical and chemical properties of borehole samples from 2101-M pond. Data include particle size distribution (Uebelacker 1980), calcium carbonate content (Nelson 1982), field pH (manufacturer's instructions), moisture content (ASTM 2216), XRF (PNL-SP-19, Rev. 0) VOA (U.S. Testing), ICP metals (U.S. Testing, and mineralogical information by energy dispersive $\mathrm{x}$-ray analysis and point count.

Chamness, M. A., T. J. Gilmore, and S. S. Teal. 1993. Savage Island Project Borehole Completion Report. PNL-8485, Pacific Northwest Laboratory, Richland, Washington. The report contains the geologist's logs for wells 699-42-E9A, 699-42-E9B, and 699-32-22B.

CH2M HILL Hanford, Inc. 2001. Borehole Completion Report - 216-Z-9 Trench Vadose Well Deepening and Characterization. BHI-01552, Rev. 0, Bechtel Hanford, Inc., Washington. This report describes deepening and reconfiguration of wells 299-W15-84 and 299-W15-95 for the 200-ZP-1 soil vapor extraction system. The geologist's logs and a graphical moisture profile are given.

Darrach, M. E. 1995. Operable Unit Borehole Summary Report for FY 1995. BHI-00470, Bechtel Hanford, Inc., Richland, Washington. The report gives the geologist's logs and some particle size distribution data for five new boreholes. The particle size distributions were measured by Westinghouse Hanford Company's Geotechnical Laboratory using procedure GEL-07.

Delaney, C. D. 1992a. Borehole Completion Data Package for the CY 1991 216-B-3 Pond Drilling Project. WHC-SD-EN-DP-046, Westinghouse Hanford Company, Richland, Washington. The report contains moisture content and calcium carbonate content from samples collected at 5 -ft intervals 
for seven new wells. In addition, 19 analyses of specific gravity, 18 analyses of moisture content, and 16 determinations of porosity were done from split spoon samples. All analyses were done by Westinghouse Hanford Company laboratories. Geologist logs are included.

Delaney, C. D. 1992b. Completion Report for the 216-B-3 Pond Drilling and Characterization Activities, FY 1988, Separations Area Ground-Water Monitoring Project. WHC-SD-EN-TI-029, Westinghouse Hanford Company, Richland, Washington. The report gives moisture data for four new RCRA wells. No discussion of methods or laboratories is given.

Delaney, C. D. 1993. Borehole Completion Data Package for the CY 1992 216-B-3 Pond Drilling Project. WHC-SD-EN-053, Westinghouse Hanford Company, Richland, Washington. The report gives moisture content (GEL-14) and calcium carbonate content (GEL-19) for 5-ft interval samples from two new RCRA boreholes. Westinghouse Hanford Company's Geotechnical Engineering Laboratory did the analyses.

Delgard, C. H. and G. S. Barney. 1983. Effects of Hanford High-Level Waste Components on Sorption of Cobalt, Strontium, Neptunium, Plutonium, and Americium of Hanford Sediments. RHORE-ST-1, Rockwell Hanford Operations, Richland, Washington. The report gives three cation exchange capacity results for Hanford Site sediments.

DOE. 1990. Grout Treatment Facility Dangerous Waste Permit Application. DOE/RL 88-27, Appendix 5, U.S. Department of Energy, Richland Field Office, Richland, Washington. The permit application gives the results of analysis of particle size distribution, calcium carbonate content, moisture content, anions, metals, cation exchange capacity, $\mathrm{pH}$, alkalinity, and specific conductance. Procedures are referenced in the text.

DOE. 1991. Liquid Effluent Retention Facility Dangerous Waste Permit Application. DOE/RL-9043, Rev.0, U.S. Department of Energy, Richland, Washington. Appendix 4F of the permit application gives particle size distribution (ASTM D-422) and moisture content (ASTM D-1557) for samples from five boreholes and 13 test pits in the LERF area. Driller's logs are also included.

DOE. 1993a. Limited Field Investigation Report for the 100-HR-3 Operable Unit. DOE/RL-93-34, U.S. Department of Energy, Richland Field Office, Richland, Washington.

DOE. 1993b. Limited Field Investigation Report for the 100-FR-1 Operable Unit. DOE/RL-93-82, U.S. Department of Energy, Richland, Washington. This report give the results of analyses for volatile organic compounds, semi-volatile organic compounds, anions, metals, and radionuclides. References are given for the CERCLA analytical procedures. 
DOE. 1993c. Hanford Site Background: Part 1, Soil Background for Nonradioactive Analytes. Volumes I and II. DOE/RL-92-24, Rev. 1, Vols. I and II. U.S. Department of Energy, Richland, Washington. Volume II of the report gives the results of chemical analyses for metals, ammonium, carbonate, and anions from more than 170 samples of Hanford (mostly) and Ringold formation sediments. The samples are from excavations and outcrops on site and from two boreholes. Analyses were done by EPA methods.

DOE. 1994a. Limited Field Investigation Report for the 100-BC-5 Operable Unit. DOE/RL-93-97, Rev. 0, U.S. Department of Energy, Richland Operations Office, Richland, Washington.

DOE. 1994b. Limited Field Investigation Report for the 100-BC-1 Operable Unit. DOE/RL-93-06, U.S. Department of Energy, Richland, Washington.

DOE. 1996. Hanford Site Background: Part 2, Soil Background for Radionuclides. DOE/RL-96-12, U.S. Department of Energy, Richland, Washington. The results of analysis for natural and manmade radionuclides in 50 vadose zone samples are presented and evaluated. The analyses were done by Quanterra, Inc. laboratories in Richland, Washington.

DOE. 2004a. Remedial Investigation Report for the 200-PW-2 Uranium-Rich Process Waste Group and the 200-PW-4 General Process Condensate Group Operable Units. DOE/RL-2004-25, Draft A. This report contains results of analyses for metals, anions, VOAs SVOAs, and radionuclides for 13 boreholes drilled in 2003.

DOE. 2004b. Remedial Investigation Report for the 200-CS-1 Operable Unit. DOE/RL-2004-17, Draft A, Department of Energy, Richland Operations Office, Richland, Washington. This report contains results of analyses for metals, anions, VOAs SVOAs, and radionuclides for four boreholes and several test pits at the 216-A-029 ditch, 216-B-63 trench, 216-S-10 pond, and 216-S-10 ditch.

DOE. 2004c. Remedial Investigation Report for the 200-CW-5 U Pond/Z Ditches Cooling Waste Group, the 200-CW-2 S Pond and Ditches Cooling Water Group, the 200-CW-4 T Pond and Ditches Cooling Water Group, and the 200-SC-1 Steam Condensate Group Operable Units. U.S. Department of Energy, Richland Operations, Richland, Washington. This report contains the analytical results for 12 samples from borehole C3808 at the 216-Z-11 ditch. Analytes include metals, anions, VOA, SVOA, and radionuclides.

Doremus, L. A. and A. W. Pearson. 1990. Borehole Completion Data Package for the Liquid Effluent Disposal Facility. WHC-MR-0235, Westinghouse Hanford Company, Richland, Washington. Report contains moisture content data from samples at 5-ft intervals in four new wells. Geologist logs are included. 
EBASCO Services Incorporated. 1992. Naval Trench 94 Soils Report. WHC-MR-0284, Westinghouse Hanford Company, Richland, Washington. The report give results of testing for lead, anions, total organic carbon, inorganic carbon, $\mathrm{pH}$, and wet and dry resistivity on 20 samples of Hanford formation from the submarine reactor compartment trench. Chemical analyses were done by Accutest Laboratories using EPA methods.

Faurote, J. M. 2001. Borehole Summary Report for the 618-11 Burial Ground Tritium Investigation. BHI-01567, Rev. 0, Bechtel Hanford, Inc., Richland Washington. This report describes the installation of four monitoring wells at the 618-11 burial ground: 699-13-1D, 699-12-2C, 699-13-2D, and 699-13-0A. Also described is the installation and decommissioning of two boreholes: C3252 and C3255. Geologist's logs are in an appendix.

Faurote, J. M. and C. S. Wright. 2001. Borehole Summary Report for Well 299-W19-43 (C2281), 200-UP-1 Operable Unit. BHI-01587, Bechtel Hanford, Inc., Richland, Washington. This report describes the installation of monitoring well 299-W19-43. The geologist's log is in an appendix.

Faurote, J. M. 2002. Borehole Summary Report for Well 299-W15-42 (C3803) and Borehole 299-W15-764 (C3494), 200-ZP-1 Operable Unit. BHI-10620, Bechtel Hanford, Inc., Richland, Washington. This report describes the installation of well 299-W15-42 and the drilling and decommissioning of borehole 299-W15-764 in the PFP protected area. Geologist's logs are in an appendix.

Fecht, K. R. and J. T. Lillie. 1982. A Catalog of Borehole Lithologic Logs from the 600 Area, Hanford Site. RHO-LD-158, Rockwell Hanford Operations, Richland, Washington. A compilation of about 800 lithologic logs from the 600 Area.

Fecht, K. R. and W. H. Price. 1977a. Granulometric Data 241-A Tank Farm Monitoring Well Sediments. RHO-LD-11, Rockwell Hanford Operations, Richland, Washington. The report gives particle size distribution and calcium carbonate content for sediments from boreholes within the 241-A tank farm. The procedures used to collect the data are discussed in the report. The data also appears in the ROCSAN database.

Fecht, K. R. and W. H. Price. 1977b. Granulometric Data 241-AX Tank Farm Monitoring Well Sediments. RHO-LD-12, Rockwell Hanford Operations, Richland, Washington. The report gives particle size distribution and calcium carbonate content for sediments from boreholes within the 241-AX tank farm. The procedures used to collect the data are discussed in the report. The data also appears in the ROCSAN database.

Fecht, K. R. and W. H. Price. 1977c. Granulometric Data 241-BX Tank Farm Monitoring Well Sediments. RHO-LD-14, Rockwell Hanford Operations, Richland, Washington. The report gives particle size distribution and calcium carbonate content for sediment from boreholes within the 241-BX tank farm. The procedures used to collect the data are discussed in the report. The data also appears in the ROCSAN database. 
Fecht, K. R. and W. H. Price. 1977d. Granulometric Data 241-BY Tank Farm Monitoring Well Sediments. RHO-LD-15, Rockwell Hanford Operations, Richland, Washington. The report gives particle size distribution and calcium carbonate content for sediment from boreholes within the 241-BY tank farm. The procedures used to collect the data are discussed in the report. The data also appears in the ROCSAN database.

Fecht, K. R. and W. H. Price. 1977e. Granulometric Data 241-C Tank Farm Monitoring Well Sediments. RHO-LD-16, Rockwell Hanford Operations, Richland, Washington. The report gives particle size distribution and calcium carbonate content for sediment from boreholes within the 241-C tank farm. The procedures used to collect the data are discussed in the report. The data also appears in the ROCSAN database.

Fecht, K. R. and W. H. Price. 1977f. Granulometric Data 241-S Tank Farm Monitoring Well Sediments. RHO-LD-17, Rockwell Hanford Operations, Richland, Washington. The report gives particle size distribution and calcium carbonate content for sediment from boreholes within the 241-S tank farm. The procedures used to collect the data are discussed in the report. The data also appears in the ROCSAN database.

Fecht, K. R. and W. H. Price. 1977g. Granulometric Data 241-SX Tank Farm Monitoring Well Sediments. RHO-LD-18, Rockwell Hanford Operations, Richland, Washington. The report gives particle size distribution and calcium carbonate content for sediment from boreholes within the 241-SX tank farm. The procedures used to collect the data are discussed in the report. The data also appears in the ROCSAN database.

Fecht, K. R. and W. H. Price. 1977h. Granulometric Data 241-T Tank Farm Monitoring Well Sediments. RHO-LD-19, Rockwell Hanford Operations, Richland, Washington. The report gives particle size distribution and calcium carbonate content for sediment from boreholes within the 241-T tank farm. The procedures used to collect the data are discussed in the report. The data also appears in the ROCSAN database.

Fecht, K. R. and W. H. Price. 1977i. Granulometric Data 241-TX Tank Farm Monitoring Well Sediments. RHO-LD-20, Rockwell Hanford Operations, Richland, Washington. The report gives particle size distribution and calcium carbonate content for sediment from boreholes within the 241-TX tank farm. The procedures used to collect the data are discussed in the report. The data also appears in the ROCSAN database.

Fecht, K. R. and W. H. Price. 1977j. Granulometric Data 241-TY Tank Farm Monitoring Well Sediments. RHO-LD-21, Rockwell Hanford Operations, Richland, Washington. The report gives particle size distribution and calcium carbonate content for sediment from boreholes within the 241-TY tank farm. The procedures used to collect the data are discussed in the report. The data also appears in the ROCSAN database. 
Fecht, K. R. and W. H. Price. 1977k. Granulometric Data 241-U Tank Farm Monitoring Well Sediments. RHO-LD-22, Rockwell Hanford Operations, Richland, Washington. The report gives particle size distribution and calcium carbonate content for sediment from boreholes within the 241-U tank farm. The procedures used to collect the data are discussed in the report. The data also appears in the ROCSAN database.

Fecht, K. R., G. V. Last, and M. C. Marratt. 1978a. Granulometric Data 216-A Crib Facilities Monitoring Well Sediments. RHO-LD-44, Rockwell Hanford Operations, Richland, Washington. The report gives particle size distribution and calcium carbonate content for sediment from boreholes at the 216-A liquid disposal facilities. The procedures used to collect the data are discussed in the report. The data also appears in the ROCSAN database.

Fecht, K. R., G. V. Last, and M. C. Marratt. 1978b. Granulometric Data 216-B Crib Facilities Monitoring Well Sediments. RHO-LD-45, Rockwell Hanford Operations, Richland, Washington. The report gives particle size distribution and calcium carbonate content for sediment from boreholes at the 216-B liquid disposal facilities. The procedures used to collect the data are discussed in the report. The data also appears in the ROCSAN database.

Fredrickson, J. K., F. J. Brockman, B. N. Bjornstad, P. E. Long, S. W. Li, J. P. McKinley, J. V. Wright, J. L. Conca, T. L. Kieft, and D. L. Balkwill. 1993. "Microbiological Characteristics of Pristine and Contaminated Deep Vadose Sediments from an Arid Region.” Geomicrobiology Journal, V. 11, pp. 95-107. The report gives results of analyses of total carbon, total organic carbon, inorganic carbon, nitrate, and moisture for a few samples from three boreholes. Methods are described in the text.

Freeman-Pollard, J. R. 1994. Engineering Evaluation of the GAO-RCED-89-157, Tank 241-T-106 Vadose Zone Investigation. BHI-00061, Bechtel Hanford Inc., Richland, Washington. The report gives analytical results from 34 samples from one borehole drilled in 1993. Analyses include VOA (EPA 8240), SVOA (EPA 8270), metals (CLP level IV procedures, anions, radionuclides, and moisture content. The geologist's log is included.

Fruchter, J. S., J. E. Amonette, C. R. Cole, Y. A. Gorby, M. D. Humphrey, J. D. Istok, F. A. Spane, J. E. Szecsody, S. S. Teel, V. R. Vermeul, M. D. Williams, and S. B. Yabusaki. 1996. In Situ Redox Manipulation Field Injection Test Report - Hanford 100-H Area. PNNL-11372, Pacific Northwest National Laboratory, Richland, Washington.

Fruchter, J. S., C. R. Cole, M. D. Williams, V. R. Vermeul, J. E. Amonette, J. E. Szecsody, J. D. Istok, and M. D. Humphrey. 2000. “Creation of a Subsurface Permeable Treatment Barrier Using In Situ Redox Manipulation.” Groundwater Monitoring and Remediation Review, p. 1-15.

Fruland, R. M, R. A. Hagan, C. S. Cline, D. J. Bates, J. C. Evans, and R. L. Aaberg. 1989. Interim Site Characterization Report and Ground-Water Monitoring Program for the Hanford Site Solid Waste Landfill. PNL-6823, Pacific Northwest Laboratory, Richland, Washington. The report gives results of testing for particle size distribution and moisture content of samples from six wells. 
Gardner, M. C. and K. D. Reynolds. 2000. SX Slant Borehole Completion Report. RPP-6917, Rev. 0, U.S. Department of Energy, Office of River Protection, Richland, Washington.

Gardner, M. G., K. D. Reynolds, and D. E. Skoglie. 2002a. Completion Report for Probe Hole C3831 (TX-107) TX Tank Farm 200 West Area. RPP-12293, Rev. 0, Prepared by Duratek Federal Services, Inc., Northwest Operations for CH2M HILL Hanford Group, Inc., Richland, Washington. The report contains the geologic and geophysical logs.

Gardner, M. G., K. D. Reynolds, and D. E. Skoglie. 2002b. Completion Report for Probe Hole C3832 (TX-104) TX Tank Farm 200 West Area. RPP-12017, Rev. 0, Prepared by Duratek Federal Services, Inc., Northwest Operations for CH2M HILL Hanford Group, Inc., Richland, Washington. The report contains the geologic and geophysical logs.

Gaylord, D. R., E. P. Poeter, and P. Townsend. 1991. Summary Report of FY90/91 Geologic and Hydrogeologic Investigations at the Old Hanford Town Site, Washington. The report gives the results of air permeameter measurements on several samples from one well. The report briefly discusses and gives reference to procedures. Unpublished report available from Duane G. Horton.

Gee, G. W. 1987. Recharge at the Hanford Site: Status Report. PNL-6403, Pacific Northwest Laboratory, Richland, Washington. The report gives moisture content for samples from four shallow boreholes at the McGee Ranch.

Gilmore, T. J, S. M. Goodwin, and D. R. Newcomer. 1989. Well Completion Report for Wells 199$N-69$ and 199- $N-70$. Unpublished Pacific Northwest National Laboratory report. This document compiles the data collected during the drilling construction and testing of these $2 \mathrm{~N}$ Area wells.

Golder. 1982. The Southeast Anticline Fault: Evaluation of Attitude and Displacement. Prepared by Golder Associates, Inc. for Washington Public Power Supply System. The report contains geologic logs of 12 boreholes in the 600 Area.

Goldstrand, P. M. 1984. Generalized Geology of the 241-AP Tank Farm. RHO-RE-EV-31, Rockwell Hanford Operations, Richland, Washington. This report give particle size distribution and calcium carbonate content for samples collected in the excavation for 241-AP tank farm. Procedures are include in appendices.

Goodwin, S. M. 1990. Borehole Completion Data Package for the 216-U-12 Crib. WHC-MR-0208, Westinghouse Hanford Company, Richland, Washington.

Goodwin, S. M. 1993. Petrography of the Coarse-Grained Facies of the Miocene-Pliocene Ringold Formation, South-Central Washington State. M. S. Thesis, Western Washington University, Bellingham, Washington. The thesis gives particle size distributions, pebble count data of gravels, point count data for sands from samples of the Ringold Formation taken from drill core on the Hanford Site and outcrops on the White Bluffs. Also includes unpublished pebble counts from Lindsey. 
Goodwin, S. M. and B. J. Bjornstad. 1990. 200-East and 200-West Areas Low-Level Burial Grounds Borehole Summary Report. WHC-MR-0204, Westinghouse Hanford Company, Richland, Washington.

Hajek, B. F. 1966. Soil Survey: Hanford Project in Benton County Washington. BNWL-243, Pacific Northwest Laboratory, Richland, Washington. The report contains a surface soils map with descriptions for the Hanford Site.

Haney, W. A. and C. E. Linderoth. 1959. Exploratory Field Study of a Ground Waste Disposal Facility. HW-60115, General Electric, Richland, Washington. The report contains beta and Sr-90 values from sediment samples in five boreholes at the 216-S-2 and 216-S-2 cribs

Haney, W. A. (ed.). 1967. Final Report on the Effects of Ben Franklin Dam on Hanford. BNWL412, Battelle Northwest Laboratory, Richland, Washington. The report gives laboratory measured radionuclide results from borehole samples near the 216-BY cribs, the BC cribs, the 216-A-8 crib, and the 216-A-5 crib in graphical form. BNWL radiological laboratories performed the analyses. The laboratory data sheets giving the numerical data can be obtained from Duane G. Horton.

Hartman, M. J. and K. A. Lindsey. 1993. Hydrogeology of the 100-N Area, Hanford Site, Washington. WHC-SD-EN-EV-027, Westinghouse Hanford Company, Richland, Washington. This report describes the hydrogeologic units beneath the 100-N Area summarizing data from previous reports, and providing chemical distribution of specific contaminants. The purpose is to serve as a reference for RCRA facility plans.

Heller, P. R., G. W. Gee, and D. A. Myers. 1984. Geotechnical Properties: Partially Saturated Zone Characteristics of the Skagit/Hanford Site. PNL-5262, Pacific Northwest Laboratory, Richland, Washington. The reports gives the results of analyses of particle size distribution (ASTM D-422), \%GSSC, CEC, calcium carbonate content, $\mathrm{pH}$, electrical conductivity, and moisture content (ASTM D-2216) from samples from five boreholes. Driller's logs are included. References to procedures are given. Particle size distribution data for sizes between $2 \mathrm{~mm}$ and 3 in. are tabulated; all other sizes are graphed only.

Heller, P. R., G. W. Gee, and D. A. Myers. 1985. Moisture and Textural Variations in Unsaturated Soils/Sediments Near the Hanford Wye Barricade. PNL-5377, Pacific Northwest Laboratory, Richland, Washington. The reports gives the results of analyses of particle size distribution (ASTM D422), \%GSSC, CEC, calcium carbonate content, $\mathrm{pH}$, electrical conductivity, and moisture content (ASTM D-2216) from samples from five un-numbered boreholes. Driller's logs are included. References to procedures are given. Particle size distribution data for sizes between $2 \mathrm{~mm}$ and $3 \mathrm{in}$. are tabulated; all other sizes are graphed only. 
Hodges, F. N. 1993. Borehole Completion Data Package for NRDWL Facility Monitoring Wells 699-25-34D and 699-26-34B. WHC-SD-EN-DP-055, Westinghouse Hanford Company, Richland, Washington. The report gives moisture content (GEL-14) and calcium carbonate content (GEL-19) for 5-ft interval samples from two new RCRA boreholes. Westinghouse Hanford Company's Geotechnical Engineering Laboratory did the analyses.

Hoffman, K. M. 1992. 200-BP-1 Borehole Summary Report for Tasks 2, 4, and 6. WHC-SD-EN-TI054, Westinghouse Hanford Company, Richland, Washington. Report contains porosity, specific gravity, calcium carbonate, bulk density, percent moisture and particle size distribution data for 13 wells and boreholes drilled in 1991 and 1992.

Horton, D. G. files Unpublished x-ray diffraction data from 150 samples of Hanford, Ringold, and Ellensburg formations from various boreholes in the 200 Areas and 100 Areas. Unpublished Dames and Moore engineering study done for Kaiser Engineers Hanford to support Hanford Waste Vitrification Project in 1989. The report contains soil engineering data from 17 boreholes. Summary lithologic logs are included. Unpublished XRF analyses of nine samples of Ringold Formation from 600 Area wells. Analyses were done by Washington State University. Data is available from Duane G. Horton.

Horton, D. G. 2000. Borehole Data Package for Wells 299-E33-334 and 299-E33-335 at Single-Shell Tank Waste Management Area B-BX-BY. PNNL-13199, Pacific Northwest National Laboratory, Richland, Washington. Report give moisture content every $5 \mathrm{ft}$ throughout one borehole. Analyses were done at the laboratory in 3720 Building, Pacific Northwest National Laboratory.

Horton, D. G. and F. N. Hodges. 1999a. Borehole Data Package for 1998 Wells Installed at SingleShell Tank Waste Management Area TX-TY. PNNL-12124, Pacific Northwest National Laboratory, Richland, Washington. Report presents several analytical results of cation exchange capacity, calcium carbonate content, alkalinity, major anions and cations, $\mathrm{pH}$, and moisture content from samples from three wells. A few particle size distributions are also given. All analyses were done in the 3720 Building laboratories, Pacific Northwest National Laboratory.

Horton, D. G. and F. N. Hodges. 1999b. Borehole Data Package for 1998 Wells Installed at SingleShell Tank Waste Management Area U. PNNL-12126, Pacific Northwest National Laboratory, Richland, Washington. Report contains analyses of cation exchange capacity, calcium carbonate content, major cations and anions, alkalinity, moisture content, electrical conductivity and $\mathrm{pH}$ for 28 samples from one well. Also, reported are four particle size distribution results from two wells. Analyses were done in the laboratories at 3720 Building, Pacific Northwest National Laboratory.

Horton, D. G. and F. N. Hodges. 1999c. Borehole Data Package for 1998 Wells Installed at SingleShell Tank Waste Management Area T. PNNL-12125, Pacific Northwest National Laboratory, Richland, Washington. Report contains analyses of major cations, major anions and alkalinity, moisture content, and inorganic carbon for four samples from one borehole. Also, a few particle size distribution results are given. Analyses were done in 3720 Building, Pacific Northwest National Laboratory. 
Horton, D. G. and S. M. Narbutovskih. 1999. Borehole Data Package for Well 299-E33-44 at Single-Shell Tank Farm Waste Management Area B-BX-BY. PNNL-12128, Pacific Northwest National Laboratory, Richland, Washington. Report contains analytical results for cation exchange capacity, calcium carbonate content, major cations and anions, alkalinity, moisture content, electrical conductivity, and $\mathrm{pH}$ for 26 samples from one borehole. Particle size distributions for nine samples are also given.

Horton, D. G. and V. G. Johnson. 2000. Borehole Data Package for Wells 200-W22-48, 299-W22-49, and 299-W22-50 at Single-Shell Tank Waste Management Area S-SX. PNNL-13200, Pacific Northwest National Laboratory, Richland, Washington. Report contains several analytical results of conductivity, $\mathrm{pH}$, and moisture content from one split-spoon cored well and conductivity, $\mathrm{pH}$, moisture content, and bulk density from on drive barrel sampled well. All analyses were done in the laboratories at the 3720 Building, Pacific Northwest National Laboratory.

Horton, D. G., B. A. Williams, and C. S. Cearlock. 2000. Borehole Data Package for the 216-S-10 Pond and Ditch Well 299-W26-13. PNNL-13198, Pacific Northwest National Laboratory, Richland, Washington. Report contains field determination of bulk density, moisture content (ASTM D-2216), pH, and particle size distribution (ASTM D-422) for three samples. The report also contains laboratory analytical results from seven samples and three QC samples for VOA, SVOA, anions, PCBs, and selected metals.

Horton, D. G. and V. G. Johnson. 2001. Borehole Data Package for Calendar Year 2000-2001 RCRA Wells at Single-Shell Tank Waste Management Area S-SX. PNNL-13589, Pacific Northwest National Laboratory, Richland, Washington. This report describes the installation of six groundwater monitoring wells at WMA S-SX: 299-W22-80, 299-W22-81, 299-W22-82, 299-W22-83, 299-W23-20, and 299-W23-21. Geologist's logs, a few particle size distributions, and spectral gamma logs are given in appendices.

Horton, D. G. and F. N. Hodges. 2001a. Borehole Data Package for Calendar Year 2000-2001 RCRA Wells at Single-Shell Tank Waste Management Area T. PNNL-13590, Pacific Northwest National Laboratory, Richland, Washington. This report describes the installation of five groundwater monitoring wells at WMA T: 299-W1-38, 299-W11-39, 299-W11-40, 299-W11-41, and 299-W11-42. Geologist's logs, a few particle size distributions, and spectral gamma logs are given in appendices.

Horton, D. G. and F. N. Hodges. 2001b. Borehole Data Package for the Calendar Year 2000 - 2001 RCRA Wells at Single-Shell Tank Waste Management Area TX-TY. PNNL-13591, Pacific Northwest National Laboratory, Richland, Washington. This report describes the installation of five groundwater monitoring wells at WMA TX-TY: 299-W10-27, 299-W14-15, 299-W14-16, 299-W14-17, and 299W15-763. Geologist's logs, a few particle size distributions, and spectral gamma logs are given in appendices. 
Horton, D. G. 2002a. Borehole Data Package for Calendar Year 2001 RCRA Well Installation at Single-Shell Tank Waste Management Area T. PNNL-13830, Pacific Northwest National Laboratory, Richland, Washington. This report describes the installation of one groundwater monitoring well, 299-W10-28, at WMA T. The geologist's log, a few particle size distributions, and spectral gamma and neutron moisture logs are given in an appendix.

Horton, D. G. 2002b. Borehole Data Package for Calendar Year 2001 RCRA Well Installation at Single-Shell Tank Waste Management Area TX-TY. PNNL-13826, Pacific Northwest National Laboratory, Richland, Washington. This report describes the installation of two groundwater monitoring wells, 299-W14-18 and 299-W15-765, at WMA TX-TY. The geologist's logs, a few particle size distributions, and spectral gamma and neutron moisture logs are given in appendices.

Horton, D. G. 2002c. Borehole Data Package for Calendar Year 2001 RCRA Wells at Single-Shell Tank Waste Management Area U. PNNL-13828, Pacific Northwest National Laboratory, Richland, Washington. This report describes the installation of three groundwater monitoring wells at WMA U: 299-W18-40, 299-W19-44, and 299-W19-45. The geologist's logs, some particle size distributions, and spectral gamma and neutron moisture logs are given in the appendices.

Horton, D. G. 2002d. Borehole Data Package for Calendar Year 2001 RCRA Wells at Single-Shell Tank Waste Management Area B-BX-BY. PNNL-13827, Pacific Northwest National Laboratory, Richland, Washington. This report describes the installation of three groundwater monitoring wells at WMA B-BX-BY: 299-E33-337, 299-E33-338, and 299-E33-339. The geologist's logs, some particle size distributions, and spectral gamma and neutron moisture logs are given in the appendices.

Horton, D. G. 2002e. Borehole Data Package for Calendar Year 2001 RCRA Wells at Single-Shell Tank Waste Management Area S-SX. PNNL-13829, Pacific Northwest National Laboratory, Richland, Washington. This report describes the installation of two groundwater monitoring wells at WMA S-SX: 299-W22-84 and 299-W22-85. The geologist's logs, some particle size distributions, and spectral gamma and neutron moisture logs are given in the appendices.

Horton, D. G., H. T. Schaef, R. J. Serne, C. F. Brown, M. M. Valenta, T. S. Vickerman, I. V. Kutnyakov, S. R. Baum, K. N. Geiszler, and K. E. Parker. 2003a. Geochemistry of Samples from Borehole C3177 (299-E24-21). PNNL-14289, Pacific Northwest National Laboratory, Richland, Washington. The report contains the results of physical and geochemical testing of 18 samples for the Integrated Disposal Facility characterization.

Horton, D. G. 2003a. Data Package for Calendar Year 2002 RCRA Groundwater Monitoring Wells at Single-Shell Tank Waste Management Area TX-TY. PNNL-14249, Pacific Northwest National Laboratory, Richland, Washington. This report describes the installation of two groundwater monitoring wells at WMA TX-TY: 299-W14-19 and 299-W15-44. The geologist's logs, some particle size distributions, and spectral gamma logs are given in THE appendices.

Horton, D. G. 2003b. Data Package for Groundwater Monitoring Well 299-W19-14 at the 200-UP-1 Operable Unit. PNNL-14248, Pacific Northwest National Laboratory, Richland, Washington. The report contains the geologist's log and the geophysical logs for one well.

$$
\text { B. } 14
$$


Horton, D. G. 2003c. Data Package for Groundwater Monitoring Well 299-W15-43 at the 200-ZP-1 Operable Unit. PNNL-14255, Pacific Northwest National Laboratory, Richland, Washington. The report contains the geologist's log and the geophysical logs for one well.

Hulstrom, L. C. 2004. 200-PW-2 and 200-PW-4 Operable Units Borehole Summary Report. CP-18666, Rev. 0, Fluor Hanford, Richland, Washington. The report contains the geologic logs, geophysical logs, particle size distributions, and moisture content from boreholes C3245, C4107, C4108, C4109, C4110, C4111, C4112, C4106, C3247, C3246, C3248, C4160, and C4113.

Isaacson, R. E., L. E. Brownell, and J. C. Hanson. 1974. Soil Moisture Transport in Arid Site Vadose Zones. ARH-2983, Atlantic Richfield Hanford Company, Richland, Washington. The report gives results of testing for $\mathrm{pH}$ and percent gravel, sand, silt and clay for 5-ft interval samples from well 699-32-49. Also included are tritium content of soil moisture for some samples from 699-19-47, 699-3249B and 699-32-49 D.

Johnson, V. G. 1993. Groundwater Impact Assessment Report for the 216-Z-20 Crib, 200 West Area. WHC-EP-0674, Westinghouse Hanford Company, Richland, Washington. The report contains Am-241 and Pu-239 laboratory results from eight samples from one well. Americium was determined by low energy gamma-ray spectrometry.

Johnson, V. M., K. A. Lindsey, R. J. Serne, R. S. Edrington, and T. H. Mitchel. 1995. N-Springs Barrier Wall Drilling Program Data Package. BHI-00135, Rev. 1, Bechtel Hanford, Inc., Richland, Washington. The report contains geologist's logs for 8 boreholes in 100-N Area. Analyses of selected soil samples include particle size distribution, gamma energy analysis, strontium-90, and tritium.

Kasper, R. B. 1981. 216-Z-12 Crib Status Report. RHO-LD-166, Rockwell Hanford Operations. Richland, Washington. Report contains the results of Pu-239 and Am-241 on sediment samples from previous studies.

Kasper, R. B. 1982. 216-Z-12 Transuranic Crib Characterization: Operational History and Distribution of Plutonium and Americium. RHO-ST-44, Rockwell Hanford Operations, Richland, Washington. The report contains analytical results for Pu-238, Pu-239/240, and Am-241 on samples from seven boreholes. Analytical laboratories were Rockwell laboratories in the 200 West Area, Eberline Instrument Corporation Laboratory, and LFE Environmental Analytical Laboratory.

Kasza, G. L. 1992. Borehole Completion Data Package for 216-A-29 RCRA Facility Monitoring Wells: Calendar Year 1991. WHC-SD-EN-DP-047, Westinghouse Hanford Company, Richland, Washington. Report contains calcium carbonate content and moisture content from samples at 5-ft intervals in four new wells. (Moisture was measured only in intervals where no water was added.) All analyses were done by Westinghouse Hanford Company laboratories. Geologist logs are included. 
Kasza, G. L. 1993. Borehole Completion Data Package for 216-A-29 RCRA Facility Monitoring Wells 299-E25-47 and 299-E25-48. WHC-SD-EN-DP-054, Westinghouse Hanford Company, Richland, Washington. The report gives moisture content (GEL-14) and calcium carbonate content (GEL-19) for 5-ft interval samples from two new RCRA boreholes. Westinghouse Hanford Company's Geotechnical Engineering Laboratory did the analyses.

Khaleel, R. 1999. Far-Field Hydrology Data Package for Immobilized Low-Activity Tank Waste Performance Assessment. HNF-4769, Rev. 1, Fluor Federal Services, Richland, Washington. This report presents the laboratory measurements on physical and hydraulic properties for soil samples at the immobilized low-activity waste disposal site.

Kious, J. J. 2001. 116-H-1 Characterization Borehole Results. BHI-01541, Rev. 0, Bechtel Hanford, Inc., Richland, Washington. This report describes characterization activities associated with drilling and sampling of borehole C3048. Moisture content data and a geologist's log are given.

Last, G. V. and T. G. Caldwell. 2001. Core Sampling in Support of the Zone Transport Field Study. PNNL-13454, Pacific Northwest National Laboratory, Richland, Washington. Borehole logs, moisture content, bromide and chloride content for three split spoon sampled holes at the Sisson and Lu site. Halide analyses were done by ion specific electrodes.

Last, G. V. and V. J. Rohay. 1993. Refined Conceptual Model for the Volatile Organic CompoundsArid Integrated Demonstration and 200 West Area Carbon tetrachloride Expedited response Action. PNL-8597, Pacific Northwest Laboratory, Richland, Washington. This report gives results of analyses for volatile organics, anions, cyanide, mercury lead, metals, and radionuclides on samples from six wells at the 216-Z-9 trench and 216-A-1A tile field.

Last, G. V, R. J. Serne, and V. L. LeGore. 1995. Field Lysimeter Studies for Performance Evaluation of Grouted Hanford Defense Wastes. PNL-10166, Pacific Northwest Laboratory, Richland, Washington. The report gives particle size analyses for samples collected from excavated soils from 241-AN tank farm.

Last, G. V, M. A. Glennon, M. A. Young, and G. W. Gee. 1987. Protective Barrier Materials Analysis: Fine Soil Site Characterization. PNL-6314, Pacific Northwest Laboratory, Richland, Washington. The report gives moisture content (ASTM D-2216) and particle size distribution (procedure developed by D. L. Uebelacker) for samples from 40 test holes at the McGee Ranch. Samples were obtained by split-spoon methods.

Last, G. V., B. N. Bjornstad, M. P. Bergeron, D. W. Wallace, D. R. Newcomer, J. A. Schramke, M. A. Chamness, C. S. Cline, S. P. Airhart, and J. S. Wilbur. 1989. Hydrogeology of the 200 Areas Low-Level Burial Grounds - An Interim Report. PNL-6820, Vol. 2, Pacific Northwest Laboratory, Richland, Washington. The report contains soil moisture content of samples from 24 new RCRA wells. Analytical procedures are discussed in the text. 
Last, G. V., D. W. Duncan, M. J. Graham, M. D. Hall, V. W. Hall, D. S. Landeen, J. G. Leitz, and R. M. Mitchell. 1994. 216-U-10 Pond and 216-Z-19 Ditch Characterization Studies. WHC-EP-0707, Westinghouse Hanford Company, Richland, Washington. Report contains analytical results for Sr-90, Sc-137, Pu-239/240, and Am-241 from several samples obtained from several boreholes. The analyses were done by Rockwell Operations Laboratories, LFE Environmental Analytical Laboratory, and Eberline Technology Laboratory.

Last, G. V. and T. G. Caldwell. 2001. Core Sampling in Support of the Vadose Zone Transport Field Study. PNNL-13454, Pacific Northwest National Laboratory, Richland, Washington. The report contains geologist's logs, moisture content, particle size distributions, and chloride content of soils for three shallow boreholes at the Sisson and Lu field site.

Last, G. V., T. G. Caldwell, and A. T. Owen. 2001. Sampling of Boreholes WL-3A through -12 in Support of the Vadose Zone Transport Field Study. PNNL-13631, Pacific Northwest National Laboratory, Richland, Washington. The report contains the geologic logs, moisture content, percent fines, and 1:1 sediment to water anion concentrations of 12 borings at the Sisson and Lu site, 200 East Area.

Laurenz, J. E. 1993. Characterization Regulatory Support Document, Project C-018H Soil Column Disposal Siting Evaluation. WHC-SC-C018H-TI-001, Westinghouse Hanford Company, Richland, Washington. The report gives the results of analyses for metals, anions gross alpha, gross beta, Cs-137, and $\mathrm{pH}$ for a small number of samples from wells 699-48-77A and 699-48-77B.

Laurenz, J. E. and L. D. Walker. 2001. In Situ Redox Manipulation Barrier Well Completion Report for the 100-HR-3 Groundwater Operable Unit. FY 2000. BHI-01381, Rev. 0, Bechtel Hanford, Inc., Richland, Washington. This report describes the installation of 16 groundwater monitoring wells in the 100-D Area (wells 199-D4-24 through 199-D4-39). Geologist's logs are in an appendix.

Lee, T. A. 1999. Well Completion Report for Fiscal Year 1999 Drilling Within the Chromium Plume West of the 100-D/DR Reactors. BHI-01300, Rev. 0, Bechtel Hanford, Inc., Richland, Washington. The report contains the geologists logs and limited particle size distribution data for twelve wells.

Liikala, T. L., R. L. Aaberg, N. J. Aimo, D. J. Bates, T. J Gilmore, E. J. Jensen, G. V. Last, P. L. Oberlander, K. B. Olsen, K. R. Oster, L. R. Roome, J. C. Simpson, S. S. Teel, and E. J. Westergard. 1988. Geohydrologic Characterization of the Area Surrounding the 183-H Solar Evaporation Basins. PNL-6728, Pacific Northwest Laboratory, Richland, Washington.

Lindberg, J. W. 1994. Geology of the McGee Ranch Site, Area B: Phase II Characterization. WHCSD-EN-TI-206, Westinghouse Hanford Company, Richland, Washington. The report gives the geologist's logs for 57 shallow boreholes drilled to assess the amount of fine-grained soil for use in surface barriers.

Lindberg, J. W., B. A. Williams, and F. A. Spane. 1997. Borehole Data Package for Well 699-3747A, PUREX Plant Cribs, CY 1996. PNNL-11515, Pacific Northwest National Laboratory, Richland, Washington. The report contains the geologist's log and minor analytical results for one borehole. 
Analytical results include x-ray diffraction for one sample of Ringold Formation lower mud and physical properties data for four samples. The physical properties include particle size distribution (ASTM D-422), specific gravity (ASTM D-854), and calcium carbonate content (ASTM D-4373).

Lindsey, K. A. 1991. Unpublished letter report containing particle size distribution data from boreholes and outcrops of Hanford formation and Ringold Formation. Outcrop locations are on the east side of the Columbia River across from the 300 Area, at Taylor Flats and on the 200 Area Plateau. Data is available from Duane G. Horton. Analyses were done by Westinghouse Hanford Company's Solids Characterization and Barriers Laboratory.

Lindsey, K. A. 1992. Geology of the Hanford Site, An Outline of Data Sources and Geologic Setting of the 100 Areas. WHC-SD-EN-TI-011, Westinghouse Hanford Company, Richland, Washington.

Lindsey, K. A. and G. K. Jaeger. 1993. Geologic Setting of the 100-HR-3 Operable Unit, Hanford Site, South-Central Washington. WHC-SD-EN-TI-132, Westinghouse Hanford Company, Richland, Washington.

Lindsey, K. A., S. E. Kos, and K. D. Reynolds. 2001. Vadose Zone Geology of Boreholes 299-E33-45 and 299-W33-46 B-BX-BY Waste Management Area Hanford Site, South-Central Washington. RPP8681, Rev. 0, U.S. Department of Energy, Office of River Protection, Richland, Washington.

Luttrell, S. P., K. R. Oster, and D. R. Newcomer. 1991. Completion Report for the 216-B-3 Pond Drilling and Characterization Activities, FY 1988, Separations Area Ground-Water Monitoring Project. WHC-SD-EN-TI-030, Westinghouse Hanford Company, Richland, Washington. The report gives particle size distributions, calcium carbonate content, and geologist's logs for four wells in the B-pond area.

Martinez, C. R. and D. C. Weekes. 2002. ISRM Barrier Well Completion Report for the 100-HR-3 Groundwater Operable Unit, Fiscal Year 2002. BHI-01638, Rev. 0, Bechtel Hanford, Inc., Richland, Washington.

Martinez, C. R. 2003. Calendar Year 2003 RCRA Groundwater Monitoring Well Summary Report. WMP-18472, Rev. 0., Fluor Hanford, Richland, Washington. The report contains the geologist's logs, the geophysical logs, and limited particle size data for two wells at WMA A-AX and five wells at WMA C.

Martinez, C. R. 2004. Fiscal Year 2004 CERCLA Groundwater Monitoring Well Summary Report for the 100-HR-3, 200-ZP-1, 200-UP-1 Operable Units. WMP-21220, Rev. 0, Fluor Hanford, Richland, Washington. The report contains the geologist's logs, the geophysical logs, and limited particle size data for nine wells. 
McHenry, J. R. 1957. Properties of Soils of the Hanford Project. HW-53218, Hanford Atomic, Products Operation, General Electric, Richland, Washington. This report gives the analytical results of testing many sediment samples from 33 wells in the 200 and 600 Areas and 28 surface locations in the Hanford Site vicinity. The sampled wells were drilled by "hard-tool." References to procedures used are given in the text. The analytical tests include percent gravel-sand-silt-clay, $\mathrm{pH}, 15$-Atmosphere moisture, calcium carbonate content, and cation exchange capacity.

Mercer, R. B. 1993a. 1992 Borehole Completion Data Package for the Low-Level Burial Grounds. WHC-SD-EN-DP-049, Westinghouse Hanford Company, Richland, Washington. Report contains calcium carbonate content and moisture content from samples at 5-ft intervals in ten new wells. All analyses were done by Westinghouse Hanford Company laboratories. Geologist logs are included.

Mercer, R. B. 1993b. 1991 Borehole Completion Data Package for the Low-Level Burial Grounds. WHC-SD-EN-DP-044, Westinghouse Hanford Company, Richland, Washington. Report contains calcium carbonate content and moisture content from samples at 5-ft intervals in 18 new wells. (Moisture was measured only in intervals where no water was added.) All analyses were done by Westinghouse Hanford Company laboratories. Geologist logs are included.

Mercer, R. B. 1994. 1993 Borehole Completion Data Package for the Low-Level Burial Grounds. WHC-SD-EN-DP-086, Westinghouse Hanford Company, Richland, Washington. The report gives moisture content (GEL-14) and calcium carbonate content (GEL-19) for 5-ft interval samples from two new RCRA boreholes. Westinghouse Hanford Company's Geotechnical Engineering Laboratory did the analyses.

Mitchell, R. M., D. L. Edwards, B. M. Markes, R. K. Price, and K. D. Reynolds. 1998. TWRS Phase 1 Privatization Site Preconstruction Characterization Report. HNF-2067, Fluor Daniel Hanford, Inc., Richland, Washington. The report provides laboratory analyses of cyanide (EPA method 9010), anions (EPA method 300.0), metals (EPA method 6010A) and radionuclides (laboratory standard operating procedures) on 76 surface soil samples from the former Grout Treatment Facility site. Analyses were done by the Hanford Site Waste Sampling and Characterization Facility and Special Analytical Services laboratories. Quanterra was the QC lab. Data are available electronically.

Moodie, C. D., R. Okazaki, H. W. Smith, and J. A. Kittrick. 1966. “A Note on the Clay Mineralogy of Four Samples from the Ringold Formation.” Northwest Science, v. 40, p. 43-45. The report gives qualitative results of $\mathrm{x}$-ray diffraction analysis of three size fractions of four samples of the Ringold Formation from the White Bluffs.

Myers, D. A., V. M. Johnson, M. Mehlhorn, and L. D. Walker. 1996. Well Summary Report: 100-HR-3 and 100-KR-4 Interim Remedial Action Wells. BHI-00953, Bechtel Hanford, Inc., Richland, Washington. 
Newcomer, D. R., L. A. Doremus, S. H. Hall, M. J. Truex, V. R. Vermeul, and R. E. Engelman. 1995. Geology, Hydrology, Chemistry, and Microbiology of the In Situ Bioremediation Demonstration Site. PNL-10422, Pacific Northwest Laboratory, Richland, Washington. The report gives the results of analyses of \%GSSC (PNL JEA-2), XRD (PNL JEA-3), total iron/ferrous iron, amorphous silica, nitrogen, phosphorus, bulk and particle density (PNL SA-9), and porosity for a few samples from one well. The results of analyses of total carbon, organic carbon and inorganic carbon are given for samples from two wells and VOC results are given for three wells. The text references the procedures used.

Opitz, B. E. 1982. A Laboratory Evaluation of the $100 \mathrm{~N}$ Disposal Basin Sediments. Prepared for the NUC Nuclear Industries by Pacific Northwest Laboratory, Richland, Washington. This report describes the laboratory testing of the chemical properties of sediments and solutions at the $100 \mathrm{~N}$ Area disposal basin. The purpose of the study was to evaluate the drainage capacity of the sediment for basin design.

Packer, D. R. 1979. “Paleomagnetism and Age Dating of the Ringold Formation and Loess Deposits in the State of Washington.” Oregon Geology 41:119-132. Presents results of paleomagnetic analyses from several stratigraphic sections of the Ringold Formation along the White Bluffs.

Packer, D. R. and J. M. Johnston. 1979. A Preliminary Investigation of the Magnetostratigraphy of the Ringold Formation. RHO-BWI-C-42, Rockwell Hanford Operations, Richland, Washington. Scoping study to determine the efficacy of paleomagnetic studies on the Ringold Formation. Results of paleomagnetic analyses from three surface outcrops along the White Bluffs (Parsons Canyon, Ringold Flat, and northern White Bluffs) as well as 6 coreholes drilled on the Hanford Site 299-W11-26 (DH-6), 299-W19-10 (DH-7), 699-54-18C (DH-9b), 299-W15-14 (DH-11), 299-W14-8 (DH-13), and 299-E19-1 (DH-17).

Pearson, A. W. 1990. Borehole Summary Report for Twelve Single-Shell Tank Wells Installed in 1989. WHC-MR-0209, Westinghouse Hanford Company, Richland, Washington. The report gives the result of analyses of gross alpha, gross beta, VOA, anions, and total organic carbon for five samples from each of twelve wells.

Peterson, R. E., R. F. Raidl, and C. W. Denslow. 1996. Conceptual Site Models for Groundwater Contamination at the 100-BC-5, 100-KR-4, 100-HR-3, and 100-FR-3 Operable Units. BHI-00917, Bechtel Hanford Company, Richland, Washington.

Pluhar, C. J., 2002. Paleomagnetism of Corehole E24-21, W10-27, W11-39, W19-43, and W23-21 Sediments, Hanford, WA. University of California, Santa Cruz, Earth Sciences Department. 1156 High St., Santa Cruz, California 95064-1077. The report gives the methods and results of 121 paleomagnetic analysis of Hanford formation and older sediments from drill ore segments. Steve Reidel, Bruce Bjornstad, and Duane Horton have copies of the report. 
Price, S. M., R. B. Kasper, M. K. Additon, R. M. Smith, and G. V. Last. 1979. Distribution of Plutonium and Americium beneath the 216-Z-1A Crib: A Status Report. RHO-ST-17, Rockwell Hanford Operations, Richland, Washington. This report contains the results of about 400 analyses of Am-241 and Pu-239/240 on samples from 16 wells drilled between 1973 and 1979. Samples were collected by split tube or drive barrel. Samples were analyzed by laboratories in the 200 West Area and Pacific Northwest Laboratory. Selected samples were analyzed by Inteelcom Radiation Technology Laboratory and LFE Laboratory.

Prater, L. S. 1984. Geology of the Area to the Northeast of the 1325-N Crib. PNL-6865, Pacific Northwest Laboratory, Richland, Washington. This report summarizes the drilling logs, provides the geophysical logs and aquifer testing results of several wells near the head end of the 1325-N Crib.

Reidel, S. P. and D. G. Horton. 1999. Geologic Data Package for 2001 Immobilized Low-Activity Waste Performance Assessment. PNNL-12257, Rev. 1, Pacific Northwest National Laboratory, Richland, Washington. This report gives the results of paleomagnetic analysis of seventeen sediment samples of the Hanford formation from well 299-E17-21.

Reynolds, K. D. 2003a. Completion Report for Probe Hole C4104 (T-106) T Tank Farm Drilling and Sampling. RPP-17275, Rev. 0, Prepared by Durateck Federal Services, Inc., Northwest Operations for CH2M HILL Hanford Group, Inc., Richland, Washington. The report contains the geologist log and the geophysical logs.

Reynolds, K. D. 2003b. Completion Report for Probe Hole C4105 (T-106) T Tank Farm Drilling and Sampling. RPP-16340, Rev. 0, Prepared by Durateck Federal Services, Inc., Northwest Operations for CH2M HILL Hanford Group, Inc., Richland, Washington. The report contains the geologist log and the geophysical logs.

Reynolds, K. D. 2003c. Completion Report for Probe Hole C3830(TX-105) TX Tank Farm 200 West Area. RPP-13741, Rev. 0, Prepared by Durateck Federal Services, Inc., Northwest Operations for CH2M HILL Hanford Group, Inc., Richland, Washington. The report contains the geologist log and the geophysical logs.

Rohay, V. J. 1995. FY 1993 Wellfield Enhancement Status Report and Data Package for the 200 West Area Carbon Tetrachloride Expedited Response Action. BHI-00105, Bechtel Hanford, Inc., Richland, Washington. This report contains the geologist's log, and data from analyses of particle size distribution, moisture content, calcium carbonate content, VOA, anions, $\mathrm{CN}, \mathrm{Hg}, \mathrm{Pb}, \mathrm{SVOA}$, and metals from samples obtained from borehole 299-W15-223.

Rohay, V. J. and D. C. Weekes. 1998. Borehole Summary Report for the 216-B-2-2 Ditch. BHI01177, Bechtel Hanford Inc., Richland, Washington. Report describes the drilling and sampling and analysis results from one characterization borehole. Laboratory chemical analyses were done by EPA approved methods; radionuclide analyses by standard laboratory procedures; specific gravity by ASTM 
D-854; cation exchange capacity by EPA 9081; organic carbon by EPA 9060, density by ASTM D-2937; particle size distribution by ASTM D-422; and percent moisture by ASTM D-2216. A geologist's log is included.

Rohay, V. J., G. V. Last, V. L. King, and L. A. Doremus. 1992. FY92 Site Characterization Status Report and Data Package for the Carbon Tetrachloride Site. WHC-SD-EN-TI-063. Westinghouse Hanford Company, Richland, Washington.

Rohay, V. J., K. J. Swett, and G. V. Last. 1994. 1994 Conceptual Model of the Carbon Tetrachloride Contamination in the 200 West Area at the Hanford Site. WHC-SD-EN-TI-248, Westinghouse Hanford Company, Richland, Washington. This report contains percent gravel-sand-silt-clay, bulk density and moisture content for 62 samples from nine boreholes determined in previous studies. Laboratory chemical analysis of VOAs are reported for five new and two deepened wells and anions, metals, SVOAs, and radionuclide activities are reported for one new well.

Rohay, V. J., K. J. Swett, V. M. Johnson, G. V. Last, D. C. Lanigan, and L. A. Doremus. 1993. FY93 Site Characterization Status Report and Data Package for the Carbon Tetrachloride Site. WHCSD-EN-TI-202, Westinghouse Hanford Company, Richland, Washington. The report contains the results of analyses of moisture content, calcium carbonate content, VOA, SVOA, metals, cations, anions, and radionuclides from samples from 6 new wells. Also included are particle size distribution, bulk density, and porosity data for samples from two wells. The Westinghouse Geotechnical Laboratory did the analyses of particle size distribution, density and porosity. Geologist's logs are included.

Routson, R. C. 1987. Impact of the Uranium Release (August 6, 1986) to the 216-U-14 Ditch. Internal Memo 65631-87-054. Westinghouse Hanford Company, Richland, Washington. Memo gives U-235 values for 5-ft interval samples from three boreholes drilled in 1987 at the 216-U-14 ditch. See Duane G. Horton for data.

Routson, R. C. and K. R. Fecht. 1979. Soil (Sediment) Properties of Twelve Hanford Wells with Geologic Interpretation. RHO-LD-82, Rockwell Hanford Operations, Richland, Washington. This report give analytical results for $\mathrm{pH}$, calcium carbonate content, cation exchange capacity, bulk density, porosity, and percent gravel-sand-silt-clay (hydrometer) for 5-ft interval samples from 12 wells. Procedures are described in the text.

Routson, R. C., W. H. Price, D. J. Brown, and K. R. Fecht. 1979. High-Level Waste Leakage from the 241-T-106 Tank at Hanford. RHO-ST-14, Rockwell Hanford Operations, Richland, Washington. The report gives $x$-ray diffraction and scanning electron microscopy mineralogy for several samples. General, representative physical property data are given for sediment in the 200 West Area.

Routson, R. C., G. S. Barney, R. M. Smith, C. H. Delegard, and L. Jensen. 1981. Fission Product Sorption Parameters for Hanford 200 Area Sediment Types. RHO-ST-35, Rockwell Hanford Operations, Richland, Washington. This report includes results of cation exchange capacity 
measurements, calcium carbonate content, texture (particle size by hydrometer), and qualitative mineralogy by x-ray diffraction from 21 samples of Hanford Site sediments collected from boreholes in the 200 Areas. See the report for references to procedures.

Schramke, J. A. 1988. Characterization of 200 Area Soil Samples. Internal Letter Report, Pacific Northwest Laboratory, Richland, Washington. The report gives the results of particle size distribution (hydrometer), XRD, XRF, total carbon, inorganic carbon, total organic carbon, and cation exchange capacity analyses of several samples from wells at low-level burial grounds. The procedures for cation exchange capacity and s-ray diffraction are given in the text.

Serkowski, J. A. 1986. Internal letter report, Rockwell Hanford Operations, Richland, Washington. The letter report give graphic moisture data and tabulated percent gravel-sand-silt-clay for five boreholes at 241-A tank farm. See Duane G. Horton for data.

Serne, R. J., H. T. Schaef, B. N. Williams, D. C. Lanigan, D. G. Horton, R. E. Clayton, V. L. LeGore, M. J. O’Hara, C. F. Brown, K. E. Parker, I. V. Kutnyakov, J. N. Serne, A. V. Mitroshkov, G. V. Last, S. C. Smith, C. W. Lindenmeier, J. M. Zachara, and D. B. Burke. 2001a. Characterization of Uncontaminated Sediments from the Hanford Reservation - RCRA Borehole Core Samples and Composite Samples. PNNL-2001-1, Pacific Northwest National Laboratory, Richland, Washington. This report presents results of detailed physical and chemical characterization of vadose zone sediments from two RCRA boreholes and four composite samples which are "standards" for the Hanford and upper Ringold formations. Analytical procedures are well documented. Analyses include moisture content, particle size distribution including hydrometer, calcium carbonate content, organic carbon content, $\mathrm{x}$-ray fluorescence, $\mathrm{x}$-ray diffraction, transmission electron microscopy composition of clay minerals, cation exchange capacity, 1:1 water extracts ( $\mathrm{pH}$, alkalinity, anions, metals), nitric acid extract (anions and metals) ammonium acetate extract (metals), and pore water composition (cations, anions, $\mathrm{pH}$, and alkalinity).

Serne, R. J, H. T. Schaef, B. N. Bjornstad, D. C. Lanigan, G. W. Gee, C. W. Lindenmeier, R. E. Clayton, V. L. LeGore, R. D. Orr, M. J. O’Hara, C. F. Brown, G. V. Last, I. V. Kutnyakov, D. B. Burke, T. C. Wilson, and B. A. Williams. 2001b. Geologic and Geochemical Data Collected from Vadose Zone Sediments from Borehole 299-W23-19 [SX-115] in the X/SX Waste Management Area and Preliminary Interpretations. PNNL-2001-3, Pacific Northwest National Laboratory, Richland, Washington. This report presents results of detailed physical and chemical characterization of vadose zone sediments from one borehole. Analytical procedures are well documented. Analyses include moisture content, particle size distribution including hydrometer, calcium carbonate content, organic carbon content, total carbon content, inorganic carbon content, gamma energy analysis, tritium, strontium-90, x-ray fluorescence, $\mathrm{x}$-ray diffraction, transmission electron microscopy composition of clay minerals, cation exchange capacity, 1:1 water extracts ( $\mathrm{pH}$, alkalinity, anions, metals), nitric acid extract (anions and metals), and pore water composition (cations, anions, $\mathrm{pH}$, and alkalinity).

Serne, R. J., G. V. Last, G. W. Gee, H. T. Schaef, D. C. Lanigan, C. W. Lindenmeier, R. E. Clayton, V. L. LeGore, R. D. Orr, M. J. O’Hara, C. F. Brown, A. T. Owen, I. V. Kutnyakov, T. C. Wilson, and D. A. Myers. 2001c. Geologic and Geochemical Data Collected from Vadose Zone Sediments from Borehole SX 41-09-39 in the S/SX Waste Management Area and Preliminary Interpretations. 
PNNL-2001-2, Pacific Northwest National Laboratory, Richland, Washington. This report presents results of detailed physical and chemical characterization of vadose zone sediments from borehole 41-0939 (299-W23-234). Analytical procedures are well documented. Analyses include moisture content, particle size distribution including hydrometer, calcium carbonate content, total carbon content, inorganic carbon content, gamma energy analysis, tectnetium-99, strontium-90, x-ray fluorescence, $\mathrm{x}$-ray diffraction, transmission electron microscopy composition of clay minerals, cation exchange capacity, $\mathrm{pH}$, alkalinity, anions, and metals. Analyzed media include sediment, pore water, 1:1 water extract and acid extract.

Serne R. J., R. E. Clayton, I. V. Kutnyakov, G. V. Last, V. L. Legore, T. C. Wilson, H. T. Schaef, M. J. O’Hara, K. B. Wagnon, D. C. Lanigan, C. F. Brown, B. A. Williams, C. W. Lindenmeier, R. D. Orr, D. S. Burke, and C. C. Ainsworth. 2002. Characterization of Vadose Zone Sediment: Slant Borehole SX-108 in the S-SX Waste Management Area. PNNL-13757-4, Pacific Northwest National Laboratory, Richland, Washington. This report gives the results of laboratory analyses of samples from the slant borehole at WMA S-SX. The types of analyses include particle size distribution, moisture content, $\mathrm{x}$-ray diffraction of bulk samples and clay separates, and particle density. Also included are analytical results of 1:1 sediment to water extracts. The analyses of the extracts include $\mathrm{pH}$, electrical conductivity, anions, major and trace metals, alkalinity, inorganic carbon, organic carbon, gamma energy, Sr-90, and tritium.

Serne, R. J., B. N. Bjornstad, D. G. Horton, D. C. Lanigan, C. W. Lindenmeier, M. J. Lindberg, R. E. Clayton, V. L. LeGore, K. N. Geiszler, S. R. Baum, M. M. Valenta, I. V. Kutnyakov, T. S. Vickerman, R. D. Orr, and C. F. Brown. 2004a. Characterization of Vadose Zone Sediments Below the T Tank Farm: Boreholes C4104, C4105, 299-W10-196, and RCRA Borehole 299-W11-39. PNNL14849, Pacific Northwest National Laboratory, Richland, Washington. This report gives the results of laboratory analyses of samples from two boreholes that penetrate sediments contaminated by the T-106 tank leak. The types of analyses include particle size distribution, moisture content, x-ray diffraction of bulk samples and clay separates, and particle density. Also included are analytical results of 1:1 sediment to water extracts. The analyses of the extracts include $\mathrm{pH}$, electrical conductivity, anions, major and trace metals, alkalinity, inorganic carbon, organic carbon, gamma energy, Sr-90, and tritium.

Serne, R. J., B. N. Bjornstad, D. G. Horton, D. C. Lanigan, C. W. Lindenmeier, M. J. Lindberg, R. E. Clayton, V. L. LeGore, R. D. Orr, I. V. Kutnyakov, S. R. Baum, K. N. Geiszler, M. M. Valenta, and T. S. Vickerman. 2004b. Characterization of Vadose Zone Sediments Below the TX Tank Farm: Boreholes C3830, C3831, C3832, and RCRA Borehole 299-W10-27. PNNL-14849, Pacific Northwest National Laboratory, Richland, Washington. This report gives the results of laboratory analyses of samples from three boreholes that penetrate sediments contaminated by the two leaks in the TX tank farm. The types of analyses include particle size distribution, moisture content, $\mathrm{x}$-ray diffraction of bulk samples and clay separates, and particle density. Also included are analytical results of 1:1 sediment to water extracts. The analyses of the extracts include $\mathrm{pH}$, electrical conductivity, anions, major and trace metals, alkalinity, inorganic carbon, organic carbon, gamma energy, Sr-90, and tritium.

Shannon \& Wilson. 1970. Report on Sampling of Soils and Laboratory Testing FFTF Site, Richland, Washington. Shannon \& Wilson, Seattle, Washington. The report gives results of testing for particle size distribution (including some hydrometer) (ASTM D-422), specific gravity (ASTM D-854), density 
(ASTM D-2049), moisture content (ASTM D-2216), and some geoengineering tests (Atterberg limits, compaction, compression) for several samples from 11 test pits in the FFTF area. See George V. Last for data.

Shannon \& Wilson. 1972. Supplementary Soils Investigation, Washington Public Power Supply System, Hanford No. 2 Nuclear Power Plant, Central Plant Facilities, Benton County, Washington. Shannon \& Wilson, Inc., Seattle, Washington. The report gives particle size distribution, densities, summary geologist's logs, field moisture content, and some geoengineering data (compaction tests and elastic and shear modulus) results for several samples from 19 borings, 4 cone penetrometer probings, and one trench in the area of WPPSS No. 2 plant. See George V. Last for data.

Singleton, K. M. and K. A. Lindsey. 1994. Groundwater Impact Assessment Report for the 216-U-14 Ditch. WHC-EP-0698, Westinghouse Hanford Company, Richland, Washington. The report contains the results of laboratory testing for calcium carbonate content and particle size on sediment samples from three new groundwater wells and two new perched water wells at $216-\mathrm{U}-14$ ditch. The report also contains results of chemical analyses for 40 CFR 264 Appendix IX constituents in samples from the five wells and three test pits and radiological analyses of samples from the wells. Samples were collected and handled according to Westinghouse procedures in WHC-CM-7-7. Physical property analyses were done according to procedures in WHC-EP-0367.

Slate, J. L. 2000. Nature and Variability of the Plio-Pleistocene Unit in the 200 West Area of the Hanford Site. BHI-01203, Bechtel Hanford Inc., Richland, Washington. This report presents detailed descriptions of Plio-Pleistocene sediments from 14 cores in and near the 200 West Area. Analytical data include bulk density, calcium carbonate content, percent gravel-sand-silt-clay and carbon and oxygen isotopic composition. (Isotopic composition from four samples.) The report also include thin-section descriptions and photomicrographs.

Smith, R. M. 1980. 216-B-5 Reverse Well Characterization Study. RHO-ST-37, Rockwell Hanford Operations, Richland, Washington. The report contains analyses for Cs-137, Sr-90, Pu-239/240, and Am-241 from six wells drilled and sampled with a split tube sampler in 1979. Rockwell Hanford Operations and Eberline Instrument Corporation laboratories did the analyses.

Smith, R. M. and M. K. Additon. 1980. Granulometric Analysis of Sediments Containing Transuranic Radionuclides. RHO-LD-123, Rockwell Hanford Operations, Richland, Washington. The report gives particle size distribution of 5-ft interval samples from two contaminated boreholes at 216-Z1A. The analyses were done by sonic sifter. The various size fractions of nine samples were analyzed for $\mathrm{Pu}$ and $\mathrm{Am}$.

Smoot, J. L, J. E. Szecsody, B. Sagar, G. W. Gee, and C. T. Kincaid. 1989. Simulations of Infiltration of Meteoric Water and Contaminant Plume Movement in the Vadose Zone at Single-Shell Tank 241-T-106 at the Hanford Site. WHC-EP-0332, Westinghouse Hanford Company, Richland, Washington. The report includes graphical representation of particle size distribution for samples obtained in the 241-AP tank farm pit. Analyses were done by method ASTM D-422. Percent gravel, sand, silt, and clay are given for each sample. 
Summers, W. K. and R. T. Hanson. 1977. Core Sample Descriptions and Summary Logs of Six Wells Within the Hanford Reservation. ARH-C-00015, Atlantic Richfield Hanford Company, Richland, Washington. The report contains geologist's descriptions of cored sections from wells 299W11-26, 299-W19-10, 699-61-55, 699-49-100A, 699-43-42, and 699-54-17C.

Sump, C. A. 2002a. Well Summary Report: 100-HR-3 Pump-and-Treat Remediation System Fiscal Year 2002 Extraction Well C3829. BHI-01637, Bechtel Hanford, Inc., Richland, Washington. This report documents the installation of extraction well 199-D8-72. Some particle size distribution analyses and the geologist's log are included.

Sump, C. A. 2002b. Well Summary Report: 100-KR-4 Pump-and-Treat Remediation System Fiscal Year 2002 CERCLA Upgrade Wells. BHI-01626, Rev. 0, Bechtel Hanford, Inc., Richland, Washington. This report documents the installation of extraction well 199-K-127 and injection well 199K-128. Some particle size distribution analyses and the geologist's logs are included.

Subrahmanyam, V. B. 1986. Internal letter report, Rockwell Hanford Operations, Richland, Washington. The letter report gives laboratory radionuclide analyses from samples collected from three boreholes at the 216-B-2-3 ditch in the 218-E12B burial ground. See Duane G. Horton for data.

Swanson, L. C. 1992. Borehole Completion Data Package for Grout Treatment Facility Well 299E25-39. WHC-SD-EN-DP-048, Westinghouse Hanford Company, Richland, Washington. The report gives moisture content and calcium carbonate content for 5 -ft interval samples from one new RCRA boreholes.

Swanson, L. C. 1993. CY 1992 Borehole Completion Data Package, Grout Treatment Facility Wells 299-E25-44 and 299-E25-45. WHC-SD-EN-DP-058, Westinghouse Hanford Company, Richland, Washington. The report gives moisture content and calcium carbonate content for 5-ft interval samples from two new RCRA boreholes.

Swanson, L. C. 1994. 1993 Borehole Completion Data Package, Grout Treatment Facility Wells 299E25-49, 299-E25-50, and 299-E25-1000. WHC-SD-EN-DP-085, Rev. 0, Westinghouse Hanford

Company, Richland, Washington. Report contains calcium carbonate content and moisture content from samples at 5-ft intervals in three new wells. (Moisture was measured only in intervals where no water was added.) All analyses were done by Westinghouse Hanford Company laboratories. Geologist logs are included.

Swanson, L. C, D. C. Weekes, S. P. Luttrell, R. M. Mitchell, D. S. Landeen, A. R. Johnson, and R. C. Roos. 1988. Grout Treatment Facility Environmental Baseline and Site Characterization Report. WHC-EP-0150, Westinghouse Hanford Company, Richland, Washington. The report gives laboratory results of analyses on 47 samples from 17 boreholes and four groundwater wells. Laboratory analyses include wet chemistry, selected metals, $\mathrm{pH}$, alkalinity, cation exchange capacity, selected anions, and radionuclides done by U.S. Testing laboratory according to standard laboratory operating procedures. Moisture content was determined according to procedures in RHO-RE-MA-20. 
Sweeney, M. D. 1993a. Borehole Completion Data Package for the 200 Areas Treated Effluent Basin - Project W-049H. WHC-SD-EN-SP-068, Westinghouse Hanford Company, Richland, Washington. Report contains calcium carbonate content (GEL-19) and moisture content (GEL-14) from samples at 5-ft intervals in three new wells. In addition, moisture content (GEL-14), specific gravity (GEL-10), porosity, and bulk density (GEL-16) were determined for 12 specific intervals. All analyses were done by Westinghouse Hanford Company's Geotechnical Laboratory. Geologist logs are included.

Sweeney, M. D. 1993b. Site Characterization Report for the Liquid Effluent Retention Facility. WHC-SD-EN-EV-024, Westinghouse Hanford Company, Richland, Washington. The report contains qualitative $\mathrm{x}$-ray diffraction analyses of 20 Hanford and Ringold formation samples and 14 XRF analyses of Ringold Formation samples from boreholes. The analyses were done by Washington State University.

Sweeney, M. D. 1994. Soil Properties Data for the Liquid Effluent Retention Facility. WHC-SD-ENDP-084, Westinghouse Hanford Company, Richland, Washington. The document summarizes the calcium carbonate, sieve, and hydrometer data for four RCRA wells at the LERF. The information supplements WHC 1990. Borehole Completion Data Package for the Liquid Effluent Retention Facility, WHC-MR-0235, Westinghouse Hanford Company, Richland, Washington.

Sweeney, M. D., D. J. Alexander, S. D. Evelo, K. A. Lindsey, V. M. Johnson, and K. M. Singleton. 1995. Groundwater Impact Assessment Report for the 216-T-1 Ditch. WHC-EP-0814, Westinghouse Hanford Company, Richland, Washington. The report contains average results of analysis for metals in sediment from three test pits and one metal analyses of sediment in one groundwater well (split spoons).

Tallman, A. M., K. R. Fecht, M. C. Marratt, and G. V. Last. 1979. Geology of the Separation Areas, Hanford Site, South-Central Washington. RHO-ST-23, Rockwell Hanford Operations, Richland, Washington. This report presents the results of 50 qualitative $\mathrm{x}$-ray diffraction analyses of various size fractions, 44 microprobe analyses, and percent basalt by size fraction from samples throughout 200 East and 200 West. All sedimentary formations are represented. Dr. Lloyd Ames of Pacific Northwest Laboratory did the x-ray and microprobe analyses.

Teel S. S. 1990. Grout Treatment Facility Borehole Completion Report for 1989. WHC-MR-0203, Westinghouse Hanford Company, Richland, Washington.

Todd, M. E. and C. A. Kahler-Royer. 2002. Borehole Summary Report for the Borehole C3102 in the 216-T-26 Crib, 200-TW-1 Scavenged Waste Group Operable Unit. BHI-01606, Rev. 0, Bechtel Hanford, Inc., Richland, Washington. This report documents the drilling and decommissioning of borehole C3102. The geologist's log and geophysical logs and some bulk density data are included.

Todd, M. E. and C. Trice. 2002. Borehole Summary Report for Boreholes C3103 and C3104 and Drive Casings C3340, C3341, C3342, C3343, and C3344 in the 216-B-38 Trench and 216-B7A Crib, 200-TW-2 Tank Waste Group Operable Unit. BHI-01607, Bechtel Hanford, Inc., Richland, Washington. This report summarizes the drilling and decommissioning of six boreholes at the 216-B-38 trench (C3104, C3340, C3341, C3342, C3343, and C3344) and one borehole (C3103) at the 216-B-7A 
crib. Geophysical logs and some bulk density data are given. Geologist's logs for C3103 and C3104 and as-built diagrams for the other boreholes are in the Hanford Well Information System database.

Trice, L. C., D. C. Weekes, and L. C. Swanson. 2001. ISRM Barrier Well Completion Report for the 100-HR-3 Groundwater Operable Unit, Fiscal Year 2001. BHI-01560, Rev. 0, Bechtel Hanford, Inc., Washington. This report documents the installation of 32 groundwater monitoring wells. The geologist's logs are in the Hanford Well Information System database.

Valenta, M. M., J. R. Moreno, M. B. Martin, R. E. Ferri, D. G. Horton, and S. P. Reidel. 2000. Particle Size Distribution Data From Existing Boreholes at the Immobilized Low-Activity Waste Site. PNNL-13328, Pacific Northwest National Laboratory, Richland, Washington. The report contains results of 79 particle size analyses from four boreholes in south-central 200 East Area. Analyses were done in the laboratories in the 3720 Building using PNNL Technical Procedure SA-2 (PNL-MA-567).

Van Alstine, D. R. 1982. Paleomagnetic Investigation of Pre-Missoula Gravels, Pasco Basin and Vicinity, Washington. Sierra Geophysics report prepared for Golder Associates, Redmond, Washington. Presents results of 42 paleomagnetic analyses performed on Pre-Missoula Gravels (i.e., Cold Creek Unit) from Skagit/Hanford corehole E-20 (699-17-26H) on the Hanford Site.

Vermeul, V. R., S. S. Teel, J. E. Amonette, C. R. Cole, J. S. Fruchter, Y. A. Gorby, F. A. Spane, J. E. Szecsody, M. D. Williams, and S. B. Yabusaki. 1995. Geologic, Geochemical, Microbiologic, and Hydrologic Characterization at the In Situ Redox Manipulation Test Site. PNL-10633, Pacific Northwest Laboratory, Richland, Washington.

Walker, L. D. 2001. Borehole Summary Report for the 2001 ILAW Site Characterization Well. BHI-01531, Bechtel Hanford, Inc., Richland, Washington. This report documents the installation of groundwater monitoring well 299-E17-21. The geologist's log is included.

Walker, L. D. 2002. Well Summary Report: 2002 Immobilized Low-Activity Waste Well Installation. BHI-01647, Bechtel Hanford, Inc., Richland, Washington. This report documents the installation of groundwater monitoring well 299-E24-21. The geologist's log is included.

WCC. 1978. Paleomagnetic Measurements of the Ringold Formation and Loess Units near Hanford, Washington and Evaluation of Dating Potential of Quaternary Deposits near Hanford, Washington. Richland, WA, subcontract report prepared for Washington Public Power Supply System, Woodward-Clyde Consultants, Walnut Creek, California. Expanded, prepublication version of Packer (1979).

WCC. 1982. Paleomagnetic Analysis of Drilling Induced Magnetization and Ringold Formation Core from DH-20, DH-23, and DH-24. Richland, WA, subcontract report prepared for Rockwell Hanford Operations by Woodward-Clyde Consultants, Walnut Creek, California. Results of paleomagnetic study to evaluate possibility and extent of drilling induced remagnetization on sediment samples. A total of 13 paleomagnetic sample pairs were analyzed from corehole 699-29-83 (DH-20), seven from 699-46-85 (DH-23), and 14 from 699-43-44 (DH-24). 
Webster, C. T. 1977. Ringold Identification Correlation, and Sampling Program: Well History DH-11 -12 -13 -13A -14 -15 -16 -17 (Continuation of ARH-C-14). RHO-LD-34, Rockwell Hanford Operations, Richland, Washington. This report gives drill logs and core records (brief lithologic descriptions for eight wells.

Weekes, D. C., S. P. Luttrell, and M. R. Fuchs. 1987. Interim Hydrogeologic Characterization Report and Groundwater Monitoring System for the Nonradioactive Dangerous Waste Landfill, Hanford Site, Washington. WHC-EP-0021, Westinghouse Hanford Company, Richland, Washington. The report soil moisture content for samples from ten wells. Analyses were done by Shannon and Wilson Inc. using procedure ASTM D-2216. Lithologic logs are included.

Weekes, D. C. and L. R. Glaman. 1995. FY95 Site Characterization Status Report and Data Package for the Carbon Tetrachloride Site. BHI-00399, Bechtel Hanford Inc., Richland, Washington. The report contains some particle size distribution data from four boreholes, particle density and porosity from three boreholes and VOA, moisture content and calcium carbonate content from one borehole.

Weekes, D. C., G. K. Jaeger, and B. H. Ford. 1995. Preoperational Baseline and Site Characterization Report for the Environmental Restoration Disposal Facility. BHI-00270, Bechtel Hanford, Inc., Richland, Washington. The report gives the results of moisture content (ASTM D-2216-90), calcium carbonate content (ASTM D-4373-84), particle size distribution (ASTM D-422-63), porosity (ASTM D698-78 and ASTM D-1557-78), specific gravity (ASTM D-854-83, ASTM C-117-87, ASTM C-127-84, and ASTM C-128-84), and bulk density (ASTM D-698-78 and ASTM D-1557-78) from samples obtained from several wells at the ERDF sight. Geologist's logs are also given. Analyses were done by the Westinghouse Hanford Company's Geotechnical Laboratory.

Williams, B. A. 1992. Borehole Completion Data Package for DOE 216-S-10 Facility, CY 1991. WHC-SD-EN-DP-045, Westinghouse Hanford Company, Richland, Washington. The report gives geologist's logs, moisture content, and calcium carbonate content of samples from three new RCRA groundwater monitoring wells. The data were generated by the Westinghouse Hanford Company's Environmental Technology Analytical Laboratory.

Williams, B. A. and D. B. Barnett. 1993. Borehole Completion Package for the 216-S-10 Facility CY 1992. WHC-SD-EN-DP-052, Westinghouse Hanford Company, Richland, Washington. The report contains calcium carbonate content (procedure GEL-19) and moisture content (procedure GEL-14) data from one new RCRA well.

Williams, B. A., R. E. Peterson, and K. B. Olsen. 2003. Soil Gas Survey and Well Installations at the 618-10 Burial Ground, 300-FF-5 Operable Unit, Hanford Site, Washington. PNNL-14320, Pacific Northwest National Laboratory, Richland, Washington. The report contains geologic logs and geophysical logs for wells 699-S6-E4K and 699-S6-E4L.

Williams, B. A. and S. M. Narbutovskih. 2003. Borehole Data Package for RCRA Wells 299-E25-93 and 299-E24-22 at Single-Shell Tank Waste Management Area A-AX, Hanford Site, Washington. 
PNNL-14538, Pacific Northwest National Laboratory, Richland, Washington. The borehole report contains the geologic and geophysical logs for wells 299-E25-93 and 299-E24-22.

Williams, B. A. and S. M. Narbutovskih. 2004. Borehole Data Package for Four CY 2003 RCRA Wells 299-E27-4, 299-E27-21, 299-E27-22, and 299-E27-23 at Single-Shell Tank, Waste Management Area C, Hanford Site, Washington. PNNL-14656, Pacific Northwest National Laboratory, Richland, Washington. The report contains the geologic and geophysical logs for four wells.

Williams, M. D., V. R. Vermeul, J. E. Szecsody, and J. S. Fruchter. 2000. 100-D Area In Situ Redox Treatability Test for Chromate-Contaminated Groundwater. PNNL-13349, Pacific Northwest National Laboratory, Richland, Washington.

Wright, J., J. L. Conca, and X. Chen. 1994. Hydrostratigraphy and Recharge Distributions from Direct Measurement of Hydraulic Conductivity Using the UFA Method. PNL-9424, Pacific Northwest Laboratory, Richland, Washington. The report gives measurements of particle size distribution and mineral composition for 50 arid soil and sediment samples from former waste disposal sites at Hanford, Washington.

Wright, J. V., J. L. Conca, P. P. Didzerekis, T. J. Mockler, and K. D. Shields. 1995. VOC-Arid Site Integrated Demonstration, R\&D Characterization and Monitoring Technologies, Subtask 3 Milestone Report, Final Report of the UFA Technology for Vadose Zone Transport Measurements. Unpublished report. The report contains the results of four analyses of particle size distribution and mineralogy by XRD from boreholes 299-W18-174 and 299-W18-96. See George V. Last for data.

Xie, Y., C. J. Murray, G. V. Last and R. Mackley. 2003. Mineralogical and Bulk-Rock Geochemical Signatures of Ringold and Hanford Formation Sediments. PNNL-14202, Pacific Northwest National Laboratory, Richland, Washington. The report pulls together much of the mineralogy and geochemistry data in one place. 


\section{Distribution}

Distributed on CD unless otherwise noted.

No. of

Copies

\section{OFFSITE}

K. A. Lindsey

KennedyJenks

719 Jadwin Avenue, \#28

Richland, WA 99352

\section{ONSITE}

\section{DOE Office of River Protection}
C. A. Babel
R. M. Yasek
H6-60
H6-60

7 DOE Richland Operations Office

B. L. Foley

J. P. Hanson

R. D. Hildebrand

J. G. Morse

K. M. Thompson

DOE Public Reading Room (2P)
No. of

Copies

9 Bechtel Hanford, Inc.

L. R. Curry

H0-19

A. G. Dada H0-23

M. A. Delamare H0-19

K. R. Fecht HO-02

B. H. Ford H0-21

M. J. Graham H0-09

M. N. Jarayssi H0-19

G. B. Mitchem H0-19

L. Soler

H0-19

7 CH2M HILL Hanford, Inc.
K. A. Bergstrom
H9-02
W. J. McMahon
$\mathrm{H} 9-03$
T. H. Mitchell
H9-02
K. M. Singleton
$\mathrm{H} 9-02$
L. C. Swanson
H9-02
D. C. Weekes
$\mathrm{H} 9-02$
C. D. Wittreich
H9-03

4 CH2M HILL Hanford Group, Inc.

M.P. Connelly

E6-35
A. J. Knepp
$\mathrm{H} 0-22$
F. M. Mann
$\mathrm{H} 0-22$
D. A. Myers
H0-22

\section{Fluor Federal Services}

R. Khaleel

B4-43

$\mathrm{P}=$ paper copy 
No. of

Copies

2 Fluor Hanford, Inc.

V. J. Rohay

H0-19

M. I. Wood

H8-44

2 MACTER-ERS

J. F. Bertsch

B2-62

R. G. McCain

B2-62

3 Duratek Federal Services, Northwest Operations
M. G. Gardner
H1-11
J. E. Meisner
H1-11
R. K. Price
H1-11

43 Pacific Northwest National Laboratory

D. B. Barnett

K6-81

B. N. Bjornstad (P)

R. W. Bryce

J. G. Bush

A. L. Bunn

K. J. Cantrell

J. L. Devary

R. L. Dirkes

M. J. Fayer

E. J. Freeman
No. of

Copies

M. D. Freshley

$\mathrm{H} 0-21$

J. S. Fruchter

K6-96

G. W. Gee

K9-33

T. J Gilmore (P)

K6-81

D. G. Horton (3P)

K6-81

C. T. Kincaid

K9-33

G. V. Last (3P)

K6-81

T. L. Liikala

K6-96

K6-81

K9-33

K6-96

K6-81

K6-81

K6-86

K6-81

K6-96

R. G. Riley K6-96

R. J. Serne

K6-81

R. M. Smith K6-96

M. D. Sweeney K6-81

P. D. Thorne K9-33

A. L. Ward

K9-33

B. A. Williams K6-81

M. D. Williams K6-36

S. B. Yabusaki K9-36

J. M. Zachara K8-96

Hanford Technical Library (2P) P8-55

$\mathrm{P}=$ paper copy 